\title{
Evaluation von Bildungsprojekten auf der Grundlage von Inventaren
}

\section{Entwicklung und Erprobung eines Ansatzes im Rahmen von europäischen Projekten}

\author{
Dissertation \\ zur Erlangung des sozialwissenschaftlichen Doktorgrades \\ der Sozialwissenschaftlichen Fakultät \\ der Georg-August-Universität Göttingen
}

vorgelegt von Jutta List-Ivankovic aus Bad Gandersheim

Göttingen 2013 
1. Gutachter: Prof.i.R. Dr. Hans-Dieter Haller

2. Gutachter: Prof.i.R. Dr. Wulf Hopf

3. Gutachter: Prof. Dr. Steffen Kühnel

Tag der mündlichen Prüfung: 21. Februar 2013 
1.1 Begriffsdefinition 11

1.2 Bereiche und Gegenstände 15

1.3 Funktionen und Ziele 16

1.4 Formen und Arten

1.4.1 Formative und summative Evaluation 23

1.4.2 Interne und externe Evaluation__ 25

1.4.3 Selbstevaluation und Fremdevaluation _ 26

1.4.4 Adressatenevaluation und Systemevaluation __ 27

2. Zur Geschichte der Evaluationsforschung

2.1 Entwicklungen in den USA

2.1.1 Die Zeit der Reform (1792-1900)

2.1.2 Die Zeit der Effizienz und des Testens $(1900-1930)$

2.1.3 Die Tylersche Zeit $(1930-1945)$

2.1.4 Die Zeit der Stagnation (1946-1957)__ 37

2.1.5 Die Zeit der Entwicklung und Expansion (1958 - 1972) 38

2.1.6 Die Zeit der Professionalisierung und Diversifizierung $\left(\overline{1973-1983)} \_42\right.$

2.1.7 Die Zeit der Expansion und Integration $(1983-2001) \_44$

2.2 Entwicklungen in Europa

2.2.1 Die Evaluationskultur in verschiedenen europäischen Nationen ___ 47

2.2.2 Die Evaluationskultur in der Europäischen Union __ 52

2.3 Aktuelle Lage der Evaluation _

3. Evaluationsansätze und Evaluationstheorien

3.1 Das Generationenmodell nach Guba und Lincoln $\quad 64$

3.1.1 Die erste Generation: Das Messen _— 65

3.1.2 Die zweite Generation: Das Beschreiben___ 65

3.1.3 Die dritte Generation: Das Bewerten _ 66

3.1.4 Die vierte Generation: Das Verhandeln __ 67

3.2 Das Baummodell nach Alkin und Christie __ 72

3.2.1 Die Wurzeln und der Stamm __ 73

3.2.2 Der Methodenast (Methods) _ 74

3.2.3 Der Wertungsast (Valuing) _ 76

3.2.4 Der Nutzungsast (Use) _ 78

3.3 Das Nutzungsmodell nach Fitzpatrick, Sanders und Worthen $\quad 82$

3.3.1 Zielorientierte Ansätze (objectives-oriented evaluation approaches)__ 83

3.3.2 Managementorientierte Ansätze (management-oriented evaluation approaches) ___ 86

3.3.3 Konsumentenorientierte Ansätze (consumer-oriented evaluation approaches) ___ 88

3.3.4 Expertenorientierte Ansätze (expertise-oriented evaluation approaches) ___ _ _ 90

3.3.5 Partizipative Ansätze (participant-oriented evaluation approaches) ___ 92 
4.1 Arbeitsfelder der Erziehungswissenschaft und pädagogische Handlungsfelder 98

4.2 Spezifika der Evaluation von Bildung und in pädagogischen Handlungsfeldern 104

4.3 Das Forschung-Praxis Dilemma 113

5. Entwicklung und Erprobung von Inventaren zur Unterstützung und Verbesserung der Evaluationspraxis in europäischen Bildungsprojekten

5.1 Fallstudie: Das Projekt eL3 121

5.1.1 Projektziele 123

5.1.2 Projektpartnerschaft 124

5.1.3 Projektablauf 131

5.2 Problemstellung und Fragestellung 136

5.3 Forschungsvorgehen und -methoden 138

5.3.1 Aktionsforschung 138

5.3.2 Triangulation

5.3.2.1 Fragebogen zur Bestandsaufnahme 146

5.3.2.2 Prozessevaluation 150

5.3.2.3 Dokumentation der Aktivitäten auf dem Lernmanagementsystem ___ 157

5.3.2.4 Fragebogen zum Abschluss des Projektes 158

6. Ergebnisse

6.1 Fragebogen zur Bestandsaufnahme 159

6.2 Prozessevaluation 168

6.3 Entwicklung der Inventare: Dokumentation der Aktivitäten auf dem Lernmanagementsystem

6.3.1 Die Inventare für Lerner

6.3.2 Das Inventar für Autoren 209

6.3.3 Das Inventar für Entscheidungsträger 226

6.4 Erprobung der Inventare: Fragebogen zum Abschluss des Projektes

6.4.1 Die Inventare für Lerner 241

6.4.2 Das Inventar für Autoren 245

6.4.3 Das Inventar für Entscheidungsträger 252

6.5 Exkurs: Weitere Daten aus einer späteren Verwendung der Inventare 254

7. Zusammenfassung und Diskussion 259

8. Fazit und Ausblick 270

Tabellenverzeichnis 274

Abbildungsverzeichnis 276

Literaturverzeichnis 277 
1. Fragebogen zur Bestandsaufnahme 292

2. Partnerprofile 304

3. Fragebogen Prozessevaluation 306

4. Information zu den blinc-Inventaren und blinc-Fragebögen 307

5. Information zur Anwendung der Inventare für Lerner 308

6. Inventare für Lerner 311

6.1 Inventar für Lerner zu Beginn eines Kurses 314

6.2 Inventar für Lerner während eines Kurses 339

6.3 Inventar für Lernen zum Abschluss eines Kurses 365

7. Inventar für Autoren und Produzenten 391

8. Inventar für Entscheidungsträger 422

9. Fragebogen zum Abschluss des Projektes 436 


\section{Einleitung}

"The Purpose of Evaluation Is Not to Prove But to Improve." (Stufflebeam 1971a)

Evaluation ist ein aktuelles Thema, vor allem im Bildungswesen wird evaluiert und kontrolliert, überprüft und verbessert. War das Wort Evaluation früher vielen fremd, „erfreut es sich heutzutage einer nie dagewesenen Beliebtheit“ (Stockmann 2007, S.11).

„Evaluation hat Hochkonjunktur” (Böttcher et al 2006, S.7), nicht nur in Deutschland (Stockmann 2006a) und Europa (Politt 1998, Leeuw 2006), sondern weltweit (Furubo, Rist \& Sandahl 2002). Die Professionalisierung der Evaluation ist in den meisten Ländern weit fortgeschritten und es hat sich eine Evaluationskultur herausgebildet, die auf fundierten Ausbildungen von Evaluatoren und einem regen wissenschaftlichen Diskurs in verschiedenen Fächern u.a. den Erziehungswissenschaften und ihren Nachbardisziplinen aufgebaut ist. In vielen Ländern wurden Evaluationsgesellschaften gegründet, Studiengänge eingerichtet und Fachzeitschriften veröffentlicht. Evaluationen finden in den verschiedensten Bereichen statt, z.B. dem Bildungswesen, der Wirtschaft, Politik und Verwaltung.

In den Erziehungswissenschaften fand in Deutschland bereits in den 1970er Jahren eine intensive Auseinandersetzung mit dem Thema Evaluation statt (z.B. Wulf 1972), wobei diese vor allem im Zusammenhang mit den zu dieser Zeit vielfältigen Reformansätzen erfolgte. Dann folgte jedoch eine Phase, in der das Thema nicht so intensiv diskutiert wurde. Heute, im Jahre 2012, sieht es wieder anders aus: Seit Mitte der 1990er Jahre ist das Thema Evaluation erneut aktuell, und inzwischen findet sich eine unüberschaubare Anzahl von Publikationen und verschiedenen Evaluationsansätzen. Die „Zeitschrift für Pädagogik“ widmete dem Thema „Qualität und Qualitätssicherung im Bildungsbereich“ im Jahre 2000 ein eigenes Beiheft (Helmke, Hornstein \& Terhart 2000), und im Jahre 2008 wurde erneut eine „aktuelle Zwischenbilanz" zur Qualitätssicherung im Bildungswesen herausgegeben (Klieme \& Tippelt 2008). Die Evaluation im Bildungssystem wird sowohl im wissenschaftlichen Umfeld (vgl. z.B. Böttcher, Holtappels \& Brohm 2006, Kuper 2005, Thonhauser \& Patry 1999), als auch in der Öffentlichkeit, der Bildungspolitik und der Bildungspraxis thematisiert. Es findet dabei eine Qualitätsdebatte statt, wobei die Begriffe Evaluation und Qualitätsentwicklung eng miteinander verknüpft sind (Stockmann 2006b). Die Frage nach der Qualität von Bildung wird insbesondere im Schulwesen, im Hochschulsystem, aber auch im gesamten Weiterbildungsbereich, intensiv diskutiert. Für diese Auseinandersetzungen spielen die jährlichen OECDBildungsberichte (OECD 2011a) und die internationalen Vergleichsstudien wie TIMMS (Baumert et al 1997, Baumert et al 2000a, Baumert et al 2000b, Bos et al 2008), PISA (Klime 2010, OECD 2011b) oder PIRLS bzw. IGLU (Mullis, Martin, Gonzales \& Kennedy 2003, Bos, Lankes, Prenzel, Schwippert, Valtin \& Walther 2003) eine zentrale Rolle. Bei diesen Studien geht es jedoch vielmehr um das Aufdecken von Qualitätsdefiziten und eine Kontrolle bzw. 
Überprüfung der Leistungen und „Outputs“ des Bildungssystems als um die Verbesserung der pädagogischen Praxis und des Schulsystems bzw. der Lernumwelten.

Im Kontext der Diskussionen über die Qualität der Bildung werden Begriffe wie Qualitätsmanagement und Qualitätssicherung für in der pädagogischen Praxis Tätige relevant, und es entsteht der Anspruch bzw. der Druck, Bildungsangebote und -institutionen zu evaluieren, um deren Qualität nachzuweisen (oder zu beweisen). Bei einem Qualitätsmanagement bzw. der Evaluation kann "von oben oder von unten" vorgegangen werden. Im Bereich der Schule herrscht eine intensive Diskussion über „Qualität von oben“, sowohl in der Politik und Öffentlichkeit als auch in der Wissenschaft (s.o.), aber über „Qualität von unten“ findet kein öffentlicher Diskurs statt, und es gibt auch kaum Modelle oder Ansätze und Definitionen, wie dies gelingen könnte (vgl. Kempfert \& Rolff 2005, S.7). Bei dem Qualitätsmanagement bzw. der Evaluation von oben geht es meist um eine Kontrolle von Leistungen, und die Auftraggeber der Evaluation sind staatliche Institutionen, Mittelgeber oder Wissenschaftliche Institute. Bei der Durchführung der Evaluationen werden dann entsprechende Standards gefordert und eingehalten. Bei der Vorgehensweise von unten ist häufig das Ziel die Verbesserung der pädagogischen Praxis, und meistens führen hierbei die Akteure selbst die Evaluationen durch. Für Schulen und Lehrer gibt es bereits unter den Stichworten Qualitätsmanagement, Evaluation und Selbstevaluation Anleitungen und Hilfestellungen für in der Praxis Tätige, wie sie selbst (also von unten bzw. von innen) aktiv werden können, um mittels Evaluationsmaßnahmen die eigene Praxis oder Schule zu optimieren. (siehe z.B. Altrichter \& Posch 2007, Buhren et al. 2002, Buhren 2007, Moser 1999, Schratz et al. 2002, von Saldern 2010). In anderen pädagogischen Handlungsfeldern wie z.B. der sozialen Arbeit finden sich ebenfalls Ansätze zur Selbstevaluation und es gibt auch eine wissenschaftliche Diskussion, die aber vor allem durch ihren Praxisbezug gekennzeichnet ist (Heiner 1988, 1994, Von Spiegel 1993, Galuske 2009) Es finden sich auch in weiteren pädagogischen Arbeitsfeldern spezielle Anleitungen oder Empfehlungen zur Selbstevaluation, so z.B. in der kulturellen Kinder- und Jugendbildung (Bundesvereinigung Kulturelle Jugendbildung e.V. 1998), in der Vorschulerziehung in Kindertagesstätten (Bertelsmann Stiftung 2007-2011), oder in der Kinder- und Jugendhilfe (http://www.qs-kompendium.de/). Das Ziel dieser Anleitungen ist, die Bildungsqualität zu steigern und den Praktikern dafür Hilfestellungen und Anregungen sowie Materialien an die Hand zu geben. Häufig sind solche Handlungsempfehlungen auf der Grundlage von Projekten entstanden, die staatlich gefördert wurden (z.B. vom Ministerium für Familie, Senioren, Frauen und Jugend, den Ländern oder von Stiftungen und Sponsoren). In vielen Bereichen der pädagogischen Praxis jedoch finden sich keine Hilfestellungen oder Anleitungen, wie gute Evaluationen gelingen können. Die Bildungspraktiker sind auf sich alleine gestellt und führen, häufig unter dem Druck, die Qualität ihrer Arbeit nachweisen zu müssen, selbstständig nach bestem Wissen und mit ihren vorhandenen (meist nicht ausreichenden) Kompetenzen Evaluationen durch. Die Evaluationen werden dabei meistens nach der Me- 
thode "quick and dirty" (Klein 1991, S.6) durchgeführt und die Ergebnisse oft nicht einmal systematisch ausgewertet und verwendet.

Es findet sich also im Themenfeld der Evaluation auf der einen Seite ein hoher Grad an Professionalisierung, der durch eine hochqualifizierte Ausbildung von Evaluatoren, die Gründung von Evaluationsgesellschaften, das Erscheinen von Fachzeitschriften und einen regen wissenschaftlichen Diskurs gekennzeichnet ist (siehe z.B. auch die Veröffentlichungen und Empfehlungen der DeGEval). Auf der anderen Seite ist ein großer Teil der Praktiker im Bildungsbereich auf sich alleine gestellt, und die Qualitäten der Evaluationen lassen zu wünschen übrig.

Bereits an dieser Stelle wird deutlich, wie komplex das Thema Evaluation ist. Evaluationen können "von oben oder von unten bzw. innen" erfolgen, und sie können dadurch auch sehr unterschiedliche Ziele und Funktionen haben. Im wissenschaftlichen Kontext gelten festgelegte Regeln und Standards, in der Praxis wird nach bestem Wissen und Gewissen vorgegangen und es finden sich kaum Hilfestellungen, um die Qualität der Evaluationen zu gewährleisten oder zu verbessern. Bei dieser Problemstellung setzt die vorliegende Arbeit an. Die Verfasserin arbeitete in dem von der Europäischen Kommission im Rahmen der ELearning Initiative geförderten Projekt eL3 (eLearning Project Cluster for Third System Organisations in Europe), das in dieser Forschungsarbeit in einer Fallstudie untersucht wird. Das Projekt erstreckte sich über einen Zeitraum von 24 Monaten. Es kamen zehn Partner aus verschiedenen Einrichtungen der Gesundheits- und Sozialwirtschaft zusammen. Das Hauptziel des Projektes eL3 war die Verbreitung von computerbasiertem Lernen und die Verbesserung der Qualität der beruflichen Bildung. Die beteiligten Akteure sollten vor allem Handlungs- und Entscheidungshilfen für den Einsatz innovativer Lernformen bekommen und sich in einem Netzwerk zusammenschließen, um Erfahrungen und Wissen auszutauschen. Zum Projektende sollte ein europäisches Netzwerk entstehen, in dem die erarbeiteten Inhalte veröffentlicht und verbreitet und damit eine Basis für einen nachhaltigen Austausch geschaffen würden. Ein zentrales Anliegen war die Evaluation und Analyse der bestehenden Weiterbildungsangebote der Projektpartner zu Beginn des Projektes.

Das Forschungsvorgehen dieser Arbeit wird der Aktionsforschung zugeordnet, die es mit sich bringt, dass die beteiligten Praxispartner in den Forschungsprozess einbezogen werden. Es stellte sich heraus, dass die Projektpartner nicht wollten, dass ihre Kurse und Weiterbildungsangebote „Von oben“ oder „von außen“ evaluiert werden. Sie wollten vielmehr hochwertiges Handwerkszeug erhalten, mit dem sie selbst ihre Maßnahmen evaluieren können. Die meisten Partner wollten auch nicht unbedingt die anderen „in ihre Karten schauen lassen“, sondern vor allem Erfahrungen und Wissen austauschen, um dann selbst zu entscheiden, wie sie die neu erworbenen Wissensinhalte und Erfahrungen für ihre Arbeit nutzen können und wollen. Ein derartiges Szenarium erfordert ein hohes Maß an Flexibilität und Offen- 
heit seitens der Forscherin (der Verfasserin) und einen großen Grad an Beteiligung seitens der Praxispartner.

Im Sinne Stufflebeams (siehe Zitat am Anfang dieser Einleitung) wird mit der vorliegenden Forschungsarbeit das Ziel verfolgt, Bildungspraktikern qualitativ hochwertige Evaluationsinstrumente zur Verfügung zu stellen, an deren Erarbeitung sie in einem partizipativen Verfahren beteiligt waren und mit denen sie selbständig ihre Angebote evaluieren und verbessern können. Die Funktion der Evaluation ist dabei nicht die Kontrolle von bestehenden Angeboten, sondern die Verbesserung der beruflichen Praxis, der Erkenntnisgewinn, die Wissenserweiterung sowie der Dialog mit anderen Bildungspraktikern. Als Evaluationsinstrumente werden Inventare entwickelt und erprobt, die den Akteuren als Grundlage zur Evaluation ihrer Bildungsprojekte dienen sollen. Im Gegensatz zu Fragebögen oder Tests sind Inventare komplexere Instrumente, die aus mehreren Bestandteilen bestehen bzw. verschiedene Skalen beinhalten und die außerdem flexibel und individuell von den Nutzern eingesetzt werden können.

In dem beschriebenen Kontext der vorliegenden Forschungsarbeit ergeben sich folgende Hauptforschungsfragen:

1. Wie ist die bisherige Evaluationspraxis der Projektpartner?

2. Können in dem transnationalen europäischen Netzwerk gemeinsam Inventare als Evaluationsinstrumente entwickelt werden?

3. Werden die entwickelten Inventare in dem transnationalen europäischen Netzwerk genutzt?

Zur Klärung dieser Fragen und der damit verbundenen Unterfragen wird die Projektpartnerschaft über den Projektverlauf von 24 Monaten wissenschaftlich begleitet. Im Sinne der Aktionsforschung wird dabei möglichst wenig eingegriffen, so dass die Forschungsmethoden zum einem angepasst an den Projektablauf und zum anderen transparent und mit den Partnern abgestimmt eingesetzt werden. Es werden mehrere quantitative und qualitative Methoden trianguliert, um ein breiteres, umfassenderes Bild von dem Untersuchungsfeld zu bekommen, als wenn nur eine Methode zum Einsatz käme. Die Ergebnisse des Forschungsvorgehens werden ebenfalls in der Projektgruppe vorgestellt, so dass die Partner einen Einblick in das haben, was auf der Basis der gemeinsamen Arbeit entstanden ist.

Nach der Beschreibung des Problemfeldes und dem Praxisprojekt, das in dieser Arbeit als Fallstudie dient, ergibt sich folgender Aufbau: 
Im ersten Kapitel wird zunächst (da das Themenfeld so komplex ist) der Begriff Evaluation geklärt. Außerdem werden Bereiche und Gegenstände von Evaluationen erläutert, Funktionen und Ziele, die sehr verschieden sein können, erörtert und unterschiedliche Formen und Arten von Evaluation dargestellt.

Im zweiten Kapitel wird auf die geschichtlichen Hintergründe der Evaluationsforschung eingegangen. Dazu werden zuerst die Entwicklungen in den USA beschrieben und dann wird auf die Entstehung der Evaluationskultur in Europa eingegangen. Zum Abschluss des Kapitels erfolgt eine Bestandsaufnahme zur aktuellen Lage der Evaluation.

Im dritten Kapitel werden verschiedene Evaluationsansätze und Evaluationstheorien dargestellt. Hierzu werden unterschiedliche Ordnungssysteme namhafter Autoren vorgestellt, die verdeutlichen, dass sich Evaluationsansätze sowohl nach ihrer historischen Entstehung als auch nach ihrer Ausrichtung im Hinblick auf die verschiedenen Ziele und Funktionen, die Evaluationen haben können, sortieren lassen. Mit diesem Kapitel wird nochmals deutlich, dass es unterschiedliche Traditionen und Verständnisse von Evaluation gibt.

Im vierten Kapitel widmet sich die Verfasserin dem Thema Evaluation im Bildungsbereich und stellt zunächst Arbeitsfelder der Erziehungswissenschaft und pädagogische Handlungsfelder dar. Außerdem werden Spezifika der Evaluation von Bildung und in pädagogischen Handlungsfeldern erläutert und das Forschung-Praxis-Dilemma beschrieben.

Im fünften Kapitel wird insbesondere auf der Grundlage des dritten und vierten Kapitels das Modell der Inventare zur Unterstützung und Verbesserung der Evaluationspraxis in europäischen Bildungsprojekten entwickelt. Dazu wird das Projekt eL3 als Fallbeispiel näher beschrieben. Darauf aufbauend werden die Problemstellung und die Fragestellung abgeleitet und das Forschungsvorgehen und die Forschungsmethoden dargestellt.

Im sechsten Kapitel werden die Ergebnisse der Entwicklungsphase und der Erprobungsphase der Inventare vorgestellt.

Im siebten Kapitel erfolgen eine Zusammenfassung der wesentlichen Ergebnisse der Forschungsarbeit und eine kritische Diskussion in Bezug auf die Fragestellungen.

Im achten Kapitel schließlich wird ein Fazit gezogen und ein Ausblick verfasst, wie die gewonnenen Erkenntnisse weiter genutzt werden können bzw. weiterführende Forschungsarbeiten anknüpfen könnten. 


\title{
1. Evaluation
}

In diesem Kapitel soll zunächst geklärt werden, was unter Evaluation verstanden werden kann und auf welche Bereiche bzw. Gegenstände sich Evaluationen beziehen. Außerdem werden Funktionen und Ziele von Evaluationen dargestellt sowie verschiedene Formen und Arten erörtert.

\subsection{Begriffsdefinition}

Der Begriff Evaluation ist sehr vielschichtig. Nach Kromrey (2001, S.105) gibt es eine "geradezu inflationäre Verwendung des Begriffs in extrem unterschiedlichen Bedeutungsvarianten“ und ein „Diskussionslabyrinth". Er unterscheidet zunächst die Verwendung und das Verständnis des Begriffs Evaluation im alltäglichen von dem im wissenschaftlichen Sprachgebrauch. Er schreibt, dass unterschiedliche Kontexte unterschiedliche Begriffsbedeutungen zur Folge haben und im alltäglichen Sprachgebrauch Evaluation nichts anderes bedeutet als „Bewertung“:

„Irgendetwas wird von irgend jemandem nach irgendwelchen Kriterien in irgendeiner Weise bewertet." (Kromrey 2001, S.106)

Demgegenüber ist im wissenschaftlichen Sprachgebrauch wesentlich präziser, was Gegenstand der Evaluation ist, von wem sie durchgeführt wird und in welcher Weise. Evaluation im empirisch-wissenschaftlichen Sinne kann wie folgt definiert werden:

\begin{abstract}
„Es handelt sich um eine besondere Form angewandter Sozialwissenschaft (nicht nur Sozialforschung). Es ist eine methodisch kontrollierte, verwertungs- und bewertungsorientierte Form des Sammelns und Auswertens von Informationen." (Kromrey 2001, S.112)
\end{abstract}

Es gehe dabei vor allem darum, wissenschaftliche Verfahren und Methoden einzusetzen, um sie für den Gegenstand der Evaluation nutzbar zu machen. Die Wissenschaft liefert hierbei „Handlungswissen für die Praxis“ (Kromrey, 2001, S.113).

\begin{tabular}{|l|l|}
\hline Alltäglicher Sprachgebrauch & Wissenschaftlicher Sprachgebrauch \\
\hline Irgendetwas wird & $\begin{array}{l}\text { Programme, Maßnahmen, Organisationen etc. wer- } \\
\text { den }\end{array}$ \\
\hline von irgend jemandem & $\begin{array}{l}\text { durch Personen, die zur Bewertung besonders befä- } \\
\text { higt sind, }\end{array}$ \\
\hline nach irgendwelchen Kriterien & $\begin{array}{l}\text { nach explizit auf den Sachverhalt bezogenen und } \\
\text { begründeten Kriterien (und ggf. Standards) }\end{array}$ \\
\hline in irgendeiner Weise bewertet. & in einem objektivierten Verfahren bewertet. \\
\hline Tabelle 1: Der Begriff Evaluation im alltäglichen und wissenschaftlichen Sprachgebrauch (erstellt \\
von der Verfasserin in Anlehnung an Kromrey 2001, S.108)
\end{tabular}

In ihrem grundlegenden Werk führen Wottawa und Thierau (2003, S.13) „Definitionsversuche (Hervorhebung durch die Verfasserin) von $<<$ Evaluation $>>$ " auf und merken ebenfalls 
an, dass es sich um einen vielfältigen Begriff handelt, für den es zahlreiche Definitionen und beschreibende Begriffserläuterungen gibt. Die „schillernde Vielfalt“ des Begriffs Evaluation veranlasst Wottawa und Thierau anstatt einer weiteren Definition, allgemeine Kennzeichen wissenschaftlicher Evaluation zu benennen:

- „Ein allgemeiner Konsens (...) liegt darin, daß alle solche Tätigkeiten etwas mit <<Bewerten >> zu tun haben. Evaluation dient als Planungs- und Entscheidungshilfe und hat somit etwas mit der Bewertung von Handlungsalternativen zu tun (...).

- Evaluation ist ziel- und zweckorientiert. Sie hat primär das Ziel, praktische Maßnahmen zu überprüfen, zu verbessern oder über sie zu entscheiden.

- Es besteht im wissenschaftlichen Sprachgebrauch ebenfalls ein Konsens darüber, daß Evaluationsmaßnahmen dem aktuellen Stand wissenschaftlicher Techniken und Forschungsmethoden angepasst sein sollten." (Wottawa und Thierau 2003, S.14)

Wie in der Definition von Kromrey (s.o.) geht es nach dieser Charakterisierung bei einer Evaluation um Bewertung, um die Anwendung von wissenschaftlichen Techniken und Methoden (Sammeln und Auswerten), die sich auf ein konkretes Ziel richten und für die Praxis Entscheidungshilfen sowie Handlungswissen liefern sollen.

Die Deutsche Gesellschaft für Evaluation (DeGEval) definiert Evaluation folgendermaßen:

„Evaluation ist die systematische Untersuchung des Nutzens oder Wertes eines Gegenstandes. Solche Evaluationsgegenstände können z.B. Programme, Projekte, Produkte, Maßnahmen, Leistungen, Organisationen, Politik, Technologien oder Forschung sein. Die erzielten Ergebnisse, Schlussfolgerungen oder Empfehlungen müssen nachvollziehbar auf empirisch gewonnenen qualitativen und/oder quantitativen Daten beruhen." (DeGEval 2004, S.13)

In diesem Ansatz wird wie in den oben zitierten das systematische Vorgehen bei einer Evaluation genannt sowie die Anwendung qualitativer und quantitativer Forschungsmethoden betont. Der Wert und Nutzen eines Gegenstandes soll durch eine Evaluation ermittelt werden und die Ergebnisse müssen nachvollziehbar (objektiv) sein.

Rossi und Freeman unterscheiden nicht zwischen den Begriffen Evaluation und Evaluationsforschung. Sie definieren Evaluation folgendermaßen:

„Evaluation research is the systematic application of social research procedures for assessing the conceptualization, design, implementation, and utility of social intervention programs. In other words, evaluation researchers (evaluators) use social research methodologies to judge and improve the ways in which human services policies and programs are conducted, from the earliest stage of defining and designing programs through their development and implementation." (Rossi und Freeman 1993, S.5)

Auch in dieser Definition von Evaluation wird deutlich, dass die systematische Verwendung von sozialwissenschaftlichen Forschungsmethoden dazu dient, Bewertungen vorzunehmen 
und die Praxis auf Basis der Ergebnisse ggf. zu verbessern. Eine Evaluation kann dabei zu jedem Zeitpunkt stattfinden und ist nach Rossi und Freeman immer nützlich:

"[...] it is useful in every stage in the conceptualization, design, planning, and implementation [...]." (Rossi und Freeman 1993, S.54)

In seinem Artikel „Evaluation als Entscheidungshilfe“ widmet sich Stufflebeam dem damals gegenwärtigen Stand der Evaluation im Bildungswesen und entwickelt alternative Ansätze der pädagogischen Evaluation. Er definiert dabei auch den Begriff der Evaluation:

„Im allgemeinen bedeutet Evaluation die Gewinnung von Informationen durch formale Mittel wie Kriterien, Messungen und statistische Verfahren mit dem Ziel, eine rationale Grundlage für das Fällen von Urteilen in Entscheidungssituationen zu erhalten." (Stufflebeam 1972, S.124)

Mit diesem Verständnis von Evaluation wird deutlich, dass es um Entscheidungen geht, die auf der Grundlage von Evaluationsergebnissen getroffen werden können. Stufflebeam sieht Evaluation nicht direkt als Bewertung wie die oben zitierten Autoren, für ihn steht das „Fällen von Urteilen“ im Zentrum. Dies beinhaltet jedoch eine Abwägung von Entscheidungsalternativen und damit auch ein Bewerten. Die "Gewinnung von Informationen“, also die Datensammlung, ist wie bei den vorher angeführten Definitionen auch für Stufflebeam zentral und erfolgt nach wissenschaftlichen Kriterien bzw. nach anerkannten Verfahren. Die Ergebnisse bieten dann eine „rationale Grundlage“ für die Entscheidungen, was die Anforderung an Objektivität unterstreicht. Das systematische Vorgehen und die Einhaltung von formalen Regeln werden in dieser Definition betont.

Um den Begriff Evaluation für diese Arbeit genauer fassen zu können, werden nun die Gemeinsamkeiten der oben zitierten Definitionen in der folgenden Tabelle abgebildet: 


\begin{tabular}{|c|c|c|c|c|c|}
\hline & $\begin{array}{l}\text { Stufflebeam } \\
\text { (1972) }\end{array}$ & $\begin{array}{l}\text { Rossi \& } \\
\text { Freeman (1993) }\end{array}$ & Kromrey (2001) & $\begin{array}{l}\text { Wottawa \& } \\
\text { Thierau (2003) }\end{array}$ & DeGEval (2004) \\
\hline Gegenstände & $\begin{array}{l}\text { Projekte, Bil- } \\
\text { dungs- } \\
\text { programme }\end{array}$ & $\begin{array}{l}\text { Programme } \\
\text { (,social interven- } \\
\text { tion programs, } \\
\text { human services } \\
\text { policies“) }\end{array}$ & $\begin{array}{l}\text { Programme, } \\
\text { Maßnahmen, } \\
\text { Organisationen } \\
\text { etc. }\end{array}$ & $\begin{array}{l}\text { Praktische Maß- } \\
\text { nahmen }\end{array}$ & $\begin{array}{l}\text { Programme, } \\
\text { Projekte, Pro- } \\
\text { dukte, Maßnah- } \\
\text { men, Leistun- } \\
\text { gen, Organisati- } \\
\text { onen, Politik, } \\
\text { Technologien, } \\
\text { Forschung }\end{array}$ \\
\hline $\begin{array}{l}\text { Ziele / Zwe- } \\
\text { cke }\end{array}$ & $\begin{array}{l}\text { Entscheidungs- } \\
\text { hilfe } \\
\text { („eine rationale } \\
\text { Grundlage für } \\
\text { das Fällen von } \\
\text { Urteilen in Ent- } \\
\text { scheidungs- } \\
\text { situationen zu } \\
\text { erhalten“) }\end{array}$ & $\begin{array}{l}\text { Bewerten und } \\
\text { Verbessern von } \\
\text { Programmen } \\
\text { (,to judge and to } \\
\text { improve the } \\
\text { ways in which } \\
\text { human services } \\
\text { policies and } \\
\text { programs are } \\
\text { conducted“) }\end{array}$ & $\begin{array}{l}\text { Bewerten } \\
\text { („Handlungs- } \\
\text { wissen für die } \\
\text { Praxis liefern“) }\end{array}$ & $\begin{array}{l}\text { Planungs- und } \\
\text { Entscheidungs- } \\
\text { hilfe } \\
\text { (,Bewertung von } \\
\text { Handlungs- } \\
\text { alternativen“) } \\
\text { überprüfen, } \\
\text { verbessern, } \\
\text { entscheiden }\end{array}$ & $\begin{array}{l}\text { Nutzen oder } \\
\text { Wert eines Ge- } \\
\text { genstandes } \\
\text { untersuchen, } \\
\text { Schluss- } \\
\text { folgerungen } \\
\text { ziehen und Emp- } \\
\text { fehlungen geben }\end{array}$ \\
\hline Methoden & $\begin{array}{l}\text { Formale Mittel: } \\
\text { Kriterien, Mes- } \\
\text { sungen, statisti- } \\
\text { sche Verfahren }\end{array}$ & $\begin{array}{l}\text { Sozialwissen- } \\
\text { schaftliche For- } \\
\text { schungs- } \\
\text { methoden } \\
\text { („social research } \\
\text { procedures and } \\
\text { methodologies“) }\end{array}$ & $\begin{array}{l}\text { Wissen- } \\
\text { schaftliche Ver- } \\
\text { fahren und Me- } \\
\text { thoden ("ange- } \\
\text { wandte Sozial- } \\
\text { wissen-schaft") }\end{array}$ & $\begin{array}{l}\text { Wissen- } \\
\text { schaftliche } \\
\text { Techniken und } \\
\text { Forschungs- } \\
\text { methoden }\end{array}$ & $\begin{array}{l}\text { Gewinnung } \\
\text { empirischer } \\
\text { Daten durch } \\
\text { qualitative } \\
\text { und/oder quanti- } \\
\text { tative Methoden }\end{array}$ \\
\hline
\end{tabular}

Tabelle 2: $\quad$ Gemeinsamkeiten unterschiedlicher Definitionen von Evaluation (erstellt von der Verfasserin)

Weitere einschlägige Definitionen und Beschreibungen von Evaluation finden sich z.B. bei. Weiss (1972, 1974), Hellstern und Wollmann (1984), Guba und Lincoln (1989, 1998), Bortz und Döring (1995), Mertens (1998) und Stockmann (2006b, 2007, 2010).

Zusammenfassend lässt sich festhalten, was unter Evaluation im wissenschaftlichen Sinne von verschiedenen Autoren im Wesentlichen übereinstimmend verstanden wird:

Evaluation richtet sich auf einen bestimmten Gegenstand, der durch die Anwendung sozialwissenschaftlicher Methoden untersucht wird. Primäres Ziel einer Evaluation ist das Bewerten nach Kriterien, die nachvollziehbar und begründet entwickelt, dargestellt und angewendet werden. Die Evaluationsergebnisse sollen eine Grundlage für Planungen und Entscheidungen bieten und zur Verbesserung der Praxis durch die Bereitstellung von Handlungswissen beitragen. Eine Evaluation

- richtet sich auf einen Gegenstand und

- bedient sich sozialwissenschaftlicher Methoden.

- Mit den Ergebnissen werden verschiedene Ziele angestrebt:

○ Bewertung, Werte, Nutzen und Qualität feststellen

- Planungs- und Entscheidungshilfen bieten

- Verbesserung, Optimierung ermöglichen

- Praxisbezug, Handlungsperspektiven und Handlungswissen bereitstellen. 
In den Definitionen wurde u.a. deutlich, dass sich Evaluationen auf Bereiche und Gegenstände beziehen. Im Folgenden soll dargestellt werden, dass diese sehr vielfältig sind.

\subsection{Bereiche und Gegenstände}

Das, was evaluiert wird, also der Evaluationsgegenstand, kann sehr unterschiedlich sein. Evaluationen beziehen sich auf z.B. auf Programme, Projekte, Maßnahmen, Organisationen, Leistungen, Produkte, Technologien oder Forschung (vgl. DeGeval 2004, S.13).

Wottawa und Thierau (vgl. 2003, S.57) unterscheiden Evaluationsbereiche und Evaluationsobjekte. Sie nennen verschiedene Praxisbereiche, in denen Evaluationen stattfinden:

- Bildungssektor

- Wirtschaft

- Agrar- und Verkehrspolitik

- Familien- und Sozialpolitik

- Justizvollzug

- Arbeitsmarkt- und Beschäftigungspolitik

- Umweltpolitik

- Gesundheitswesen

- Städtebau- und Wohnungspolitik

- Militärischer Bereich

Die Autoren weisen darauf hin, dass insbesondere im Bildungssektor ab den 1980er Jahren zahlreiche Evaluationen und Begleitforschungen durchgeführt wurden. Besonders bekannt seien dabei die Studien zum Gesamtschulsystem und der Vergleich mit dem dreigliedrigen Schulsystem.

Als Evaluationsobjekte, also wer oder was evaluiert wird, kommen verschiedene Möglichkeiten in Betracht. Es können sowohl einzelne Personen, Personengruppen, aber auch Projekte, Produkte oder Programme im Zentrum einer Evaluation stehen. Wottawa und Thierau (2003, S.59) listen folgende Evaluationsobjekte auf:

- Personen

- Umwelt-/Umgebungsfaktoren

- Produkte

- Techniken/Methoden

- Zielvorgaben

- Programme

- Projekte

- Systeme/Strukturen

- Forschungsergebnisse/Evaluationsstudien

An diesen Auflistungen wird bereits deutlich, dass Evaluationen sich auf die unterschiedlichsten Bereiche und Objekte bzw. Gegenstände beziehen können. Außerdem können sie ver- 
schiedene Funktionen und Ziele haben, die im nächsten Unterkapitel näher betrachtet werden.

\subsection{Funktionen und Ziele}

Bei Evaluationen geht es, wie oben beschrieben, immer um eine Bewertung des Evaluationsgegenstandes und um Planungs- und Entscheidungshilfen, die auf der Basis der Ergebnisse getroffen werden können. Evaluationen sind nach Wottawa und Thierau (2003, S.14) aber auch immer „ziel- und zweckorientiert“. Primäre Ziele von Evaluation seien die Überprüfung und Verbesserung von praktischen Maßnahmen sowie das Entscheiden über diese (vgl. ebenda 2003, S.14).

Die Ziele und Motive einer Evaluation können für verschiedene Beteiligte sehr unterschiedlich sein. Bei der Evaluation einer Weiterbildungsmaßnahme beispielsweise differieren die Ziele und auch der Nutzen der Ergebnisse für die Seminarteilnehmer von denen der Trainer / Lehrer und von denen der Vorgesetzten bzw. der Unternehmens- oder Schulleitung.

\begin{tabular}{|c|c|}
\hline $\begin{array}{l}\text { Evaluationsbeteiligte bei einer Wei- } \\
\text { terbildungsmaßnahme }\end{array}$ & Ziele und Motive \\
\hline Seminarteilnehmer & \begin{tabular}{|l|} 
Lernerfolgsnachweis \\
Karriereförderung \\
Individuelles Feedback \\
Lernmotivation \\
\end{tabular} \\
\hline Trainer / Lehrer / Dozenten & $\begin{array}{l}\text { Lernerfolgsnachweis } \\
\text { Bildungsbedarfshinweis } \\
\text { Feedback durch Teilnehmer } \\
\text { Ressourcen - Gewinnung } \\
\end{array}$ \\
\hline Vorgesetzte & $\begin{array}{l}\text { Berichterstattung, Dokumentation und persönliche Eindrücke } \\
\text { Entscheidungshilfen bei Personalfragen } \\
\text { Beurteilung der Traineraktivitäten }\end{array}$ \\
\hline Unternehmensleitung / Schulleitung & $\begin{array}{l}\text { Beurteilung der Trainer / Lehrer / Dozenten } \\
\text { Beurteilung der Teilnehmer / Schüler } \\
\text { Ressourcen - Bemessung } \\
\text { Rechenschaftslegung } \\
\text { Effizienz-Nachweis }\end{array}$ \\
\hline
\end{tabular}

Die Ziele des Evaluators und der Evaluierten können sehr verschieden sein, was auch zu Konflikten führen kann. Die Ziele einer Evaluation sollten deshalb definiert und die unterschiedlichen Interessen erläutert werden (etwa zwischen Auftraggebern, Evaluatoren und Evaluierten). Wottawa und Thierau (vgl. 2003, 83ff) beschreiben, wie bei der Zielexplikation und der Festlegung von Bewertungskriterien vorgegangen werden kann.

Stockmann (vgl. 2006a, S.19f) benennt vier grundlegende Funktionen von Evaluation, wobei er betont, dass diese nicht immer trennscharf voneinander abgegrenzt werden können. Die Ausrichtung der Evaluation, Design und Methoden sowie Erwartungen richten sich nach ihrer 
jeweiligen Funktion. Die Funktionen sind nach Stockmann: Erkenntnisfunktion, Kontrollfunktion, Dialog-/Lernfunktion und Legitimierungsfunktion.

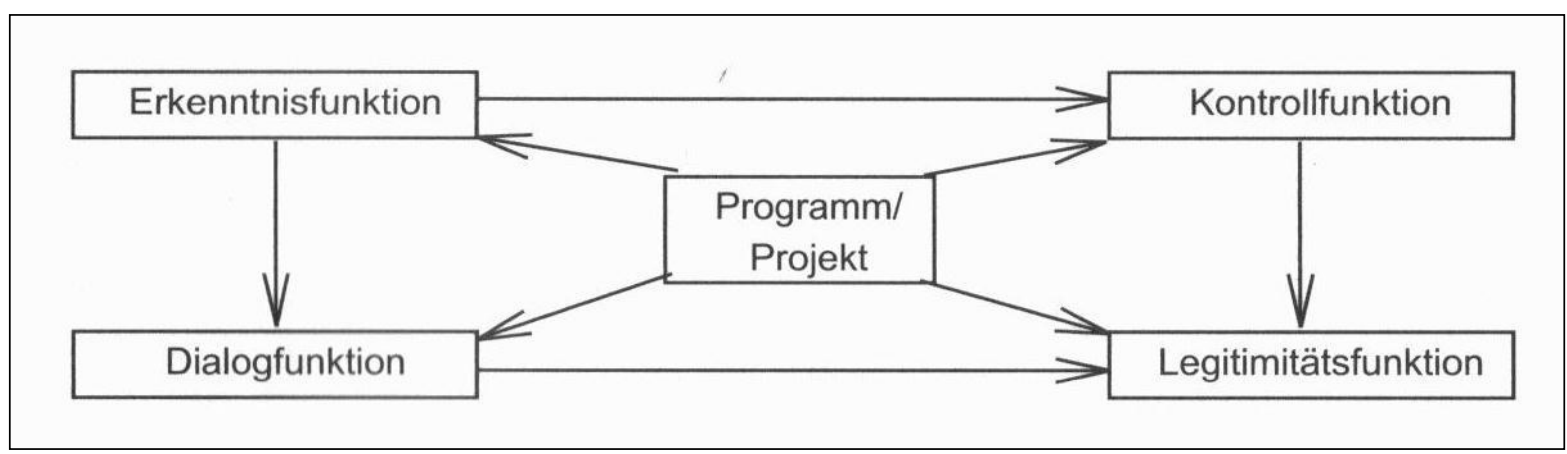

Abbildung 1 Funktionen von Evaluation (Quelle: Stockmann 2006a, S.21)

\section{Erkenntnisfunktion}

Bei der Erkenntnisfunktion werden entscheidungsrelevante Daten gesammelt, mit denen z.B. ermittelt wird, welche Bedarfe eine Zielgruppe hat, ob die Maßnahme die Zielgruppe erreicht hat und in welchem Ausmaß (z.B. Akzeptanz, Erfolg), welche Zusammenhänge und Kausalbeziehungen zwischen verschiedenen Variablen bestehen. Die gesammelten Daten werden ausgewertet und als Basis für Steuerungsentscheidungen benutzt.

\section{Kontrollfunktion}

Die Kontrollfunktion von Evaluation begründet Stockmann damit, dass direkt oder indirekt durch die Sammlung von Informationen und Daten eine Form von Kontrolle gegeben ist, da Defizite aufgedeckt werden können und deutlich wird, ob alle Beteiligten ihre Aufgaben erfüllen und in welcher Weise. Die Kontrollfunktion kann sich auch darauf beziehen, dass man wissen möchte, wie das Programm läuft, um ggf. eingreifen zu können.

\section{Dialog-/Lernfunktion}

Die systematisch gesammelten, ausgewerteten und bereitgestellten Informationen können als Basis für den Dialog zwischen verschiedenen Beteiligten genutzt werden. Die Ergebnisse halten fest bzw. machen transparent, wie eine Zusammenarbeit (z.B. ein Projekt) verlief und zeigen Stärken und Schwächen auf. Für eine weitere Zusammenarbeit können dann Konsequenzen gezogen werden und Optimierungen erfolgen. Auf diese Weise kann gemeinsam gelernt werden, wofür die Evaluationsergebnisse eine wichtige Voraussetzung bieten.

\section{Legitimitätsfunktion}

Durch eine Evaluation können die Wirkungen eines Programms aufgezeigt bzw. nachgewiesen werden. Mittelgeber und Durchführungsorganisationen können beispielsweise belegen, wie sie Finanzmittel verwendet haben und was sie mit ihrem Programm oder Projekt erreicht 
haben. Es wird so legitimiert, wie gearbeitet wurde, und die Erfolge einer Maßnahme werden belegt.

Stockmann (vgl. 2006a, S.20) beschreibt weiterhin, dass Evaluationen häufig auch eine taktische Funktion haben, z.B. um bestimmte politische Entscheidungen (manchmal sogar nachträglich) zu legitimieren oder um sie einfach besser erscheinen zu lassen. Er sieht diese Verwendung von Evaluation aber als konträr zu ihren eigentlichen Zwecken an und deshalb gehört sie für inn nicht in das Aufgabenfeld von Evaluation.

Chelimsky (1997a) beschreibt ebenfalls unterschiedliche Ziele (purposes) von Evaluationen und entwickelt drei konzeptionelle Rahmen, die ihrer Meinung nach jeweils andersartige Methoden mit sich bringen:

"The purposes (...) fall naturally in three conceptual frameworks of evaluation:

- Evaluation for accountability (e.g. measuring results or efficiency)

- Evaluation for development (e.g. providing evaluative help to strengthen institutions) and

- Evaluation for knowledge (e.g. obtaining a deeper understanding in some specific area or policy field)." (Chelimsky 1997a, S.100)

Diese Klassifizierungen von Chelimsky werden im Folgenden näher beschrieben:

\section{Evaluation mit dem Ziel der Kontrolle bzw. Rechenschaft}

Es geht bei diesem Typ von Evaluation vor allem um die Erfolgskontrolle eines Programms oder einer Maßnahme nach bestimmten Erfolgskriterien wie z.B. Effektivität, Effizienz und Akzeptanz. Der Fokus liegt darauf, was mit einem Programm erreicht wurde (outcome und output) oder wie es implementiert wurde. Die Programmdurchführenden und / oder die Mittelgeber entscheiden, welchen Informationsbedarf sie haben und welche Kriterien für Erfolg sie zugrunde legen.

\footnotetext{
„For governmental sponsors of evaluations, for donors to international agencies, and for heads of private enterprises as well, evaluation is conducted to establish accountability to the public for funds received. For evaluators, this typically involves providing information about effectiveness and efficiency to decisionmakers." (Chelimsky 1997a, S.100f)
}

Manchmal geht es nur darum nachzuweisen, ob eine Veränderung eingetreten ist oder nicht, nachdem etwas Neues ausprobiert oder eingeführt wurde. Ziel ist primär, Wirkungen einer Maßnahme oder eines Programms nachzuweisen. Evaluatoren müssen bei dieser Art von Evaluation in erster Linie Effekte herausarbeiten: 
„accountability evaluators are faced with cause- and effect questions." (Che-

limsky 1997a, S.101)

Im Hinblick auf das Ziel der Kontrolle werden häufig eher quantitative als qualitative Methoden eingesetzt bzw. gefordert.

\title{
Evaluation mit dem Ziel der Entwicklung
}

Bei der Evaluation mit dem vorrangigen Ziel der Entwicklung geht es um eine Verbesserung und Optimierung von vorhandenen Strukturen und Prozessen bzw. um die Stärkung von positiven Aspekten und Potenzialen. Die Effektivität der Arbeit in Organisationen, Erfolgsfaktoren aber auch Hemmnisse für Zusammenarbeit werden identifiziert und auf Basis der Ergebnisse bewusst gestaltet.

\begin{abstract}
„For reformers of government, for the new public managers, for capacity builders and for others, evaluation is conducted to improve institutional performance. It is used both prospectively and retrospectively to do a number of things: to set a research agenda for an agency; to improve the design of new projects based on past evaluation findings; to develop the indicators and performance targets needed to improve organizational effectiveness and responsiveness; to determine what data systems are required for understanding what is happening in a program; to examine successes and failure of cooperation in a collaborative program involving independent agencies; to find out how program beneficiaries feel about its services; to check on whether spending is going according to plan and so on." (Chelimsky 1997a, S.101)
\end{abstract}

Es geht bei dieser Form von Evaluation vor allem um Fragen danach, wie auf Basis des Wissens und der Ergebnisse Prozesse bestmöglich gestaltet werden können. Es kommen häufig qualitative Methoden zum Einsatz wie z.B. Fallstudien, aber es werden auch des Öfteren verschiedene Methoden kombiniert. Evaluationen mit dem Ziel der Entwicklung bzw. Verbesserung von Programmen oder Maßnahmen werden meistens intern durchgeführt, in enger Zusammenarbeit mit den Akteuren. Die Rolle des Evaluators ist eher die eines Partners, Helfers oder Beraters als die eines unabhängigen Gutachters. Das Ziel ist vor allem eine Optimierung von Programmen und Abläufen, sowie eine Stärkung des Potenzials von den Beteiligten:

„They want to empower people, not establish merit or worth." (Chelimsky 1997a, S.101)

Die Einhaltung von (wissenschaftlichen) Gütekriterien, Unabhängigkeit bzw. Objektivität und Signifikanz sind eher zweitrangig bei dieser Form der Evaluation. Im Zentrum steht vor allem die Verbesserung der Praxis.

\section{Evaluation mit dem Ziel der Wissensgenerierung bzw. des Erkenntnisgewinns}

Im Kontext wissenschaftlichen Arbeitens dient Evaluation vor allem der Wissensgenerierung:

„For many researchers working independently in universities and for other evaluators in scientific institutions, evaluation is done to generate understanding and explanation. The specific questions are up to the evaluator: it is 
he or she who decides what will be asked in a study, and the topic generally follows from his or her prior research." (Chelimsky 1997a, S.102)

Es geht bei der „knowledge perspective“ darum, einen tieferen Einblick in das Evaluationsfeld zu bekommen und ein besseres Verständnis („explanatory insights“) über ein Themenfeld wie z.B. soziale Programme oder Interventionsmaßnahmen zu bekommen. Je nach Forschungsfeld und Forschungsfragen wählen die Evaluatoren ein passendes methodisches Design für die Datenermittlung und bemühen sich, wissenschaftlichen Gütekriterien so gut wie möglich gerecht zu werden.

Bei der Systematik von Chelimsky wird deutlich, dass bei jeder der drei Formen zum einen unterschiedliche Zielgruppen genannt werden (sowohl die Evaluatoren als auch die Auftraggeber und beteiligten Institutionen variieren) und zum anderen verschiedene Ziele und Perspektiven, wie die Ergebnisse von Evaluationen zu verwenden sind, damit verbunden sind. Außerdem richtet sich die Auswahl der Methoden auch nach dem Kontext und dem Verwendungszweck der Evaluation. In der Einteilung von Chelimsky wird die Vielfalt der Möglichkeiten im Feld der Evaluation deutlich.

Kromrey (2001, S.114) greift die Einteilung von Chelimsky auf und benennt drei Paradigmen der Evaluation:

- Das Forschungsparadigma der Evaluation

- Das Kontrollparadigma der Evaluation

- Das Entwicklungsparadigma der Evaluation.

An dieser Stelle wird das Leitkonzept für das Entwicklungsparadigma der Evaluation, wie Kromrey (2001) es beschreibt, aufgegriffen, da es für den Forschungsansatz dieser Arbeit besonders relevant ist:

\section{Das Entwicklungsparadigma der Evaluation}

Im Kontext des Entwicklungsparadigmas ist eine Evaluation dadurch gekennzeichnet, dass durch sie kein „fertiges Programm“ überprüft wird, sondern eher Ergebnisse von Anfang an zur Gestaltung systematisch zusammengestellt werden.

„Am Beginn steht nicht ein bereits realisiertes oder in der Implementationsphase befindliches oder zumindest ausformuliertes Programm. Vielmehr geht es darum, Konzepte und Vorstellungen zu entwickeln, die Fähigkeit von Organisationen zur Problemwahrnehmung und -bewältigung zu stärken, mitzuwirken retrospektiv und prospektiv Politikfelder zu strukturieren. Im Falle der Entwicklung und Erprobung von Programmen bedeutet dies: Die Evaluation ist in die gesamte Programm-Historie eingebunden [...]." (Kromrey 2001, S. 115) 
Die Evaluation findet dabei während der Planung, Entwicklung und Durchführung von Programmen oder Maßnahmen statt und begleitet so den Arbeitsprozess. Durch die Ergebnisse der Evaluation können Prozesse optimiert und Hemmnisse rechtzeitig erkannt werden (Evaluation als "Frühwarnsystem“"1). Die Ergebnisse können zur Gestaltung genutzt werden und sichern die Qualität der Arbeit:

"Evaluation unter solchen Bedingungen ist im wörtlichen Sinne „formativ“, also programmgestaltend. Sie ist wesentlicher Bestandteil des Entwicklungsprozesses, in welchem ihr die Funktion der Qualitätsentwicklung und Qualitätssicherung zukommt." (Kromrey 2001, S. 115)

Bei dem prozesshaften Vorgehen einer Evaluation mit dem Ziel der Entwicklung und Verbesserung von Programmen und Maßnahmen werden außerdem die Beteiligten explizit einbezogen (vgl. Kromrey 2001, S. 128). Die Ergebnisse und Informationen, die durch eine Evaluation ermittelt werden, sollen primär für den Handlungskontext nützlich sein. Die methodologischen Standards wissenschaftlicher Untersuchungen sind eher zweitrangig.

Nach Kromrey werden Methoden der Aktionsforschung eingesetzt:

„Methodisch verfährt die Evaluation dieses Typus häufig ähnlich wie ein Forschungskonzept, das Aktionsforschung (Handlungsforschung, action research) genannt wird. Ihr Ablauf ist iterativ, schleifenartig, ist ein fortwährendes Fragenstellen, Antworten, bewerten, Informieren und Aushandeln." (Kromrey 2001, S. 129)

Die Rolle des Evaluators ist die eines Beraters oder Helfers, der sich am Prozess beteiligt und mit den Akteuren kooperiert:

„Evaluatoren in diesem Konzept verstehen sich als Moderatoren im Diskurs der am Projekt beteiligten Gruppen (Informationssammler und -manager, 'Übersetzer' unterschiedlicher Fachsprachen und Argumentationsmuster, Koordinatoren und Konfliktregulierer, Vermittler von Fachwissen, Berater). Man kann daher mit Recht in diesem Zusammenhang von einem 'Helferund Beratermodell' sprechen." (Kromrey 2001, S. 129)

Bei dem Entwicklungsparadigma der Evaluation sind die Ziele der Evaluation und die Rollen des Evaluators anders als bei dem Forschungsparadigma und dem Kontrollparadigma. Es wird deutlich, dass Evaluationen unterschiedliche Funktionen haben können, die vom Kontext und der Zielsetzung abhängen. Zusammenfassend können insgesamt, unabhängig vom Kontext (Zielgruppen, Auftraggebern, Evaluatoren, Evaluationsgegenständen), folgende Primärfunktionen von Evaluation definiert werden:

a) Überprüfen und Kontrollieren,

b) Verbessern, Entwickeln und Lernen,

c) Entscheiden, Erkenntnisse gewinnen und Wissen generieren.

\footnotetext{
${ }^{1}$ Ein Wort, das Jürgen Habermas in seiner gesellschaftspolitischen Bedeutung gern zu verwenden scheint, so in einer Rede bei der Entgegennahme des Bruno-Kreisky-Preises am 9.3.2006, vgl. http://www.information-philosophie.de/?a=1\&t=222\&n=2\&y=1\&c=1, Zugriff am 20.8.2012).
} 
Neben diesen Primärfunktionen werden von einigen Autoren auch weitere Funktionen von Evaluation benannt. Will und Blickhan (1987) nennen beispielsweise als Nebenfunktionen u.a. Dokumentation, Intervention, Integration und Förderung der Kommunikation, Weiterbildung, Taktik und Ablenkung. Bei Dorenkamp (1999) finden sich als Funktionen von Evaluation u.a. mehr Effektivität und Effizienz, Vermeidung von Fehlinvestitionen, Versachlichung, Straffung von Curricula, Aufschluss über Qualitätsstand und Identifizierung von „best practice".

Die folgende Tabelle gibt einen Überblick über die von verschiedenen Autoren genannten Funktionen von Evaluation:

\begin{tabular}{|c|c|}
\hline Autoren & Primär- und Nebenfunktionen von Evaluation \\
\hline Wottawa und Thierau (2003) & $\begin{array}{ll}\text { - } & \text { Bewertung / Überprüfung } \\
\text { - } & \text { Verbesserung / Optimierungsgrundlage } \\
\text { - } & \text { Durcheheidungsgrundlage } \\
\text { - } & \text { Verantwortungshilfe } \\
\text { - } & \text { Kontrolle der Zielerreichung } \\
\end{array}$ \\
\hline Stockmann (2006a) & $\begin{array}{ll} & \text { Erkenntnis } \\
\text { - } & \text { Kontrolle } \\
\text { - } & \text { Dialog / Lernen } \\
\text { - } & \text { Legitimierung } \\
\text { - } & \text { Taktik }\end{array}$ \\
\hline Chelimsky (1997) & $\begin{array}{ll}\text { - } & \text { Kontrolle, Rechenschaft („Accountability“) } \\
\text { - } \quad \text { Entwicklung (,Development“) } \\
\text { - Wissensgenerierung, Erkenntnisgewinn (,Knowledge“) }\end{array}$ \\
\hline Kromrey (2001) & $\begin{array}{ll}\text { - } & \text { Kontrolle } \\
\text { - } & \text { Entwicklung / Gestaltung } \\
\text { - } & \text { Forschung / Erkenntnisgewinn }\end{array}$ \\
\hline Dorenkamp (1999) & $\begin{array}{l}\text { - } \quad \text { Steigerung der Effektivität und Effizienz } \\
\text { - Vermeidung von Fehlinvestitionen } \\
\text { - } \quad \text { Versachlichung } \\
\text { - } \quad \text { Straffung von Curricula } \\
\text { - } \quad \text { Identifizizierung von „,best practice“ } \\
\end{array}$ \\
\hline Will und Blickhan (1987) & $\begin{array}{l}\text { - } \quad \text { Steuerung und Optimierung } \\
\text { - } \text { Bewertung und Beurteilung } \\
\text { - } \text { Intervolle und Disziplinierung } \\
\text { - } \quad \text { Dokumention } \\
\text { - } \quad \text { Förderung der Kommunikation } \\
\text { - Takterbildung } \\
\end{array}$ \\
\hline Hellstern und Wollmann (1984) & $\begin{array}{l}\text { - } \quad \text { Analyse, Erkenntnis, Forschung } \\
\text { - Legitimation } \\
\text { - } \quad \text { Entscheidungshilfe, Argumentationsgrundlage } \\
\text { - Steuerungsinstrument, Eingriffschance } \\
\text { - Stärkung } \\
\text { - } \quad \text { Rückkoppontrolle, Selbstüberprüfung } \\
\text { - "Jobs“, Aufgaben und Forschungsfelder, Materialbasis } \\
\text { - Lerninstrument }\end{array}$ \\
\hline
\end{tabular}

Tabelle 4: $\quad$ Funktionen von Evaluation nach verschiedenen Autoren (erstellt von der Verfasserin) 
Mit dieser Zusammenstellung wird nochmals die Vielfalt der Funktionen von Evaluationen deutlich, die sich durch unterschiedlichste Kontexte und Interessen, Zielgruppen, Auftraggeber, Evaluatoren, Evaluationsgegenstände etc. ergeben kann. Darüber hinaus lässt sich Evaluation auch nach verschiedenen Formen und Arten unterscheiden, die im Folgenden näher beschrieben werden.

\title{
1.4 Formen und Arten
}

Je nachdem zu welchem Zeitpunkt eine Evaluation durchgeführt und von wem sie geleistet wird, kann zwischen verschiedenen Formen und Arten von Evaluation unterschieden werden. In der Literatur haben sich die Begriffe formative und summative sowie interne und externe Evaluation durchgesetzt. Außerdem kann zwischen Selbstevaluation und Fremdevaluation sowie zwischen Adressaten- und Systemevaluation unterschieden werden. In diesem Kapitel werden diese Unterscheidungen näher beschrieben.

\subsubsection{Formative und summative Evaluation}

Bei Evaluationen kann je nach Zeitpunkt und Zielsetzung zwischen formativer und summativer Evaluation unterschieden werden. Im Anschluss an Cronbach (1963), der sich für den Einsatz von Evaluationen bereits während des Entwicklungsprozesses von Curricula und nicht nur für die Evaluation abgeschlossener Curricula oder Produkte aussprach, wurden die Begriffe „formative“ und „summative“ von Scriven im Jahre 1967 eingeführt.

\begin{abstract}
„As a matter of terminology, I think that novel terms are worthwhile here, to avoid inappropriate connotations, and I propose to use the terms 'formative' and 'summative' to qualify evaluation in these roles." (Scriven 1967, S.43)
\end{abstract}

Scriven unterscheidet verschiedene Ziele von Evaluation. Je nach Ziel können mit einer Evaluation unterschiedliche Aufgaben erfüllt werden.

\section{Formative Evaluation}

Das Ziel einer formativen Evaluation ist die Optimierung des Evaluationsgegenstandes (z.B. eines Curriculums, eines Projektes oder eines Bildungsproduktes), während dieser sich noch in Entwicklung befindet. Die formative Evaluation ist prozessorientiert und ihre Rolle entspricht der eines Begleiters oder Beraters, der die Entwicklung unterstützt. Der Begriff „formativ“ drückt aus, dass die Entwicklung noch nicht abgeschlossen ist und die Ergebnisse für die Gestaltung (Formung) und zur Optimierung genutzt werden können. Bei der Entwicklung von Curricula können beispielsweise durch eine begleitende Evaluation Unzulänglichkeiten ermittelt und ggf. korrigiert werden. Der Entwicklungsprozess wird konstruktiv unterstützt, 
indem die Ergebnisse der Evaluation zur Optimierung beitragen. Formative Evaluationen können auch mehrfach während des Entwicklungsprozesses durchgeführt werden.

„Formative evaluation is contrasted with summative evaluation. It is typically conducted during the development or improvement of a program or product (or person, and so on) and it is conducted, often more than once, for the inhouse staff of the program with the intend to improve. The reports normally remain in-house [...]." (Scriven 1991, S.168f)

Die formative Evaluation kann auch als "Frühwarnsystem" genutzt werden, da Schwächen oder Hemmnisse frühzeitig erkannt und beseitigt werden können. Eine formative Evaluation liefert Zwischenergebnisse, die zur Bewertung und Kontrolle und ggf. zur Korrektur genutzt werden können.

"(... ) it is helpful to keep in mind that one of the most useful kinds of formative evaluation is `early-warning summative` [...]." (Scriven 1991, S.169)

Die Ergebnisse der Evaluation werden in der Regel intern genutzt und sind nicht unbedingt für Außenstehende vorgesehen. Die formative Evaluation kann aber auch genutzt werden, um darzustellen, dass bereits während des Entwicklungsprozesses auf Qualitätssicherung Wert gelegt wurde. Eine formative Evaluation wird in der Literatur oft auch als Prozessevaluation bezeichnet, wobei ein Prozess auch summativ evaluiert und bewertet werden könnte (vgl. z.B. Stufflebeam 1972, S.135).

\section{Summative Evaluation}

Das Ziel einer summativen Evaluation ist die Kontrolle und Bewertung des Evaluationsgegenstandes (z.B. eines Projektes, Programms oder Produktes) nachdem dieser fertig gestellt bzw. abgeschlossen wurde. Die Wirksamkeit oder Tauglichkeit wird am Ende der Laufzeit oder der Herstellung bzw. Entwicklung beurteilt. Das Produkt oder Ergebnis steht im Zentrum und nicht der Prozess. Die summative Evaluation ist ergebnisorientiert und die Aufgaben des Evaluators entsprechen denen eines Kontrolleurs oder Gutachters, der die Ergebnisse beurteilt. Der Begriff „summativ“ drückt aus, dass abschließend ein Resümee gezogen wird (ein Fazit in der Summe aller Dinge wird ermittelt, die Wirksamkeit überprüft). Die Evaluationsergebnisse dieser Art von Evaluation richten sich häufig an die (interessierte) Öffentlichkeit oder Entscheidungsträger, die wissen wollen, ob ein Produkt oder Programm gut ist.

„Summative evaluation of a programm (or other evaluand) is conducted after completion of the program (for ongoing programs, that means after stabilization) and for the benefit of some external audience or decision-maker (for example, funding agency, oversight office, historian, or future possible users), though it may be done by either internal or external evaluators or a mixture. "(Scriven 1991, S.340)

Die summative Evaluation wird in der Literatur auch als Produktevaluation oder Ergebnisevaluation bezeichnet (vgl. z.B. Stufflebeam 1972, S.136). 
Formative und summative Evaluationen haben jeweils einen anderen Fokus, und es ist nach Scriven sinnvoll, beide Formen zu nutzen.

„Doch zum Glück braucht man sich nicht für eine der beiden Rollen der Evaluation zu entscheiden. Bei pädagogischen, besonders jedoch bei curricularen Projekten muß man versuchen, beide Rollen der Evaluation zu erfüllen." (Scriven 1972, S.64)

Weiterhin können interne von externen Evaluationen unterschieden werden, die im nächsten Abschnitt erörtert werden.

\subsubsection{Interne und externe Evaluation}

Bei Evaluationen wird je nachdem, wer die Evaluation durchführt, zwischen interner und externer Evaluation unterschieden. Beide Formen haben Vor- und Nachteile.

\section{Interne Evaluation}

Formative Evaluationen (s.o.) werden meistens von internen Evaluatoren durchgeführt und die Ergebnisse werden intern verwendet, um den Gestaltungsprozess zu optimieren. Die regelmäßige Überprüfung und (Zwischen-)Ergebniskontrolle kann gewollter Bestandteil eines Programms sein und zur Qualitätssicherung genutzt werden. Der Evaluator ist ein Mitglied des Projektteams, gehört zumindest zum Personal, auch wenn er explizit nur mit der Evaluation betraut ist.

\footnotetext{
„Internal evaluators (or evaluations) are those done by project staff, even if they are special evaluation staff - that is, even if they are external to the production/writing/teaching/ - service part of the project. Usually internal evaluation is part of the formative evaluation effort [...]." (Scriven 1991, S.197)
}

Der Vorteil einer internen Evaluation ist, dass der Evaluator Zugang zu Informationen hat und bei der Erarbeitung von Produkten dabei ist. Er genießt in der Regel das Vertrauen der Beteiligten, da er zur Optimierung der Prozesse beitragen kann, Hinweise gibt und keine Kontrolle oder abschließende Bewertung vornimmt. Der Nachteil kann sein, dass ein interner Evaluator nicht objektiv genug ist, um die Prozesse und Ergebnisse zu beurteilen.

\section{Externe Evaluation}

Summative Evaluationen (s.o.) werden meistens von externen Evaluatoren durchgeführt, und die Ergebnisse werden verwendet, um ein Projekt, Programm oder Produkt für Außenstehende zu bewerten. Die Ergebnisse werden beispielsweise dem Mittelgeber oder Entscheidungsträgern vorgelegt, können aber auch von interessierten Nutzern oder Fachleuten verwendet werden. Der Evaluator ist in der Regel kein Mitglied des Projektteams, sondern eine außenstehende, unabhängige Person, die den Evaluationsgegenstand möglichst objektiv beurteilt. 
„An external evaluator is someone who is at least not on the project or program`s regular staff, or someone - in the case of personnel evaluation other than the individual being evaluated, or their family or staff. It is better if they are not previously known to or paid by any entity with a commitment to the success or failure of the project." (Scriven 1991, S.159)

Der Vorteil einer externen Evaluation ist, dass der Evaluator meistens ein Evaluationsexperte ist, professionell d.h. mit einem gewissen Abstand auf die Ergebnisse schaut und wissenschaftliche Standards einhält. Ein Nachteil kann sein, dass der externe Evaluator die Prozesse bei der Gestaltung nicht berücksichtigt, sondern nur das Endergebnis beurteilt.

Außerdem kann zwischen Selbst- und Fremdevaluation differenziert werden, wie im nächsten Abschnitt verdeutlicht wird.

\title{
1.4.3 Selbstevaluation und Fremdevaluation
}

Je nachdem, wer eine Evaluation durchführt, kann zwischen einer Selbstevaluation und einer Fremdevaluation unterschieden werden. Die Deutsche Gesellschaft für Evaluation definiert Selbstevaluation wie folgt:

\begin{abstract}
„Unter Selbstevaluation werden systematische, datenbasierte Verfahren der Beschreibung und Bewertung verstanden, bei denen die praxisgestaltenden Akteure identisch sind mit den evaluierenden Akteuren. Selbstevaluatorinnen und Selbstevaluatoren sind stets Mitglieder der Organisation, des Netzwerkes oder der sozialen Gemeinschaft, welche die zu evaluierenden Programme, Maßnahmen usw. tragen. Der Gegenstand der Evaluation ist dabei die eigene Praxis." (DeGeval 2004)
\end{abstract}

Im Zentrum einer Selbstevaluation steht demnach die eigene Praxis, das eigene berufliche Handeln. Vor allem in den Praxisfeldern von Schule und sozialer Arbeit werden Selbstevaluationen durchgeführt und auch als Bestandteil pädagogischer Professionalität verstanden. Im Bereich der sozialen Arbeit führte Heiner $(1988,1994)$ theoretisch fundierte und zugleich praxisbezogene Konzepte der Selbstevaluation ein. Außerdem sind die Arbeiten von Beywl und Bestvater (1998) sowie von König (2007) einschlägig. Das Bundesministerium für Familie, Senioren, Frauen und Jugend hat zudem zwischen 1995 und 2001 eine 36 Bände umfassende Reihe herausgegeben, um die Arbeit und Selbstevaluation in der Kinder- und Jugendhilfe mit dem Ziel der Qualitätssicherung zu unterstützen (http://www.qskompendium.de/, Zugriff am 25.9.2012). Bei der Selbstevaluation ist es „der Sozialarbeiter, der als "Forscher" in eigener Sache den Verlauf und die Ergebnisse seines beruflichen Handelns untersucht" (Heiner 1988, S.7). Entscheidend ist, dass die Praktiker die Verantwortung für den Evaluationsprozess haben, was nicht heißt, dass sie die Evaluation alleine durchführen müssen. Sie können durchaus Unterstützung von professionellen Evaluatoren oder Sozialwissenschaftlern bekommen.

Im Bereich Schule scheint Selbstevaluation in den letzten Jahren zum festen Bestandteil der Entwicklung von Schulen geworden zu sein. Es finden sich einige Publikationen, die sich an 
Schulen und Lehrer richten, um deren (Selbst-)Evaluationstätigkeiten mittels Leitfäden, Praxisanleitungen und Fallbespielen zu unterstützen und zu fördern (Buhren et al. 2002, Buhren 2007, Moser 1999, Schratz et al. 2002, von Saldern 2010). Schulentwicklungsprozesse und kontinuierliche Überprüfung, Reflexion und ggf. Verbesserung von Schulprogrammen und dem Lehrangebot sollen durch die Selbstevaluation eigenständig und selbst organisiert erfolgen. Die Weiterentwicklung und Qualitätsverbesserung der schulischen Arbeit steht dabei im Zentrum.

Die Begriffe Selbstevaluation und interne Evaluation werden häufig synonym verwendet. Kennzeichnend für die Selbstevaluation ist, dass die Praxisakteure ihre Arbeit selbst evaluieren, maßgeblich gestalten und definieren, was mit welchen Methoden untersucht wird und welche Ziele die Evaluation hat. Eine interne Evaluation liegt dann vor, wenn Evaluierende einer Organisation angehören, die evaluiert wird (beispielsweise könnte ein Lehrer einer Schule den Unterricht eines Kollegen oder einer Kollegin evaluieren, dann läge eine interne Evaluation vor. Untersucht ein Lehrer seinen eigenen Unterricht, so kann von einer Selbstevaluation gesprochen werden). Die Grenzen zwischen interner Evaluation und Selbstevaluation können jedoch fließend sein.

Eine Fremdevaluation bedeutet, dass die Evaluatoren nichts mit dem Gegenstand der Evaluation zu tun haben, sondern ihre Tätigkeit sich lediglich auf die professionelle Durchführung der Evaluation bezieht. Eine "fremde“, außenstehende Person führt dabei eine Evaluation durch und ist nicht Mitglied der Organisation und auch kein Teil des Systems, das evaluiert wird.

Michael Scriven (1967, S.44) unterscheidet in diesem Kontext „professional versus amateur evaluation“. Diese Unterscheidung verdeutlicht noch einmal, dass bei einer Selbstevaluation die Praktiker nicht unbedingt eine systematische Ausbildung genossen haben, um eine Evaluation professionell durchzuführen. Die Deutsche Gesellschaft für Evaluation (DeGEval 2004) hat Empfehlungen zur Anwendung der Standards für Evaluation im Handlungsfeld der Selbstevaluation herausgegeben, um die Qualität der Selbstevaluationen zu erhöhen und Hilfestellungen im Sinne eines Orientierungsrahmens für Praktiker zu bieten.

\subsubsection{Adressatenevaluation und Systemevaluation}

Bei Evaluationen kann zwischen einer Adressaten- und einer Systemevaluation unterschieden werden. Diese Unterscheidung wurde von Flechsig und Haller (vgl.1975, S.135) eingeführt. Bei einer Adressatenevaluation stehen die Personen im Zentrum, an die sich z.B. eine Bildungsmaßnahme richtet. Im Bereich der Schule sind die Adressaten die Schüler. Individuelle Leistungsmessungen, Überprüfung des Gelernten sowie Eignungstests können hier durchgeführt werden und als Adressatenevaluation bezeichnet werden. Verschiedene Ad- 
ressatenmerkmale werden dabei erfasst und ausgewertet bzw. bewertet. Die Ergebnisse der Evaluation können, je nachdem wann sie erfolgt, verwendet werden, um Vorkenntnisse, Fertigkeiten, Lernvoraussetzungen und Interessen zu ermitteln, aber auch, um Lernerfolge und Leistungen zu erfassen und zu messen.

Bei einer Systemevaluation hingegen können der Unterricht, das Schulsystem oder das Schulverwaltungssystem, aber auch ökonomische Kontextbedingungen im Zentrum stehen. Es werden beispielsweise Lernbedingungen und die Qualität der Lernangebote sowie institutionelle Rahmenbedingungen mittels einer Systemevaluation betrachtet. Flechsig und Haller identifizieren verschiedene „Handlungsebenen der Didaktik“ (1975, S.14). Systemevaluationen können sich auf diese unterschiedlichen Ebenen des Systems beziehen: Institutionelle, ökonomische, personelle und konzeptionelle Rahmenbedingungen (A-Ebene), Lehrplan- und Schulkonzepte (B-Ebene), Lernbereiche und Unterrichtskonzepte (C-Ebene), Unterrichtseinheiten (D-Ebene) und Lehr-/Lernsituationen (E-Ebene).

Zusammenfassend lässt sich sagen, dass zwischen verschiedenen Arten und Formen von Evaluation unterschieden werden kann, je nachdem zu welchem Zeitpunkt, von wem und mit welchem Zweck und Fokus eine Evaluation durchgeführt wird. Vor allem die Bezeichnungen formative und summative Evaluation, interne und externe Evaluation, Fremdevaluation und Selbstevaluation sowie die Unterscheidung zwischen Adressaten- und Systemevaluation finden sich in der Literatur. 


\section{Zur Geschichte der Evaluationsforschung}

In diesem Kapitel soll darauf Bezug genommen werden, dass Evaluationen vom Prinzip und Ansatz her mit Forschungen verbunden sein können; sei es dass eine Evaluation als Forschungsvorhaben verstanden und durchgeführt wird, sei es, dass eine Evaluation auf Ergebnisse, Verfahren, Instrumente und Erfahrungen von Forschungen zurückgreift.

Die Entwicklung der Evaluationsforschung kann nicht isoliert betrachtet werden, vielmehr müssen historische Rahmenbedingungen und Prozesse gesehen und verstanden werden, die Einfluss hatten auf die heutigen Ansätze, Konzepte, Verfahren und Methoden der Evaluation.

Die Evaluationsforschung ist eingebettet in gesamtgesellschaftliche Entwicklungen und eng verbunden mit der Politik und ihren Institutionen. Nach Stockmann ist die Evaluation sogar „politikgetrieben“ (Stockmann 2010, S.23). Die Aufgabenstellungen und Funktionen, die Evaluationen zugeschrieben werden, sind abhängig von den Interessen der jeweiligen Regierenden, ihrer Schwerpunkte und Zielsetzungen, sowie der wirtschaftlichen Situation.

Zum Ursprung der Evaluationsforschung wird in der Literatur meist auf die Entwicklungen in den USA in den 1930er und 1940er Jahren hingewiesen. Einige Autoren betonen aber auch, dass die Evaluationsforschung weiter zurückreichende Wurzeln hat. Hellstern und Wollmann (vgl. 1984, S.20) führen beispielsweise die staatsphilosophischen und politikwissenschaftlichen Diskussionen über die Wirkungsweise und Wirksamkeit des Staates im 19.Jhd. an und verweisen auf Schriften von Wilhelm von Humboldt. Die kritische Reflexion und Diskussion im Kontext von Staat, Politik, Verwaltung und Wissenschaft hat demnach eine lange Tradition.

Nach Stockmann lässt sich sogar sagen, dass „Evaluation so alt ist wie die Welt" (Stockmann 2010, S.23), da in der Schöpfungsgeschichte bereits erzählt wird, wie Gott sein geschaffenes Werk betrachtet und für gut befindet. Außerdem beschreibt Stockmann alle menschlichen Versuche, herauszufinden und zu bewerten, was gut und was weniger gut ist, als Evaluationstätigkeiten (Stockmann 2010, S.23f). Evaluation als ein „systematisches Verfahren, das bestimmten wissenschaftlich vorgegebenen Regeln folgt“" (Stockmann 2010, S.24) sei jedoch ein relativ modernes Phänomen.

Im Folgenden werden einige historische Entwicklungslinien der Evaluationsforschung beschrieben, dabei wird der Fokus zunächst auf die USA gerichtet, da die dortigen Entwicklungen maßgeblich diejenigen in Europa beeinflusst haben. 


\subsection{Entwicklungen in den USA}

Die Ursprünge systematischer Evaluationen in den USA werden von verschiedenen Autoren im frühen 19. Jahrhundert gesehen (vgl. Madaus \& Stufflebeam 2000, Fitzpatrick, Sanders \& Worthen 2004, Chelimsky 2006, Mertens 2006). Es gibt verschiedene Einteilungen in historische Phasen, die sich u.a. im Bezug auf ihre Differenziertheit unterscheiden. In der Übersicht zur historischen Entwicklung der Programm Evaluationen von Madaus und Stufflebeam (2000) werden sieben Phasen identifiziert, die im Folgenden dargestellt werden. Diese Einteilung wird in dieser Arbeit aufgegriffen, da sie einen direkten Bezug zur Evaluation von Bildung, Bildungsmaßnahmen und Bildungsinstitutionen sowie zu weiteren Programmen im humanitären Bereich hat. Die folgende Tabelle gibt einen Überblick über die Phasen, die dann im Einzelnen beschrieben werden:

\begin{tabular}{|l|l|}
\hline Phase & Zeitraum \\
\hline The Age of Reform & $1792-1900$ \\
\hline The Age of Efficiency and Testing & $1900-1930$ \\
\hline Tylerian Age & $1930-1945$ \\
\hline The Age of Innocence & $1946-1957$ \\
\hline The Age of Development & $1958-1972$ \\
\hline The Age of Professionalization & $1973-1983$ \\
\hline The Age of Expansion and Integration & $1983-2000$ \\
\hline \multicolumn{1}{|c|}{ Tabelle 5: Phasen von Programm Evaluation nach Madaus und Stufflebeam (vgl. 2000, S.3) }
\end{tabular}

\subsubsection{Die Zeit der Reform (1792 - 1900)}

Madaus und Stufflebeam beschreiben diese Phase als Ära der Industriellen Revolution, die durch gravierende Veränderungen im Bereich der Ökonomie und der Technologie sowie einen gesamtgesellschaftlichen Wandel gekennzeichnet ist:

„The 19th century was the era of the Industrial Revolution with all of its attendant economic and technological changes. The very structure of society was transformed. Major social changes occurred. There was drastic change in physical and mental health and outlook, in social life and social conscience, and in the structures of social agencies." (Madaus und Stufflebeam 2000, S.4)

Die ersten Evaluationen von Programmen seien eher "informal and impressionistic" (Madaus und Stufflebeam 2000, S.4) gewesen. Die Evaluationen wurden geleitet von „presidential commissions“ und richteten sich neben "human service programs“ auch auf Schulen. Die erste, systematische, 'formale Evaluation`wurde in Amerika 1845 in Boston durchgeführt, mit dem Ziel, die Leistungen (performance) der Schulen zu überprüfen. Nach Madaus und 
Stufflebeam ist dies ein Meilenstein in der Entwicklung der Evaluationsforschung, da eine lange Tradition von Schülertests und der Untersuchung zur Effektivität von Schulen begann:

„[...] it began a long tradition of using pupil test scores as a principal source of data to evaluate the effectiveness of a school or instructional program." (Madaus und Stufflebeam 2000, S.5)

Die Evaluationen an Schulen mit dem Ziel der Überprüfung und Kontrolle der Schulen, aber auch zum Vergleich verschiedener Schulen sowie Leistungsmessungen bezogen auf Schüler und die Eignung von bestimmten Unterrichtsmethoden, wurden dann Ende des 19.Jahrhunderts eingeführt. Dafür waren nach Madaus und Stufflebeam die Arbeiten von Joseph Rice bedeutend:

\begin{abstract}
„Between 1887 and 1898, Joseph Rice conducted what is generally recognized as the first formal educational program evaluation in America. He carried out a comparative study on the value of drill in spelling instruction across a number of school districts." (Madaus und Stufflebeam 2000, S.6)
\end{abstract}

Außerdem sehen Madaus und Stufflebeam die Gründung einer Akkreditierungsgesellschaft Ende des 19.Jahrhunderts als bedeutsam an, die damals zwar noch keinen besonderen Status besaß, aber doch bemerkenswert sei, da in den 1930er Jahren weitere sechs regionale Akkreditierungsgesellschaften verteilt über ganz Amerika eingerichtet wurden. Der Weg für die Etablierung von systematischen Schulevaluationen wurde damit geebnet.

\title{
2.1.2 Die Zeit der Effizienz und des Testens (1900 - 1930)
}

In dieser Phase sehen Madaus und Stufflebeam zunächst die Ideen und Arbeiten von Frederick Taylor (1856-1915) als prägend. Sie erwähnen vor allem den "Scientific Management" Ansatz, der von Taylor begründet und auch unter dem Begriff „Taylorismus“ bekannt wurde. Es ging bei diesem Ansatz um Zeit- und Arbeitsstudien an Arbeitern mit dem Ziel der Optimierung von Arbeitsabläufen in Betrieben. Nach Madaus und Stufflebeam ist dies „an early form of personnel evaluation“ (2000, S.6), und es entstand auf der Basis der Ideen von Taylor eine Bewegung, die nachhaltigen Einfluss im Erziehungswesen und der Industrie hatte, wobei sich der Fokus vor allem auf die Steigerung von Effektivität richtete.

„Taylors ideas became a powerful force in administrative theory in educational and industrial circles (...). The emphasis of this movement was on systemization, standardization, and, most importantly, efficiency." (Madaus und Stufflebeam 2000, S.7)

Im Bereich des Schulwesens wurden in dieser Phase Begutachtungen und Tests in großem Umfang eingeführt. Zum einen standen dabei die Schulen im Zentrum der Ermittlungen und zum anderen die Lehrer, wobei verschiedene Kriterien zur Bewertung verwendet wurden. Bei diesen Evaluationen wurden neu entwickelte Tests eingesetzt, die objektiv und sehr genau 
messen sollten, was untersucht wurde. Die Tests bezogen sich meist auf einen bestimmten Bezirk.

\begin{abstract}
„A number of these surveys employed the newly developed "objective" tests in arithmetic, spelling, handwriting, and English composition to determine the quality of teaching. [...] These tests were often developed in large districts by a bureau or department set up specifically to improve the efficiency of the district." (Madaus und Stufflebeam 2000, S.7)
\end{abstract}

In dieser Zeit wurden jedoch auch Tests von verschiedenen Forschern entwickelt, die überregional eingesetzt und mit deren Hilfe genau festgelegte Ziele überprüft werden konnten.

„In addition to these locally developed tests there were a number of tests developed by researchers like Courtis, Ayers, Thorndike, and others, which were geared to measure very precise set of instructional objectives." (Madaus und Stufflebeam 2000, S.7)

Die Phase der "Effizienz und des Testens" ist demnach gekennzeichnet von Bestrebungen, mit neu entwickelten Instrumenten (Tests) möglichst präzise und objektiv zu messen, wie effizient bestimmte Methoden des Lehrens und Lernens bzw. Arbeitens waren. Mit den ermittelten Ergebnissen konnte dann das Ziel der Optimierung verfolgt werden. Die Geschichte der Evaluationsforschung ist dadurch mit dem Bereich des Testens eng verbunden.

„Throughout its history, the field of evaluation has been closely linked to the field of testing. Test data have often been the principal data source in evaluations [...]." (Madaus und Stufflebeam 2000, S.8)

Die Untersuchungen bezogen sich in dieser Phase auf bestimmte Schulbezirke, während später, in den 1950er und 1960er Jahren der Fokus auf überregionale Curriculumentwicklungen gerichtet wurde. Vor 1930 lag die Durchführung von Tests und Evaluationen vor allem in der Hand der Lehrer oder eines Komitees von Lehrern, deren Fragestellungen sich auf regionale Zusammenhänge bezogen.

In den Jahren zwischen 1920 und 1930 wurden erste universitäre Institute gegründet, die spezialisiert waren auf Begutachtungen und Bewertungen von Schulen in bestimmten Bezirken. Madaus und Stufflebeam sehen diese Institute als Vorläufer der Zentren an Universitäten, die in den 1960ern und 1970ern entstanden und die speziell auf Evaluationen ausgerichtet waren.

\footnotetext{
„During the late 1920s and 1930s, university institutes specializing in field studies were formed and conducted surveys for local districts. [...] These institutes could be considered the precursors of the university centers dedicated to evaluation that grew up in the 1960s and 1970s." (Madaus und Stufflebeam 2000, S.8)
}

Insgesamt ist diese Phase der Effizienz und des Testens bedeutsam für die Geschichte der Evaluationsforschung, weil Ansätze entwickelt wurden, die als Basis für die moderne Evaluationsforschung gesehen werden können. 


\title{
2.1.3 Die Tylersche Zeit (1930 - 1945)
}

Für die moderne Evaluation sind die Arbeiten von Ralph W. Tyler bahnbrechend, und sein Einfluss hat die Evaluation von Bildung nach Madaus und Stufflebeam maßgeblich beeinflusst. Ralph W. Tyler wird auch als „Vater der Bildungsevaluation“ bezeichnet. Er führte eine neue und innovative Sichtweise in Bezug auf Curricula und Evaluation ein:

\begin{abstract}
„Ralph W. Tyler has had enormous influence on education in general and educational evaluation and testing in particular. He is often referred to, quite properly we feel, as the father of educational evaluation. Tyler began by conceptualizing a broad and innovative view of both curriculum and evaluation." (Madaus und Stufflebeam 2000, S.8)
\end{abstract}

In den 1930er Jahren kam es insgesamt zu gravierenden sozialen Umbrüchen in der Gesellschaft. Vor dem Hintergrund der "Großen Depression" entstanden im Rahmen des „New Deal“, der von Präsident Roosevelt eingeführt wurde, zahlreiche Reformprogramme zur Verminderung der Arbeitslosigkeit, zur Verbesserung der sozialen Sicherheit und zu Optimierungen im Bildungswesen. In diese Zeit fällt auch der Höhepunkt einer pädagogischen Erneuerungsbewegung, die unter dem Namen "Progessive Education Movement" bekannt wurde, an der John Dewey und ebenso Ralph W. Tyler beteiligt waren.

Tyler übernahm 1929 an der Ohio State University die Aufgabe, das Lehren und Lernen zu optimieren. Einer der Gründe war, dass viele Studierende ihr Studium in den ersten zwei Jahren abbrachen. Der Direktor der Universität war der Meinung, dass durch systematische Forschung und den Einsatz von Tests und Testverfahren die Lehre und das Lernen verbessert werden könnten bzw. müssten. Tyler war damit beauftragt, dies zu tun, und begann seine Arbeit in Biologiekursen. Er arbeitete mit den Lehrenden zusammen und stellte fest, dass die gebräuchlichen Tests nicht gut geeignet waren, um den Lernzuwachs der Studierenden zu erfassen:

\begin{abstract}
„I found that the instructors were using tests that demanded only that students recall specific information. None of the test exercises required the more complex behaviours that the courses were planned to help students learn. This was typical of the tests commonly in use that time. The most widely used tests in high school and college appraised only recall of information on specific skills in mathematics and reading. Using tests of this sort, the instructors were unable to assess objectively the progress of their students in learning what the courses were designed to help them learn. Furthermore, the use of tests of recall gave the students the wrong notion of what they were expected to learn." (Tyler 2000, S.88)
\end{abstract}

Mit diesem Zitat wird deutlich, dass die bis dahin üblichen Tests nicht nur ungeeignet waren zu überprüfen und zu messen, was die Studierenden gelernt hatten, sie legten auch nahe, dass es darauf ankäme, lediglich bestimmtes Faktenwissen mehr oder weniger auswendig zu lernen. An dem Ansatz von Tyler war auch neuartig, dass er mit den Lehrenden zusammen arbeitete. Er fragte sie, was ihre Lernziele waren, um dann gemeinsam geeignete Methoden zur Messung und Erreichung dieser Ziele zu finden: 
„As I worked with the instructors, asking them what they expected students to be learning in and from their courses, I found that the first answers were usually very general and often vague. For example, 'I am trying to teach them to think,', 'I am teaching the scientific method', 'I want them to develop skills in the laboratory.' Since the fundamental purpose of education is to help students acquire new ways of thinking, feeling, and acting, objectives should be defined clearly enough to indicate what the educational program is intended to help students develop - what kinds of behaviour, what ways of thinking, feeling, or acting; and with what content." (Tyler 2000, S.88)

Auf dieser Grundlage wurde deutlich, dass zunächst Lernziele (objectives) der Dozenten definiert werden mussten, wobei auch zwischen angestrebtem Verhalten (behavior) und Wissensinhalten (content) unterschieden wurde. Nach der Definition der Ziele mussten neue Methoden und Testverfahren gefunden oder entwickelt werden, die eine Überprüfung ermöglichten. Tyler setzte von 1930-1934 seine Arbeiten in anderen Kursen z.B. in den Fächern Chemie, Mathematik, Philosophie, Geschichte und Wirtschaft fort. Seine Erfahrungen fasste er in dem Artikel „A General Technique for Constructing Achievement Tests“ zusammen (Tyler 1934). Er entwickelte ein siebenstufiges Verfahren für die Erstellung von Lernzuwachstests, das folgende Schritte umfasst (Tyler 2000, S.90):

1. Identifying the objectives of the educational program.

2. Defining each objective in terms of behavior and content.

3. Identifying situations where objectives are utilized.

4. Devising ways to present situations.

5. Devising ways to obtain a record.

6. Deciding on the terms to use in appraisal.

7. Devising means to get a representative sample.

1934 wurde Tyler Leiter der so genannten "Eight Year Study“, einer Studie zur Effizienz und Leistungsfähigkeit verschiedener Schulsysteme. Es wurde dabei u.a. ein Vergleich der traditionellen Highschool mit der "progressiven" Sekundarschule vorgenommen. Der Hintergrund war, dass zu Beginn der 1930er Jahre viele junge Menschen an die Hochschulen gingen, ohne konkrete Ziele zu haben. Im Zusammenhang mit der "Großen Depression" gab es wenig Perspektiven für die Schulabgänger, die dann vermehrt ein Studium begannen. Die Hochschulen waren zum einen, was ihre Curricula und Lernangebote anbelangte, seit den 1920er Jahren unverändert geblieben, und die Strukturen waren so starr, dass Veränderungen schwierig erschienen. Zum anderen verlangten die Studierenden selbst mehr Freiheiten und Möglichkeiten zu einem selbst gesteuerten Lernen.

"The high schools of 1930 were still much like those of 1920, particularly in terms of curriculum content and learning activities. High school staff felt that they were prevented from making improvements because of the rigidity of college entrance requirements and of state accreditation regulations. Pressures for change were mounting, coming in part of the students themselves. Many of the young people entering high school came from elementary 
schools that had given them greater freedom and more opportunities for selfdirection in learning than they were permitted as high school students." (Tyler 2000, S.91)

Die "Progressive Education Association" ging dieses Problem an. Eine Kommission erteilte den Auftrag, das Verhältnis von Schule und Hochschule genauer zu untersuchen, die Missstände bei dem Übergang auszuräumen und die Konflikte zu lösen. Es wurde 1933 mit einer Pilotstudie begonnen, bei der ausgewählte Modellschulen untersucht wurden. Sie sollten, begleitet von der Kommission, Lehrangebote erarbeiten und durchführen, die ihrer Meinung nach passend für die Studierenden wären. Es war die Aufgabe der Schulen, im Zeitraum der acht Jahre Informationen zu sammeln und darüber zu berichten, was die Schüler lernten. Die Kommission sollte sicherstellen, dass das Pilotprogramm evaluiert und die Ergebnisse dokumentiert würden. Es wurde schnell deutlich, dass die Schulen Unterstützung brauchten, sowohl was die Entwicklung der Curricula als auch die Evaluation anbelangte (vgl. Tyler 2000, S.92).

Tyler wurde Leiter der Evaluationsgruppe und war damit beauftragt, die Prozeduren und Instrumente für die Studie zu entwickeln. Außerdem gab es eine Gruppe, deren Fokus auf der Curriculumentwicklung lag. Beide Gruppen arbeiteten eng mit den Lehrenden zusammen. Es stellte sich bald heraus, dass die Entwicklung neuer Curricula eine komplexere Angelegenheit war, als zuerst angenommen. Es begann eine intensive Zeit der Entwicklung neuer Ideen und Verfahren, die bei zahlreichen Treffen diskutiert und erarbeitet wurden:

\begin{abstract}
„It soon became apparent that the development of the curriculum and instructional program and the plan of evaluation required more time than was available on weekends, when the working committees met during the first year-and-a-half of the program. It was then we devised the summer workshops, in which representatives teachers from all of the schools worked together with the study staffs and subject-matter consultants for six weeks each summer to developed what was needed." (Tyler 2000, S.93)
\end{abstract}

Im Rahmen der "Eight-Year-Study” wurde deutlich, dass die Evaluationsgruppe mit der „rationale for testing" eine gute Grundlage hatte, um ihre Arbeit zu strukturieren. Der Curriculumgruppe jedoch fehlte eine entsprechende Anleitung. Dies veranlasste Tyler dazu, sein Werk „Basic Principles of Curriculum and Instruction“ (1949) zu schreiben, in dem er seine Gedanken, Erfahrungen und sein Wissen zusammenfasste und das zu einem Bestseller wurde. Die so genannte "Tyler Rationale" kann für eine strukturierte und systematische Planung von Curricula verwendet werden und besteht aus vier Basisfragen (vgl. Tyler 1949):

1. What educational purposes should the school seek to attain? (Defining appropriate learning objectives.)

2. How can learning experiences be selected which are likely to be useful in attaining these objectives? (Introducing useful learning experiences.)

3. How can learning experiences be organized for effective instruction? (Organizing experiences to maximize their effect.)

4. How can the effectiveness of learning experiences be evaluated? (Evaluating the process and revising the areas that were not effective.) 
Aus der Sicht von Madaus und Stufflebeam hat "die Eight-Year-Study" die Evaluationsforschung - insbesondere im Bildungsbereich - entscheidend geprägt. Tyler führte den Begriff "educational evaluation" (Madaus und Stufflebeam 2000, S.8) ein und lenkte den Fokus von dem Ansatz des Testens und Messens auf die Begleitung von Entwicklungsprozessen. Er ebnete den Weg von einer individuenzentrierten Leistungsmessung, die in der Zeit der „Effizienz und des Testens" (siehe Abschnitt 2.2.2) zentral war, zu einer auf Lernergebnisse (outcomes) fokussierten Forschung. Tyler versteht Evaluation als Prozess, bei dem herausgefunden wird, ob die zuvor definierten Lernziele erreicht wurden bzw. welche Veränderungen im Verhalten der Lernenden stattfanden:

"The process of evaluation is essentially the process of determining to what extend the educational objectives are actually being realized by the program of curriculum and instruction. However, since educational objectives are essentially changes in human beings, that is, the objectives aimed at are to produce certain desirable changes in the behavior patterns of the student, then evaluation is the process for determining the degree to which these changes in behavior are actually taking place." (Tyler 1949, S.105f)

Die Arbeiten von Tyler erweiterten die Sicht auf die Evaluation von Bildung und die Methoden zur Erforschung ihrer Ergebnisse.

„The Eight-Year Study introduced educators throughout Amerika to a new and broader view of educational evaluation than which had been in vogue during the age of efficiency and testing. Tyler conceptualized evaluation as a comparison of intended outcomes with actual outcomes." (Madaus und Stufflebeam 2000, S.9)

Zusammenfassend lässt sich festhalten, was das Besondere und Innovative an den Ansätzen von Tyler in dieser Zeit war:

- Evaluation wird auf die Entwicklung von Curricula bezogen.

- Der Fokus richtet sich nun von der individuellen Leistungsmessung durch Tests auf die Lernerfolgsmessung durch Evaluation.

- Lernen wird als Prozess gesehen.

- Lehrer werden bei der Definition von Zielen sowie der Entwicklung von Instrumenten zur Erforschung und Evaluation beteiligt.

- Systematische pädagogische Strategien werden mit der Hilfe von Leitfragen entwickelt.

- Die Arbeit ist gekennzeichnet durch ein zyklisches Vorgehen: Vorher-Nachher-Tests, auf deren Basis Stärken und Schwächen beschrieben werden und auf die dann entsprechende Modifikationen des Curriculums vorgenommen werden - dann beginnt wieder ein Evaluationsprozess.

Die Arbeiten von Tyler haben viele nachfolgende Forscher inspiriert und beeinflusst: 
"Clearly by the middle of the 1940s Tyler had, through his work and writing, laid the foundation for his enormous influence on the educational scene in general and on testing and evaluation in particular during the next 25 years." (Madaus und Stufflebeam 2000, S.9)

Bis heute sind die Ansätze und Arbeiten von Ralph W. Tyler bedeutend und prägen auch aktuelle Evaluationsvorgehensweisen.

\title{
2.1.4 Die Zeit der Stagnation (1946 - 1957)
}

Nach dem zweiten Weltkrieg begann in der Gesellschaft eine Phase des Aufbaus im Bildungsbereich. Zahlreiche Angebote und Einrichtungen wurden geschaffen, wobei der Bedarf nach systematischen Evaluationen kaum vorhanden war. Die in der Bildung Tätigen mussten nicht die Effizienz und Effektivität ihrer Angebote beweisen, es wurde der Schwerpunkt auf Neuschaffung und Expansion gelegt.

\begin{abstract}
„Throughout American society, the late 1940s and 1950s were a time to forget the war, leave the depression behind, build and expand capabilities, acquire resources, and engineer and enjoy "a good life". This general scene in society and education was reflected in educational evaluation. While there was great expansion of education there was no particular interest on the part of society in solving social and education problems and holding educators accountable. There was little call for educators to demonstrate the efficiency and effectiveness of any of the many developmental efforts." (Madaus und Stufflebeam 2000, S.10)
\end{abstract}

Madaus und Stufflebeam (2000, S.9) nennen diese Phase "The Age of Innocence", führen aber aus, dass sie diese Zeit ebenso als "The Age of Ignorance" bezeichnen könnten, da der Weiterentwicklung von Evaluationsansätzen und -methoden kaum Beachtung geschenkt wurde. Dennoch fanden Entwicklungen im Bereich der Evaluation statt, vor allem technische Neuerungen wurden eingeführt. Es gab einen Zuwachs von standardisierten Testverfahren, der sich u.a. in der Veröffentlichung zahlreicher standardisierter Tests zeigte. Im Jahr 1947 wurde der "Educational Testing Service" eingerichtet, was diese Entwicklungen ebenfalls zum Ausdruck bringt. ${ }^{2}$

„During this period there was considerable development of some of the technical aspects of evaluation; this was consistent with the then-prevalent expansion of all sorts of technologies. Chief among these developments was the growth in standardized testing. Many new nationally standardized tests were published during this period." (Madaus und Stufflebeam 2000, S.10)

Um die Aktivitäten im Bereich der Testentwicklungen zu regulieren, wurden Mitte der 1950er Jahre einige Veröffentlichungen geschaffen, die für in den 1970er Jahren entwickelte Stan-

\footnotetext{
${ }^{2}$ Damit verfügten die USA über eine zentrale Forschungs- und Entwicklungseinrichtung im Bereich der Bildungsevaluation, die zahlreichen Forscherinnen und Forschern eine Plattform bot, so z.B. Herman Witkin für seine Untersuchungen zu Feldabhängigkeit/Feldunabhängigkeit. ETS beschäftigt ca. 2500 Personen und hatte im Jahre 2003 einen Jahresetat von 630 Millionen US-Dollar (http://www.fundinguniverse.com/company-histories/educational-testing-service-history/, Zugriff am 20.8.2012).
} 
dards die Basis darstellen wie z.B. die „Standards for Educational and Psychological Tests“ (vgl. Madaus und Stufflebeam 2000, S.10).

Die Ansätze von Ralph W. Tyler (vgl. Kap. 2.1.3) wurden ebenfalls aufgegriffen und um technische Aspekte erweitert. Es stellte sich heraus, dass Lehrende, um ihre Angebote zu optimieren, Unterstützung bei der Formulierung ihrer Lernziele brauchten. Es wurden Techniken wie z.B. Taxonomien entwickelt, die helfen sollten, Ziele klar zu definieren. Madaus und Stufflebeam (vgl. 2000, S.11) erwähnen dazu die Arbeiten von Bloom (1956) und Krathwohl et al. (1964). Außerdem wurden Tylers Arbeiten verwendet, um Lehrer im Bereich der Testentwicklung zu trainieren.

Insgesamt sind die späten vierziger und fünfziger Jahre jedoch durch ein relativ geringes Interesse an spezifisch pädagogisch ausgerichteten Evaluationen gekennzeichnet. Trotz der wachsenden Finanzmittel war das politische Interesse an Evaluationen kaum vorhanden. Es lag in der Verantwortung der einzelnen lokalen Institute bzw. Organisationen, Evaluation in Auftrag zu geben und zu finanzieren, was sich in der nächsten Phase deutlich ändern sollte.

\subsubsection{Die Zeit der Entwicklung und Expansion (1958 - 1972)}

Am 4.Oktober 1957 startete in der Sowjetunion der erste künstliche Erdsatellit "Sputnik 1“ erfolgreich ins All, und diese technologische Leistung hatte den so genannten "Sputnikschock" in den USA zur Folge. Der Westen glaubte sich in einer überlegenen Situation und meinte eine Vorrangstellung in den wissenschaftlich-technischen Gebieten zu haben. Die Tatsache, dass die Sowjetunion den USA im Weltraum nun einen Schritt voraus war, überraschte und schockierte die amerikanische Öffentlichkeit zutiefst. Die Bildungskatastrophe wurde ausgerufen, und es wurden Forderungen nach einer grundlegenden Reform des Bildungssystems gestellt. Insbesondere in den Naturwissenschaften, der Mathematik und in den Fremdsprachen kam es zu umfangreichen und landesweiten Programmen u.a. durch den im Jahr 1958 eingeführten "National Defence Education Act".

In der Evaluationsforschung hatte der Sputnikschock ebenfalls Konsequenzen. Finanzielle und personelle Ressourcen für Curriculumentwicklungen und Evaluationen wurden bereitgestellt. Die Programme waren oft landesweit angelegt, während Tyler noch lokal gearbeitet und sich auf einzelne Schulen bezogen hatte. Nach Madaus und Stufflebeam wurden alle bis dahin entwickelten Evaluationsansätze genutzt: Der Ansatz von Tyler (Lernzieldefininition und Zielerreichungsgradüberprüfung), Testverfahren (standardisierte Tests), Beurteilungsansätze (Effizienzmessungen) und klassische Experimente.

\footnotetext{
„All four of the approaches to evaluation discussed so far were represented in the evaluations done during this period. First, the Tyler approach was used to help define objectives for the new curricula and to assess the degree to which the objectives were later realized. Second, new nationally standardized tests were created to better reflect the objectives and content of the new curricula. Third, the professional-judgement approach was used to rate pro-
} 
posals and to check periodically on the efforts of contractors. Finally, many evaluators evaluated curriculum development efforts through the use of field experiments. " (Madaus und Stufflebeam 2000, S.11f)

Die Evaluationen in dieser Zeit dienten vor allem dazu nachzuweisen, was in den Programmen getan wurde (Verwendungsnachweis) und sollten Wirkungen dokumentieren bzw. beweisen (Legitimationsfunktion).

Ein weiteres Ereignis hat in dieser Phase die Evaluationsgeschichte beeinflusst. Mitte der sechziger Jahre rief Präsident Lyndon B. Johnson den „War on Poverty“ aus. Die Armut in der Bevölkerung sollte bekämpft, Chancengleichheit und soziale Versorgung unterstützt werden. Die "Great Society“ sollte u.a. durch Reformen im Gesundheits-, Sozial- und Bildungssystem verwirklicht werden. Senator Robert Kennedy und seine Kollegen verabschiedeten den „Elementary and Secondary Education Act“ (ESEA) im Jahre 1964, in dem sie festlegten, dass bei allen Programmen Evaluationen durchgeführt werden mussten, u.a. um zu gewährleisten, dass die verwendeten Mittel gut eingesetzt wurden (vgl. Madaus und Stufflebeam 2000, S.12). Evaluation sollte dabei nach Hellstern und Wollmann (1984, S. 27) „Zeuge, Rechtfertigungsinstrument und Stimulator für eine Politik werden, durch die erstmals ein gezielter Angriff auf die Ursachen der Armut unternommen werden sollte". Mit dem „Community Action Program“ wurden verschiedene Angebote für die Bevölkerung realisiert. Zu den bekanntesten Programmen, die auch für die Entwicklung der Evaluationsforschung bedeutsam sind, gehört „Head Start“, das 1965 startete (vgl. Hellstern und Wollmann 1984, S. 29). Die Ziele von Head Start waren, Kindern aus bildungsferneren Schichten eine Vorschulerziehung unter Beteiligung der Eltern anzubieten und dadurch Bildungsbenachteiligungen abzubauen und die Chancengleichheit zu erhöhen. Außerdem sollte sozialen Problemen frühzeitig entgegengewirkt werden. Das Programm erfuhr eine sehr gute Resonanz, und es beteiligten sich fünfmal so viele Familien wie zunächst geplant. Es wurden enorme Mittel aufgebracht, um das Programm entsprechend zu realisieren. Dieser Erfolg führte dazu, umfangreiche Evaluationen durchzuführen, mit dem Ziel, die Wirksamkeit nachzuweisen, die Angemessenheit der eingesetzten Mittel zu rechtfertigen und die Effizienz der Programme zu verdeutlichen.

Head Start hatte aber auch Gegner und Kritiker, zu denen vor allem Nixon gehörte, der die Programme abbauen wollte. Durch inn kamen vorzeitig Ergebnisse an die Öffentlichkeit, die Head Start in keinem besonders guten Licht dastehen ließen, und dies löste „eine intensive sozialwissenschaftliche Diskussion über Forschungsmethoden, Evaluierungskriterien und Forschungsstrategien zur Evaluierung von staatlichen Programmen aus" (Hellstern und Wollmann 1984, S. 29). Es kam zu zahlreichen Veröffentlichungen und heftigen Debatten, neue Evaluierungen wurden gestartet. Letztlich wurde das Programm erhalten, doch blieben Folgen nicht aus. Die Sommerprogramme (Sommerlager) waren besonders kritisiert worden und wurden nach und nach abgebaut. In der Evaluierungsdiskussion wurde gefordert, dass 
Wirkungen differenzierter betrachtet werden müssten und neue Methoden (z.B. auch Langzeitstudien) eingeführt werden sollten (vgl. Hellstern und Wollmann 1984, S. 30f).

\begin{abstract}
„Während sich die wissenschaftliche Diskussion über die Ergebnisse dieser großangelegten Sozialexperimente erst abzuzeichnen beginnt, wurde jedoch schon bald deutlich, daß der ursprünglich fast naturwissenschaftlich zu nennende Denkansatz die Erkenntnismöglichkeiten in den Testprogrammen stark limitierte. Kreativere, qualitativere Evaluierungsformen gewannen an Bedeutung, die weniger strenge Bedingungen an eine Konstanz der Umweltbedingungen und Programmvariablen erforderten. Auch erschien es wichtiger, mehrfache Evaluierungen durchzuführen, um unterschiedliche Aspekte eines Programms zu erfassen." (Hellstern und Wollmann 1984, S. 31f)
\end{abstract}

Am Beispiel von Head Start wird deutlich, dass es zwar gute finanzielle Bedingungen für Evaluationen gab und sie zu den Programmen dazu gehörten, weil sie bei der Planung schon vorgesehen wurden, weshalb diese Phase als Zeit der Entwicklung und Expansion der Evaluationstätigkeiten und der Evaluationsforschung bezeichnet werden kann. Es gab jedoch auch schon Anfang der 1960er Jahre Kritik an den bisherigen Evaluationsmethoden und -ansätzen. Die Methoden der Evaluation richteten sich bisher vor allem darauf, Endergebnisse (outcomes) zu betrachten, und waren weniger geeignet, die Optimierung der Praxis im laufenden Prozess zu unterstützen. Das Zielerreichungsmodell von Tyler, standardisierte Leistungstests und Experimente waren nicht besonders hilfreich bzw. nicht ausreichend, um die Wirkungen eines Curriculums umfassend zu analysieren. Cronbach (1963) wies in seinem einschlägigen Artikel „Course Improvement Through Evaluation“ darauf hin, dass neue Techniken nötig seien:

„Old habits of thought and long-established techniques are poor guides to the evaluation required for course improvement." (Cronbach 2000, S.247)

Er lenkte den Fokus darauf, dass Evaluationen genutzt werden sollten, um Entscheidungen über Curricula auch schon während ihrer Laufzeit zu treffen, mit dem Ziel, sie zu verbessern. Für Cronbach dient Evaluation dazu, Informationen zu sammeln und auszuwerten, um Entscheidungen über ein Bildungsprogramm treffen zu können:

„we may define evaluation broadly as the collection and use of information to make decisions about an educational program." (Cronbach 2000, S.235)

Er kritisierte, dass die bis dahin üblichen Evaluationsmethoden nicht geeignet seien, um Kurse bzw. Curricula zu verbessern und genügend über Wirkungen der Kurse zu erfahren. Für Cronbach (vgl. 2000, S.236) hat Evaluation den Zweck, Entscheidungen in drei Bereichen fällen zu können: 1. Kursoptimierung, 2. Einzelpersonen betrachten und 3. Administrative Bewertungen. Er schlägt in diesem Kontext vor, neue Methoden, und zwar eine Vielfalt von quantitativen und qualitativen Ansätzen, in der Evaluation einzusetzen. Seiner Meinung nach sollten dafür Messverfahren entwickelt werden, die eine neue Form der Qualität haben sollten im Vergleich zu dem, was bis dahin an Methoden zur Verfügung stand. 
„The three purposes - course improvement, decisions about individuals, and administrative regulation - call for measurement procedures having somewhat different qualities." (Cronbach 2000, S.240)

Für ihn gehörte Evaluation außerdem zum festen Bestandteil der Curriculumentwicklung und der Nutzen sollte auf die Praxis ausgerichtet sein. Das Ziel ist die Optimierung von Lernangeboten, die Unterstützung der Lehrenden, damit sie einen „besseren Job machen können“.

„Evaluation is a fundamental part of curriculum development, not an appendage. Its job is to collect facts the course developer can and will use to do a better job and facts from which a deeper understanding of the educational process will emerge." (Cronbach 2000,S.247)

Mit den Ansätzen von Cronbach wird deutlich, dass sowohl neue Evaluationsmethoden entwickelt werden sollten, als auch der Fokus dahin verschoben werden müsste, schon während der Laufzeit Programme zu evaluieren und nicht erst am Ende im Sinne einer Ergebnisevaluation - Scriven (1967) führte dazu später die Begriffe „formative und summative Evaluation" ein (vgl. Abschnitt 1.4.1).

Der Aufsatz von Michael Scriven „The Methodology of Evaluation“ aus dem Jahre 1967 trug ebenfalls erheblich zur Weiterentwicklung der Evaluationsforschung und der wissenschaftlichen Diskussion bei. Scriven fokussiert die Evaluation von Curricula, betont aber, dass seine Ausführungen auch auf andere Bereiche der Evaluation bzw. Evaluation im Allgemeinen übertragen werden könnten. Er kritisiert die bis dahin gebräuchlichen Verfahren und Methoden der Evaluation und führt wesentliche Begriffe, Unterscheidungen und Diskussionspunkte ein.

"Current conceptions of the evaluation of educational instruments (e.g. new curricula, programmed texts, inductive methods, individual teachers) are still inadequate both philosophically and practically." (Scriven 1967, S.39)

Die Unterscheidung zwischen den Zielen einer Evaluation und deren Rollen, sowie die Kennzeichnung verschiedener Formen und eine Reflexion der Methoden machen den Artikel von Scriven zu einem wesentlichen Beitrag für die Weiterentwicklung der Evaluationsforschung. Er spricht sich deutlich für den Wert von Evaluationen im Bildungsbereich aus und betont, dass pädagogische Forschung mit komplexen Fragestellungen zu tun hat und deshalb aufwendig und auch kostspielig sein muss. Der Nutzen guter Forschung sei aber für die Gesellschaft langfristig sehr hoch und deshalb lohnenswert.

\footnotetext{
„If we want to know the answers to the questions that matter about new teaching instruments, we have to do experiments which will yield those answers. The educational profession is suffering from a completely inappropriate conception of the cost scale for educational research. (...) When we are dealing with a teaching instrument such as a new curriculum or classroom procedure, with its extreme dependence upon highly variable operators and recipients, we must expect considerably more expense. The social pay-off is enormously more important, and the society can. In the long run, afford the expense." (Scriven 1967, S.83)
} 
Weitere wichtige Beiträge und Grundlagenwerke in dieser Phase stammen von Stake (1967), Suchman (1967) und Campbell \& Stanley (1966). Insgesamt wurden in dieser Phase neue Modelle von Evaluation eingeführt, die sich deutlich von den Ansätzen, die es zuvor gab, unterschieden. Durch die neuen Konzepte wurden wesentliche Neuerungen geschaffen:

„These conceptualizations recognized the need to evaluate goals, look at inputs, examine implementation and delivery of services, as well as measure intended and unintended outcomes of the program. They also emphasized the need to make judgements about the merit or worth of the object being evaluated." (Madaus und Stufflebeam 2000, S.14)

Die 1960er und 1970er Jahre sind gekennzeichnet durch einen großen Zuwachs von Evaluationstätigkeiten und eine intensive Diskussion um Evaluationsmethoden (Ziele, Funktionen, Formen). Durch negative Evaluationsergebnisse, wie etwa in dem Beispiel des Programmes Head Start (s.o.), entstand aber auch eine Diskussion über Evaluation und deren Möglichkeiten und Grenzen im Allgemeinen (vgl. Madaus und Stufflebeam 2000, S.14). In der nächsten Phase sind daher Professionalisierungsbestrebungen und eine Diversifizierung von Evaluationsansätzen charakteristisch.

\title{
2.1.6 Die Zeit der Professionalisierung und Diversifizierung (1973 - 1983)
}

Anfang der 1970er Jahre war eine Zeit des Umbruchs in der Evaluationsforschung. Durch negative Erfahrungen und Enttäuschungen sowie angefangene Debatten und Diskussionen, was Evaluation überhaupt leisten kann, und welche Methoden für welche Zwecke adäquat sein würden, gab es eine Krise. Häufig wurden die Erwartungen, die an Evaluationen geknüpft waren, nicht erfüllt, und das Selbstverständnis der Personen, die Evaluationen durchführten war heterogen. Madaus und Stufflebeam beschreiben die Situation folgendermaßen:

\begin{abstract}
„Before this period evaluators faced an identity crisis. They were not sure whether they should try to be researchers, testers, administrators, teachers, philosophers, or iconoclasts. It was unclear what special qualifications, if any, they should possess. There was no professional organization dedicated to evaluation as a field, nor were there specialized journals through which evaluators could exchange information about their work. There was essentially no literature about program evaluation except unpublished papers that circulated through an underground network of practitioners. There was a paucity of pre-service and inservice training opportunities in evaluation. Articulated standards of good practice were confined to educational and psychological tests. The field of evaluation was amorphous and fragmented [...]." (Madaus und Stufflebeam 2000, S.14f)
\end{abstract}

Im Laufe der 1970er Jahre setzte dann eine Entwicklung ein, die Madaus und Stufflebeam als Professionalisierung der Evaluation bezeichnen und die sich in verschiedenen Aspekten zeigte (vgl. S.15). Es erschienen eine Reihe von Publikationen, Büchern und Monographien zum Thema Evaluation und es wurden Fachzeitschriften gegründet (z.B. Evaluation Review, New Directions for Program Evaluation, Studies in Educational Evaluation und Evaluation 
and Program Planning oder Evaluation News). Außerdem wurden Berufs- und Interessenverbände gegründet, Netzwerke, in denen sich Möglichkeiten für einen Austausch zwischen im Bereich der Evaluation Tätigen boten, entstanden (z.B. Evaluation Network, Evaluation Research Society und Division H of AERA).

Darüber hinaus wurden an Universitäten eigene Kurse in „Evaluationsmethodologie“ angeboten als Ergänzung zur sonst üblichen sozialwissenschaftlichen Methodenausbildung, und eine Vielfalt von professionellen Organisationen offerierten Workshops und Seminare zu verschiedenen Evaluationsthemen. Somit wurden eigene Ausbildungs- und Fortbildungsgänge geschaffen, die erheblich zu einer Professionalisierung beitrugen. Darüber hinaus entstanden die „Standards für Evaluation“ (Joint Committee 1981), die bis in die heutige Zeit weiterentwickelt und verfeinert werden.

Neben der eben beschriebenen Professionalisierung ist die Zeit der 1970er und 1980er Jahre aber auch gekennzeichnet durch eine Diversifizierung der Evaluationspraxis. Es entstand eine Fülle von Evaluationsansätzen und Evaluationsmodellen, von denen viele heute, im Jahre 2012, noch als aktuell gelten können. Viele dieser Modelle wurden in den folgenden Jahren weiter entwickelt.

Einige wichtige Werke sind das CIPP-Modell von Stufflebeam (1983), das Konzept der zielfreien Evaluation von Scriven (1973), das Modell der Responsiven Evaluation von Stake (1975) und der Ansatz naturalistischer Evaluation von Guba und Lincoln (1981).

In der Zeit der 1970er und 1980er Jahre wurden auch einschlägige Lehrbücher zum Thema Evaluation veröffentlicht, wie etwa Rossi, Freeman und Wright (1979) und Weiss (1972), die heute noch zu den Standardwerken zählen und zum Teil auch in aktualisierten Neuauflagen vorliegen (z.B. Rossi, Lipsey und Freeman 2004).

Die Entwicklungen in der Evaluationsforschung und -praxis in Richtung Professionalisierung und Diversifizierung hatte verschiedene Auswirkungen. Zum einen gab es mehr wissenschaftlichen Austausch im Feld der Evaluation, es gab aber auch eine Menge „Rederei“ „an enormous amount of chatter" (Cronbach 1980, zitiert nach Madaus und Stufflebeam 2000, S.16). Zum anderen zeigten sich Fortschritte in der Ausbildung und Qualifizierung von Evaluatoren, die aber auch mit Sorgen betrachtet wurden, weil man fürchtete, Evaluation könnte dadurch zu etwas sehr Exklusivem, Elitärem werden (vgl. Madaus und Stufflebeam 2000, S.16). Außerdem zeichnete sich eine Polarisation zwischen verschiedenen Lagern in der evaluationstheoretischen Auseinandersetzung ab. Auf der einen Seite gab es eher positivistisch-quantitativ orientierte Ansätze und auf der anderen Seite phänomenologischqualitativ ausgerichtete Konzepte. Letztlich hat sich nach Madaus und Stufflebeam die Praxis der Evaluation seit dieser Phase aber nicht wesentlich geändert:

"[...] in spite of growing search for appropriate methods, increased communication and understanding among the leading methodologists, and the development of new techniques, the actual practice of evaluation changed very little in the great majority of settings." (Madaus und Stufflebeam 2000, S.16) 
Insgesamt zeichnet sich die Zeit der 1970er und 1980er Jahre durch intensive Diskussionen um die Möglichkeiten und Grenzen sowie um den Nutzen der Evaluation aus. Die beschriebenen Professionalisierungstendenzen sowie die Diversifizierung von Evaluationsmodellen sind charakteristisch für diese Zeit. In der folgenden Phase erfolgte eine Art Neuorientierung bzw. eine Zeit der Integration verschiedener Ansätze.

\subsubsection{Die Zeit der Expansion und Integration (1983 - 2001)}

Diese Phase begann nach Madaus und Stufflebeam (vgl. 2000, S.16f) zunächst mit Einschneidungen im Bereich der Evaluation. Ronald Reagan wurde 1981 Präsident der Vereinigten Staaten, und es kam zu umfangreichen Veränderungen in der Sozial- und Bildungspolitik sowie in der Wissenschaftspolitik. Finanzielle Mittel für Evaluationen wurden gekürzt und die Funktion von Evaluation wurde vor allem in der Ermittlung von Kosten und Nutzen gesehen. Ergebnisse von Evaluationen sollten in diesem Kontext vor allem zu ökonomischen Entscheidungszwecken dienen, z.B. Einsparungsmaßnahmen und Mittelkürzungen rechtfertigen und unterstützen, oder dazu verwendet werden, um Programme vorzeitig abzubrechen, falls sie sich als nicht effektiv genug zeigen sollten.

Obwohl ökonomische Betrachtungsweisen und Kosten-Nutzen-Abwägungen in den Vordergrund traten, wuchs und expandierte das Feld der Evaluation. Die Expansion zeigt sich besonders in der Entwicklung und Etablierung von professionellen Evaluationsinstitutionen („evaluation societies“) sowie der Vernetzung, dem Austausch und der Kollaboration von Evaluatoren aus unterschiedlichen Fachdisziplinen (vgl. Madaus und Stufflebeam 2000, S.17).

Die Phase zeichnete sich ebenfalls durch Integrationsbestrebungen aus. Der Methodenstreit zwischen verschiedenen Lagern (qualitative und quantitative bzw. positivistische und phänomenologische Ansätze) geriet in den Hintergrund bzw. verlor an Bedeutung. Es ging in dieser Phase nicht mehr so sehr um die Wahl der einen richtigen Methode, sondern eher darum, die angemessenen Methoden für einen bestimmten Evaluationsgegenstand auszuwählen. Durch die Schaffung von Evaluationsnetzwerken wurden Ideen ausgetauscht und Methoden weiterentwickelt:

„The Evaluation Research Society, which was composed mainly of evaluators from the social sciences, amalgamated with the Evaluation Network, comprised mainly of educational evaluators, to form the new "ecumenical" American Evaluation Association (AEA). The result has been an integration and cross-fertilization of evaluation ideas and methods across the disciplines." (Madaus und Stufflebeam 2000, S.17)

Ein weiteres Zeichen für die Expansion der Evaluation sind die Gründung von Interessenverbänden und Gemeinschaften (evaluation associations) in der ganzen Welt, die 
Entstehung zahlreicher Zeitschriften (evaluation journals) sowie die Schaffung und Nutzung professioneller Standards für Evaluation wie die Program Evaluation Standards des Joint Committee on Standards for Educational Evaluation (Joint Committee 1994).

Dieser kurze Abriss der Geschichte der Evaluationsforschung in den USA zeigt, dass sich Evaluation und Evaluationsforschung im Kontext von Bildung im letzten Jahrhundert und vor allem in den letzten Jahrzehnten stark entwickelt haben. Diese Entwicklungen sind jedoch noch nicht abgeschlossen, so dass Madaus und Stufflebeam (2000, S.18) insgesamt zu dem Schluss kommen, Evaluation als dynamische, noch nicht fertige (unreife) Profession zu bezeichnen („,a dynamic, yet immature, profession“).

Sie fordern auch, dass Forschung und Ausbildung im Feld der Evaluation verbessert und weiterentwickelt werden müssen:

\begin{abstract}
„There is a need to improve research, training and financial support for program evaluation. Leaders of the evaluation profession must ensure that efforts to improve their profession are geared to the service needs of their clients, not merely designed to serve their private or corporate needs. Ultimately the value of program evaluation must be judged in terms of its actual and potential contributions to improving learning, teaching and administration, health care and health, and in general the quality of life in our society and others." (Madaus und Stufflebeam 2000, S.18)
\end{abstract}

Auch die Ausrichtung auf in der Praxis entstehende Bedarfe, d.h. eine anwendungs- und umsetzungsorientierte Evaluationstätigkeit, wird als wichtig betrachtet. Schließlich soll Evaluation dazu beitragen, Lernen und Lehren zu verbessern und insgesamt die Lebensqualität in der Gesellschaft zu erhöhen.

Die beschriebene historische Entwicklung der Evaluationsforschung in den USA macht deutlich, dass Evaluation eingebunden ist in gesamtgesellschaftliche Prozesse und die Politik, von denen Richtungen und Schwerpunktsetzungen vorgegeben werden. Evaluation kann nicht isoliert betrachtet werden, sondern es müssen die Kontextbedingungen mit beachtet werden. Die Entwicklungen in den USA haben die in Europa beeinflusst. Da es in dieser Arbeit um die Evaluierung europäischer Bildungsprojekte geht, werden im folgenden Unterkapitel die Entwicklungen der Evaluationsforschung in Europa näher betrachtet.

\title{
2.2 Entwicklungen in Europa
}

Die moderne Evaluationsforschung in Europa beginnt nach Stockmann (vgl. 2006a, S.27) im Vergleich zu den USA später. Ende der 1960er Jahre gewinnt sie durch politische Reformprogramme, die in dieser Zeit aufkommen, an Bedeutung und Profil. Bei einer Betrachtung der historischen Entwicklung der Evaluationsforschung in Europa ist es im Gegensatz zu den USA schwerer, eine allgemeine, für alle Länder geltende Systematisierung zu finden. Die 
einzelnen Länder sind relativ unabhängig voneinander und haben unterschiedliche nationale Rahmenbedingungen, die für die Entwicklung ihrer Evaluationskultur prägend sind. Dies führt innerhalb von Europa zu einer Unterschiedlichkeit und Vielfalt von Evaluationskulturen in den verschiedenen Nationen.

Mit der Gründung der Europäischen Wirtschaftsgemeinschaft im Jahre 1957 in Rom und der 1967 auf dieser Basis entstandenen Europäischen Gemeinschaft - später Europäische Union genannt - verpflichteten sich die Mitgliedsländer, eng zusammen zu arbeiten und gemeinsame wirtschaftliche wie rechtliche Regeln einzuführen. Im Gegensatz zu den USA, wo auf der Grundlage einer gemeinsamen Verfassung die Staatsgründung stattfand, ist es in der Europäischen Union umgekehrt: Unterschiedliche Nationen mit eigenen Rechts- und Verwaltungssystemen werden nach und nach aneinander angeglichen und harmonisiert. Im Jahre 2004 entstand eine gemeinsame Europäische Verfassung, die von den damals 25 Mitgliedsstaaten erarbeitet wurde ${ }^{3}$. Obwohl die EU-Staaten keine gemeinsame Regierung haben, gibt es Institutionen, die Politik und Wirtschaft in der Europäischen Union regeln. Mit dem Europäischen Rat, dem Europäischen Ministerrat, der Europäischen Kommission und dem Europäischen Parlament wurde ein System geschaffen, mit dem das Miteinander der verschiedenen Nationen systematisch organisiert bzw. unterstützt und gefördert wird.

Im Hinblick auf die Entwicklung der Evaluationskultur in Europa muss unterschieden werden, zwischen der Entwicklung in einzelnen Nationen und der in der Europäischen Union. Wie Stockmann (2010, S.55) feststellt, ist „die Rolle der Evaluation in der Gesellschaft in hohem Umfang politikbestimmt und die Entwicklung der Evaluationsgeschichte politikgetrieben“. Es ist daher wichtig, zum einen die Entwicklung der Evaluation in den einzelnen Ländern Europas zu betrachten unter Berücksichtigung der jeweiligen nationalen Rahmenbedingungen, und zum anderen die Entstehung der Evaluationskultur in der Europäischen Union zu verfolgen.

In diesem Kapitel werden deshalb zunächst Entwicklungslinien der Evaluationskultur in verschiedenen europäischen Nationen thematisiert. Anschließend werden die Entwicklungen beschrieben, die sich in der Europäischen Union vollzogen haben. Ziel ist zum einen zu zeigen, dass sich die Evaluationsforschung auch im europäischen Raum auf unterschiedlichen Ebenen entwickelt hat, und zum anderen herauszuarbeiten, welchen besonderen Herausforderungen eine Evaluationspraxis gegenübersteht, die sich an Europäische Bildungsprojekte richtet.

\footnotetext{
${ }^{3} \mathrm{Da}$ aber nach gescheiterten Referenden in Frankreich und den Niederlanden nicht alle Mitgliedstaaten den Vertrag ratifiziert hatten, erlangte er keine Rechtskraft. Deshalb beschlossen die beteiligten europäischen Staats- und Regierungschefs im Jahre 2007 den Vertrag von Lissabon, in welchem immerhin eine Reihe von Regelungen zur künftigen Zusammenarbeit vereinbart wurde.
} 


\subsubsection{Die Evaluationskultur in verschiedenen europäischen Nationen}

Einen allgemeinen Überblick zur Entwicklung der Evaluation in Europa hat Leeuw (2006) vorgelegt. Er beschreibt in seinem Artikel die Entwicklungen in den einzelnen Nationen und bezieht sich dabei auf die Untersuchungen von Furubo, Rist und Sandahl (2002) sowie auf die Entstehung von Institutionen, insbesondere Evaluationsgesellschaften, in verschiedenen europäischen Ländern als Indikatoren für die Professionalisierung von Evaluation. Ende der 1980er Jahre wurden nach Leeuw (vgl. 2006, S.67) die ersten Evaluationsgesellschaften gegründet, und im Jahr 2006 gab es bereits 10 Evaluationsgesellschaften im europäischen Raum. Außerdem betrachtet Leeuw die Angebote von Evaluationen auf dem europäischen Markt und beschreibt die besonderen Bedingungen der „Evaluationsindustrie“ (vgl. ebenda, S.68f).

Als Vorläufer der Zusammenstellung von Furubo, Rist und Sandahl (2002) erschien im Jahr 1990 das Buch „Program evaluation and the management of government: Patterns and prospects across eight nations“, das von Rist herausgegeben wurde. In diesem Werk wird die Evaluationskultur in acht Nationen beschrieben (Canada, Deutschland, England, USA, Dänemark, Niederlande, Norwegen und Schweiz). Das Ziel ist nach Rist (vgl. 1990, S.3), den aktuellen Stand der Evaluation im Hinblick auf Institutionalisierung und Nutzanwendung zu geben („a detailed and up-to-date account of both the organization and uses of evaluation“). Derlien (vgl. 1990, S.153) arbeitet drei verschiedene Trends der Entwicklungen in den verschiedenen Ländern heraus, kommt aber zu dem Schluss, dass der Stand der Evaluationsforschung in vielen der betrachteten Länder noch nicht besonders gefestigt sei: „[...] the summary reflects the still-shaky state of evaluation in many of the countries considered here" (Derlien 1990, S.174). Die Evaluationskultur scheint demnach in den verschiedenen Ländern recht unterschiedlich zu sein.

Auf der Basis weiterführender umfassender Untersuchung zur Evaluationskultur unter globaler Perspektive haben Rist, Furubo und Sandahl im Jahr 2002 dann den „International Atlas of Evaluation“ herausgegeben. Es werden in diesem „Atlas“ 21 Nationen beschrieben, wobei jeder Artikel folgende Punkte thematisiert bzw. enthält (vgl. ebenda, S.6):

- Einen historischen Überblick zum Angebot und zur Nachfrage von Evaluationen, sowie institutionelle Rahmenbedingungen.

- Eine Beschreibung des Grades der Professionalisierung von Evaluation (z.B. Ausbildungsmöglichkeiten und eine nationale Evaluationsgesellschaft).

- Eine Beschreibung von Evaluation in der Exekutive.

- Eine Beschreibung von Evaluation in der Legislative.

- Eine Zusammenfassung von Beobachtungen zum aktuellen Stand der Evaluation in einer Nation und deren Zukunftsperspektiven. 
Furubo, Rist und Sandahl (vgl. 2002, S.7f) arbeiten neun Indikatoren für die Bestimmung der Evaluationskultur einer Nation heraus, auf deren Grundlage sie eine Rangreihe erstellen. Sie geben jedem Land, das sie bewerten, entweder keinen, einen oder zwei Punkte für die jeweiligen Indikatoren, woraus sich dann die „Höhe“ bzw. der Grad der Evaluationskultur ergibt:

1. Evaluation findet in verschiedenen Bereichen statt („Evaluation takes place in many policy domains”).

2. Ausgebildete Evaluatoren sind verfügbar

("There is a supply evaluators specializing in different disciplines who have mastered different evaluation methods and who conduct evaluations").

3. Nationaler Diskurs zum Thema Evaluation

("There is a national discourse concerning evaluation").

4. Evaluation als Profession

("There is a profession with its own societies or frequent attendance at meetings of international societies").

5. Institutionelle Verankerung innerhalb des Staates

("Institutional arrangements in the government for conducting evaluations and disseminating their results to decision makers").

6. Vorhandensein von institutionellen Bedingungen im Parlament, die die Durchführung von Evaluationen und die Verwendung von Ergebnissen begünstigen ("Institutional arrangements are present in the Parliament for conducting evaluations and disseminating them to decision makers").

7. Pluralismus von Institutionen und Evaluatoren

("An element of pluralism exists, that is, within each policy domain there are different people or agencies commissioning and performing evaluations").

8. Übergeordnete Audit-Institutionen führen Evaluationen durch (“Evaluation activities within the Supreme Audit Institution").

9. Fokus von Evaluationen / Proportion von "output evaluation" und "process evaluation"

("The evaluation done should not just be focused on the relation between inputs/outputs or technical production").

Im Austausch mit den Autoren der jeweiligen Kapitel zu den einzelnen Nationen vergaben Furubo, Rist und Sandahl die Punkte für die neun Indikatoren, wodurch ein Ranking entstand (vgl. ebenda, S.9). Die folgende Tabelle zeigt die Ergebnisse: 


\begin{tabular}{|l|c|c|c|c|c|c|c|c|c|c|}
\hline & \multicolumn{10}{|c|}{ Ranking of Countries on the Indicators of an Evaluative Culture } \\
\hline & I & II & III & IV & V & VI & VII & VIII & IX & Total \\
\hline United States & 2 & 2 & 2 & 2 & 2 & 2 & 2 & 2 & 2 & $\mathbf{1 8}$ \\
\hline Canada & 2 & 2 & 2 & 2 & 2 & 1 & 2 & 2 & 2 & $\mathbf{1 7}$ \\
\hline Australia & 2 & 2 & 2 & 2 & 1 & 1 & 2 & 2 & 2 & $\mathbf{1 6}$ \\
\hline Sweden & 2 & 2 & 2 & 1 & 2 & 1 & 2 & 2 & 2 & $\mathbf{1 6}$ \\
\hline Netherlands & 2 & 2 & 2 & 1 & 2 & 1 & 2 & 2 & 1 & $\mathbf{1 5}$ \\
\hline United Kingdom & 2 & 2 & 2 & 2 & 1 & 1 & 2 & 1 & 2 & $\mathbf{1 5}$ \\
\hline Germany & 2 & 2 & 1 & 2 & 1 & 1 & 2 & 1 & 1 & $\mathbf{1 3}$ \\
\hline Denmark & 2 & 2 & 2 & 1 & 1 & 0 & 2 & 1 & 1 & $\mathbf{1 2}$ \\
\hline Korea & 1 & 1 & 2 & 2 & 2 & 0 & 2 & 1 & 1 & $\mathbf{1 2}$ \\
\hline Norway & 2 & 1 & 1 & 1 & 2 & 1 & 1 & 2 & 1 & $\mathbf{1 2}$ \\
\hline France & 2 & 1 & 1 & 2 & 2 & 1 & 1 & 1 & 0 & $\mathbf{1 1}$ \\
\hline Finland & 2 & 1 & 1 & 1 & 1 & 1 & 1 & 1 & 1 & $\mathbf{1 0}$ \\
\hline Israel & 1 & 1 & 1 & 2 & 1 & 0 & 1 & 1 & 1 & $\mathbf{9}$ \\
\hline Switzerland & 1 & 1 & 2 & 2 & 0 & 0 & 2 & 0 & 0 & $\mathbf{8}$ \\
\hline New Zealand & 1 & 0 & 1 & 2 & 0 & 0 & 1 & 1 & 1 & $\mathbf{7}$ \\
\hline Ireland & 1 & 1 & 1 & 0 & 1 & 0 & 1 & 1 & 1 & $\mathbf{7}$ \\
\hline Italy & 1 & 1 & 1 & 2 & 0 & 0 & 1 & 1 & 0 & $\mathbf{7}$ \\
\hline China & 1 & 1 & 0 & 0 & 2 & 0 & 1 & 0 & 1 & $\mathbf{6}$ \\
\hline Spain & 1 & 0 & 1 & 2 & 1 & 0 & 0 & 0 & 0 & $\mathbf{5}$ \\
\hline Zimbabwe & 1 & 1 & 0 & 0 & 1 & 0 & 0 & 1 & 0 & $\mathbf{4}$ \\
\hline Japan & 1 & 0 & 0 & 1 & 1 & 0 & 0 & 0 & 0 & $\mathbf{3}$ \\
\hline Total & $\mathbf{3 2}$ & $\mathbf{2 6}$ & $\mathbf{2 7}$ & $\mathbf{3 0}$ & $\mathbf{2 6}$ & $\mathbf{1 1}$ & $\mathbf{2 8}$ & $\mathbf{2 3}$ & $\mathbf{2 0}$ & \\
\hline
\end{tabular}

Tabelle 6: $\quad$ Evaluationskultur in verschiedenen Ländern (Quelle: Furubo, Rist und Sandahl 2002, S.10)

I. Evaluation takes place in many policy domains

II. Supply of domestic evaluators in different disciplines

III. National discourse concerning evaluation

IV. Professional organizations

V. Degree of institutionalization - Government

VI. Degree of institutionalization - Parliament

VII. Pluralism of institutions or evaluators performing evaluation within each policy domain

VIII. Evaluation within the Supreme Audit Institution

IX. Proportion of outcome evaluations in relation to output and process evaluations

Anschließend wurden die Nationen nach weiteren Kriterien geclustert (vgl. ebenda S.20f) und in einer Matrix dargestellt. Es wird dabei festgelegt, ob die Evaluationskultur einer Nation eher intern oder extern betrieben wird und ob der Druck, Evaluationen durchzuführen, eher stark oder schwach ist. 


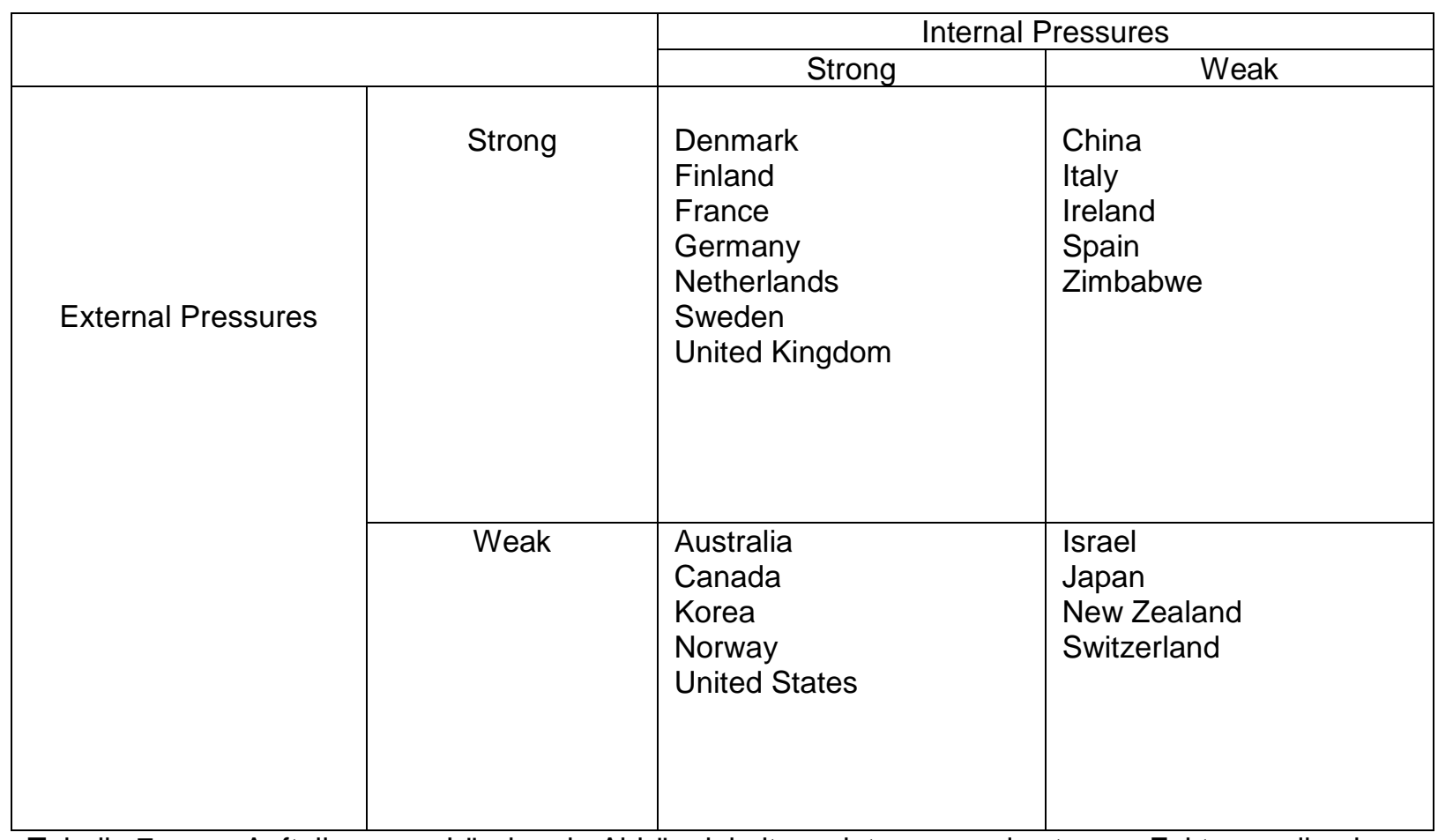

Tabelle 7: $\quad$ Aufteilung von Ländern in Abhängigkeit von internen und externen Faktoren, die einen starken oder schwachen Einfluss auf die Entwicklung der Evaluationskultur haben (Quelle: Furubo Rist und Sandahl 2002, S.21)

Die Nationen im Quadranten 1 werden als Pioniere im Hinblick auf ihre Evaluationskultur bezeichnet. Sie gehören zu "first- and second-wave countries“ (vgl. ebenda, S.22). Nach Furubo, Rist und Sandahl haben einige Länder aufgrund des hohen Drucks durch die Europäische Union während der 1990er Jahre Evaluationen in vielen Bereichen eingeführt.

„During the 1990s several countries have also been exposed to very strong external pressures to implement various evaluation activities. The source of this external pressure has mainly been organizations like the European Union (influencing almost every area of policy in the member countries) and (to some extend) the World Bank." (Furubo, Rist und Sandahl, 2002, S.21)

Zu den Evaluationsaktivitäten in Europa berichten Leeuw, Toulemonde und Brouwers (1999) in einem Artikel über einen Workshop, der während einer Konferenz im Oktober 1998 in Rom zum Thema „Evaluation as a business“ stattfand. Sie reflektieren den damaligen aktuellen Stand der Evaluationsaktivitäten in Europa und stellen die Frage, ob die Zunahme von Mitgliedern in der European Evaluation Society als Zeichen der wachsenden Bedeutung von Evaluation gesehen werden kann und ob "wether we are at the beginning of a rising curve or at the brink of a mature market" (Leeuw et al. 1999, S.487). Sie beschreiben, dass die Evaluationstätigkeiten in Europa stark zugenommen haben, was sich in der hohen Anzahl von Beratungs- und Evaluationsfirmen sowie in Professionalisierungstendenzen zeigt (vgl. ebenda, S.488). Außerdem arbeiten sie heraus, dass die Evaluationsaktivitäten in Europa sehr heterogen sind, es gebe keine einheitlichen (gemeinsamen) Kriterien und Methoden oder Definitionen und Regeln innerhalb der EU, an die sich Evaluatoren halten müssten. Darüber hinaus habe jedes Land einen eigenen "Markt“ an Evaluationsangeboten und es gebe ver- 
schiedene Hindernisse für die Durchführung von Evaluationen über die Ländergrenzen hinaus:

„Evaluation activities in Europe seem even more fragmented when we look at national (sub) markets. One can easily understand that foreign evaluators face high barriers when trying to enter into a given national market, especially for reasons of language, knowledge of institutional settings and familiarity with administrative culture." (Leeuw et al. 1999, S.489)

Insgesamt kommen Leeuw at al. zu dem Schluss, dass Evaluation und das „Evaluationsgeschäft" ein Feld seien, in dem es noch viel zu tun gebe. Sie bezeichnen Evaluation als „still an infant industry“ (ebenda, S.493) und plädieren für die Vernetzung und den Austausch innerhalb Europas, die durch die European Evaluation Society und andere Nationale Evaluationsgesellschaften gefördert bzw. organisiert werden könnten.

„We suggest, that the EES, together with national evaluation societies, should continue to investigate evaluation activities, possibly on a more permanent basis. Topical workshops could be organized in various European countries under the same heading, like 'recording and circulating lessons from managing evaluations`. Though small it may help." (Leeuw et al. 1999, S.494)

Pollitt (1998) betrachtet Evaluationstätigkeiten in Europa und die Wirkungen, die mit Evaluationsergebnissen erzielt werden, kritisch. In seinem Artikel „Evaluation in Europe. Boom or Bubble?” konstatiert er: „These are grand days for European Evaluators“ (Pollitt 1998, S.214). Er beschreibt verschiedene Indikatoren für den "Boom" und den Erfolg von Evaluation. Es würden weltweit zahlreiche Publikationen und „guidelines“ für Evaluationen herausgegeben, Evaluation würde in verschiedensten Bereichen eingesetzt und inzwischen als selbstverständlich bzw. obligatorisch dazu gehören, außerdem hätten sich zahlreiche Evaluationsgesellschaften in verschiedenen Nationen gegründet - und für Europa insgesamt die European Evaluation Society, und schließlich gebe es unzählige Konferenzen und Seminare zum Thema Evaluation.

Dennoch kritisiert Pollitt, dass Evaluationen im Bereich der Politik häufig nicht genutzt würden, um Entscheidungen systematisch zu begründen. Vielmehr gebe es eine „sichere Distanz" (vgl. S.214), oder Evaluationen würden von Externen Evaluatoren durchgeführt und die Ergebnisse nicht dazu genutzt, den Prozess der demokratischen Regierungsführung zu unterstützen. Politische Entscheidungsträger seien sensibel, was Evaluationen anbelangt - oder sogar ignorant (vgl. ebenda S.215): Damit Evaluationen und deren Ergebnisse in der Politik genutzt werden könnten, müsste vielmehr berücksichtigt werden, wie Entscheidungen in der Politik getroffen werden und auf welche Weise die Evaluationen diese unterstützen könnten. Insgesamt kommt er zu dem Schluss, dass es zwar einen Evaluationsboom in Europa gibt, aber Evaluationen zur Seifenblase werden können, wenn sie als „Dekoration“ und Zeichen von Modernität benutzt würden, oder dazu, die Entscheidungen, die ohnehin schon getroffen waren, zu legitimieren und zu bestärken. 
„There are temptations for politicians to use evaluations as baubles or as bolsters - as decorative symbols of modernity or simply as reinforcements for courses of action they had already decided upon for other reasons. If these tendencies come to predominate then the evaluation boom could quickly become a bubble - for the moment colourful, but essentially fragile and ephemeral." (Pollitt 1998, S.223)

Pollitt plädiert dafür, dass Evaluatoren selbst dazu beitragen sollten, damit aus dem Boom von Evaluation keine Seifenblase wird. Sie müssten an Standards für Evaluationen und ihren Fähigkeiten (besonders der Methodenkompetenz) arbeiten, außerdem sei es sehr wichtig, dass sie die Bedarfe der Politiker und Auftraggeber verstehen und beachten, damit die Ergebnisse auch tatsächlich genutzt werden.

„Evaluators themselves can do something to reduce the probability of the boom becoming a bubble. They can work hard to develop their own standards and skills, and work even harder to try to understand the needs of the politicians and senior officials who commission them." (Pollitt 1998, S.223)

\subsubsection{Die Evaluationskultur in der Europäischen Union}

Die Entwicklungen innerhalb der Europäischen Union werden in einem eigenen Kapitel im „International Atlas of Evaluation“ (Furubo, Rist und Sandahl 2002) von Summa und Toulemonde (2002) beschrieben. Die Entstehung einer Evaluationskultur in der Europäischen Kommission beginnt nach Summa und Toulemonde (vgl.2002, S.409) in den frühen 1980er Jahren. Eine Studie von Sensi und Cracknell (1991) zeigt, dass in den meisten Direktionen der Europäischen Kommission zu dieser Zeit zumindest einige Evaluationstätigkeiten durchgeführt wurden. Insbesondere in drei Politikbereichen waren Evaluationen etabliert: Entwicklungsarbeit, Forschungs- und Technologiepolitik und regionale Strukturpolitik. Das Ziel war eher, mit den Evaluationen Verbesserungen möglich zu machen, als Rechenschaft abzulegen oder Kontrolle auszuüben:

\footnotetext{
„Those in charge of the evaluation units mainly considered their work as having internal utility and leading to an improvement in the performance of European policies, rather than being a tool for accountability toward the council or the Parliament. However, several practitioners agreed that evaluation had developed rapidly because of pressure from outside the commission, especially from the council. Apart from the commission itself, the first addresses of the evaluation reports were said to be the council, the Parliament and the member states." (Summa und Toulemonde 2002, S.409)
}

Mit diesem Zitat wird deutlich, dass es doch einen gewissen Druck gegeben hat, Evaluationen einzuführen, und dass sich die Ergebnisse bzw. Berichte neben der Europäischen Kommission selbst vor allem an den Europäischen Rat, das Europäische Parlament sowie die Mitgliedsstaaten richteten. Summa und Toulemonde (vgl. ebenda) beschreiben auch, dass es bereits eine lange Tradition von systematischen Evaluierungen für Projekte gibt, bei der Ziele überprüft werden. Die Instrumente, mit denen Projekte entwickelt, begleitet und 
überwacht werden, sind traditionelle Projektmanagementinstrumente wie „Project Cycle Management“ und "Logical Framework“. Evaluationen und Projektbegleitungen (Monitoring) wurden für die Europäische Kommission von externen, unabhängigen Experten durchgeführt (vgl. ebenda, S.410).

Einige der einflussreichsten EU-Maßnahmen sind auf Regulationen ausgerichtet und haben keinen Programmcharakter, z.B. Verbraucher- und Umweltschutz oder Marktregulierungsmaßnahmen. Obwohl viele Evaluationen in diesem Bereich durchgeführt wurden, stellen Summa und Toulemonde fest, dass die EU-Institutionen nicht mit einem formalen Evaluationssystem ausgestattet sind:

„(...) EU-institutions are not equipped with a formal evaluation system nor even with a database that would record the amount of work undertaken within the various directorates general. The council and Parliament pass a small number of "sunset" regulations that include a formal clause with a given deadline (...). Most regulations simply include an obligation to "report" about the results, generally after three years. Usually these reports do not have the name nor the substance of evaluations." (Summa und Toulemonde 2002, S.409)

Eine „Berichtspflicht“ bestand, aber diese hatten nicht den Charakter von Evaluationen.

Mitte der 1990er Jahre weitete sich die EU durch den Beitritt neuer Länder weiter aus und auch das EU-Budget vergrößerte sich. Es wurden zahlreiche Förderprogramme geschaffen und Gelder in einem weiten Feld investiert, vor allem in den Bereichen Umwelt, Forschung, Technologie, Transport, Bildung, Kultur und Entwicklungshilfe (vgl. ebenda, S.412). Nicht zuletzt durch die Skepsis einiger Länder gegenüber der Effektivität und Effizienz der Programme und die Initiative des Europäischen Parlaments und des Europäischen Rechnungshofes ging die Europäische Kommission dazu über, Evaluationen vermehrt durchzuführen bzw. das Evaluationsfeld auszuweiten. Das Ziel war u.a. nachzuweisen, für was die Gelder ausgegeben wurden, und dass die Mittel sinnvoll verwendet wurden.

„To a large extend, the concerns for better accountability and value-formoney in European policies and programs were particulary directed to the commission as the initiator and executor of community policies. The European Parliament as well as the Court of Auditors also played an important role in pushing the commission to better account for what it spends." (Summa und Toulemonde 2002, S.409)

Nach Summa und Toulemonde (vgl. 2002, S.412) gibt es noch eine Reihe anderer Faktoren, die dazu beigetragen haben, dass sich der Fokus nun stärker darauf richtete, mittels Evaluationen zu überprüfen, ob Ressourcen gut eingesetzt wurden. Sie zählen dazu beispielsweise den Beitritt skandinavischer Länder zur EU, in denen es bereits eine Tradition des Controllings (accountability) und des ergebnisorientierten Managements gab, sowie Bestrebungen der OECD (Organisation for Economic Co-operation and Development), die Reformpolitik mit einem starken Fokus auf Ergebnisse, Effektivität und Kontrolle (accountability) voranzutreiben. 
Die sogenannte „Santer Commision“ führte ebenfalls Mitte der 1990er Jahre weit reichende Reformmaßnahmen ein, mit dem Ziel, in den Bereichen Finanzen und Rechnungslegung systematischer als bisher zu evaluieren. Zwei Initiativen wurden besonders bekannt, die „SEM 2000“ (Sound and Efficiency Management) und „MAP 2000“ (Modernization of Administration and Personnel Policies) (vgl. Summa und Toulemonde 2002, S.412f). Im Jahre 1995 wurde ein neues Evaluationsvorgehen eingeführt, dass jährliche Monitoringverfahren und regelmäßige Begutachtungen umfasste:

\begin{abstract}
„In 1995, a new evaluation scheme was adopted which combines a rolling system of annual monitoring exercises and periodic five-year assessments of all research programs as well as of the framework program. These assessments can be understood as a combination of an ex post evaluation of the previous program, an intermediate evaluation of the current program, and ex ante appraisal of future activities." (Summa und Toulemonde 2002, S.410)
\end{abstract}

Im Vergleich zu dem früheren Evaluationsvorgehen, bei denen überwiegend Zwischenevaluationen oder summative Evaluationen durchgeführt wurden, sollten nun durch ex ante Evaluationen Informationen und Ergebnisse früher zur Verfügung stehen, damit sie in Entscheidungsprozesse einbezogen werden konnten.

Bei der Initiative, systematischere Evaluationen in allen Bereichen der EU-Politik und des Finanzmanagements und der Personalverwaltung einzuführen wurde 1996 ein Memorandum verfasst (European Commission 1996). Es enthält konkrete Schritte zur Verbesserung der Evaluationspraxis wie etwa die Festlegung, dass jede Direktion einen Verantwortlichen für Evaluation hat, ein jährlicher Evaluationsplan erstellt wird, der die Programme benennt, die evaluiert werden sollen, dass ein Netzwerk von Evaluatoren entstehen soll, um den internen und informellen Austausch mit dem Ziel der Verbreitung guter Praxis zu fördern, sowie Trainings- und Handlungsanleitungen zur Unterstützung der Evaluationstätigkeiten. Es wurde ein dezentrales Modell eingeführt, nach dem die Direktionen für die Durchführung ihrer Evaluationen selbst verantwortlich sind:

„The starting point in the commission's approach to improving evaluation practices was a decentralized model in which the operational directorates general are responsible for establishing systematic evaluation procedures for the programs they are executing. The directorates general for budgets and for financial control were to assist and supervise the spread of best practices, to improve evaluation techniques, and to exercise overall quality control." (Summa und Toulemonde 2002, S.413)

Die Evaluationen unterliegen dabei hinsichtlich ihrer Inhalte und des Verfahrens keinen festen Regeln oder standardisierten Vorgaben, sondern es ist eine Vielfalt von Evaluationskonzepten möglich. Wichtig scheint der Kommission jedoch die „Budget-Perspektive“, d.h. mit den Ergebnissen der Evaluationen sollen Entscheidungen getroffen werden können, die für Budgetverteilungen und Mittelzuweisungen relevant sind (vgl. ebenda, S.415).

Im Jahre 1999 wurde erneut ein Memorandum verfasst (European Commission 1999a), in dem eine Bestandsaufnahme zu den Entwicklungen der Evaluationstätigkeiten erfolgte und 
die nächsten Schritte thematisiert wurden. Ziel der Bestandsaufnahme war, zu prüfen, ob die Ziele aus dem Jahre 1996 erreicht wurden. Es wurde festgestellt, dass die Evaluationskultur sich erheblich weiterentwickelt hat. Deutlich wird dies vor allem an der gestiegenen Anzahl von Evaluationsprojekten und -berichten, wobei nahezu alle Politikbereiche eingeschlossen sind (vgl. Summa und Toulemonde 2002, S.416). Die Evaluationsergebnisse sind nach der Kommission hilfreich und werden für Entscheidungsprozesse genutzt:

\begin{abstract}
„It concludes that the evaluation systems that are now in place have been fairly successful in providing support for program management and that they contribute to some extend to improved accountability as an increasing number of evaluation reports and an annual evaluation review are published." (Summa und Toulemonde 2002, S.415)
\end{abstract}

Charakteristisch für die Evaluationstätigkeiten der Europäischen Kommission ist, dass eher formative Evaluationen durchgeführt werden als summative und dass es nicht üblich ist, Akteure einzubeziehen (vgl. ebenda, S.417).

Die meisten Evaluationen innerhalb der Europäischen Kommission werden an externe Evaluatoren abgegeben, einerseits, weil die personellen Ressourcen fehlen, andererseits, um die Unabhängigkeit der Ergebnisse sicher zu stellen (vgl. ebenda, S.421). Diese Evaluationspraxis hat aber Folgen für den Grad der Professionalisierung:

\begin{abstract}
„The practice of externalizing most evaluation projects has certain consequences for the process of professionalization of evaluation. It does not support the development of high-level specialist knowledge in evaluation approaches and methods within the commission, but on the other hand it creates a demand for developing know-how in the management of evaluation projects." (Summa und Toulemonde 2002, S.420)
\end{abstract}

Die Fähigkeit, Evaluationen zu organisieren, wird von der Europäischen Kommission unterstützt durch interne Evaluationstrainings und Publikationen wie Handlungsanleitungen und Empfehlungen (vgl. European Commission 1997 und 1999b). Durch die Vergabe von Evaluationsaufträgen an externe Evaluatoren entstand nach Summa und Toulemonde (vgl. 2002, S.421) ein schnell wachsender Evaluationsmarkt. Die Anzahl von Beratungs- und Dienstleistungsfirmen wuchs stark an in allen Nationen, wobei in manchen Ländern quasi bei Null angefangen wurde (z.B. in Griechenland und Portugal). Viele der Evaluatoren stammen aus einem universitären Kontext oder außeruniversitären Forschungseinrichtungen und viele Teams von Evaluatoren gründeten in Brüssel Firmen bzw. Dienststellen. Ein Problem sehen Summa und Toulemonde (2002, S.421) darin, dass „This quantitative development has been much faster than that of professionalization.“. Außerdem sind viele der Evaluatoren nicht hauptberuflich mit Evaluationen beschäftigt, sondern bieten diese neben anderen Dienstleistungen an und sind dadurch nicht so sehr Experten, wie es zu wünschen sei (vgl. ebenda, S.421f). Es besteht u.a. deshalb die Notwendigkeit, die Entwicklung der Evaluationskultur zu unterstützen. 1995 wurde die European Evaluation Society gegründet, die z.B. Konferenzen organisiert und durchführt, um die Vernetzung und den Austausch in Europa zu fördern. Au- 
Berdem wurde das MEANS-Programm initiiert, mit dem die Qualität von Evaluationen in den Mitgliedsstaaten gesichert werden sollte (European Commission 1999c). Einen großen Bedarf sehen Summa und Toulemonde in dem Ausbau der Angebote von Evaluationstrainings (vgl. 2002, S.422).

Die Spezifika sowie Schwierigkeiten und Herausforderungen bei Evaluationen im Rahmen der EU begründen sich vor allem durch die Komplexität von Entscheidungsprozessen, die Beteiligung vieler Gremien, nur vage definierten Zielen und Zielgruppen sowie Unklarheiten in Bezug auf die Durchführung von Maßnahmen:

\begin{abstract}
„EU policies and programs result from complex and long negotiation processes and are implemented through multiple chains of authorities linking the center to the ground. This makes the evaluability of European policies and programs generally poor. This common problem is worsened by the fact that decision making usually involves more negotiation in Brussels than in national political systems. The costs of compromises often include vague definition of objectives, poorly specified target populations, or lack of agreed clear indicators of performance." (Summa und Toulemonde 2002, S.42f)
\end{abstract}

Aufgrund dieser Rahmenbedingungen wird in einem Großteil der Evaluationsberichte vor allem beschrieben, wie Programme umgesetzt und implementiert werden, wofür Manager interviewt, Experten befragt und sekundäre Daten zusammengefasst werden. Eine Analyse "im Feld", die Untersuchung der Auswirkungen der Programme in der Praxis oder die Reaktionen der Zielgruppen werden selten durchgeführt (vgl. ebenda, S.423). Trotzdem hat sich in der EU in relativ kurzer Zeit eine Evaluationskultur entwickelt, die sich auf nahezu alle Politikbereiche ausgedehnt hat und die eine Ausweitung von Evaluationstätigkeiten in der ganzen Welt beeinflusst hat:

„On the whole, the EU experience of attempts to establish an evaluation culture shows that it is possible to achieve - in a relatively short period of time a considerable increase in evaluation activities across a wide range of policy areas. The EU has been successful in institutionalizing evaluation at least as a procedural form, and it has influenced the growth of evaluation practices across the whole continent." (Summa und Toulemonde 2002, S.423)

Mit diesem Kapitel wird deutlich, dass Evaluationen, die im europäischen Raum durchgeführt werden sollen, besonderen Herausforderungen gegenüberstehen. Zum einen gibt es durchaus unterschiedliche Evaluationskulturen in den einzelnen Ländern Europas, die berücksichtigt werden müssen. Zum anderen kann es sprachliche Barrieren geben. Außerdem sind das Wissen sowie die Erfahrung und Vertrautheit mit institutionellen Rahmenbedingungen und der Verwaltungskultur eines Landes ebenfalls wichtige Faktoren für erfolgreiche Evaluationen, deren Ergebnisse auch tatsächlich genutzt werden.

Darüber hinaus müssen die Entwicklungen im Kontext der zunehmenden europäischen Integration beachtet werden. Mit einer einheitlichen Verfassung in der Europäischen Union und der Forderung nach systematisch durchgeführten Evaluationen würden auch die Ansprüche an professionelle Evaluationen und die Qualifizierung der Evaluatoren steigen. Durch die Gründung der European Evaluation Society und andere Evaluationsgesellschaften in mehre- 
ren Nationen sowie durch die Schaffung von Standards der Evaluation wird eine Professionalisierung und Vereinheitlichung sowie die Einhaltung von Standards angestrebt. Der Austausch und die Vernetzung von Evaluatoren in Europa werden dadurch gefördert und Qualitätsmaßstäbe entwickelt, die für alle gelten. Diese Entwicklung ist jedoch noch nicht abgeschlossen und sollte nach den zitierten Autoren durch europaweite Konferenzen, Seminare, Aktivitäten und Publikationen weiterhin unterstützt werden.

\subsection{Aktuelle Lage der Evaluation}

Die Evaluationsforschung hat sich im Laufe der Zeit entwickelt, wie in den vorangegangenen Kapiteln gezeigt wurde. Fitzpatrick, Sanders und Worthen haben zur aktuellen Lage der Evaluation und zur Zukunft Trends herausgearbeitet und Prognosen erstellt. Sie gehen davon aus, dass die Bedeutung der Evaluation weltweit zunimmt, die Einsatzfelder sich ausweiten und die Professionalisierung und Institutionalisierung weiter voranschreitet:

„We believe that evaluation will continue to spread rapidly around the globe, until there are few countries, territories, provinces, states and locales in which program evaluations are not at least an occasional occurence." (Fitzpatrick, Sanders und Worthen 2004, S.508)

Sie sehen Evaluationen vor allem als nützlich für die Verbesserung von Programmen und Optimierungen in der Politikgestaltung, als Unterstützung im Bereich des Qualitätsmanagement sowie in der Förderung gesellschaftlicher Entwicklungen (vgl. ebenda).

Nach Stockmann (vgl. 2010, S.32) wird die internationale Evaluationsforschung stark von den USA beeinflusst. Sowohl Themensetzungen und Trends, aber auch theoretische und methodologische Diskussionen würden von den USA ausgehen, was ihn dazu veranlasst, die USA als das „Mutterland der Evaluation“ (Stockmann 2010, S.32) zu bezeichnen. Der Grad der Professionalisierung sei in den USA am höchsten, wozu er eine Reihe von Indikatoren aufzählt (vgl. ebenda, S.32f): Die starke institutionelle Verankerung der Evaluation in der Gesetzgebung, der weltweit mitgliederstärkste und einflussreichste Fachverbund - die American Evaluation Society, zahlreiche Publikationen und lebhafte Diskurse, die Schaffung von methodischen und theoretischen Evaluationsansätzen und -modellen sowie den am stärksten ausgeprägten Aus- und Weiterbildungsmarkt für Evaluation.

Auch in der Studie von Furubo, Rist und Sandahl (2002), in der die Evaluationskultur in verschiedenen Ländern der Welt untersucht, beschrieben und bewertet wurde (siehe Abschnitt 2.2.1), erreichen die USA den Spitzenplatz. In dem Ranking bekommen nur die USA in allen Bereichen die maximale Punktzahl und heben sich damit von allen anderen Ländern ab. Mit der Studie wird aber auch deutlich, dass in vielen Ländern eine Evaluationskultur vorhanden ist, die weiter ausgebaut und vorangebracht werden muss. In Europa ist der Stand der Evaluationskultur recht unterschiedlich, es gibt einige „frontrunners“ (Leeuw, 2006, S.67), Länder, in denen die Evaluationskultur recht ausgeprägt ist und die bereits ab den 1960er Jah- 
ren mit dem Einsatz von systematischen und professionellen Evaluationen begonnen haben. In vielen anderen Ländern setzen diese Entwicklungen später ein, nach und nach nimmt aber in allen Nationen die Bedeutung der Evaluation zu und der Grad der Professionalisierung steigt (siehe Abschnitt 2.2.1 und 2.2.2). Evaluationen werden seitens der Politik und Regierungen eingefordert und die Entwicklung der Evaluationskultur in Europa wird dadurch vorangetrieben.

\begin{abstract}
"Generell ist aber speziell in Europa eine hohe Dynamik hinsichtlich der Professionalisierungsentwicklung in den letzten beiden Jahrzehnten spürbar gewesen. Wie schon ausgeführt, stellen dabei die Europäische Kommission und ihre einzelnen Departments die stärksten Kräfte dar welche auf eine Ausweitung und Vereinheitlichung der Evaluation in den einzelnen Ländern Europas hinwirken. Auch Länder, in denen es bisher keinerlei Evaluationskultur gab, müssen nach und nach Evaluationskapazitäten aufbauen, um die mit der Durchführung von EU-Programmen verbundenen Evaluationsvorgaben erfüllen zu können." (Stockmann 2010, S.34)
\end{abstract}

Insgesamt ist eine Zunahme an Evaluationsaktivitäten, an Politik- und Programmevaluationen und eine Ausweitung in verschiedenste Praxisfelder zu beobachten. In Europa, aber auch weltweit, haben die Bedeutung und der Einsatz von Evaluationen deutlich zugenommen und werden auch weiter steigen.

Für den aktuellen Stand der Evaluation sowie deren Zukunft stellen Fitzpatrick, Sanders und Worthen (vgl. 2004, S.508ff) einige Trends zusammen. Sie unterscheiden dabei zwischen der „Profession Evaluation“ und der Praxis der Evaluation. Auch Stockmann fasst Indikatoren für die weltweite Bedeutung der Evaluation zusammen (vgl. 2010, S.35ff), und Datta (2006) widmet sich den aktuellen Herausforderungen und neuen Richtungen der Evaluationsforschung.

Im Folgenden werden sieben Bereiche beschrieben, an denen sich der aktuelle Stand der Evaluationskultur betrachten lässt:

\title{
Evaluation als Bestandteil von Politik und gesamtgesellschaftlichen Entwicklungen
}

Evaluationsaktivitäten sind heute, im Jahre 2012, fester Bestandteil von Gestaltungsprozessen in der Politik und auch ein Steuerungsinstrument im Rahmen von Entwicklungsprogrammen im öffentlichen Bereich. Nach Fitzpatrick, Sanders und Worthen wird Evaluation zu einer treibenden Kraft bei der Verbesserung von Programmen in den verschiedensten Feldern und Fächern:

„Evaluation will become an increasingly useful force in our society, in improving programs and policies in public, nonprofit and the private sector. [...] Evaluation will expand into other fields or disciplines, beyond its now venerable role in education and psychology to expanded roles in housing, social welfare, environmental programs, city planning, transportation, health, criminal justice, biotechnology, recreation and management programs in many organizations" (Fitzpatrick, Sanders und Worthen 2004, S.508). 


\title{
Gründung von Evaluationsgesellschaften
}

Mit der Gründung von Evaluationsgesellschaften wird die Professionalisierung und Institutionalisierung der Evaluation gefördert. Weltweit hat die Anzahl der Evaluationsgesellschaften zugenommen (vgl. Stockmann 2010, S.36). Die American Evaluation Society ist die mitgliederstärkste und einflussreichste Evaluationsgesellschaft. In Europa gibt es ebenfalls zahlreiche Evaluationsgesellschaften, die seit den 1980er Jahren zuerst in wenigen Ländern und später in vielen europäischen Nationen entstanden (vgl. Leeuw 2006, S.67). Die European Evaluation Society wurde 1994 gegründet und vernetzt Evaluatoren in ganz Europa. Das Ziel ist vor allem, die Qualität von Evaluationen zu fördern (Theorie, Praxis und Nutzen von Evaluationen), indem die Vernetzung in ganz Europa unterstützt und ein Forum für den Austausch von Erfahrungen geschaffen wird.

\begin{abstract}
"The goal of the European Evaluation Society is thus to stimulate and promote theory, practice, and utilization of high quality evaluation especially, but not exclusively, within Europe. This goal is obtained by bringing together academics and practitioners from all over Europe and from different professional sectors, thus creating a forum where all participants can benefit from the co-operation and bridge building opportunities of the EES." (http://www.europeanevaluation.org, Zugriff am 31.8.2012)
\end{abstract}

Die "Kellog Foundation" (https://www.wkkf.org) investierte Fördermittel in die Gründung der "International Organization for Cooperation in Evaluation" (IOCE), die das Ziel hat, Evaluationen weltweit zu verbessern (http://www.ioce.net). Die IOCE versteht sich als loser Zusammenschluss von regionalen und nationalen Evaluationsorganisationen, die zusammen arbeiten, um sich zu vernetzen, Materialien zu verbreiten und den Austausch zu fördern:

„IOCE is a loose alliance of regional and national evaluation organisations (associations, societies and networks) from around the world that collaborate to:

- build evaluation leadership and capacity in developing countries,

- foster the cross-fertilisation of evaluation theory and practice around the world,

- address international challenges in evaluation, and

- $\quad$ assist the evaluation profession to take a more global approach to contributing to the identification and solution of world problems."

(http://www.ioce.net/overview/general.shtml, Zugriff am 31.8.2012l)

Die IOCE hat auch eine Zusammenstellung aller Evaluationsgesellschaften auf regionaler und internationaler Ebene zusammen gestellt

(http://www.ioce.net/members/reg_intl_organizations.shtml, Zugriff am 19.9.2012) und auBerdem nationale Evaluationsgesellschaften aufgelistet (http://www.ioce.net/members/national_organizations.shtml, Zugriff am 19.9.2012). Mit diesen Sammlungen wird deutlich, dass Evaluation weltweit institutionalisiert ist und zum einen regional bzw. national und zum anderen aber auch international organisiert wird. 
Vierzehn Mitglieder der IOCE haben in Fallstudien über ihre Organisationen berichtet, die in einer gemeinsamen Publikation "Creating and Developing Evaluation Organizations: Lessons learned from Africa, Americas, Asia, Australasia and Europe." veröffentlicht wurden, welche frei zugänglich ist (http://www.ioce.net/resources/case_studies.shtml, Zugriff 19.9.2012). Mit dem zur Verfügung stellen solcher Materialien wird ebenfalls die Professionalisierung der Evaluation unterstützt.

\section{Standards für Evaluation}

Im Kontext der Professionalisierung der Evaluationsforschung entstanden in den 1980er Jahren in den USA die ersten Bestrebungen, Evaluationsstandards zu entwickeln. Ziel war dabei, die Qualität der Evaluation zu sichern und einen Orientierungsrahmen für professionelles Verhalten und Arbeiten von Evaluatoren zu gewährleisten (vgl. Stockmann 2010, S.181). Im Jahr 1981 gab das "Joint Commitee on Standards for Educational Evaluation“ die ersten Standards für den erziehungswissenschaftlichen Bereich heraus (Joint Commitee 1981). Danach wurden die Standards auf immer mehr Themenbereiche angewendet und 1994 gab das „Joint Commitee“ eine überarbeitete Fassung der Standards heraus mit dem Titel: „The Program Evaluation Standards“ (Joint Commitee 1994). Sie standen nach Stockman (2010, S.329) „Pate für eine Vielzahl mittlerweile verabschiedeter Evaluationsstandards anderer nationaler Verbände weltweit“. In den einzelnen Nationen sind die Evaluationsgesellschaften treibende Kräfte in der Schaffung und Umsetzung von Evaluationsstandards, wie etwa in Deutschland die Deutsche Gesellschaft für Evaluation (DeGEval), die 1997 gegründet wurde und im Jahre 2002 die „Standards für Evaluation“ (DeGEval 2002) herausgab. Die Verbreitung der Standards ist eine Aufgabe, der sich die DeGEval u.a. widmet. Die Standards des Joint Commitee sind auch in deutscher Sprache erschienen (Joint Commitee 2006).

\section{Aus- und Weiterbildungsmöglichkeiten}

Weltweit hat das Angebot an Ausbildungs- und Weiterbildungsmöglichkeiten im Feld der Evaluation zugenommen. In den USA bestanden zuerst zahlreiche Möglichkeiten des Trainings und der Aus - und Weiterbildung, aber auch in Europa entstand eine Palette von Möglichkeiten und ein Markt, der weiter expandiert (vgl. Stockmann 2010, S.37). Die Entwicklungen in Europa werden aber z.B. von Leeuw kritisiert. Er ist der Meinung, dass hier noch viel zu tun sei: „Rather slowly one can see developments regarding training and education“ (Leeuw 2006, S.83). Datta sieht den Bereich der Aus- und Weiterbildung ebenfalls als verbesserungswürdig. Er beschreibt, dass zwar viele Fortschritte gemacht wurden, es aber an Studien zur Qualität der Angebote und zu den Ergebnissen (z.B. den Kompetenzen der Evaluatoren) mangeln würde:

„Progress has been made developing training programs using formal academic settings, internships, pre-conference workshops, short sessions, the internet, journals and a Niagara of evaluation texts. There is an extensive, 
but somewhat nervous literature on assessing the quality of these offerings, touching as these discussions must, on the raw nerves of accreditation and credentialing. A lot of talk is good; a paucity of empirical study is not good. Even less progress has been made examining the results of such training in terms of evaluator competencies." (Datta, 2006, S.428f)

Der Bedarf an Evaluationen nimmt zu und damit auch der Bedarf an Evaluatoren. Fitzpatrick, Sanders und Worthen sehen gute und steigende berufliche Chancen für Evaluatoren in der Zukunft, Evaluationskompetenzen würden immer wichtiger. In diesem Zusammenhang sind qualitativ hochwertige Ausbildungsmöglichkeiten bedeutsam und wichtig. Sie gehen davon aus, dass bestehende Angebote von Universitäten und Instituten weiter ausgebaut werden, sind aber auch der Meinung, dass dies nicht reichen wird. Viele Evaluatoren würden ihre „Trainings“ weiterhin in ihrem beruflichen Umfeld bekommen, weshalb es einen zunehmenden Bedarf an guten Weiterbildungsangeboten gäbe:

„The need for in-service education in evaluation will expand dramatically as educators, public and nonprofit administrators, corporate officers, and those in a variety of other roles are asked to assume some responsibility for carrying out evaluation studies alongside their other professional duties." (Fitzpatrick, Sanders und Worthen 2004, S.509)

Nach Datta (vgl. 2006, S.429) ist es dabei eine besondere Herausforderung, die Qualität und Professionalität der Angebote sicher zu stellen - übliche ein bis zwei Tage Training ergänzt durch „do-it-yourself guidebooks“ sieht er als nicht ausreichend an.

Insgesamt wird deutlich, dass es im Bereich der Aus- und Weiterbildung noch viel Entwicklungspotenzial gibt.

\section{Erweiterung des Angebotsmarktes}

Durch die zunehmende Bedeutung von Evaluation und der daraus folgenden Nachfrage ist ein breiter Angebotsmarkt entstanden, der weiter wächst (siehe Kapitel 2.2.1). Leeuw, Toulemonde und Brouwers (1999, S.493) sehen den Evaluationsmarkt als „still an infant industry“. Auch Summa und Toulemonde (vgl. 2002, S.421) beschreiben für die Europäische Union die Entstehung eines Evaluationsmarktes, der stetig wächst. Die Anzahl von Beratungsfirmen und Kleinunternehmen hat stark zugenommen, wobei in einigen Ländern wie etwa Griechenland oder Portugal quasi von Null angefangen wurde (hier war in den 1990er Jahren die Evaluationskultur noch nicht besonders ausgeprägt, der Bedarf nach Evaluation aber vorhanden). Viele private Firmen wurden gegründet, es gibt aber auch eine Reihe von Forschungseinrichtungen und Universitäten, die ihre Angebote auf dem Evaluationsmarkt anbieten. Summa und Toulemonde (vgl. ebenda) bemängeln, dass die quantitative Entwicklung sich viel schneller vollzog als die qualitative und dass viele Evaluationsteams Evaluationen nur als Teil ihrer Dienstleistungsangebote anbieten, neben vielen anderen Dingen. Es mangele häufig an Professionalität und Kompetenz. Insgesamt ist der Angebotsmarkt für Evaluationen ein vielversprechender Markt, dessen Bedeutung weiter zunehmen wird. 


\section{Verbreitung von Evaluationsergebnissen}

Durch die Entwicklung der Informations- und Kommunikationstechnologien können heute Evaluationsergebnisse viel leichter verbreitet werden. Leeuw (vgl. 2006, S. 80f) sieht in diesem Bereich ein großes Potenzial für den Transfer von Wissen und den Nutzen von Evaluationsergebnissen in vielen Feldern der Gesellschaft, da mehr Transparenz gegeben ist. Fitzpatrick, Sanders und Worthen (vgl. 2004, S.511) beschreiben für die Praxis der Evaluation ebenfalls vielfältige Möglichkeiten. Sie sehen zum einen Vorteile in der vereinfachten Verbreitung von Evaluationsergebnissen und zum anderen in den Methoden, Daten zu erheben und zu analysieren.

Nach Stockmann (vgl. 2010, S.37) hat zwar die Verbreitung von Evaluationsergebnissen durch die modernen Technologien stark zugenommen, in welcher Weise dies genutzt wird für Programmgestaltungen bzw. die Planung und Durchführung von Evaluationen, ist aber nicht oder nur kaum abzusehen. Teilweise würden aber Evaluationsergebnisse von den Medien aufgegriffen, wie z.B. bei der PISA-Studie, wenn das Thema politisch brisant oder interessant sei.

Für die Zukunft der Evaluation bieten die Informations- und Kommunikationstechnologien in jedem Fall viele Möglichkeiten zur Verbreitung von Ergebnissen, die auch schon genutzt werden (z.B. auf den zahlreichen Internetplattformen).

\section{Literatur und Publikationen}

Sowohl die Verbreitung von Evaluationsergebnissen nimmt zu (siehe Punkt 6) als auch die Anzahl der Veröffentlichungen zum Thema Evaluation. Dabei muss unterschieden werden zwischen Literatur, die sich auf die praktische Anwendung von Evaluationen bezieht, und der, die sich auf Evaluationsforschung und -theorie richtet. Fitzpatrick, Sanders und Worthen gehen davon aus, dass empirisch gesichertes Wissen nur langsam zunimmt. Es wird immer mehr publiziert, aber nur wenig davon bezieht sich auf Forschungen zum Prozess der Evaluation selbst. Hier sehen Fitzpatrick, Sanders und Worthen einen Bedarf für die Zukunft:

\footnotetext{
„Evaluation literature will increase in both quantity and quality, but relatively little of it will be based on research into the process of evaluation itself. (...) As evaluation expands, there is a critical need for more research on what occurs in evaluations, what works and what doesn 't, in reference to politics, practice, participation, and use." (Fitzpatrick, Sanders und Worthen 2004, S.510)
}

Insgesamt wird mit diesem Kapitel deutlich, dass viel Entwicklung im Bereich der Evaluation stattfindet. Sowohl die Nachfrage nach Evaluationen und deren Bedeutung sind gestiegen, sie sind heute Bestandteil von Politikgestaltung und ein gängiges Steuerungsinstrument, als auch damit verbunden der Angebotsmarkt von Evaluationstätigkeiten und -dienstleistungen. Auch auf dem Aus- und Weiterbildungsmarkt sind viele Entwicklungen zu verzeichnen, wobei hier ebenfalls eine Expansion in den nächsten Jahren zu erwarten ist. Die Verbreitung von Evaluationsergebnissen ist heute durch moderne Informations- und Kommunikationstechno- 
logien erheblich erleichtert, was zu mehr Transparenz führt. Die Qualität von Evaluationen wird mit der Schaffung von Standards unterstützt, und auch die Gründungen von Evaluationsgesellschaften in der ganzen Welt kennzeichnen die Professionalisierungsbestrebungen. Durch die Institutionalisierung der Evaluation in Form von Gesellschaften und Assoziationen werden die Vernetzung, der Austausch und Diskurse in Veranstaltungen, Publikationen und Zeitschriften vorangetrieben. Nach Phasen der Stagnation (siehe Abschnitt 2.1.4) ist nun eine Phase großen Wachstums zu beobachten, die sich an den hier aufgeführten Indikatoren zeigen lässt. Nach Stockmann kann von einem zweiten „Evaluationsboom“ gesprochen werden:

\footnotetext{
„Diese weltweit zu beobachtenden Entwicklungen lassen den Schluss zu, dass nach den Expansionsjahren in den 60ern und 70ern ein zweiter Evaluationsboom zu konstatieren ist, der allerdings weitaus globaler angelegt ist." (Stockmann 2010, S.38)
}

Mit diesem Zitat wird deutlich, dass Evaluationen heute weltweit an Bedeutung gewonnen haben und weiter zunehmen werden. Evaluationen finden in nahezu allen Bereichen statt (siehe auch Unterkapitel 1.2) und werden von Fachleuten ebenso wie von Laien (z.B. in Form von Selbstevaluationen) durchgeführt. Hierbei ist zu beachten, dass Evaluationen verschiedene Konzepte zu Grunde liegen können. Nicht nur die Funktionen von Evaluationen können unterschiedliche sein (siehe Unterkapitel 1.3), es gibt auch diverse Ansätze und Traditionen, in deren Kontext Evaluationen stattfinden. Im nächsten Kapitel werden verschiedene Evaluationsansätze und Evaluationstheorien dargestellt. 


\section{Evaluationsansätze und Evaluationstheorien}

In der Literatur findet sich eine Fülle von Evaluationsansätzen, Evaluationstheorien und Evaluationsmodellen. Wie in dem vorangegangenen Kapitel gezeigt wurde, hat sich die Evaluationsforschung historisch entwickelt, wobei sich auch unterschiedliche Herangehensweisen und Schwerpunkte bzw. Zielrichtungen ergeben haben.

Verschiedene Autoren haben den Versuch unternommen, die Vielfalt der Evaluationsansätze zu strukturieren und zu ordnen, wobei jeweils unterschiedliche Kriterien für eine Systematisierung verwendet wurden. In diesem Kapitel werden drei Systematisierungen beschrieben: Der Generationenansatz von Guba und Lincoln (1989), das Baummodell von Alkin und Christie (2004) und das Zweck- und Nutzenorientierte Modell von Fitzpatrick, Sanders und Worthen (2004).

\subsection{Das Generationenmodell nach Guba und Lincoln}

Guba und Lincoln (1989) beginnen ihre Arbeit über Evaluation mit einer Betrachtung der historischen Entwicklung von Evaluation, wobei sie ein Modell entwerfen, das vier verschiedene Generationen umfasst. Sie konstruieren und beschreiben zunächst rückblickend drei Generationen und erarbeiten auf dieser Grundlage eine vierte Generation, die im Jahre 1989 aktuelle und zukunftsweisende Themen und Herausforderungen aufgreift. Guba und Lincoln (vgl. 1989, S.7) betrachten Evaluation dabei nicht rein aus historischer Perspektive, sie beziehen sich auch nicht auf verschiedene Modelle der Evaluation, sie sehen nicht die technischen Aspekte im Vordergrund, und sie behandeln Evaluation ebenfalls nicht als einen vornehmlich wissenschaftlichen Prozess. Sie beschreiben Evaluation vielmehr als einen sich entwickelnden Ansatz, der menschlich, politisch, sozial und kulturell bedingt wird und auf den außerdem eine Fülle von Kontextbedingungen Einfluss haben:

„It is our intention to define an emergent but mature approach to evaluation that moves beyond mere science - just getting the facts - to include the myriad human, political, social, cultural, and contextual elements that are involved." (Guba und Lincoln, 1989, S.8)

Guba und Lincoln verzichten auf eine Definition von Evaluation und beschreiben in den ersten drei Generationen die jeweiligen Schwerpunkte und Zielsetzungen, die in dieser Zeit für Evaluationen aus ihrer Sicht charakteristisch waren. Sie konstatieren, dass die jeweils nachfolgende Generation fortschrittlicher sei und die vorhergehenden Generationen ablöste.

„Instead we will begin by sketching briefly the changed meanings that have been ascribed to evaluation for the past hundred years, ascriptions that have reflected the existing historical context, the purpose that people had in mind for doing evaluations, and the philosophic assumptions that evaluators, theoreticans, and practicioners alike have been willing to make. We will argue that, over time, the construction of evaluation has become more informed 
and sophisticated, until, at this present time, we are in a position to devise a radical new construction, which we characterize as fourth generation evaluation." (Guba und Lincoln, 1989, S.22)

Guba und Lincoln arbeiten Charakteristika für die verschiedenen Generationen heraus, wobei diese aufeinander aufbauen und jeweils neue Entwicklungen umfassen. Im Folgenden werden die einzelnen Generationen näher beschrieben:

\subsubsection{Die erste Generation: Das Messen}

Evaluationsaktivitäten entstanden nach Guba und Lincoln aus einer Reihe verschiedener Einflüsse. Im Bildungsbereich gebe es bereits seit einigen Hundert Jahren Schultests, mit denen ermittelt werden sollte, was die Schüler konnten bzw. wussten. Guba und Lincoln (vgl.1989, S.23) verweisen auch auf die Arbeiten von Joseph Mayer Rice, der Ende des 19. Jahrhunderts „spelling tests“ in amerikanischen Schulen einführte, um zu ermitteln, wie effektiv der Unterricht war. Sie nennen noch eine Reihe weiterer Beispiele für die Popularität von Testverfahren und neuen Techniken des Messens und führen auch an, dass beispielsweise der IQ-Test zu einem festen Bestandteil von Personalauswahlverfahren wurde. Unter anderem im Kontext des Ersten Weltkrieges wurden vermehrt Persönlichkeitstests eingesetzt, um Armeepersonal auszuwählen. Außerdem erfuhren die Sozialwissenschaften einen Aufschwung und zahlreiche neue Ansätze zu Methoden und Messverfahren wurden entwickelt (vgl. ebenda, S.24f). Schultests, Persönlichkeitstests sowie Intelligenztests waren stark gefragt, und es wurde nicht besonders unterschieden zwischen den Begriffen Evaluation und Messen. Die Rolle des Evaluators wird als eher technisch beschrieben und besteht in dieser Generation darin, vorhandene Evaluations- und Messinstrumente zu kennen bzw. bei Bedarf neue Verfahren zu entwickeln und einzusetzen.

\footnotetext{
"Thus, the first generation of evaluation can legitimately be called the measurement generation. The role of the evaluator was technical; he or she was expected to know the full panoply of available instruments, so that any variable for investigation could be measured. If appropriate instruments did not exist, the evaluator was expected to have the expertise necessary to create them." (Guba und Lincoln, 1989, S.26)
}

Guba und Lincoln weisen auch darauf hin, dass die Generation des Messens bis heute besteht.

\subsubsection{Die zweite Generation: Das Beschreiben}

Nach Guba und Lincoln (vgl. 1989, S.27) wurde bereits nach dem Ersten Weltkrieg deutlich, dass Schulcurricula erneuert und verbessert und auch die Methoden zur Evaluation überdacht werden müssten. Die Anzahl der Schüler stieg stetig an, da viele einen höheren Bil- 
dungsabschluss als Chance ansahen, bessere soziale und ökonomische Möglichkeiten zu erlangen. Die Schulen und Universitäten waren auf so einen großen Andrang nicht ausgerichtet und die Curricula veraltet. Mit der „Eight Year Study“, die 1933 begann und von Ralph W. Tyler geleitet wurde (siehe auch Abschnitt 2.1.3), sollten vorhandene Curricula untersucht und ggf. neue, adäquatere und zeitgemäßere entwickelt werden. Acht Jahre sollten ein Zeitraum sein, um eine Kohorte von Schülern zu begleiten (während der "Secondary School" und dem "College"). Der Fokus wurde nun nicht mehr auf das reine Messen der Ergebnisse bzw. der Schülerleistungen gerichtet, sondern es ging darum, zunächst Lernziele (objectives) zu definieren und zu beschreiben, um dann zu überprüfen, ob die Schüler das gelernt hatten, was ihre Lehrer beabsichtigten, das sie lernen sollten. Es wurden Stärken und Schwächen der Curricula beschrieben und während der Laufzeit der Studie Verbesserungen und Revisionen durchgeführt. Dieser Ansatz war nach Guba und Lincoln (vgl. 1989, S.28) neu und wurde später unter dem Begriff der „formativen Evaluation“ (siehe auch Abschnitt 1.4.1) bekannt. Die Ergebnisse wurden bei dieser Art von Evaluation bereits während des Prozesses genutzt und nicht erst am Ende. Guba und Lincoln bezeichnen die zweite Generation als die des Beschreibens, in der ein Evaluator vor allem Stärken und Schwächen im Hinblick auf bestimmte, vorher festgelegte Ziele herausarbeitet:

\begin{abstract}
„Thus there emerged what we now choose to call second generation evaluation, an approach characterized by description of patterns of strengths and weaknesses with respect to certain stated objectives. The role of the evaluator was that of describer, although the earlier technical aspects of that role were also retained. Measurement was no longer treated as the equivalent of evaluation but was redefined as one of several tools that might be used in its service." (Guba und Lincoln, 1989, S.28)
\end{abstract}

Tyler wurde später als „Vater der Evaluation“ bezeichnet (Joint Commitee, 1981, zitiert nach Guba und Lincoln 1989, S.28) und prägte auch den Begriff der Programmevaluation. Er beeinflusste zahlreiche nachfolgende Evaluationsansätze.

\title{
3.1.3 Die dritte Generation: Das Bewerten
}

Nach Guba und Lincoln hatte die zweite Generation des Beschreibens einige Schwachstellen, die insbesondere in der Zeit nach dem Sputnik-Schock (siehe dazu auch Abschnitt 2.1.5) deutlich wurden. Mit dem reinen Beschreiben von Lernzielen und dem Ermitteln von Stärken und Schwächen wurden Charakteristika von Programmen nur benannt. Es sollten nun aber Programme evaluiert werden mit dem Ziel, Defizite im Bildungssystem in Amerika zu identifizieren, um darauf aufbauend handeln und neue, bessere Angebote machen zu können. Evaluatoren wollten zunächst Lernziele (objectives) definiert bekommen, um dann die Bildungsangebote daraufhin zu untersuchen und zu beschreiben, ob diese auch erreicht wurden. Damit wurden Ergebnisse erst relativ spät verwendbar, häufig erst zum Ende von Programmen, wenn sich nichts mehr ändern ließ. Nach Guba und Lincoln (vgl. 1989, S.30) 
wies Stake (1967) in einem bedeutenden Artikel auf die „andere Seite“ der Evaluation hin und zwar auf die der Bewertung. Demnach ist die eine Seite von Evaluation das Beschreiben von Bedingungen im Evaluationsfeld (wie etwa die Lernumgebung, die Begabung der Lernenden oder die Ausführung eines Programms), und die andere die der Bewertung, die insbesondere für die Beteiligten (wie Lehrer und Administratoren oder Entscheidungsträger) eine wichtige Rolle spielt.

Guba und Lincoln nennen deshalb die dritte Generation die des Bewertens:

\begin{abstract}
„The call to include judgement in the act of evaluation marked the emergence of third generation evaluation, a generation in which evaluation was characterized by efforts to reach judgements, and in which the evaluator assumed the role of judge, while retaining the earlier technical and descriptive functions as well." (Guba und Lincoln, 1989, S.30)
\end{abstract}

Der Bedarf nach Beurteilungen und Bewertungen von Programmen wurde immer eindringlicher formuliert, und dies wurde auch in der Fachliteratur aufgegriffen. Guba und Lincoln (vgl. 1989, S.30) nennen beispielsweise die Arbeit von Scriven (1967), der u.a. anmerkt, dass auch Lernziele selbst kritisch betrachtet und evaluiert werden müssten. Außerdem wurde die Forderung nach Standards als Richtlinien, anhand derer Bewertungen vorgenommen werden könnten, gestellt. Das Problem war in dieser Zeit nach Guba und Lincoln (vgl. ebenda), dass viele Evaluatoren sich nicht in der Rolle von „Bewertern“ bzw. „Beurteilern“ sahen. Schließlich konnte aber diese Seite der Evaluation und die Forderung danach nicht ignoriert werden, und es entstand eine Reihe von Evaluationsmodellen, die u.a. diese Herausforderung aufgriffen.

„All of these post-1967 models agreed on one point, however: Judgement was an integral part of evaluation. All urged, more or less explicitly, that the evaluator be judge" (Guba und Lincoln 1989, S.31).

Das Markenzeichen dieser dritten Generation ist demnach das Bewerten und Beurteilen.

Obwohl nach Guba und Lincoln jede nachfolgende Generation ein Fortschritt bedeutete, sehen sie einige tiefgreifende Probleme und Defizite (vgl. 1989, S.31ff). Sie entwickeln auf der Basis ihrer Kritik einen alternativen Ansatz und eine so genannte vierte Generation - die des Verhandelns.

\title{
3.1.4 Die vierte Generation: Das Verhandeln
}

Die vierte Generation geht über die ersten drei Generationen hinaus, und das Kernelement ist die Verhandlung:

„We have called this new approach fourth generation evaluation to signal our construction that this form moves beyond previously existing generations, characterizable as measurement-oriented, descricption-oriented, and judge- 
ment-oriented, to a new level whose key dynamic is negotiation." (Guba und Lincoln, 1989, S.8)

Guba und Lincoln (vgl. ebenda) stellen das Modell in ihrem Buch ausführlich vor. An dieser Stelle werden nur wesentliche Charakteristika des Ansatzes bzw. Grundannahmen, die dem Modell zugehörig sind, dargestellt. Guba und Lincoln beschreiben folgende Merkmale bzw. Grundsätze der vierten Generation von Evaluation (vgl. 1989, S.8ff):

- Evaluationsergebnisse sind keine Fakten und stellen keine ultimativen Wahrheiten dar, sondern sie sind Konstruktionen der am Evaluationsprozess Beteiligten.

- Diese Konstruktionen sind geprägt durch die Werte derjenigen, die sie gebildet haben (also Evaluatoren und am Prozess beteiligte Personen oder Gruppen).

- Die Konstruktionen stehen in Verbindung mit den physischen, psychologischen, sozialen und kulturellen Kontexten, die sie beeinflussen und in denen sie entstehen.

- Evaluationen und deren Ergebnisse können beteiligte Personen oder Gruppen unterschiedlich beeinflussen - sie können entrechten und entmachten oder Rechte geben bzw. sie unterstützen, ihnen Handlungsräume geben und sie stärken. Bei dem Ansatz der vierten Generation ist es zentral, Akteure (stakeholder) einzubeziehen in den Prozess der Evaluation und sie an Entscheidungen zu beteiligen.

- Evaluation muss eine Handlungsorientierung haben. Sie soll nicht nur die Akteure einbeziehen, sondern vor allem in deren Handlungsfeldern nützlich sein. Evaluationsergebnisse bestehen häufig aus Empfehlungen und Berichten, die weit entfernt von den Bedürfnissen der Evaluierten sind. Evaluationen der vierten Generation beziehen Akteure in den Evaluationsprozess in einer Weise ein, dass sie an der Erarbeitung von Ergebnissen Anteil haben und diese dann auch nutzen.

- Die Art der Verhandlung ist gekennzeichnet durch einen wertschätzenden Umgang mir den Akteuren, deren Würde, Integrität und Privatsphäre respektiert wird. Sie werden als gleichwertige und gleichberechtigte Verhandlungspartner geschätzt und behandelt, so dass am Ende des Evaluationsprozesses gemeinsam erarbeitete und verhandelte Ergebnisse herauskommen. Die Evaluierten sind keine Gegenstände, sondern Menschen.

Mit dem Modell der vierten Generation sind einige weitere Grundsätze und Verfahrensweisen verbunden. Zum einen betrifft dies die Art und Weise, wie ein Evaluationsthema betrachtet bzw. angegangen wird, und zum anderen die Methoden, die eingesetzt werden, um zu Informationen und Aussagen zu gelangen. Guba und Lincoln beschreiben zwei Elemente des Ansatzes:

„Fourth generation evaluation, (...), rests on two elements: responsive focusing - determining what questions are to be asked and what information is to be collected on the basis of stakeholder inputs - and constructivist method- 
ology - carrying out the inquiry process within the ontological and epistemological presuppositions of the constructivist paradigm." (Guba und Lincoln, 1989, S.11)

Guba und Lincoln (vgl. 1989, S.38) greifen das Modell der Responsiven Evaluation von Robert Stake (1975) auf, nennen aber ihr eigenes Modell „responsive constructivist evaluation“. Sie beziehen den Konstruktivismus als Paradigma ein und erwähnen aber auch andere „Namen“: „(...) we choose to call it the constructivist but is has many other names including interpretive and hermeneutic" (Guba und Lincoln 1989, S.39). Sie beziehen sich auf die Leitideen dieser Ansätze und nennen die Vorgehensweise in der vierten Generation „The responsive mode of focusing“ (ebenda).

Bei dieser Herangehensweise werden gemeinsam mit den Akteuren zuerst die Fragen besprochen bzw. verhandelt, die im Kontext der Evaluation relevant sind, wobei die Ansprüche (claims), Interessen (concerns) und Fragen/Themen (issues) der Akteure entscheidend sind (vgl. Guba und Lincoln 1989, S.39f). In dem Verhandlungsprozess, der vom Evaluator geleitet bzw. moderiert wird, werden gemeinsam alle relevant erscheinenden Informationen und Aspekte thematisiert und verhandelt: (...) the evaluator prepares an agenda for negotiation, taking the leadership in setting up and moderating a negotiation session." (Guba und Lincoln 1989, S.41). Die „responsive evaluation“ besteht aus vier Phasen, die sich überlappen können und ggf. auch wiederholt werden (vgl. ebenda, S.42):

1. Identifizierung der Akteure und Erfragung ihrer Interessen, Fragen und Probleme, die im Rahmen der Evaluation zentral sein werden.

2. Die Interessen und Themen aller beteiligten Akteure werden vorgestellt und diskutiert (alle Kommentare und Bewertungen sind erlaubt). Dabei können sich schon Klärungen oder Lösungen ergeben.

3. Die Themen und Probleme, die noch nicht gelöst wurden, kommen ins Zentrum der Evaluation, und es werden gezielt Informationen gesammelt, die dazu beitragen können, das Problem zu lösen. Je nach Thema werden passende Methoden gewählt quantitative und qualitative Verfahren können dabei zum Einsatz kommen.

4. Unter der Leitung des Evaluators, der die Rolle eines Moderators hat, werden Verhandlungsprozesse der am Evaluationsprozess Beteiligten durchgeführt. Das Ziel ist dabei, zu einem gemeinsamen Konsens und zu Lösungen zu kommen bei allen Themen, die zur Debatte stehen und diskutiert werden. Wenn nicht alle Themen und Probleme gelöst werden, sollen sie bei der nächsten Evaluation ins Zentrum gerückt werden, wenn zeitliche und personelle Ressourcen dies erlauben.

Diese Vorgehensweise verlangt bestimmte Methoden, die von Guba und Lincoln als "constructivist methodology" bezeichnet wird (ebenda, S.43). Entscheidend ist dabei die Grundannahme, dass es keine objektiven Wahrheiten gibt, sondern dass Realitäten soziale 
Konstruktionen von Menschen sind und es so viele Wahrheiten (bzw. Konstruktionen) gibt, wie es Individuen gibt. Bei der Evaluation der vierten Generation werden die Ergebnisse reflektiert und nicht als absolute Wahrheiten angesehen, sondern als Konstruktionen, die in diesem Kontext, mit diesen Beteiligten und zu einem bestimmten Zeitpunkt gemeinsam in einen dialektischen Prozess erarbeitet (konstruiert) wurden. Der Evaluator hat nicht die Kontrolle über alle Faktoren und Beteiligten, sondern ist selbst Teil des Systems und muss sich dessen bewusst sein. Er gestaltet und moderiert den Prozess der Evaluation.

\begin{abstract}
„Methodologically, and in consequence of the ontological and epistemological assumptions already made, the naturalistic paradigm rejects the controlling, manipulative (experimental) approach that characterizes science and substitutes for it a hermeneutic/dialectic process that takes full advantage, and account, of the observer/observed interaction to create a constructed reality that is as informed and sophisticated as it can be made at a particular point in time." (Guba und Lincoln 1989, S.44)
\end{abstract}

Die folgende Abbildung zeigt die Elemente und die Methodologie bei dem konstruktivistischen Verfahren im Rahmen der Evaluation der vierten Generation: 


\section{THE METHODOLOGY OF CONSTRUCTIVIST INQUIRY}

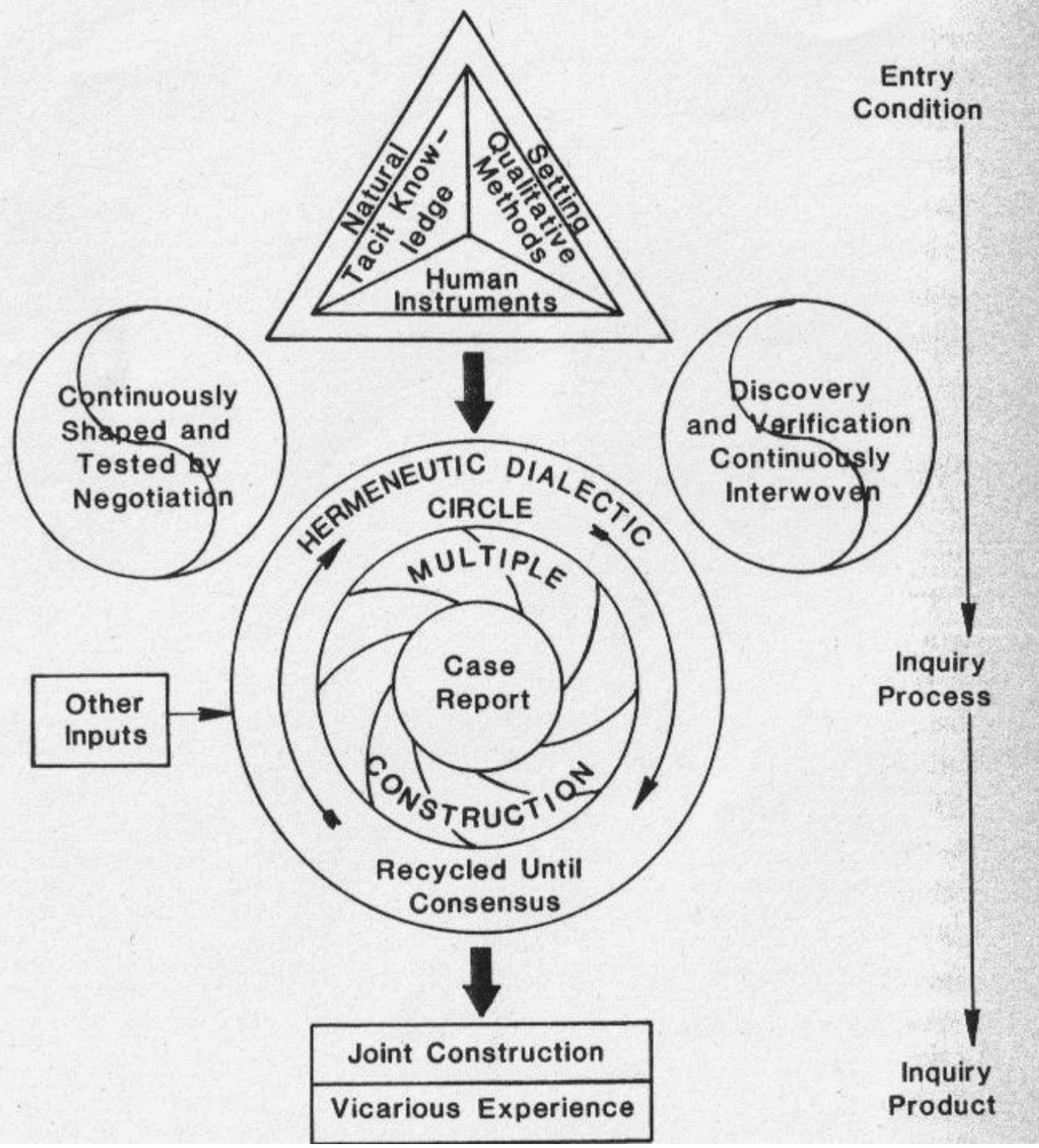

Abbildung 2: Methodisches Vorgehen nach dem Modell der Vierten Generation nach Guba und Lincoln (1989)

Am Ende des Evaluationsverfahrens kommen die Evaluatoren und alle am Verhandlungsprozess beteiligten Akteure zu gemeinsamen Konstruktionen und Erfahrungen, die für diese Gruppe, zu diesem Zeitpunkt und zu den gewählten Themen Lösungen bieten.

Zusammenfassend soll festgehalten werden dass, in dem Modell von Guba und Lincoln die Einteilung in Generationen nachvollziehbar dargestellt ist und auch die jeweiligen Schwerpunkte verschiedener Zeiten (oder Moden) logisch herausgearbeitet wurden. Kritisch anzumerken ist jedoch, dass das Modell auch eine gewisse Wertung der verschiedenen Ansätze enthält. Eine Generation folgt auf die nächste und die vierte Generation, die von Guba und Lincoln als fortschrittlichste Art der Evaluation entwickelt und beschrieben wird, ist demnach den anderen Generationen überlegen (auch wenn z.B. die erste Generation des Messens bis heute existiert). Nach Meinung der Verfasserin gibt es nicht bessere oder schlechtere Ansät- 
ze oder Schwerpunkte von Evaluation, sondern einfach nur verschiedene. Mit dem Ansatz von Guba und Lincoln werden aber zentrale Tätigkeiten, die bei Evaluationen eine Rolle spielen, deutlich, und es steht außer Frage, dass in unterschiedlichen Evaluationsansätzen oder -kontexten diese Aspekte in unterschiedlicher Form zur Geltung kommen.

\subsection{Das Baummodell nach Alkin und Christie}

Alkin betrachtet in seinen Arbeiten etwa seit den 1970er Jahren die Entwicklung von Evaluationstheorien bzw. Evaluationsmodellen und die Debatten rund um diese Themen. In seinem Buch aus dem Jahre 2004 (Alkin 2004a) entwirft er zusammen mit Christie ein Baummodell, in dem verschiedene theoretische Strömungen und Theoretiker dargestellt werden (vgl. Alkin \& Christie 2004). Außerdem kommen 22 ausgewählte Theoretiker zu Wort, die ihre eigenen Ansätze und Sichtweisen auf Evaluation beschreiben, ihre Position im Baummodell kommentieren und darauf eingehen, welche Einflüsse ihre Sicht auf bzw. von Evaluation geprägt haben.

In Bezug auf das Baummodell beschreibt Alkin (vgl. 2004b, S.4ff) zunächst, wie er und Christie vorgingen bei der Auswahl und Zuordnung von Evaluationstheorien bzw. Ansätzen und Modellen und wie sie die Autoren und deren Beiträge zur Evaluationsforschung in ihrem System kategorisiert haben. In dem Artikel von Alkin und Christie (2004) werden dann das Baummodell und die einzelnen „Hauptäste“, sowie „Nebenäste“ genauer beschrieben, d.h. die dazugehörigen Theoretiker mit ihren Beiträgen ausführlich erörtert und gewürdigt. Es wird jeweils begründet, warum die Ansätze gerade dieser Theoretiker nach Meinung der Autoren prägend für einen „Ast“ bzw. einen Schwerpunkt oder eine Strömung sind. Im Folgenden wird das Modell näher beschrieben. 


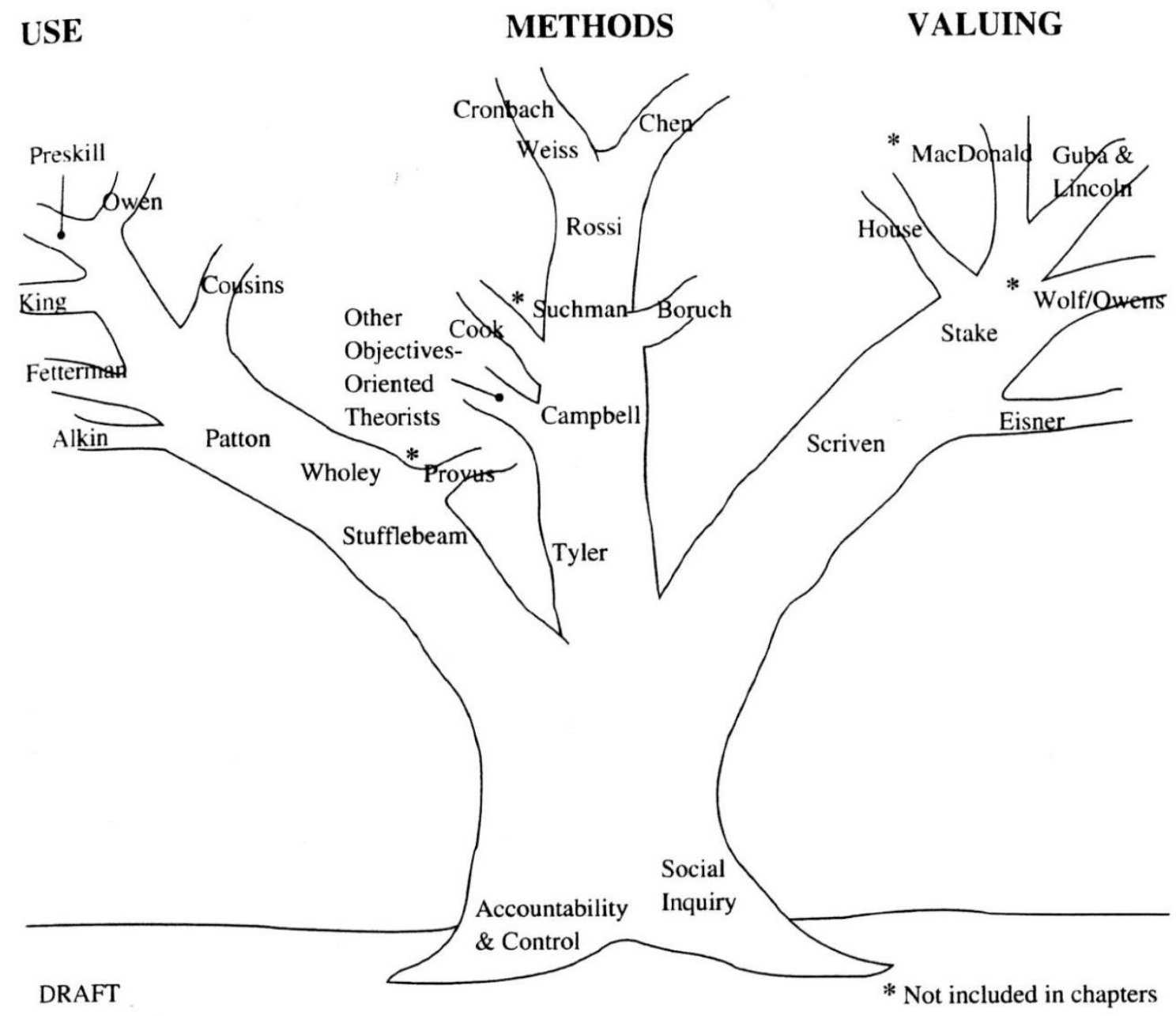

Abbildung 3: Der Evaluationsbaum nach Alkin und Christie (2004)

\subsubsection{Die Wurzeln und der Stamm}

Wichtige Ausgangsfaktoren (Wurzeln) für die Entwicklung der Evaluationsansätze bzw. richtungen („Äste“) sehen Alkin und Christie zum einen in der Rechenschaftspflicht (accountability) und Kontrollbestrebungen bzw. Buchführungstraditionen und Berichtspflichten, die mit Projekten oder Programmen verbunden sind, und zum anderen in der Sozialforschung (social inquiry). Der Bedarf nach Rechenschaft und Kontrolle begründe den Bedarf nach Evaluation und die Sozialforschung spiegle das Interesse an systematischen und vertretbaren bzw. angemessenen Methoden, um Berichte und Forschungsergebnisse guter Qualität zu gewährleisten.

„The trunk is built on a dual foundation of accountability and systematic social inquiry. These two areas have supported the development of the field in different ways. The need and desire for accountability presents a need for evaluation. (...) The social inquiry root of the tree emanates from a concern for employing a systematic and justifiable set of methods for determining accountability. While accountability provides the rationale, it is primarily from social inquiry that evaluation models have been derived." (Alkin \& Christie 2004, S.12) 
Die Rechenschaftspflicht und die empirische Sozialforschung sind demnach die Basis für die Entstehung von Evaluationstheorien und Evaluationsmodellen. Bei der Rechenschaftspflicht (accountability) geht es darum, dass nachgewiesen werden muss, wie mit Ressourcen (z.B. Fördermitteln) umgegangen wurde, dass dokumentiert, analysiert und berichtet wird, wie Prozesse gestaltet und Ziele erreicht und was für Ergebnisse produziert wurden. Und im Kontext der Sozialforschung werden von Alkin und Christie (vgl. 2004, S.16) verschiedene Forscher und Theoretiker benannt, die mit ihren Fragen und Ideen dazu beigetragen haben, dass mittels verschiedener Methoden in systematischen Studien das Verhalten von Menschen untersucht wird. Sie beziehen sich dabei auf die Sozialwissenschaften im Allgemeinen und legen sich nicht auf bestimmte Fächer oder Disziplinen fest. Auf der Grundlage dieser Faktoren entstanden nach Alkin und Christie (vgl. 2004, S.12f) drei Hauptäste, und zwar der Methodenast, der Wertungsast und der Nutzungsast, die nun näher beschrieben werden.

\title{
3.2.2 Der Methodenast (Methods)
}

Der Methodenast geht vor allem aus der empirischen Sozialforschung hervor. Alkin und Christie beschreiben verschiedene Ansätze bzw. Arbeiten von Autoren, denen sie zentrale Bedeutung für die Entwicklung von Methoden zumessen bzw. in deren Veröffentlichungen besonderer Wert auf empirische Methoden gelegt wird.

\begin{abstract}
„In the beginning there was research. And the methods of research dominated the conduct of studies. While most evaluation theorists have methodological concerns and view research as the genesis of program evaluation, one group of theorists has been steadfast in clinging to that orientation. In the social sciences and psychology, this emphasis on research depends on welldesigned experimental studies and other controls." (Alkin \& Christie 2004, S.17)
\end{abstract}

Als ersten Vertreter in diesem Ast benennen Alkin und Christie Ralph Tyler, da er mit seinen Arbeiten in den 1940er Jahren im Bereich der Evaluationsmethoden und der Theorieentwicklung Meilensteine gesetzt habe. Vor allem die im Rahmen der „Eight Year Study“ (vgl auch Abschnitt 2.1.3) eingesetzten, neuartigen Methoden sowie grundlegende Überlegungen zu einer zielorientierten Evaluation (objectives-oriented-evaluation) und der Fokus auf Ergebnisse (outcome measurement) haben nachfolgende Arbeiten besonders im Bildungsbereich wesentlich beeinflusst. Sowohl für die Methodenentwicklung als auch für die Theorieentwicklung sehen nicht nur Alkin und Christie (vgl. 2004, S.18) eine herausragende Bedeutung in den Arbeiten von Tyler.

Als nächster bedeutsamer Autor im Methodenast findet sich Donald Campbell, der wesentliche Impulse für die Methodenentwicklung mit seinen Arbeiten zum Design von Evaluationsstudien vorlegte. Besonders wichtig und einschlägig seien hierbei der Artikel zum Thema Validität in sozialen (Untersuchungs-)Feldern (Campbell 1957) sowie der Beitrag „Experimental and Quasi-Experimental Designs for Research“ (Campbell \& Stanley 1966). Zunächst 
werden Bedingungen beschrieben, unter denen Experimente durchgeführt werden müssten, um wissenschaftliche Kriterien zu erfüllen, und dann werden verschiedene Schwachstellen aufgezeigt, u.a. wird postuliert, dass „echte“ Experimente in den meisten Situationen nicht durchgeführt werden könnten. Als Alternative werden so genannte „quasi-experimentelle Designs“ vorgeschlagen. Die Ansätze und Ideen von Campbell und Stanley zählen heutzutage zu den Basisthemen in nahezu jedem Seminar über Forschungsmethoden (vgl. Alkin \& Christie 2004, S.19).

Als weiterer einflussreicher Autor wird auf dem Methodenast Edward Suchmann genannt. Seinen Beitrag sehen Alkin und Christie vor allem in der Unterscheidung zwischen dem Gebrauch des Wortes Evaluation im alltäglichen Sprachgebrauch und im wissenschaftlichen Kontext. Im Alltag werde allgemein unter Evaluation verstanden, dass in einem sozialen Prozess etwas oder jemand bewertet oder beurteilt wird, wohingegen es im wissenschaftlichen Kontext der Evaluation darum gehe, bestimmte Forschungsmethoden und -techniken einzusetzen, um zu aussagekräftigen Ergebnissen zu kommen. Das Hauptwerk von Suchmann stammt aus dem Jahre 1967 und trägt den Titel "Evaluative Research" (Suchmann 1967). Es geht hierin vor allem um die wissenschaftliche Vorgehensweise und Designfragen, wobei die Ausführungen und Ideen von Suchmann wiederum andere Autoren beeinflusst haben (vgl. Alkin \& Christie 2004, S.21f).

Auf dem „Hauptast Methoden“ wird als nächster Autor Peter Rossi benannt. Nach Alkin und Christie (vgl. 2004, S. 25f) bezieht er sich auf die Arbeiten von Cambell und Stanley (1966) sowie Suchmann (1967) bzw. führt diese fort und richtet seinen Fokus auf Evaluationsforschung - insbesondere Evaluationsmethoden. Im Jahre 1979 erscheint das Buch „Evaluation: A Systematic Approach“ (Rossi, Freemann \& Wright 1979), das bis heute gut bekannt, einschlägig und mittlerweile in der siebten Auflage erschienen ist (Rossi, Lipsey \& Freeman 2004). Nach Alkin und Christie (vgl.2004, S.26) sieht Rossi Evaluation als Sozialwissenschaftliche Forschung (social research) und widmet sich wissenschaftlichen Gütekriterien wie der internen und externen Validität von Evaluationsstudien. Rossi habe auch, zusammen mit Chen und Weiss, wertvolle und prägende Ideen für die theoriegeleitete Evaluation (theory-driven evaluation) geliefert. Insgesamt werde in seinen Arbeiten seine Vorliebe für die Reinheit und den an wissenschaftlichen Kriterien orientierten Einsatz von experimentellen und quasi-experimentellen Designs deutlich.

Auf dem Methodenast befindet sich als nächster Autor Chen, der nach Alkin und Christie (vgl. 2004, S.27) den größten Einfluss auf die Entwicklung des Konzeptes der theoriegeleiteten Evaluation (theory-driven evaluation) hatte. Er geht davon aus (nach Christie und Alkin, vgl. ebenda S.28), dass mittels der theoriegeleiteten Evaluation jede Art von Validität am besten erfüllt würde. Chen veröffentlichte zusammen mit Rossi einschlägige Artikel zu diesem Themengebiet (Chen \& Rossi 1983, 1987) sowie ein eigenes Buch (Chen 1990). Chen richtet seinen Fokus nicht nur auf die Einbettung von Evaluationen in den Kontext sozialwissenschaftlichen Wissens, sondern vor allem auch auf traditionelle Forschungsmethoden, und 
ordnet die Evaluation damit in die Tradition der sozialwissenschaftlichen Forschung im Allgemeinen ein (vgl. Alkin \& Christie 2004, S.28).

Des Weiteren befindet sich Weiss auf dem Methodenast, sie wird von Alkin und Christie (vgl. 2004, S.28) aufgrund ihrer Arbeiten zum Thema Evaluationsforschung hier eingeordnet. Sie habe sich zu Beginn ihrer Beschäftigung mit dem Thema Evaluation vor allem mit Forschungsmethoden beschäftigt, was sich in ihrer ersten Veröffentlichung in diesem Bereich zeige (Weiss 1972). Die Worte „Evaluation Research“ waren für Alkin und Christie eine Art Codewörter, um Autoren auf dem Methodenast zu platzieren. Weiss widmet sich auch der Beziehung zwischen Evaluation und Politik (Weiss 1973) und beschreibt als eine der ersten, dass Evaluationen nicht in einem politischen Vakuum entstünden.

Als besonders wichtigen und einflussreichen Autor sehen Alkin und Christie (vgl. 2004, S. 30) Cronbach, den sie ebenfalls auf dem Methodenast eingeordnet haben. Er habe durch seine Arbeiten wesentlich zu der Methodenentwicklung und-diskussion beigetragen, z.B. durch seinen "coefficient alpha, generalizability theory and notions about construct validity" (Alkin \& Christie 2004, S. 30). Cronbach war ein Schüler Tylers und orientierte sich bei seinen Arbeiten an Methodenfragen und generellen Fragen zur sozialwissenschaftlichen Forschung. Seine Publikation „Toward Reform of Program Evaluation“ (Cronbach \& Associates 1980) sowie seine Überlegungen zum Design von Evaluationen im Rahmen sozialer Programme und im Bildungsbereich (Cronbach 1982) gelten als einschlägig.

\subsubsection{Der Wertungsast (Valuing)}

Der Wertungsast geht ebenfalls aus der empirischen Sozialforschung hervor. Alkin und Christie beschreiben verschiedene Ansätze bzw. Arbeiten von Autoren, die sich darauf fokussieren, dass mittels Evaluationen Wertungen und Beurteilungen vorgenommen werden. Bei den Autoren, die auf dem Wertungsast platziert sind, gehört es zu den wesentlichen Aufgaben von Evaluatoren, dass diese auf der Basis ihrer Untersuchungen und Ergebnisse Werturteile abgeben müssen, was sie von anderen Forschern unterscheidet:

"Out of the trunk of social inquiry has grown a branch of evaluators who focus on concerns related to valuing or the making of judgements. Theorists on this branch believe that what distinguishes evaluators from other researchers is that evaluators must place value on their findings and, in some cases, determine which outcomes to examine." (Alkin \& Christie 2004, S.32)

Als ersten und wichtigsten Vertreter nennen Alkin und Christie (vgl. 2004, S.32) Scriven, der besonderen Wert darauf legt, dass es zu einer Evaluation gehört, dass der Evaluator entscheidet, was "gut“ und was "schlecht“ ist, wobei transparent gemacht wird, welche Entscheidungskriterien dem Urteil und den Bewertungen zugrunde liegen. Nach Shadish et al (1991, zitiert nach Alkin \& Christie 2004, S.32) hat Scriven sogar als erster und einziger Autor eine generelle und allgemeine "Theorie des Wertens" (theory of valuing) entwickelt. In der 
viel zitierten Definition von Scriven wird ebenfalls deutlich, dass die Bewertung zu den wesentlichen Elementen einer Evaluation gehört: „Evaluation is the process of determining the merit, worth and value of things, and evaluations are the product of that process." (Scriven 1991, S.1). Ein Evaluator handelt nach Scriven im öffentlichen Interesse und hat nicht nur die Aufgabe, für die Entscheidungsträger Informationen zu sammeln, aufzubereiten und zu beurteilen, sondern er muss sich von den Zielsetzungen eines Programms lösen und „zielfrei“ forschen. Scriven $(1973,1974)$ entwickelt dafür das Modell der „zielfreien Evaluation“ (goalfree evaluation), wonach bei einer Evaluation nach allen Effekten gesucht werden muss, die ein Programm auslöst - auch wenn diese nicht beabsichtigt waren bzw. zu den Zielen gehörten. Nebeneffekte und Nebenwirkungen sowie nicht intendierte Folgen können auf diese Weise entdeckt und bewertet werden ${ }^{4}$. Außerdem ist es bei der zielfreien Evaluation nach Scriven wichtig, dass die Bedürfnisse der Zielgruppe („needs“) berücksichtigt werden. Nachdem der Zielgruppenbedarf ermittelt wurde, sollen Wirkungen einer Maßnahme oder eines Programms mit den Bedürfnissen verglichen und dazu eine Bewertung vorgenommen werden. Nach Alkin und Christie (2004, S.34) ist Scriven der "leading theorist of the valuing perspective and provides the 'spiritual guidance' and direction for others depicted on this branch".

Auf den Nebenästen benennen Alkin und Christie Eisner, Wolf und Owens, House sowie Mc Donald. Auf dem Wertungshauptast folgt nach Scriven Robert Stake, der für die beiden nicht einfach einzuordnen war, da er zu jedem der drei Äste passen würde (vgl. 2004, S.37). Stake $(1972,1975,1980)$ führte das Konzept der „responsiven Evaluation“ (responsive Evaluation) ein, mit dem er im Baummodell sowohl zu dem Bewertungsast aber auch zu dem Nutzungsast passen könnte. Nach House (vgl. 2001, zitiert nach Alkin \& Christie 2004, S. 37) sind die zentralen Aspekte des Modells der responsiven Evaluation, die Grundannahme, dass a) Wissen kontextgebunden ist und es keine allgemeingültige Wahrheit gibt, b) die Sichtweisen von Akteuren ein wichtiger Bestandteil einer Evaluation sind und c) Fallstudien die beste Methode für die Erforschung und Darstellung der Meinungen und Sichtweisen von Akteuren sowie für die Berichterstattung im Rahmen einer Evaluation sind. Bei der responsiven Evaluation nach Stake sind die Bedarfe der Akteure, in deren Feld die Evaluation stattfindet, besonders wichtig und es gibt kein im Vorfeld festgelegtes Evaluationsdesign. Viel-

\footnotetext{
${ }^{4}$ Dass gerade Bildungsmaßnahmen ganz andere als die erhofften Wirkungen erzielen können, hat in der deutschen Pädagogik Eduard Spranger 1962 in die Diskussion gebracht. Der ehemalige Generalsekretär des Stifterverbandes für die Deutsche Wissenschaft, Manfred Ehrhardt, hat darauf in einer kritischen Würdigung der Bildungsreformen in Deutschland zurückgegriffen: "'Heterogonie der Wirkungen' hat Eduard Spranger jene Diskrepanz von Wollen und Vollbringen genannt, bei der insbesondere im Bereich von Bildung und Erziehung selbst die bestgemeinten Absichten durch die vom Handeln tatsächlich hervorgebrachten Folgen durchkreuzt werden. Für die Gültigkeit dieses ,Gesetzes der ungewollten Nebenwirkungen' liefern die Schul- und Hochschulreformen der letzten 30 Jahre Beweise im Überfluß. Sonst stünden wir heute nicht vor der Notwendigkeit einer nachhaltigen Reform der Reformen." (http://www.forschung-und-lehre.de/archiv/06-01/erhardt.html, Zugriff am 31.8.2012) SPRANGER, E.: Das Gesetz der ungewollten Nebenwirkungen in der Erziehung. Quelle und Meyer, Heidelberg, 1962.
} 
mehr wird im Evaluationsprozess auf die sich jeweils ergebenden Erfordernisse (responsiv) reagiert. Stake geht davon aus, dass es mehrere Realitäten/Wahrheiten gibt und plädiert deshalb dafür, die Sichtweisen und Standpunkte der Akteure in eine Evaluation einzubeziehen. Die Vielfalt soll erfasst und abgebildet werden, es ist jedoch die Aufgabe des Evaluators, den Evaluationsbericht zu schreiben, die Ergebnisse zusammenzufassen und zu bewerten, weshalb Alkin und Christie (vgl. 2004, S. 38) Stake auf dem Wertungsast eingeordnet haben.

Auf dem Wertungshauptast befinden sich als weitere Vertreter Guba und Lincoln, die das Generationenmodell der Evaluation und darin enthalten eine vierte Generation der Evaluation entwarfen (Guba und Lincoln 1989 - siehe auch Kapitel 3.1). Guba und Lincoln sehen nach Alkin und Christie (vgl. 2004, S. 42) vor allem die Akteure als beteiligt, wenn es um Wertungen geht, die im Rahmen von Evaluationen entstehen, und nicht nur den Evaluator. Guba und Lincoln bauen auf den Arbeiten von Stake auf und übernehmen Teile des Modells der responsiven Evaluation. Auch sie gehen davon aus, dass es mehrere Realitäten / Wahrheiten gibt, die auf den Interessen und Wahrnehmungen der betroffenen Akteure beruhen. Die Rolle des Evaluators sei es, diese Vielfalt zu erfassen und gemeinsam mit den Akteuren in einem Verhandlungsprozess zu reflektieren, um zu Lösungen für vorher definierte Probleme zu gelangen. In dem Modell von Guba und Lincoln ist der Evaluator wie ein Moderator, der den Prozess leitet und die verschiedenen Ansichten (gemeinsam mit den Akteuren) sortiert. Guba und Lincoln beziehen auch den "hermeneutischen Zirkel“ als Vorgehensweise mit in ihr Modell ein, worin sich Datensammlung und Datenanalyse abwechseln und nach und nach Erkenntnisse und Lösungen herauskommen.

Auf dem Wertungsast sind Autoren platziert, die auf verschiedene Weise vertreten, wie es zu Wertungen bei Evaluationen kommt - einmal ist der Evaluator derjenige, der die Beurteilung (judgement) oder Wertung (valuing) übernimmt, ein anderes Mal sind es die Akteure, die gemeinsam mit dem bzw. angeleitet vom Evaluator Wertungen vornehmen.

\subsubsection{Der Nutzungsast (Use)}

Auf dem Nutzungsast des Evaluationsbaumes haben Alkin und Christie Autoren eingeordnet, die vor allem Wert darauf legen, dass Evaluationen einen Nutzen haben müssen, und zwar besonders im Hinblick darauf, Entscheidungen treffen zu können. Als besondere Zielgruppe sehen diese Autoren zumeist Entscheidungsträger, die im Rahmen von Maßnahmen und Programmen von den Ergebnissen der Evaluation profitieren können, indem sie eine Informationsgrundlage für Entscheidungen geliefert bekommen. Evaluationen müssen nach diesen Ansätzen einen konkreten Nutzen und Einfluss bzw. Auswirkungen in der Praxis haben.

„The use branch began its growth with what are often referred to as 'decision-oriented theories'. Decision-oriented theorists felt it was critical to con- 
duct evaluations that were designed specifically to assist key program stakeholders in program decision making. (...) This class of theories is concerned with designing evaluations that are intended to inform decision making, but it is not their only function to ensure that evaluation results have direct impact on program decision making and organizational change." (Alkin \& Christie 2004, S.44)

Als ersten und besonders wichtigen Vertreter des "Nutzenansatzes" nennen Alkin und Christie (vgl. 2004, S.44) Stufflebeam und sein CIPP-Modell, das er im Jahre 1971 entwickelt hat (Stufflebeam 1971, 1983). Die Buchstaben CIPP stehen für: Context, Input, Process and Product. Stufflebeam unterscheidet in dem Modell vier Arten von Evaluation: Kontext-, Input-, Prozess- und Produktevaluation. Die Kontextevaluation dient dazu, Bedarfe zu erkennen bzw. zu identifizieren und auf dieser Grundlage Zielsetzungen eines Programms zu entwickeln bzw. festzulegen (über sie zu entscheiden). Die Inputevaluation unterstützt Entscheidungen zum Design und zur Strategie eines Programms. Mittels einer Prozessevaluation werden Stärken und Schwächen eines Programms herausgearbeitet, um die Implementation von Maßnahmen zu verbessern und zu optimieren. Bei einer Produktevaluation werden Ergebnisse und Wirkungen (outcomes) untersucht, um die Nachhaltigkeit von Maßnahmen zu fördern und ggf. Verbesserungsoptionen zu entwickeln, womit die Effektivität und Effizienz von Programmen gesichert werden soll.

Nach Stufflebeam ist Evaluation ein zyklischer Prozess, und der Evaluator müsse eine gewisse Flexibilität mitbringen, um die Evaluation an die Bedarfe im Feld bei der Entwicklung des Evaluationsdesigns anzupassen. Er liefere dann den Akteuren (Entscheidungsträgern) kontinuierlich Informationen, um sicher zu stellen, dass innerhalb des Programms Angebote und Maßnahmen möglichst optimal entwickelt werden können (vgl. Alkin \& Christie 2004, S.44). Die Evaluation sei demnach gekennzeichnet durch einen interaktiven Prozess zwischen dem Evaluator und den Akteuren (Entscheidungsträgern). Die Evaluation habe so Dienstleistungscharakter, da sie sich den Bedürfnissen der Zielgruppe anpassen soll und immer wieder sicher gestellt werden muss, dass die Ergebnisse dem Informationsinteresse entsprechen und die Entscheidungsfindung unterstützen.

Als nächsten Vertreter des „Nutzungsansatzes“ sehen Alkin und Christie Provus, der 1971 ein Buch mit dem Titel „Discrepancy Evaluation“ veröffentlichte (Provus 1971). Für Provus ist Evaluation ein kontinuierlicher Prozess, mit dem Programmverantwortliche unterstützt werden im Hinblick auf die optimale Entwicklung und Gestaltung eines Programms. Er benennt vier Entwicklungsphasen und eine optionale Phase: 1. Definition (um die spezifischen Ziele, Prozesse und Ressourcen festzulegen), 2. Installation (um Diskrepanzen bei der Implementation eines Programms zu identifizieren), 3. Prozess (um das Ausmaß der Zielerreichung und die Wirkungen zu ermitteln), 4. Produkt (um den Umfang der endgültigen Zielerreichung zu erfassen) und 5. Kosten-Nutzen Analyse. Kennzeichnend für den Ansatz von Provus ist eine starke interaktive Komponente der Evaluation, da Programmakteure aktiv in den Pro- 
zess einbezogen werden. Mit den Ergebnissen der Evaluation sollen der Managementprozess unterstützt und Entscheidungsfindungen optimiert werden.

Auch bei dem nächsten Autor, der von Alkin und Christie auf dem Nutzungsast genannt wird, gehört die Unterstützung des Managementprozesses sowie dessen Verbesserung zu den Hauptzielen der Evaluation. Wholey stellt die Bedarfe von Managern und (politischen) Entscheidungsträgern in den Mittelpunkt. Evaluation ist für Wholey ein Prozess, um effektives Management zu stimulieren und zu fördern (vgl. Alkin \& Christie 2004, S.47). Wholey entwirft ein vierstufiges Verfahren für die Beschaffung von Informationen: 1. „Evaluability Assessment“ (initiale Erfassung der Evaluationsmöglichkeiten und Ermittlung des Ausmaßes, in dem die Ergebnisse genutzt werden können, um das Programm zu verbessern und das Management zu unterstützen), 2. „Rapid Feedback Evaluation“ (bestehende und leicht zu bekommende Informationen werden gesammelt), 3. „Performance Monitoring" (die Ausführung des Programms wird verglichen mit den vorher festgelegten Zielen) und 4. „Intensive Evaluation“ (Vergleichs- und Kontrollgruppen werden betrachtet und untersucht, um die Effektivität eines Programms beurteilen zu können). Da Wholey vor allem den Nutzen für Manager von Programmen und (politische) Entscheidungsträger als wichtig bei einer Evaluation erachtet, wurde er dem Nutzungsast zugeordnet.

Des Weiteren befindet sich Patton auf dem Nutzungsast. In seinem Ansatz geht es nicht nur darum, Entscheidungsträgern mittels Evaluation Entscheidungen zu erleichtern und sie zu unterstützen, sondern es ist die Aufgabe, sogar die Pflicht des Evaluators herauszufinden, wer von den Ergebnissen profitieren kann und einen Nutzen davon hat. Der Evaluator hat die Verantwortung dafür, dass die Evaluationsergebnisse nützlich sind und eine Nutzung (utilization) tatsächlich stattfindet (vgl. Alkin \& Christie 2004, S. 48). Der bekannteste theoretische Ansatz mit dem Fokus auf die Nutzenanwendung von Evaluation stammt nach Alkin und Christie von Patton (vgl. ebenda), der das Modell der "utilization-focused evaluation“ entwickelte (Patton 1978, 1986, 1997). In dem Modell gibt es fünf Phasen: 1. Identifikation der Nutzer (der Evaluator soll proaktiv danach suchen und sie auch benennen), 2. Einbeziehung der Nutzer (der Fokus der Evaluation wird gemeinsam festgelegt), 3. Beteiligung der Nutzer bei der Auswahl von Methoden, 4. Engagement und Dialog mit den Nutzern bei der Interpretation der Ergebnisse und bei den Bewertungen und 5. Gemeinsame Entscheidungen über die weitere Vorgehensweise. Mit diesen Phasen wird deutlich, dass mit dem Ansatz der „utilization-focused evaluation“ sehr flexibel auf die individuelle Situation eingegangen wird, da mit den Akteuren gemeinsam verhandelt und geforscht wird. Der Evaluator hilft den Nutzern, sozusagen herauszufinden, was sie brauchen, und was sie von der Evaluation bekommen können, indem er die Möglichkeiten (z.B. Ziele, Nutzen, Methoden) erörtert und darstellt. Es wird dann zusammen, in einem interaktiven Prozess entschieden, was die Nutzer benötigen und wie sie von den Evaluationsergebnissen in ihrer individuellen Situation profitieren können. Patton prägte nach Alkin und Christie (vgl. 2004, S.49) außerdem den Begriff „developmental evaluation“, womit gemeint ist, dass der Evaluator Teil des Teams 
oder einer Organisation wird und an Entscheidungen beteiligt ist. Er ist kein „Außenseiter“, sondern hat eher eine Beratungsfunktion innerhalb eines Programms, Teams oder einer Organisation. Im Jahre 2010 veröffentlichte Patton dazu auch ein Buch (Patton 2010).

Ein weiterer Vertreter des Nutzenansatzes ist Alkin. Auch er richtet den Fokus darauf, dass Evaluationen nützlich sein sollen, um Entscheidungsprozesse zu unterstützen. Sein Ansatz weist eine Nähe zu dem Modell von Stufflebeam auf (siehe oben), da es einige Gemeinsamkeiten zwischen den Sichtweisen auf Evaluation gibt. Alkin selbst nennt seinen Ansatz „useroriented evaluation" und plädiert stark für die Zusammenarbeit des Evaluators mit den Nutzern, wobei in einem interaktiven Prozess gemeinsam Bewertungssysteme erarbeitet werden sollen (vgl. Alkin \& Christie 2004, S. 50).

Auf dem Nutzungsast findet sich als nächster Autor Cousins, der zusammen mit Earl einen Ansatz entwickelte, bei dem die Akteure in starkem Maße einbezogen werden, um die Möglichkeiten des Nutzens der Evaluation und der Umsetzung bzw. Anwendung der Evaluationsergebnisse zu erhöhen. Sie nennen den Ansatz „participatory evaluation“ (Cousins \& Earl 1995). Nach Cousins findet die Anwendung und Nutzung (utilization) innerhalb des Kontextes einer Organisation statt und wird am besten erreicht, indem sie als Teil der Organisationsentwicklung gesehen wird. Die Nutzer sind bei diesem Ansatz „Mitarbeiter“ im Evaluationsprozess. Die Evaluation wird sehr praxisbezogen durchgeführt und auch als „practical participatory evaluation“ bezeichnet. Das Modell ist eingebettet in ein System, in dem es um Organisationslernen geht und die Lern- und Entwicklungsmöglichkeiten einer Organisation durch Evaluation gefördert und unterstützt werden sollen. Cousins geht davon aus, dass der Wunsch nach Beteiligung bei der Verbesserung von Programmen und Strukturen Nutzer dazu anregt, sich bei der Evaluation einzubringen und sich zu engagieren, was wiederum die Umsetzung von Ergebnissen und den Nutzen der Evaluation erhöht, da sich die Beteiligten als Teil der Evaluation sehen. Evaluator und Nutzer tragen die Verantwortung für den Evaluationsprozess und die Ergebnisse gemeinsam, wobei der Evaluator die Rolle eines Beraters hat, der Möglichkeiten aufzeigt und die technischen (methodischen) Kompetenzen mitbringt und den Nutzern beibringt, sie anleitet bzw. „trainiert“. Der Evaluator ist eher ein Begleiter, der für bestimmte Aktivitäten wie die Entwicklung des Evaluationsdesigns, Datenanalyse und Berichterstattung zuständig ist (vgl. Alkin \& Christie 2004, S. 51).

Einen verwandten Ansatz entwickelte Preskill, die Evaluation ebenfalls als Teil des Organisationslernens sieht. Sie geht davon aus, dass der Nutzen von Evaluationsergebnissen, der schon im Evaluationsprozess entstehen und deutlich sein soll, ein Mittel dazu ist, um „transformatives Lernen“ zu ermöglichen (vgl. Alkin \& Christie 2004, S. 51). Nach Preskill und Torres (2001) geht es bei dem „transformal learning“ darum, dass Individuen, Teams oder Organisationen Informationen identifizieren, inspizieren und begutachten, um sie zu verstehen und mit ihren Zielen abzugleichen. Der Evaluator soll diesen (Lern-)Prozess unterstützen und moderieren. 
Auf dem Nutzungsast werden von Alkin und Christie (vgl. 2004, S. 53f) noch King und Owen genannt, die wie die Autoren zuvor großen Wert auf die Interaktion und Kommunikation zwischen Evaluatoren und Nutzern legen und Evaluation als Teil von Organisationsentwicklung sehen. Außerdem befindet sich Fetterman mit seinem Ansatz der „empowerment evaluation“ auf dem Nutzungsast, bei dem Akteure angeregt und bestärkt werden, Selbstevaluationen durchzuführen. In dem Modell von Fetterman $(1996,1998)$ dient der Evaluator als Berater oder Moderator, der bei dem Evaluationsprozess hilft und unterstützt, indem er sein Wissen und Instrumente (Methoden) zur Verfügung stellt. Evaluatoren „trainieren“ und ermächtigen gewissermaßen die Praktiker, ihre eigenen Evaluationen durchzuführen und bestärken sie dadurch. Das Modell der „empowerment evaluation“ und das der „participatory evaluation“ (siehe oben) weisen im Hinblick auf die Beteiligung von Akteuren und den Fokus auf den Nutzen in der Praxis eine gewisse Nähe auf. Es gibt nach Alkin und Christie (vgl. 2004, S. 56) jedoch auch einen grundsätzlichen Unterschied: Bei der „participatory evaluation“ geht es vor allem darum, den Nutzen in der Praxis zu erhöhen (utilization) und im Modell der „empowerment evaluation“ ist das Hauptziel, Nutzer zu emanzipieren, indem sie befähigt werden, selbstständig ihre Evaluationen durchzuführen.

Zusammenfassend lässt sich sagen, dass das Ordnungsmodell von Alkin und Christie klar, übersichtlich und nachvollziehbar darstellt, wie sich unterschiedliche Schwerpunkte und Sichtweisen von Evaluation entwickelt haben. Die Vielfalt der Evaluationsansätze wird deutlich und sichtbar. Die drei „Hauptäste“ Methode, Wertung und Nutzen, die sich auf der Basis (dem Boden und den Wurzeln) der Rechnungslegung und empirischen Sozialforschung herausgebildet haben, ist schlüssig und hilft, Evaluation in einen größeren Kontext einzuordnen. Was die Zuordnung einzelner Autoren anbelangt, ließe sich diskutieren, ob manche nicht auch auf anderen „Ästen“ hätten eingeordnet werden können. Alkin und Christie selbst reflektieren dies aber auch und lassen in ihrem Buch die Autoren zu Wort kommen, die zu ihrer „Position“ auf dem Evaluationsbaum Stellung nehmen können. Ein Hinweis sei jedoch an dieser Stelle noch gegeben: Die Zusammenstellung von Alkin und Christie bezieht sich auf die englischsprachige Welt und berücksichtigt nicht die Entwicklungen in Europa bzw. einzelnen Ländern in Europa. Die „Evaluationslandschaft“ der Welt müsste vielleicht in einem Bild mit mehreren Bäumen oder Pflanzen abgebildet werden.

\subsection{Das Nutzungsmodell nach Fitzpatrick, Sanders und Worthen}

Ein weiteres Ordnungsmodell, um die Fülle und Verschiedenartigkeit von Evaluationsansätzen darzustellen, wurde von Fitzpatrick, Sanders und Worthen (2004) vorgelegt. Zunächst beschreiben sie, wie sich nach ihrer Einschätzung vor den unterschiedlichen Hintergründen und Weltanschauungen verschiedener Autoren die Vielfalt von Evaluationsansätzen heraus- 
gebildet hat, die zu spezifischen Orientierungen und Vorlieben, z.B. bei der Methodenwahl und den Auswertungstechniken, geführt haben (vgl. Fitzpatrick, Sanders und Worthen 2004, S.59). Danach stellen sie ihr Ordnungssystem vor, wobei sie betonen, dass sie verschiedene Evaluationsansätze (approaches) klassifiziert haben und sich dabei nicht auf Evaluationstechniken beziehen. Sie ordnen die Evaluationsansätze nach ihrer praktischen Ausrichtung sowie nach den Zielen und dem beabsichtigten Nutzen, die mit der Evaluation verbunden sind:

\begin{abstract}
„Our classification is based on what we see as driving force behind doing the evaluation: the major questions to be addressed and/or the major organizer(s) that underlie each approach (e.g., objectives or management decisions, increasing the participation or power of stakeholders)." (Fitzpatrick, Sanders und Worthen 2004, S.68f)
\end{abstract}

Fitzpatrick, Sanders und Worthen unterscheiden fünf verschiedene Ansätze von Evaluation:

- Zielorientierte Ansätze (objectives-oriented evaluation approaches)

- Managementorientierte Ansätze (management-oriented evaluation approaches)

- Konsumentenorientierte Ansätze (consumer-oriented evaluation approaches)

- Expertenorientierte Ansätze (expertise-oriented evaluation approaches)

- Partizipative Ansätze (participant-oriented evaluation approaches)

Im Folgenden werden diese Ansätze näher beschrieben.

\title{
3.3.1 Zielorientierte Ansätze (objectives-oriented evaluation approaches)
}

Im Rahmen der zielorientierten Ansätze geht es bei der Evaluation vor allem darum, Ziele (goals, purposes and objectives) eines Programms oder einer Maßnahme zu definieren und zu überprüfen. Mit der Evaluation werden zunächst Ziele herausgearbeitet und spezifiziert, um dann zu ermitteln und zu kontrollieren, ob und in welcher Form bzw. in welchem Ausmaß diese Ziele erreicht wurden. Die Ergebnisse können dazu genutzt werden, ggf. Ziele zu modifizieren, neu zu formulieren oder die Prozesse so zu gestalten, dass die ursprünglichen Ziele erreicht werden können. Die Evaluationsgegenstände können dabei sehr verschieden sein und kurze Maßnahmen oder Ereignisse betreffen oder aber komplexe Programme oder Vorhaben umfassen:

„The distinguishing feature of an objectives-oriented evaluation approach is that the purposes of some activity are specified, and then evaluation focuses on the extent to which those purposes are achieved. In education the activity could be as short as a one-day classroom lesson or as complex as the whole schooling enterprise. In health and human services it is often a service or intervention. In business, it again could be as simple as a one-day meeting or as complex as a corporation's five year strategic plan. The information gained from an objectives-oriented evaluation could be used to reformulate the purposes of the activity, the activity itself, or the assessment procedures and devices used to determine the achievement of the purpose." (Fitzpatrick, Sanders und Worthen 2004, S.71) 
Zu den zielorientierten Ansätzen von Evaluation haben nach Fitzpatrick, Sanders und Worthen (vgl. 2004, S. 72) viele Personen beigetragen, aber als ersten und wichtigsten Vertreter benennen sie Ralph W. Tyler, der diesen Ansatz ab den 1930er Jahren konzipiert und wesentlich geprägt hat. Seine Arbeiten waren bahnbrechend und haben viele andere beeinflusst. Im Kontext der Eight-Year Study entwickelte Tyler nicht nur einen neuen Evaluationsansatz, sondern prägte auch den Einsatz neuer Methoden. Das Besondere und Neue bei den Arbeiten von Tyler war, dass zunächst die (Lern-)Ziele definiert bzw. klar gestellt werden mussten, um dann zu überprüfen, ob diese auch erreicht wurden (siehe auch Abschnitt 2.1.3). Tyler war im Bildungsbereich tätig (an Schulen und Universitäten in Amerika), aber seine Ideen und Ansätze hatten großen Einfluss auf verschiedene Bereiche von Evaluation.

Weitere Autoren, die Beiträge zu den zielorientierten Ansätzen lieferten sind nach Fitzpatrick, Sanders und Worthen (vgl. 2004, S.73f) Sanders und Cunningham. Sie betonten, dass mittels einer Evaluation die Ziele einer Maßnahme sowohl empirisch untersucht, aber auch auf ihre logische Struktur hin betrachtet werden können, wie z.B. die Kohärenz der Ziele untereinander bzw. die Übereinstimmung mit übergeordneten Werten.

Das Modell der „Discrepancy Evaluation“, das von Provus (1971) entwickelt wurde, sehen Fitzpatrick, Sanders und Worthen (vgl. 2004, S.75) als einen weiteren Ansatz in der Tradition der Arbeiten von Tyler. In dem „Discrepancy Evaluation Model“ nach Provus geht es darum, Differenzen zwischen den zuvor definierten Zielen und der tatsächlichen Erreichung dieser Ziele festzustellen. Provus geht davon aus, dass ein Programm vier Phasen durchläuft, mit denen verschiedene Evaluationsaufgaben verbunden sind. Zunächst werden in der Definitions- und Entwicklungsphase Ziele definiert, Prozesse, Aktivitäten und Ressourcen dargestellt und Standards formuliert, nach denen mittels der Evaluation Bewertungen vorgenommen werden können. Dann werden in der Installationsphase die zuvor definierten Ziele und Standards bei der Implementierung überprüft, d.h. es wird durch Übereinstimmungsprüfungen (congruency tests) verfolgt, ob es bei den erwarteten Folgen und tatsächlichen Wirkungen zu Diskrepanzen kommt bzw. kommen könnte (dann wären Änderungen noch möglich). Darauf folgt die Prozessphase, in der mittels der Evaluation vor allem Daten gesammelt werden, um den Fortschritt des Programms bzw. der Programmteilnehmer zu ermitteln und zu messen, ob sich Leistungen verbessern bzw. das Verhalten sich so verändert, wie es durch die Maßnahme oder das Programm beabsichtigt war. Schließlich wird in der Produkt- oder Produktionsphase überprüft, ob die langfristigen Programmziele erreicht wurden. Provus unterscheidet zwischen direkten Ergebnissen bzw. Zielen (direct outcomes or terminal objectives) und langfristigen Folgen bzw. Zielen (long-term-outcomes or ultimative objectives), die in dieser Phase mit der Evaluation erfasst werden sollen (z.B. auch durch Follow-upStudien). Eine fünfte (optionale) Phase fügt Provus noch mit der Kosten-Nutzen Analyse hinzu, in der die Ergebnisse, die Kosten und der Nutzen eines Programms mit anderen, ähnlichen Programmen verglichen werden sollen. Das „Discrepancy Evaluation Model“ wurde vor allem im Schulbereich eingesetzt, aber auch auf andere Bereiche übertragen. Elemente die- 
ses Evaluationsansatzes finden sich nach Fitzpatrick, Sanders und Worthen (vgl. 2004, S.77) auch heute noch in vielen Evaluationen wieder.

Fitzpatrick, Sanders und Worthen (vgl. 2004, S.77f) führen noch eine Reihe weiterer Autoren bzw. deren Arbeiten auf, die sich in die Tradition der zielorientierten Ansätze einordnen lassen, und beschreiben dann, wie diese genutzt wurden. Die zielorientierten Ansätze haben nach Fitzpatrick, Sanders und Worthen (vgl. 2004, S.80) das Denken und die Entwicklung von Evaluation nicht nur in Amerika maßgeblich dominiert.

Im Bildungsbereich sind hierbei noch die Arbeiten von Bloom et al. $(1956,1964)$ einschlägig, die Taxonomien entwickelten, mit denen Ziele im Bildungsbereich überprüft werden können. Die Taxonomien sollten als Instrumente dienen, mit denen im Bildungsbereich Tätige eine Art Werkzeug erhalten, um ihre Lehre zu überprüfen und ggf. zu verbessern - ganz im Sinne des Ansatzes von Tyler.

Auch die Arbeiten von Cronbach (1963) stehen in der Tradition Tylers (Cronbach arbeitete u.a. mit Tyler während der Eight-Year Study zusammen). In dem Ansatz von Cronbach geht es darum, Messtechniken einzusetzen und Evaluationen durchzuführen, um Ziele zu überprüfen und Kurse sowie Curricula auf der Basis der Ergebnisse ggf. zu verbessern.

Zu den Stärken der zielorientierten Ansätze gehört nach Fitzpatrick, Sanders und Worthen deren Einfachheit. Sie seien leicht zu verstehen und einzusetzen, außerdem sähen Programmverantwortliche solche Ansätze, bzw. die Informationen, die mit einer Evaluation in dieser Tradition verbunden sind, als wertvoll an. Im Kontext dieser Vorgehensweisen seien auch eine Vielzahl von methodischen Entwicklungen und Innovationen angeregt worden:

„Probably the greatest strength and appeal of the objectives-oriented approach to evaluation lies in its simplicity. It is easily understood, easy to follow and implement, and produces information that program directors generally agree is relevant to their mission. This approach has stimulated so much technological development over the years that the process of specifying objectives and developing or finding appropriate measurement procedures and instruments have been finely honed." (Fitzpatrick, Sanders und Worthen 2004, S.82)

Des Weiteren wird den zielorientierten Ansätzen zu Gute gehalten, dass Programmverantwortliche und an der Umsetzung von Projekten Beteiligte über die Ziele von Programmen und Projekten sowie damit verbundene Intentionen nachdenken und reflektieren.

Zu den Schwächen zählen Fitzpatrick, Sanders und Worthen (vgl. 2004, S. 82f) u.a., dass es in einigen Fällen nicht einfach möglich sei, Ziele präzise zu definieren, da diese häufig eher verschwommen sein könnten oder sich im Laufe eines Programms auch änderten. Es komme weiterhin vor, dass offizielle Ziele, wie sie sich etwa in allgemeinen Programmbeschreibungen finden, sich mit den tatsächlich verfolgten Zielen nicht decken. Zielvorgaben können sich an politischen Interessen orientieren und die tatsächlichen Programmabläufe dann doch anders gestaltet werden - insbesondere wenn verschiedene Interessensgruppen betroffen seien, werde es schwierig. Wer bestimmt die Ziele einer Maßnahme? Viele Ergebnisse bzw. 
Folgen im Rahmen von Programmen seien außerdem schwer nachzuweisen oder nicht leicht auf bestimmte Maßnahmen zurückzuführen, besonders im sozialen Bereich, so dass u.U. gar nicht genau ermittelt werden könne, ob die Ziele mittels des Programms tatsächlich erreicht wurden (hohe Variabilität). Insbesondere wenn verschiedene Akteure beteiligt seien werde es nahezu unmöglich, mit einem zielorientierten Evaluationsansatz allen gerecht zu werden. Die Bewertung, ob die Ziele erreicht wurden, werde dann eine Frage des Standpunktes.

\subsubsection{Managementorientierte Ansätze (management-oriented evaluation approaches)}

Bei den managementorientierten Ansätzen ist es das Hauptziel der Evaluation, Entscheidungsträgern Informationen zu liefern, damit sie gute und auf den Ergebnissen der Evaluation begründete rationale Entscheidungen treffen können. Die Belange, Informationsbedarfe und Interessen der "Manager“, d.h. von Programmverantwortlichen und Projektmanagern sowie Entscheidungsträgern stehen deshalb im Vordergrund. Mittels der Evaluation und der erarbeiteten Informationen werden deren Entscheidungsprozesse unterstützt:

\footnotetext{
„The management-oriented evaluation approach is meant to serve decision makers. Its rationale is that evaluative information is an essential part of good decision making and that the evaluator can be most effective by serving administrators, managers, policy makers, boards, practitioners, and others who need good evaluative information." (Fitzpatrick, Sanders und Worthen 2004, S. 87)
}

Wie auch bei den zielorientierten Ansätzen (s.o.) haben viele Autoren zu den managementorientierten Ansätzen beigetragen. Als besonders wichtige Beiträge sehen Fitzpatrick, Sanders und Worthen (vgl. 2004, S. 89f) die Arbeiten von Stufflebeam, der das CIPP-Modell entwickelte $(1971,1983)$ und das UCLA-Modell von Alkin (1969). Das CIPP-Modell wurde bereits im Abschnitt 3.2.4 dieser Arbeit beschrieben. Das Ziel ist, mit diesem Ansatz Entscheidungsträger zu unterstützen, gute Entscheidungen zu treffen. Stufflebeam unterscheidet darin vier Arten von Evaluation, die je nach Projekt bzw. Projektphase zum Einsatz kommen. Die Buchstaben CIPP stehen für Context Evaluation, Input Evaluation, Process Evaluation und Product Evaluation.

Das UCLA-Modell von Alkin weist einige Paralellen zum CIPP-Modell auf. Die Buchstaben UCLA stehen für „University of California, Los Angeles“, an der Alkin als Direktor des „Center for the Study of Evaluation" zu der Zeit arbeitete, als er das Modell entwickelte. Das UCLAModell enthält fünf Typen von Evaluation: 1. Systembewertung (system assessment), um Informationen über das System zu bekommen, das im Zentrum der Evaluation steht, 2. Programmplanung (program planning), um die Auswahl von Programmen zu erleichtern, die bestimmte Bedarfe unterstützen, 3. Programmimplementation (program implementation), um Informationen darüber zu erhalten, ob das Programm in der beabsichtigten Weise und für die 
angestrebte Zielgruppe umgesetzt wurde, 4. Programmverbesserung (program improvement), um Informationen zusammenzustellen wie das Projekt funktioniert, ob Zwischenziele erreicht wurden und ob unerwartete Ergebnisse bzw. Effekte auftreten, und 5. Programmzertifizierung (program certification), um Informationen darüber zu gewinnen, welchen Wert das Projekt hat und das Potenzial für den Nutzen bzw. die Anwendungsmöglichkeiten in anderen Bereichen zu ermitteln. Die Evaluation dient also dazu, Informationen zu sammeln und sie Entscheidungsträgern bereit zu stellen, damit sie gute Entscheidungen treffen können. Dafür müssen die Evaluationsergebnisse für die Zielgruppe der Entscheidungsträger entsprechend aufbereitet bzw. dargestellt werden.

Weitere wichtige Beiträge im Bereich der managementorientierten Ansätze stammen nach Fitzpatrick, Sanders und Worthen (vgl. 2004, S. 93f) von Provus, der das „Discrepancy Evaluation Modell“ entwickelte (1971), und von Patton, der die "Utilization-Focused Evaluation“ prägte $(1978,1986,1997)$. Diese beiden Modelle wurden im Baummodell von Alkin und Christie (vgl. Unterkapitel 3.2.) dem Nutzungsast zugeordnet (vgl. Abschnitt 3.2.4). Der Nutzen der Evaluation soll demnach darin bestehen, dass die gewonnenen Informationen eine praktische Verwendung finden.

Als eine besondere Stärke von managementorientierten Ansätzen sehen Fitzpatrick, Sanders und Worthen (vgl. 2004, S. 95), dass die Evaluation einen klaren Fokus habe, da sie sich nach den Informationsbedürfnissen der Entscheidungsträger richte. Außerdem stehe der Nützlichkeitsaspekt der Evaluation im Vordergrund, was ebenfalls als Stärke bewertet wird. Darüber hinaus hätten managementorientierte Ansätze dazu beigetragen, dass Managern deutlich wurde, dass Evaluationen auch schon frühzeitig stattfinden können, zu Beginn und im Verlauf eines Projektes, und nicht nur bzw. erst am Ende. Nicht ausschließlich die Zielerreichung sei relevant, sondern auch die Programmplanung, -entwicklung und implementation, die mit einer Evaluation unterstützt werden kann.

Was als Stärke gesehen wird, kann zugleich eine Schwäche sein: Die Ausrichtung der Evaluation auf die Bedarfe der Entscheidungsträger, die zu einem klaren Fokus führt, kann auch beinhalten, dass andere bzw. wichtige Perspektiven außen vor bleiben. So könnten Bedarfe und Interessen von anderen Beteiligten unberücksichtigt bleiben oder sogar bewusst ausgeblendet werden. Es bestehe auch die Gefahr, dass Evaluatoren sich zu sehr von den Entscheidungsträgern und deren Interessen vereinnahmen oder sogar manipulieren lassen. Eine Verengung des Blickwinkels könne zur Verfälschung von Evaluationsergebnissen oder zumindest zu einer sehr eingeschränkten Darstellung führen, wenn wichtige Meinungen und Perspektiven von beteiligten Akteuren keine Berücksichtigung fänden. Die Komplexität von Programmen dürfe nicht unterschätzt werden, und auch wenn ein managementorientierter Ansatz von Evaluation umgesetzt werde, sollten verschiedene Akteure sowie deren Perspektiven und Meinungen einbezogen werden. 


\title{
3.3.3 Konsumentenorientierte Ansätze (consumer-oriented evaluation approaches)
}

Die Zielgruppen, die von Evaluationsergebnissen profitieren sollen, sind bei den konsumentenorientierten Ansätzen die Konsumenten, die Entscheidungshilfen bekommen, um Bildungsprodukte und deren Qualität beurteilen zu können. Nach Fitzpatrick, Sanders und Worthen (vgl. 2004, S. 100f) führte der stetig wachsende Markt von Bildungsangeboten ab den 1960er Jahren dazu, dass für Konsumenten unübersichtlich wurde, welche Angebote, Dienstleistungen, Produkte und Materialien gut seien. Zu Bildungsangeboten gehören z.B. Kurs- und Weiterbildungsprogramme, Curricula, Medien, Trainings und Bücher. Einige Evaluatoren begannen deshalb damit, unabhängige Bewertungssysteme zu entwickeln und bereit zu stellen wie z.B. Checklisten, mit denen Konsumenten selbstständig Produkte überprüfen konnten. Außerdem entstanden zahlreiche Veröffentlichungen und (Evaluations-) Berichte, in denen Produkte beurteilt bzw. bewertet wurden. Meistens wurden dabei summative Evaluationen durchgeführt (zu den Begriffen summative und formative Evaluation siehe Abschnitt 1.4.1).

\begin{abstract}
„As competition has grown in the educational and human services product industry, marketing strategies have become creative, but often they are not calculated to serve the best interest of the consumer or client. For this reason, some evaluators have actively urged consumer education, independent reviews of products patterned after the Consumer Union approach, and requirements for objective evidence of product effectiveness. Checklists for rating products and product evaluation reports are two typical outgrowths of this approach. The consumer-oriented approach to evaluation is predominantly a summative evaluation approach." (Fitzpatrick, Sanders und Worthen 2004, S. 101)
\end{abstract}

Als besonders wichtigen und einflussreichen Vertreter des konsumentenorientierten Ansatzes sehen Fitzpatrick, Sanders und Worthen (vgl. 2004, S. 101) Scriven, der eine Vielzahl von Checklisten und Bewertungskriterien entwickelte und zur freien Verfügung stellte. Mit seinen Arbeiten und Beiträgen (Scriven 1967, 1974, 1991b, 2002, 2007a) habe er wesentliche Beiträge geleistet, die der Bewertung von Bildungsprodukten dienen. Unter http://www.wmich.edu/evalctr/archive_checklists/papers/logic\&methodology_dec07.pdf (Zugriff 17.9.2012) beschreibt Scriven (2007b), wie Checklisten zu verstehen und anzuwenden sind, und unter http://www.wmich.edu/evalctr/archive_checklists/kec_feb07.pdf (Zugriff 17.9.2012) steht beispielsweise die „Key Evaluation Checklist“ bereit, um von Konsumenten verwendet zu werden (Scriven 2007a). In dem Evaluationsraster finden sich Kriterien, die als unverzichtbare Prüfkriterien formuliert wurden, um die Qualität von Bildungsprodukten zu überprüfen und zu erfassen. Auf den Webseiten des „Western Michigan University Evaluation Center" www.wmich.edu/evalctr/checklists (Zugriff 17.9.2012) finden sich zahlreiche Checklisten, die kostenlos verwendet werden können und sozusagen als „free service“ zur Verfügung stehen. Der konsumentenorientierte Ansatz von Evaluation wird in den USA ins- 
besondere von staatlichen Einrichtungen sowie unabhängigen verbraucherorientierten Einrichtungen verwendet.

Als weitere Bewertungssysteme und -angebote, die Verbrauchern bzw. Konsumenten Entscheidungshilfen über die Qualität von Bildungsprodukten bieten, nennen Fitzpatrick, Sanders und Worthen (vgl. 2004, S. 103) noch den „Educational Products Information Exchange“ (EPIE), der Mitte der 1960er Jahre von Komoski gegründet wurde und dessen Angebote bis heute bestehen. Auf der Webseite www.epie.org (Zugriff 17.9.2012) finden sich Informationen zu der Organisation EPIE, die als „nonprofit organisation“ Schulen und privaten Konsumenten Informationen und wissenschaftlich ermitteltes Wissen zur Verfügung stellt, um Lernmaterialien und Bildungsangebote besser beurteilen zu können. Auch neuere Technologien werden seit den 1990er Jahren einbezogen, und so finden sich unter http://www.elearningspace.org/ (Zugriff 17.9.2012) auch Informationen, Empfehlungen und Bewertungsmöglichkeiten für e-Learning Angebote. Darüber hinaus beschreiben Fitzpatrick, Sanders und Worthen (vgl. 2004, S. 103f) noch andere Checklisten und Systeme, die von Evaluatoren entwickelt und Konsumenten zur Verfügung gestellt werden.

Zu den Stärken der konsumentenorientierten Ansätze zählen Fitzpatrick, Sanders und Worthen (vgl. 2004, S. 108) vor allem, dass Evaluationsinstrumente wie Checklisten und Evaluationskriterien für Produkte und Dienstleistungen frei zur Verfügung gestellt werden für Personen (Konsumenten), die vielleicht nicht die Zeit oder das Wissen für eine fundierte Evaluation, die Entwicklung von Instrumenten oder Bewertungskriterien haben. Außerdem habe die Bereitstellung von Checklisten und Berichten dazu geführt, dass Konsumenten kritischer wurden und eine gute Informationsbasis haben, um Entscheidungen bzw. eine Auswahl auf dem Markt zu treffen. Die Anbieter von Bildungsprodukten und Dienstleistungen könnten Instrumente und damit ermittelte Ergebnisse auch zur Qualitätsverbesserung verwenden. Als Schwäche oder Kritik an den konsumentenorientierten Ansätzen äußern Fitzpatrick, Sanders und Worthen (vgl. 2004, S. 109), dass die Kosten für gute Produkte steigen könnten, da Produkttests aufwändig und mit Kosten verbunden sein könnten, die auf die Verbraucher zurückfallen. Außerdem könnten festgelegte Standards und Bewertungskriterien die Entwicklung von neuen, kreativen Angeboten hemmen oder von regionalen Produkten, die durch entstehende Kosten und den hohen Aufwand von Evaluationen nicht überprüft werden könnten und deshalb durch überregionale, getestete Produkte verdrängt werden könnten. Die Schwächen von konsumentenorientierten Ansätzen bewerten Fitzpatrick, Sanders und Worthen (vgl. 2004, S. 109) aber als sehr gering. Sie weisen darauf hin, dass durch Tests und Checklisten der Druck für Anbieter nicht zu groß werden sollte, sondern diese durch solche Instrumente auch unterstützt werden könnten, gute Produkte und Dienstleistungen zu entwickeln, die den Bedürfnissen der Konsumenten gerecht werden. 


\subsubsection{Expertenorientierte Ansätze (expertise-oriented evaluation approa- ches)}

Bei den expertenorientierten Ansätzen von Evaluation beurteilen Experten oder ein Team von Experten eine Institution, ein Programm oder Produkt oder aber auch eine Aktivität.

„The expertise-oriented approach to evaluation, probably the oldest and most widely used, depends primarily on professional expertise to judge an institution, program, product or activity." (Fitzpatrick, Sanders und Worthen 2004, S. 112)

Obwohl in allen bisher beschriebenen Ansätzen professionelle Beurteilungen von Experten eine Rolle gespielt haben, betonen Fitzpatrick, Sanders und Worthen (vgl. 2004, S. 113), dass bei den expertenorientierten Ansätzen die direkte und primäre Evaluationsstrategie von den Experten festgelegt und umgesetzt werden. Sachverhalte werden prioritär von Experten oder Teams bewertet. Sie nennen verschiedene Varianten und Beispiele solcher Evaluationsprozesse, in denen Experten Beurteilungen treffen (vgl. ebenda), wie z.B. auch Promotionsverfahren, die von einem Komitee begleitet werden. Um diese Vielfalt zu ordnen, entwickeln Fitzpatrick, Sanders und Worthen (vgl. 2004, S. 113) vier Typen von expertenorientierten Evaluationen: 1. Formale Review-Verfahren, 2. Informelle Review-Verfahren, 3. Ad-hocPanel-Reviews und 4. Ad-hoc-Individual-Reviews und differenzieren sie nach fünf Kategorien, wie die folgende Tabelle zeigt:

\begin{tabular}{|l|l|l|l|l|l|}
\hline $\begin{array}{l}\text { Typ expertenorien- } \\
\text { tierter Evaluation }\end{array}$ & $\begin{array}{l}\text { Vorhandene } \\
\text { Struktur } \\
\text { Standards } \\
\text { Reröffentlichte }\end{array}$ & $\begin{array}{l}\text { Festgelegter } \\
\text { Rhythmus } \\
\text { Verfahren }\end{array}$ & $\begin{array}{l}\text { Meinung ver- } \\
\text { schiedener } \\
\text { Experten }\end{array}$ & $\begin{array}{l}\text { Beeinflussbarkeit } \\
\text { durch Ergebnis- } \\
\text { se }\end{array}$ \\
\hline $\begin{array}{l}\text { Informelle Review- } \\
\text { Verfahren }\end{array}$ & $\mathrm{Ja}$ & $\mathrm{Ja}$ & $\mathrm{Ja}$ & Ja & Meistens \\
\hline $\begin{array}{l}\text { Ad-hoc-Panel- } \\
\text { Reviews }\end{array}$ & Nein & Nein & Nein & Ja & Meistens \\
\hline $\begin{array}{l}\text { Ad-hoc-Individual- } \\
\text { Reviews }\end{array}$ & Nein & Nein & Nein & Nein & Manchmal \\
\hline
\end{tabular}

Tabelle 8: $\quad$ Typen Expertenorientierter Evaluation nach Fitzpatrick, Sanders und Worthen (übersetzt von der Verfasserin)

Formale Review-Verfahren sind nach Fitzpatrick, Sanders und Worthen (vgl. 2004, S. 114) dadurch gekennzeichnet, dass 1. Eine Struktur oder Organisation vorhanden ist, um Reviews periodisch durchzuführen, 2. Standards veröffentlicht sind (und mögliche Instrumente vorhanden sind), die in den Reviews gelten bzw. verwendet werden, 3. Ein festgelegter Rhythmus vereinbart ist, wann die Reviews durchgeführt werden (z.B. alle fünf Jahre), 4. Meinungen von verschiedenen Experten zusammengeführt werden, um zu einem Gesamt- 
werturteil zu kommen und 5. Die Ergebnisse einen Effekt bzw. Auswirkungen auf den Sachverhalt (oder die Institution) haben, der bzw. die evaluiert wird.

Als bekannteste formale Review-Verfahren nennen Fitzpatrick, Sanders und Worthen (vgl. ebenda) Akkreditierungsverfahren, durch die Institutionen wie etwa Schulen, Universitäten oder Krankenhäuser Auszeichnungen oder Befürwortungen (approval) erfahren. Akkreditierungsverfahren und Organisationen, die diese durchführen, gibt es bereits seit den späten 1800er Jahren. Im Bildungsbereich haben Akkreditierungsverfahren eine lange Tradition und sind auch in der heutigen Zeit hochaktuell (beispielsweise das Hochschulakkreditierungssystem in Deutschland, aber auch europaweit und international betrachtet haben Akkreditierungssysteme eine große Bedeutung).

Informelle Review-Verfahren haben nicht so klare Regelvorgaben und Standards als Grundlage wie formale Review-Verfahren. Es finden sich keine zuvor festgelegten Ablaufpläne oder es sind keine periodisch wiederkehrenden Verfahren vorgesehen. Fitzpatrick, Sanders und Worthen (vgl. 2004, S. 118) nennen als Beispiel einen Evaluationsauftrag, der von der Landesregierung an das "state department of education“ vergeben wurde und in dessen Rahmen über einen Zeitraum von zwei Dekaden alle Programme und Projekte an Schulen evaluiert werden sollten, die eine spezielle Förderung erhielten. Das Evaluationsteam entwickelte ein eigenes Evaluationssystem, Evaluationsverfahren und Standards ohne zuvor festgelegte Strukturen oder Vorgaben. Als weiteres Beispiel informeller Review-Verfahren führen Fitzpatrick, Sanders und Worthen (vgl. ebenda) Promotionskollegs an, in denen selbst gesteuerte Reviews - also Besprechungen und Klärungen sowie Bewertungen und Beurteilungen von Experten - stattfinden sowie „peer reviews“, in denen Fachleute gegenseitig beispielsweise Artikel oder Manuskripte begutachten bzw. bewerten.

Bei "Ad-hoc-Panel-Reviews" finden häufig nur einmal Evaluationen statt, die von Experten durchgeführt werden für einen bestimmten Informationsbedarf oder anstehende Entscheidungen. Es liegen dabei keine festen (institutionellen) Rahmenbedingungen oder Standards für die Evaluation vor. Beispielsweise bei der Vergabe von Fördermitteln finden solche Begutachtungen von Projektanträgen oder Ideen statt, die von einem Ausschuss oder einem Komitee geleitet bzw. durchgeführt werden. Experten, die aufgrund ihrer Erfahrung und ihres Wissens ausgewählt wurden, führen dann die Evaluation durch.

Zu „Ad-hoc-Individual-Reviews” zählen Fitzpatrick, Sanders und Worthen (vgl. 2004, S. 120) Begutachtungen oder Kritiken von einzelnen Experten, die in einem bestimmten Kontext ihr Fachurteil abgeben wie z.B. Theaterkritiken, Kunst- oder Literaturkritiken. Auch bei der Evaluation von Bildungsprodukten kann die Meinung und das Fachwissen einzelner Experten dazu eingesetzt oder gefordert werden, um etwa Trainingsprogramme, Medien oder Tests zu bewerten.

Zu den Stärken der expertenorientierten Evaluationsansätze gehört nach Fitzpatrick, Sanders und Worthen (vgl. 2004, S. 123) die Betonung darauf, dass für die Durchführung von 
Evaluationsprozessen Erfahrung und (Experten-)Wissen erforderlich und die Entwicklung von Standards und Beurteilungskriterien wichtig sind. Außerdem seien bei Akkreditierungsverfahren häufig auch anteilig Selbstevaluationen vorgesehen, die den Beteiligten eine intensive Auseinandersetzung mit den eigenen Strukturen und Prozessen erlauben. Meistens kämen formative und summative Evaluationen vor, mit deren Ergebnissen die Stärken und Schwächen deutlich würden, und die es ermöglichten, Verbesserungen zu planen und umzusetzen.

Als Schwächen von expertenorientierten Ansätzen sehen Fitzpatrick, Sanders und Worthen (vgl. 2004, S. 123f) vor allem, dass den Experten ein Wissen und Kompetenzen unterstellt werden, das bzw. die sie womöglich gar nicht besitzen. Außerdem können Experten auch „Fehler machen“ oder zu falschen Einschätzungen oder Beurteilungen kommen oder eigene Interessen verfolgen, die nicht im Sinne der Auftraggeber sind, für die sie arbeiten oder deren Interessen sie vertreten sollten. Darüber hinaus wird kritisiert, dass Expertenreviews sehr kosten- und zeitintensiv sein können, insbesondere, wenn mehrere Evaluatoren (Expertenteams) zum Einsatz kommen. Nicht alle Institutionen könnten sich dies leisten.

\title{
3.3.5 Partizipative Ansätze (participant-oriented evaluation approaches)
}

Den Hintergrund der Entstehung von partizipativen Ansätzen bildet nach Fitzpatrick, Sanders und Worthen (vgl. 2004, S. 130) eine bereits Ende der 1960er Jahre aufkommende Kritik an Methoden und Verfahren, die in den Sozialwissenschaften zu verzeichnen ist. In der Erziehungswissenschaftlichen Forschung waren Methoden verbreitet, die in der Evaluationsforschung einfach übernommen wurden und genau dies wurde von einigen Autoren bemängelt bzw. hinterfragt. Sie waren der Meinung, dass Evaluatoren so sehr damit beschäftigt seien, Ziele zu klassifizieren, aufwändige Evaluationsdesigns zu entwerfen, Instrumente zu entwickeln und technisch anspruchsvolle und komplizierte Berichte zu verfassen, dass sie weit entfernt von den Realitäten der Programme, deren Teilnehmern und Interessen waren:

\begin{abstract}
„As early as 1967, several evaluation theorists began to react to what they considered to be the dominance of mechanistic and insensitive approaches to evaluation in the field of education. These theorists expressed concerns that evaluators were largely preoccupied with stating and classifying objectives, designing elaborate evaluation systems, developing technically defensible objective instrumentation, and preparing long technical reports, with the result that evaluators were distracted from what was really happening in the programs they evaluated." (Fitzpatrick, Sanders und Worthen 2004, S. 130)
\end{abstract}

Die Einwände führten zu einer umfangreichen Diskussion in der Literatur und zur Entwicklung neuer Ansätze zur Evaluation im Bildungsbereich und auch in anderen Gebieten (z.B. in der Entwicklungsforschung). Es entstand geradezu eine neue Bewegung, in der die Praxisnähe bzw. der direkte Bezug zu den erforschten und evaluierten Feldern, d.h. Programmen, Maßnahmen und Aktivitäten und Menschen, als sehr wichtig oder sogar unabdingbar ange- 
sehen wurde. Die Evaluatoren sollten bei ihrer Arbeit möglichst umfassend die Perspektiven aller Beteiligten beobachten, erfassen und einbeziehen, um dann ein ganzheitliches „Bild“ zu zeichnen, wobei sie intuitiv und nicht nach einem festgelegten Plan vorgehen sollten:

„The evaluator portrays the different values and needs of individuals and groups served by the program, weighing and balancing plurality of judgements and criteria in a largely intuitive fashion." (Fitzpatrick, Sanders und Worthen 2004, S. 130)

Die partizipativen Ansätze von Evaluation wurden nach Fitzpatrick, Sanders und Worthen (vgl. 2004, S. 130) am intensivsten in der Literatur diskutiert, und es findet sich eine Vielfalt von Herangehensweisen in diesem Rahmen. Das Spektrum reicht dabei von Ansätzen, bei denen verschiedene Beteiligte (participants) in die Evaluation einbezogen werden, bis hin zu Ansätzen, in denen die Teilnehmenden eines Programms oder die Zielgruppen befähigt werden, eigenständig Evaluationen durchzuführen. Die Unterscheidung von „partizipativen Ansätzen der Evaluation“ bis hin zu „participant-oriented approaches“ ist dabei wichtig, denn der Grad der Beteiligung ist nicht in allen Ansätzen gleich hoch.

Als ersten und wichtigsten Vertreter sehen Fitzpatrick, Sanders und Worthen (vgl. 2004, S. 131) Stake und sein Modell der „Responsiven Evaluation“ (siehe auch Abschnitt 3.1.4). Mit seinem Artikel „The Countenance of Educational Evaluation“ (Stake 1967) gab er einen wesentlichen Impuls zur Diskussion und leistete damit einen wichtigen Beitrag zur Entstehung von Konzeptionen und Prinzipien neuer Evaluationsansätze. Auch mit seinen späteren Arbeiten trug er zur Weiterentwicklung von Verfahren bei, bei denen Beteiligte bzw. am evaluierten Programm Teilnehmende einbezogen wurden (Stake 1969, 1972, 1975b, 1978, 1980, 1994, 1995, 2000). In dem Modell der „Responsiven Evaluation“ geht es darum, die Interessen und Bedürfnisse der an einem Programm Beteiligten zu erfassen und ihnen gerecht zu werden. Der Evaluator gibt nicht einfach vor, was und mit welchen Methoden oder Instrumenten evaluiert wird, sondern verhält sich „responsiv“, d.h. er reagiert auf die Teilnehmer und deren Belange, stellt sich darauf ein und entwickelt gemeinsam mit innen ein Evaluationsdesign, das zum Praxisfeld (den Menschen, Aktionen und Maßnahmen) passt. Er richtet sich nach der Programmrealität und den direkt Beteiligten und nicht nach den in Berichten oder Beschreibungen formulierten Zielen. Es kommen dabei vor allem qualitative Methoden zum Einsatz, wie etwa Fallstudien. Hierbei bilden kleinere Fallzahlen, die dafür aber ausführlicher analysiert werden, die Grundlage für ein umfassendes Bild (portray) vom Evaluationsfeld sowie ein Feedback an die beteiligten Akteure. Es sei besonders wichtig, dass der Evaluator eine gewisse Flexibilität mitbringe, um sich auf das Praxisfeld, das er evaluiert, einzulassen. Zur Methodologie von Fallstudien hat Stake (vgl. 1978, 1981, 1988, 1994, 1995) ebenfalls einige Diskussionsbeiträge geliefert. In den 1980er Jahren finden sich auch eine Reihe von Beiträgen zur „naturalistischen Evaluation“ (vgl. z.B. Guba und Lincoln 1981, 1989, Lincoln \& Guba 1985), die sich an die Arbeiten von Stake anlehnen bzw. diese aufgreifen. Der Evaluator wird in diesen Ansätzen als "Lerner" gesehen und die Evaluierten als 
"Lehrer", die dem Evaluator beibringen, was in dem evaluierten Feld wichtig ist. Die Evaluierten geben dem Evaluator die wichtigen Informationen zum Evaluationsgegenstand, die dieser sammelt, zusammenstellt und auswertet. Auf diese Weise „verstehe“ der Evaluator nach und nach das Feld oder den Gegenstand, den er evaluiert, lerne etwas über die Perspektiven und die „Welt“ der Programmteilnehmenden kennen. Bei dieser Vorgehensweise werden Forschungsmethoden eingesetzt, die zum Evaluationsfeld passen, der primäre Fokus liegt nicht auf Gütekriterien wie Validität oder Reliabilität, sondern eher darauf, dass die Methoden angemessen und passend sind. Bevorzugt werden qualitative Methoden und nicht-reaktive Verfahren (unobtrusive measurement) verwendet. Häufig kommen auch mehrere Methoden zum Einsatz, und die erhobenen Daten werden miteinander kombiniert (cross-checking und Triangulation). Zu dem Ansatz von Guba und Lincoln siehe auch Unterkapitel 3.1 bzw. Abschnitt 3.1.4.

Als weitere Autoren, die Beiträge zu partizipativen Evaluationsansätzen geliefert haben, zählen Fitzpatrick, Sanders und Worthen (vgl. 2004, S. 141f) Cousins und Earl $(1992,1995)$ und deren Modell der „Participatory Evaluation“. Dieses Modell wurde bereits in Abschnitt 3.2.4 beschrieben. Das Wesentliche an diesem Ansatz ist, dass geschulte Evaluatoren und Entscheidungsträger aus der Praxis (dem evaluierten Programm) quasi eine Partnerschaft eingehen, zusammen arbeiten und so eine Praxisrelevanz und ein Nutzen der Ergebnisse gewährleistet werden soll. Vor allem bei formativen Evaluationen, die das Ziel der Verbesserung von Programmen haben, lohne sich dieses Vorgehen. Es würden mittels der Evaluation Probleme gelöst und optimale Lösungen gefunden. Es gehe dabei nicht um Theorieentwicklung oder die Stärkung einzelner Programmbeteiligter, sondern vor allem darum, eine bestmögliche Qualität von Projekten zu unterstützen, indem Entscheidungen von den Evaluatoren und bestimmten, ausgewählten Programmbeteiligten bzw. Programmverantwortlichen oder Projektmanagern auf der Basis der Evaluationsergebnisse getroffen werden.

Außerdem nennen Fitzpatrick, Sanders und Worthen (vgl. 2004, S. 143) das Modell der „Utilization-Focused Evaluation“ von Patton bei den Beispielen für partizipative Evaluationsansätze. Der Nutzen und die Verwendbarkeit der Evaluationsergebnisse seien hier klare Ziele, wobei die potenziellen Nutzer in die Planung der Evaluation, die Auswahl von Erhebungsmethoden, die Erhebung von Daten sowie die Auswertung einbezogen würden. Die Evaluatoren hätten die Verantwortung für den Prozess der Evaluation. Das Modell von Patton wurde ebenfalls in Abschnitt 3.2.4 bereits beschrieben.

Als einen Ansatz der „participant oriented approaches“ beschreiben Fitzpatrick, Sanders und Worthen (vgl. 2004, S. 143) die „Empowerment Evaluation“, die u.a. von Fetterman (1994, 2000, 2004) sowie Fetterman, Kaftarian und Wandersman (1996) entscheidend geprägt wurde. Dieser Ansatz geht über die Beteiligung von Akteuren, wie sie bei den partizipativen Ansätzen gefordert und vorgesehen ist, hinaus. Das Konzept der „Empowerment Evaluation“ hat seine Wurzeln in der Aktionsforschung, die in den 1980er Jahren als ein intensiv diskutiertes neues Paradigma in verschiedenen Disziplinen eingeführt wurde. Erforschte sollten 
dabei nicht nur beteiligt, sondern gleichberechtigt in die Forschung einbezogen werden. Im Kontext von Evaluation sollte der Evaluator als Vertreter oder sogar Anwalt der Evaluierten (Benachteiligten, Minderheiten) fungieren und den Akteuren zu mehr Selbstbestimmung und Selbstverantwortung verhelfen:

\begin{abstract}
"[...] evaluators should not only facilitate citizen partizipation in evaluation, but also become advocates for societies` disenfranchised and voiceless minorities. Concerns of advocacy-inclined evaluators about involvement, participation, and empowerment echo the strong concerns expressed in other disciplines and social sectors over stakeholders' participation, advocacy, social actions, and teamwork." (Rappaport, 1987, zitiert nach Fitzpatrick, Sanders \& Worthen 2004, S. 143)
\end{abstract}

So geht es bei der "Empowerment Evaluation" auch vor allem darum, die Kompetenzen der Beteiligten so zu stärken, dass sie befähigt werden, ihre Situation selbst zu verbessern (Hilfe zur Selbsthilfe). Die klassischen Gütekriterien Validität, Reliabilität und Objektivität rücken dabei in den Hintergrund. Es wird davon ausgegangen, dass Wissenschaft und insbesondere Evaluationen sowieso nicht „neutral“ sein können und es eine allgemeingültige Wahrheit auch nicht gebe. Vielmehr sind die Perspektiven und Belange der Akteure, deren Probleme und Interessen bedeutsam. Die Evaluatoren unterstützen die Akteure mittels ihres Wissens und ihrer Erfahrungen, selbst bestimmt und verantwortungsbewusst zu neuen Erkenntnissen zu gelangen und Verbesserungen zu bewirken. Es kommen verschiedene Evaluationsmethoden (qualitative und quantitative gleichermaßen) zum Einsatz. Meistens geht es um die Evaluation von Programmen.

\begin{abstract}
„Empowerment evaluation is the use of evaluation concepts, techniques, and findings to foster improvement and self-determination. It employs both qualitative and quantitative methodologies. Although it can be applied to individuals, organizations (at both intra- and extraorganizational levels), communities, and societies or cultures, the focus is on programs." (Fetterman 1996, S. 4)
\end{abstract}

Die Facetten des Empowerment Ansatzes nach Fetterman (1996, S. 9f) lassen sich wie folgt zusammenfassen:

\title{
1. Ausbildung (training)
}

Die Evaluatoren bringen den Akteuren bei, selbstständig Evaluationen durchführen zu können, wodurch diese neben dem Wissenserwerb und Kompetenzzuwachs auch mehr Selbstvertrauen erhalten und autark werden. Evaluation wird „entmystifiziert“ und kann zu einem festen, selbstverständlichen Teil von Programmplanung und Programmdurchführung werden. 


\section{Förderung (facilitation)}

Die Evaluatoren wirken als Helfer oder Berater und helfen den Akteuren, selbstständig Evaluationen durchzuführen. Sie fördern und befähigen die Programmbeteiligten, indem sie ihnen Informationen geben, Wissen und Erfahrungen vermitteln.

\section{Fürsprache (advocacy)}

Evaluatoren dienen darüber hinaus als „Anwälte“ der Akteure oder Gruppen, die sie begleiten und helfen ggf. bei der Vertretung ihrer Interessen und Äußerung ihrer Belange (z.B. bei Benachteiligten oder Minderheiten).

\section{Erkenntnisgewinn (illumination)}

Durch den Prozess der Evaluation und das Streben nach Verbesserung gelangen die Beteiligten zu neuen Erkenntnissen und Perspektiven. Sie erhalten neue Einblicke und haben die Möglichkeit zu lernen, Strukturen, Rollen und die Dynamik von Programmen zu verstehen. Dies kann eine vollkommen neue Erfahrung sein: „Illumination is an eye-opening, revealing, and enlightning experience“ (Fetterman 1996, S. 15).

\section{Befreiung (liberation)}

Durch den Erkenntnisgewinn wird die Selbstbestimmung unterstützt. Die eigene Rolle und die Rollen von anderen werden reflektiert. Es besteht dadurch die Möglichkeit, dass sich die Beteiligten aus bestehenden Rollen lösen und von damit verbundenen Erwartungen befreien. Sie können Verantwortung für sich selbst übernehmen, ihre Ressourcen in einem neuen Licht sehen und ihre Identität und zukünftige Rolle neu definieren.

Nach Fitzpatrick, Sanders und Worthen (vgl. 2004, S. 146) wurden die partizipativen bzw. teilnehmerorientierten Evaluationsansätze besonders heftig und kontrovers diskutiert. Die Gegner bemängeln die geringen Ansprüche an Wissenschaftlichkeit (z.B. die Berücksichtigung und Einhaltung von Gütekriterien), und die Befürworter betonen die Flexibilität und Vielfalt der Möglichkeiten sowie die Fokussierung auf die Bedürfnisse von Programmbeteiligten in der Praxis.

Zu den Stärken des Ansatzes werden die Beachtung von „menschlichen Komponenten“ und die Einbeziehung der Vielfalt von Perspektiven, die der Komplexität in Evaluationsfeldern gerecht werden, gezählt. Außerdem komme eine große Bandbreite von Methoden zum Einsatz. Obwohl die Vertreter der partizipativen Ansätze betonen, dass qualitative und quantitative Methoden verwendet werden, liegt der Schwerpunkt doch meist auf qualitativen Verfahren. Der Dialog mit den Praxisakteuren werde gesucht und gepflegt, wodurch das voneinander Lernen und die Einsicht in unterschiedliche Perspektiven gefördert werden. Bei den Em- 
powerment Ansätzen werde die Selbstbestimmtheit und Autonomie der Programmbeteiligten gestärkt und die Kompetenzbildung unterstützt.

Als Schwächen werden die Vernachlässigung von wissenschaftlichen Standards und Regeln (z.B. Gütekriterien) und die hohe Subjektivität bei der Vorgehensweise gesehen. Die Evaluatoren würden ihrer Aufgabe, Wertungen vorzunehmen, nicht nachkommen und die Verantwortung stattdessen an die Programmbeteiligten abgeben. Die Vielfalt von möglichen Rollen des Evaluators könne zu Unklarheiten und Verwirrungen führen. Außerdem seien mit Evaluationen dieser Art oft hohe Kosten verbunden, monetär wie zeitlich gesehen. Der Evaluator müsse viel Präsenz zeigen und ein Programm von Anfang bis Ende begleiten, was zu hohen Kosten führen könne. Deshalb würden häufig nur kleine Studien mit geringer Fallzahl durchgeführt, was die Aussagekraft der Ergebnisse einschränkt und eine geringe oder sogar fehlende Repräsentativität der Befunde zur Folge habe. Darüber hinaus können Evaluatoren politisch motiviert oder befangen sein und z.B. bestimmte Gruppen oder Minderheiten vertreten, wobei sie dann ihre Neutralität, die sie als Forscher eigentlich haben müssten, aufgeben. Wenn Programmbeteiligte, wie beispielsweise in dem Empowerment Modell vorgesehen, befähigt werden, Evaluationen selbstständig durchzuführen, besteht nach Auffassung der Kritiker teilnehmerorientierter Ansätze die Gefahr, dass Evaluationen zu sehr vereinfacht und unreflektiert durchgeführt werden könnten.

Zusammenfassend kann festgehalten werden, dass Fitzpatrick, Sanders und Worthen in ihrem Ordnungsmodell verschiedene Evaluationsansätze nach deren primärem Nutzen, nach ihrer praktischen Ausrichtung und den Zielen, die damit verbunden sind, ordnen. Sie stellen die fünf verschiedenen Ansätze nebeneinander dar, wobei sie keine Wertung vornehmen, sondern jeder Ansatz für sich stehen kann. Sie arbeiten zwar auch die Stärken und Schwächen der einzelnen Ansätze heraus, aber bleiben dabei neutral. Dennoch bleibt offen, wie trennscharf die Einteilung ist, ob die einzelnen Ansätze sich wirklich immer so eindeutig voneinander unterscheiden lassen, oder ob es nicht auch Mischformen in Bezug auf den Nutzen einer Evaluation gibt. 


\section{Evaluation im Bildungsbereich}

In den vorangegangenen Kapiteln wurde dargestellt, wie vielschichtig der Begriff der Evaluation ist (Unterkapitel 1.1), dass Evaluationen in sehr unterschiedlichen Bereichen stattfinden und sich auf vielfältige Gegenstände beziehen können (Unterkapitel 1.2), dass Evaluationen verschiedene Funktionen haben und die Ziele variieren können (Unterkapitel 1.3 ), sowie dass es mehrere Formen und Arten von Evaluation gibt - je nach Zeitpunkt der Durchführung, den Evaluierenden und dem Betrachtungsfokus (Unterkapitel 1.4). Außerdem wurde die historische Entwicklung der Evaluationsforschung in den USA und in Europa beschrieben und der aktuelle Stand der Evaluation erläutert (Kapitel 2). Anschließend wurden verschiedene Evaluationsansätze und -theorien aufgegriffen und erörtert (Kapitel 3).

Mit den vorangegangenen Kapiteln wird deutlich, wie komplex das Praxisfeld Evaluation ist, und wie vielfältig die theoretischen Hintergründe sind. Es zeigt sich auch, dass es sehr unterschiedliche Verständnisse von Evaluation gibt in Bezug auf deren Funktionen und Ziele. Evaluationen finden immer in einem bestimmten Kontext statt, der verschiedene Vorgehensweisen, Methoden und Spezifika wie z.B. auch den Umgang mit Ergebnissen mit sich bringt. In den unterschiedlichen Bereichen finden sich entsprechende Fachliteratur und Diskussionen (siehe auf nationaler Ebene beispielsweise die Arbeitskreise der DeGEval: http://www.degeval.de/arbeitskreise, Zugriff 25.9.2012).

In diesem Kapitel wird nun der Bezug zur Pädagogik und zum Bildungsbereich hergestellt. Mit dem Begriff „Bildungsbereich“ ist das Bildungssystem gemeint, d.h. die Gesamtheit der Einrichtungen und Möglichkeiten der Bildung (Kindergarten, Schule, Hochschule und Ausund Weiterbildung). Im Zentrum der vorliegenden Arbeit stehen Bildungsprojekte, und deshalb soll dieser Bezugsrahmen im Folgenden näher betrachtet werden. Zunächst werden Arbeitsfelder der Erziehungswissenschaft und pädagogische Handlungsfelder dargestellt (Unterkapitel 4.1) und die Spezifika der Evaluation von Bildung bzw. in pädagogischen Handlungsfeldern erörtert (Unterkapitel 4.2). Anschließend wird das Theorie-Praxis Dilemma erläutert (Unterkapitel 4.3), um darauf aufbauend im Kapitel 5 das Modell von Inventaren als Instrumente zur Unterstützung und Verbesserung der Evaluationspraxis in Bildungsprojekten zu entwickeln und zu erproben.

\subsection{Arbeitsfelder der Erziehungswissenschaft und pädagogische Handlungsfelder}

In diesem Unterkapitel wird zunächst der Frage nachgegangen, welche Arbeitsfelder sich in der Erziehungswissenschaft bzw. der Pädagogik finden. Es scheint eine Fülle von Themen und Gebieten zu geben, wenn man in Einführungswerken zur Erziehungswissenschaft oder 
zur Pädagogik die Inhaltsverzeichnisse anschaut. Zuerst fällt auf, dass einmal von Erziehungswissenschaft und ein anderes Mal von Pädagogik gesprochen wird. Alltagssprachlich betrachtet sei dies nach Lenzen nur mit unterschiedlichen Assoziationen zu den Begriffen verbunden:

„Im alltäglichen Sprachgebrauch hat sich für den Unterschied zwischen Erziehungswissenschaft und Pädagogik eine markante Differenz herausgebildet. So assoziieren mit <Erziehungswissenschaft> viele Zeitgenossen: Forschung, Präzision, dicke Bücher, Langeweile und Irrelevanz. Dementsprechend steht <Pädagogik > für manche besser da: Wirklichkeit, Leben, Praxis, Wohltat und Engagement im Gegensatz zu Nüchternheit und Interessenlosigkeit. Diese Unterscheidung ist nicht zufällig, aber sie ist gleichzeitig auch <nur> alltagssprachlich." (Lenzen 1994, S.13)

Betrachtet man die Entwicklung des Fachs im deutschen Sprach- und Kulturraum, so ist diese Unterscheidung nach Lenzen (vgl. 1994, S.14) jedoch historisch entstanden. Das Fach habe seit seiner Entstehung im 18. Jahrhundert immer den Namen Pädagogik gehabt, bis zu Beginn des 20. Jahrhunderts die Bezeichnung Erziehungswissenschaft aufkam, um sich dann in den 1960er Jahren endgültig durchzusetzen. Mit dem Begriff der Erziehungswissenschaft verbinde sich das Vorhaben, mittels empirischer Methoden die „Erziehungswirklichkeit“ zu untersuchen, um die Ergebnisse für die „pädagogische Praxis“ zur Verfügung zu stellen, während es bei der Pädagogik eher darum gehe, das pädagogische Handeln anzuleiten, zu reflektieren und zu diskutieren, wobei eher hermeneutische Methoden zum Einsatz kommen.

\begin{abstract}
„Historisch betrachtet, stehen sich also in den beiden Bezeichnungen für das Fach zwei grundlegend verschiedene Programme gegenüber: auf der einen Seite die ältere Pädagogik mit ihrem zumindest teilweise vertretenen Anspruch, pädagogisches Handeln anleiten zu wollen oder doch zumindest die Normen, Orientierungen, Standards dieses Handelns der Reflexion, Diskussion und Auseinandersetzung zuzuführen; auf der anderen Seite die Erziehungswissenschaft, deren programmatisches Selbstverständnis gerade nicht auf eine normative Beeinflussung pädagogischen Handelns aus sein sollte, sondern der es um die Untersuchung von <Gesetzmäßigkeiten> erzieherischer Prozesse ging." (Lenzen 1994, S.14)
\end{abstract}

Mit diesen Zitaten wird bereits deutlich, dass es zum einen das Nachdenken über Erziehung und damit verbundene Prozesse, das Erforschen von „Erziehungswirklichkeit“ mittels empirischer Methoden sowie die Theoriebildung zu deren Gesetzmäßigkeiten gibt. Dieser „Theoriebereich“ umfasst eine Vielzahl von Themengebieten und kann auch als „Arbeitsfelder der Erziehungswissenschaft“ bezeichnet werden. Zum anderen gibt es die „pädagogischen Handlungsfelder", in denen es konkret und ganz praktisch um die Erziehung und das Lernen von Kindern, Jugendlichen und Erwachsenen geht. Auch Giesecke weist darauf hin, dass der Begriff Pädagogik zwei Aspekte beinhaltet:

„Der Begriff 'Pädagogik' ist doppeldeutig; er meint einmal eine Praxis, nämlich das Handeln derjenigen, die mit Kindern und Jugendlichen umzugehen haben. Er meint aber auch das Nachdenken über dieses Handeln." (Giesecke 1994, S.9) 
Mit diesem Zitat wird deutlich, dass zwischen Theorie und Forschung auf der einen und Handlungen, Praxis auf der anderen Seite differenziert werden kann.

In der Zeitschrift für Pädagogik werden „Forschungs- und Handlungsfelder der Pädagogik“ behandelt (vgl. Fatke 1997). Auch hier zeigt sich, dass Forschung und Handlung, Theorie und Praxis in der Pädagogik eng verbunden sind. Die „Theorie-Praxis-Diskussion“ ist in der Erziehungswissenschaft schon lange vorhanden (vgl. z.B. dazu auch Blankertz 1978) und immer noch aktuell:

„Eines der zentralen Themen der Erziehungswissenschaft ist das Theorie-

Praxis-Verhältnis." (Kuper 2005, S.14)

Vielfach wird die Pädagogik auch als eine "Theorie der Praxis“ (Kümmel, 1978, S.121) bezeichnet und als „Reflexions- und Handlungswissenschaft“ (ebenda, S.121).

An dieser Stelle soll nun nicht auf die historische Entwicklung des Faches und die theoretischen Strömungen eingegangen werden (siehe dazu z.B. Krüger 1999), vielmehr wird hier auf die Vielfalt der Themenfelder hingewiesen, um zu verdeutlichen, wie breit das Spektrum der Pädagogik bzw. der Erziehungswissenschaft ist. Nach Lenzen (1994, S.37) ist die gegenwärtige Situation „nicht nur durch einen Pluralismus der Theorien, sondern auch durch eine Vielfalt der Fachrichtungen gekennzeichnet". Lenzen (ebenda, S.38) stellt die Struktur des Fachs in drei unterschiedlichen Ebenen dar: Subdisziplinen, Fachrichtungen und Praxisfelder. Die Subdisziplinen sind „Fachelemente, die seit vielen Jahrzehnten bestehen, über eigene Institute verfügen, häufig über eigene Studiengänge, für die es eigene Lehrbefähigungen der Professoren gibt, zum Teil eigene wissenschaftliche Gesellschaften“. Die Fachrichtungen sind ,jünger“ und mit innen werde versucht, auf länger anhaltende gesellschaftliche Probleme zu reagieren. Teilweise gibt es zu diesen Fachrichtungen bereits Subdisziplinen oder diese entstehen noch. Die dritte Ebene der Praxisfelder ist die „Anwendungsebene“. Teilweise gibt es zu den Praxisfeldern passende Fachrichtungen oder sogar Subdisziplinen. In den pädagogischen Praxisfeldern werden „professionelle Pädagogen“ tätig.

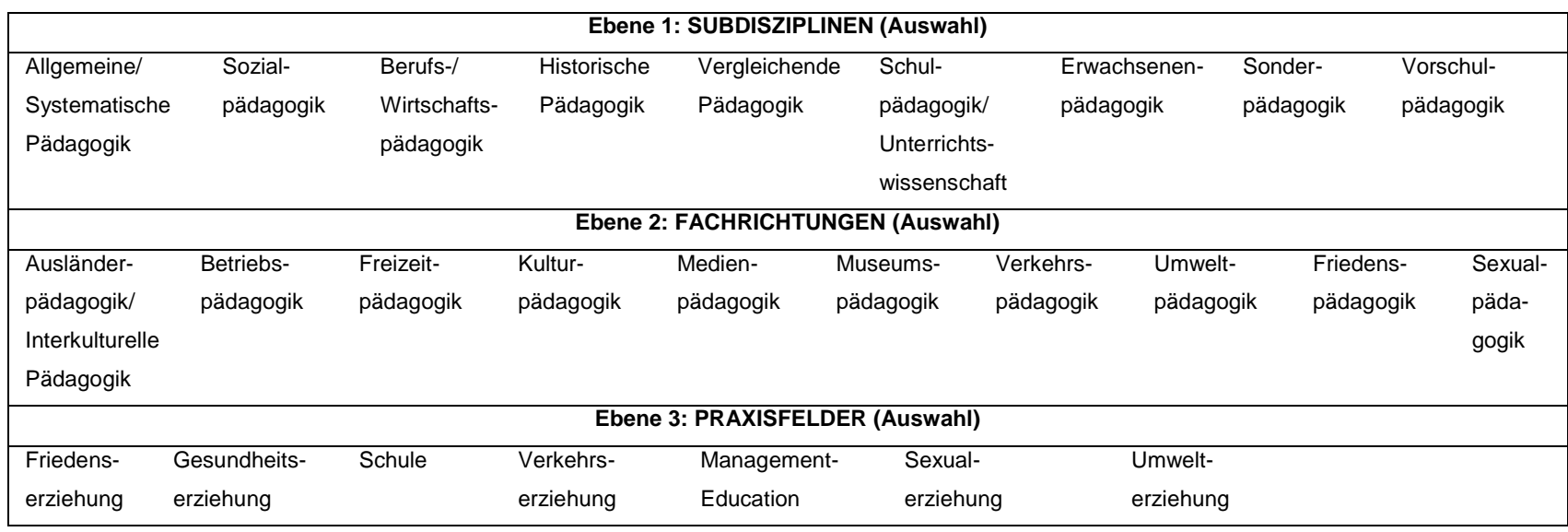

Abbildung 4: Struktur des Fachs Erziehungswissenschaft (erstellt von der Verfasserin in Anlehnung an Lenzen 1994, S.38) 
Die Struktur des Fachs nach Lenzen zeigt bereits, wie breit das Feld der Erziehungswissenschaften ist. Verschiedenste Bereiche des Lernens und Lehrens (z.B. Schule, Beruf, Freizeit) sind darin enthalten und alle Altersgruppen kommen vor (Vorschule bis Erwachsenenalter). In der „Einführung in die Arbeitsfelder der Erziehungswissenschaft“ (vgl. Krüger \& Rauschenbach 1997, S.7f) werden sechzehn Bereiche benannt:

1. Familie: Elternhaus, Familienhilfen, Familienbildung

2. Schule: Regelschulen, Reformschulen, Privatschulen

3. Berufsbildung: Betriebliche Berufsbildung, berufliche Schulen, Weiterbildung

4. Öffentliche Kindererziehung: Kinderkrippe, Kindergarten, Hort

5. Kinder- und Jugendarbeit: Freizeitzentren, Jugendbildungsstätten, Aktions- und Erholungsräume

6. Erwachsenenbildung: Volkshochschulen, Verbände, Initiativen, Bildungsstätten

7. Mädchen- und Frauenarbeit: Mädchenbildung, Frauenselbsthilfe, Frauenprojekte

8. Medien- und Kulturpädagogik: Medienerziehung, Kulturarbeit, jugendkulturelle Bildung

9. Beratung: Lebenswelt, Netzwerk, Institutionen

10. Gesundheitsförderung: Gesundheitserziehung, Gesundheitsberatung, Gesundheitsdienste

11. Hilfe für behinderte Menschen: Sonderschulen, Rehabilitation, Prävention, integrative Einrichtungen

12. Stationäre Erziehungshilfen: Heim, Wohngruppe, Pflegefamilie

13. Soziale Randgruppenarbeit: Obdachlose, Nichtsesshafte, Jugenddelinquenz

14. Interkulturelle Arbeit: Migranten, Einwanderungsgesellschaft, interkulturelle Pädagogik

15. Pädagogische Aus-, Fort- und Weiterbildung: Fachschule, Fachhochschule, Universität

16. Erziehungswissenschaftliche Forschung: Hochschulen, außeruniversitäre Forschungseinrichtungen, Praxisforschung

Mit dieser Darstellung der Arbeitsfelder der Erziehungswissenschaft wird ebenfalls deutlich, wie umfassend der Bereich der Theorie und Praxis ist: Von der Familie und dem Kindergarten bzw. vorschulischer Erziehung über sämtliche Schultypen, sozialpädagogische Einrichtungen, Beratung und Gesundheitsförderung, interkulturelle Arbeit und Erwachsenenbildung sowie die berufliche Bildung, Hochschulen und Fachhochschulen bis zur erziehungswissenschaftlichen Forschung und Praxisforschung.

In der Zeitschrift für Pädagogik (Fatke 1997) werden folgende sieben „Forschungs- und Handlungsfelder der Pädagogik" benannt: 
1. Jugendforschung - Jugendpädagogik

2. Sozialisation, Entwicklung und Erziehung im Kindesalter

3. Schule und Schultheorie

4. Interkulturelle Bildung

5. Berufsbildung

6. Reformpädagogik

7. Allgemeine Pädagogik

Bei dieser Aufstellung wird sichtbar, dass die Pädagogik den Zeitraum der Kindheit, Jugend und des Erwachsenenalters umfasst und dass sie sich sowohl auf die Praxis pädagogischen Handelns bezieht (Erziehung im Kindesalter, Schule, Jugendpädagogik, Berufsbildung, Interkulturelle Bildung) als auch auf die Forschung und Entwicklung von Ideen und Modellen zum gesamten Bereich der Erziehung und Bildung und die Fachentwicklung an sich (Reformpädagogik, Allgemeine Pädagogik).

Krüger und Helsper (1998, S.7f) thematisieren neun „Grundbegriffe pädagogischen Handelns":

1. Bildung

2. Erziehung

3. Sozialisation

4. Unterricht

5. Lernen

6. Diagnose

7. Beratung

8. Hilfe

9. Organisation, Management und Planung

Außerdem werden sechs „Räume pädagogischen Handelns“ beschrieben:

1. Familie

2. Schule

3. Vor- und außerschulische sozialpädagogische Einrichtungen

4. Betrieb

5. Erwachsenenbildung/Weiterbildung

6. Die „nichtpädagogische Welt“ (erziehen, bilden lernen und lehren außerhalb von Institutionen) 
Bemerkenswert ist bei dieser Aufstellung, dass auch die „nichtpädagogische Welt“ genannt wird - also all die Bereiche, in denen Lernen stattfindet, die nicht institutionalisiert sind. Diese Form des Lernens wird auch als „informelles Lernen“ bezeichnet (zum Thema informelles Lernen siehe Livingstone 1999, 2006, Overwien 2005, 2009 und Zürcher 2007). Die Bedeutung informellen Lernens wird häufig unterschätzt, jedoch gewinnt der Begriff zunehmend an Bedeutung (vgl. Dohmen 2001 und Overwien 2006). Außerdem wird erneut die Bandbreite der Pädagogik deutlich und es zeigt sich, dass nahezu alle Lebensbereiche des Menschen angesprochen werden - Lernen z.B. findet überall statt. Nicht zuletzt mit dem Thema des lebenslangen Lernens ist auch eine zeitliche Dimension vorhanden. Zum einen ist die Pädagogik also sehr breit angelegt was ihre Themenvielfalt anbelangt, sie betrifft zum anderen auch das gesamte Leben.

Nach Giesecke (vgl. 1996, S.7) gibt es fünf „Grundformen pädagogischen Handelns“:

1. Unterrichten

2. Informieren

3. Beraten

4. Arrangieren

5. Animieren

Das Ziel all dieser pädagogischen Handlungen ist das Lernen:

„Pädagogisches Handeln hat zum Ziel, Menschen Lernen zu ermöglichen.“ (Giesecke 1996, S.29)

Giesecke betrachtet in seinem Buch die „Pädagogik als Beruf“ und beschreibt die Tätigkeiten von „professionellen Pädagogen“ in pädagogischen Handlungsfeldern im Gegensatz zu nicht-planmäßig stattfindenden Lernprozessen. „Professionelle Pädagogen“ handeln an spezifischen, bekannten Orten geplant und mit klaren Zielen:

\begin{abstract}
„Professionelle Pädagogen sind 'Lernhelfer', die planmäßig und zielorientiert vorgehen, und dies an bestimmten, öffentlich bekannten Orten tun (Institutionen), dies unterscheidet sie von allen anderen Lernhelfertätigkeiten, die sich im alltäglichen Leben sowieso ergeben." (Giesecke 1996, S.76)
\end{abstract}

Betrachtet man einmal alle Institutionen und Orte, an denen planmäßig und zielorientiert gelernt wird (wie etwa Schule, Hochschule, Berufsbildung, Weiterbildung), so wird erneut deutlich, wie groß das Feld des Lernens und der Pädagogik ist.

Zusammenfassend soll festgehalten werden, dass es „Arbeitsfelder der Erziehungswissenschaft" und „pädagogische Handlungsfelder“ gibt, und dass diese Theorie- und Praxisfelder in besonderer Weise aufeinander bezogen sind bzw. eng zusammen hängen. Außerdem sollen mittels der Forschung und Theorien Ergebnisse und Wissen erarbeitet und für die pä- 
dagogische Praxis zur Verfügung gestellt werden. Das Wissen soll in der Praxis nutzbar sein.

Pädagogik ist eine „Handlungs- und Reflexionswissenschaft“ (s.o.), d.h. der Fokus richtet sich auf pädagogische Handlungen und die Reflexion ist wesentlicher Bestandteil der pädagogischen Arbeits- und Forschungsweise.

Des Weiteren wird deutlich, dass der Bildungsbereich das gesamte Leben des Menschen betrifft - alle Lebensbereiche und die gesamte Lebensspanne - und es eine große Vielfalt von Themenfeldern in der Pädagogik gibt. Dabei geht es nicht nur um Lernen, Bildung und Sozialisation innerhalb von Institutionen, sondern auch außerhalb formaler Strukturen und Institutionen (informelles Lernen), was die Vielfältigkeit der Themengebiete noch erhöht. In allen Bereichen der Pädagogik und der Erziehungswissenschaft, des Lernens und der Bildung ist Evaluation möglich. Im nächsten Unterkapitel soll näher betrachtet werden, was die Spezifika der Evaluation von Bildung bzw. in pädagogischen Handlungsfeldern sind.

\subsection{Spezifika der Evaluation von Bildung und in pädagogischen Handlungsfeldern}

Evaluationen können in verschiedensten Bereichen stattfinden (siehe Unterkapitel 1.2) und erhalten durch den jeweiligen Evaluationskontext eine eigene Note. Eine Evaluation eines technischen Ablaufes oder Produktes verläuft vollkommen anders als eine im Humandienstleistungsbereich. In pädagogischen Handlungsfeldern ergeben sich bei einer Evaluation Spezifika, die in diesem Unterkapitel näher betrachtet werden. Dazu wird u.a. der Qualitätsbegriff thematisiert und die Frage gestellt, was gute Qualität von Bildungsmaßnahmen ausmacht bzw. wie diese ermittelt und gesichert werden kann. Außerdem werden verschiedene Evaluationsansätze bzw. -traditionen dargestellt, die sich aus erziehungswissenschaftlicher Sicht ergeben.

Nach Beywl und Bestvater (vgl. 1998, S.34) ist eine Evaluation bzw. sind "datenbasierte Überprüfungen“ für Humandienstleistungen nicht selbstverständlich und auch nicht einfach, was an ihrer Eigenart liegt. Sie erklären dies am Beispiel der Evaluation eines Fahrrades, eines Krankentransportes und von Unterricht und stellen dabei drei Fragen:

1. Wie hoch ist der Fachkonsens über Qualitätsmaßstäbe?

2. Wie einig sind sich Fachleute über die Ursachen von Wirkungen?

3. Wie stabil ist der Evaluationsgegenstand über die Zeit?

Beim Fahrrad ist erstens der Fachkonsens über Qualitätskriterien recht hoch: Es gibt DINNormen für Produkteigenschaften und sogar gesetzliche Vorgaben. Ingenieure, Verkäufer 
und Verbrauchertester sind sich relativ einig über Qualitätsmaßstäbe. Die Mängel des Fahrrades, technische Defekte oder Fehlfunktionen sind zweitens meistens eindeutig auf bestimmte Ursachen zurückzuführen (z.B. Material- oder Konstruktionsfehler). Und drittens verändert sich ein Fahrrad über die Zeit relativ wenig (z.B. beträgt die Garantiezeit für den Rahmen 10 Jahre).

Beim Krankentransport ist erstens der Fachkonsens darüber, was gute Qualität ist, vergleichsweise hoch. Es gibt Qualitätsmaßstäbe, die jedoch u.U. nicht eingehalten werden, was dazu führen kann, dass im Falle eines Fehlers oder einer Schädigung des Kranken herausgefunden werden muss, was dazu geführt hat. Die Frage nach den Ursachen ist im Gegensatz zum Fahrrad schwieriger, da zweitens die Faktoren, die diese Wirkungen verursacht haben können, nicht immer ganz eindeutig sind (technische Mängel oder auch menschlicher Einfluss sind vorstellbar, wenn z.B. beim Krankentransport der Patient von der Liege fällt oder die Wirbelsäule geschädigt wird). Das Meiste wird jedoch nachvollziehbar sein, da auch eine Dokumentations- und Aufzeichnungspflicht besteht. Und drittens ist die Stabilität über die Zeit bei dem Krankentransport nicht so hoch wie beim Fahrrad. Zum einen kommt es darauf an, wer transportiert wird, und zum anderen darauf, wer den Wagen fährt (z.B. können je nach Tagesform oder Dienstdauer Unterschiede in der Qualität auftauchen).

Beim Unterricht gibt es erstens nur einen geringen Fachkonsens darüber, was "guter Unterricht" ist und an welchen Qualitätskriterien dies festzumachen ist. Es bestehen unterschiedliche oder sogar sich widersprechende Meinungen dazu, welche Methoden die besten sind. Diese Verschiedenartigkeit der Auffassungen ist nach Beywl und Bestvater (vgl. 1998, S.36) charakteristisch für pädagogische oder sozialpädagogische Arbeitsfelder. Zweitens sind die Ursachen für Lernerfolg und -misserfolg nicht so einfach zu erkennen. Die Einflussfaktoren weisen eine hohe Variabilität auf: Liegt es an dem Lerner selbst (Vorerfahrungen, Lernstil, Lernvoraussetzungen), oder am Lehrer (Persönlichkeit des Lehrenden, Unterrichtsstil), oder an den Unterrichtsmaterialien (gutes Lehrbuch, Medien)? Es ist nicht leicht und eindeutig zu erkennen, was die Ursache für den Erfolg oder Misserfolg ist. Und drittens sind „pädagogische Dienstleistungen“ (ebenda) nicht stabil über die Zeit, da die beteiligten Personen einen wesentlichen Einfluss auf die Qualität des Unterrichts haben: Kommt ein "neuer Lehrer" z.B. in eine Klasse, können sich die Schülerleistungen sehr stark verändern, außerdem verläuft kein Unterricht wie der andere, kein Unterrichtstag und keine Unterrichtsstunde wie zuvor. Die folgende Grafik verdeutlicht noch einmal die Evaluation von Fahrrädern, Krankentransporten und Unterricht im Vergleich: 


\section{Evaluation von Fahrrädern, Krankentransporten, Unterricht ...}

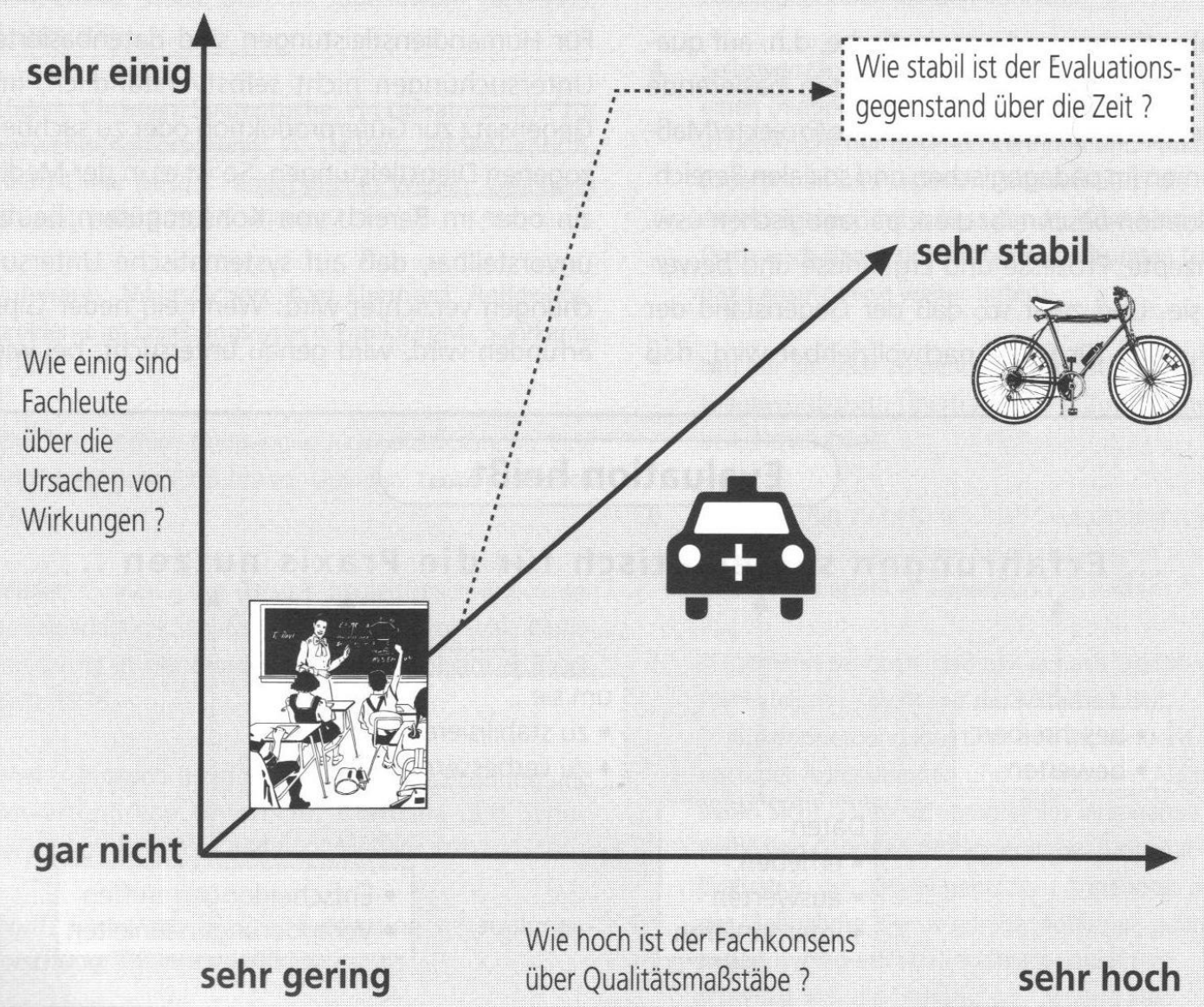

Abbildung 5: Evaluation von Fahrrädern, Krankentransporten und Unterricht (Quelle: Beywl \& Bestvater 1998, S.34)

Insgesamt wird mit diesem Beispiel deutlich, was die Spezifika und zugleich Schwierigkeiten bei der Evaluation von Bildungsprozessen sind: Ein geringer Konsens über Qualitätsmaßstäbe und -kriterien, eine Vielfalt von Ursachen für Erfolg und Misserfolg sowie eine hohe Instabilität über die Zeit. Insgesamt lässt sich sagen:

"[...] sind pädagogische oder sozialpädagogische Leistungsprozesse gekennzeichnet von ihrer hohen Abhängigkeit von den konkret am Leistungsprozeß beteiligten Menschen, vom chronischen Fehlen klarer, in der Fachwelt akzeptierter Bewertungsmaßstäbe und von einem ausgeprägtem Dissens darüber, auf welche Ursachen positive oder negative Ergebnisse pädagogischer Prozesse letztlich zurückzuführen sind." (Beywl \& Bestvater 1998, S.37)

Nach Beywl und Bestvater ist es bei pädagogischen Handlungen besonders schwer, deren Qualität zu bestimmen, weil es verschiedene, zum Teil sich widersprechende Meinungen darüber gibt, was gute Qualität ist oder ausmacht (s.o.). Was nun aber bedeutet überhaupt „gute Qualität"? Wie und an was kann man sich z.B. als Lehrender orientieren, wenn man ein „qualitativ hochwertiges Lernangebot" schaffen möchte, wie kann man für „gute Qualität“ sorgen und wie kann man diese evaluieren bzw. sicher stellen? 
Dazu soll nun zuerst einmal der Qualitätsbegriff näher betrachtet werden. Nach Helmke, Hornstein und Terhart (vgl. 2000a, S.7) kennzeichnet kaum ein anderer Begriff seit den 90er Jahren die Diskussion in den Bildungswissenschaften, der Bildungs- und Sozialpolitik wie "Qualität“. Auch zahlreiche Wortverbindungen und -schöpfungen weisen darauf hin, dass "Qualität“ einen besonderen Stellenwert hat, wie etwa „Qualitätsprüfung“, „Qualitätsmanagement“, „Qualitätsstandards“, „Qualitätskontrolle“, Qualitätsverbesserung“ und „Qualitätssicherung“. Im Wissenschaftsbereich gibt es inzwischen sogar die „Qualitätswissenschaften“. Im gesamten Bildungs- und Sozialbereich ist die Frage nach der "Qualität" sehr zentral. Die Ursachen sehen Helmke, Hornstein und Terhart (vgl. 2000a, S.8) u.a. im immensen Wachstum von sozialen Berufen, Einrichtungen und Bildungsangeboten bei gleichzeitig immer geringer zur Verfügung stehenden Mitteln in den „öffentlichen Kassen“. Dies habe zu einem „Weltbildwechsel im Denken über staatliche Sozial- und Bildungsleistungen geführt" (ebenda, S.9). Neben dem Streben nach Effektivität und Effizienz wird auch die „Qualität“ von Prozessen und Angeboten genauer betrachtet bzw. deren Nachweis oder Sicherung gefordert. Der Aufwand und der Ertrag von Bildungs- und Sozialleistungen werden analysiert und "Marktkräfte" scheinen dabei eher zur Geltung zu kommen als das Vertrauen in den Staat. Das gesamte Spektrum des Bildungswesens und der sozialen Strukturen in unserer Gesellschaft sind davon betroffen:

\begin{abstract}
„Die Frage nach den Voraussetzungen, Prozessen und Kontrollmöglichkeiten von Qualität betrifft dabei den gesamten Bildungs- und Sozialbereich. Von der Kinderkrippe über Kindergärten, Primar- und Sekundarschulen, sozialpädagogische Einrichtungen, Hochschulen, öffentliche und private Formen der Erwachsenenbildung, der Weiterbildung, der inner- und außerbetrieblichen Berufsbildung etc., über Institutionen und Einrichtungen der Jugend- und Sozialhilfe, der Betreuung von Randgruppen bis hin zu den Einrichtungen der Seniorenbildung und -betreuung, der Pflege kranker und alter Menschen - alle im Bildungs- und Sozialbereich angesiedelten Institutionen, letztlich alle sozialstaatlichen Infrastrukturleistungen (...), ihre Administration und ihr Personal sehen sich der kritischen Frage nach ihrer Qualität gegenüber, müssen sich um Qualität bemühen, müssen Qualität dokumentieren, müssen sich hinsichtlich ihrer Qualität überprüfen lassen und müssen schließlich - wie könnte es anders sein - ihre Qualität kontinuierlich verbessern." (Helmke, Hornstein und Terhart 2000a, S.9f)
\end{abstract}

Obwohl der Begriff der Qualität omnipräsent ist, scheint eine Definition und inhaltliche Präzisierung schwierig zu sein. In zwei Beiheften der Zeitschrift für Pädagogik widmen sich zahlreiche namhafte Autoren dem Thema Qualität und Qualitätssicherung im Bildungsbereich (vgl. Helmke, Hornstein und Terhart 2000b und Klieme und Tippelt 2008). Während es im 41. Beiheft aus dem Jahre 2000 um eine begriffliche Fassung und die Bereiche Schule, Sozialpädagogik und Hochschule geht, wird im 53. Beiheft aus dem Jahre 2008 das gesamte Bildungswesen thematisiert: Der Elementarbereich, Schulen, das allgemeinbildende Schulsystem, Sozialpädagogik, berufliche Bildung, Weiterbildung, betriebliche Weiterbildung, Hochschulen und Erziehungswissenschaft. Dies zeigt, dass das Thema Qualität, Qualitätssicherung und Qualitätsverbesserung heute umso mehr eine große Bedeutung hat. 
Nach Klieme und Tippelt (vgl. 2008, S.7) hat sich das deutsche Bildungswesen grundlegend durch die Einführung von Qualitätssicherungsverfahren geändert. Heutzutage richteten sich Zielvorgaben, Strukturen und Prozesse der "institutionalisierten Bildung“ nach deren Effektivität und Effizienz. Außerdem haben Evaluation und Empirie einen sehr hohen Stellenwert. Für die "Qualitätsdebatte" benennen Klieme und Tippelt (vgl. 2008, S.10) unterschiedliche Wurzeln: Im Bereich der Schule sind die Bemühungen, Untersuchungen und großangelegten Studien wie etwa TIMSS, PISA, PIRLS/IGLZ eine „Antwort auf Schwächen des Bildungssystems“ (ebenda). Im außerschulischen Bildungsbereich kommen hingegen eher „betriebswirtschaftliche Konzepte“ zum Einsatz, die übernommen bzw. angepasst wurden und der Optimierung von Prozessen dienen.

Klieme und Tippelt (vgl. 2008, S.8) merken an, dass der Begriff Qualitätssicherung in dem Beiheft der Zeitschrift für Pädagogik von keinem der Autoren explizit definiert werde. Vielmehr werde der Begriff „Qualität“ umschrieben und Teilaspekte würden beleuchtet. Insgesamt werde deutlich, dass

"[...] es nicht die eine Skala für Bildungsqualität geben kann, sondern bereichs- und zielabhängig unterschiedliche Kriterien und Indikatoren benannt werden müssen." (Klieme und Tippelt 2008, S.9)

Harvey und Green betrachten den Qualitätsbegriff genauer und kommen zu dem Schluss:

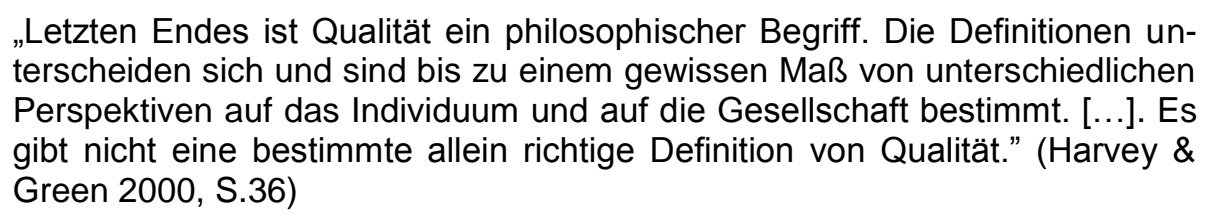

Außerdem komme hinzu, dass verschiedene an einem Prozess oder Bildungsangebot Beteiligte die Qualität unterschiedlich beurteilen, da sie unterschiedliche Interessen und Blickwinkel haben. Die Kursteilnehmer beispielsweise werden die Qualität eines Kurses anders definieren (und verschiedene Teilnehmer können die Qualität auch noch unterschiedlich bewerten) als der Dozent oder die Dozentin, und auch anders als Außenstehende oder z.B. Auftraggeber. Harvey und Green fassen zusammen:

„Definition von Qualität ist interessenabhängig. [...]. Aus diesen Gründen kann Qualität nicht als einheitliches Konzept verstanden werden; man muß statt dessen von unterschiedlichen Qualitäten sprechen. (Harvey \& Green 2000, S.36)

Die Tatsache, dass es unterschiedliche Verständnisse von Qualität gibt und es nicht „richtig oder falsch“ gibt bzw. niemand „Recht oder Unrecht“ hat, solle nun nicht dazu führen, dass es aufgegeben wird, sich um eine "gute Qualität“ zu bemühen. Harvey und Green schlagen eine pragmatische Haltung vor:

„Der pragmatische Ansatz formuliert eine Reihe von Kriterien, die sich auf der Basis des vorhandenen Wissens verantwortlich benennen lassen, und 
wählt dementsprechend praktikable Meßverfahren aus, um Qualität zu quan-

tifizieren." (Harvey \& Green 2000, S.36)

Der Einsatz von Messverfahren könnte aber auch zum Gegenteil führen und die „Glaubwürdigkeit des Messens“ (ebenda) benutzt werden, um Qualität zu versichern, obwohl diese gar nicht vorhanden ist. Wichtig sei die Offenlegung und Betrachtung der verschiedenen Interessen, Verständnisse und Sichtweisen auf bzw. von Qualität.

Wie kann nun im Kontext von Bildung für "gute Qualität“ gesorgt werden? Welche Konzepte und Verfahren gibt es bzw. welche eignen sich, um die Qualität zu kontrollieren, zu verbessern oder sicherzustellen?

Dem Thema Evaluation und Qualitätsentwicklung widmet sich Stockmann intensiv (siehe z.B. Stockmann 2006c). Nach Stockmann (vgl. 2006d, S.23) gibt es zwei Konzepte zur Sicherstellung bzw. Steigerung der Qualität im Bildungsbereich, die zunehmend zum Einsatz kommen: Qualitätsmanagement und Evaluation. Beide Konzepte seien bisher kaum miteinander verbunden, könnten sich aber ergänzen (vgl. ebenda, S.38). Stockmann vergleicht Qualitätsmanagement und Evaluation und erarbeitet Gemeinsamkeiten und Unterschiede.

„Die zentrale Gemeinsamkeit zwischen Total Quality Management (TQM) und Evaluationskonzepten ist vor allem darin zu sehen, dass beide darauf ausgerichtet sind, zur Qualitätsverbesserung beizutragen. Beide Konzepte wollen - wenn auch in unterschiedlicher Weise - rationale Entscheidungsgrundlagen für das Unternehmens- oder Programmmanagement schaffen." (Stockmann 2006d, S.34)

Im Hinblick auf Umsetzungsprobleme (wie z.B. geringe Akzeptanz bei Betroffenen oder geringe Ergebnisnutzung nach einem aufwändigen Verfahren) gibt es aber auch grundlegende Unterschiede zwischen Evaluations- und Qualitätsmanagementkonzepten (vgl. Stockmann 2006d, S.34f). Zunächst haben TQM und Evaluation verschiedene wissenschaftstheoretische Wurzeln. TQM entstammt aus den Betriebswissenschaften, während Evaluation sich an den Sozialwissenschaften ausrichtet und die eingesetzten Methoden aus diesem Kontext entstehen. Außerdem kommen TQM und Evaluation auch in unterschiedlichen Praxisfeldern zum Einsatz:

„TQM wird eher in gewinnorientierten Unternehmen, die auf prinzipiell offenen Märkten konkurrieren und die über eine klare Produzenten-KundenBeziehung verfügen, verwendet, während Evaluation eher von öffentlichen Einrichtungen und Non-Profit-Organisationen genutzt wird, für die deutlich andere organisationale und situative Bedingungen gelten." (Stockmann 2006d, S.35)

Des Weiteren unterscheiden sich Qualitätsmanagement und Evaluation dadurch, „dass TQM ein allumfassendes (totales) aufeinander abgestimmtes System der Qualitätssicherung darstellt“, während Evaluation „vor allem eine Informationsbeschaffungs- und Bewertungsfunktion hat" (Stockmann 2006d, S.35). Bei der Evaluation liegen „die Handlungsentscheidungen und ihre Umsetzung [...] außerhalb des Evaluationsverfahrens“ (ebenda). Außerdem ist eine 
Evaluation nach Stockmann (vgl. ebenda) eher partizipativ angelegt und bietet mehr Handlungsoffenheit als TQM (wobei auch TQM der Zustimmung von Mitarbeitern bedarf, was bei Evaluation nicht unbedingt der Fall sein muss). Ein weiterer Unterschied liegt in der „Kundenorientierung" beider Verfahren:

„TQM stellt den Kunden und Evaluation die Beteiligten (Stakeholder) in den Vordergrund." (Stockmann 2006d, S.35)

Evaluationen sind nach Stockmann (ebenda, S. 36) dem „stakeholder value“ verpflichtet. Die Beteiligten bzw. die Zielgruppe soll einen möglichst hohen Nutzen aus den Ergebnissen ziehen. Nach Stockmann (ebenda) handelt es sich bei TQM und Evaluation eher um „sich ergänzende, als sich gegenseitig ausschließende Konzepte mit unterschiedlichen Leistungspotenzialen“.

Bei dem Vergleich von TQM und Evaluation fallen einige Charakteristika des jeweiligen Konzeptes auf. In dieser Arbeit geht es vor allem um Evaluationsverfahren, deshalb soll noch einmal zusammengefasst werden, was das Besondere im Kontext von Qualitätssicherung ist: Evaluationen sollen dazu beitragen, die Qualität eines Programms oder einer Maßnahme zu verbessern bzw. sicherzustellen, wobei die Kriterien, nach denen eine Bewertung erfolgt, sehr verschieden sein können. Die Bewertungskriterien werden im Rahmen der Evaluation von verschiedenen Beteiligten festgelegt. Evaluationsverfahren sind eher partizipativ angelegt und weisen eine gewisse Handlungsoffenheit auf, da die Ergebnisse frei zur Verfügung gestellt werden und darauf aufbauende Handlungsentscheidungen von den Beteiligten getroffen werden können und müssen. Die "Stakeholder" stehen im Vordergrund. Bemerkenswert ist an dieser Stelle aber auch, dass Evaluationen sehr unterschiedliche Funktionen haben können (siehe Unterkapitel 1.3) und es sehr verschiedene Formen von Evaluation gibt (siehe Unterkapitel 1.4).

Der Fokus dieser Arbeit richtet sich auf Evaluation im Bildungsbereich bzw. im Kontext der Pädagogik und Erziehungswissenschaft. Wie schon in den vorangegangenen Kapiteln deutlich wurde, gibt es verschiedene Evaluationstraditionen, die zum einen historisch begründet sind oder sich durch verschiedene wissenschaftstheoretische Herangehensweisen ergeben. Verschiedene Autoren vertreten verschiedene Evaluationsansätze und stammen häufig auch aus unterschiedlichen Fachrichtungen (z.B. Psychologie, Soziologie, Pädagogik, Wirtschaftswissenschaften, Politik). Evaluationsmethoden und -ziele variieren entsprechend der Fachtraditionen, womit sich zeigt, um was für ein komplexes Feld es sich handelt, wenn man Evaluation betrachtet.

An dieser Stelle wird nun auf Evaluation im Kontext von Pädagogik und Erziehungswissenschaft näher eingegangen.

Kuper (2005, S.50f) widmet sich dem Thema Evaluation aus „erziehungswissenschaftlicher Sicht" und identifiziert drei Ansatzpunkte: 
1. Evaluation als Gegenstand erziehungswissenschaftlicher Forschung.

2. Die (zunehmende) Evaluation der Erziehungswissenschaft selbst.

3. Evaluation als eine durch erziehungswissenschaftliche Theorie geleitete Praxis.

Nach Kuper (vgl. 2005, S.51) gibt es zwei unterschiedliche Traditionen in der deutschsprachigen Erziehungswissenschaft, in denen Evaluationsforschung stattfindet: Die pädagogischpsychologische Diagnostik und die Handlungsforschung. In den zwei Traditionen wird Evaluation unterschiedlich definiert, die Ziele und eingesetzten Methoden variieren und das Grundverständnis ist verschieden.

In der „Tradition der Diagnostik“ steht vor allem die Leistungsbeurteilung durch Lehrer im Zentrum. Auf der Basis von empirischen Daten und Bewertungsnormen werden Urteile gefällt, wobei nicht nur einzelne Schülerleistungen, sondern auch Leistungen von Gruppen beurteilt und bewertet werden können. Entscheidend ist, dass die Standards empirischer Forschung berücksichtigt und erfüllt werden, um die Objektivität der Ergebnisse zu gewährleisten (vgl. Kuper 2005, S.52).

Leutner (vgl.1999) vertritt diesen Ansatz und beschreibt Evaluation aus Sicht der kritischempirischen Forschung. Bei Evaluationen im Bildungsbereich müssen danach empirische Methoden zum Einsatz kommen und die Standards empirischer Forschung eingehalten werden. Es geht nicht darum, irgendwie irgendwelche Daten zu erheben und diese irgendwie zu interpretieren, vielmehr müssen die Daten mit den richtigen Methoden erhoben, mit den richtigen Verfahren ausgewertet und die richtigen Schlussfolgerungen daraus gezogen werden. Leutner betrachtet „Evaluation im Dienste der Verbesserung von Qualität im Bildungsbereich“ (Leutner 1999, S.121) und beschreibt die Charakteristika dieses Ansatzes wie folgt:

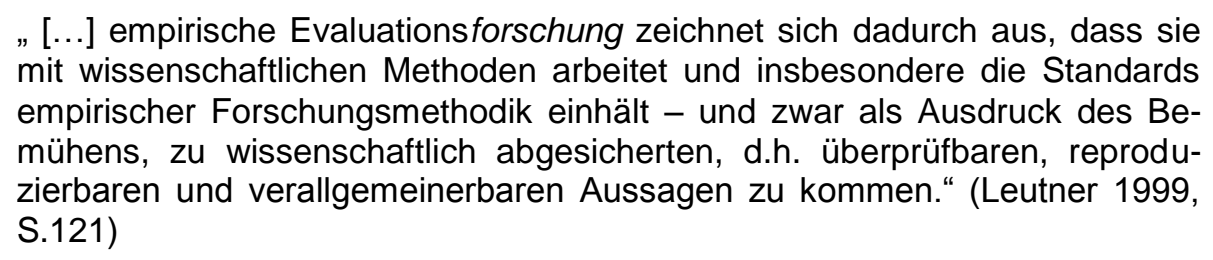

In fünf Thesen stellt Leutner dar (1999, S.123ff), worauf es bei der Evaluation im Bildungsbereich ankommt:

1. Evaluation im Bildungsbereich erfordert empirische Methoden.

2. Qualitätsverbesserung erfordert die Verwendung empirischer Methoden für pädagogische Interventionen.

3. Relevanz der Standards empirischer Forschung in allen Phasen und zu allen Teilaspekten.

4. Die Standards empirischer Forschung sind im Bildungsbereich grundsätzlich realisierbar. 
5. Die Nicht-Realisierung der Standards empirischer Forschung erhöht die Wahrscheinlichkeit von Fehlsteuerungen.

Nach Kuper (2005, S.53) wird in der Tradition der pädagogisch-psychologischen Diagnostik eine "Strategie der konsequenten Minimierung von Komplexität" verfolgt, d.h. bestimmte Aspekte werden durch den Einsatz diagnostischer Instrumente (empirischer Methoden) erfasst und interpretiert. Die Rahmenbedingungen würden dabei häufig ungeachtet bleiben.

In der „Tradition der Handlungsforschung“ ist der Umgang mit Komplexität anders:

„Anstelle einer objektivierbaren Aussage über die Wirkung pädagogischer Intervention zielt die Evaluation auf das handlungspraktische Wissen von Akteuren. Der Ausgangspunkt der Evaluation in der Praxis ist die Professionalität der Akteure im pädagogischen Handlungsfeld." (Kuper 2005, S.54)

Altrichter (vgl.1999) widmet sich diesem Ansatz. Er hält die „Förderung von Selbstreflexivität im Alltag, die Förderung einer reflektierten Alltagskultur, für einen gesellschaftlichen Wert.“ und stellt dieses Evaluationskonzept in den Kontext der „action research Bewegung“ (ebenda, S.106). Es gehe darum, selbstevaluative Fähigkeiten der Akteure im Praxisfeld zu stärken, um „Spielraum gegenüber Routinen und vorgegebenen Strukturen zu gewinnen“ (ebenda). Die Charakteristika der Aktionsforschung bzw. eines Evaluationsansatzes nach diesen Prinzipien stellt Altrichter (1999, S.106ff) in sechs Prämissen dar:

1. PraktikerInnen als ForscherInnen; Einbeziehung von anderen 'Betroffenen' in den Forschungsprozess und Regelung ihrer Beziehung durch einen `ethischen Code`.

2. Problemorientierung, Interdisziplinarität und Kontextualisierung.

3. Handlungsorientierung und längerfristige, `zyklische` Prozesse`von Reflexion und Aktion.

4. Konfrontation verschiedener Perspektiven.

5. Einbettung der individuellen Forschung in eine professionelle Gemeinschaft.

6. Veröffentlichung von Praktikerwissen.

Dieser Evaluationsansatz wird im nächsten Kapitel nochmals aufgegriffen, da er zu der Vorgehensweise in dieser Forschungsarbeit passt. An dieser Stelle sollte nur gezeigt werden, dass es unterschiedliche Traditionen und Herangehensweisen innerhalb der Erziehungswissenschaften gibt, die jeweils ihre Befürworter und ihre Berechtigung haben. Sie stehen im Kontext von unterschiedlichen wissenschaftlichen Herangehensweisen. 
Zusammenfassend soll festgehalten werden, dass es bei der Evaluation von Bildung bzw. Humandienstleistungen schwierig ist, deren Qualität zu beurteilen. Dies liegt u.a. daran, dass es unterschiedliche Ansichten darüber gibt, was „gute Qualität“ ist, je nachdem, wer die Qualität bewertet. Die Frage nach guter Qualität ist jedoch heutzutage im gesamten Bildungsbereich sehr zentral, nahezu alle Institutionen im Bildungs- und Sozialbereich müssen sich bemühen und die Qualität ihrer Arbeit nachweisen, dokumentieren und ständig verbessern. Zur Verbesserung bzw. Sicherung der Qualität eignen sich Qualitätsmanagement und Evaluation als Verfahren. Im Bereich von öffentlichen Einrichtungen und Non-Profit-Organisationen werden eher Evaluationen durchgeführt, während Qualitätsmanagementansätze vor allem im wirtschaftlichen Bereich zum Einsatz kommen. Bei einer Evaluation werden die Bewertungskriterien für die Qualität von verschiedenen Beteiligten gemeinsam festgelegt. Die Partizipation von "Stakeholdern" bei Evaluationsverfahren ist Bestandteil der Evaluation. Die Ergebnisse werden frei zur Verfügung gestellt, so dass darauf aufbauende Handlungsentscheidungen von den Beteiligten getroffen werden können.

Bei der Evaluation in pädagogischen Handlungsfeldern bzw. aus erziehungswissenschaftlicher Sicht gibt es unterschiedliche Ansätze und Traditionen. Zum einen die pädagogischpsychologische Diagnostik, bei der vor allem die Standards empirischer Forschung berücksichtigt werden müssen, und zum anderen die Handlungsforschung mit dem Hintergrund der Aktionsforschung, bei der die selbstevaluativen Fähigkeiten der beteiligten Akteure gestärkt werden sollen und Praktiker zu Forschern werden bzw. am Forschungsprozess maßgeblich beteiligt sind.

\subsection{Das Forschung-Praxis Dilemma}

In den vorangegangenen Kapiteln wurde erläutert, dass die Pädagogik eine „Handlungs- und Reflexionswissenschaft" ist (Kümmel, 1978, S.121), und dass Theorie und Praxis in besonderer Weise miteinander verbunden sind (siehe Kapitel 4.1). Das Wissen, das in der „Theorie“ vorhanden ist bzw. durch Forschung erarbeitet wird, soll für die pädagogische Praxis zur Verfügung gestellt werden und dort nutzbar sein (vgl. Lenzen 1994). Nun gelten aber in der Theorie und Forschung auf der einen Seite und der Praxis auf der anderen Seite unterschiedliche Regeln, außerdem sind verschiedenartige Anforderungen und Denkweisen vorhanden. In diesem Unterkapitel soll näher betrachtet werden, welche Bedingungen und Funktionen in den jeweiligen Bereichen vorherrschen, zu was das im Zusammenhang mit Evaluationsmaßnahmen führt und welche Lösungsmöglichkeiten es dafür gibt.

Theoretiker und Forscher denken anders als Praktiker, sie "leben in unterschiedlichen Welten“, könnte man sagen. Es gelten unterschiedliche Regeln, die Bedarfe und Arbeitsweisen sind verschieden. Diese Annahme verlangt keine empirischen Beweise. Im Kontext von Eva- 
luation ist es wichtig, die Unterschiedlichkeiten zu kennen und zu berücksichtigen bzw. sie bewusst in das Evaluationskonzept und den Evaluationsprozess einzubeziehen. Insgesamt lässt sich sagen,

"[...] dass in Forschung und Praxis unterschiedliche Denkweisen vorherrschen, die nicht unbedingt auf einen Nenner zu bringen sind. Mehr noch: Forschung und Praxis stehen unter unterschiedlichen Funktionen und Bedingungen im Hinblick auf Evaluationsmaßnahmen und folglich auch im Hinblick auf Evaluationskonzepte und -ansprüche." (Haller 2005, S.4)

Haller führt weiter aus, was die wesentlichen Unterschiede zwischen Forschung und Praxis sind und was sie für die Evaluation im Bildungsbereich bedeuten:

\begin{abstract}
„Evaluation ist im Forschungssinn eine Reihe komplexer und oftmals sehr aufwendiger Verfahren, um Wirkungen oder Nebenwirkungen von Maßnahmen im Bildungswesen zu überprüfen und um Entscheidungen vorzubereiten bzw. zu treffen. [...] Für praktische Bedürfnisse jedoch, wie sie in den Bildungseinrichtungen selbst gegeben sind, die unter dem fortwährenden Anspruch der Leistungsbewertung stehen, haben sich Verfahren und Messpraktiken ergeben, die den wissenschaftlichen Ansprüchen einer standardisierten Testkonstruktion nicht gerecht werden können: hier entstand der hausgemachte Kleintest für den Praktiker." (Haller 2005, S.4)
\end{abstract}

Im Rahmen der Forschung müssen die Standards empirischer Sozialforschung eingehalten werden, die Forschungsmethoden sind klar definiert und Vorgehensweisen festgelegt. Die Gütekriterien sozialwissenschaftlicher Forschung müssen berücksichtigt und erfüllt werden, so dass Verfahren oft sehr zeitintensiv sind, außerdem eine hohe Expertise und sehr spezielle Kompetenzen erfordern.

Die Konstruktion eines Tests ist beispielsweise unter den Voraussetzungen und der Einbehaltung wissenschaftlicher Kriterien eine sehr intensive Prozedur, bei der u.a. die Gütekriterien wie Objektivität, Reliabilität und Validität eingehalten werden müssen. Außerdem muss ein Test im wissenschaftlichen Sinne normiert werden (vgl. Lienert \& Raatz 1998 und Rost 2004). Das Verfahren braucht Zeit und meistens arbeiten mehrere Personen daran. Haller (vgl. 2005, S.5) schätzt beispielsweise, dass bei der Erstellung eines Schulleistungstests, mit dem das Pensum eines Faches für ein Jahr erfasst werden soll, ein Team von drei ausgebildeten Personen ein bis zwei Jahre braucht, bis der Test vorliegt und eingesetzt werden kann. Noch komplizierter und aufwändiger wird es, wenn Erwartungen und Zufriedenheiten von Lernern gemessen bzw. Einstellungen erfasst werden sollen.

Wird ein Fragebogen als Methode der Datenerhebung für die Evaluation ausgewählt, so erfordert auch dies Expertise und die Beherrschung der Itemkonstruktion. Für die korrekte Auswahl und Formulierung von Antwortformaten bzw. Skalierungen müssen wiederum die Regeln wissenschaftlicher Forschung eingehalten werden (vgl. Borg \& Staufenbiel 2007, Rohrmann 1978 und Rost 2004). Die Formulierung von Fragebogen-Fragen erfordert entsprechende Kompetenz und Erfahrung, da es zum Teil auf jedes einzelne Wort einer Frage ankommt bzw. die Antworten durch einzelne Worte beeinflusst und verändert werden können 
(vgl. Rost 2000). Zur Fragebogenmethode siehe auch Bühner 2006, Kirchhoff, Kuhnt, Lipp \& Schlawin 2006, Mummendey 1987 und Porst 2008).

Werden andere Verfahren der Datenerhebung ausgewählt - qualitative oder quantitative Methoden - so gibt es jeweils Richtlinien, Vorgaben und Regeln, die eingehalten werden müssen, damit die Ergebnisse als repräsentativ und aussagekräftig im wissenschaftlichen Sinne gelten können.

Anders sieht es in der Praxis aus: Evaluationen verlaufen in der Praxis nach Klein (1991, S.6) häufig "quick and dirty“, wissenschaftliche Standards werden dabei nicht berücksichtigt und die Erhebungen werden meist in Eigenregie durchgeführt. In der Regel besteht ein gewisser Zeit- und Handlungsdruck. Die Ergebnisse sind nicht repräsentativ und liefern eher exploratives Material als aussagekräftige Daten für Entscheidungen. Fragebögen, die beispielsweise in Kursen zur Evaluation eingesetzt werden, sind meistens „selbstgestrickt“, d.h. die Praktiker entwerfen nach bestem Wissen ein paar Fragen, um eine Rückmeldung von den Teilnehmern zu bekommen, den Kurs auszuwerten bzw. die Qualität zu sichern oder nachzuweisen, dass sie sich darum bemühen. Häufig gehen die Fragen nicht in die Tiefe und die Ergebnisse sollen (wenn überhaupt) direkt verwendbar sein.

Wie sollen Evaluatoren nun aber den Ansprüchen und Pflichten im Rahmen der Forschung und den Anforderungen und Bedarfen der Praxis bei einer Evaluation gerecht werden? Wie können Ergebnisse ermittelt werden, die in der Praxis nutzbar sind und als Entscheidungsgrundlagen dienen, aber gleichzeitig den Vorgaben der wissenschaftlichen Forschung Rechnung tragen? Dies ist oft nicht möglich, es entsteht ein Dilemma, das von Haller (vgl. 2005) als „Forschung-Praxis Dilemma“ bezeichnet wird.

Zusammenfassend soll hier festgehalten werden, dass in der Forschung einerseits und der Praxis andererseits unterschiedliche Denkweisen, Bedarfe und Regeln herrschen. Theoretiker bzw. Forscher (oder Wissenschaftler) denken anders als Praktiker. Im Forschungsbereich müssen wissenschaftliche Standards berücksichtigt und eingehalten werden, was aber aufwändiger Verfahren bedarf. In der Praxis besteht häufig Zeit- und Handlungsdruck, so dass Erhebungsmethoden eingesetzt werden, die nicht den Kriterien der Forschung gerecht werden und Ergebnisse entstehen, die relativ oberflächlich bleiben und nicht verallgemeinerbar und aussagekräftig sind. Beiden Seiten gerecht zu werden, scheint unmöglich - es entsteht das Forschung-Praxis Dilemma.

Für Evaluation bedeutet dies, dass seitens der Forschung andere Ansprüche und Standards bestehen als in der Praxis, was sich auf das Evaluationskonzept, die Vorgehensweise bei der Datenerhebung und die Evaluationsziele auswirkt. Die Funktion der Evaluation kann sehr verschieden sein, je nachdem in welchem Kontext sie stattfindet. 
Im folgenden Kapitel 5 wird ein Lösungsansatz entwickelt, mit dem Theorie und Praxis verbunden werden sollen, der an Wissenschaftlichkeit und Forschung einerseits und Praxisbedarfen und -arbeitsweisen andererseits ansetzt. 


\section{Entwicklung und Erprobung von Inventaren zur Unter- stützung und Verbesserung der Evaluationspraxis in euro- päischen Bildungsprojekten}

Aufbauend auf dem in Kapitel 4 beschriebenen Forschung-Praxis Dilemma wird in diesem Kapitel ein Lösungsansatz entwickelt, um die Evaluationspraxis, die häufig mit „selbstgestrickten Instrumenten" stattfindet und dadurch nicht besonders hochwertig ist, in Bildungsprojekten zu unterstützen und zu verbessern. Es wird davon ausgegangen, dass Praktiker in europäischen Bildungsprojekten in der Regel selbst erstellte Instrumente zur Evaluation verwenden, die nicht den Standards wissenschaftlicher Forschung und Vorgehensweisen entsprechen. In der beruflichen Praxis von Bildungsanbietern ist meistens nicht die Zeit und auch nicht die Kompetenz vorhanden, Evaluationsmethoden und Evaluationsinstrumente zu entwickeln und einzusetzen, deren Qualität sehr gut ist. Die Ergebnisse können deshalb häufig nicht längerfristig verwendet werden und taugen oft auch nicht zur Weiterentwicklung der eigenen Praxis. Evaluationen haben in diesem Fall häufig nur die Funktion der Legitimation, dass die eigene Arbeit (zumindest scheinbar unter dem Siegel der Evaluation) eine gute Qualität aufweist (siehe zu verschiedenen Funktionen von Evaluation Unterkapitel 1.3). Andererseits ist die Entwicklung von Instrumenten (z.B. Fragebögen) unter der Beachtung wissenschaftlicher Regeln meistens ein derart aufwändiges Verfahren, das viel Zeit und personellen Aufwand erfordert und ein hohes Maß an Wissen und Kompetenz voraussetzt, dass dies in der Praxis nicht geleistet werden kann. Im Rahmen dieser Forschungsarbeit wird ein Modell entwickelt und erprobt, das genau an dieser Stelle ansetzt:

Im Projekt eL3 (eLearning Project Cluster for Third System Organisations in Europe) sollten in der Gruppe aus verschiedenen Akteuren gemeinsam Evaluationsinstrumente entwickelt und erprobt werden, die zur Unterstützung und Verbesserung der Evaluationspraxis der Projektpartner dienen. Praktiker aus der Krankenhauswirtschaft sowie Bildungsanbieter und Partner mit universitärem Hintergrund sollten hierbei zusammenarbeiten. Auf diese Weise sollte gewissermaßen eine Brücke zwischen Theorie und Praxis entstehen - die Partner mit universitärem Kontext sollten ihr Wissen einbringen (z.B. zum Thema Test- und Fragebogenentwicklung, Evaluation, Didaktisches Design) und die Praktiker mit ihren Praxiserfahrungen und „Alltagserfahrungen“ aus Kursen und Weiterbildungsmaßnahmen ebenfalls entscheidende Impulse geben, damit die Instrumente praxistauglich und handhabbar würden. Das Ziel bei diesem Vorgehen war nicht, standardisierte Instrumente nach wissenschaftlichen Kriterien zu entwickeln, diesem Anspruch könnte in dem Projektkontext nicht gerecht werden, es sollten aber schon Instrumente entwickelt werden, die hochwertiger waren als „selbstgebastelte Fragebögen“, die häufig in der Praxis verwendet werden. Es stand der Nutzen im Vordergrund und nicht primär die Gütekriterien (wie Validität, Reliabilität und Ob- 
jektivität), die bei Testverfahren sonst zentral sind. Es sollten vielmehr zunächst einmal Instrumente geschaffen werden, die auf der Basis des Wissens und der Erfahrungen für die Akteure wichtig, hilfreich und nützlich waren, damit sie die Qualität der beruflichen Bildung in ihren Arbeitsfeldern sichern und ggf. verbessern können (zu den Projektzielen siehe Abschnitt 5.1.1 und zu Prämissen der Aktionsforschung siehe Abschnitt 5.3.1). Durch den Gebrauch der Instrumente (die Erprobung) sollten dann Erfahrungen und Wissen gebündelt werden, und im Netzwerk der Partner sollte ein Austausch stattfinden zu zentralen Themen rund um Weiterbildungsmaßnahmen bzw. e-Learning und Blended Learning (das Projekt eL3 wurde von der Europäischen Kommission im Rahmen der e-Learning-Initiative gefördert und sollte u.a. zur Verbesserung der beruflichen Praxis in diesem Bereich beitragen, siehe dazu auch Unterkapitel 5.1). Längerfristig könnte so eine Erfahrungs- und Wissensbasis aufgebaut werden, die europaweit bzw. über das Internet weltweit auch anderen Akteuren zur Verfügung steht.

Im Zentrum dieser Arbeit steht die Idee von Inventaren als Instrumente zur Unterstützung und Verbesserung der Evaluationspraxis in europäischen Bildungsprojekten. Inventare sind im Gegensatz zu Tests oder Fragebögen meist umfassendere, komplexere Instrumente, die aus mehreren Bestandteilen bestehen oder verschiedene Skalen beinhalten. Für die Begriffe Fragebogen und Inventar gibt es keine abgegrenzte Definition, häufig werden sie synonym verwendet. Ein Test kann hingegen folgendermaßen definiert werden:

„Ein Test ist ein wissenschaftliches Routineverfahren zur Untersuchung eines oder mehrerer empirisch abgrenzbarer Persönlichkeitsmerkmale mit dem Ziel einer möglichst quantitativen Aussage über den relativen Grad der individuellen Merkmalsausprägung." (Lienert 1998, S.1)

Mit dieser Definition wird deutlich, dass der Begriff Test relativ eingeschränkt ist und sich vor allem auf einen wissenschaftlichen Anspruch bezieht. Bei einem Test müssen wissenschaftliche Kriterien streng eingehalten werden und einzelne Kriterien, die empirisch klar abgegrenzt sind und zu denen es einen theoretischen Hintergrund gibt, werden mit dem Ziel untersucht, zu quantitativen Aussagen zu kommen. Es werden so relativ stabile und konstante Merkmale von Personen mittels eines Tests erfasst, so dass Aussagen über die getesteten Personen, z.B. zu deren Verhalten oder Wissen, getroffen werden können.

Der Begriff des Fragebogens ist nicht klar definiert - auch ein selbst erstellter Fragebogen ist ein Fragebogen - es handelt sich einfach um eine Zusammenstellung von Fragen zu einem bestimmten Themenfeld oder in Bezug auf eine bestimmte Maßnahme.

Der Begriff des Inventars hingegen kann weiter gefasst werden. Von der Bedeutung des Wortes her betrachtet, kann unter einem Inventar nach dem Online Etymology Dictionary eine Liste oder ein Verzeichnis aller Vermögenswerte oder Gegenstände bezeichnet werden, die in einem bestimmten Kontext zu finden sind: 
„inventory (n.): early 15c., from O.Fr. inventoire 'inventory, detailed list of goods, catalogue,' from M.L. inventorium (L.L. inventarium) 'list of what is found,' from L. inventus, pp. of invenire "to find" (see invention). The verb is first recorded c.1600."

(http://www.etymonline.com/index.php?term=inventory\&allowed_in_frame=0, Zugriff 31.8.2012)

Der Begriff des Inventars wird häufig im wirtschaftlichen Kontext verwendet und bezeichnet hier die Gesamtheit der Objekte bzw. Besitztümer eines Unternehmens, einer Firma oder Institution:
"in business, any item of property held in stock by a firm, including finished goods ready for sale, goods in the process of production, raw materials, and goods that will be consumed in the process of producing goods to be sold." (http://www.britannica.com/search?query=inventory, Zugriff 31.8.2012)

Außerdem beinhaltet der Begriff Inventar noch weitere Aspekte, wie mit der folgenden Definition deutlich wird:

\author{
"Definition of INVENTORY \\ 1 a: an itemized list of current assets: as (1) : a catalogue of the property of \\ an individual or estate (2) : a list of goods on hand $b:$ a survey of natural \\ ressources $c:$ a list of traits, preferences, attitudes, interests, or abilities \\ used to evaluate personal characteristics or skills \\ 2: survey, summary \\ 3: the quantity of goods or materials on hand : stock \\ 4: the act or process of taking an inventory" \\ (http://www.merriam-webster.com/dictionary/inventory, Zugriff 31.8.2012)
}

Zum einen kann ein Inventar aus einer Auflistung von Gegenständen und Produkten bestehen, z.B. mit dem Ziel einer Vermögensaufstellung, es können aber auch natürliche Ressourcen, Einstellungen oder Fähigkeiten systematisch erfasst bzw. zusammengefasst werden. Als Inventarisierung kann dann das Aufstellen bzw. der Prozess der Erfassung aller relevanten Objekte oder Aspekte bezeichnet werden; „Inventur machen“ bedeutet z.B., alle Vermögenswerte einer Firma oder Person aufzulisten.

In der psychologischen Diagnostik kann zwischen einem Inventar und einem Test unterschieden werden. Während Tests sich meistens auf die Messung bestimmter klar abgegrenzter Merkmale beziehen, für die es Vorgaben gibt, was als "richtig“ und als "falsch“ gilt, geht es beispielsweise bei Persönlichkeitsinventaren um die Erfassung komplexerer Konstrukte, für die es keine „richtigen oder falschen Antworten“ gibt:

„The term 'test' most frequently refers to devices for measuring abilities or qualities for which there are authoritative right and wrong answers. Such a test may be contrasted with a personality inventory, for which it is often claimed that there are no right or wrong answers."

(http://www.britannica.com/search?query=inventory+versus+test,

Zugriff 31.8.2012) 
Für die zentrale Idee dieser Arbeit, Inventare als Instrumente zur Unterstützung und Verbesserung der Evaluationspraxis in europäischen Bildungsprojekten zu entwickeln und zu erproben, zeigen die aufgeführten Definitionen verschiedene Charakteristika von Inventaren, die in diesem Kontext eine Bedeutung haben:

Die Inventare der vorliegenden Arbeit sind als komplexe Instrumente zu verstehen, die nicht ein klar abgegrenztes Konstrukt erfassen sollen, sondern vielmehr die Reflexion anhand von Schlüsselfragen zu einem bestimmten Themengebiet anregen sollen. Außerdem werden die Inventare aus mehreren Teilen bestehen und können somit auch unterschiedliche Skalen enthalten. Darüber hinaus werden die Inventare, die im Rahmen dieser Forschungsarbeit und des Praxisprojektes eL3 entwickelt und erprobt werden, als eine Art Bestandsaufnahme angelegt, eine Zusammenstellung aller vorhandenen und relevanten Fragen und Punkte (,list of items"), die im Kontext von e-Learning und Blended Learning eine Rolle spielen können. In diesem Sinn wird statt des Begriffs des Fragebogens der Begriff des Inventars als passender gesehen. Für die weitere Verwendung der Instrumente ist der Begriff des Inventars mit einem weiteren Vorteil verbunden und mit folgender Idee verknüpft: Aus einem Inventar können die Praktiker einzelne Items auswählen und sie zu einem individuellen Fragebogen zusammenstellen. Aus der Gesamtheit der Items können so diejenigen ausgewählt werden, die in dem jeweiligen Kontext relevant und passend sind. Dies kann als ein entscheidender Vorteil betrachtet werden, da die zu evaluierenden Maßnahmen der Projektpartner sich zum Teil deutlich voneinander unterscheiden (z.B. verschiedene Zielgruppen und Inhalte, unterschiedliche Dauer der Kurse und Weiterbildungsmaßnahmen). Trotzdem wird davon ausgegangen, dass es Fragen gibt, die generell und unabhängig vom Kurskontext eine Rolle bei der Evaluation von Weiterbildungsmaßnahmen spielen und die deshalb auch quasi als immer wieder verwendbare Items in verschiedenen Szenarien eingesetzt werden können.

Der Inventaridee liegt weiterhin ein bestimmtes Verständnis von Evaluation zu Grunde. Es geht vor allem darum, Erkenntnisse zu gewinnen, Wissen zu generieren und den Dialog zwischen verschiedenen Praxispartnern zu fördern, sowie darum, Praktikern Entscheidungshilfen zur Weiterentwicklung und Optimierung ihrer beruflichen Praxis zu bieten und nicht darum, Instrumente zur Kontrolle oder Legitimierung zur Verfügung zu stellen (siehe zu verschiedenen Funktionen und Zielen von Evaluation auch Kapitel 1.3). Es handelt sich weiterhin um einen partizipativen Evaluationsansatz, d.h. die Praktiker sollen zum einen beteiligt werden bei der Erarbeitung der Evaluationsinstrumente und dem gesamten Verfahren, und zum anderen sollen die Materialien und Vorgehensweisen im Sinne des EmpowermentAnsatzes die Akteure befähigen, die Evaluationen selbstständig durchzuführen. Die Evaluationsinstrumente sollen flexibel sein, um an verschiedene Kontexte angepasst werden zu können, und die erhobenen Daten bzw. Ergebnisse der Evaluationsaktivitäten sollen nützlich und handhabbar sein. Die Kompetenzen der Beteiligten sollen gestärkt werden, so dass sie 
selbst fähig sind, ihre Weiterbildungsmaßnahmen zu evaluieren und auf der Grundlage der Ergebnisse zu verbessern.

Schließlich war mit der Inventaridee die Perspektive verbunden, dass bei einer Rückmeldung von empirischen Daten aus den einzelnen Projekten die Möglichkeit entsteht, die einzelnen Items mit Kennziffern zu versehen, so dass für spätere Verwendungen auch Hinweise entstehen würden, wie die betreffenden Items tendenziell bewertet würden. Es wäre somit auch eine Perspektive für Standardisierung und Benchmarking entstanden. Weiter fortgesetzt, würde ein fortwährender Verbesserungsprozess für die Inventare möglich werden. ${ }^{5}$

In dem folgenden Unterkapitel wird das Projekt eL3, in dessen Rahmen die vorliegende Forschungsarbeit entstanden ist, beschrieben. Es werden die Projektziele und die beteiligten Partner sowie der Ablauf des Projektes dargestellt. Darauf aufbauend wird die Problemstellung geschildert und die Fragestellungen werden entwickelt. Anschließend wird die vorliegende Arbeit eingeordnet in den Typus der Aktionsforschung (bzw. Handlungsforschung) und die eingesetzten Methoden werden erläutert.

\subsection{Fallstudie: Das Projekt eL3}

Die Idee für die vorliegende Forschungsarbeit entstand im Rahmen des von der Europäischen Kommission im Rahmen der E-Learning Initiative geförderten Projektes eL3 (eLearning Project Cluster for Third System Organisations in Europe). Die Autorin arbeitete als wissenschaftliche Mitarbeiterin in diesem Projekt, das einen Zeitraum von 24 Monaten von Januar 2004 bis Dezember 2005 umfasste, und in dem zehn europäische Projektpartner aus unterschiedlichen Einrichtungen des Dritten Systems (Gesundheits- und Sozialwirtschaft) zusammen arbeiteten. In den nächsten Abschnitten werden die Projektziele, die Projektpartnerschaft sowie der Ablauf des Projektes näher beschrieben. Das Projekt eL3 dient in dieser Arbeit als Fallstudie (genauer: Einzelfallstudie), d.h. es soll erprobt und untersucht werden, ob im Rahmen dieses europäischen Bildungsprojektes der Ansatz von Inventaren realisierbar ist. Damit ist verbunden, dass keinerlei Anspruch auf Verallgemeinerbarkeit der hier gewonnenen Erfahrungen und Einsichten erhoben werden kann. Im Unterschied zu Fallstudien, wie sie in der Literatur oft vorzufinden sind (siehe dazu auch Hamel 1993 und Yin 2009), besteht auch kein Anspruch auf Exemplarizität oder irgend eine Besonderheit dieses Falles. Man könnte auch sagen, dass eine Pilotstudie durchgeführt wird, die im Wesentlichen in Form einer Fallstudie aufgebaut ist, d.h. sie bezieht sich auf dieses eine Projekt und diese eine Projektgruppe.

\footnotetext{
${ }^{5}$ Um es gleich zu sagen: Diese Perspektive konnte in dem eL3-Projekt aus verschiedenen Gründen nicht realisiert werden.
} 
Der Unterschied zwischen Fallstudien und Pilotstudien besteht darin, dass letztere oft zunächst der Erkundung dienen und umfangreichere Studien (Fallstudien oder quantitative Untersuchungen) auf der Basis der gewonnenen Erkenntnisse durchgeführt werden können.

Friedrichs beschreibt Pilotstudien folgendermaßen:

„Weniger deskriptiv als analytisch, wenngleich auf zu kleinen Stichproben beruhend, sind explorative Studien oder pilot studies. Nach den Definitionen oben sind sie Exploration und Pretest zugleich. Derartige Studien sind dann sinnvoll, wenn Kenntnisse und Hypothesen zu einem Problem vorliegen, dem aber nur eine umfangreiche Studie gerecht würde; die umfangreiche Studie vorzubereiten ist Aufgabe einer explorativen Studie (wenngleich oft die Hauptuntersuchung im Anschluß gar nicht erfolgt)." (Friedrichs 1990, S.155f)

Bei Fallstudien wird ein exemplarischer Fall ausgewählt, wobei ein Fall eine Person oder eine Gemeinschaft sein kann, auf die sich die Untersuchung bezieht. Ragin und Becker (1992) befassen sich genauer damit, was ein Fall ist oder sein kann. An dieser Stelle sei nur die Definition von Flick wiedergegeben, die verdeutlicht, worum es in Fallstudien geht:

„Fallstudien zielen auf die genaue Beschreibung oder Rekonstruktion eines Falls ab [...]. Das Fallverständnis ist dabei eher weit gefasst - neben Personen können auch soziale Gemeinschaften (z.B. Familien), Organisationen und Institutionen (z.B. ein Pflegeheim) Gegenstand einer Fallanalyse werden. Dabei ist das entscheidende Problem die Identifikation eines für die Fragestellung der Untersuchung aussagekräftigen Falls, die Klärung, was zum Fall noch dazugehört und welche methodischen Zugänge seine Rekonstruktion erfordert [...]." (Flick 2008a, S.253f)

Mit diesem Zitat wird u.a. deutlich, dass verschiedene Methoden bei einer Fallstudie zum Einsatz kommen können. Meistens werden dabei qualitative Methoden, wie etwa Interviews, Tagebücher oder Beobachtungen, eingesetzt bzw. kombiniert. Es können auch qualitative und quantitative Methoden kombiniert werden, wobei berücksichtigt werden sollte, dass sie sich in wesentlichen Aspekten voneinander unterscheiden (vgl. Flick, von Kardoff \& Steinke 2008, S.24). Bei der qualitativen Forschung geht es oft „um die Erschließung eines bislang wenig erforschten Wirklichkeitsbereichs“ (ebenda, S.25).

Ein Vorteil von Fallstudien ist deren Überschaubarkeit und Begrenzung, so dass sie sich besonders zur Erkundung eines Themengebietes eignen:

\begin{abstract}
„Einzelfallstudien zeichnen sich gegenüber Stichprobenuntersuchungen mit vielen Untersuchungsteilnehmern durch eine bessere Überschaubarkeit des Untersuchungsfeldes und damit durch eine bessere Kontrollierbarkeit potentieller Störvariablen aus; sie eignen sich besonders zur Erkundung psychologischer, medizinischer, pädagogischer o.ä. Hypothesen.“ (Bortz \& Döring 1995, S.8)
\end{abstract}

Bei Einzelfallstudien kann nach Bortz und Döring (vgl. 1995, S.8) entweder ein Anspruch auf Allgemeingültigkeit gestellt werden, der dann durch hypothesenprüfende Untersuchungen an repräsentativen Stichproben bestätigt oder widerlegt werden kann, es kann aber auch bei der Betrachtung eines Einzelfalls bleiben und die Hypothesen werden durch eine systematische Untersuchung desselben Falls bestätigt oder wiederlegt. 
Insgesamt lässt sich festhalten, dass Einzelfallstudien bzw. Pilotstudien verschiedene Vorteile mit sich bringen und sich insbesondere eignen, um eine explorative Studie durchzuführen, an die sich dann ggf. weitere Untersuchungen anschließen können.

\subsubsection{Projektziele}

Das Hauptziel des Projekts eL3 war die Verbreitung von computerbasiertem Lernen in Betrieben der Gesundheits- und Sozialwirtschaft. Durch die Projektaktivitäten sollte eL3 zur Verbesserung der Qualität in der beruflichen Bildung beitragen und den Akteuren in diesen Berufsfeldern Handlungs- und Entscheidungshilfen für einen Einsatz von innovativen Lernformen liefern. Das Konzept für das Projekt eL3 umfasste drei ineinander greifende Arbeitspakete:

\section{Analyse und Evaluation}

2. Netzwerkbildung

3. Kommunikation und Verbreitung

Im ersten Arbeitspaket sollten computerbasierte Lernangebote im europäischen Gesundheits- und Sozialwesen erfasst, analysiert und evaluiert werden. Insbesondere die methodisch-didaktischen Konzepte, das Lernerverhalten und Implikationen für internes und externes Lehrpersonal sowie der Nutzen für die Einrichtungen sollten dabei ermittelt werden.

Das Ziel des zweiten Arbeitspaketes war die Vernetzung von Organisationen des dritten Sektors, die e-Learning und Blended Learning anbieten. Es sollte eine stabile Organisationsform für das Netzwerk gefunden und im Laufe des Projekts eine nachhaltige Entwicklung eingeleitet werden, so dass ein Fortbestehen des Netzwerks auch nach der Projektförderungsphase möglich wäre.

Im dritten Arbeitspaket war das Ziel, ein leistungsfähiges, erweiterbares Open-Source Webportal zu entwickeln, das neben verschiedenen Kommunikationsdiensten ein Lernmanagement- und ein Contentmanagement-System beinhalten sollte. Mittels dieses Webportals sollte es den Netzwerkmitgliedern möglich sein, Wissensinhalte abzurufen und bereitzustellen. Ferner sollten die Netzwerkpartner ihre Produkte und Dienstleistungen (z.B. Kurse und Beratungsangebote) anbieten können. Auf diese Weise sollte eine Plattform entstehen, über die zum einen die verschiedenen Angebote der Mitglieder präsentiert und zum anderen der Austausch über Erfahrungen mit e-Learning und Blended Learning unter den Netzwerkmitgliedern möglich wäre (vgl. http://www.blinc-eu.org/konzept_el3/?L=1, Zugriff 5.9.2012). 


\subsubsection{Projektpartnerschaft}

Im Projekt eL3 waren Partnereinrichtungen aus Deutschland, Schottland, Italien und Frankreich, die aus verschiedenen Bereichen des Dritten Systems stammten, beteiligt. Die einzelnen Partner waren die folgenden:

1. Asklepios Harzkliniken $\mathrm{GmbH}$, Goslar, Deutschland

2. BUPNET - Bildung und Projekt Netzwerk GmbH, Göttingen, Deutschland

3. Pädagogisches Seminar der Georg August Universität Göttingen, Deutschland

4. Robert Gordon University Aberdeen, CenPrad, Schottland, Vereinigtes Königreich

5. Tetraktys Localization Agency, Spoleto, Italien

6. Unita Sanitaria Locale 7, Gesundheitsagentur, Siena,Italien

7. Centre Hospitalier Universitaire de Bordeaux, Frankreich

8. Institut Paoli Calmettes, Marseille, Frankreich

9. GIP FIPAN, Institut des französischen Bildungsministeriums, Nizza, Frankreich

10. INSUP Formation, Bildungsagentur Bordeaux, Frankreich

Die Projektpartner sind in verschiedenen Bereichen angesiedelt, die sich wie folgt einteilen lassen:

\section{a) Gesundheits- und Krankenhauswirtschaft}

- Asklepios Harzkliniken GmbH, Goslar, Deutschland

- Unita Sanitaria Locale 7, Siena, Italien

- Institut Paoli Calmettes, Marseille, Frankreich

\section{b) Bildungsanbieter}

- BUPNET - Bildung und Projekt Netzwerk GmbH, Göttingen, Deutschland

- Tetraktys Localization Agency, Spoleto, Italien

- GIP FIPAN, Nizza, Frankreich

- INSUP Formation, Bordeaux, Frankreich

\section{c) Universitäten}

- Pädagogisches Seminar der Georg August Universität, Göttingen, Deutschland

- Robert Gordon University Aberdeen, CenPrad, Schottland

- Centre Hospitalier Universitaire de Bordeaux, Frankreich

Im Folgenden werden die einzelnen Projektpartner und deren Arbeitsschwerpunkte genauer beschrieben. 


\section{Asklepios Harzkliniken $\mathrm{GmbH}$, Goslar, Deutschland}

Die Asklepios Kliniken wurden 1984 gegründet und zählen zu den größten privatwirtschaftlich tätigen Klinikunternehmen in Deutschland. Zur Gruppe der Asklepios Kliniken gehören im Eigentum geführte Häuser, im Management betriebene Einrichtungen sowie Beteiligungen an Gesundheitseinrichtungen. Die Einrichtungen des Konzerns befinden sich in fast allen deutschen Bundesländern. Außerdem zählt zur Gruppe die Pacific Health Corporation mit 6 Akuthäusern in den USA. Das Unternehmen betreibt allein in Deutschland 89 Einrichtungen und beschäftigt insgesamt 34.500 Mitarbeiter. Die Asklepios Harzkliniken $\mathrm{GmbH}$ ist Teil der Asklepios-Gruppe und unterhält drei Krankenhäuser in Goslar, Bad Harzburg und Clausthal-Zellerfeld (http://www.asklepios.com, Zugriff 5.9.2012). In den Harzkliniken sind etwa 1100 Mitarbeiter beschäftigt, deren kontinuierliche Fortbildung als ein Grundpfeiler fortschrittlicher Personalentwicklung verstanden wird. Den ca. 700 Pflegekräften, 250 Mitarbeitern in verschiedenen Wirtschaftsbereichen und der Verwaltung sowie 150 Medizinern steht ein umfangreiches Aus- und Fortbildungsangebot zur Verfügung, wobei in zunehmendem Maße e-Learning als Methode eingesetzt wird. In den Schulungsbereichen EDV (Microsoft Office Anwendungen, Word, Excel, Power Point), Management (EMAS, Marketing) und verschiedenen regelmäßig zu wiederholenden Pflichtveranstaltungen (z.B. Brandschutz) wird eLearning bereits genutzt. Die Asklepios Harzkliniken $\mathrm{GmbH}$ verfügt über ein eigenes Bildungszentrum und eine angeschlossene Pflegeschule.

Im Projekt el3 hatte die Asklepios Harzkliniken $\mathrm{GmbH}$ die Aufgabe des zentralen Projektmanagements, was die Organisation der transnationalen Treffen, die Planung und Überwachung der Arbeitsabläufe sowie die Dokumentation und Berichterstattung der Projektergebnisse umfasste.

\section{BUPNET - Bildung und Projekt Netzwerk GmbH, Göttingen, Deutschland}

Das Bildungs- und Beratungsunternehmen BUPNET (Bildung und Projekt Netzwerk GmbH) wurde 1985 gegründet und umfasst drei Standorte in Deutschland (Göttingen, Lüneburg, Potsdam). BUPNET bietet Weiterbildungen und berufliche Qualifizierungen auf nationaler und europäischer Ebene an und führt Bildungs- und Beratungsprojekte durch. Das Unternehmen entwickelt e-Learning und Blended Learning Systeme und webgestützte Dienste für den betrieblichen Einsatz und für EU-Projekte und berät seine Kunden in den Bereichen Bildungs-, Informations-, Wissens- und Projektmanagement (http://www.bupnet.de/profil.html, Zugriff 5.9.2012).

Zu den Zielgruppen von BUPNET gehören Unternehmen und Institutionen aus verschiedenen Branchen und deren Mitarbeiter sowie Arbeitsuchende. Zu den Spezialgebieten von BUPNET zählen vor allem Gesundheits- und Sozialwirtschaft, Tourismus, Umweltwirtschaft und Informationstechnologien. Im Mittelpunkt der Maßnahmen, die von BUPNET entwickelt und realisiert werden, stehen die Förderung kleiner und mittlerer Unternehmen und die damit verbundene Arbeitsplatzsicherung und Karriereförderung der Beschäftigten und/oder die 
berufliche Reintegration von Arbeitslosen. Das interdisziplinäre Mitarbeiterteam von BUPNET konzipiert und realisiert seit Ende der 90er Jahre von der Europäischen Kommission geförderte Projekte und ist in verschiedenen transnationalen Netzwerken tätig.

Im Rahmen des Projektes eL3 war die BUPNET GmbH vor allem für die Entwicklung und Betreuung der im Projekt genutzten Lernplattform sowie für den Aufbau des Webportals zuständig. Aufgrund der Erfahrungen mit europäischen Projekten und Netzwerken war das Team von BUPNET auch maßgeblich für den Aufbau des blinc-Netzwerkes, das auf der Basis des eL3 Projektes gegründet werden sollte, verantwortlich.

\section{Pädagogisches Seminar der Georg August Universität Göttingen, Deutschland}

Das Pädagogische Seminar ist Teil der Sozialwissenschaftlichen Fakultät der Georg-AugustUniversität Göttingen (http://www.uni-goettingen.de/de/sh/29951.html, Zugriff 5.9.2012). Prof. Dr. Hans-Dieter Haller arbeitet hier seit den 70er Jahren in der pädagogischen Forschung und Lehre im Bereich der Entwicklung didaktischer Modelle, der Entwicklung und Erprobung von Curricula („Kinder und ihre natürliche Umwelt“) und der Übertragung von innovativen Lernformen in die schulische und berufliche Praxis. Zu seinen Schwerpunkten gehören neben der Medien- und Webdidaktik auch Aspekte interkulturellen Lernens, Lernstilforschung, didaktische Sozialisation und Jugendarbeit (http://www.uni-goettingen.de/de/34402.html, Zugriff 5.9.2012).

Im Projekt eL3 übernahm das Team des Pädagogischen Seminars, bestehend aus Prof. Dr. Hans-Dieter Haller und der Verfasserin, die wissenschaftliche Leitung. Zu dem Aufgabenspektrum gehörte laut Projektplanung der Untersuchungsaufbau innerhalb der Projektpartnerschaft, d.h. die Konzeption eines Forschungsdesigns für das Projekt eL3 und das aufzubauende Lernnetzwerk. Zum Ende des Projektes eL3 sollte eine Institutionsgründung (blinc blended learning institition`s cooperative) erfolgen, die begleitet und evaluiert werden sollte. Außerdem gehörte es zu den Aufgaben des Teams vom Pädagogischen Seminar, Ergebnisbeschreibungen aus den Untersuchungen im Rahmen von eL3 anzufertigen und Handlungsempfehlungen für verschiedene Akteure des Dritten Systems in Europa zu geben. Mittels Publikationen sollten Informationen und Ergebnisse aus der Projekt- bzw. Netzwerkarbeit aufbereitet und einer breiteren Öffentlichkeit zugänglich gemacht werden.

Die wissenschaftliche Leitung des Projektes eL3 umfasste zunächst vor allem die Bereitstellung von Wissen, d.h. Theorien und Forschungsergebnissen zum Thema e-Learning, Blended Learning, Evaluation, Evaluationsmethoden und Didaktisches Design. Die Vermittlung bzw. Bereitstellung von Wissen sollte über Vorträge während der transnationalen Treffen sowie Texte, Materialien und Lerneinheiten auf der projektinternen Lernplattform realisiert werden. Die wissenschaftliche Leitung beinhaltete auch die individuelle Beratung von Projektpartnern hinsichtlich der Entwicklung bzw. Verbesserung ihrer e-Leaning und Blended Learning-Angebote. 
Das Team vom Pädagogischen Seminar hatte einerseits die Aufgabe, das Projekt intern zu evaluieren mit dem Ziel, die Zusammenarbeit in der Projektpartnerschaft zu dokumentieren und zu optimieren, und andererseits extern eine Bestandsaufnahme von e-Learning und Blended Learning-Angeboten bzw. Produkten im Dritten Sektor in Europa zu machen. Ferner sollte am Ende des Projektes eine summative Evaluation erfolgen, um die Ergebnisse und Produkte, die im Rahmen von eL3 erarbeitet wurden, darzustellen und abschließend zu bewerten.

\section{Robert Gordon University Aberdeen, CeNPRaD, Schottland, Vereinigtes Königreich}

Das CeNPRaD (Centre for Nurse Practice Research and Development) ist Teil der Schule für Krankenpflege und Geburtshilfe (School of Nursing and Midwifery) an der Robert Gordon Universität in Aberdeen und gehört zur Fakultät für Gesundheits- und Sozialwesen (Faculty of Health and Social Care) (http://www.rgu.ac.uk/nursing, Zugriff 5.9.2012). Im CeNPRaD werden Studierenden von der Phase des Studienbeginns über das gesamte Studium bis nach der Dissertationsphase Blended Learning-Angebote zur Verfügung gestellt. Das Spektrum umfasst Lehrangebote zu verschiedenen Forschungsmethoden (z.B. qualitative Methoden, Survey Methoden), zur systematischen Literaturrecherche, zum Thema Evaluationsdesign und -methoden, zu klinischen Prüfungsverfahren, Curriculum-Entwicklung sowie Erstellung und Implementierung von klinischen Richtlinien. Das CeNPRaD wird geleitet von Dr. Bernice West, die Krankenschwester, Psychologin und promovierte Sozialwissenschaftlerin ist. Dr. Bernice West lehrt an der Robert Gordon Universität in den Bereichen Gesundheitspflege für Familien (Family health nursing), Forschungsmethoden, Infektionskrankheiten bei der Gesundheitsfürsorge (Health care aquired infections) und Implementierung von klinischen Richtlinien. An der Universität in Aberdeen ist man um eine enge Zusammenarbeit mit verschiedenen Institutionen aus der Praxis bemüht (z.B. industrielle Organisationen, Pflegeeinrichtungen, Regierungsstellen und Forschungsinstitute). Der Großteil der Veranstaltungen kann neben der Arbeit in Praxiseinrichtungen durchgeführt werden, und eine steigende Zahl von Kursen ist den Studenten als e-Learning bzw. Blended Learning-Angebot über den Virtuellen Campus der Universität zugänglich.

Im Projekt eL3 sollte das Mitarbeiterteam vom CeNPRaD um Dr. Bernice West Erfahrungen mit e-Learning und Blended Learning und mit dem Virtual Campus in das Netzwerk einbringen. Außerdem war eine Mitarbeit bei dem Untersuchungsaufbau sowie der Erstellung von Handlungsempfehlungen und Publikationen vorgesehen. Das CeNPRaD sollte auch eine Brückenfunktion zu Pflegeeinrichtungen erfüllen und so zur Verbreitung von im Projekt eL3 erarbeiteten Materialien beitragen.

\section{Tetraktys Localization Agency, Spoleto, Italien}

Die Tetraktys Localization Agency bietet Weiterbildungen und verschiedene Dienstleistungen im Bereich der Internationalisierung von Produkten und Services an. Tetraktys ist speziali- 
siert auf Übersetzungen, Software-Internationalisierung, Webinhalte, Content Management und Beratungen im Projekt Management (http://www.tetraktys.it ${ }_{2}$ Zugriff 5.9.2012).

Bei der Internationalisierung von Produkten und Services geht es nicht nur darum, Texte zu übersetzen, sondern sie an den Zielmarkt anzupassen durch einen Prozess, der Lokalisierung genannt wird. Die Tetraktys Localization Agency bietet hierfür Beratung und Unterstützung an. Durch die Vermittlung von Wissen zum Thema Projekt- und Qualitätsmanagement möchte Tetraktys ihre Klienten unterstützen, Kunden optimal zu erreichen und eine hohe Kundenzufriedenheit zu erzielen. Webinhalte und Produkte können nicht einfach nur übersetzt werden, sondern müssen an verschiedene Sprachen, Zielgruppen und Kulturen angepasst werden. Diesen Prozess unterstützt oder übernimmt die Tetraktys Localization Agency. Außerdem bietet Tetraktys Beratungstätigkeiten und Weiterbildungsmöglichkeiten für Projekt-Management an. Es werden Kurse und Trainings in Italienisch, Französisch und Englisch sowie individuelle Beratungen angeboten.

Für das Projekt eL3 sollte die Tetraktys Localization Agency ihre Erfahrungen und Fähigkeiten mit der Übersetzung und Implementierung (Lokalisierung) von Webinhalten sowie eLearning und Blended Learning einbringen.

\section{Unita Sanitaria Locale 7, Gesundheitsagentur, Siena,Italien}

Die Unita Sanitaria Locale 7 (USL 7) ist eine staatliche Gesundheitsagentur mit einem eigenen Ausbildungszentrum. Verschiedene Einrichtungen aus dem Gesundheitswesen (drei Krankenhäuser, verschiedene ambulante Dienste und eine Krankenpflegeschule) sind in der Unita Sanitaria Locale 7 zusammengeschlossen. Die Gesundheitsagentur wurde 1995 gegründet und hat insgesamt 2300 Beschäftigte (http://www.usl7.toscana.it, Zugriff 5.9.2012). Innerhalb der Unita Sanitaria Locale 7 wird die kontinuierliche Weiterbildung von Mitarbeitern als Schlüssel für das Qualitätsmanagement eines Unternehmens angesehen. In zunehmendem Maße werden e-Learning Angebote eingesetzt, insbesondere um längere Ausfallzeiten des Personals zu vermeiden und dieses nicht aus dem Arbeitsprozess herausnehmen zu müssen. Das Lernen soll während der Arbeitszeiten möglich sein bzw. in die Freizeit der Mitarbeiter verlagert werden. Die Gesundheitsagentur realisiert bereits zahlreiche eLearning-Kurse, bietet aber auch Blended Learning an. Zu den Zielgruppen gehören Ärzte, Krankenpflegekräfte, Verwaltungsangestellte und sämtliches Personal der beteiligten Einrichtungen aus dem Dritten Sektor in der Region der Toskana. Bisher werden e-Learning und Blended Learning-Kurse u.a. zu folgenden Themen durchgeführt: Management von Budget und Kontrollsystemen innerhalb der Unita Sanitaria Locale 7, Nutzung verschiedener Computer-Programme (z.B. Microsoft Office Applikationen), Umweltmanagement im Krankenhaus und Datenschutz im Gesundheitswesen. Die Unita Sanitaria Locale 7 arbeitet ebenfalls eng mit der Universität Siena zusammen.

Im Projekt eL3 sollte das Team der Unita Sanitaria Locale 7 und der Universität Siena seine Erfahrungen mit e-Learning und Blended Learning einbringen und bei den Handlungsemp- 
fehlungen und Publikationen mitarbeiten. Außerdem sollten die Kontakte in verschiedene Institutionen des Gesundheitswesens für den Aufbau des Netzwerkes genutzt werden.

\section{Centre Hospitalier Universitaire de Bordeaux, Frankreich}

Das Centre Hospitalier Universitaire de Bordeaux ist ein universitäres Krankenhaus und eine Körperschaft des öffentlichen Rechts aus dem Gebiet des Gesundheitswesens (http://www.chu-bordeaux.fr, Zugriff 5.9.2012).

Professor Dr. André Taytard ist spezialisiert auf das Respirationssystem und unterrichtet Studenten, Krankenschwestern und Physiotherapeuten in diesem Themenbereich. Seine Lehre am Centre Hospitalier Universitaire de Bordeaux unterstützt und ergänzt Professor Dr. André Taytard durch ein umfangreiches Angebot auf einer Internetplattform, das er seit 1998 kontinuierlich erweitert (www.respir.com, Zugriff 5.9.2012). Die etwa 700 bis 800 Studenten nutzen diese frei zugänglichen und kostenlosen online-Kurse zur Vorbereitung für ihre $A b$ schlussprüfungen. Die Kurse enthalten Vorträge, Wissensinhalte in Form von Texten und Audioaufnahmen sowie Selbsttests, die einen individuell gesteuerten Lernprozess ermöglichen.

Im Projekt eL3 sollte Professor Dr. André Taytard seine Erfahrungen mit e-Learning sowie dem e-Consulting von Patienten einbringen. Es war außerdem vorgesehen, dass er bei der Erstellung von Handlungsempfehlungen und Publikationen mitarbeitet. Als Universitätsangehöriger sollte er bei dem Untersuchungsaufbau mitwirken und seine Kontakte zu verschiedenen Pflegeeinrichtungen und Krankenhäusern für die Netzwerkarbeit nutzen.

\section{Institut Paoli Calmettes, Marseille, Frankreich}

Das Institut Paoli Calmettes ist eine gemeinnützige Organisation im öffentlichen Dienst und gehört zu einem nationalen Bündnis von 20 Einrichtungen zum Kampf gegen Krebs mit insgesamt 15.000 Mitarbeitern. Zum Institut Paoli Calmettes gehört auch ein universitäres Krankenhaus (www.institutpaolicalmettes.fr, Zugriff 5.9.2012). Es werden vor allem vier Ziele im Zusammenhang mit Krebskrankheiten verfolgt: Prävention, Vorsorge, Forschung und Lehre. Das IPC ist besonders spezialisiert auf Krebs im Verdauungssystem, Brustkrebs und Blutkrebs. Im Institut Paoli Calmettes sind etwa 1200 Mitarbeiter beschäftigt. Eine kontinuierliche Weiterbildung des Personals und der medizinischen Mitarbeiter wird als elementar angesehen, wobei e-Learning und Blended Learning zunehmend genutzt werden.

Im Projekt eL3 sollte das Team vom Institut Paoli Calmettes seine Erfahrungen mit eLearning einbringen und bei Handlungsempfehlungen und Publikationen mitarbeiten. Außerdem sollte das Team seine Kontakte zu verschiedenen Einrichtungen des Dritten Systems für die Netzwerkarbeit nutzen. 
GIP FIPAN, Nationales Bildungsinstitut des französischen Bildungsministeriums, Nizza, Frankreich

Die GIP FIPAN (Groupement d'Interet Public, Formation et Insertion Professionnelles, Academie de Nice) ist ein nationales Bildungsinstitut, das dem französischen Bildungsministerium unterstellt ist. Die öffentliche Interessengruppe hat zur Aufgabe, auf akademischer Ebene Institute des Bildungswesens bei der Entwicklung von Ausbildungsinhalten und deren Anpassung an sich ständig verändernde gesellschaftliche Anforderungen zu unterstützen. GIP FIPAN beteiligt sich nach Bedarf an der Erstellung komplexer Trainingskurse und arbeitet eng mit Instituten zusammen, die für die Aus- und Weiterbildung von Lehrern verschiedener Schulformen verantwortlich sind. Ferner kooperiert GIP FIPAN mit lokalen Bildungsanbietern und trägt zur Reflexion angemessener und erforderlicher Lehrinhalte bei. Zu GIP FIPAN gehört u.a. das Centre Académique Formation Continue (CAFOC) in Nizza, das alle Institute der nationalen Bildung im Bereich der Erwachsenenbildung und Ausbildung bündelt. GIP FIPAN soll Bildungsangebote überwachen und Innovationen anregen (http://www.acnice.fr, Zugriff 5.9.2012).

Im Projekt eL3 sollte das Mitarbeiterteam von GIP FIPAN seine Erfahrungen mit der Entwicklung komplexer Weiterbildungsangebote einbringen und sich bei der Erstellung von Handlungsempfehlungen beteiligen. Außerdem war vorgesehen, dass sie ihre Kontakte zu Akteuren auf ministerieller Ebene nutzen und in das Netzwerk einbringen. Ferner sollte GIP FIPAN die Institutionalisierung des Netzwerkes unterstützen.

\section{INSUP Formation, Bildungsagentur Bordeaux, Frankreich}

Die INSUP Formation wurde 1978 gegründet und ist eine gemeinnützige Bildungsagentur. Der Hauptsitz befindet sich in Bordeaux, zusätzlich gibt es fünfzehn Filialen in der Region von Aquitaine. Die INSUP Formation bietet berufliche Aus- und Weiterbildungen für Jugendliche und Erwachsene im Südwesten von Frankreich an. Das Hauptziel der Organisation ist, die Lern- und Trainingsbedarfe für den regionalen Arbeitsmarkt zu ermitteln und Bürger zu befähigen, sich in diesen zu integrieren. Die Zielgruppen, mit denen die INSUP Formation arbeitet, sind Jugendliche und junge Erwachsene ohne Schulabschluss bzw. Berufsausbildung, Arbeitssuchende, Versorgungsempfänger von Wiedereingliederungsmaßnahmen, Erwerbsunfähige und die Arbeiterschaft im Allgemeinen (http://www.insup.org, Zugriff 5.9.2012).

INSUP bietet Kurse zum Ausbau von Kompetenzen und Fähigkeiten (z.B. Sprachkurse, Schlüsselkompetenzen), Karriereberatung, Hilfe bei der Arbeitssuche sowie Lernangebote zur Qualifizierung auf dem Arbeitsmarkt (z.B. Fähigkeiten für den Umgang mit dem Computer, Buchführung, Sicherheitstraining, Umweltschutz, Hygiene, Marketing und Verkauf) an. Die Kurse, die INSUP bereitstellt, werden angeboten in Kooperation mit den Behörden vor Ort, dem Arbeitsamt und sozialen Organisationen, die zum Auftrag haben, benachteiligte Gruppen zu fördern. E-Learning und Blended Learning werden dabei bereits seit Anfang der 
90er Jahre genutzt, wobei vor allem die Überwindung von räumlichen Distanzen als großer Vorteil gesehen wird. Die Lernenden müssen aus den ländlichen Regionen nicht in die gröBeren Städte kommen, um zu lernen, sondern können mittels der e-Learning-Angebote im Kontakt mit Tutoren von zu Hause aus lernen bzw. müssen bei Blended Learning-Kursen nur von Zeit zu Zeit anreisen. Außerdem werden IT-Kompetenzen vermittelt.

Im Projekt eL3 sollte das Mitarbeiterteam von INSUP seine Erfahrungen mit e-Learning und Blended Learning einbringen und sich an der Erstellung von Handlungsempfehlungen beteiligen. Für die Netzwerkarbeit sollten die Kontakte zu verschiedenen Institutionen und Bildungsträgern auf nationaler Ebene genutzt werden. Außerdem sollte das Team von INSUP an Publikationen mitwirken.

\subsubsection{Projektablauf}

Das Projekt eL3 erstreckte sich über einen Zeitraum von 24 Monaten von Januar 2004 bis Dezember 2005. Es fanden fünf transnationale Treffen in verschiedenen Städten und Ländern der beteiligten Projektpartner statt. In der übrigen Zeit kommunizierten die Partner über E-Mail und eine eigens dafür bereitgestellte Lernplattform. Die Projektarbeit wurde durch die drei Arbeitspakete strukturiert, die ineinander griffen, wobei die Übergänge zum Teil fließend waren. Die folgende Abbildung zeigt den Projektablauf mit den einzelnen Arbeitspaketen, dem zeitlichen Rahmen und den ausgeführten Aktivitäten: 


\begin{tabular}{|c|c|c|}
\hline Arbeitspaket & Zeitraum & Aktivitäten \\
\hline Analyse & $\begin{array}{l}9 \text { Monate } \\
\text { Januar - } \\
\text { September } 2004\end{array}$ & $\begin{array}{l}\text { Kontakt einzelner Partner mit der Projektleitung } \\
\text { Erstes Kennenlernen per E-Mail } \\
\text { 1.Transnationales Treffen in Goslar (22.-24.03.2004) } \\
\text { Vorstellung der Partner mittels eigener Präsentationen } \\
\text { Vorstellung des Evaluationskonzeptes } \\
\text { Bedarfsklärungen } \\
\text { Abstimmung gemeinsamer Ziele und Möglichkeiten } \\
\text { Planung der weiteren Vorgehensweise } \\
\text { Fragebogenentwicklung für die Synopsis der eL3-Partner } \\
\text { Fragebogeneinsatz } \\
\text { Auswertung der Ergebnisse } \\
\text { Erstellung von Profilen der Partner als Netzwerkbasis } \\
\text { Recherche zu und Sammlung von Evaluationsinstrumenten (Fragebögen) für } \\
\text { e-Learning } \\
\text { 2. Transnationales Treffen in Aberdeen (02.-04.09.2004) } \\
\text { Präsentation der Befragungsergebnisse, Bedarfe und Partnerprofile } \\
\text { Theoretische Einführung zum Thema Evaluation } \\
\text { Gründung von Arbeitsgruppen für die Entwicklung der Inventare }\end{array}$ \\
\hline Netzwerkbildung & $\begin{array}{l}8 \text { Monate } \\
\text { Oktober } 2004 \text { - } \\
\text { Mai } 2005\end{array}$ & $\begin{array}{l}\text { Entwicklung der Inventare innerhalb der Arbeitsgruppen über das Lernma- } \\
\text { nagementsystem und E-Mails } \\
\text { 3. Transnationales Treffen in Göttingen (10.-13. 02. 2005) } \\
\text { Präsentation der Ergebnisse der Arbeitsgruppen } \\
\text { Theoretische Einführung zum Thema Blended Learning Design } \\
\text { Vereinbarung der weiteren Vorgehensweise } \\
\text { 4. Transnationales Treffen in Siena (11.-14.05.2005) } \\
\text { Präsentation der gemeinsam entwickelten Inventare } \\
\text { Planung der Erprobung der Inventare in den Partnereinrichtungen } \\
\text { Vereinbarungen zur Übersetzung und Auswertung von Daten } \\
\text { Ideen zur Verwendung der Ergebnisse (Publikationen, Beratungen) }\end{array}$ \\
\hline $\begin{array}{l}\text { Kommunikation und } \\
\text { Verbreitung }\end{array}$ & $\begin{array}{l}7 \text { Monate } \\
\text { Juni - } \\
\text { Dezember } \\
2005\end{array}$ & $\begin{array}{l}\text { Übersetzung der Inventare } \\
\text { Erprobung der Inventare in Einrichtungen der Projektpartner } \\
\text { Auswertung von Daten } \\
\text { Befragung der Projektpartner zur Webseite } \\
\text { Fertigstellung des Webportals } \\
\text { 5. Transnationales Treffen in Marseille (16.-19.11.2005) } \\
\text { Blinc Conference } \\
\text { Zusammenfassung und Bericht zur Erprobung der Inventare } \\
\text { Überarbeitung der Inventare nach der Erprobungsphase } \\
\text { Verbreitung über blinc } \\
\text { Projektabschlussbericht }\end{array}$ \\
\hline
\end{tabular}

Tabelle 9: $\quad$ Übersicht über den Projektverlauf

\section{Analyse (Januar - September 2004)}

Zu Beginn des Projektes kannten sich noch nicht alle Partner, so dass ein Kennenlernen zunächst im Vordergrund stand. In den ersten drei Monaten des Projektes fand der Kontakt und Austausch hauptsächlich zwischen der Projektleitung und einzelnen Partnern per E-Mail 
statt. Seitens des Pädagogischen Seminars wurde ein Evaluationskonzept nach den Antragsunterlagen ausgearbeitet.

Das erste transnationale Treffen fand in Goslar (Deutschland) im März 2004 statt. Hier trafen die Projektpartner nun alle das erste Mal zusammen und hatten die Gelegenheit, sich und ihre Einrichtungen mittels Präsentationen vorzustellen. Das Evaluationskonzept wurde ebenfalls vorgestellt und diskutiert. Es wurde thematisiert, welche Erwartungen, Bedarfe und Wünsche vorlagen und welche Umsetzungsmöglichkeiten im Rahmen des Projektes gegeben waren. Es wurden Vereinbarungen getroffen, wie die weitere Vorgehensweise sein sollte und welche Ziele gemeinsam erreichbar wären. Bereits während dieses Projekttreffens wurde deutlich, dass die Partner doch sehr heterogen waren und sehr unterschiedliche Bedürfnisse und Ansprüche hatten. Als erstes sollte deshalb eine systematische Bestandsaufnahme gemacht werden, welche Gemeinsamkeiten und Unterschiede es zwischen den Partnern gab. Hierzu sollte das Team vom Pädagogischen Seminar einen Fragebogen einsetzen, um wesentliche Informationen zu den Bildungsangeboten der Projektpartner zu sammeln und eine Synopse der eL3 Partner zu erstellen. Es wurde ein Fragebogen entwickelt, mit dem eruiert wurde, was die einzelnen Partner bis zu diesem Zeitpunkt an Angeboten in eLearning und Blended Learning entwickelt hatten. Mit den Antworten der Partner sollte eine einheitliche Beschreibung der jeweiligen Bildungsangebote möglich sein. Der Fragebogen enthielt zum einen Fragen zur Beschreibung der e-Learning-Projekte und den Kontexten und zum anderen Fragen zu Erfahrungen mit e-Learning-Projekten und "Visionen". Auf der Basis der Antworten wurden vom Team des Pädagogischen Seminars Profile für jeden Projektpartner erarbeitet, und auf dem zweiten transnationalen Treffen im September 2004 in Aberdeen (Schottland) der Projektgruppe präsentiert. Außerdem zeigte sich durch die Befragung, dass ein großer Bedarf an Informationen und Handlungsempfehlungen zum Thema Evaluation von e-Learning vorhanden war. Ein Teil der Partner äußerte den Wunsch nach einer grundlegenden Einführung zu Verfahrensweisen und bewährten Instrumenten, ein anderer Teil war eher an einem Austausch von Erfahrungen und Evaluationsergebnissen interessiert. Die Erfahrungen und Vertrautheit mit der Theorie und Praxis von Evaluationen waren sehr unterschiedlich. Während des transnationalen Treffens erfolgte deshalb eine theoretische Einführung zum Thema Evaluation und es wurde vorgeschlagen, dass gemeinsam Inventare für verschiedene Akteure im Kontext von e-Learning entwickelt werden sollten. Das Wissen der Partner aus unterschiedlichen Bereichen des Dritten Sektors sollte dabei zusammengebracht, und wissenschaftliche mit praxisorientierten Ansätzen verbunden werden. Es wurden drei Arbeitsgruppen gegründet, die für die weitere Entwicklung der Inventare zuständig waren.

Netzwerkbildung (Oktober 2004 - Mai 2005)

Ab Oktober 2004 erfolgte die Entwicklung der Inventare innerhalb der drei Arbeitsgruppen. Die Partner kommunizierten dazu per E-Mail und konnten auch das projektinterne Lernma- 
nagementsystem nutzen. Hier konnten Materialien für alle einsehbar gesammelt und dokumentiert werden. Jede Gruppe hatte eine Leitungsperson, die für die Strukturierung der Arbeit, die Bereitstellung der Materialien, die Zusammenfassung von Ergebnissen der Diskussionen und die Umsetzung in eine gemeinsame Version eines Inventars für die jeweiligen Zielgruppen verantwortlich war.

Auf dem transnationalen Treffen im Februar 2005 in Göttingen (Deutschland) präsentierten die Arbeitsgruppen ihre Ergebnisse. Sie berichteten über ihre Arbeit und zeigten der Gruppe, was sie gemeinsam entwickelt hatten. Während dieses Treffens gab es auch eine theoretische Einführung zum Thema Blended Learning-Design, da sich herausgestellt hatte, dass hierfür ein großer Bedarf vorhanden war. Die Projektpartner vereinbarten die weitere Vorgehensweise in Bezug auf die Weiterentwicklung der Inventare, da diese noch nicht einsatzbereit waren.

Das vierte transnationale Treffen fand im Mai 2005 in Siena (Italien) statt. Bis zu diesem Zeitpunkt sollte eine Version der Inventare in den Arbeitsgruppen entwickelt worden sein, die dann von allen Projektpartnern in ihren jeweiligen Einrichtungen erprobt werden könnte. Während des Treffens wurde die aktuelle Version der Inventare präsentiert und geplant, welche Partner sie in welchen Kontexten erproben würden. Es wurde schriftlich fixiert, welche Partnereinrichtungen wie viele Entscheidungsträger, Autoren und Lerner (die drei Zielgruppen der Inventare) in welchen Zeitrahmen befragen würden. Zunächst mussten die Inventare jedoch ins Französische, Italienische und Deutsche übersetzt werden, da die Arbeitssprache im Projekt Englisch war und die Inventare dementsprechend auch nur in dieser Sprache vorlagen. Den Partnern wurde angeboten, dass die Auswertung der Daten, die mit den Inventaren erhoben werden sollten, durch das Team des Pädagogischen Seminars erfolgen könnte, falls sie dies wünschten. Es wurde den Projektmitgliedern freigestellt, ob sie ihre Daten selbst auswerten wollten oder ob dieses für sie durchgeführt werden sollte. Während des Treffens wurde auch darüber diskutiert, wie die Befragungsergebnisse genutzt werden könnten. Einige Partner wollten gerne vor allem Publikationen auf der Basis der erhobenen Daten erarbeiten oder das Wissen in irgendeiner Form über die Internetseiten (das Webportal) einer breiteren Öffentlichkeit zugänglich machen. Andere Projektmitglieder wollten in erster Linie Dienstleistungen in Form von Beratungen oder professionellen Evaluationen anbieten, in denen die Erfahrungen mit den Inventaren und damit erhobenen Daten genutzt werden könnten. Innerhalb der Projektpartnerschaft wurde zu diesem Zeitpunkt vereinbart, dass zunächst die Erprobung der Inventare erfolgen sollte und danach entschieden werden könnte, in welcher Form sie weiter genutzt würden.

\section{Kommunikation und Verbreitung (Juni - Dezember 2005)}

Ab Juni 2005 wurden die bis dahin gemeinsam entwickelten Inventare übersetzt und dann in den verschiedenen Einrichtungen der Projektpartner erprobt. Die erhobenen Daten wurden zum größten Teil von den Partnern selbst ausgewertet und nicht an das Team vom Pädago- 
gischen Seminar geschickt. Es musste den Partnern freigestellt werden, ob sie die erhobenen Daten in das Netzwerk geben wollten oder nicht.

Das Team aus der BUPNET GmbH hatte die Aufgabe, während des Projektes eL3 das Webportal zu entwickeln, das vor allem auch nach der Projektlaufzeit eine Basis für das Netzwerk von Akteuren des Dritten Sektors in Europa sein sollte. Das Webportal wurde kontinuierlich entwickelt und die Bedürfnisse der eL3-Partner fanden dabei Berücksichtigung. Um diesen Prozess systematisch zu begleiten und zu unterstützen, wurden die Partner mit einem eigens dafür entwickelten Webseitenfragebogen befragt. Auf diese Weise sollte ermittelt werden, wie die eL3 Partner das entstehende Webportal bewerteten und in welchen Bereichen sie ggf. Verbesserungsvorschläge oder Änderungswünsche hatten.

Im Oktober 2005 wurde die blinc eG in Göttingen (Deutschland) gegründet. Damit erfuhr das Netzwerk eine offizielle Institutionsgründung.

Auf dem fünften und letzten transnationalen Treffen im November 2005 in Marseille (Frankreich) wurden die Erfahrungen mit den Inventaren zusammengetragen. Jeder einzelne Partner berichtete über seine individuelle Nutzungsweise der Inventare und die damit erhobenen Daten. Die Ergebnisse und Erfahrungen wurden in der Gruppe diskutiert und weitere Möglichkeiten der Anwendung thematisiert. Die Inventare sollten nun nach der Erprobungsphase überarbeitet und dann über das Webportal von blinc verbreitet werden. Innerhalb der Projektpartnerschaft bestand nicht die Möglichkeit, ein dynamisches und automatisches Softwaresystem zu entwickeln und auf europäischer Ebene zur Verfügung zu stellen. Es wurde deshalb beschlossen, dass die Inventare als frei zugängliches Material auf den Webseiten von blinc einer breiteren Öffentlichkeit zugänglich gemacht werden sollten. Zum einen wurden die Inventare als pdf-Dokument hinterlegt, damit sie als Einheit angeschaut werden können, und andererseits als Word-Dokument, damit potenzielle Nutzer in der Lage sind, es nach ihren individuellen Bedürfnissen zu bearbeiten.

Im Anschluss an das Projekttreffen fand die erste blinc-Konferenz in Marseille statt, zu der verschiedene europäische Partner aus dem Dritten Sektor in Europa geladen waren. Das Thema der Konferenz war die „Verbreitung und Valorisierung von innovativen Lernformen in Europa“. Zuerst präsentierten sich die unterschiedlichen Partnereinrichtungen und die Strategie der EU-Kommission „Verbreitung und Valorisierung“6 ${ }^{\text {“6 }}$ wurde erläutert. Anschließend erarbeiteten die Anwesenden in drei Workshops Konzepte zu Vernetzungsmöglichkeiten und Ideen, wie Erfahrungen auf europäischer Ebene über blinc gebündelt und genutzt werden könnten. Die Partner wurden angeworben, der blinc-Gemeinschaft beizutreten, um das europäische Netzwerk aufzubauen und zu nutzen. Im Dezember 2005 ging das Projekt eL3 zu Ende und es wurde noch ein Abschlussbericht für die EU-Kommission geschrieben.

\footnotetext{
${ }^{6}$ „Valorisierung“ war zu dieser Zeit eine neu entstandene Zielsetzung in der Politik der EUProjektförderung, es ist damit letztlich eine pekuniäre Wertschöpfung gemeint.
} 


\subsection{Problemstellung und Fragestellung}

Im Projekt eL3 kamen sehr unterschiedliche Partner aus verschiedenen Einrichtungen zusammen. Einige Partner gehörten Universitäten an, fühlten sich vor allem der wissenschaftlichen Arbeitsweise verbunden und hatten dementsprechende Interessen wie z.B. Publikationen erarbeiten, wissenschaftliche Kriterien einhalten, Theorieentwicklung, klare Definitionen von Begriffen. Andere Partner waren ausgesprochene „Praktiker“ und wollten vor allem Handwerkszeug für ihre alltägliche Arbeit bekommen, das möglichst „abgesichert“ und praktikabel sein sollte, d.h. die Qualität sollte gut und der Einsatz nicht besonders kompliziert sein, schnelle Ergebnisse liefern und nach außen (z.B. gegenüber anderen Praktikern "auf dem Markt") einen gewissen Wettbewerbsvorteil einbringen.

Für die Autorin, die als wissenschaftliche Hilfskraft im Projekt arbeitete, stellten sich einige weitere Fragen: Wie konnten nun diese Unterschiedlichkeiten in der Projektarbeit berücksichtigt werden? Wie könnten Materialien und Instrumente entwickelt werden, die beiden Seiten bzw. den verschiedenen Partnern gerecht würden und die für alle nützlich sein könnten?

Als Fragestellungen für die vorliegende Forschungsarbeit wurden Fragenkomplexe gebildet, die in mehreren Etappen beantwortet werden sollten, und die eine gewissen Offenheit zulassen, da nicht genau absehbar war, wie sich das Projekt bzw. die Zusammenarbeit der Partner entwickeln würde (siehe dazu Charakteristika der Aktionsforschung im Abschnitt 5.3.1).

Drei zentrale Fragenkomplexe, die sich orientiert am Projektverlauf ergaben, leiteten das Forschungsvorgehen:

1. Wie ist die Evaluationspraxis der Partner?

Was tun die Partner zur Evaluation ihrer Lernangebote?

Welche Methoden setzen sie ein?

Findet sich das Forschung-Praxis-Dilemma (siehe Kapitel 4.3)?

2. Können in einem transdisziplinären europäischen Netzwerk gemeinsam Inventare als Evaluationsinstrumente entwickelt werden?

Wie kann diese Entwicklung erfolgen?

Welche Form der Zusammenarbeit bietet sich an und wird akzeptiert?

Wie gestaltet sich der Prozess der gemeinsamen Arbeit?

Welche positiven Effekte hat die Zusammenarbeit?

Welche Schwierigkeiten und Herausforderungen ergeben sich dabei? 
3. Werden die entwickelten Inventare als Evaluationsinstrumente in dem transnationalen europäischen Netzwerk genutzt?

Wer nutzt die Inventare und in welcher Weise?

Welche positiven Effekte entstehen dabei?

Welche Schwierigkeiten und Herausforderungen ergeben sich?

Ist das Modell der Inventare praktikabel? Oder was muss noch getan werden, damit es praktikabel ist?

Zur Klärung dieser Fragen wurden verschiedene Methoden ausgewählt, die jedoch den Projektablauf nicht beeinflussen sollten. Die Methoden sollten vielmehr in Abstimmung mit den Partnern eingesetzt werden. Das Forschungsvorgehen sollte transparent für die Projektpartner sein, da sie nicht als Objekte erforscht werden sollten, sondern als gleichwertige Partner angesehen wurden (siehe dazu den Ansatz der Aktionsforschung bzw. Handlungsforschung im Abschnitt 5.3.1). Es sollten vor allem der Prozess der Entstehung der Inventare sowie deren erster Einsatz (die Erprobung) begleitet werden. 


\subsection{Forschungsvorgehen und -methoden}

Die Autorin war als wissenschaftliche Mitarbeiterin des Pädagogischen Seminars der Universität Göttingen im Projekt eL3 tätig. Es bestanden klare Arbeitsaufträge im Rahmen des Projektes, gleichzeitig war es möglich, auf die Bedarfe der Projektpartner zu reagieren bzw. gemeinsam zu analysieren, wo Handlungs- und Unterstützungsnotwendigkeiten bestanden, um Lösungen zu entwickeln. Das Forschungsvorgehen wird der Aktionsforschung zugeordnet. Es wurden verschiedene qualitative Forschungsmethoden eingesetzt bzw. kombiniert, um das Feld zu analysieren und systematisch sowie wissenschaftlich fundiert Strategien zu entwickeln, die den Projektpartnern helfen sollten, ihre Praxis zu verbessern. Im Folgenden wird das Konzept der Aktionsforschung beschrieben, anschließend das Modell der Triangulation erörtert.

\subsubsection{Aktionsforschung}

Der Begriff Aktionsforschung (,action research“) wurde von Kurt Lewin geprägt (Lewin 1946, 1953), der in verschiedenen Beiträgen auch als Begründer dieser Forschungsart benannt wird (vgl. z.B. Bortz/Döring 2006, Heinze 2001, Wellhöfer 1997). Die „Aktionsforschung als Strategie der empirischen Sozialforschung“ (Pieper 1977, S. 7) kann jedoch auch in einen größeren historisch-gesellschaftlichen Zusammenhang eingebettet werden, so dass die Entwicklung dieser Konzeption auf unterschiedliche Ursprünge zurückgeht. Kurt Lewin ist aber nach Pieper (1977, S. 8) derjenige, der dieser Strategie den Namen gegeben hat.

Kurt Lewin war ein deutsch-jüdischer Psychologe, der ab den 1920er Jahren in Berlin lehrte und 1933 nach Amerika emigrierte. Er führte zahlreiche experimentelle Studien durch, wobei sein Interesse vor allem konkreten sozialen Problemen galt. Er erforschte soziale Prozesse im Kontext von Arbeit und Leistung im industriellen Milieu (z.B. kleine Gruppen von Arbeitern und Bedingungen von Produktionssteigerungen), die Dynamik des Gruppenlebens (z.B. wie entsteht die Gruppenatmosphäre, wie kommt es zu Gruppenentscheidungen, wie verständigen sich Gruppenmitglieder miteinander, wie etablieren sich Gruppenstandards) und Prozesse im kommunalen Bereich von Politik und Demokratie (z.B. Minderheitenforschung, Judenthematik, Integrationsansätze). Sein Ziel war vor allem die Verbesserung der sozialen Praxis (zum Leben und Werk von Kurt Lewin siehe Lück 2001 und Marrow 2002).

\footnotetext{
„Die für die soziale Praxis erforderliche Forschung lässt sich am besten als eine Forschung im Dienste sozialer Unternehmungen oder sozialer Technik kennzeichnen. Sie ist eine Art Tat-Forschung ('action research'), eine vergleichende Erforschung der Bedingungen und Wirkungen verschiedener Formen des sozialen Handelns und eine zu sozialem Handeln führende Forschung. Eine Forschung, die nichts anderes als Bücher hervorbringt, genügt nicht." (Lewin, 1953, S.280)
} 
In diesem Zitat wird deutlich, dass Lewin daran gelegen war, die Forschung in der konkreten sozialen Praxis zu nutzen. Forschung darf nicht nur sich selbst genügen bzw. nicht nur theoretisches Wissen (z.B. in Form von Büchern) produzieren, sondern soll dazu dienen, das soziale Handeln zu verstehen und auch zu verbessern. Es wird eine Verbindung von wissenschaftlicher Forschung und alltäglicher sozialer Praxis angestrebt. Nach Pieper (1977, S. 8) kann die Aktionsforschung auch als „Reaktion von Sozialwissenschaftlern auf die Trennung von Wissenschaft und gesellschaftlicher Praxis“ verstanden werden.

Im deutschsprachigen Raum wurde die Aktionsforschung in den 1970er Jahren aktuell (z.B. Haag et al. 1972, Moser 1975) und teilweise auch als Handlungsforschung (Klafki 1973, Heinze et al. 1975) oder Entwicklungsforschung (Wellenreuther 1982, S. 114f) bezeichnet.

Der Aktionsforschungsansatz wurde in Deutschland in den 1970ern im Zusammenhang gesellschaftspolitischer Reformbestrebungen sowie der Studentenbewegung aufgegriffen (vgl. Bortz/Döring 2006, S.341). Der „Aufbruch in den Sozialwissenschaften“ und der Ursprung des Aktionsforschungsansatzes hatte nach Heinze (vgl. 2001, S.79) zwei Gründe: Zum einen die interaktionistisch orientierte amerikanisch-englische Human-Relation-Bewegung der 1940er und 1950er Jahre, und zum anderen den Positivismusstreit, der in den Studentenbewegungen aktualisiert wurde. Die Aktionsforschung wurde als „kritische Theorie der Sozialwissenschaften“ (Moser 1975) diskutiert und sogar als „eigenständiges, wissenschaftstheoretisch fundiertes Paradigma“ postuliert (Moser 1975). Der Aktionsforschungsansatz wurde als Gegenpol zu empirischen eher quantitativ orientierten Forschungsansätzen dargestellt.

\begin{abstract}
„Aktionsforschung ('action research') versteht sich als ein von klassischempirischer Sozialforschung sich distanzierender sozialwissenschaftlicher Forschungsansatz, in dem die Fragen nach dem Verhältnis von Theorie und Praxis, Theorie und Empirie sowie der Interaktion von Forscher und Erforschten neu aufgeworfen werden." (Heinze 2001, S. 79)
\end{abstract}

Eine der zentralen Forderungen waren der Praxisbezug der Forschung und die Auflösung der Distanz zwischen Forschern und Erforschten bzw. der Wissenschaft und der sozialen Praxis.

\begin{abstract}
„Im konzeptionell reinen Fall soll Handlungsforschung sowohl den praktischen Bedürfnissen der Personen, die sich in einer unmittelbar problematischen Situation befinden, als auch den Zielen der Wissenschaft dienen, d.h. neues valides Wissen produzieren." (Skowronek/Schmied 1977, S.259)
\end{abstract}

Die Anwendungsgebiete bzw. Forschungsthemen der Aktionsforschung sind demnach vor allem in pädagogischen Praxisfeldern zu finden wie z.B. der Sozialarbeit, der Jugendhilfe, der Sonderpädagogik und der Rehabilitation (Moser 1975), sowie in der Schule und den Lebenswelten von Arbeiterkindern (Heinze et al. 1975). In dem Beitrag von Haag et al. (1972) finden sich die Aktionsforschungsfelder Institutionsberatung, Hochschuldidaktik, Gruppendynamik in Arbeitsgruppen, Randgruppenforschung, Strafvollzug, Sozialisation von Gastarbeiterkindern und politische Bildung. Nach Friedrichs (1990, S. 771) liegen Anwendungsbereiche der Aktionsforschungsstrategie z.B. „in der Arbeit mit Bewohnern einer Neubausiedlung 
oder eines Sanierungsgebiets, dem Aufbau einer sozialtherapeutischen Anstalt, im Bereich der Vorschulerziehung, in der Lösung sozialer Konflikte in einem Jugendfreizeitheim, bei Konflikten zwischen verschiedenen ethnischen Gruppen oder der Integration von Gastarbeitern." Bei Heinze (2001) werden vor allem das pädagogische Praxisfeld von Arbeitswelt und Berufsalltag, aber auch Schule und Lehrer als Anwendungsbereiche genannt. Die erwähnten Themenfelder der Aktionsforschung zeigen die Vielfalt und die Breite der Möglichkeiten, es wird aber auch deutlich, dass dieser Forschungsansatz immer einen Bezug zur sozialen Praxis bzw. zu Praxisproblemen hat.

Im deutschsprachigen Raum finden sich heute vor allem in dem Praxisfeld von Schule Beiträge zum Thema Aktionsforschung (z.B. Altrichter/Posch 2007, Juna 2002), wobei dieser Ansatz durchaus auch kritisch diskutiert bzw. reflektiert wird (Altrichter 1990). In Deutschland scheint es mittlerweile eine gewisse Vorliebe für empirisch-quantitative Untersuchungen zu geben, die sich in der Popularität von Studien wie z.B. PISA und TIMMS (siehe Einleitung) zeigt. In der internationalen "scientific community" wird die Aktionsforschung aber durchaus ernst genommen, eingesetzt, diskutiert und weiter entwickelt (vgl. z.B. Beiträge im International Journal of Action Research und Greenwood/Levin 2007).

Im Folgenden sollen einige Charakteristika der Aktionsforschung erläutert werden, die für die vorliegende Forschungsarbeit eine Bedeutung haben. Gstettner (1995) beschreibt drei Prämissen der Handlungsforschung:

1. Methodische Offenheit des Forschers beim Einstieg in das Feld

2. Kontinuierliches, aber zeitlich begrenztes Forschungsengagement

3. Erweiterung der Kompetenzen aller im gemeinsamen Forschungsprozess beteiligten Akteure als Ziel.

Mit „methodischer Offenheit“ ist gemeint, dass zu Beginn des Forschungsprozesses noch kein vorgefertigtes methodisches Design, keine klare Fragestellung und Hypothesen vorhanden sind, wie es z.B. bei eher empirisch-quantitativ orientierter Forschung der Fall wäre. Der Forscher lässt sich relativ unvoreingenommen auf das Feld ein, reflektiert aber sein Denken und Handeln systematisch.

„Die Handlungsforschenden begeben sich methodisch ungeschützt in das
Forschungsfeld und nützen zur wissenschaftlichen Erkenntnisgewinnung
ausschließlich inre über subjektive Erfahrung hergestellte, intendierte und
objektiv stattfindende Verflochtenheit mit dem Forschungsfeld [...]. 'Unge-
schützt' soll heißen, dass im Stadium des Eintauchens ins Feld kein metho-
disches Instrumentarium eingesetzt wird, das in irgendeiner Weise zur Defi-
nition von Forschungssituationen, zum agitatorischen Abbau von Angst bei
den Forschenden bzw. zur Herstellung von herrschaftsstabilisierender Dis-
tanz dienlich ist. Die Forschenden müssen daher in erster Linie sensibel für
ihr eigenes Vorgehen werden und die alltäglichen Reflexionsmöglichkeiten 
methodisch verfeinern [...]. Die Verflochtenheit des Forschers mit dem Untersuchungsfeld muß als Faktum theoretisch, aber auch als Prozeß des Sicheinlassens strategisch reflektiert werden." (Gstettner 1995, S. 267)

Charakteristisch für den Aktionsforschungsansatz ist außerdem, dass die Zeit, in der sich der Forscher in dem Feld engagiert, begrenzt ist. Er wird nicht dauerhaft Teil der sozialen Praxis werden, sondern nach einer gewissen Zeit das Feld auch wieder verlassen.

„Handlungsforschung ist ein kontinuierliches, aber zeitlich befristetes Forschungsengagement in einem Praxisfeld und steht als solches im Gegensatz zum reinen Inspizieren (Modell 'neugierige Touristen') oder kulturellen Missionieren (Modell 'verkappte Kolonisierung').“ (Gstettner 1995, S. 268)

Das Ziel der Forschung ist, wie bereits oben beschrieben, die Verbesserung der sozialen Praxis aber auch die Gewinnung von neuen und validen Erkenntnissen für die Wissenschaft. Demnach erweitern sich die Kompetenzen und das Wissen aller am Forschungsprozess beteiligten Personen.

\begin{abstract}
„Handlungsforschung zielt darauf ab, dass sich die Kompetenzen aller im gemeinsamen Forschungsprozeß erweitern. Für die Betroffenen steigen die Chancen, sich auch faktisch von den vorgegebenen Inhalten und Personen, d.h. von der Expertenkultur, zu emanzipieren. Der Aufbau von tragfähigen organisatorischen Infrastrukturen [...] ist deshalb ebenso integrierter Bestandteil von Handlungsforschungskonzepten wie die Kontaktaufnahme zu möglichen "Bündnispartnern" in den bestehenden Organisationen [...]." (Gstettner 1995, S. 268)
\end{abstract}

In diesem Zitat wird deutlich, dass nicht nur die Verbesserung der sozialen Praxis im Sinne von neuen Erkenntnissen angestrebt wird. Die konkrete Schaffung von Strukturen und Netzwerken zur eigenständigen Kommunikation und Pflege von Kontakten außerhalb bzw. nach dem zeitlich begrenzten Forschungsprozess ist ein klares Ziel von Aktionsforschungskonzepten.

Bortz/Döring (2006, S.342) benennen drei weitere Grundsätze der Aktionsforschung:

1. Forscher und Beforschte sind gleichberechtigt

2. Untersuchungsthemen sind praxisbezogen und emanzipatorisch

3. Der Forschungsprozess ist ein Lern- und Veränderungsprozess

In der Aktionsforschungskonzeption sind die Menschen im Forschungsfeld keine Untersuchungsobjekte, sondern Subjekte, die mitentscheiden, welche Ziele die Forschung hat bzw. haben soll und welche Methoden dafür gewählt werden. Alle am Forschungsprozess beteiligten Personen sind gleichberechtigt.

Außerdem sollen die Untersuchungsthemen einen unmittelbaren praktischen Bezug und praktische Relevanz haben. Das Ziel ist, die Ergebnisse direkt in der Praxis zu nutzen und 
soziale sowie politische Probleme aktiv zu lösen. Aktionsforschung greift in die Praxis ein, und Veränderungen der Praxis sind gewollt.

Der Forschungsprozess läuft dabei nicht linear ab, sondern ist gekennzeichnet durch die „dialogische Wahrheitsfindung“ (Bortz/Döring 2006, S.342). Dies bedeutet, dass der Forschungsprozess ein offener Lern- und Veränderungsprozess ist, in dem sich die Beteiligten über neue Erkenntnisse und Ergebnisse durch Rückkoppelung und gemeinsame Reflexion austauschen. Dieser Prozess des „kooperierenden Dialogs“ (Heinze 2001, S.84) hat dabei explorative, aber auch reflektorische Anteile.

Die "Exploration" und "Inspektion" sind auch wesentliche Etappen des Aktionsforschungsprozesses:

\begin{abstract}
"Typisch für die explorative Phase des Aktionsforschungsprozesses ist eine flexible Vorgehensweise des Forschers im Hinblick auf die Verwendung von Untersuchungsmethoden zur Gewinnung möglichst reichhaltiger Erkenntnisse über die Handlungsabläufe oder die Berichte über Handlungen in ihrem situativen Kontext. Weiterhin typisch für diese Phase ist eine relative Breite und Unstrukturiertheit des theoretischen Vorverständnisses des Forschers, das im Verlaufe des Forschungsprozesses zunehmend eingeengt und gleichzeitig differenziert wird. Ziel der Exploration ist eine Konkretisierung von Forschungsfragen und Forschungsmethoden aufgrund von Erfahrungen, die in einem ersten Zugang im Feld ermittelt worden sind." (Heinze 2001, S.83)
\end{abstract}

Dieses Zitat verdeutlicht Charakteristika des Aktionsforschungsprozesses: Zunächst die Offenheit des Forschers beim Eintauchen in das Feld, die relative Breite von theoretischen Bezügen und Möglichkeiten sowie eine gewisse Unstrukturiertheit. Erst mit bzw. nach der Exploration des Feldes können eine Konkretisierung der Fragestellung und die Auswahl adäquater Forschungsmethoden erfolgen. Bei der Wahl der Methoden dürfen die am Forschungsprozess Beteiligten mitwirken (siehe oben). Sie sind gleichberechtigte Partner, die mitentscheiden, welche Ziele die Forschung haben soll und welche Methoden dafür geeignet sind.

Für den weiteren Forschungsprozess gilt das Prinzip der „Dialogischen Wahrheitsfindung“ (siehe oben), d.h. es erfolgt ein wechselseitiges Miteinander zwischen Forschern und allen Beteiligten. Es wird durch Austausch und Reflexion gemeinsam am Forschungsgegenstand gearbeitet und nach Lösungen gesucht, die zur Verbesserung der sozialen Praxis beitragen. Das Gelingen dieses Prozesses hängt dabei von allen Beteiligten ab.

Das Konzept und das Vorgehen in dieser Forschungsarbeit entsprechen den Prinzipien der Aktionsforschung, weil folgende Punkte kennzeichnend für die Situation im Feld waren:

- Gewisse Offenheit beim Eintauchen in das Feld

Zu Beginn des Projektes eL3 war zwar sowohl ein Evaluationskonzept nach den Antragsunterlagen ausgearbeitet worden als auch ein umfangreiches theoretisches Vorverständnis in Bezug auf verschiedene Aspekte von e-Learning und Blended Learning vorhanden (z.B. Di- 
daktik, Evaluation, Virtuelle Kommunikation), aber die Möglichkeiten und Ziele mussten erst mit den Projektpartnern gemeinsam diskutiert und gefunden werden. Am Anfang des Projektes eL3 gab es eine Fülle von Möglichkeiten sowohl in Bezug auf diese Forschungsarbeit (Fragestellung, Erkenntnisinteresse, Methoden), als auch auf die praktischen Ziele und Umsetzungsmöglichkeiten (Was kann tatsächlich gemeinsam erreicht werden?).

- Kontinuierliches, aber zeitlich begrenztes Forschungsengagement

Das Projekt eL3 war zunächst auf eineinhalb Jahre angelegt und wurde dann auf zwei Jahre erweitert. Es war jedoch von Anfang an klar, dass dies die begrenzte Zeit einer Finanzierung und auch des Forschungsengagements war. Es sollten zwar im Projekt Strukturen und Formen einer nachhaltigen Vernetzung entwickelt werden, diese müssten dann aber auf freiwilliger Basis weiter gepflegt werden.

- Erweiterung der Kompetenzen aller beteiligten Akteure

Das Ziel von Aktionsforschung ist die Erweiterung der Kompetenzen aller beteiligten Akteure in einem gemeinsamen Forschungsprozess. In diesem Sinne ging es darum, die Bedarfe der eL3-Partner zu ermitteln und an deren konkreten individuellen „Praxis-Problemen“ anzusetzen. Der Wissensstand, die Informationsbedarfe und Kompetenzen in Bezug auf e-Learning und Blended Learning mussten dabei berücksichtigt werden. Das Ziel war eine Förderung und Erweiterung jedes einzelnen Partners und auch ein gemeinsames „Weiterkommen“ in der Projektgruppe.

- Forscher und Beforschte sind gleichberechtigt

In dem Forschungsprozess sind nach den Grundsätzen der Aktionsforschung die Forscher und die Beforschten gleichberechtigt. Im Projekt eL3 bedeutete dies, dass den Partnern nicht vorgegeben wurde, in welche Richtung das Forschungsvorhaben genau gehen musste. Es wurden stattdessen Vorschläge gemacht und gemeinsam entschieden, welche Wege und Methoden geeignet schienen, zu Erkenntnissen und Lösungen zu kommen. Dies vollzog sich in einem „kooperierenden Dialog“ (Heinze 2001, S.84), in einem wechselseitigen Miteinander.

- Untersuchungsthemen sind praxisbezogen und emanzipatorisch

Die Untersuchungsthemen sollen in der Aktionsforschung einen unmittelbaren praktischen Bezug und praktische Relevanz haben. Das Ziel ist eine Verbesserung der Praxis, bzw. Ergebnisse direkt in der Praxis nutzen zu können. Im Projekt eL3 bedeutete dies, dass unmittelbar an den „Praxis-Problemen“ der beteiligten Partner angesetzt wurde. Das Ziel war eine Verbindung von Wissenschaft und Praxis, theoretische Konzepte sollten auf einer praktischen Ebene nutzbar gemacht werden und zur Verbesserung der e-Learning und Blended Learning-Angebote der Partner in einem umfassenden Sinne beitragen. Der Begriff „emanzi- 
patorisch“ kennzeichnet, dass darauf abgezielt wird, die Akteure zu befähigen, eigenständig in ihren Feldern zu handeln, dass sie einen Zugewinn an Freiheit bekommen und eigenständig die Ergebnisse nutzen können. Eine Stärkung der Kompetenzen und der Eigenständigkeit in Bezug auf die Gestaltung und Evaluation von e-Learning und Blended Learning im europäischen Gesundheits- und Sozialwesen war stand deshalb im Zentrum.

- Der Forschungsprozess ist ein Lern- und Veränderungsprozess

Der Aktionsforschungsprozess läuft nicht linear ab, sondern zeichnet sich aus durch die „dialogische Wahrheitsfindung“ (Bortz/Döring 2006, S.342). Der Forschungsprozess ist dabei ein offener Lern- und Veränderungsprozess, und die Beteiligten tauschen sich regelmäßig durch Rückkoppelung und gemeinsame Reflexion von Ergebnissen und Erkenntnissen aus. Im Projekt eL3 erfolgte dieser Austausch hauptsächlich während der transnationalen Treffen, die alle paar Monate stattfanden. Dies waren die einzigen Gelegenheiten, zu denen die gesamte Gruppe zusammentraf und alle Beteiligten „Zeit hatten“, sich ganz auf das Projekt eL3 zu konzentrieren. Nach Heinze (2001, S.84) hat der Prozess des „kooperierenden Dialogs“ dabei sowohl explorative als auch reflektorische Anteile. Im Projekt zeigte sich dies dadurch, dass auf den Treffen immer wieder entdeckt und abgestimmt werden musste, wie weiter vorgegangen werden konnte.

Für die Aktionsforschung ist eine gewisse Offenheit und Unstrukturiertheit in Bezug auf die Auswahl von Forschungsmethoden und das theoretische Vorverständnis am Anfang der Forschungsarbeit charakteristisch. Im Projekt eL3 stellte sich zu Beginn die Frage, was die konkreten „praktischen Probleme“ der Projektpartner sind, welche Bedarfe vorliegen und welche Lern- und Lösungsmöglichkeiten gemeinsam erreicht werden könnten. Dies ist ein wesentlicher Aspekt der Forschungsarbeit, da ein zentrales Ziel von Aktionsforschung die Verbesserung der Praxis ist. Die zuvor recht allgemein formulierten Fragestellungen aus dem wissenschaftlichen Kontext wurden demzufolge im Projekt eL3 gemeinsam mit den Projektpartnern konkretisiert. Außerdem musste gemeinsam entschieden werden, welche Forschungsmethoden aus wissenschaftlicher Perspektive geeignet schienen und von den Partnern (praktisch) akzeptiert würden. Nicht die Forscherin entschied allein, welche Methoden geeignet wären, sondern alle Beteiligten konnten mitbestimmen. Beim „Eintauchen in das Feld“ standen eine Fülle von Methoden zur Verfügung, die im weiteren Projektverlauf mit den Projektpartnern in einem „kooperierenden Dialog“, d.h. einem gleichberechtigten, wechselseitigen Miteinander spezifiziert und ausgewählt wurden.

Die Aktionsforschung zeichnet sich in ihrer Konzeption vor allem durch eine Vielfalt an Methoden aus, die eingesetzt werden können, um empirische Daten im Untersuchungsfeld zu sammeln. Dabei können sowohl qualitative als auch quantitative Methoden eingesetzt oder kombiniert werden. 
„Gemäß dieser methodologischen Prämissen erfährt das traditionelle Instrumentarium empirischer Sozialforschung im Kontext von Aktionsforschung eine neue Gewichtung und teilweise Modifikation. Oberster Leitsatz ist die weitestmögliche Integration unterschiedlicher Methoden [...]. Ziel von Aktionsforschung muss der Versuch sein, qualitative und quantitative Datengruppen und Untersuchungsinstrumente in eine systematische Beziehung zu setzen." (Heinze 2001, S.83)

Dementsprechend standen am Anfang des Projektes eL3 verschiedene Methoden zur Auswahl, die geeignet schienen. Die Autorin entschied sich für eine Kombination unterschiedlicher Methoden, die mit den Partnern abgestimmt wurden. Dieses Vorgehen wird auch als Triangulation bezeichnet und im nächsten Abschnitt beschrieben.

Das Forschungsvorgehen wurde der Aktionsforschung zugeordnet (siehe Unterkapitel 5.3 bzw. Abschnitt 5.3.1), hat aber auch Züge von Entwicklungsforschung. Bei der Aktionsforschung geht es vor allem um die Erforschung von Prozessen und das soziale Handeln von Personen. Das Vorgehen orientiert sich an den Bedarfen von den Personen, die im Zentrum stehen und die auch am Forschungsprozess beteiligt werden. Die Ergebnisse der Forschung sollen in der Praxis nützlich sein und zu einer Verbesserung der Praxis beitragen bzw. die Kompetenzen der Beteiligten stärken.

Bei der Entwicklungsforschung stehen vor allem Produkte im Zentrum der Untersuchungen. In den 1960 und 1970er Jahren war die Entwicklungsforschung im Kontext der Entwicklung von Curricula sowie der Erstellung bzw. Gestaltung von Lehrmedien wie z.B. Unterrichtsfilmen besonders häufig. In seinem Aufsatz „Forschungsschwerpunkte im Bereich der Unterrichtstechnologie“ differenziert Flechsig $(1975$, S.152) drei Arten von unterrichtstechnologischer Forschung: „Praxisbegründende, praxisentwickelnde und praxisevaluierende Forschung“. Im Rahmen der vorliegenden Forschungsarbeit wird diese Einteilung aufgenommen und das Vorgehen der praxisentwickelnden Forschung zugeordnet. Flechsig führt dazu aus:

\footnotetext{
„Praxisentwickelnde Forschung' ist weitgehend identisch mit dem Begriff des 'developmental research'. Ziel dieser Forschung ist die Entwicklung 'prototypischer Praxis' unter kontrollierten und zumeist auch günstigen Bedingungen. [...]

Im Unterschied zur 'bloßen', auf mehr oder weniger intuitiver Grundlage entwickelten (innovativen) Praxis zeichnet sich praxisentwickelnde Forschung einerseits dadurch aus, daß sie sich um die Begründung ihrer Konzepte durch wissenschaftliche Argumentation bemüht, zum anderen dadurch, daß sie den Entwicklungsprozeß durch Aktivitäten der (formativen) Evaluation begleitet und auf diese Weise schon während des Entwicklungsprozesses Evaluationskriterien entwickelt und anwendet, die auf erfahrungswissenschaftlich zu sichernde Daten bezogen werden können." (Flechsig 1975, S.153)
}

Mit diesem Zitat wird deutlich, dass bei dem Vorgehen in der vorliegenden Forschungsarbeit und im Rahmen des Projektes eL3 wesentliche Kriterien praxisentwickelnder Forschung erfüllt wurden. Zum einen war das Ziel die Entwicklung einer prototypischen Praxis, die dann 
auf andere Projekte bzw. Zusammenhänge hätte übertragen werden können. Zum anderen waren die Konzepte begründet durch eine wissenschaftliche Argumentation, und schließlich wurde der Entwicklungsprozess der Inventare sowie deren Erprobung durch eine formative Evaluation (die Prozessevaluation - siehe Unterabschnitt 5.3.2.2) begleitet.

Die Zuordnung der Forschungsarbeit zur Aktionsforschung wie auch zu der Entwicklungsforschung ergänzt sich nach Meinung der Autorin, da es sowohl um die Erforschung sozialer Prozesse und Handlungen geht (die Evaluationspraxis und das Miteinander) als auch um die Produkte (die Inventare).

\subsubsection{Triangulation}

In den Sozialwissenschaften wird die Kombination unterschiedlicher Methoden der Datengewinnung auch unter dem Begriff der Triangulation diskutiert. Der Begriff stammt ursprünglich aus der Landvermessung und Geodäsie, wo die Triangulation ein Verfahren darstellt, um Vermessungsarbeiten durchzuführen (Erdmessungen, Landesaufnahme). In den Sozialwissenschaften bedeutet Triangulation, „dass ein Forschungsgegenstand von (mindestens) zwei Punkten aus betrachtet [...] wird“ (Flick 2008b, S.11). Im Zusammenhang der sozialwissenschaftlichen Forschung kann Triangulation folgendermaßen definiert werden:

„Triangulation beinhaltet die Einnahme unterschiedlicher Perspektiven auf einen untersuchten Gegenstand oder allgemeiner: bei der Beantwortung von Forschungsfragen. Diese Perspektiven können sich in unterschiedlichen Methoden, die angewandt werden, und/oder unterschiedlichen gewählten theoretischen Zugängen konkretisieren, wobei beides wiederum mit einander in Zusammenhang steht bzw. verknüpft werden sollte. Weiterhin bezieht sie sich auf die Kombination unterschiedlicher Datensorten jeweils vor dem Hintergrund der auf die Daten jeweils eingenommenen theoretischen Perspektiven. Diese Perspektiven sollten so weit als möglich gleichberechtigt und gleichermaßen konsequent behandelt und umgesetzt werden. Durch die Triangulation [...] sollte ein prinzipieller Erkenntniszuwachs möglich sein, dass also bspw. Erkenntnisse auf unterschiedlichen Ebenen gewonnen werden, die damit weiter reichen, als es mit einem Zugang möglich wäre." (Flick, 2008b, S.12)

In dieser Definition wird deutlich, dass es unterschiedliche Formen von Triangulation (DatenTriangulation, Theorien-Triangulation, Methoden-Triangulation) gibt, und dass es vor allem um eine Erkenntniserweiterung bei der Beantwortung von Forschungsfragen geht.

Das Konzept der Triangulation wurde von Norman Denzin eingeführt (1970, 1989), es finden sich jedoch bereits davor theoretische Ansätze und Diskussionen zu dieser Herangehensweise qualitativer Forschung (Campbell und Fiske 1959 sowie Webb et al. 1966). 
Das Modell von Denzin $(1970,1989)$ wurde besonders intensiv diskutiert und vielfach aufgegriffen. Er unterscheidet vier verschiedene Formen der Triangulation:

1. Daten-Triangulation

(Einbeziehung verschiedener Datenquellen)

2. Investigator-Triangulation

(unterschiedliche Beobachter oder Interviewer werden eingesetzt)

3. Theorien-Triangulation

(Einbeziehung verschiedener theoretischer Ansätze)

4. Triangulation von Methoden

(Einsatz unterschiedlicher Forschungsmethoden)

An dieser Stelle soll es um die Triangulation von Methoden gehen, da im Rahmen dieser Forschungsarbeit dieser Typus von Triangulation gewählt wurde, um die Forschungsfragen zu beantworten. Denzin unterscheidet zwischen der „within-method“ (Triangulation innerhalb einer Methode) und der „between-method“ (Einsatz verschiedener Methoden).

In der vorliegenden Arbeit findet sich die „between-method“, d.h. es werden verschiedene Methoden kombiniert, um ein breiteres, umfassenderes Bild von dem Untersuchungsfeld zu bekommen, als wenn nur eine Methode zum Einsatz käme. Das Ziel ist, möglichst vielfältige Daten zu erhalten und damit eine bessere Sicht auf den Forschungsgegenstand. Es geht allerdings nicht bloß um die Summation von Methoden, um einen „objektiven Tatbestand“ zu ermitteln, sondern es geht um eine überlegte, reflektierte Kombination von angemessenen Methoden mit denen tiefergehende und umfassendere Einblicke möglich werden. Es geht auch nicht um eine Überprüfung bzw. Validierung von Ergebnissen, „vielmehr soll die Triangulation unterschiedlicher methodischer (qualitativer) Zugänge das interessierende Phänomen in seiner Vielschichtigkeit aus unterschiedlichen Perspektiven erfassen“ (Flick 2008b, S.43). Die folgende Abbildung zeigt den Einsatz unterschiedlicher Methoden zur Untersuchung eines Forschungsgegenstandes ("between-method"): 


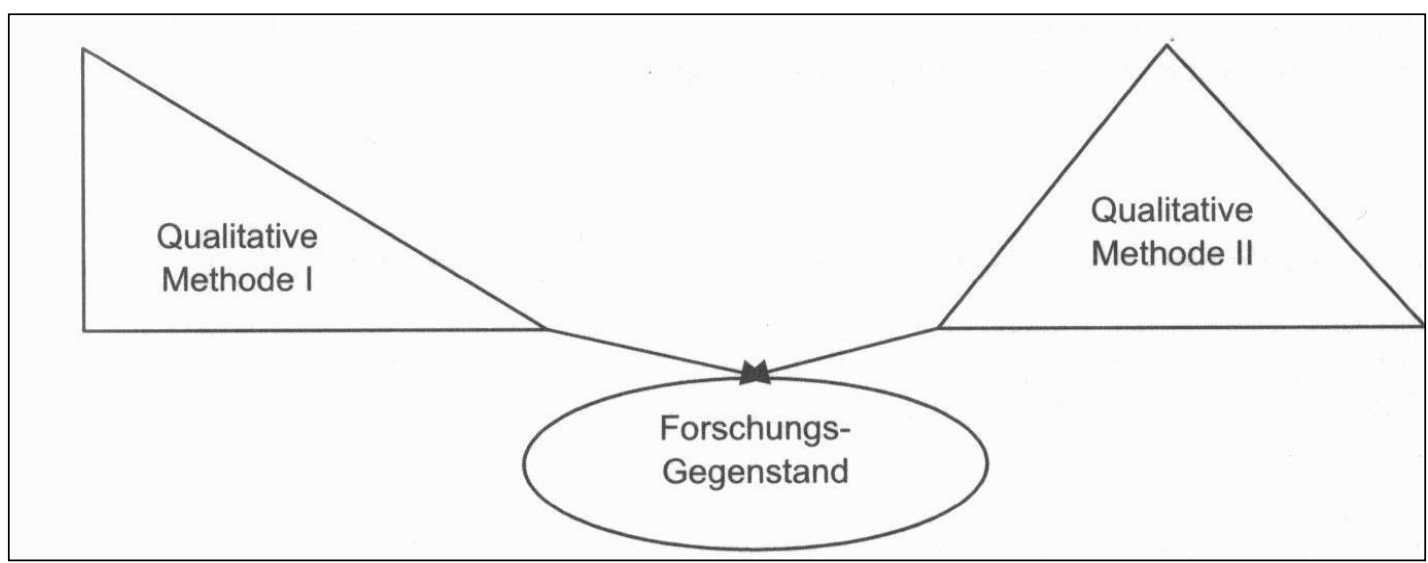

Abbildung 6: Triangulation verschiedener qualitativer Methoden (Quelle: Flick 2008b S.41)

Bei der Kombination von Methoden können sowohl qualitative als auch quantitative Methoden verwendet werden. Nach Flick (vgl. 2008b, S.75) ist seit einigen Jahren der Trend zu beobachten, dass qualitative und quantitative Forschungsmethoden kombiniert werden. Die Ergebnisse unterschiedlicher Methoden bzw. Forschungsweisen können einander ergänzen, indem sie sinnvoll aufeinander abgestimmt zum Einsatz kommen. Miles und Huberman (1994, S.41, zitiert nach Flick 2008b, S.79) entwerfen vier Basisdesigns für die Verbindung von qualitativen und quantitativen Forschungsmethoden:

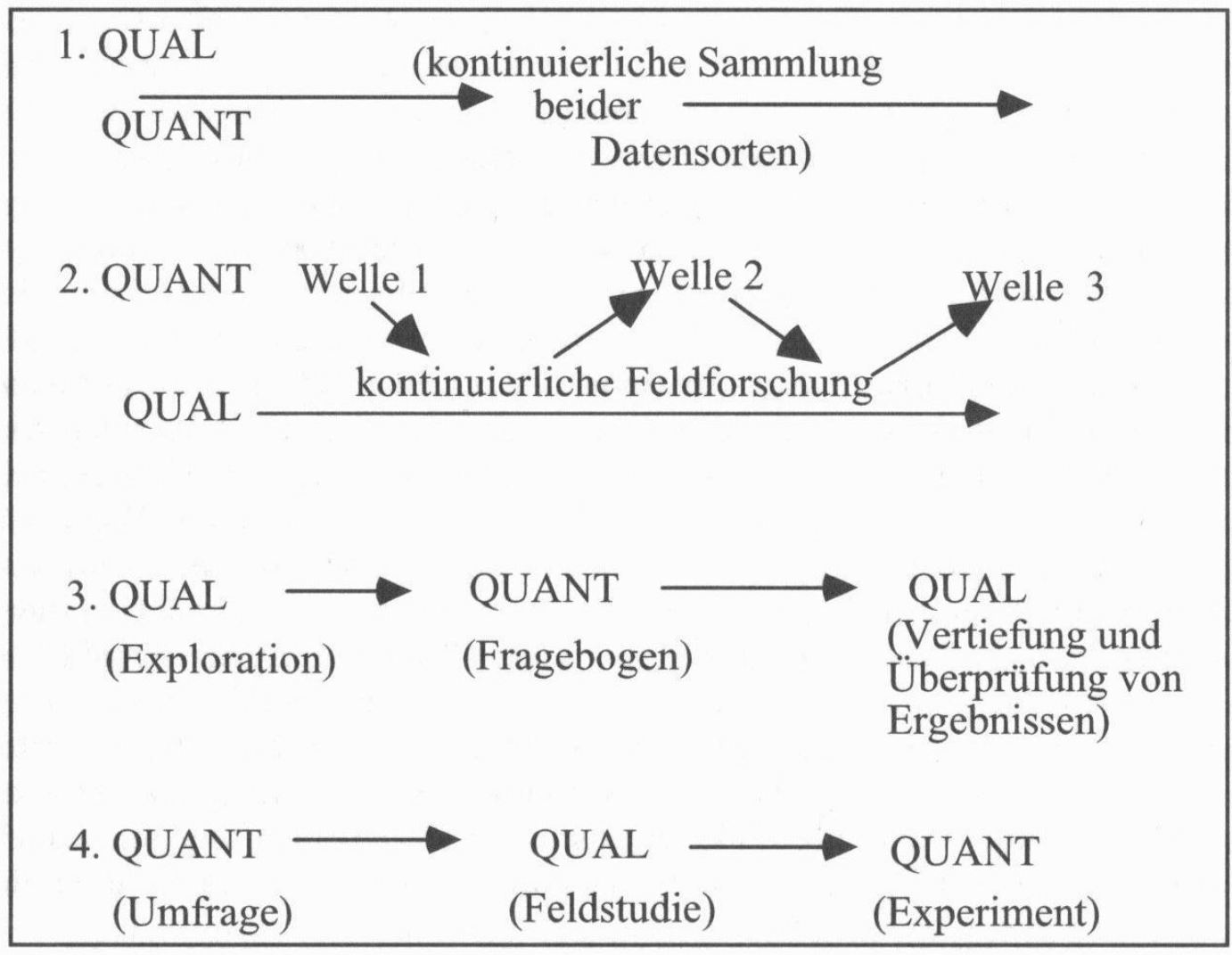

Abbildung 7: Basisdesigns zur Verbindung von qualitativer und quantitativer Forschung (Quelle: Miles und Huberman 1994, S.41 in Flick 2008b, S.80) 
In der vorliegenden Forschungsarbeit werden quantitative und qualitative Methoden kombiniert und es ergibt sich folgendes Design:

1. Quantitatives Verfahren: Fragebogen mit dem Ziel der Exploration zu Beginn des Projektes

2. Quantitatives Verfahren: Fragebogen Prozessevaluation über den gesamten Projektverlauf von zwei Jahren

3. Qualitatives Verfahren: Dokumentation der Aktivitäten auf dem Lernmanagementsystem (nichtreaktives Verfahren)

4. Quantitatives Verfahren: Fragebogen Überprüfung und Resümee zum Abschluss

Folgende Fragen sollten mit den jeweiligen Methoden beantwortet werden:

\section{Fragebogen zur Bestandsaufnahme}

Was tun die Partner genau?

Welche Lernangebote haben sie?

Welche Evaluationsmethoden kommen zum Einsatz?

Was für Ziele haben die Partner?

Wo sind Probleme und Handlungsbedarfe?

\section{Fragebogen zur Prozessevaluation}

Wie erleben die Partner das Projekt und die Zusammenarbeit?

Was erachten sie als wertvoll? Was gefällt den Partnern?

Was finden die Partner problematisch?

\section{Dokumentation von Aktivitäten auf dem Lernmanagementsystem}

Wie gestaltet sich die Zusammenarbeit in den Arbeitsgruppen?

Was wird gemeinsam erarbeitet?

\section{Fragebogen zum Abschluss des Projektes}

Wurden die Inventare benutzt?

Waren die Inventare nützlich?

Was sind Schwierigkeiten?

Welche Potenziale ergeben sich?

Im Folgenden werden die einzelnen Methoden, die in dieser Arbeit zum Einsatz kommen, genauer beschrieben. 
Es sei an dieser Stelle darauf hingewiesen, dass für die vorliegende Forschungsarbeit zusätzlich zu den hier benannten und im Folgenden beschriebenen Methoden Diskussionen berücksichtigt wurden, die während der transnationalen Treffen stattfanden. Die Verfasserin verfasste Notizen zu den Diskussionen, die nicht in den offiziellen Protokollen enthalten sind und auf die bei der Ergebnisdarstellung zurückgegriffen wird.

\subsubsection{Fragebogen zur Bestandsaufnahme}

Zu Beginn des Projektes eL3 stellte sich auf dem ersten gemeinsamen Treffen in der Gruppendiskussion heraus, dass die e-Learning bzw. Blended Learning-Angebote sowie die Interessen und Bedürfnisse der Partner doch heterogener waren als zunächst angenommen. Es wurde deshalb vorgeschlagen, zuerst eine systematische Bestandsaufnahme zu den individuellen Projekten der Partner zu machen, um Gemeinsamkeiten und Unterschiede zu identifizieren. Die eL3-Partner lehnten es jedoch ab, dass ein Expertenteam des Pädagogischen Seminars eine externe Evaluation ihrer e-Learning bzw. Blended Learning-Angebote durchführt. Es wurde dann gemeinsam entschieden, dass stattdessen ein Fragebogen entwickelt werden sollte, mit dessen Hilfe zentrale Informationen zu den Angeboten der Partner gesammelt würden. In der Auswertung sollten zum einen Bedarfe (Interessen, Informationsbedarfe, Entwicklungsmöglichkeiten sowie praktische Probleme) herausgearbeitet und zum anderen gemeinsame Themenfelder ermittelt werden (z.B. technische Aspekte, soziale Komponenten, e-Learning-Didaktik). Die Ergebnisse sollten während des nächsten transnationalen Treffens vorgestellt werden und einer Synopse der Partnerprojekte dienen. Außerdem sollten auf der Basis der Exploration und Inspektion der Partnerprojekte Ziele festgelegt werden, die im Rahmen der Projektpartnerschaft und der vorliegenden Forschungsarbeit erreicht werden könnten.

Im Rahmen dieser Forschungsarbeit sollte als ein Aspekt der e-Learning-Angebote die Evaluationspraxis der Partner mit dem Fragebogen zur Bestandsaufnahme erfasst werden. Hierbei stand im Zentrum, welche Methoden eingesetzt bzw. kombiniert werden. Außerdem sollte erforscht werden, ob sich Unterschiede zwischen den Evaluationsansätzen der Partner zeigen, je nachdem, ob sie eher einen wissenschaftlichen oder einen eher praxisorientierten Hintergrund haben (Forschung-Praxis-Dilemma siehe Unterkapitel 4.3).

Der Fragebogen wurde per E-Mail an die Partner verschickt, die Ergebnisse wurden ausgewertet und den Partnern auf dem nächsten transnationalen Treffen präsentiert. Es wurden u.a. auf der Basis der Antworten Profile erstellt, die zur Transparenz beitragen sollten, was die einzelnen Projektpartner tun, bzw. dazu dienen sollten, dass die Partner als Basis für eine Zusammenarbeit mehr voneinander erfahren. 


\subsubsection{Prozessevaluation}

Die Projektpartner in eL3 arbeiteten über einen Zeitraum von zwei Jahren zusammen. Es fanden in dieser Zeit fünf transnationale Treffen statt, zu denen die Projektgruppe real zusammen kam. Während der Treffen wurden die aktuellen Entwicklungen besprochen und das weitere Vorgehen vereinbart. Zwischen den transnationalen Treffen waren die Partner weitestgehend auf sich gestellt und die Kommunikation der Gruppe untereinander erwies sich als eine Herausforderung für alle Beteiligten. Die Ziele eines EU-Projektes sind u.a. die Kooperation und Vernetzung unterschiedlicher Partner und die Erarbeitung von gemeinsamen Konzepten, Produkten und Wissensinhalten. Die Projektgruppe soll im Idealfall zu einem Team werden, in dem sich alle gleichberechtigt einbringen können und gemeinsam die vereinbarten Ziele erreichen. Es gibt verschiedene Möglichkeiten, die Kooperations- und Vernetzungsprozesse innerhalb der Partnerschaft zu optimieren und die Qualität des Projektes sicher zu stellen. Eine Methode ist eine regelmäßige Evaluation während der Projektlaufzeit. Eine Prozessevaluation kann an dieser Stelle zur Unterstützung und Optimierung der transnationalen Kooperation beitragen. Darüber hinaus können mit einer Prozessevaluation die Projektfortschritte dokumentiert und sichtbar gemacht werden.

\section{Prozessevaluation als Ansatz zur Optimierung der transnationalen Kooperation}

Eine Prozessevaluation ist eine begleitende Evaluation, die während einer Maßnahme durchgeführt wird (vgl. Wottawa \& Thierau, 2003, S. 32). Es werden Prozesse systematisch erfasst, dokumentiert und ausgewertet. Ziel einer Prozessevaluation ist es, Störungen oder Schwachstellen im System aufzudecken und zu minimieren, indem konkrete Ansatzpunkte zur Verbesserung der Abläufe herausgearbeitet werden. Es ist auch das Ziel einer Prozessevaluation, Stärken und Potenziale zu erkennen und diese zu fördern. Die Prozessevaluation dient zum einen als Frühwarnsystem, um eventuelle Probleme rechtzeitig zu erkennen, und zum anderen als Verfahren, um positive Aspekte zu identifizieren und diese auszubauen. Eine Prozessevaluation kann genutzt werden, um den Vernetzungsprozess der Partner zu unterstützen und die Kooperation innerhalb der Projektgruppe zu optimieren. Durch die regelmäßige Evaluation und die Rückkoppelung der Ergebnisse kann die Zusammenarbeit der Projektgruppe effektiv unterstützt werden.

\section{Prozessevaluation als interne und formative Evaluation}

Eine Prozessevaluation kann als interne Evaluation durchgeführt werden. Dies bedeutet, dass eine Person aus der Projektgruppe die Evaluation übernimmt (siehe dazu auch Abschnitt 1.4.2).

"Internal evaluators (or evaluations) are those done by project staff, even if they are special evaluation staff - that is, even if they are external to the pro- 
duction/writing/teaching/ - service part of the project. Usually internal evaluation is part of the formative evaluation effort [...]." (Scriven 1991, p. 197)

Dieses Vorgehen hat den Vorteil, dass eine projektinterne Person mit den Abläufen vertraut ist und auch die einzelnen Partner kennt, so dass eine Vertrauensbasis vorhanden ist. Es muss von den Beteiligten nicht befürchtet werden, dass ihre Mitarbeit durch die Evaluation kontrolliert oder überprüft wird, da das Ziel die Optimierung der Zusammenarbeit ist.

Eine Prozessevaluation wird als formative Evaluation durchgeführt, d.h. während des Projektverlaufes (siehe dazu auch Abschnitt 1.4.1).

"Formative evaluation is contrasted with summative evaluation. It is typically conducted during the development or improvement of a program or product (or person, and so on) and it is conducted, often more than once, for the inhouse staff of the program with the intend to improve." (Scriven 1991, p. 168f)

Durch die regelmäßigen Rückmeldungen der Projektpartner kann der gesamte Projektverlauf begleitet und dokumentiert werden. Bei Bedarf sind Interventionsmaßnahmen möglich, da das Projekt noch nicht abgeschlossen ist.

„Formative Evaluation ist definitionsgemäß besonders 'praxisrelevant'. Andererseits ist es besonders schwer, ihre Resultate im Sinne von Erfolgs- oder Wirkungskontrolle zu interpretieren, da die Forschung den Gegenstand der Bewertung selbst fortlaufend beeinflusst und verändert. Besonders geeignet ist sie dagegen als Instrument der Qualitätsentwicklung und/oder Qualitätssicherung.“ (Kromrey, 2001, S.118)

Eine Prozessevaluation kann in diesem Sinne vor allem der Optimierung und Qualitätssicherung eines EU-Projektes dienen.

\section{Ziele einer Prozessevaluation}

Eine Prozessevaluation kann über den gesamten Projektverlauf wiederholt durchgeführt werden und richtet sich insbesondere auf die Qualitätsentwicklung und -sicherung, die Stärkung der Arbeitskultur, die Förderung der Beteiligung aller Projektpartner sowie die Selbstreflexion und Selbstkontrolle der geleisteten Arbeit. Die folgende Tabelle gibt einen Überblick über die Ziele: 


\begin{tabular}{|c|c|}
\hline $\begin{array}{l}\text { Qualitätsentwicklung und } \\
\text { Qualitätssicherung }\end{array}$ & $\begin{array}{l}\text { - Monitoring des Entwicklungsprozesses } \\
\text { - Stärken erkennen und ausbauen } \\
\text { - Schwächen aufdecken und beseitigen } \\
\text { - Kompetenzen nutzen und stärken }\end{array}$ \\
\hline Arbeitskultur stärken & $\begin{array}{l}\text { - Sensibilisierung für unterschiedliche Bedürfnisse und Sichtweisen } \\
\text { innerhalb der Projektgruppe } \\
\text { - Klärung und Konkretisierung von Qualitätsvorstellungen } \\
\text { - Gemeinsame Ziele herausarbeiten }\end{array}$ \\
\hline Beteiligungsförderung & $\begin{array}{l}\text { - Förderung der Kommunikation und Kooperation innerhalb der Pro- } \\
\text { jektgruppe } \\
\text { - Verstärkung des Bewusstseins über die eigene Mitverantwortung } \\
\text { - } \quad \text { Tür das Gelingen des Projektes } \\
\text { - Einbeziehung aller Partner }\end{array}$ \\
\hline $\begin{array}{l}\text { Selbstreflexion und } \\
\text { Selbstkontrolle }\end{array}$ & $\begin{array}{l}\text { - } \text { Reflexion des gemeinsamen Handelns } \\
\text { - Bestandsaufnahme in verschiedenen Projektphasen } \\
\text { - } \text { Aufdeckung von Entwicklungspotenzialen } \\
\text { - Dokumentation von Entwicklungsphasen und Erfolgen aber auch } \\
\text { von Herausforderungen }\end{array}$ \\
\hline
\end{tabular}

Tabelle 10: Ziele einer Prozessevaluation

Mit einer Prozessevaluation soll die Qualität der Partnerschaft und Zusammenarbeit gefördert werden. Evaluation dient so der Begleitung und Stärkung der transnationalen Kooperation innerhalb eines EU-Projektes.

\section{Methodisches Vorgehen bei der Prozessevaluation des Projektes eL3}

Im Projekt eL3, das als Fallstudie in dieser Arbeit dient, wurde von der Verfasserin eine Prozessevaluation durchgeführt. Die Ziele der Prozessevaluation sowie die Vorgehensweise wurden zunächst mit der Projektleitung besprochen. Der Projektgruppe wurde mitgeteilt, was mit der Prozessevaluation bezweckt wurde und wie die Ergebnisse erhoben, ausgewertet und präsentiert werden sollten.

Ein bis zwei Wochen nach jedem transnationalen Treffen erfolgte eine Befragung der Projektpartner mittels Fragebögen. Die Befragung hätte auch während der Treffen stattfinden können, um die Rücklaufquote zu erhöhen und die Auswertungen ggf. gleich nutzen zu können. Hierbei war aber zu bedenken, dass die "Stimmung eines Treffens" (alle Partner sind zusammen, es wird gemeinsam gearbeitet und möglicherweise viel geschafft) die Ergebnisse verfälschen könnte. Es wurde deshalb entschieden, die Erhebungen eine Weile nach einem Treffen im Kontext des normalen Alltags der Projektpartner durchzuführen. Auf diese 
Weise konnte eine "nüchternere Einschätzung" der Projektarbeit und des Projektfortschritts erfolgen.

Das Ziel war, mit den Befragungen ein Feedback aller Partner im Hinblick auf positive wie negative Aspekte der Projektarbeit zu erhalten, um es dann in die Gruppe zurück zu spiegeln und für die weitere Arbeit nutzen zu können. Hierfür wurde ein Fragebogen entwickelt, der von den Projektpartnern auf der gemeinsamen Internetseite bzw. einem internen LMS (Learning Management System) ausgefüllt werden konnte. Zusätzlich wurde der Fragebogen per E-Mail an die Partner verschickt. Die Beantwortung der Fragen war freiwillig, und alle Beteiligten hatten sechs Wochen Zeit ihr Feedback einzureichen. In dieser Zeit wurden die Partner einige Male daran erinnert, ihr Feedback abzugeben.

Mit dem Fragebogen sollten die Zufriedenheit mit dem Projektfortschritt sowie subjektiv erlebte positive und negative Aspekte der Zusammenarbeit im Projekt erfasst und auch Verbesserungsvorschläge gesammelt werden. Individuelle Bedarfe und Probleme sollten ebenso ermittelt werden, wie der Nutzen der bisherigen Projektarbeit für die eigene Arbeit und Erwartungen bzw. Wünsche für die zukünftige Zusammenarbeit. Folgende Fragen wurden den Partnern gestellt (der Originalfragebogen befindet sich im Anhang):

- How do you judge the project progression? Contacts with partners, Project management, Transnational Meeting, Online Meetings

- Considered all in all are you satisfied with the project?

- What have you liked so far in this project?

- What have you disliked so far in this project?

- In your opinion what needs improvement?

- What are possible solutions or ways for this improvement?

- Which perspectives have you gained from the network which you can use or adopt for your own projects?

- The working language in the network is English...how do you get along?

- What do you expect or wish for the further work of the network?

- Do you know what your partners are doing and in what areas they work?

- Do you want to have any more information?

- Please indicate in brief additional critic, comments or opinions that you have with regard to the entire project.

Nach der Befragungsphase folgte die Auswertungsphase. Der Fragebogen bestand aus geschlossenen und offenen Fragen. Die geschlossenen Fragen wurden quantitativ und die offenen Fragen inhaltsanalytisch ausgewertet. Die Antworten der Partner wurden in einzelnen Aspekten zusammengefasst. 


\begin{tabular}{|l|l|l|l|}
\hline No. & Statement & Aspects \\
\hline 1 & $\begin{array}{l}\text { Meeting with other partners and learning of } \\
\text { their work }\end{array}$ & $\begin{array}{l}\text { Meeting with partners } \\
\text { (a) }\end{array}$ & $\begin{array}{l}\text { Learning from others } \\
\text { (b) }\end{array}$ \\
\hline 2 & Meeting the range of people involved & $\begin{array}{l}\text { Meeting the range of } \\
\text { people (a) }\end{array}$ & \\
\hline 3 & $\begin{array}{l}\text { Interchange of know-how and personal e- } \\
\text { learning experiences }\end{array}$ & $\begin{array}{l}\text { Exchange of know- } \\
\text { how and experiences } \\
\text { (c) }\end{array}$ & \\
\hline 4 & $\begin{array}{l}\text { The different approaches brought by the } \\
\text { experience of the different partners. That } \\
\text { was our interest in the project: being able } \\
\text { to judge the partners situations and their } \\
\text { exchange of developments and experi- } \\
\text { ence. }\end{array}$ & $\begin{array}{l}\text { Different approaches } \\
\text { (d) }\end{array}$ & $\begin{array}{l}\text { Exchange of experi- } \\
\text { ences (c) }\end{array}$ \\
\hline 5 & $\begin{array}{l}\text { The opportunities to exchange good ideas } \\
\text { and best practice, mainly on methodology } \\
\text { about blended learning and e-learning }\end{array}$ & $\begin{array}{l}\text { Opportunity to ex- } \\
\text { change ideas and best } \\
\text { practice (c) }\end{array}$ & \\
\hline
\end{tabular}

Tabelle 11: Beispiel für die Zusammenfassung von Antworten der Projektpartner auf die Frage "What have you liked so far in this project?"

Im nächsten Schritt wurden die Aspekte in Kategorien gefasst, um ähnliche Aussagen zu bündeln und quantitativ auszuwerten.

\begin{tabular}{|l|l|l|l|l|}
\hline & Category / Key words & Frequency & Partner & Aspects of the statement \\
\hline a) & Meeting & $2 \mathrm{x}$ & 1 & $\begin{array}{l}\text { Meeting with other partners } \\
\text { Meeting the range of people involved }\end{array}$ \\
\hline b) & Learning & $1 \mathrm{x}$ & 1 & Learning from others \\
\hline c) & Exchange & $3 \mathrm{x}$ & 3 & $\begin{array}{l}\text { Exchange of know-how and experiences } \\
\text { Exchange of experiences } \\
\text { Opportunity to exchange ideas and best } \\
\text { practice }\end{array}$ \\
\hline & & 4 & 5 & Different approaches \\
\hline
\end{tabular}

Tabelle 12: Beispiel für die Zusammenfassung der Antworten in Kategorien

Dieses Auswertungsverfahren orientierte sich an der Methode der qualitativen Inhaltsanalyse nach Mayring (vgl. Mayring 2000).

Für jeden Erhebungszeitpunkt wurde ein Bericht und eine Zusammenfassung der zentralen Ergebnisse geschrieben, den zuerst die Projektleitung erhielt. Außerdem wurden die Berichte auf der Lernplattform für alle einsehbar hinterlegt und auf dem nächsten transnationalen Treffen der gesamten Gruppe präsentiert. Es lassen sich drei verschiedene Phasen bei der Prozessevaluation kennzeichnen: 


\section{Präsentation der Ergebnisse}

Darstellung und

Diskussion während eines

Treffens

\section{Befragung der}

Projektpartner

\author{
Online Fragebogen \\ und per E-Mail
}

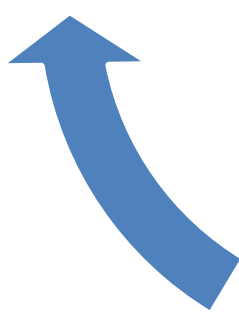

Auswertung der

Ergebnisse

Evaluationsbericht

Zusammenfassung

zentraler

Ergebnisse

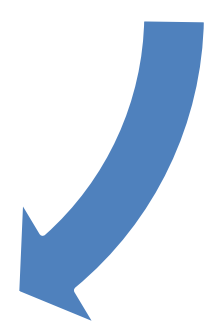

Abbildung 8: $\quad$ Phasen der Prozessevaluation im Projektverlauf

Diese Phasen verliefen zyklisch über die gesamte Projektlaufzeit, da die Prozessevaluation mehrfach zu unterschiedlichen Zeitpunkten erfolgte. Durch die periodischen Rückmeldungen der Partner wurden diese in die laufenden Planungsprozesse einbezogen. Die Meinungen der Projektpartner waren so für alle transparent, und Verbesserungsvorschläge konnten direkt für die weitere Zusammenarbeit genutzt werden.

Im Hinblick auf die Fragestellungen dieser Forschungsarbeit (vgl. Unterkapitel 5.2 ) werden die Ergebnisse der Prozessevaluation genutzt, um den Prozess der Zusammenarbeit der Projektpartner genauer zu betrachten bzw. zu überprüfen, welche Äußerungen die Partner machen im Hinblick auf positive und negative Aspekte, die sich aus ihrer Perspektive in eL3 ergeben. Die Auswertung der Rückmeldungen der Partner soll Aussagen zur Qualität der Zusammenarbeit ermöglichen und zeigen, was die Partner wertschätzten, und was sie für schwierig hielten. Ein Fokus bei der Auswertung soll auch auf die Inventarentwicklung in den Arbeitsgruppen gerichtet werden, wobei die Fragen im Fragebogen zur Prozessevaluation bewusst zum größten Teil offen formuliert wurden, um möglichst individuelle Äußerungen der Partner zu erhalten. Der Prozess der gemeinsamen Arbeit sollte durch die Befragung so wenig wie möglich beeinflusst bzw. gestört werden, und die Ergebnisse sollten auch für die (praktische) Projektarbeit direkt nützlich sein (siehe Prinzipien und Gütekriterien von Aktionsforschung im Abschnitt 5.3.1). 


\subsubsection{Dokumentation der Aktivitäten auf dem Lernmanagementsystem}

Im Projekt el3 wurde durch die Bestandsaufnahme zu Beginn des Projektes und die Gruppendiskussion während des zweiten transnationalen Treffens das Interesse an und der Bedarf nach Evaluationsinstrumenten deutlich. Daraufhin wurde der Vorschlag zur gemeinsamen Entwicklung von Inventaren gemacht und von allen Partnern angenommen. Zunächst wurden die Zielgruppen identifiziert, die bei einer Evaluierung der e-Learning und Blended Learning-Angebote der Partner vorhanden waren. Dann wurde vorgeschlagen, dass die Inventare in Arbeitsgruppen erarbeitet werden sollten, wobei die Partner je nach Interesse an einem der Inventare mitwirken konnten. Es wurden drei Arbeitsgruppen gegründet für die Zielgruppen:

\section{Lerner}

2. Autoren/Produzenten

3. Entscheidungsträger.

In jeder Arbeitsgruppe waren sowohl „Praktiker“ aus Bildungseinrichtungen als auch „Theoretiker" mit wissenschaftlichem Hintergrund vertreten. Die Inventare sollten theoretisch fundiert und praxistauglich sein.

Für die vorliegende Forschungsarbeit sollte die Zusammenarbeit der Partner und der Entwicklungsprozess der Inventare verfolgt werden. Dabei entschied sich die Verfasserin für ein nichtreaktives Verfahren (vgl. Webb et al 1975), um den Entstehungsprozess nicht zu beeinflussen oder zu stören. Für die Zusammenarbeit der Kleingruppen stand den Partnern ein Lernmanagementsystem zur Verfügung, auf dem z.B. Dokumente, die von allen angeschaut und bearbeitet werden sollten, hinterlegt werden konnten, und auf dem die Gruppenleiter Arbeitsschritte definierten, um den Prozess der Inventarentwicklung voranzutreiben. Es gab auch ein Forum, in dem die Partner Fragen diskutieren konnten, die sich bei der gemeinsamen Arbeit ergaben.

An den Inventaren wurde während der transnationalen Treffen gearbeitet, wenn alle Partner zusammen kamen und in der übrigen Zeit über das Lernmanagementsystem. So sollte die räumliche Entfernung der Partner überbrückt werden.

Bei dem für diese Forschungsarbeit gewählten Beobachtungsverfahren wurden die Aktivitäten und Spuren auf dem Lernmanagementsystem dokumentiert. Auf diese Weise sollte die „Natürlichkeit der Situation“ (Webb et al 1975, S.181) nicht beeinflusst werden. 


\subsubsection{Fragebogen zum Abschluss des Projektes}

Am Ende des Projektes eL3 sollten alle Partner die zuvor gemeinsam entwickelten Inventare benutzt haben, so dass erste Erfahrungen damit gemacht wurden und ggf. Verbesserungen vorgenommen werden könnten. Außerdem sollten erste Daten als Vergleichsgrundlage für darauf folgende Untersuchungen bzw. Verwendungen vorliegen. Das Problem bei einmalig eingesetzten Fragebögen ist in der Praxis häufig, dass keine Vergleichsmaßstäbe vorliegen. Mit den Inventaren sollten eine Grundlage geschaffen und Datenpools gebildet werden, damit Partner, die die Instrumente dann ebenfalls benutzen, sich besser einordnen können: Wenn beispielsweise in einem e-Learning-Kurs 70\% der Teilnehmer zufrieden sind, sagt das noch nichts darüber aus, ob das nun gut oder bedenklich ist; liegen allerdings Vergleichsgruppen bzw. Vergleichsdaten vor, lassen sich die Ergebnisse leichter interpretieren, und man könnte z.B. zu dem Schluss kommen, dass dies durchaus „normal“ oder sogar zufriedenstellend ist.

Durch den wiederholten Einsatz der Inventare sollte eine Datenakkumulation möglich sein, so dass die Ergebnisse in ihrer Wertigkeit eingeordnet werden können, da ein Bewertungsmaßstab vorliegt bzw. nach und nach entsteht. Durch die Nutzung der Inventare von möglichst vielen Partnern auf europäischer Ebene sollte so ein „open source“-Modell entwickelt und angeboten werden, und die damit entstehenden Ergebnisse und Wissensinhalte sollten nicht als "Geheimwissen“ eines Institutes oder einer Einrichtung dienen, sondern allen Nutzern zur Verfügung stehen (vgl. Haller 2005, S.5).

Zum Abschluss von eL3 sollte mittels eines Fragebogens erfasst werden, welche Partner welche Inventare benutzt hatten. Außerdem sollten die Kontexte, in denen die Inventare verwendet wurden, ermittelt und die Erfahrungen der Partner abgefragt werden. Die Rückmeldungen der Partner wurden als wichtig erachtet, um nach dem ersten Einsatz - sozusagen dem Pretest der Inventare - ggf. Schwachstellen ausfindig zu machen, aber auch positive Erfahrungen zu dokumentieren. Der „open source-Ansatz“ sollte danach realisiert werden, indem die Inventare über das Netzwerk „blinc“ bzw. die Internetpräsenz frei verfügbar sein würden. Die Erprobungsphase der Inventare sollte mittels des Fragebogens ausgewertet werden 


\section{Ergebnisse}

In diesem Kapitel werden die Ergebnisse, die mit den unterschiedlichen Methoden erhoben wurden, dargestellt. Zunächst wird die Bestandsaufnahme mittels eines Fragebogens (siehe Anhang) ausgewertet bzw. nur der Teil der Befragung, der im Hinblick auf die Evaluationspraxis der Partner zu Beginn des Projektes als Ausgangslage für die Entwicklung der Inventare relevant ist. Daran anschließend werden die Ergebnisse der Prozessevaluation geschildert, die über den gesamten Projektverlauf mit vier Erhebungszeitpunkten durchgeführt wurde. Auch hier werden vor allem die Ergebnisse beschrieben, die für die Entwicklung und Erprobung der Inventare von Interesse sind (der Fragebogen befindet sich ebenfalls im Anhang). Außerdem wird der Entwicklungsprozess der Inventare anhand der Dokumentation der Aktivitäten auf dem Lernmanagementsystem, das den Partnern für die Zusammenarbeit außerhalb der transnationalen Treffen zur Verfügung stand, rekonstruiert. Die Erprobungsphase wurde mit einer Befragung abgeschlossen (Fragebogen siehe Anhang). Zum Ende des Kapitels werden die Ergebnisse im Hinblick auf die Fragestellungen der vorliegenden Forschungsarbeit zusammengefasst.

\subsection{Fragebogen zur Bestandsaufnahme}

Die Bestandsaufnahme mittels eines Fragebogens, eine Status-Quo-Evaluation zu Beginn des Projektes, diente eher der Exploration des Feldes als der intensiven Erforschung der Angebote der Projektpartner (der Fragebogen befindet sich im Anhang). Es sollte ein erstes Bild davon entstehen, was die Projektpartner an e-Learning und Blended Learning-Kursen entwickelt hatten, um eine gemeinsame Arbeitsbasis zu haben. Im Projekt eL3 wurden auf der Basis der Befragung Profile erarbeitet, die die Zusammenarbeit erleichtern sollten im Sinne eines Kennenlernens und einer Transparenz, was die anderen tun (ein Profilbogen befindet sich ebenfalls im Anhang). Ein Ziel der Befragung war auch, die Evaluationspraxis der Partner genauer zu betrachten. Die (Selbst)-Evaluationspraxis der Projektpartner sollte u.a. mit der Frage erfasst werden, welche Methoden und Verfahren sie zur Evaluation bzw. Auswertung ihrer Projekte (Bildungsangebote) bisher nutzten, als Antwortmöglichkeiten gab es vier Kategorien (siehe auch nachfolgende Tabelle).

1. „Tests/Selbsttests/Leistungsnachweise innerhalb ihrer Projekte“

2. „Prüfungen/Leistungsnachweise außerhalb ihres Projektes“

3. "Feedback von anderen"

4. „Formale Befragung der Lerner (z.B. Fragebögen oder Interviews)“ 


\section{What kind of evaluation monitoring processes have been used so far in your pro- ject? (Please tick all the relevant answers)}

- Informal feedback from learners

If yes, is this usually done verbally or in written form?

- Tests/self-tests/efficiency statements within the project

If yes, which?

- Exams/efficiency statements outside the project

If yes, which?

- Feedback of others (e.g. superiors/charges/other institutions)

If yes, which?

- Formal questioning of learners

If yes, in what way? (Questionnaire, Interviews, Others...)

Are the results already available? (Yes, No)

(If yes, please, give details...)

Tabelle 13: $\quad$ Frage zur Bestandsaufnahme der Evaluationspraxis zu Beginn des Projektes eL3: "What kind of evaluation monitoring processes have been used so far in your project?"

Insgesamt wurden neun eL3 Partner befragt (es waren zehn Partner, einer davon war das Pädagogische Seminar der Georg-August-Universität Göttingen. Das Team vom Pädagogischen Seminar führte die Erhebung durch und kommt deshalb nicht in den Antworten vor). Von den neun befragten Partnern beantwortete ein Partner die Frage nicht. In der betreffenden Einrichtung (einem Krankenhaus in Frankreich) wurden verschiedene e-Learning-Kurse geplant, die aber noch nicht realisiert waren. Die folgende Tabelle zeigt alle Antworten der Partner auf die Frage, welche Evaluationsmethoden sie bisher eingesetzt bzw. praktiziert haben (Mehrfachantworten waren möglich):

What kind of evaluation monitoring processes have been used so far in your project?

\begin{tabular}{|l|l|l|l|l|}
\hline $\begin{array}{l}\text { Informal feed- } \\
\text { back from learn- } \\
\text { ers }\end{array}$ & $\begin{array}{l}\text { Tests/self- } \\
\text { tests/efficiency } \\
\text { statements within } \\
\text { the project }\end{array}$ & $\begin{array}{l}\text { Exams/efficiency } \\
\text { statements out- } \\
\text { side the project }\end{array}$ & $\begin{array}{l}\text { Feedback of oth- } \\
\text { ers (e.g. superi- } \\
\text { ors/charges/other } \\
\text { institutions) }\end{array}$ & $\begin{array}{l}\text { Formal question- } \\
\text { ing of learners }\end{array}$ \\
\hline $\begin{array}{l}8 \mathrm{x} \\
(6 \mathrm{x} \text { verbally and } \\
\text { in written form } \\
2 \mathrm{x} \text { verbally) }\end{array}$ & $4 \mathrm{x}$ & $2 \mathrm{x}$ & $2 \mathrm{x}$ & $5 \mathrm{x}$ \\
\hline
\end{tabular}

Tabelle 14: $\quad$ Antworten der eL3- Partner auf die Frage:" What kind of evaluation monitoring processes have been used so far in your project?" $(n=9)$

Die Befragungsergebnisse zur Evaluationspraxis der Partner am Anfang des Projektes zeigen, dass alle Partner zumindest irgendwelche Evaluationsaktivitäten durchführen (bis auf den einen Partner, der zu diesem Zeitpunkt noch keine Kurse entwickelt hatte). Die Befragten gaben alle an, dass sie "informelles Feedback der Lerner" bekommen, die meisten in schriftlicher und in mündlicher Form, manche auch nur in mündlicher Form. Vier der acht 
Partner, die Evaluationen durchführten, verwendeten innerhalb ihrer Kurse „Tests/Selbsttests/Leistungsnachweise“, wobei hier vor allem Angebote zur Selbstkontrolle des Lernfortschritts für Lerner gemeint sind (dies wird aus den Erläuterungen dazu deutlich siehe unten). Zwei Partner gaben an, dass es auch außerhalb des Kurses „Prüfungen/ Leistungsnachweise" gibt, um den Lernerfolg bzw. die Qualität des Lernangebotes zu ermitteln und abzusichern bzw. nachzuweisen. Ebenfalls zwei Partner gaben an, „Feedback von anderen" zu bekommen, wobei in den Erläuterungen deutlich wird, dass darunter verschiedene „andere Personen“ gemeint sind (siehe unten). Fünf Partner führten eine „formale Befragung der Lerner" durch, wobei verschiedene Methoden zum Einsatz kommen (siehe unten).

Zu den Evaluationsmethoden, die von den Partnern genutzt wurden, konnten noch Erläuterungen gegeben werden. Die Antworten verdeutlichen, dass durchaus unterschiedliche Dinge gemeint sein können, wenn die Partner eine Methode zur Evaluation nutzten. Die folgenden Tabellen zeigen jeweils die Ausführungen der Partner, die eine Methode als eigene Evaluationspraxis angegeben haben. Bei der Kategorie „Informelles Feedback der Lerner“ wurde jeweils nur angekreuzt, ob es in mündlicher oder schriftlicher Form bzw. in beiden Formen erhoben wurde. Es wurden keine weiteren Einzelheiten erläutert. Bei den anderen Kategorien hingegen wurden Ausführungen gegeben. Bei der Antwortmöglichkeit „Tests/selftests/efficiency statements within the project" führten die Partner Folgendes aus:

\begin{tabular}{|c|c|}
\hline Partner & Tests/self-tests/efficiency statements within the project \\
\hline 4 & $\begin{array}{l}\text { Each content is divided into } 3 \text { stages: an introducing stage that shows the training } \\
\text { contents, a working stage that shows up some notions, etc. The last stage is an } \\
\text { assessment based on the training modules. Some assessments can be done many } \\
\text { times ( } 10 \text { questions are chosen haphazardly), others can not be done more than } \\
\text { once. Those who created the modules thought about this possibility. }\end{array}$ \\
\hline 7 & $\begin{array}{l}\text { The mode of assessment is by continuous course work which takes the form of } \\
\text { essays, presentations, and poster production. }\end{array}$ \\
\hline 8 & $\begin{array}{l}\text { Test of knowledge about contents inside the eLearning: questions with multiple } \\
\text { choice of answers. }\end{array}$ \\
\hline 9 & Multiple Choice Questions \\
\hline & $\begin{array}{l}\text { Erläuterungen der Partner zur verwendeten Evaluationsmethode } \\
\text { „Tests/Selbsttests/Leistungsnachweise innerhalb des Projektes“ }\end{array}$ \\
\hline
\end{tabular}

Vier Partner gaben an „Tests/Selbsttests/Leistungsnachweise innerhalb ihrer Projekte“ zur Evaluation zu verwenden. In den Erläuterungen wird deutlich, dass es darunter verschiedene Möglichkeiten gibt. Drei Partner scheinen „Multiple Choice Questions” innerhalb ihrer eLearning Kurse zu verwenden, mit denen die Lerner ihren Lernfortschritt selbst kontrollieren können. Partner Nr. 7 hingegen nutzte zum Nachweis des Lernfortschritts bzw. für einen Nachweis kontinuierlicher Arbeit in dem Kurs Essays, Präsentationen und Poster, die von den Lernern entwickelt werden müssen. Partner Nr. 7 bot vor allem Blended Learning an und verknüpft so e-Learning und Präsenzveranstaltungen. 


\begin{tabular}{|l|l|}
\hline Partner & Exams/efficiency statements outside the project \\
\hline 7 & $\begin{array}{l}\text { Fulfillment of University regulations for the stages leading to submission of a PhD in } \\
\text { accordance with academic regulations. }\end{array}$ \\
\hline 9 & Multiple Choice Questions, frontal exams \\
\hline Tabelle 16: & $\begin{array}{l}\text { Erläuterungen der Partner zur Evaluationsmethode „Prüfungen/Leistungsnachweise } \\
\text { außerhalb des Projektes“ }\end{array}$
\end{tabular}

Zwei Partner gaben an, „Prüfungen/Leistungsnachweise außerhalb ihres Projektes“ als Evaluationsmethode zu nutzen. Bei Partner Nr. 7 müssen universitäre Richtlinien in verschiedenen Phasen des Projektes erfüllt werden, die dazu führen, dass die Lerner (Studenten) den akademischen Grad eines Doktors erreichen. Bei Partner Nr. 9 wurden „Multiple Choice Questions" und Prüfungen verwendet, um den Lernerfolg der Lerner (Studenten) zu ermitteln.

\begin{tabular}{|l|l|}
\hline Partner & Feedback of others (e.g. superiors/charges/other institutions) \\
\hline 1 & $\begin{array}{l}\text { E-learning is regarded as time saving and very good addition to traditional training } \\
\text { methods. Presence times can be reduced and costs are saved. }\end{array}$ \\
\hline 7 & $\begin{array}{l}\text { Critical evaluation of programme by course management team and external exam- } \\
\text { iner }\end{array}$ \\
\hline
\end{tabular}

Das "Feedback von anderen" wurde von zwei Partnern zur Evaluation bzw. Bewertung der eigenen Projekte genutzt. Bei Partner Nr.1, einem Krankenhaus, sind die Bildungsverantwortlichen der Meinung gewesen, dass e-Learning Aus- und Weiterbildungszeiten verkürzt, also Weiterbildungszeiten reduziert und traditionelle Methoden sinnvoll ergänzt. Präsenzzeiten werden weniger und damit Kosten gespart. Bei Partner Nr.7, einer Universität, wird unter „Feedback von anderen“ verstanden, dass eine kritische Evaluation des gesamten Programmes durchgeführt wird, und zwar sowohl von dem Team, das für die Kursplanung verantwortlich ist, als auch von einem externen Prüfer.

\begin{tabular}{|l|l|}
\hline Partner & Formal questioning of learners \\
\hline 3 & Interviews \\
\hline 4 & Questionnaire \\
\hline 7 & Questionnaire \\
\hline 8 & We have the feedback of traking for learners in excel files for each course \\
\hline 9 & Questionnaire \\
\hline Tabelle 18: & Erläuterungen der Partner zur Evaluationsmethode „Formale Befragung der Lerner“
\end{tabular}

Fünf Partner gaben an, eine „Formale Befragung der Lerner“ durchzuführen. Drei Partner verwendeten dazu Fragebögen, ein Partner nutzte Interviews als Methode und einer zeichnete die Aktivitäten der Lerner auf (z.B. was, wann, wie lange genutzt wurde innerhalb des eLearning-Kurses, also eine Dokumentation der Tätigkeiten der Lerner).

Die eL3 Partner verstanden unter den einzelnen Methoden nicht nur Verschiedenes, sie verwendeten ihre Evaluationsmethoden außerdem in unterschiedlichen Kombinationen. 
Welche Methoden bzw. Methodenkombinationen zur Evaluation von den einzelnen Partnern genutzt wurden, veranschaulicht die folgende Tabelle:

What kind of evaluation monitoring processes have been used so far in your project?

\begin{tabular}{|l|l|l|l|l|l|}
\hline & $\begin{array}{l}\text { Informal } \\
\text { feedback } \\
\text { from learners }\end{array}$ & $\begin{array}{l}\text { Tests/ } \\
\text { self-tests/ } \\
\text { efficiency } \\
\text { statements }\end{array}$ & $\begin{array}{l}\text { Exams/ } \\
\text { efficiency } \\
\text { statements } \\
\text { outside of the } \\
\text { programme }\end{array}$ & $\begin{array}{l}\text { Feedback of } \\
\text { others (e.g. } \\
\text { superiors/ } \\
\text { charges/other } \\
\text { institutions) }\end{array}$ & $\begin{array}{l}\text { Formal ques- } \\
\text { tioning of } \\
\text { learners }\end{array}$ \\
\hline Partner 1 & $\mathrm{X}$ & - & - & $\mathrm{X}$ & - \\
\hline Partner 2 & $\mathrm{X}$ & - & - & - & - \\
\hline Partner 3 & $\mathrm{X}$ & - & - & - & $\mathrm{X}$ \\
\hline Partner 4 & $\mathrm{X}$ & $\mathrm{X}$ & - & - & $\mathrm{X}$ \\
\hline Partner 5 & - & - & - & - & - \\
\hline Partner 6 & $\mathrm{X}$ & $\mathrm{X}$ & $\mathrm{X}$ & $\mathrm{X}$ & $\mathrm{X}$ \\
\hline Partner 7 & $\mathrm{X}$ & $\mathrm{X}$ & $\mathrm{X}$ & $\mathrm{X}$ & $\mathrm{X}$ \\
\hline Partner 8 & $\mathrm{X}$ & $\mathrm{X}$ & - & - & $\mathrm{X}$ \\
\hline Partner 9 & $\mathrm{X}$ & $\mathrm{X}$ & $\mathrm{X}$ & - & \\
\hline
\end{tabular}

Tabelle 19: Übersicht über die Kombination von Methoden bei der Evaluationspraxis der einzelnen Projektpartner im Projekt eL3 $(n=9)$

Mit diesen Ergebnissen wird deutlich, dass die Evaluationspraxis der Projektpartner sehr unterschiedlich ist. Es sind sowohl die Anzahl als auch die Kombination der verwendeten Methoden zur Evaluation verschieden. Das Spektrum reicht von der Nutzung einer Methode bis zur Kombination aller zur Auswahl angebotenen Methoden: Ein Partner (Nr.2) bekommt nur „informelles Feedback der Lerner" und ein anderer (Nr.7) gibt an, alle Maßnahmen durchzuführen. Das Ausmaß der Nutzung von verschiedenen Methoden zeigt sich mit diesen Ergebnissen als sehr individuell. An dieser Stelle lässt sich noch nichts über die Qualität der verwendeten Methoden und Instrumente sagen und auch noch nichts über die Professionalität der Evaluationen. Es wird lediglich deutlich, dass alle Projektpartner bestrebt sind, ihre Kurse in irgendeiner Form auszuwerten und dies wohl auch tun. Es scheint, dass alle Partner Evaluationsaktivitäten durchführen (bis auf einen, der noch keine Kurse realisierte).

Die eL3-Projektpartnerschaft besteht aus verschiedenen Einrichtungen des Dritten Systems, die sich grob in Universitäten, Bildungsanbieter und Krankenhäuser einteilen lassen. Die jeweiligen Rahmenbedingungen, Anforderungen, Ziele, Interessen und „Traditionen“ im Kontext von Evaluationen sind dadurch unterschiedlich. Im Praxisfeld und "täglichen Geschäft“ eines Bildungsanbieters oder Krankenhauses gelten andere Regeln als im universitären Umfeld. Um zu überprüfen, ob es tatsächlich bestimmte Charakteristika hinsichtlich der Evaluationspraxis im Zusammenhang mit der Art der Einrichtung gibt, werden die eL3 Partner im Folgenden zu Gruppen gebündelt: 


\begin{tabular}{|l|l|l|l|l|l|}
\hline Bildungsanbieter & \multicolumn{3}{|l|}{ Evaluationsmethoden } \\
\hline & $\begin{array}{l}\text { Informal feed- } \\
\text { back from learn- } \\
\text { ers }\end{array}$ & $\begin{array}{l}\text { Tests/ } \\
\text { self-tests/ } \\
\text { efficiency state- } \\
\text { ments }\end{array}$ & $\begin{array}{l}\text { Exams/ } \\
\text { efficiency state- } \\
\text { ments outside } \\
\text { the project }\end{array}$ & $\begin{array}{l}\text { Feedback of } \\
\text { others (e.g. } \\
\text { superiors/ } \\
\text { charges/other } \\
\text { institutions) }\end{array}$ & $\begin{array}{l}\text { Formal } \\
\text { questioning } \\
\text { of learners }\end{array}$ \\
\hline $\begin{array}{l}\text { Partner 2 } \\
\text { Deutschland }\end{array}$ & $\begin{array}{l}\mathrm{X} \\
\text { (verbally and in } \\
\text { written form) }\end{array}$ & - & - & - & - \\
\hline $\begin{array}{l}\text { Partner 4 } \\
\text { Frankreich }\end{array}$ & $\begin{array}{l}\mathrm{X} \\
\text { (verbally and in } \\
\text { written form) }\end{array}$ & $\mathrm{X}$ & - & - & $\begin{array}{l}\text { X } \\
\text { (Question- } \\
\text { naire) }\end{array}$ \\
\hline $\begin{array}{l}\text { Partner 6 } \\
\text { Frankreich }\end{array}$ & $\begin{array}{l}\text { (verbally and in } \\
\text { written form) }\end{array}$ & - & - & - \\
\hline
\end{tabular}

Tabelle 20: $\quad$ Verwendete Evaluationsmethoden in der Gruppe der Bildungsanbieter im Projekt eL3

Von den Bildungsanbietern nutzen alle „Informelles Feedback der Lerner“ in mündlicher und schriftlicher Form. Für zwei der drei Partner ist dies die einzige Methode der Evaluation ihrer Kurse. Ein Partner aus der Gruppe der Bildungsanbieter gibt an, auch „Tests/Selbsttests/ Leistungsnachweise“ und eine „Formale Befragung von Lernern“ zu nutzen. Bei diesem Partner (Partner Nr.4) muss angemerkt werden, dass es sich dabei um ein Bildungsinstitut handelt, das dem französischen Bildungsministerium unterstellt ist. Die öffentliche Interessensgruppe hat zur Aufgabe, auf akademischer Ebene Institute des Bildungswesens bei der Entwicklung von Ausbildungsinhalten und deren Anpassung an sich ständig verändernde gesellschaftliche Anforderungen zu unterstützen (siehe Punkt 1.3 Projektpartnerschaft GIP FIPAN). Diese Anmerkung spielt deshalb an dieser Stelle eine Rolle, da deutlich wird, dass auch innerhalb der Gruppe von Bildungsanbietern durchaus Unterschiede vorherrschen. Manche Bildungsanbieter arbeiten als Unternehmen rein wirtschaftlich, bieten Kurse und Projekte an und „leben davon“, andere Bildungsanbieter haben eine Anbindung an Institutionen wie z.B. Universitäten oder Ministerien, wie in diesem Fall Partner Nr.4. Diese Kontexte müssen mit berücksichtigt werden, da sie eine Auswirkung auf die Auswahl von Methoden zur Evaluation haben könnten und sich eventuell auch Rückschlüsse auf die Kompetenzen bzw. den Grad der Professionalität im Handlungsfeld der Evaluation ziehen lassen. Es liegt nahe, dass in einem Bildungsinstitut, das die Aufgabe hat, auf akademischer Ebene andere Institute zu unterstützen, gewisse Maßstäbe und Anforderungen vorherrschen, die bei einem privaten Bildungsanbieter vielleicht nicht gegeben sind.

Insgesamt werden in der Gruppe der Bildungsanbieter im Projekt eL3 ein bis drei Methoden zur Evaluation von e-learning und Blended Learning-Kursen genutzt. 


\begin{tabular}{|l|l|l|l|l|l|}
\hline Krankenhäuser & \multicolumn{4}{l|}{ Evaluationsmethoden } \\
\hline & $\begin{array}{l}\text { Informal feed- } \\
\text { back from learn- } \\
\text { ers }\end{array}$ & $\begin{array}{l}\text { Tests/ } \\
\text { self-tests/ } \\
\text { efficiency state- } \\
\text { ments }\end{array}$ & $\begin{array}{l}\text { Exams/ } \\
\text { efficiency state- } \\
\text { ments outside } \\
\text { the project }\end{array}$ & $\begin{array}{l}\text { Feedback of } \\
\text { others (e.g. } \\
\text { superiors/ } \\
\text { charges/other } \\
\text { institutions) }\end{array}$ & $\begin{array}{l}\text { Formal } \\
\text { questioning } \\
\text { of learners }\end{array}$ \\
\hline $\begin{array}{l}\text { Partner 1 } \\
\text { Deutschland }\end{array}$ & $\begin{array}{l}\mathrm{X} \\
\text { (verbally and in } \\
\text { written form) }\end{array}$ & - & - & $\mathrm{X}$ & - \\
\hline $\begin{array}{l}\text { Partner 5 } \\
\text { Frankreich }\end{array}$ & - & - & - & - & - \\
\hline $\begin{array}{l}\text { Partner 8 } \\
\text { Italien }\end{array}$ & $\begin{array}{l}\text { X } \\
\text { (verbally) }\end{array}$ & $\mathrm{X}$ & - & - & $\mathrm{X}$ \\
\hline Tabelle 21: & - & - & & - \\
\hline
\end{tabular}

Tabelle 21: $\quad$ Verwendete Evaluationsmethoden in der Gruppe der Krankenhäuser im Projekt eL3

In der Gruppe der Krankenhäuser wird „Informelles Feedback der Lerner“ zur Evaluation verwendet. Ein Partner nutzt es in mündlicher und schriftlicher Form und ein Partner nur in mündlicher Form. Ein Partner hat noch keine e-Learning-Kurse realisiert und auch im Vorfeld noch keine Evaluationsaktivitäten durchgeführt. In dem Krankenhaus aus Deutschland wird neben dem „Informellen Feedback der Lerner“ auch „Feedback von anderen“, in diesem Fall von den Weiterbildungsverantwortlichen, gesammelt. Bei dem Partner aus Italien werden neben „Informellem Feedback der Lerner“ in mündlicher Form auch „Tests/Selbsttests/ Leistungsnachweise" angeboten, außerdem liegen Daten in Form von Protokollen zum Nutzerverhalten über die Kurse vor (z.B. was wurde angeklickt, wie lange wurde das Programm genutzt etc.). Insgesamt werden in der Gruppe der Krankenhäuser im Projekt eL3 zwei bis drei Methoden zur Evaluation von e-Learning und Blended Learning-Kursen genutzt.

\begin{tabular}{|c|c|c|c|c|c|}
\hline \multirow[t]{2}{*}{ Universitäten } & \multicolumn{5}{|c|}{ Evaluationsmethoden } \\
\hline & $\begin{array}{l}\text { Informal feed- } \\
\text { back from learn- } \\
\text { ers }\end{array}$ & $\begin{array}{l}\text { Tests/ } \\
\text { self-tests/ } \\
\text { efficiency state- } \\
\text { ments }\end{array}$ & $\begin{array}{l}\text { Exams/ } \\
\text { efficiency state- } \\
\text { ments outside } \\
\text { the project }\end{array}$ & $\begin{array}{l}\text { Feedback of } \\
\text { others (e.g. } \\
\text { superiors/ } \\
\text { charges/other } \\
\text { institutions) }\end{array}$ & $\begin{array}{l}\text { Formal } \\
\text { questioning } \\
\text { of learners }\end{array}$ \\
\hline $\begin{array}{l}\text { Partner } 3 \\
\text { Frankreich }\end{array}$ & $\begin{array}{l}\text { X } \\
\text { (verbally) }\end{array}$ & - & - & - & $\begin{array}{l}\text { X } \\
\text { (Interviews) }\end{array}$ \\
\hline $\begin{array}{l}\text { Partner } 7 \\
\text { Schottland }\end{array}$ & $\begin{array}{l}\mathrm{X} \\
\text { (verbally and in } \\
\text { written form) }\end{array}$ & $\mathrm{X}$ & $\mathrm{X}$ & $\mathrm{X}$ & $\begin{array}{l}\text { X } \\
\text { (Question- } \\
\text { naire) }\end{array}$ \\
\hline $\begin{array}{l}\text { Partner } 9 \\
\text { Italien }\end{array}$ & $\begin{array}{l}\text { X } \\
\text { (verbally and in } \\
\text { written form) }\end{array}$ & $\mathrm{X}$ & $\mathrm{X}$ & - & $\begin{array}{l}\text { X } \\
\text { (Question- } \\
\text { naire) } \\
\end{array}$ \\
\hline
\end{tabular}

Tabelle 22: $\quad$ Verwendete Evaluationsmethoden in der Gruppe der Universitäten im Projekt eL3

Von allen eL3 Partnern, die in Universitäten beschäftigt sind, werden zur Evaluation der eLearning und Blended Learning Kurse verschiedene Methoden kombiniert. Die Befragten geben an, „Informelles Feedback der Lerner“ zu erheben, zwei Partner in mündlicher und schriftlicher und ein Partner nur in mündlicher Form. Partner Nr.3 verwendet zusätzlich zum „Informellen Feedback der Lerner“ in mündlicher Form Interviews. Partner Nr.9 verwendet alle Methoden außer „Feedback von anderen“ (z.B. externen Evaluatoren), und Partner Nr.7 nutzt alle Evaluationsmethoden. Bei diesem Partner sind die Evaluationsaktivitäten beson- 
ders stark ausgeprägt, was die Anzahl der verwendeten Methoden anbelangt und die Vielfalt der Personengruppen, die in die Evaluationen einbezogen werden (Lerner, Lehrende, externe Evaluatoren). Es fällt auf, dass auch in der Gruppe der Universitäten Unterschiede vorhanden sind. Partner Nr.3 stammt aus dem medizinischen Bereich einer Universität in Frankreich und nutzt e-Learning zur Ergänzung seiner Präsenzlehre. Partner Nr.7 entwickelt eLearning und Blended Learning-Programme als eigenständige Kurse, um den Studenten innovative Lernformen anzubieten. Die Evaluation dieser Kurse hat u.a. auch aus der Perspektive der Universitätsleitung einen ganz anderen Stellenwert als Kurse eines Lehrenden, der seine Lehre durch e-Learning ergänzt. Diese Kontexte und Interessen müssen bei dieser Darstellung mit bedacht werden. Insgesamt werden in der Gruppe der Universitäten im Projekt eL3 zwei bis fünf Methoden zur Evaluation von e-Learning und Blended Learning-Kursen genutzt.

Bei dem Vergleich der drei Gruppen fällt auf, dass die Evaluationspraxis in den verschiedenen Kontexten unterschiedlich ist. Für die Gruppe der Bildungsanbieter spielt Evaluation eine Rolle, es werden aber deutlich weniger Methoden eingesetzt als in den anderen beiden Gruppen. In der Gruppe der Universitäten scheint Evaluation zum einen durch die Vielfalt der eingesetzten Methoden, aber auch durch die Einbeziehung verschiedener Akteursgruppen sehr umfangreich zu sein. Es wird durch die Status Quo-Evaluation zur Evaluationspraxis der eL3 Projektpartner auch deutlich, dass es innerhalb der Gruppen von Bildungsanbietern, Krankenhäusern und Universitäten Unterschiede gibt.

Zusammenfassend kann mit den Ergebnissen der Bestandsaufnahme im Hinblick auf die Fragestellung, besonders Fragenkomplex eins (siehe Unterkapitel 5.2), Folgendes festgehalten werden: Die Evaluationspraxis der Partner zeigt sich als ein weites Feld. Es werden unterschiedliche Methoden eingesetzt und kombiniert, wobei nicht immer alle Partner unter einer Methode dasselbe verstehen. Wie professionell die Evaluationen durchgeführt werden, lässt sich mit dieser Erhebung nicht sagen, deutlich wird aber, dass alle Partner Evaluationsaktivitäten durchführen und damit wohl für wichtig erachten. Die Gründe und Ziele der Evaluationen sind dabei verschieden, und auch die Auswertung und Nutzung der Ergebnisse werden unterschiedlich gehandhabt.

In den Gruppendiskussionen zu Beginn des Projektes wurde mehrfach der Wunsch nach Austausch zur Evaluationspraxis und nach Hilfestellungen zur Auswahl von Instrumenten geäußert. Einige wollten auch einfach Anregungen für die eigene Praxis bekommen und vor allem Wissensinhalte erhalten bzw. Erfahrungen der anderen nutzen. Zu Beginn des Projektes war aber noch keine gute Vertrauensbasis vorhanden, so dass sich auch einige Partner nicht „in die Karten schauen lassen wollten“, was sich z.B. darin zeigte, dass das Team vom Pädagogischen Seminar nicht die e-Learning und Blended Learning-Angebote der Partner untersuchen durfte (weshalb dann die Befragung mittels Fragebogen durchgeführt wurde). 
Im Hinblick auf das Forschung-Praxis-Dilemma wird durch die Befragung, aber auch durch die Diskussionen bei den transnationalen Treffen, bei denen die Ergebnisse vorgestellt und besprochen wurden, deutlich, dass in der Praxis tatsächlich weniger Evaluationsmethoden eingesetzt werden, und diese auch nach eigenem besten Wissen und nach eigenen Regeln eingesetzt werden. In der Praxis können wissenschaftliche Standards nicht eingehalten werden, da aufwändige Verfahren „Zeit und Geld“ kosten. Grundsätzlich wollen die Praktiker ihre Kurse verbessern und sehen in Evaluationstätigkeiten auch die Chance, zu Ergebnissen zu kommen, mit denen sie ihre Angebote auswerten können, häufig geht es ihnen aber lediglich um einen Nachweis, dass sie "gute Kurse" anbieten (dann hat die Evaluation vor allem eine „Legitimationsfunktion“ - siehe Unterkapitel 1.3). Sie sehen Evaluation als Methode, um nach außen (also z.B. Mittelgebern, Vorgesetzten, potenziellen Kunden gegenüber) zu zeigen, dass sie erfolgreich sind und „gute Praxis“ abliefern. Eine bloße Reflexion der eigenen Tätigkeiten wird als wenig wertvoll erachtet, vielmehr werden konkrete und „nützliche“ Ergebnisse gebraucht. Im beruflichen Alltag ist keine Zeit für Erhebungen, Auswertungen und Diskussionen vorhanden. Es besteht aber der Wunsch nach der Vereinfachung von Evaluationsverfahren, nach Erleichterung der Arbeit und nach dem Austausch von „best practice“ bzw. einer Einsicht in die Praxis der Arbeit von den anderen Partnern. Evaluation soll nicht "quick and dirty“ aber „quick and easy“ sein und die Qualität der eigenen Arbeit hervorheben. Bei den Partnern mit wissenschaftlichem Hintergrund zeigt sich vor allem das Interesse am Austausch von Erfahrungen und Wissensinhalten bzw. theoretischen Hintergründen. Das Reflektieren zur eigenen Evaluationspraxis, zu Methoden und Inhalten wird als sehr anregend und wertvoll erachtet. Es zeigt sich auch eine kritische Haltung zu den Inventaren im Hinblick auf die Einhaltung wissenschaftlicher Standards, aber der Ansatz wird als lohnenswert gesehen und das Projekt eL3 als Basis, in der eine erste Version erarbeitet werden kann. Es besteht aber auch der Wunsch nach einer Auswertung der Erprobungsphase und nach dem Austausch über Erfahrungen bei dem Einsatz in den unterschiedlichen Kontexten. Im universitären Feld besteht viel deutlicher die Bereitschaft, auch aufwändige Verfahren auszuprobieren bzw. einzusetzen. Es wird aber auch danach verlangt, diese Erprobungsphase abzuschließen, zu analysieren und die Ergebnisse umzusetzen, und eine Arbeitsweise für die weitere Verwendung und/oder Zusammenarbeit zu finden.

Es zeigt sich, dass "in der Praxis" und im wissenschaftlichen Kontext unterschiedliche Bedarfe, Interessen und Erwartungen vorherrschen, die nicht unbedingt auf einen Nenner zu bringen sind. Die Heterogenität der Partner als Basis für die Entwicklung und Erprobung der Inventare stellt sich als eine Stärke und zugleich als eine Schwäche der Projektgruppe dar. 


\subsection{Prozessevaluation}

Die Ergebnisse der Prozessevaluation über den Projektverlaufszeitraum von zwei Jahren sollen in dieser Arbeit genutzt werden, um den Entwicklungsprozess und die Erprobungsphase der Inventare im Spiegel der Rückmeldungen der eL3 - Projektpartner zu betrachten. Insgesamt wurden vier Erhebungen durchgeführt, wobei jedes Mal dieselben Fragen gestellt wurden (Fragebogen siehe Anhang).

Insgesamt zeigt sich, dass die Zufriedenheit der Partner mit dem Projektfortschritt über den gesamten Projektverlauf hinweg relativ positiv ist. Lediglich bei der ersten Erhebung im November 2004 bewertet ein großer Teil der Gruppe (42\% der Befragten) den Kontakt zwischen den Partnern als "weniger gut“. Dabei muss beachtet werden, dass das Projekt zu diesem Zeitpunkt schon 11 Monate lief. Ein kontinuierlicher Kontakt, Austausch und eine regelmäßige Kommunikation war eine der zentralen Herausforderungen in der Zusammenarbeit - dies spiegelt sich auch in diesem Ergebnis. Der Entwicklungsprozess der Inventare dauerte wesentlich länger als antizipiert und geplant. Die Kooperation der eL3-Projektpartner brauchte eine lange Anlaufphase - immerhin die Hälfte der Projektlaufzeit.

Die Ergebnisse zeigen auch, dass die Partner die transnationalen Treffen sehr positiv bewerten. Während der Treffen „passierte viel“. Die Partner kamen zusammen, es wurde diskutiert, Ergebnisse wurden vorgetragen und weitere Arbeitsschritte vereinbart. Jedes transnationale Treffen brachte im Hinblick auf den Projektfortschritt entscheidende Impulse. Ein „Weiterkommen" wurde für die Partner spürbar und konkrete Arbeitsergebnisse lagen vor. Die folgenden Tabellen zeigen die Antworten der Projektpartner auf die Frage „How do you like the progression of the eL3-network?" im Hinblick auf drei Aspekte: Den Kontakt mit den Partnern, das Management/Projektmanagement und die transnationalen Treffen: 
How do you like the progression of the eL3-network?

Contact with partners

\begin{tabular}{|l|c|c|c|c|c|}
\hline Feedback & Very good & Good & Less good & Bad & No data \\
\hline Nov $2004 \mathrm{n}=12$ & - & $7(58 \%)$ & $5(42 \%)$ & - & - \\
\hline Mar $2005 \mathrm{n}=12$ & $5(42 \%)$ & $5(42 \%)$ & $2(17 \%)$ & - & - \\
& & & & - & - \\
\hline June $2005 \mathrm{n}=10$ & $6(60 \%)$ & $3(30 \%)$ & $1(10 \%)$ & - & - \\
\hline Nov $2005 \mathrm{n}=9$ & $3(33 \%)$ & $5(55 \%)$ & $1(11 \%)$ & - & - \\
\hline
\end{tabular}

Management/Project management

\begin{tabular}{|c|c|c|c|c|c|}
\hline Feedback & Very good & Good & Less good & Bad & No data \\
\hline Nov 2004 n=12 & $3(25 \%)$ & $8(67 \%)$ & $1(8 \%)$ & - & - \\
\hline Mar $2005 n=12$ & $3(25 \%)$ & $7(58 \%)$ & $1(8 \%)$ & - & $1(8 \%)$ \\
\hline June $2005 \mathrm{n}=10$ & $2(20 \%)$ & $8(80 \%)$ & - & - & - \\
\hline Nov $2005 \mathrm{n}=9$ & $4(44 \%)$ & $5(55 \%)$ & - & - & - \\
\hline \multicolumn{6}{|c|}{ Transnational Meeting } \\
\hline Feedback & Very good & Good & Less good & Bad & No data \\
\hline Nov $2004 n=12$ & $7(58 \%)$ & $5(42 \%)$ & - & - & - \\
\hline Mar $2005 \mathrm{n}=12$ & $5(42 \%)$ & $6(50 \%)$ & - & - & $1(8 \%)$ \\
\hline June $2005 n=10$ & $3(30 \%)$ & $6(60 \%)$ & - & - & $1(10 \%)$ \\
\hline Nov $2005 n=9$ & $4(44 \%)$ & $5(55 \%)$ & - & - & - \\
\hline
\end{tabular}

Tabelle 23: Ergebnisse der Prozessevaluation auf die Frage „How do you like the progression of the eL3-network?"

Mit den Antworten zu der Frage "Considered all in all are you satisfied with the eL3 project?" wird ebenfalls deutlich, dass die allgemeine Zufriedenheit mit dem Projekt über den gesamten Projektverlauf gegeben war. Im Juni 2005 war die Zufriedenheit besonders groß, zu diesem Zeitpunkt waren die Inventare bereits entwickelt worden, und die Gruppe hatte gemeinsam „viel geschafft“. Es wurden Pläne für die letzten sechs Monate des Projektes gemacht und die Gründung des Netzwerkes „blinc“ (blended learning institutions cooperative), das die Nachhaltigkeit und Zukunftsfähigkeit der erarbeiteten Materialien sowie der Zusammenarbeit sichern sollte, stand kurz bevor.

Considered all in all are you satisfied with the eL3 project?

\begin{tabular}{|l|c|c|c|c|c|c|}
\hline Feedback & $\begin{array}{c}\text { Very satis- } \\
\text { fied }\end{array}$ & Satisfied & O.K. & $\begin{array}{c}\text { Not satis- } \\
\text { fied }\end{array}$ & $\begin{array}{c}\text { Not at all } \\
\text { satisfied }\end{array}$ & No data \\
\hline Nov $2004 \mathrm{n}=12$ & - & $10(83 \%)$ & $2(17 \%)$ & - & - & - \\
\hline Mar $2005 \mathrm{n}=12$ & $1(8 \%)$ & $8(67 \%)$ & $3(25 \%)$ & - & - & - \\
\hline June $2005 \mathrm{n}=10$ & $3(30 \%)$ & $4(40 \%)$ & $3(30 \%)$ & - & - & - \\
\hline Nov $2005 \mathrm{n}=9$ & $1(11 \%)$ & $7(77 \%)$ & $1(11 \%)$ & - & - & - \\
\hline
\end{tabular}

Tabelle 24: Ergebnisse der Prozessevaluation auf die Frage "Considered all in all are you satisfied with the eL3 project?"

Im Folgenden werden weitere Fragen der Prozessevaluation und die Antworten der Partner zu den vier Erhebungszeitpunkten dargestellt. Bei den zumeist offenen Fragen bildete die Verfasserin nach der Methode der Inhaltsanalyse nach Mayring (2000) Kategorien, um die Essenz der Aussagen der Partner zu zeigen. Hinter den Kategorien steht jeweils, wie viele der Befragten etwas zu diesem Aspekt geäußert haben, d.h. wie oft etwas zu diesem Thema in den Antworten der Partner vorkam. Diese Kategorien variieren je nach Erhebungszeitpunkt. Für die Auswertung wurden ähnliche bzw. gleiche Kategorien nebeneinander gestellt, 
um zu analysieren, welche Aspekte durchgehend, häufiger bzw. nur einmal genannt wurden. Es zeigen sich so Themen, die über den gesamten Projektverlauf für die Partner eine Bedeutung hatten, aber auch einzelne Themen, die nur zu bestimmten Phasen relevant und charakteristisch waren. Die Rückmeldungen der Partner sollen in dieser Arbeit vor allem in Bezug auf den Entwicklungsprozess sowie die Erprobung der Inventare als Instrumente zur Verbesserung der Evaluationspraxis im Kontext von e-Learning und Blended Learning ausgewertet werden.

Auf die Frage, was den Partnern bisher im Projekt gefallen hat, wurden zu jedem Erhebungszeitpunkt der Aspekt des Treffens von verschiedenartigen Menschen, der Austausch von Wissen und Erfahrungen sowie das Voneinander-Lernen genannt. Bei drei von vier Erhebungszeitpunkten wurde erwähnt, dass neue Ideen für das eigene Projekt entstanden sind und der Einblick in die Arbeit von anderen als wertvoll erachtet wird. Auch die Zusammenarbeit und die Verschiedenartigkeit bzw. Vielfältigkeit der Partner wurde genannt. Außerdem gefiel den Befragten das Teilen von Ideen sowie die Entstehung von Ideen und das Reflektieren. Alle anderen Aspekte, die genannt wurden, tauchten nur einmal während der vier Erhebungszeitpunkte auf.

Insgesamt lässt sich sagen, dass der Kontakt zu und das Treffen von unterschiedlichen Partnern, die in einem ähnlichen Arbeitsfeld arbeiten, als positiv bewertet wurde, und auch das Teilen von Ideen, der Austausch und die Einsicht in die Arbeit der anderen sowie das Lernen von anderen wertgeschätzt wurde. Die Partner scheinen mit der Zusammenarbeit zufrieden gewesen zu sein und schätzten auch die Vielfalt der beteiligten Partner. Diese Ergebnisse lassen darauf schließen, dass von Anfang an und bis zum Ende des Projektes eine gute Basis für die Zusammenarbeit und die gemeinsame Entwicklung von Inventaren vorhanden war. Das Interesse an den anderen und die Bereitschaft, das eigene Wissen und die eigenen Erfahrungen zu teilen bzw. gemeinsam zu erweitern, zeichnen sich in den Antworten der Partner ab. Die folgende Tabelle zeigt alle Antworten im Überblick: 


\begin{tabular}{|c|c|c|c|}
\hline \multicolumn{4}{|c|}{ What have you liked so far? } \\
\hline Nov 2004 n=12 & Mar 2005 n=12 & Jun 2005 n=10 & Nov $2005 n=9$ \\
\hline $\begin{array}{l}\text { Meeting the "range of } \\
\text { people" } 4 \times(33 \%)\end{array}$ & $\begin{array}{l}\text { Meeting different part- } \\
\text { ners, fruitful contacts } 6 x \\
(50 \%)\end{array}$ & $\begin{array}{l}\text { Meeting, contact } 5 x \\
(50 \%)\end{array}$ & $\begin{array}{l}\text { Meeting new partners, } \\
\text { contact } 3 \times(33 \%)\end{array}$ \\
\hline $\begin{array}{l}\text { Exchange of know how, } \\
\text { ideas and experiences } 5 x \\
(42 \%)\end{array}$ & $\begin{array}{l}\text { Exchange of know-how } \\
1 \times(8 \%)\end{array}$ & $\begin{array}{l}\text { Exchange of know-how } \\
\text { and experiences } 3 x \\
(30 \%)\end{array}$ & $\begin{array}{l}\text { Exchanging and inter- } \\
\text { changing } 2 \times(22 \%)\end{array}$ \\
\hline $\begin{array}{l}\text { Learning from others } 1 \mathrm{x} \\
(8 \%)\end{array}$ & $\begin{array}{l}\text { Learning from others } 2 x \\
(17 \%)\end{array}$ & $\begin{array}{l}\text { Learning, Increasing of } \\
\text { knowledge } 2 x(20 \%)\end{array}$ & Learning $1 \times(11 \%)$ \\
\hline $\begin{array}{l}\text { New ideas and influences } \\
\text { for the own project } 2 x \\
(17 \%)\end{array}$ & $\begin{array}{l}\text { Insight in other projects } \\
1 \times(8 \%)\end{array}$ & - & $\begin{array}{l}\text { Finding out what others } \\
\text { are doing, knowing about } \\
\text { experiences } 2 \times(22 \%)\end{array}$ \\
\hline Collaboration 3x (25\%) & Collaboration $1 \times(8 \%)$ & - & Collaboration 2x (22\%) \\
\hline $\begin{array}{l}\text { The group consists of } \\
\text { partners from different } \\
\text { countries } 1 \times(8 \%)\end{array}$ & $\begin{array}{l}\text { Variety/Different nature of } \\
\text { partners } 1 \times(8 \%)\end{array}$ & - & Diversity $1 \times(11 \%)$ \\
\hline- & Sharing of ideas $3 \times(25 \%)$ & - & Sharing $2 \times(22 \%)$ \\
\hline- & Concrete results $1 \times(8 \%)$ & - & Project results $1 \times(11 \%)$ \\
\hline- & $\begin{array}{l}\text { Thinking/Reflections } 1 \mathrm{x} \\
(8 \%)\end{array}$ & $\begin{array}{l}\text { Spreading new ideas } 1 \mathrm{x} \\
(10 \%)\end{array}$ & Stimulation $1 \times(11 \%)$ \\
\hline $\begin{array}{l}\text { Work of single partners } \\
2 x(17 \%)\end{array}$ & - & - & - \\
\hline Positive feedback 1x (8\%) & - & - & - \\
\hline ( & $\begin{array}{l}\text { Potenzial of ideas } 2 x \\
(17 \%)\end{array}$ & - & - \\
\hline- & $\begin{array}{l}\text { Transnational Meeting 2x } \\
(17 \%)\end{array}$ & - & - \\
\hline- & Perspectives 1x (8\%) & - & - \\
\hline- & - & $\begin{array}{l}\text { Integration of all partners } \\
1 \times(10 \%)\end{array}$ & - \\
\hline- & - & $\begin{array}{l}\text { Partners follow the same } \\
\text { objectives } 1 \times(10 \%)\end{array}$ & - \\
\hline- & - & $\begin{array}{l}\text { Project progression } 2 x \\
(20 \%)\end{array}$ & - \\
\hline- & - & $\begin{array}{l}\text { Processevaluation } 1 \mathrm{x} \\
(10 \%)\end{array}$ & - \\
\hline- & - & $\begin{array}{l}\text { Involvement in evaluation } \\
\text { process } 1 \times(10 \%)\end{array}$ & - \\
\hline- & - & $\begin{array}{l}\text { Clearer view from another } \\
1 \times(10 \%)\end{array}$ & - \\
\hline- & - & $\begin{array}{l}\text { Project management } 1 \mathrm{x} \\
(10 \%)\end{array}$ & - \\
\hline- & - & $\begin{array}{l}\text { Aspiration of the project } \\
1 \times(10 \%)\end{array}$ & - \\
\hline- & - & $\begin{array}{l}\text { Achievements to date } 1 \mathrm{x} \\
(10 \%)\end{array}$ & - \\
\hline
\end{tabular}

Tabelle 25: $\quad$ Ergebnisse der Prozessevaluation auf die Frage „What have you liked so far?“

Eine weitere Frage bei der Prozessevaluation war, was den Partnern im Projekt bisher nicht gefallen hat. Zu jedem Zeitpunkt wurde genannt, dass die Partner unterschiedliche Ziele hätten oder die Ziele und Rollen unklar seien. Außerdem wurde jedes Mal als negativ bemerkt, dass wenige Aktivitäten außerhalb der transnationalen Treffen stattfänden, wenig Interaktion und Beteiligung bzw. Kommunikation in der Gruppe vorhanden sei. Es wurde auch erwähnt, dass Zeit verschwendet bzw. vergeudet werde, z.B. durch Wiederholungen. Die Webseite wurde ebenfalls bemängelt und zwar zu jedem Erhebungszeitpunkt. Im ersten Projektjahr wurde auch die Arbeit einzelner Partner bzw. deren mangelnde Erfahrung als negativ bewertet bzw. dass der Aspekt der Zusammenarbeit eher im Hintergrund stehe. Nach dem ersten Projektjahr schien einigen Partnern zu viel diskutiert zu werden (besonders auf theoreti- 
schem Niveau) und es schien schwierig zu sein, zu Ergebnissen zu kommen bzw. Ergebnisse zu nutzen. Außerdem wurde eine Lücke zwischen Theorie und Praxis genannt und das Vorgehen der Partner als wenig synchron erlebt. Einige Partner beteiligten sich zu wenig in den Augen der anderen.

Insgesamt wird deutlich, dass es auch mit Schwierigkeiten verbunden ist, in einer so heterogenen Gruppe zusammenzuarbeiten und gemeinsame Ziele zu verfolgen. Einige Partner schienen sich mehr zu beteiligen als andere, was zu Unzufriedenheit führte. Außerdem wurde nach Meinung einiger Partner zu viel diskutiert bzw. zu viel auf theoretischem Niveau besprochen, wobei konkrete Ergebnisse fehlten oder ausblieben. Die Ziele, Bedarfe und Intentionen schienen verschieden zu sein, was eine intensive Beteiligung aller und die Erfüllung der anstehenden Aufgaben erschwerte oder unmöglich machte. Entwicklungen dauerten nach Auffassung einiger Partner lange und es ginge dabei viel Zeit verloren. Technische Probleme bzw. die Webseite sorgten ebenfalls für Unmut. In den Äußerungen der Partner spiegelte sich, dass die Entwicklung der Inventare in der Gruppe bzw. in den Kleingruppen keinesfalls eine leichte Aufgabe war und dass wohl auch die Beteiligung einiger Partner zu wünschen übrig ließ. Die folgende Tabelle zeigt alle Antworten in der Übersicht: 


\begin{tabular}{|c|c|c|c|}
\hline \multicolumn{4}{|c|}{ What have you disliked so far? } \\
\hline Nov $2004 n=12$ & Mar $2005 \mathrm{n=12}$ & Jun $2005 \mathrm{n}=10$ & Nov $2005 \mathrm{n}=9$ \\
\hline Different aims 1x (8\%) & $\begin{array}{l}\text { Different needs, aims and } \\
\text { intentions } 3(25 \%)\end{array}$ & No clear aim $1 \times(10 \%)$ & $\begin{array}{l}\text { No clear targets in some } \\
\text { workgroups } 1 \times(11 \%)\end{array}$ \\
\hline $\begin{array}{l}\text { Confusion about purpos- } \\
\text { es / different aims } 1 \mathrm{x} \\
(8 \%)\end{array}$ & & & $\begin{array}{l}\text { Unclarity of purpose, roles } \\
\text { and commitment } 1 \times(11 \%)\end{array}$ \\
\hline \multirow{2}{*}{$\begin{array}{l}\text { Less interactivity, no/poor } \\
\text { communication, no con- } \\
\text { tact (with the group) } 6 \mathrm{x} \\
(50 \%)\end{array}$} & $\begin{array}{l}\text { Not much interaction } 1 \mathrm{x} \\
(8 \%)\end{array}$ & $\begin{array}{l}\text { Activity only during the } \\
\text { meetings } 2 x(20 \%)\end{array}$ & $\begin{array}{l}\text { Non-fulfilment of tasks, } \\
\text { missing commitment } 2 x \\
(22 \%)\end{array}$ \\
\hline & Lost of time $2(17 \%)$ & $\begin{array}{l}\text { Lost of time and repeti- } \\
\text { tions } 3 \times(30 \%)\end{array}$ & Waste of time $1 \times(11 \%)$ \\
\hline \multirow[t]{2}{*}{$\begin{array}{l}\text { Technical problems } 1 x \\
(8 \%)\end{array}$} & Blinc website $2(17 \%)$ & The website1x (10\%) & $\begin{array}{l}\text { Use of Blinc-website } \\
\text { (technical problems) } 1 \mathrm{x} \\
(11 \%)\end{array}$ \\
\hline & & $\begin{array}{l}\text { Some partners respond } \\
\text { very slowly } 1 \times(10 \%)\end{array}$ & $\begin{array}{l}\text { Very slow development } \\
\text { processes } 1 \times(11 \%)\end{array}$ \\
\hline \multirow{3}{*}{$\begin{array}{l}\text { Work of single partners } \\
\text { (less contribution and few } \\
\text { experiences of single } \\
\text { partners) } 3 \times(25 \%)\end{array}$} & $\begin{array}{l}\text { The collaborative aspect } \\
\text { stands back } 1 \times(8 \%)\end{array}$ & & \\
\hline & $\begin{array}{l}\text { Difficulties to use outputs } \\
1 \times(8 \%)\end{array}$ & & $\begin{array}{l}\text { Difficulty to reach concrete } \\
\text { results, results are limited } \\
2 \times(22 \%)\end{array}$ \\
\hline & No synchronism 1x (8\%) & & $\begin{array}{l}\text { Poor synchronism 1x } \\
(11 \%)\end{array}$ \\
\hline \multicolumn{4}{|l|}{$\begin{array}{l}\text { No way to handle differ- } \\
\text { ent aims } 1 \times(8 \%)\end{array}$} \\
\hline \multicolumn{4}{|l|}{$\begin{array}{l}\text { Language problems } 1 \mathrm{x} \\
(8 \%)\end{array}$} \\
\hline \multicolumn{4}{|l|}{$\begin{array}{l}\text { Lack of information } 1 \mathrm{x} \\
(8 \%)\end{array}$} \\
\hline & $\begin{array}{l}\text { Theory and discussions } \\
4 \times(33 \%)\end{array}$ & & \\
\hline & $\begin{array}{l}\text { Gap between theory and } \\
\text { practice } 1 \times(8 \%)\end{array}$ & & \\
\hline & $\begin{array}{l}\text { Difficulties to reach } \\
\text { agreements } 1 \times(8 \%)\end{array}$ & & \\
\hline & $\begin{array}{l}\text { Complaining instead of } \\
\text { contribution } 1 \times(8 \%)\end{array}$ & & \\
\hline & Poor output $2 \times(17 \%)$ & & \\
\hline & $\begin{array}{l}\text { No whole group commu- } \\
\text { nication } 1 \times(8 \%)\end{array}$ & & \\
\hline & & & $\begin{array}{l}\text { No deeper insight } 1 \mathrm{x} \\
(11 \%)\end{array}$ \\
\hline
\end{tabular}

Tabelle 26: $\quad$ Ergebnisse der Prozessevaluation auf die Frage „What have you disliked so far?“

Eine weitere Frage bei der Prozessevaluation war, was nach Meinung der Partner verbesserungswürdig sei. Zu fast jedem Erhebungszeitpunkt wurde die Art bzw. die Organisation der Diskussion genannt. Insbesondere bei der ersten Erhebung, gegen Ende des erstens Projektjahres, waren 58\% der Partner der Meinung, dass die Kommunikation häufiger und regelmäßiger sein müsste und die Zusammenarbeit verbessert werden sollte. Außerdem sind die Partner der Meinung, dass sich alle bzw. einige Partner mehr beteiligen und einbringen müssten. Verbessert werden müsste nach den Äußerungen der Partner auch die Klarheit der Aufgaben, aber auch die Eindeutigkeit einzelner Partner, sich beteiligen zu wollen. All dies werde nach den Antworten der Partner zu einer Verbesserung der Zusammenarbeit im Projekt beitragen. Die Webseite wurde fast jedes Mal als verbesserungswürdig genannt, und auch die Kompetenz der Partner, Typo 3 zu benutzen (ein Contenmanagementsystem, mit 
dem die Partner Inhalte auf der Webseite selbst gestalten und einbringen konnten), müsste nach Meinung der Partner weiter ausgebaut bzw. verbessert werden. Bei den letzten zwei Erhebungen wurden auch die Aspekte genannt, dass die Verbreitung von Materialien, mehr Aktivitäten auf der blinc-Webseite und der Transfer der theoretischen Ansätze in die alltägliche Praxis erfolgen sollten.

Insgesamt zeigt sich, dass vor allem die Kommunikation, Interaktion und Partizipation der Partner - zumindest einiger Partner - durchweg verbesserungswürdig war. Außerdem war es ein Anliegen der Partner, dass die Webseite optimiert würde und auch die Partner kompetenter würden, die Inhalte selbst zu gestalten. Die folgende Tabelle zeigt alle genannten Aspekte der vier Erhebungszeitpunkte: 


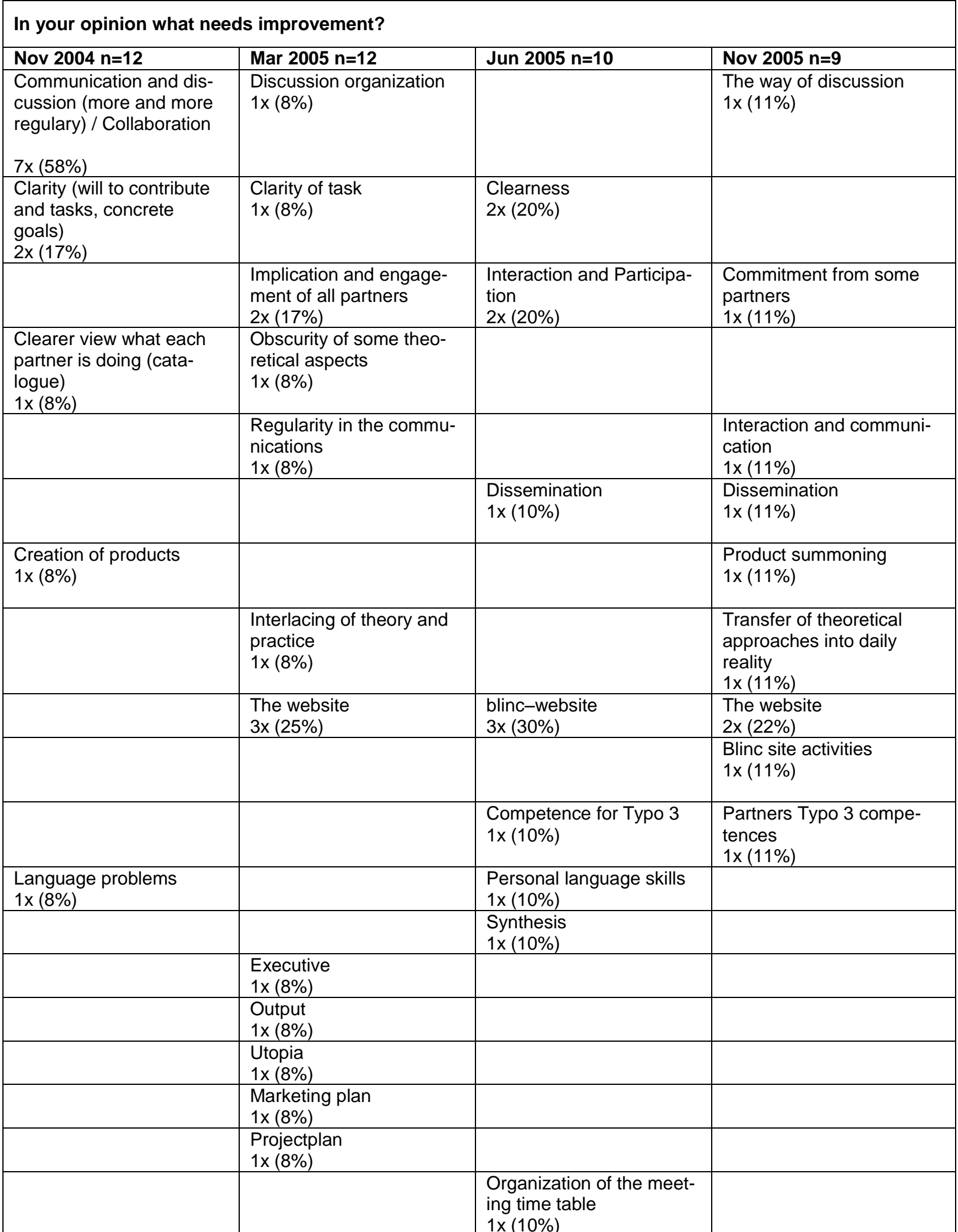

Tabelle 27: Ergebnisse der Prozessevaluation auf die Frage „In your opinion what needs improvement?"

Auf die Frage, welche positiven Aspekte das eL3-Netzwerk für die Partner habe, wurden sehr unterschiedliche Aspekte genannt. Dies zeigt, dass die Interessen und Bedarfe ebenfalls sehr verschieden waren - die einen fanden es positiv, neue Sichtweisen kennen zu lernen und neues Wissen zu erarbeiten bzw. ihr Wissen zu erweitern, die anderen fanden es wertvoll, sich auszutauschen und Ideen zu teilen, und andere wiederum fanden einfach den 
Kontakt zu unterschiedlichen Partnern gut. Manche erwähnten auch positive Rückmeldungen der Europäischen Kommission bzw. einer Grundtvig Agentur (also positive Rückmeldungen „Von außen“). Für einige Partner war auch das Netzwerken (vor allem der Austausch von Erfahrungen und das Entwickeln von Ideen) ein positiver Effekt des Projektes eL3.

Bemerkenswert im Hinblick auf die Inventarentwicklung ist eine Antwort bei dem dritten Erhebungszeitpunkt im Juni 2005 (also nach zwei Dritteln der Projektlaufzeit): Ein einziger Partner erwähnte, dass der Evaluationsprozess in der eigenen Institution angeregt wurde. Man kann daraus schließen, dass die Arbeit an den Inventaren die Evaluationstätigkeiten angeregt oder bereichert hat - allerdings nur bei einer von 10 Personen. Ansonsten wurden weder die Inventare noch die Evaluationspraxis erwähnt, woraus abzulesen ist, dass sie von den meisten Partnern nicht als besonders hilfreich oder bemerkenswert erlebt wurden (tatsächlich hatten die meisten Partner kein besonders großes Interesse an den Inventaren die Partner mit theoretischem bzw. wissenschaftlichem Hintergrund waren vor allem an den Wissensinhalten und theoretischen Hintergründen interessiert, und die „Praktiker“ wollten vor allem „schnell praktikable Instrumente haben, die nicht aufwendig sind“). Die folgende Tabelle zeigt alle genannten Aspekte: 


\begin{tabular}{|c|c|c|c|}
\hline \multicolumn{4}{|c|}{ What positive effects has the eL3 network for you until now? } \\
\hline $\begin{array}{l}\text { Contact with others } \\
2 \times(17 \%)\end{array}$ & $\begin{array}{l}\text { New points of view } \\
2 x(17 \%)\end{array}$ & $\begin{array}{l}\text { Improvement of our vision } \\
1 \times(10 \%)\end{array}$ & $\begin{array}{l}\text { Increasing knowledge (about } \\
\text { what is going on in Europe } \\
\text { /about different approaches, } \\
\text { new experiences/ideas) } 4 x \\
(44 \%)\end{array}$ \\
\hline $\begin{array}{l}\text { Acquiring knowledge (see } \\
\text { and understand what others } \\
\text { are doing, "Wide Vision of } \\
\text { opportunities") } \\
4 \times(33 \%)\end{array}$ & $\begin{array}{l}\text { Ideas about evalaution } \\
2 \times(17 \%)\end{array}$ & $\begin{array}{l}\text { Blinc is gaining speed } \\
1 \times(10 \%)\end{array}$ & $\begin{array}{l}\text { Interchanging of know-how } \\
\text { and experiences } \\
1 \times(11 \%)\end{array}$ \\
\hline $\begin{array}{l}\text { Extension of knowledge } \\
\text { (activation, thinking, new } \\
\text { ideas and perspectives) } \\
5 \times(42 \%)\end{array}$ & $\begin{array}{l}\text { Insight } \\
1 \times(8 \%)\end{array}$ & $\begin{array}{l}\text { Positive feedback from } \\
\text { Grundtvig National Agency } \\
1 \times(10 \%)\end{array}$ & $\begin{array}{l}\text { Identification of services for } \\
\text { own institution } \\
1 \times(11 \%)\end{array}$ \\
\hline $\begin{array}{l}\text { International collaboration, } \\
\text { cooperative working } \\
2 \times(17 \%)\end{array}$ & $\begin{array}{l}\text { Increase and enrichment of } \\
\text { knowledge and practical } \\
\text { experience } \\
3 \times(25 \%)\end{array}$ & $\begin{array}{l}\text { Interaction } \\
1 \times(10 \%)\end{array}$ & $\begin{array}{l}\text { Networking, Network of con- } \\
\text { tacts, new transnational part- } \\
\text { ners } \\
3 \times(33 \%)\end{array}$ \\
\hline $\begin{array}{l}\text { Positive feedback from the } \\
\text { European Commission } \\
1 \times(8 \%)\end{array}$ & $\begin{array}{l}\text { Learning } \\
2 \times(17 \%)\end{array}$ & $\begin{array}{l}\text { Increasing knowledge } \\
3 \times(30 \%)\end{array}$ & $\begin{array}{l}\text { Sharing of ideas } \\
1 \times(11 \%)\end{array}$ \\
\hline \multirow[t]{14}{*}{$\begin{array}{l}\text { Involvement of colleagues } \\
1 \times(8 \%)\end{array}$} & $\begin{array}{l}\text { Confrontation and collabora- } \\
\text { tion with different partners } \\
3 \times(25 \%)\end{array}$ & $\begin{array}{l}\text { Stimulation of evaluation } \\
\text { process in the own institu- } \\
\text { tion } \\
1 \times(10 \%)\end{array}$ & $\begin{array}{l}\text { Confrontation with different } \\
\text { approaches, productions and } \\
\text { uses } \\
1 \times(11 \%)\end{array}$ \\
\hline & $\begin{array}{l}\text { New projects } \\
1 \times(8 \%)\end{array}$ & $\begin{array}{l}\text { Learning about partners } \\
1 \times(10 \%)\end{array}$ & $\begin{array}{l}\text { Quality of exchanges } \\
1 \times(11 \%)\end{array}$ \\
\hline & $\begin{array}{l}\text { Stimulating curiosity } \\
1 \times(8 \%)\end{array}$ & $\begin{array}{l}\text { Interesting cooperative } \\
\text { platform } \\
1 \times(10 \%)\end{array}$ & $\begin{array}{l}\text { Visit of an eL3-partner } \\
1 \times(11 \%)\end{array}$ \\
\hline & $\begin{array}{l}\text { Sharing the concept of eL3 } \\
1 \times(8 \%)\end{array}$ & $\begin{array}{l}\text { Idea of a virtual community } \\
1 \times(10 \%)\end{array}$ & $\begin{array}{l}\text { In-depth analysis of network- } \\
\text { ing } \\
1 \times(11 \%)\end{array}$ \\
\hline & $\begin{array}{l}\text { Building a network of con- } \\
\text { tacts } \\
1 \times(8 \%)\end{array}$ & $\begin{array}{l}\text { The e-learning library is } \\
\text { taking shape } \\
1 \times(10 \%)\end{array}$ & $\begin{array}{l}\text { New project proposals and } \\
\text { one granted project } \\
1 \times(11 \%)\end{array}$ \\
\hline & $\begin{array}{l}\text { Dissemination } \\
1 \times(8 \%)\end{array}$ & $\begin{array}{l}\text { Insight in/Overview about } \\
\text { different approaches } \\
\text { and products for learners in } \\
\text { Europe } \\
1 \times(10 \%)\end{array}$ & $\begin{array}{l}\text { Backing and encouragement } \\
\text { from the EU-Commission } \\
1 \times(11 \%)\end{array}$ \\
\hline & $\begin{array}{l}\text { Meeting of interesting people } \\
\text { with fresh ideas } \\
1 \times(8 \%)\end{array}$ & $\begin{array}{l}\text { Validation of own e-learning } \\
\text { solutions } \\
1 \times(10 \%)\end{array}$ & \\
\hline & & $\begin{array}{l}\text { New software to upgrade e- } \\
\text { learning } \\
1 \times(10 \%)\end{array}$ & \\
\hline & & $\begin{array}{l}\text { New input in English lan- } \\
\text { guage } \\
1 \times(10 \%)\end{array}$ & \\
\hline & & $\begin{array}{l}\text { Acquirement of new ways to } \\
\text { work } \\
1 \times(10 \%)\end{array}$ & \\
\hline & & $\begin{array}{l}\text { Good international links } \\
1 \times(10 \%)\end{array}$ & \\
\hline & & $\begin{array}{l}\text { Thinking about collaborative } \\
\text { working } \\
1 \times(10 \%)\end{array}$ & \\
\hline & & $\begin{array}{l}\text { Finding ways of evaluating } \\
\text { blended learning } \\
1 \times(10 \%)\end{array}$ & \\
\hline & & $\begin{array}{l}\text { In-depth analysis of network- } \\
\text { ing } \\
1 \times(10 \%)\end{array}$ & \\
\hline
\end{tabular}

Tabelle 28: $\quad$ Ergebnisse der Prozessevaluation auf die Frage „What positive effects has the eL3 network for you until now?"

Ein anderes Bild im Hinblick auf die Inventarentwicklung zeigt sich bei der Frage, ob die Partner neue Perspektiven oder Impulse bekommen hätten, die sie für ihre eigenen Bildungsprojekte nutzen konnten. Während der gesamten Projektlaufzeit antworteten etwa 60 
bis $90 \%$ der Partner mit „Ja“, und keiner der Partner gab zum Ende des Projektes an, keine Perspektiven oder Impulse durch das Projekt eL3 bekommen zu haben (siehe nachfolgende Tabelle).

\section{Do you have any perspectives or a fresh impetus which you can use or realize for your own project?}

\begin{tabular}{|l|c|c|c|}
\hline Feedback & Yes & No & No data \\
\hline Nov $2004 n=12$ & $7(58 \%)$ & $4(33 \%)$ & $1(8 \%)$ \\
\hline Mar $2005 n=12$ & $9(75 \%)$ & $2(17 \%)$ & - \\
\hline June $2005 n=10$ & $7(70 \%)$ & $3(30 \%)$ & $1(11 \%)$ \\
\hline Nov $2005 n=9$ & $8(88 \%)$ & - & $(8 \%)$ \\
\hline
\end{tabular}

Tabelle 29: $\quad$ Ergebnisse der Prozessevaluation auf die Frage „Do you have any perspectives or a fresh impetus which you can use or realize for your own project?”

Bei der Konkretisierung der Perspektiven und Impulse, die die Partner bisher im Projekt eL3 bekommen hatten, nannten bei dem ersten Erhebungszeitpunkt etwa 30\% die Fragebögen (Inventare), die Ideen und Reflexionen dazu. Bei dem dritten Erhebungszeitpunkt, also Mitte des zweiten Projektjahres, nannten 30\% direkt die Fragebögen, die sie zur Evaluation ihrer eigenen Projekte auf der Basis der Inventare benutzten, sowie die Evaluationsansätze, pädagogische Techniken (hierbei war die Art der Reflexion gemeint, wie sich bei der Ergebnispräsentation und der Gruppendiskussion herausstellte) und die Evaluation der eigenen Plattform mit den Inventaren. Zum Ende des Projektes gaben wiederum etwa 30\% der Partner an, dass sie die Evaluationsinstrumente (also die Inventare) als neue Impulse für ihre Arbeit und ihre eigenen Bildungsprojekte erlebten. Außerdem wurde die kritische Reflexion über die eigenen Blended Learning-Angebote sowie die Bewusstheit der Bedeutsamkeit von Evaluation und die Evaluation der eigenen Plattform genannt, was ebenfalls im direkten Zusammenhang mit der Entwicklung und Erprobung der Inventare gesehen werden kann.

Insgesamt zeigt sich bei den Antworten der Partner, dass die Inventare und dadurch angeregte Diskussionen und Denkprozesse als neue und bemerkenswerte Impulse erlebt wurden. Es spiegeln sich dabei zwei Aspekte: Zum einen gaben die Partner an, dass sie die Fragebögen bzw. Inventare praktisch nutzten, und zum anderen, dass sie über ihre Blended Learning-Angebote kritisch nachdenken würden und eine Bewusstheit entstünde, dass Evaluation wichtig und nützlich ist. Bei den Gruppendiskussionen während der transnationalen Treffen zeigte sich auch, dass Evaluation für die meisten Partner eher ein Kontrollinstrument oder ein notwendiges Übel war (z.B. als lästige Pflicht dem Mittelgeber gegenüber gesehen wurde), und nicht ein Weg der Erkenntniserweiterung oder Optimierung der eigenen Angebote. Nach den Rückmeldungen der Partner scheinen die gemeinsame Arbeit an den Inventaren sowie die theoretischen Hintergründe, die vorgestellt wurden, etwas verändert und in Bewegung gesetzt zu haben - zumindest bei einigen der Partner. Die folgende Tabelle zeigt alle Antworten in der Übersicht: 
If yes, please describe in short?

\begin{tabular}{|c|c|c|c|}
\hline Nov 2004 n=12 & Mar $2005 \mathrm{n}=12$ & Jun $2005 n=10$ & Nov $2005 \mathrm{n}=9$ \\
\hline $\begin{array}{l}\text { a) Questionnaires / } \\
\text { tools (ideas, reflections) } \\
4 \times(33 \%)\end{array}$ & $\begin{array}{l}\text { a) Growing the elearning } \\
\text { process } \\
1(8 \%)\end{array}$ & $\begin{array}{l}\text { a) To get on } \\
1 \times(10 \%)\end{array}$ & a) Networking \\
\hline $\begin{array}{l}\text { b) New project proposals } \\
1 \times(8 \%)\end{array}$ & $\begin{array}{l}\text { b) Ideas for collaboration } \\
\text { and own work } \\
2(17 \%)\end{array}$ & $\begin{array}{l}\text { b) Using questionnaires } \\
3 \times(30 \%)\end{array}$ & $\begin{array}{l}\text { b) Sharing of prices } \\
1 \times(11 \%)\end{array}$ \\
\hline $\begin{array}{l}\text { c) Integration of new } \\
\text { partners } \\
1 \times(8 \%)\end{array}$ & $\begin{array}{l}\text { c) Clarification of own } \\
\text { position } \\
1(8 \%)\end{array}$ & $\begin{array}{l}\text { c) Ideas for new courses } \\
\text { and services } \\
1 \times(10 \%)\end{array}$ & $\begin{array}{l}\text { c) Evaluation instru- } \\
\text { ments } \\
3 \times(33 \%)\end{array}$ \\
\hline \multirow[t]{8}{*}{$\begin{array}{l}\text { d) Potenzial ideas } \\
1 \times(8 \%)\end{array}$} & $\begin{array}{l}\text { d) Evaluation theory } \\
3(25 \%)\end{array}$ & $\begin{array}{l}\text { d) Evaluation ap- } \\
\text { proaches } \\
1 \times(10 \%)\end{array}$ & $\begin{array}{l}\text { d) New techniques } \\
1 \times(11 \%)\end{array}$ \\
\hline & $\begin{array}{l}\text { e) New projects } \\
1(8 \%)\end{array}$ & $\begin{array}{l}\text { e) Networking } \\
1 \times(10 \%)\end{array}$ & $\begin{array}{l}\text { e) Critical reflections } \\
\text { about blended learning } \\
1 \times(11 \%)\end{array}$ \\
\hline & $\begin{array}{l}\text { f) Collaborative links for } \\
\text { future projects } \\
1(8 \%)\end{array}$ & $\begin{array}{l}\text { f) Pedagogic techniques } \\
1 \times(10 \%)\end{array}$ & $\begin{array}{l}\text { f) Awareness about the } \\
\text { importance of evaluation } \\
1 \times(11 \%)\end{array}$ \\
\hline & $\begin{array}{l}\text { g) Dissemination } \\
1(8 \%)\end{array}$ & $\begin{array}{l}\text { g) Evaluation of own } \\
\text { platform } \\
1 \times(10 \%)\end{array}$ & $\begin{array}{l}\text { g) Idea to visit other part- } \\
\text { ners } \\
1 \times(11 \%)\end{array}$ \\
\hline & & $\begin{array}{l}\text { h) Implementation of own } \\
\text { platform } \\
1 \times(10 \%)\end{array}$ & $\begin{array}{l}\text { h) Evaluation and im- } \\
\text { plementation of platform } \\
1 \times(11 \%)\end{array}$ \\
\hline & & & $\begin{array}{l}\text { i) New areas of work } \\
1 \times(11 \%)\end{array}$ \\
\hline & & & $\begin{array}{l}\text { j) New work relations with } \\
\text { Universities } \\
1 \times(11 \%)\end{array}$ \\
\hline & & & $\begin{array}{l}\text { k) New part time job } \\
1 \times(11 \%)\end{array}$ \\
\hline & & & $\begin{array}{l}\text { I) Integration of final bene- } \\
\text { ficiaries under the roof of } \\
\text { blinc } \\
1 \times(11 \%)\end{array}$ \\
\hline
\end{tabular}

Tabelle 30: $\quad$ Ergebnisse der Prozessevaluation auf die Frage „Do you have any perspectives or a fresh impetus which you can use or realize for your own project? - If yes, please describe in short?"

Eine weitere Frage der Prozessevaluation war, ob die Partner mehr Informationen haben wollten (z.B. wenn etwas unklar sein sollte). Zu Beginn des Projektes war der Informationsbedarf sehr hoch (83\%), aber auch gegen Ende des Projektes zeigte sich, dass immer noch Informationsbedarfe bestanden (44\%). Die folgende Tabelle zeigt alle Ergebnisse im Überblick:

\begin{tabular}{|l|c|c|c|}
\hline \multicolumn{2}{|l|}{ Do you want to have any more information? } & No & No data \\
\hline Feedback & Yes & $1(8 \%)$ & - \\
\hline Nov $2004 \mathrm{n}=12$ & $10(83 \%)$ & $7(58 \%)$ & - \\
\hline Mar $2005 \mathrm{n}=12$ & $5(42 \%)$ & $6(60 \%)$ & $1(11 \%)$ \\
\hline June $2005 \mathrm{n}=10$ & $4(40 \%)$ & $4(44 \%)$ & \\
\hline Nov $2005 \mathrm{n}=9$ & $4(44 \%)$ & & \\
\hline
\end{tabular}

Tabelle 31: $\quad$ Ergebnisse der Prozessevaluation auf die Frage "Do you want to have any more information?" 
Bei der Konkretisierung wird deutlich, dass die Informationsbedarfe der Partner sehr verschieden waren und sich auch über die Projektlaufzeit veränderten. Zu Beginn ging es vor allem darum zu erfahren, was die einzelnen Partner genau taten, was für Kurse sie im Detail anboten und welche Erfahrungen sie hatten. Außerdem bestand der Bedarf nach Klärung der Ziele beteiligter Partner sowie nach der Ermittlung der Bereitschaft, sich zu beteiligen. Nach dem ersten Projektjahr ging es eher darum zu erfahren, wie sich die verschiedenen Ansätze verbinden ließen, wie erarbeitete Wissensinhalte in die Praxis transferiert werden könnten, wie die Zusammenarbeit gelingen könnte, und was zum Projekterfolg beitragen könnte. Außerdem bestand der Bedarf nach mehr Transparenz darüber, was die einzelnen Partner taten. Bei dem dritten Erhebungszeitpunkt hatten die Partner Bedarf nach Klärung oder Informationen zur weiteren Entwicklung des Projektes bzw. von Projekten über eL3 hinaus, und es bestand der Wunsch, die vorhandenen (erarbeiteten) Produkte zu typisieren. Außerdem war ein Informationsbedarf vorhanden zur Intention der Partner, Ergebnisse miteinander zu teilen. Zum letzten Erhebungszeitpunkt, gegen Ende des Projektes, wollten die Partner mehr Einblick in die neuesten Entwicklungen und Produkte haben und waren interessiert am Wissen und den Erfahrungen der anderen Partner. Im Hinblick auf die Entwicklung und Erprobung der Inventare zeigte sich nur zum Ende des Projektes eine Frage, und zwar danach, wie die Partner die Fragebögen (Inventare) konkret nutzten. Die folgende Tabelle zeigt alle Antworten im Überblick:

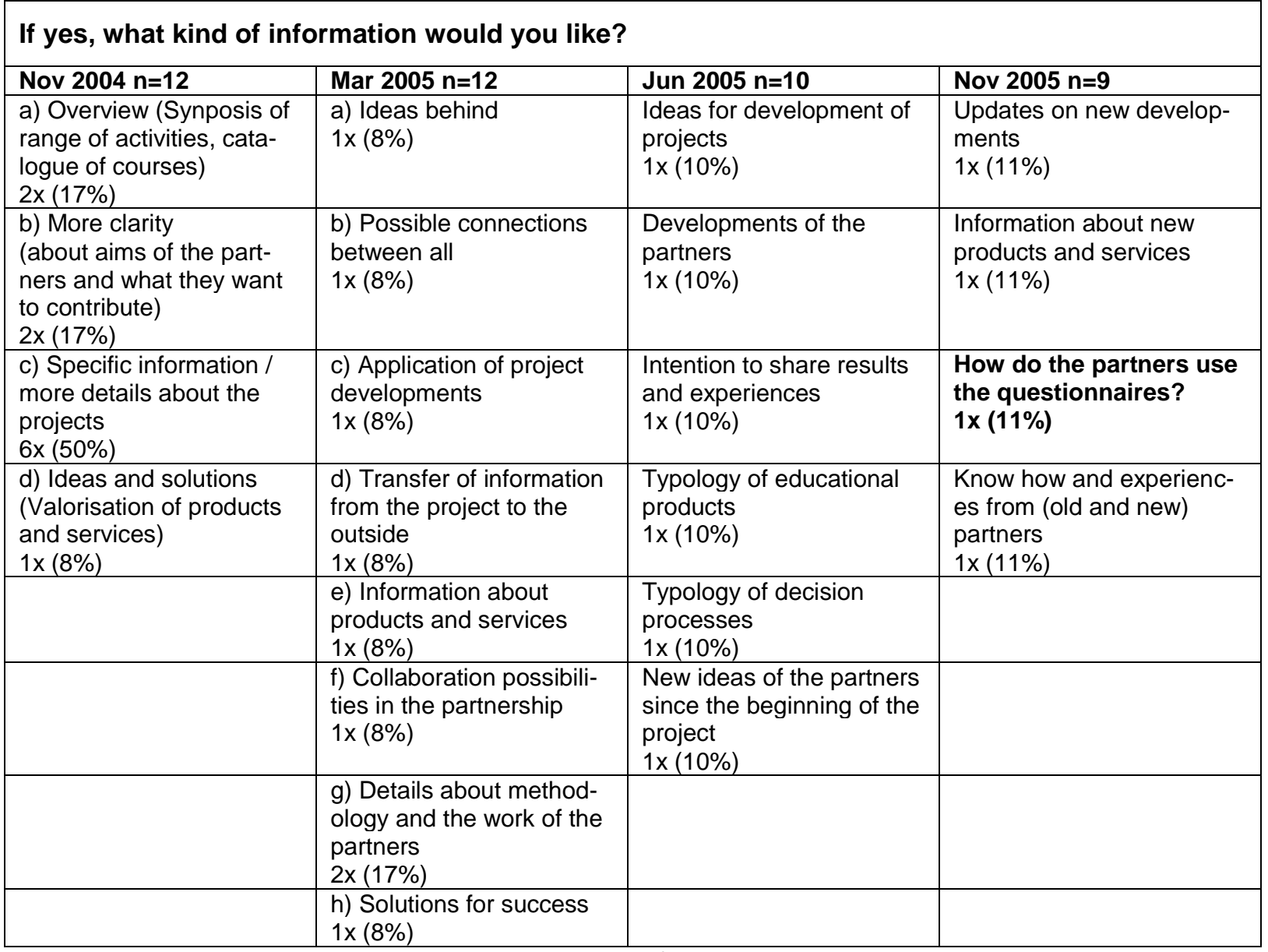

Tabelle 32: Ergebnisse der Prozessevaluation auf die Frage „Do you want to have any more information? - If yes, what kind of information would you like?" 
Bei der Frage nach den Erwartungen der Partner für die weitere Arbeit im gemeinsamen Netzwerk zeigt sich, dass diese sehr verschieden waren und sich außerdem über den Projektverlauf veränderten. Bei dem ersten Erhebungszeitpunkt wird deutlich, dass ein Teil der Partner an gemeinsamer Forschungsarbeit und einer größeren wissenschaftlichen Gemeinschaft Interesse hatte, während andere Partner Produkte (z.B. Kurse) verbreiten und Erfahrungen „zu Geld machen“ wollten. Andere Aspekte, die zu diesem Zeitpunkt genannt wurden, beziehen sich vor allem auf die Zusammenarbeit innerhalb des Projektes. So bestand der Wunsch nach mehr Intensität bei dem Austausch, mehr Klarheit und Beteiligung sowie nach der konsequenten Nutzung der Plattform.

Zum zweiten Erhebungszeitpunkt äußerten die Partner vor allem den Wunsch, Dokumente und Produkte zu erarbeiten, angefangene Arbeiten weiter zu bringen und dass sich alle Partner beteiligen und zusammen arbeiten bzw. stärker engagieren und besser kommunizieren. Außerdem wurde gewünscht, dass das Netzwerk erweitert werde und neue Partner hinzukämen.

Bei dem dritten Erhebungszeitpunkt, also nach zwei Dritteln der Projektlaufzeit, ging es den Partnern vor allem darum, die Projektziele zu erreichen bzw. das Projekt in die nächste Phase zu bringen, die blinc-Idee (ein gemeinsames Netzwerk) weiter voranzutreiben - was auch Marketingaspekte beinhaltete - zusammen zu arbeiten und den Austausch über e-LearningMethodologie aufrecht zu erhalten. Die Partner wollten das Netzwerk erweitern und die Ergebnisse der bisherigen Arbeit sollten in der Praxis nützlich sein. Für die Zukunft wünschten sie sich, „in Kontakt zu bleiben“.

Zum Ende des Projektes (bei dem vierten Erhebungszeitpunkt) erwarteten die Partner für die Zukunft vor allem die Verbreitung von Materialien und Ergebnissen, eine Erweiterung des Netzwerkes und das Wachstum der Gemeinschaft. Sie bemerkten, dass die Entwicklung der Webseite erforderlich sei und wünschten sich Interaktionen zwischen allen Partnern und dass Materialien und Erfahrungen weiterhin geteilt würden. Die Zusammenarbeit solle fortgesetzt werden - auch über die Projektlaufzeit hinaus.

Im Hinblick auf die Inventarentwicklung zeigte sich zu allen Erhebungszeitpunkten, dass die Partner Interesse an der Zusammenarbeit und Weiterentwicklung der angefangenen Arbeiten hatten. Es zeigte sich auch, dass die Interessen der Partner verschieden waren - einige waren an den Inventaren auch bzw. vor allem aus wissenschaftlicher und theoretischer Perspektive interessiert, und andere sahen vor allem den praktischen Nutzen bzw. die Verbreitung und Vermarktung im Vordergrund. Die unterschiedlichen Ziele und Intentionen der beteiligten Partner zeigten sich bereits bei anderen Fragen der Prozessevaluation (s.o.). Die Unterschiedlichkeit der Partner und die verschiedenen Interessen und Ziele waren zum einen eine Stärke bei der Zusammenarbeit - im Sinne der Vielfältigkeit - und andererseits eine Schwäche - im Sinne des gemeinsamen Vorwärtskommens. Im Hinblick auf die Erprobungsphase zeigte sich zum letzten Erhebungszeitpunkt, dass eine weitere Zusammenarbeit und Fortsetzung der Arbeit erwartet wurde und auch ein Bericht über den Einsatz der erar- 
beiteten Instrumente (Inventare) bzw. deren Evaluation erfolgen sollte. Die folgende Tabelle zeigt alle Antworten in der Übersicht:

\begin{tabular}{|c|c|c|c|}
\hline Nov $2004 \mathrm{n}=12$ & Mar $2005 \mathrm{n}=12$ & Jun 2005 n=10 & Nov $2005 n=9$ \\
\hline $\begin{array}{l}\text { Collaborative research, } \\
\text { create a large scientific } \\
\text { community } \\
2 x(17 \%)\end{array}$ & $\begin{array}{l}\text { Documents and products } \\
3 \times(25 \%)\end{array}$ & $\begin{array}{l}\text { Achievement of goal } \\
1 \times(10 \%)\end{array}$ & $\begin{array}{l}\text { Dissemination, enlarge- } \\
\text { ment, growth } \\
3 \times(33 \%)\end{array}$ \\
\hline $\begin{array}{l}\text { More clarity and sharing } \\
1 \times(8 \%)\end{array}$ & $\begin{array}{l}\text { Further development } \\
2 \times(17 \%)\end{array}$ & $\begin{array}{l}\text { Progression and enrich- } \\
\text { ment of blinc } \\
2 \times(20 \%)\end{array}$ & $\begin{array}{l}\text { Interaction with all partners } \\
1 \times(11 \%)\end{array}$ \\
\hline $\begin{array}{l}\text { More intensity in the ex- } \\
\text { changes } \\
1 \times(8 \%)\end{array}$ & $\begin{array}{l}\text { Implication and contribu- } \\
\text { tion of all partners } \\
3 \times(25 \%)\end{array}$ & $\begin{array}{l}\text { Collaborations } \\
2 \times(20 \%)\end{array}$ & $\begin{array}{l}\text { Development of the web- } \\
\text { site } \\
1 \times(11 \%)\end{array}$ \\
\hline $\begin{array}{l}\text { Distribute some prod- } \\
\text { ucts/coursers, capitalising } \\
\text { on the experience } \\
2 x(17 \%)\end{array}$ & $\begin{array}{l}\text { Collaboration } \\
3 \times(25 \%)\end{array}$ & $\begin{array}{l}\text { Maintenance of exchang- } \\
\text { es and brainstorming on } \\
\text { e-learning methodology } \\
1 \times(10 \%)\end{array}$ & $\begin{array}{l}\text { Further Collaborations, } \\
\text { Continuation of collabo- } \\
\text { rations, and the work } 3 x \\
(33 \%)\end{array}$ \\
\hline $\begin{array}{l}\text { Suggestions for the own } \\
\text { project } \\
1 \times(8 \%)\end{array}$ & $\begin{array}{l}\text { Exchanging of know-how } \\
1 \times(8 \%)\end{array}$ & $\begin{array}{l}\text { Networking } \\
2 x(20 \%)\end{array}$ & $\begin{array}{l}\text { Sharing and developing of } \\
\text { material with eL3-partners } \\
1 \times(11 \%)\end{array}$ \\
\hline $\begin{array}{l}\text { Consequent usage of the } \\
\text { platform } \\
1 \times(8 \%)\end{array}$ & $\begin{array}{l}\text { More engagement } \\
1 \times(8 \%)\end{array}$ & $\begin{array}{l}\text { Advertisement of the blinc } \\
\text { idea } \\
1 \times(10 \%)\end{array}$ & $\begin{array}{l}\text { News } \\
1 \times(11 \%)\end{array}$ \\
\hline $\begin{array}{l}\text { Contributions of the part- } \\
\text { ners in the following work } \\
\text { packages } \\
1 \times(8 \%)\end{array}$ & $\begin{array}{l}\text { Communication } \\
2 \times(17 \%)\end{array}$ & $\begin{array}{l}\text { Results for practical use } \\
1 \times(10 \%)\end{array}$ & $\begin{array}{l}\text { Report about evaluation } \\
\text { of instruments } \\
1 \times(11 \%)\end{array}$ \\
\hline $\begin{array}{l}\text { Establish links with pro- } \\
\text { jects in partner institutions } \\
1 \times(8 \%)\end{array}$ & $\begin{array}{l}\text { Help } \\
1 \times(8 \%)\end{array}$ & $\begin{array}{l}\text { Taking the project into the } \\
\text { next phase } \\
1 \times(10 \%)\end{array}$ & \\
\hline $\begin{array}{l}\text { Closer communication } \\
1 \times(8 \%)\end{array}$ & $\begin{array}{l}\text { Less ignorance } \\
1 \times(8 \%)\end{array}$ & $\begin{array}{l}\text { Keeping in touch during } \\
\text { the time } \\
1 \times(10 \%)\end{array}$ & \\
\hline \multirow[t]{4}{*}{$\begin{array}{l}\text { Find a way to go on with } \\
\text { the work we are doing } \\
1 \times(8 \%)\end{array}$} & $\begin{array}{l}\text { More potenzial partners } \\
1 \times(8 \%)\end{array}$ & $\begin{array}{l}\text { Increasing number of } \\
\text { partners } \\
1 \times(10 \%)\end{array}$ & \\
\hline & $\begin{array}{l}\text { Creating a durable com- } \\
\text { munity } \\
1 \times(8 \%)\end{array}$ & & \\
\hline & $\begin{array}{l}\text { Serious discusssion of } \\
\text { the co-operative concept } \\
\text { and ways of marketing } \\
1 \times(8 \%)\end{array}$ & & \\
\hline & $\begin{array}{l}\text { Identification of our origi- } \\
\text { nality and uniqueness in } \\
\text { an already highly compet- } \\
\text { itive market } \\
1 \times(8 \%)\end{array}$ & & \\
\hline
\end{tabular}

Tabelle 33: $\quad$ Ergebnisse der Prozessevaluation auf die Frage „What do you expect or wish for the further work of the eL3-network?"

Zusammenfassend kann auf der Grundlage der Ergebnisse der Prozessevaluation im Hinblick auf die Fragestellungen der vorliegenden Forschungsarbeit bzw. die zugrunde liegenden Fragenkomplexe zwei und drei (siehe Unterkapitel 5.2) Folgendes festgestellt werden: In der Projektgruppe scheint eine gute Basis vorhanden gewesen zu sein, um gemeinsam Inventare als Evaluationsinstrumente zu entwickeln, da die Partner sehr zufrieden mit dem Projekt und der Zusammenstellung der Gruppe waren. Es war also möglich, die Inventare in 
dem transdisziplinären Netzwerk zu erarbeiten, wobei sich eine Reihe positiver Effekte ergaben, sich aber auch Schwierigkeiten bzw. Herausforderungen zeigten und zu meistern waren. In den Antworten der Prozessevaluation spiegelt sich der Prozess der gemeinsamen Arbeit. Es wird deutlich, dass es eine relativ lange Anlaufphase brauchte, bis der Kontakt zwischen den Partnern eine Qualität erreicht hatte, die für eine gemeinsame Arbeit nötig war. Die Partner mussten sich erst kennen lernen, es musste Vertrauen aufgebaut werden, Rollen, Aufgaben und Ziele mussten definiert bzw. geklärt werden. Als das Projekt richtig in Gang gekommen war, galt es vor allem, die Kontinuität der Zusammenarbeit zu erhalten, einige Partner beteiligten sich intensiver als andere und manchmal fehlte wohl die Verbindlichkeit bzw. es war nicht klar, wer sich wirklich und in welcher Weise einbringen und „in die Karten schauen lassen“ wollte. Außerhalb der transnationalen Treffen fand wenig Interaktion und Kommunikation statt, auf den Treffen hingegen passierte sehr viel, und die Gruppe kam mit anstehenden Arbeiten insgesamt sehr viel weiter. Für die Inventarentwicklung war bei jedem Treffen eine gesonderte Zeit eingeplant, in der sich die Arbeitsgruppenmitglieder treffen konnten. Hier wurde viel diskutiert, Wissen und Erfahrungen ausgetauscht.

Als besonders positive Elemente der Zusammenarbeit nannten die Partner den Kontakt zu und das Treffen von anderen europäischen Partnern, den Austausch und das VoneinanderLernen sowie den Einblick in die Arbeit der anderen. Die Vielfalt der beteiligten Partner wurde als wertvoll erlebt. Die Unterschiedlichkeit der Partner war einerseits bereichernd für die Gruppe und andererseits eine Hürde, da die Partner unterschiedliche Bedarfe, Ziele und Ansprüche hatten. Schwierigkeiten entstanden auch aufgrund der Webseite, die von den Partnern von Anfang bis Ende des Projektes bemängelt wurde und keine gute Basis für die transdisziplinäre Zusammenarbeit darzustellen schien. Hier wären wohl eine andere Form oder andere technische Voraussetzungen hilfreich gewesen.

Im Hinblick auf die Erprobungsphase der Inventare zeigt sich, dass die Arbeit an den Inventaren durchaus die Evaluationspraxis einiger Partner veränderte bzw. das Nachdenken über die Bedeutsamkeit und Wichtigkeit von Evaluation in Gang gebracht hat. Die „Theorie zur Evaluation“ (Hintergrundwissen, das den Partnern vorgestellt bzw. von innen selbst erarbeitet wurde) hat anscheinend Reflexionsprozesse aktiviert, wie sich im Spiegel der Rückmeldungen zeigt. Für die Partner neuartige „pädagogische Techniken“ wurden eingesetzt und stimulierten Denkprozesse und die bisherige Evaluationspraxis. Die Inventare wurden von den Partnern nach ihren Angaben genutzt, jedoch nicht von allen gleichermaßen. Einige Partner konnten mit dem Ansatz anscheinend mehr anfangen als andere. Für manche blieb die Frage offen, wie sie die Inventare nutzen könnten bzw. wie die anderen sie benutzten. Es bestand der Wunsch nach Austausch, weiterer Zusammenarbeit und einem abschließenden Bericht bzw. einer Evaluation der entwickelten Instrumente. Der Ansatz der Inventare schien praktikabel, doch müssten noch einige Dinge geklärt bzw. ausgewertet werden. Am Ende des Projektes stand die Frage im Raum, wie die Zusammenarbeit fortgesetzt werden könnte - in welcher Form und mit welchen Zielen und wer sich in welcher Weise einbringen könne 
und wolle. Die Inventare wurden gemeinsam entwickelt und standen allen gleichermaßen zur Verfügung, sie konnten von allen genutzt werden, jedoch blieb die weitere Handhabung jedem selbst überlassen. Diese Offenheit (oder auch Freiheit) wurde unterschiedlich erlebt für die einen waren die Wissensinhalte bzw. -hintergründe interessant und wurden auf kreative Weise genutzt (z.B. um eigene bereits zuvor bestehende Instrumente zu erweitern oder auch zu ersetzen, aber auch um völlig neue Fragen zu stellen und die methodische und inhaltliche Herangehensweise bei der Evaluation zu verändern), für andere blieb die Frage, wie sie denn nun mit diesen Instrumenten umgehen sollten, wie sie die Inventare bzw. daraus erstellte Fragebögen konkret einsetzen sollten. Insgesamt stellte sich für das Netzwerk die Frage, wie es in Zukunft gestaltet werden könnte, und wie die Partner weiter in Kontakt und im Austausch bleiben könnten, wie und wo Ergebnisse, Erfahrungen und Wissen geteilt werden könnten.

\subsection{Entwicklung der Inventare: Dokumentation der Aktivitäten auf dem Lernmanagementsystem}

In diesem Kapitel wird die Entwicklung der Inventare aus zwei Perspektiven betrachtet. Zum einen wurden die Aktivitäten auf dem Lernmanagementsystem dokumentiert, das der Projektgruppe und den Arbeitsgruppen als „Raum“ zur Zusammenarbeit außerhalb der transnationalen Treffen diente. Zum anderen flossen Notizen zu den Gruppendiskussionen zum Fortschritt der Arbeitsgruppen auf den transnationalen Treffen sowie die Protokolle mit in diese Auswertung ein. Auf dieser Basis wird die Entwicklung der Inventare rekonstruiert, in verschiedene Phasen eingeteilt und genauer beschrieben:

Die Entwicklung der Inventare war eingebettet in den Projektverlauf von eL3 und die Entstehung des Netzwerkes „blinc" (blended learning institutions cooperative). Die Idee, gemeinsam auf europäischer Ebene Evaluationsinstrumente zu entwickeln, die von allen Projektpartnern genutzt und mit denen Ergebnisse und Erfahrungen im Kontext von e-Learning und Blended Learning im dritten Sektor gesammelt, verglichen und ausgewertet werden könnten, fand in der Projektgruppe großen Anklang. Die Erfahrungen der Beteiligten aus der Praxis und das Wissen der Partner mit wissenschaftlichem Hintergrund schienen eine Erfolg versprechende Mischung zu sein, um Instrumente zu entwickeln, die sowohl praxistauglich als auch theoretisch fundiert sein sollten. Zunächst wurde davon ausgegangen, dass gemeinsam Fragebögen entwickelt werden, die von allen genutzt werden könnten. Es wurde jedoch relativ schnell deutlich, dass die Kurse der Partner und deren Kontexte so heterogen waren, dass dies nicht möglich war und sich deshalb Inventare als Basis für individuelle Fragebögen besser eignen würden. 
Zunächst wurden von den Projektpartnern in einer Gruppendiskussion drei Zielgruppen für die Evaluation von e-Learning und Blended Learning Kursen identifiziert:

1. Lerner, die an e-Learning und Blended Learning-Kursen teilnehmen.

2. Autoren, die e-Learning und Blended Learning-Kurse entwickeln.

3. Entscheidungsträger, die entscheiden, ob e-Learning und Blended Learning-Kurse in ihre Einrichtung implementiert werden.

Es wurde geplant, dass für jede dieser drei Zielgruppen Inventare entwickelt werden sollten, die dann im Netzwerk je nach Bedarf genutzt würden. Es wurde vorgeschlagen, dass zunächst mit einer Recherche nach bereits vorhandenen Instrumenten zur Evaluation von eLearning und Blended Learning und nach relevanter Literatur begonnen werden sollte, auBerdem sollten Materialien der Projektpartner gesammelt werden, um die Erfahrungen der Partner zu nutzen. Zu einem späteren Zeitpunkt sollten entsprechend den Zielgruppen drei Arbeitsgruppen gegründet werden, die für die weitere Entwicklung der Inventare verantwortlich sein würden. Die Ergebnisse der Arbeitsgruppen sollten auf den transnationalen Projekttreffen vorgestellt und die weitere Vorgehensweise sollte mit der gesamten Gruppe abgestimmt werden. Es wurde vereinbart, zum Ende des Projektes eL3 auf dem letzten Projekttreffen die Ergebnisse zusammenzutragen und eine Bilanz zu ziehen.

Die an diese Planung anschließende Entwicklung der Inventare erfolgte insgesamt über einen Zeitraum von fast zwei Jahren. Auf der Grundlage der Dokumentationen zur Arbeit in der Projektgruppe lassen sich fünf Entwicklungsphasen kennzeichnen:

Phase 1: Recherche und Sammlung.

Phase 2: Diskussion und Austausch.

Phase 3: Gemeinsamer Entwurf und Übersetzung.

Phase 4: Erprobung und Ergebnisbericht.

Phase 5: Gemeinsame Endversion und Verbreitung.

Zunächst übernahm ein Projektpartner mit wissenschaftlichem Hintergrund die Recherche und Sammlung von Literatur und Instrumenten über e-Learning und Blended Learning und stellte die Ergebnisse der Projektgruppe als Basis für die weitere Arbeit zur Verfügung. Nach der Gründung der Arbeitsgruppen im September 2004 wurde über das projektinterne Lernmanagementsystem (LMS) gearbeitet. Es wurde ein Kurs für jede Gruppe angelegt, der die verschiedenen Arbeitsschritte verdeutlichte. Für jede Gruppe gab es einen Hauptverantwortlichen, der die Aktivitäten planen und dokumentieren sollte. Außerdem gehörte es zu seinen Aufgaben, Ergebnisse zusammenzufassen und der gesamten Projektgruppe während der transnationalen Treffen zu präsentieren. Die Arbeit mittels des LMS schien geeig- 
net, um die Gruppenarbeit zu strukturieren, indem verschiedene Arbeitsschritte festgelegt wurden. Gleichzeitig erfolgte auf diese Weise eine Dokumentation der verschiedenen Arbeitsphasen bzw. des Fortschritts. Das LMS bot den weiteren Vorteil für die Arbeitsgruppe, dass Materialien (Texte, Fragebögen, Dokumente) für alle Mitglieder hinterlegt werden konnten, so dass jederzeit darauf zugegriffen werden konnte. Für die Diskussion der Materialien war für jede Arbeitsgruppe ein eigenes Forum vorgesehen, in dem Beiträge hinterlassen werden konnten. Während der transnationalen Projekttreffen hatten die Arbeitsgruppen Zeit für Diskussionen und Austausch und die weitere Entwicklung der Inventare.

Eine gemeinsame Endversion der Inventare für Lerner, Autoren und Entscheidungsträger in vier Sprachen wurde im Dezember 2005 vorgelegt und über die Internetseiten des gemeinsamen Netzwerkes "blinc" einem breiteren Publikum zugänglich gemacht. Die folgende Tabelle zeigt die Phasen der Entwicklung der Inventare als Übersicht:

\begin{tabular}{|l|l|l|}
\hline Zeitraum & Zeitdauer & Phasen und Aktivitäten \\
\hline $\begin{array}{l}\text { März - September 2004 } \\
\text { September 2004 - Mai 2005 } \\
\downarrow\end{array}$ & 6 Monate & $\begin{array}{l}\text { 1. Recherche und Sammlung } \\
>\text { Literatur und Instrumente der Partner }\end{array}$ \\
\hline $\begin{array}{l}\text { Mai - Juni 2005 } \\
\downarrow\end{array}$ & 2 Monate & $\begin{array}{l}\text { 3. Gemeinsamer Entwurf und Übersetzung } \\
\text { 2. Diskussion und Austausch }\end{array}$ \\
\hline $\begin{array}{l}\text { Juli - November 2005 } \\
\downarrow\end{array}$ & 5 Monate & $\begin{array}{l}\text { 4. Erprobung und Ergebnisbericht } \\
>\text { Einsatz in verschiedenen Einrichtungen }\end{array}$ \\
\hline $\begin{array}{l}\text { Ab Dezember 2005 } \\
\downarrow\end{array}$ & 1 Monat & $\begin{array}{l}\text { 5. Gemeinsame Endversion und Verbreitung } \\
>\text { Bilanz und Verbreitung über blinc }\end{array}$ \\
\hline $\begin{array}{l}\text { Tabelle 34: Überblick über den Entwicklungszeitraum und die Entwicklungsphasen der Inventare } \\
\text { im Projekt eL3 }\end{array}$
\end{tabular}

Die gemeinsame Erarbeitung der Inventare stellte eine Herausforderung für die Arbeitsgruppen dar, da die Kontexte der Projektpartner sehr verschieden waren. Es sollte dennoch versucht werden, unabhängig vom institutionellen und kulturellen Hintergrund sowie verschiedenen Kursinhalten Inventare zu entwickeln, die von allen genutzt werden könnten. Das Ziel war, in dem Expertennetzwerk allgemeingültige und wieder verwendbare Grundfragen über e-Learning und Blended Learning zu finden und herauszuarbeiten, die dann je nach Kontext individuell angepasst und verwendet werden könnten. Die folgende Tabelle gibt einen Überblick über die Entwicklungsschritte der Inventare für Lerner, Autoren und Entscheidungsträger. 


\begin{tabular}{|c|c|}
\hline \multicolumn{2}{|c|}{ Phase 1: Recherche und Sammlung } \\
\hline $\begin{array}{l}\text { März } 2004 \\
\text { Transnationales Treffen Goslar }\end{array}$ & $\begin{array}{l}\text { Vereinbarung, gemeinsam innerhalb des Projektes eL3 Eva- } \\
\text { luationsinstrumente für e-Learning und Blended Learning- } \\
\text { Kurse zu entwickeln, erste Ideensammlung }\end{array}$ \\
\hline April - Juni 2004 & $\begin{array}{l}\text { Recherche nach vorhandenen Evaluationsinstrumenten in der } \\
\text { wissenschaftlichen Literatur und im Internet }\end{array}$ \\
\hline Juli - August 2004 & $\begin{array}{l}\text { Erstellung eines Fragebogenentwurfs für die Zielgruppen } \\
\text { Lerner, Autoren und Entscheidungsträger unter Berücksichti- } \\
\text { gung der Projekte der eL3-Partner } \\
\text { auf der Basis der Profile }\end{array}$ \\
\hline \multicolumn{2}{|c|}{ Phase 2: Diskussion und Austausch } \\
\hline $\begin{array}{l}\text { September } 2004 \\
\text { Transnationales Treffen Aberdeen }\end{array}$ & $\begin{array}{l}\text { Gründung von drei Arbeitsgruppen für die Weiterentwicklung } \\
\text { der Inventare } \\
\text { Diskussion und Austausch während des transnationalen Tref- } \\
\text { fens }\end{array}$ \\
\hline Oktober - November 2004 & $\begin{array}{l}\text { Revision der Inventare auf der Basis der Diskussionen in den } \\
\text { Arbeitsgruppen } \\
\text { Beginn der Kurse für jede der drei Arbeitsgruppen auf dem } \\
\text { Learning Management System (LMS) }\end{array}$ \\
\hline Dezember 2004 & $\begin{array}{l}\text { Austausch und Dokumentation von Instrumenten der Projekt- } \\
\text { partner zur Evaluation ihrer e-Learning und Blended Learn- } \\
\text { ing- Angebote }\end{array}$ \\
\hline Januar 2005 & $\begin{array}{l}\text { Diskussionen auf dem LMS im Forum } \\
\text { Austausch via E-Mail }\end{array}$ \\
\hline $\begin{array}{l}\text { Februar } 2005 \\
\text { Transnationales Treffen Göttingen }\end{array}$ & $\begin{array}{l}\text { Präsentation und Zwischenbilanz der von den Arbeitsgruppen } \\
\text { bis dahin erarbeiteten Inventare }\end{array}$ \\
\hline Februar - April 2005 & $\begin{array}{l}\text { Weiterarbeit an den Inventaren in den Arbeitsgruppen, Ver- } \\
\text { feinerungen, sprachliche Veränderungen }\end{array}$ \\
\hline \multicolumn{2}{|c|}{ Phase 3: Gemeinsamer Entwurf und Übersetzung } \\
\hline $\begin{array}{l}\text { Mai } 2005 \\
\text { Transnationales Treffen Siena }\end{array}$ & $\begin{array}{l}\text { Präsentation einer gemeinsamen Version der Inventare für } \\
\text { die drei Zielgruppen } \\
\text { Vereinbarung, wer welche Inventare nutzen wird } \\
\text { Auftrag zur Übersetzung der Inventare }\end{array}$ \\
\hline \multicolumn{2}{|c|}{ Phase 4: Erprobung und Ergebnisbericht } \\
\hline Juni - Juli 2005 & $\begin{array}{l}\text { Erprobung der Inventare in verschiedenen Einrichtungen der } \\
\text { eL3-Partner }\end{array}$ \\
\hline August - September 2005 & $\begin{array}{l}\text { Interne Evaluation zur Erprobungsphase der Inventare mittels } \\
\text { Fragebögen zur Nützlichkeit und Anwendbarkeit der Inventa- } \\
\text { re }\end{array}$ \\
\hline Oktober 2005 & $\begin{array}{l}\text { Auswertung der Erfahrungen aus der Erprobungsphase } \\
\text { Revision der Inventare auf der Basis von Feedback }\end{array}$ \\
\hline November 2005 & Präsentation der Ergebnisse aus der Erprobungsphase \\
\hline Transnationales Treffen Mars & $\begin{array}{l}\text { Diskussion und Planung zur weiteren Verwendung der Inven- } \\
\text { tare (Bedürfnisse und Zukunftsperspektiven) }\end{array}$ \\
\hline \multicolumn{2}{|c|}{ Phase 5: Gemeinsame Endversion \& Verbreitung } \\
\hline Ab Dezember 2005 & $\begin{array}{l}\text { Gemeinsame Endversion der Inventare in vier Sprachen } \\
\text { Verbreitung über die Internetseite von blinc }\end{array}$ \\
\hline
\end{tabular}

Tabelle 35: Übersicht über die Entwicklungsschritte der Inventare im Rahmen von eL3

Im Folgenden wird die Entwicklung der einzelnen Inventare (Lerner, Autoren und Entscheidungsträger) näher beschrieben.

\subsubsection{Die Inventare für Lerner}

Zu Beginn der Planungen zur Entwicklung von Evaluationsinstrumenten für e-Learning und Blended Learning-Kurse wurden drei Zielgruppen identifiziert, wobei die Lerner eine davon 
sind (s.o.). Lerner wurden definiert als Personen, die an e-Learning und Blended LearningKursen teilnehmen. Zu dieser Personengruppe gehören im Kontext des eL3-Projektes Mitarbeiter verschiedener Einrichtungen des Dritten Sektors wie etwa Krankenschwestern und Pflegepersonal, Studenten verschiedener Universitäten im medizinischen Bereich und in der Gesundheitspflege sowie Kunden oder Klienten verschiedener Bildungseinrichtungen, wie z.B. Jugendliche aus ländlichen Regionen oder Arbeitslose, die sich durch Weiterbildungen qualifizieren wollen. Die Zielgruppe der Lerner ist demnach sehr heterogen hinsichtlich Alter, Bildungshintergrund und Beruf. Die Lerner stammen außerdem aus unterschiedlichen europäischen Ländern (Deutschland, Frankreich, Italien und Schottland).

\section{Phase 1: Recherche und Sammlung}

Es erfolgte zuerst eine Recherche, welche Evaluationsinstrumente es bereits für e-Learning gibt. In der Literatur wurde nach veröffentlichten Studien und Fragebögen gesucht, in denen Lerner im Zentrum standen. Im deutschsprachigen Raum fanden sich vor allem die Arbeiten von Schulmeister (2003) und Ehlers (2004). Außerdem wurde im Internet nach bestehenden Fragebögen gesucht, die von Universitäten, Bildungseinrichtungen, Krankenhäusern und sozialen Einrichtungen im dritten Sektor zur Evaluation von e-Learning Verwendung fanden. Diverse Fragebögen von unterschiedlicher Qualität wurden gefunden, gesammelt und analysiert.

Die Arbeiten von Schulmeister und Ehlers sowie die Sammlung von Fragebögen aus dem Internet dienten als Grundlage, um zentrale Themenbereiche für einen Fragebogen herauszuarbeiten, der für möglichst viele verschiedene Einrichtungen verwendbar sein sollte. Die Grundfrage war: Was sind Fragen bzw. Themenbereiche, die sich wiederholen, und die unabhängig vom Kontext, d.h. Kursinhalt und Institution, relevant sind? Welche Fragen sind generell für die Evaluation von e-Learning in Bezug auf die Lerner wichtig?

Es wurden zunächst fünf Fragenbereiche herausgearbeitet, die sich in vorhandenen Fragebögen und Studien fanden:

- Demographische Daten der Lerner

- Allgemeine Einschätzung des Lernprogramms

- Benutzung des Lernprogramms

- Benutzbarkeit des Lernprogramms

- Nützlichkeit und Angemessenheit des Lernprogramms

Auf der Basis dieser fünf Themenbereiche wurde ein erster Entwurf für ein Lernerinventar entwickelt, wobei Fragen aus vorhandenen Fragebögen übernommen sowie auch eigene 
formuliert wurden. Anschließend erfolgte eine Übersetzung, da die Arbeitssprache im Projekt eL3 Englisch war.

\section{Phase 2: Diskussion und Austausch}

Während des Projekttreffens im September 2004 wurde eine Arbeitsgruppe gegründet, die bis zum Ende des Projektes speziell für die Entwicklung des Inventars für Lerner zuständig sein sollte. Die Gruppe bestand aus acht Personen, die aus Schottland, Frankreich, Italien und Deutschland stammten und von ihrem beruflichen Kontext her in der Lehre und Gestaltung von Kursen in Universitäten und Bildungseinrichtungen tätig waren und auch Erfahrung mit der Evaluation ihrer eigenen Kurse hatten.

Zunächst diskutierte die Arbeitsgruppe während des Projekttreffens in Aberdeen über den ersten Entwurf des Inventars aus Phase 1 (siehe oben). Für die weitere Entwicklung konnte die Arbeitsgruppe das projektinterne Lernmanagementsystem nutzen. Eine Person übernahm die Führung und Koordination der Gruppe, formulierte verschiedene Arbeitsschritte und stellte auf dem Lernmanagementsystem die Materialien bereit, die für die weitere Arbeit benötigt wurden. Die Arbeitsgruppe sollte innerhalb von neun Monaten eine gemeinsame Version der Inventare für Lerner entwickeln. Der Entwicklungsprozess umfasste folgende Arbeitsschritte:

1. Präsentation eines ersten gemeinsamen Entwurfes des Inventars für Lerner nach der Diskussion der Arbeitsgruppe in Aberdeen.

2. Diskussion über die neue Version im Forum und Sammlung von Instrumenten der beteiligten Partner.

3. Präsentation einer überarbeiteten Version des Inventars.

4. Diskussion und Austausch im Forum.

5. Abschluss und gemeinsame Endversion (Masterversion in Englisch).

6. Endversion in verschiedenen Sprachen.

Das folgende Bild gibt einen „screenshot“ aus dem Lernmanagementsystem wieder: 


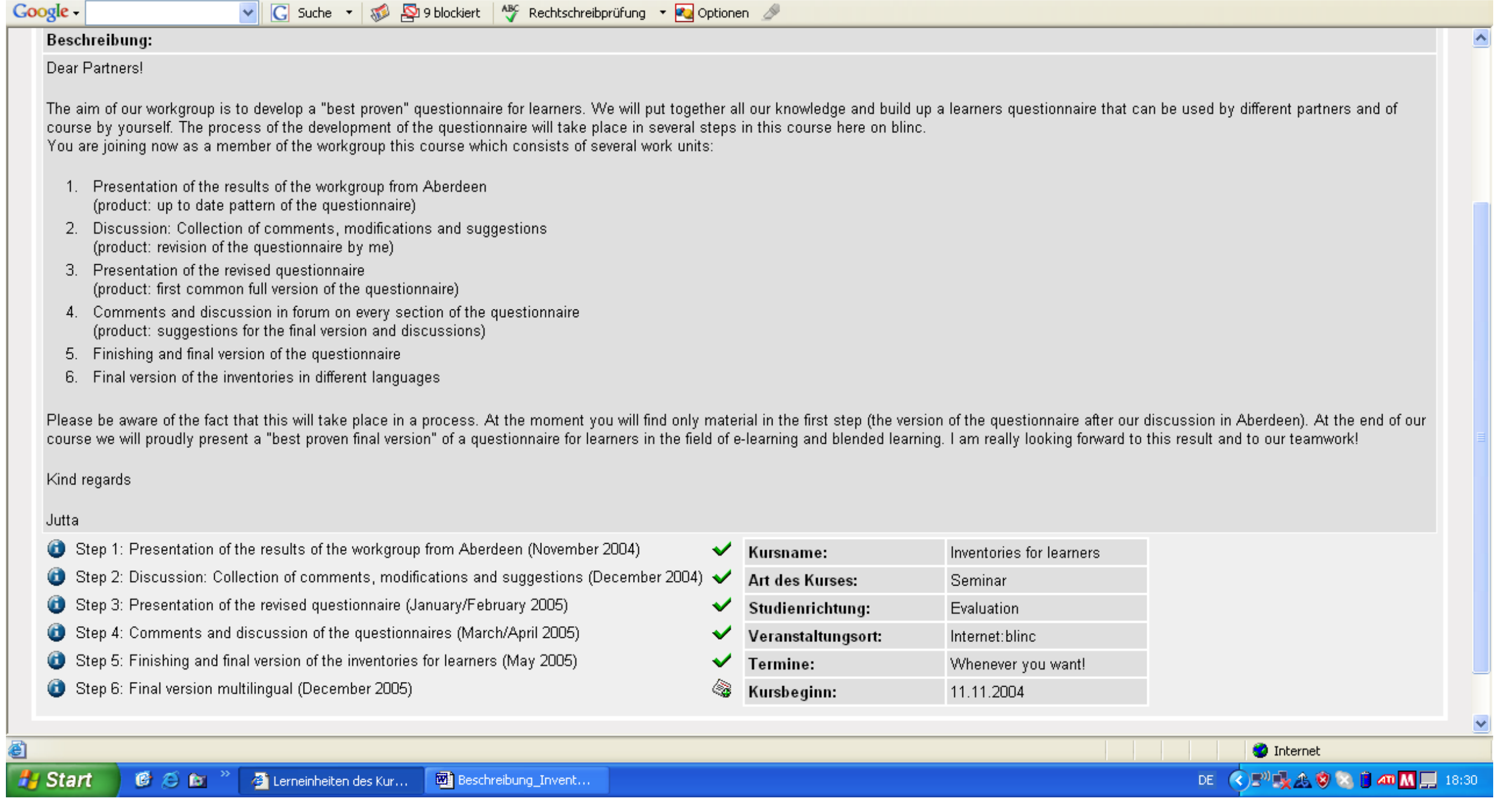

\section{Abbildung 9: Übersicht des Kurses für die Arbeitsgruppe „Inventare für Lerner“ auf der Lernplattform}


Zu Beginn wurde der Entwurf des Fragebogens aus Phase 1 hinterlegt, damit er für alle einsehbar war. Danach wurden die Evaluationsinstrumente der Arbeitsgruppenmitglieder gesammelt, die bisher in den verschiedenen Einrichtungen Verwendung fanden, wodurch alle einen Einblick in die Evaluationspraxis ihrer Projektpartner bekommen konnten. Die Arbeitsgruppe bekam den Auftrag, die vorhandenen Instrumente anzuschauen und in einem Forum auf dem Lernmanagemensytsem ihre Kommentare und Vorschläge für eine gemeinsame Version abzugeben. Die Meinungen und Erfahrungen der Gruppe sollten über das Forum ausgetauscht werden, und die Hauptverantwortliche der Arbeitsgruppe sollte die Vorschläge und Ideen in ein gemeinsames Dokument einarbeiten. Es gab durch diese Vorgehensweise mehrere Zwischenversionen, die wiederum zur Diskussion bereitgestellt wurden. So sollte nach und nach eine gemeinsame Version entstehen. Die folgende Abbildung zeigt die Sammlung verschiedener Instrumente der Partner

\section{Step 2: Discussion: Collection of comments, modifications and suggestions (December 2004) - Microsoft Internet Explorer}

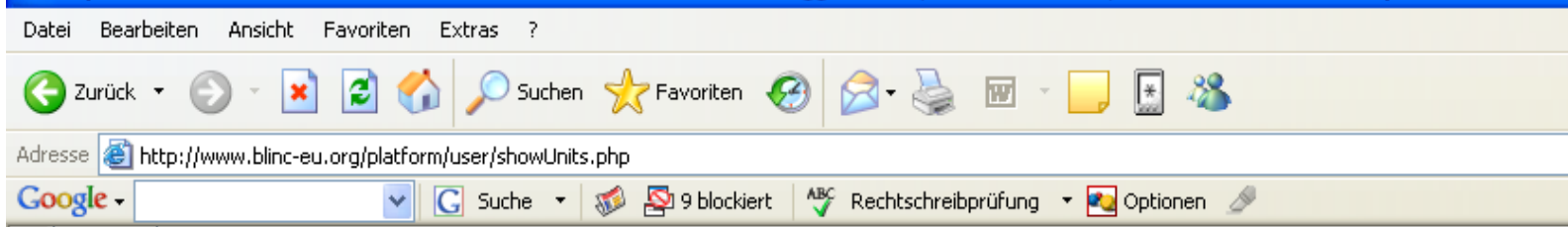

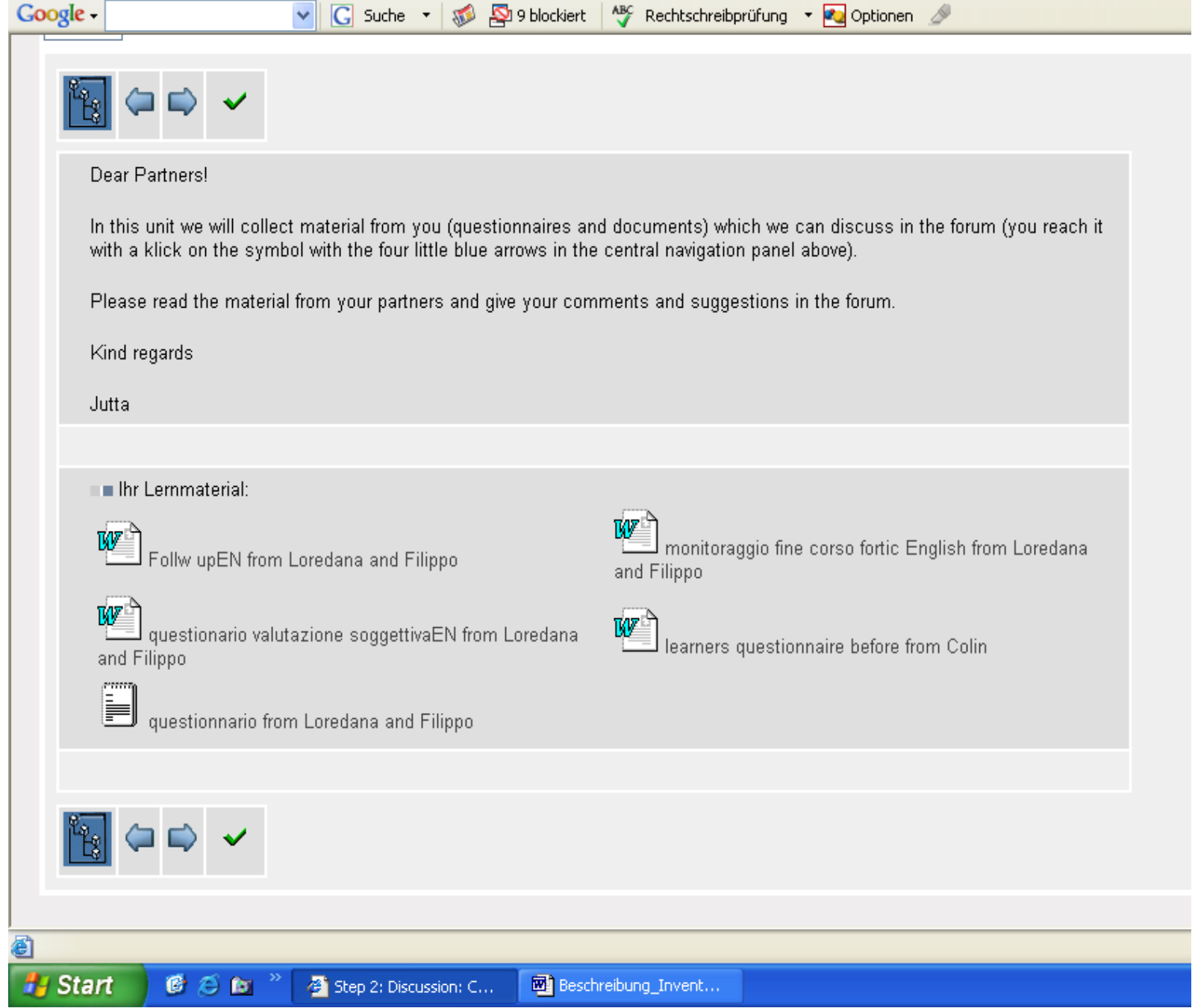

Abbildung 10: Sammlung von Instrumenten der Partner im Kurs für die Arbeitsgruppe der Inventare für Lerner 
Die Inventare für Lerner wurden zu diesem Zeitpunkt noch „learners questionnaire“ genannt. Die Versionen wurden mit dem Datum der letzten Bearbeitung versehen, um zu verdeutlichen, welches die letzte aktuelle Version war, in der alle Änderungs- und Verbesserungsvorschläge enthalten waren. Im Januar 2005 gab es eine „Vorher“ und „nachher“-Version des Inventars für Lerner, d.h. ein Inventar, das zur Evaluation vor Beginn des Kurses verwendet werden kann und eines für die Befragung am Ende des Kurses. In der folgenden Abbildung wird die Arbeitsphase auf der Lernplattform gezeigt. Ein Fragebogen eines Arbeitsgruppenmitgliedes wurde ebenfalls hinterlegt, damit die Partner sich das Dokument anschauen und ggf. Fragen oder Fragenbereiche davon als Anregung für die gemeinsame Version der Lernerinventare finden konnten.

\section{S Step 3: Presentation of the revised questionnaire (January/February 2005) - Microsoft Internet Explorer}

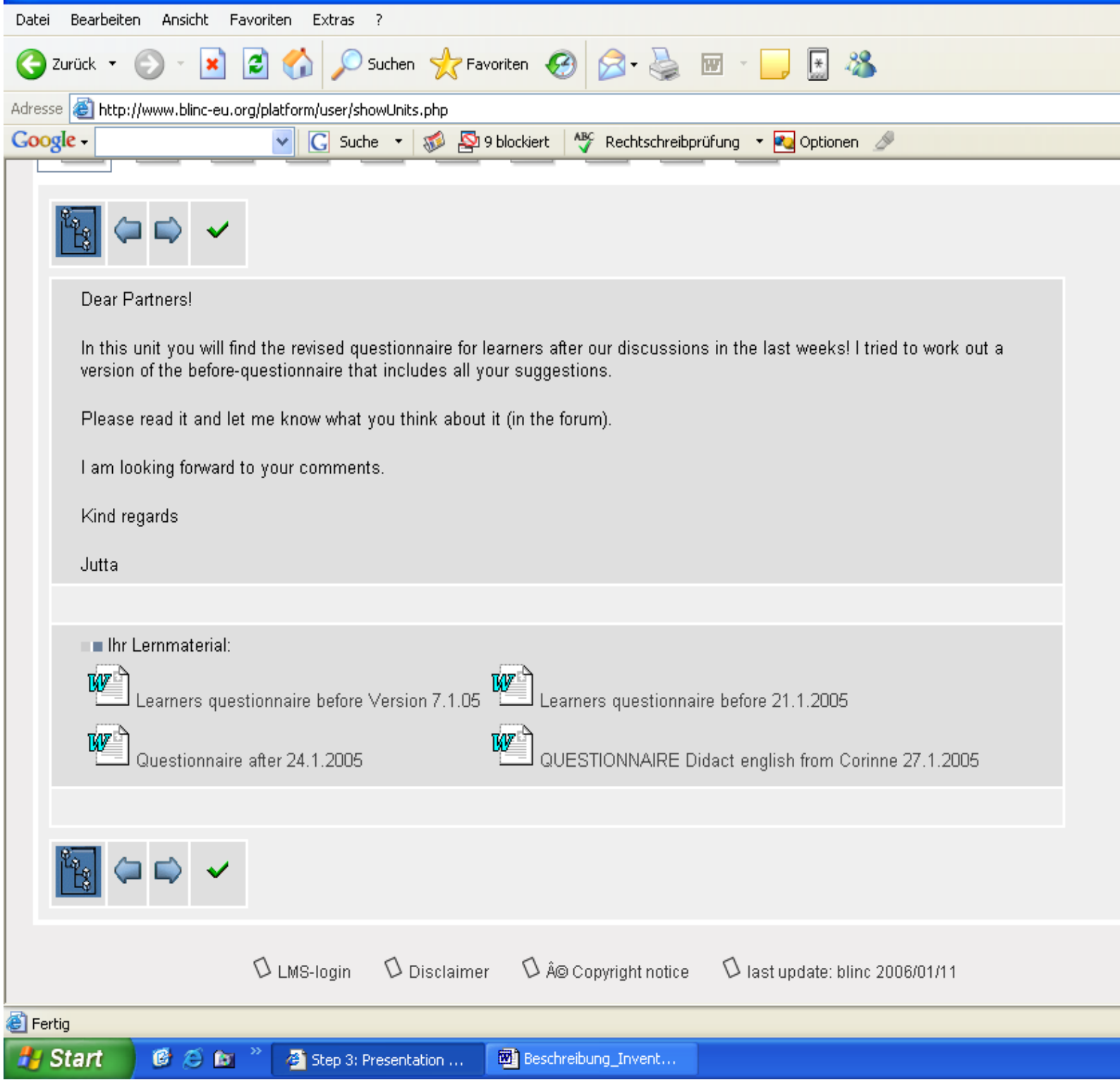

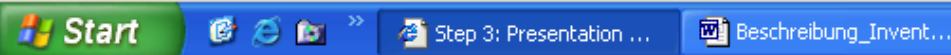

Abbildung 11: Verschiedene Versionen des Lernerinventars im Kurs auf dem Lernmanagementsystem 
Die Partner bekamen von der Arbeitsgruppenleiterin per E-Mail und auf dem Lernmanagementsystem die Aufgabe, sich die hinterlegten Materialien anzuschauen und ihre Meinung dazu im Forum zu äußern. Es konnten Kommentare, Veränderungs- und Verbesserungsvorschläge zu dem Inventar für Lerner im Forum hinterlassen werden. Die folgende Abbildung zeigt einen Ausschnitt aus den Beiträgen der Arbeitsgruppenmitglieder:

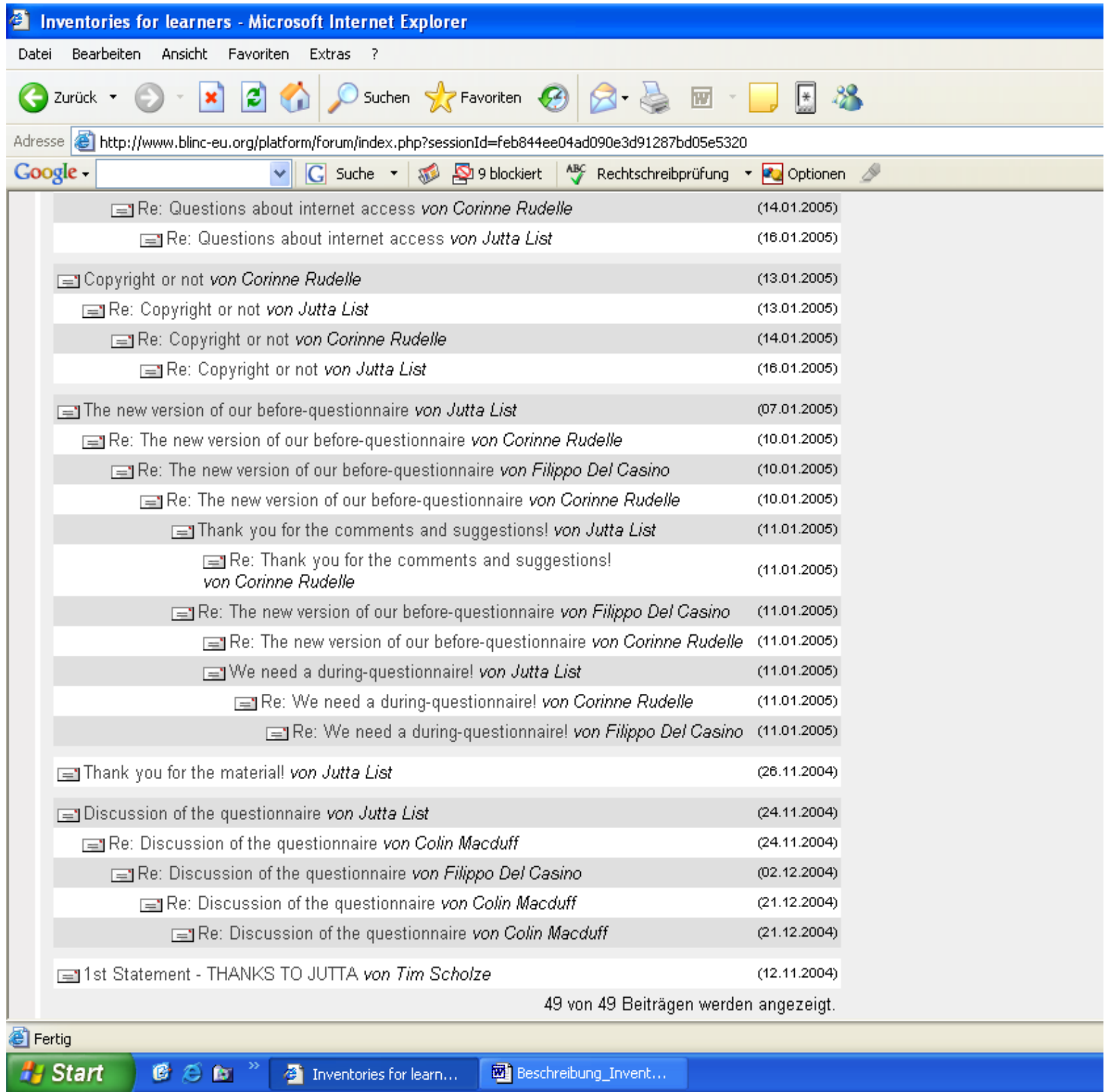

Abbildung 12: Ausschnitt aus den Beiträgen der Arbeitsgruppenmitglieder zum Inventar der Lerner im Forum

Neben der Arbeit über das Lernmanagementsystem wurde per E-Mail kommuniziert. Außerdem gab es während der Projekttreffen Zeiten für die Arbeitsgruppe, in denen an den Inventaren gearbeitet werden konnte. Die Diskussion und der Austausch über einzelne Fragen und Themenbereiche aus den Inventaren, aber auch der Austausch über Erfahrungen mit bestimmten Fragen der Evaluation im Allgemeinen erfolgte in dieser Phase der Inventarentwicklung über die Lernplattform, E-Mails und Projekttreffen. Die Projekttreffen waren jeweils 
Meilensteine in der Entwicklung der Inventare, da sowohl die Arbeitsgruppe einige Stunden real zusammen sein konnte als auch die Ergebnisse der gesamten Projektgruppe vorgestellt wurden. Die gemeinsame „Präsenzzeit“ war besonders effektiv im Gegensatz zu den Zeiten, in denen die Projektgruppe über E-Mail kommunizierte bzw. sich über die Lernplattform austauschte. Die Phase „Diskussion und Austausch“ dauerte insgesamt neun Monate, in denen sich die Arbeitsgruppe neben inhaltlichen Fragen, die für eine Befragung von Lernern relevant schienen, mit folgenden Themen beschäftigte:

- Befragungszeitpunkte

- Inventare für reines e-learning und/oder Blended Learning

- Papierbasierte Inventare versus online Befragung

- Begriffe: Kurs, Seminar, Programm, Bildungsmaßnahme

- Kursinhalt und Lernmethoden

- Kulturelle Unterschiede bei der Befragung

- Schriftgröße und Layout

- Umfang der Inventare bzw. der individuellen Fragebögen

- Formulierung von Antwortvorgaben versus offene Fragen

- Copyright

Die Inhalte und Ergebnisse der Diskussionen werden im Folgenden beschrieben:

\section{Befragungszeitpunkte}

Die Partner waren der Meinung, dass es sinnvoll sei, die Lerner zu unterschiedlichen Zeiten zu befragen. Zu Beginn des Kurses oder bereits davor sollte erhoben werden, wie viel Erfahrung die Lerner im Umgang mit dem Computer hatten bzw. wie vertraut sie mit e-Learning waren. Außerdem sei es wichtig zu wissen, wie sehr die Lerner daran gewöhnt waren, selbständig zu arbeiten (z.B. Zeitmanagement für das eigenverantwortliche Lernen). Diese Informationen würden es den Kursleitern ermöglichen, den Kurs optimal auf die Lernergruppe abstimmen zu können. Nach der Erfahrung einiger Arbeitsgruppenmitglieder hätten viele Lerner (z.B. Personal) Schwierigkeiten im Umgang mit dem Computer, was eine Barriere für e-Learning darstellte. Andere Arbeitsgruppenmitglieder berichteten, dass ihre Lerner (z.B. Studenten) keine Probleme mit dem Computer hätten, sondern im Gegenteil bereits auf einem sehr hohen Niveau selbständig mit Computer und Internet arbeiten könnten. Das Zeitmanagement für e-Learning, z.B. Festlegen von Lernzeiten sowie eigenständiges Bearbeiten von Arbeitspaketen, stellte nach Erfahrung der Arbeitsgruppe ebenfalls für viele Lerner eine Herausforderung dar. Mit einer Erhebung zu Beginn des Kurses könne erfasst werden, ob die Lernergruppe Bedarf hatte, zu diesem Thema Unterstützung zu bekommen. 
Am Ende des Kurses sollte vor allem die Zufriedenheit mit dem Kurs insgesamt erfragt werden. Eine abschließende Bewertung des Kurses, der Materialien, der Dozenten bzw. Tutoren und der Methode e-Learning sei sinnvoll. Die Rückmeldungen der Lerner könnten vor allem genutzt werden, um zukünftige Kurse zu optimieren, aber auch, um die Qualität des Kurses zu dokumentieren und zu sichern.

Zusätzlich zu Befragungen am Anfang und am Ende eines Kurses, die nach Meinung der Arbeitsgruppe unbedingt durchgeführt werden sollten, sei es sinnvoll und nützlich, eine Befragung während des Kurses durchzuführen, um die Qualität des Kurses zu überprüfen und ggf. nachbessern zu können. Insbesondere für einen e-Learning-Kurs sei eine Überprüfung, ob die Lerner mit dem Angebot gut zurechtkommen, wichtig, da kaum Kontakt zwischen den Lernern untereinander bzw. zu den Dozenten bestünde. Die hohe Abbruchquote beim eLearning könne durch eine Befragung reduziert werden, da die Ergebnisse genutzt werden könnten, um den Kurs auf die Bedürfnisse der Lerner abzustimmen.

Es lohne sich nach der Auffassung der Arbeitsgruppe auch, eine Weile nach Ende des Kurses eine Befragung durchzuführen (follow-up-Erhebung), um die Zufriedenheit mit dem Kurs bzw. die Einschätzung der Lerner etwas losgelöst vom Kurs zu erfassen, z.B. wenn der Prüfungsdruck nicht mehr vorhanden sei.

Eine umfassende und den gesamten Kurs betreffende Evaluation würde nach den Vorstellungen der Arbeitsgruppe wie folgt aussehen:

\begin{tabular}{|l|l|l|}
\hline Befragungszeitpunkt & Art der Evaluation & Ziel der Evaluation \\
\hline $\begin{array}{l}\text { Zu Beginn des Kurses } \\
\downarrow\end{array}$ & Vorab-Evaluation & $\begin{array}{l}\text { Voraussetzungen und Erwartungen } \\
\text { der Lerner }\end{array}$ \\
\hline$\downarrow$ & Formative Evaluation & $\begin{array}{l}\text { Wie nehmen die Lerner den Kurs an? } \\
\text { Kommen sie gut mit dem Angebot } \\
\text { zurecht? }\end{array}$ \\
\hline Am Ende des Kurses & Summative Evaluation & $\begin{array}{l}\text { Erfolgskontrolle } \\
\text { Qualitätsüberprüfung } \\
\text { Qualitätsdokumentation Qualitätssi- } \\
\text { cherung } \\
\text { Bilanz }\end{array}$ \\
\hline Nach dem Kurs & & Bewährung des Kurses \\
& & Nützlichkeit im Alltag \\
& & Nachträgliche Bewertung \\
\hline
\end{tabular}

Tabelle 36: Möglicher Evaluationsverlauf für die Befragung von Lernern 
Inventare für reines e-Learning und/oder Blended Learning

Der erste Entwurf der Inventare war auf e-Learning ausgerichtet und in der Arbeitsgruppe wurde diskutiert, ob er speziell für Blended Learning abgestimmt werden sollte.

Die meisten Projektpartner strebten längerfristig an, eher Blended Learning-Kurse anzubieten als e-Learning. Für manche Kursinhalte biete sich reines e-Learning an, aber für die gröBere Anzahl von Kursthemen bzw. Bildungsinhalten eigne sich nach Meinung und Erfahrung der Arbeitsgruppenmitglieder eher Blended Learning. Dies hatte Konsequenzen für die Auswahl der Themenbereiche und Fragen, da die technischen Aspekte des e-Learning nicht mehr so sehr im Zentrum standen, sondern vielmehr die Kombination von verschiedenen Lernmethoden (Präsenzunterricht, e-Learning, Projektlernen) und wie Lerner damit umgehen bzw. gut lernen können. In der Arbeitsgruppe wurde beschlossen, die Inventare für Blended Learning zu entwickeln, da der Verwendungszusammenhang größer schien. Für die Projektpartner, die reines e-Learning anbieten, sollten Fragenbereiche, die andere Lernmethoden betreffen, ausgelassen werden können.

\section{Papierbasierte Inventare versus online-Befragung}

In der Arbeitsgruppe wurde diskutiert, ob es sich eher eignen würde, eine papierbasierte Befragung durchzuführen, oder ob die Inventare bzw. individuelle Fragebögen online, d.h. elektronisch von den Lernern ausgefüllt werden sollten. Einerseits wurde argumentiert, dass sich für einen reinen e-Learning-Kurs eine online-Befragung anbieten würde, da die Fragebögen in die Lerneinheiten integriert bzw. ans Ende gestellt werden könnten. Das Lernen würde am Computer durchgeführt, so dass sich auch eine Befragung mit diesem Medium eigne. Andererseits wurde konstatiert, dass manche Lerner mit einer online-Befragung vor allem zu Beginn eines Kurses Schwierigkeiten haben könnten, falls sie im Umgang mit dem Computer noch nicht vertraut wären. Die Arbeitsgruppenmitglieder diskutierten auch darüber, ob es sinnvoll wäre, mit Rücksicht auf die Lerner und deren Vorerfahrungen mit dem Computer am Anfang eines Kurses eine papierbasierte Befragung anzubieten und die späteren Erhebungen online durchzuführen. Es wurde ebenfalls überlegt, ob die Befragungsergebnisse unterschiedlich ausfallen würden, wenn sie ausschließlich papierbasiert bzw. ausschließlich elektronisch von den Lernern ausgefüllt würden. Eine Erhebung mit papierbasierten Fragebögen, die ausgeteilt, handschriftlich ausgefüllt und dann eingesammelt werden, könnte zu anderen Ergebnissen führen als eine Befragung mit einem online-Fragebogen.

In der Arbeitsgruppe wurde auch überlegt, welche Konsequenzen eine papierbasierte gegenüber einer online-Befragung für die Auswertung der Ergebnisse hätte. Während bei der papierbasierten Auswertung alle Antworten gelesen und in ein Auswertungsprogramm eingegeben werden müssten, könnten die Daten einer online-Befragung direkt in eine Datenbank einfließen. Sie könnten sowohl für die Lernergruppe eines einzelnen Kurses ausgewertet, aber auch mit den Daten anderer europäischer Projektpartner verglichen werden. Für 
das blinc-Netzwerk würde idealerweise ein dynamisches Inventar für Lerner angeboten werden, das die Gesamtmenge der entwickelten Fragen enthielte. Die Projektpartner würden dann durch die Auswahl der für ihren Kurszusammenhang relevanten Fragen ihren individuellen Fragebogen zusammenstellen bzw. automatisch generiert bekommen. Die Daten der Lerner würden dann in eine Datenbank einfließen, und es könnte sowohl eine individuelle Ergebnisauswertung stattfinden als auch ein Vergleich mit anderen Daten aus anderen Kurszusammenhängen. So könnten sukzessive Vergleichsdaten aufgebaut werden und sowohl die einzelnen Partner als auch die blinc-Gemeinschaft könnten von den Ergebnissen profitieren. 
Dynamische Inventare für Lerner: Pool mit allen Items

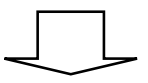

Individueller Fragebogen: Auswahl von Fragen für einen speziellen Kurs

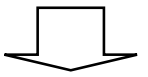

Lerner füllen am Computer einen Fragebogen innerhalb ihres Kurses aus

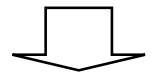

Auswertung der Daten mittels einer Datenbank

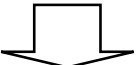

Daten eines speziellen Kurses

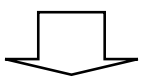

Ergebnis und Nutzen für die Partner:

- Evaluation ihres Kurses

- Qualitätsdokumentation

- Qualitätssicherung

- Vereinfachte Datensammlung und automatische Auswertung

- Qualität der Fragebögen (Wiederverwendbarkeit, Professionalität)

- Reflexion des eigenen beruflichen Handelns

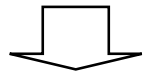

Daten aller Kurse, in denen die Inventare genutzt wurden

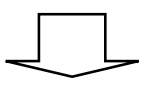

Ergebnis und Nutzen für das blinc-Netzwerk:

- Aufbau von Vergleichsdaten

- Aufbau von Wissen über Lerner im Kontext von Blended Learning im Dritten Sektor in Europa

- Sukzessive Eichung bzw. Optimierung der Inventare durch deren Einsatz

- Synergieeffekte: Teilen von „,best-proven-practice“

Abbildung 13: Mögliche Onlineform von dynamischen Inventaren für Lerner mit einer Datenbank im Hintergrund 
Eine gemeinsame Datenbank wäre nach Meinung der Arbeitsgruppe wünschenswert, jedoch schien eine Programmierung von dynamischen Inventaren durch die Projektpartner innerhalb des Projektes nicht möglich. Außerdem stellte sich die Frage, ob alle Projektpartner ihre Daten in eine gemeinsame Datenbank geben würden bzw. dürften (z.B. Datenschutz, Erlaubnis durch die jeweiligen Verantwortlichen der Bildungseinrichtungen und Institutionen). Es wurde außerdem gefordert, dass jeder Partner selbst entscheiden können müsse, ob er die individuellen Fragebögen seinen Lernern papierbasiert oder in elektronischer Form anbieten wollte. Die Daten könnten dann zwar ggf. nachträglich in die Datenbank eingegeben werden, um eine gemeinsame Wissensbasis aufbauen zu können, dies würde aber einen erheblichen zeitlichen und personellen Aufwand bedeuten.

In der Projektgruppe wurde entschieden, dass alle Projektpartner für die Erprobung der Inventare selbst entscheiden sollten, in welcher Form sie die Erhebungen durchführen wollten, je nach Lernergruppe bzw. eigenen Möglichkeiten der Auswertung. Es sollten zunächst vor allem Erfahrungen und Ergebnisse mit den Lernerinventaren im Allgemeinen gesammelt werden. Eine gemeinsame Datenbank könnte dann zu einem späteren Zeitpunkt mit der Endversion der Inventare angelegt werden. Den Projektpartnern wurde freigestellt, ob sie die Auswertung selbst durchführen wollten, oder ob sie die Auswertung von einem Projektpartner abgenommen bekommen wollten. Es wurde angeboten, dass die ausgefüllten Fragebögen an das Pädagogische Seminar geschickt werden konnten und eine Auswertung individuell erstellt werden würde. Auf diese Weise wäre eine Dateneingabe und Auswertung an einer zentralen Stelle möglich und den Projektpartnern würde eine zeitintensive Eingabe der Daten erspart bleiben. Manche Partner hatten zudem keine bzw. kaum Erfahrung mit der Eingabe bzw. Auswertung von Daten (z.B. Codierung, statistische Auswertung). Die Projektgruppe einigte sich darauf, dass das Programm Excel zur Auswertung verwendet werden sollte, da die meisten Partner im Gegensatz zum Programm SPSS einigermaßen vertraut damit waren.

\section{Begriffe: Kurs, Seminar, Programm, Bildungsmaßnahme}

In der Arbeitsgruppe wurde bei der Entwicklung von Fragen darüber diskutiert, welcher Begriff für einen Blended Learning-Kurs angemessen sei. Manche Projektpartner boten Weiterbildungskurse für ihre Mitarbeiter an, so dass sich der Begriff „Kurs“ eignete z.B. „Wie hat Ihnen der Kurs gefallen?“. Andere Projektpartner hatten aber ganze Bildungsprogramme oder ein Seminar für Studenten über ein halbes Jahr oder sogar noch länger als Blended Learning-Angebot für ihre Lerner entwickelt. In diesem Zusammenhang würde sich eher anbieten, die Lerner zu fragen: „Wie hat Innen die Bildungsmaßnahme, das Programm bzw. das Seminar gefallen?“. 
Die Inventare sollten von möglichst vielen verschiedenen Partnern in Europa genutzt werden können, ohne dass zu viele Veränderungen oder Umformulierungen der Fragen vorgenommen werden müssten. Die Arbeitsgruppe einigte sich auf den Begriff „Kurs“, da er in die verschiedenen Sprachen gut übersetzt werden konnte und außerdem nahe an der Alltagssprache der Lerner war.

\section{Kursinhalt und Lernmethoden}

Die Idee der Inventare beinhaltete, dass möglichst viele verschiedene Partner die Inhalte in ihren Kursen nutzen können sollten. Die Projektpartner arbeiteten zwar alle im Dritten Sektor, jedoch waren die Kursinhalte sehr verschieden (z.B. Englischkurs, Hygiene im Krankenhaus, Arbeitsplatzsuche). Es war vor diesem Hintergrund unmöglich, inhaltliche Aspekte der Kurse zu berücksichtigen. Das Gemeinsame aller Kurse der Projektpartner war jedoch die Methode Blended Learning. Alle Projektpartner boten ihren Lernern an, die Kursinhalte durch eine Kombination verschiedener Lernmethoden zu lernen. Charakteristisch für alle Kurse ist demnach der Wechsel von Präsenz- und Onlinephasen. Der Fokus bei der Entwicklung der Inventare für Lerner richtete sich deshalb auf die Methodik des Blended Learning an sich.

\section{Kulturelle Unterschiede bei der Befragung}

Es stellte sich heraus, dass es nationale Unterschiede bei der Befragung von Lernern gab, die u.U. sogar auf kulturelle Unterschiede zurückzuführen waren. In Italien ist es z.B. nach Meinung der Arbeitsgruppenmitglieder aus diesem Land nicht üblich, nach dem Familienstand der Lerner bzw. Mitarbeiter zu fragen, da dieses Privatsphäre sei. An dieser Stelle schien die Idee der Inventare besonders sinnvoll, da die Frage nach dem familiären Hintergrund individuell ausgelassen werden kann. Bei der weiteren Arbeit der Projektgruppe traten kulturelle Unterschiede jedoch in den Hintergrund und man konzentrierte sich mehr auf Gemeinsamkeiten, die für die Befragung von Lernern nach den bisherigen Erfahrungen und dem Wissen der Projektgruppe relevant schienen.

\section{Schriftgröße und Layout}

In der Arbeitsgruppe wurde über die Gestaltung der Inventare diskutiert. Die Fragen sollten für die Lerner vor allem gut lesbar sein, d.h. die Schriftgröße sollte etwa 10-12 pt betragen. Die Befragten sollten nicht mit zu kleiner oder enger Schrift konfrontiert werden, da dies abschreckend wirken könnte und es ggf. zu lückenhafter Beantwortung bzw. zur Verweigerung führen würde. Es sollte ebenfalls eine Schriftart gewählt werden, die gut lesbar ist. Das Layout der Inventare war nach Meinung der Arbeitsgruppe wichtig, um die Bereitschaft der Befragten zu erhöhen, sich zu beteiligen (z.B. Übersichtlichkeit, Textblöcke). Außerdem wurde überlegt, dass die Inventare für Lerner und die anderen Inventare, die in anderen Arbeitsgruppen entwickelt wurden, ein einheitliches Design haben sollten, um der Zusammengehö- 
rigkeit der Inventare auch visuell Rechnung zu tragen. Für die potenziellen Anwender sollte es aber auch möglich sein, Fragen bzw. einen individuellen Fragebogen so zu gestalten, dass er zu der Einrichtung passt, in der er eingesetzt wird (z.B. Firmenlogo, Corporate Identity eines Unternehmens). Die Inventare sollten zum einen in einer Endversion „sofort einsetzbar" sein, und zum anderen sollten die Nutzer die Inhalte verwenden können, wie es ihren Bedürfnissen und ihrem Kontext entspricht.

\section{Umfang der Inventare bzw. der individuellen Fragebögen}

Nach den Erfahrungen der Arbeitsgruppe ist die Motivation seitens der Lerner, Fragebögen auszufüllen, nicht besonders groß. Ein Fragebogen sollte deshalb im Umfang nicht zu groß sein, d.h. er sollte möglichst wenige Seiten umfassen. Zwischen der Länge bzw. Kürze des Fragebogens einerseits und der Schriftgröße andererseits müsse ein gutes Maß gefunden werden. Im individuellen Fragebogen der Projektpartner sollten nur die Fragen enthalten sein, die unbedingt für die Evaluation des Kurses benötigt würden, da die Zeit für das Beantworten möglichst kurz gehalten werden solle, um die Lerner nicht unnötig zu belästigen bzw. innen zu viel abzuverlangen.

\section{Formulierung von Antwortvorgaben versus offene Fragen}

Die Arbeitsgruppe war sich einig, dass Antwortvorgaben zu einer besseren Vergleichbarkeit der Ergebnisse unterschiedlicher europäischer Projektpartner führen würden. Eine Quantifizierung der Ergebnisse würde durch Antwortvorgaben erleichtert. Es wurde aber auch diskutiert, dass in manchen Bereichen offene Fragen sinnvoll seien, da bisher wenige Erkenntnisse über das Lernerverhalten bzw. deren subjektive Sichtweise vorliegen (z.B. „Was hat Ihnen am Blended Learning gefallen?“ oder „Womit hatten Sie Schwierigkeiten?“). Einerseits würden eigenständig formulierte Antworten der Lerner inhaltlich mehr bringen und es könnte Wissen im blinc-Netzwerk zusammengetragen und ausgetauscht werden, andererseits würde dies eine Auswertung erschweren, zumal die Lerner in unterschiedlichen Muttersprachen befragt würden.

Es wurden möglichst viele Antwortvorgaben formuliert, aber in einigen Bereichen wurden auch offene Fragen entwickelt. Es wurde entschieden, dass zu einem späteren Zeitpunkt aus den Antworten der Lerner Kategorien oder Skalen entwickelt werden könnten, wenn man mehr über das Spektrum der Antworten wüsste.

\section{Copyright}

Es wurde diskutiert, ob die Inventare, die innerhalb des Projektes eL3 entwickelt wurden frei verfügbar auf der Internetseite von blinc angeboten werden sollten, und wer der Autor der Inventare sein sollte (z.B. die betreffende Arbeitsgruppe oder die gesamte Projektgruppe?). Eine weitere Frage war, ob die Ergebnisse, die mit den individuellen Fragebögen erhoben 
würden, auf der Internetplattform öffentlich einsehbar sein sollten oder nicht. Zum einen wurde argumentiert, die Ergebnisse könnten in anonymisierter Form öffentlich sein, da jeder, der die Inventare benutzen möchte, auch zu deren Optimierung beitragen sollte. Zum anderen betonten manche Partner, dass die Verantwortlichen ihrer Einrichtungen sicher nicht erlauben würden, interne Daten öffentlich einsehbar darzustellen. Eine Anonymisierung sei zwar möglich, aber u.U. könnten Rückschlüsse auf einzelne Partner gezogen werden. Einige Partner merkten an, dass z.B. im Krankenhaussektor Konkurrenz zwischen verschiedenen Häusern herrsche und dass mit „Betriebsgeheimnissen“ (Erfolgen wie Misserfolgen) sehr sensibel umgegangen werden müsse.

In Bezug auf das geistige Eigentum (Copyright) der Inventare kam die Arbeitsgruppe zu folgendem Ergebnis: In einer Einführung zum Entstehungszusammenhang der Inventare sollten auf der ersten Seite diejenigen genannt werden, die maßgeblich die Inhalte der Inventare erarbeitet hatten. Jeder Partner, der die Inventare verwenden wollte, sollte darauf verweisen, woher er die Inhalte hatte (Internetseite von blinc, Autoren der Inventare und Jahresangabe). Darüber hinaus sei eine kommerzielle Vermarktung der Inventare über die Internetseite von blinc möglich. Dort könnte die Evaluation von Kursen mit den Inventaren, die im Projekt eL3 entwickelt wurden, angeboten werden. In diesem Fall würden ebenfalls die Autoren (die Arbeitsgruppe) entsprechend benannt.

\section{Phase 3: Gemeinsamer Entwurf und Übersetzung}

Die Arbeitsgruppe hatte nach neun Monaten Austausch und Diskussion von September 2004 bis Mai 2005 eine gemeinsame Version der Lernerinventare erarbeitet. Auf einem Treffen wurden die Inventare der gesamten Projektgruppe vorgestellt. Insgesamt standen nun drei Inventare für Lerner zur Verfügung, die zu unterschiedlichen Befragungszeitpunkten im Verlauf eines Blended Learning-Kurses eingesetzt werden konnten:

1. Inventar für Lerner zu Beginn eines Blended Learning Kurses

2. Inventar für Lerner während eines Blended Learning Kurses

3. Inventar für Lerner am Ende eines Blended Learning Kurses

Die Arbeitsgruppe beschrieb auf den ersten Seiten der Inventare die Fragenbereiche und deren Zweck für eine Kursevaluation, wodurch den potenziellen Nutzern verständlich gemacht werden sollte, was die Arbeitsgruppe sich bei der Konstruktion der Inventare bzw. einzelner Fragenbereiche gedacht hatte. Außerdem wurde ein Einleitungstext für den Fragebogen entworfen, mit dem die Lerner über den Sinn der Befragung informiert werden sollten und Hinweise auf Datenschutz und Verwendung der Daten gegeben wurden. Ein Einleitungstext war nach Erfahrung der Arbeitsgruppe für Befragte sehr wichtig, um deren Motivation zu erhöhen, sich an der Befragung zu beteiligen. Alle drei Inventare umfassten sechs 
Themenbereiche, wobei es für den ersten Einsatz noch einen siebten Bereich gab, in dem die Befragten Rückmeldungen zum Fragebogen eintragen konnten. Die Themenbereiche, die von der Arbeitsgruppe erarbeitet wurden waren:

- Kontext des Kurses.

- Demographische Daten der Lerner und Lernvoraussetzungen für e-Learning.

- Lernerfahrungen mit verschiedenen Lehrmethoden und Vorwissen.

- Ressourcen für e-Learning, Fähigkeiten und Wissen im Umgang mit dem Computer.

- Persönliche Motivation, an dem Kurs teilzunehmen, Erwartungen und Ziele.

- Platz für Vorschläge und Kommentare.

- Platz für Kommentare zum Fragebogen.

Die Inventare wurden im Rahmen des Projektes eL3 in Englisch erarbeitet, da dies die vereinbarte Arbeitssprache war. Es wurde eine Masterversion der Inventare auf Englisch entwickelt, die dann in die verschiedenen Sprachen der Projektmitglieder übersetzt werden sollte. Die Übersetzung sollte im Juni 2005 abgeschlossen sein, damit die Inventare dann in den verschiedenen Sprachen eingesetzt werden konnten. Grundsätzlich war dies möglich, es ist jedoch anzumerken, dass es für einige Partner recht schwierig war, bestimmte Begriffe oder Formulierungen zu übersetzen. Dies zeigte sich schon in den Diskussionen während der Projekttreffen. Eine Verständigung in der Arbeitssprache Englisch war möglich, jedoch zum Teil mit Verständigungsproblemen verbunden. Bei der Übersetzung der Inventare durch die Partner könnten sich Fehler einschleichen, die nicht bemerkt würden, da nicht alle Projektmitglieder die verschiedenen Sprachen beherrschen. Es könnte passieren, dass eine Frage aus den Inventaren bei der Übersetzung verfälscht würde und etwas anderes gefragt würde als in der Masterversion vorgesehen war. Die Ergebnisse könnten u.U. aus testtheoretischer Sicht nicht miteinander verglichen werden. Üblicherweise würde die Masterversion von unabhängigen und professionellen Personen übersetzt und rückübersetzt werden und erst, wenn beide Versionen übereinstimmten, könnten die Fragen eingesetzt werden. Diese Vorgehensweise war jedoch im Projekt eL3 nicht möglich, da keine Mittel dafür vorhanden waren. Die Inventare wurden von den Projektpartnern selbst übersetzt. Für die Nachprüfbarkeit der Übersetzungen wurden alle Sprachen in einem Dokument gesammelt (multilinguale Version), damit die Übersetzungen für alle einsehbar waren. Außerdem konnten so zu einem späteren Zeitpunkt noch andere Sprachen ergänzt werden, wenn das Netzwerk vergrößert würde. 


\begin{tabular}{|l|l|}
\hline 2b) Wie alt sind Sie? & $\ldots \ldots$ Jahre \\
\hline 2b) How old are you? & $\ldots$. years \\
\hline 2b) Quanti anni ha? & $\ldots \ldots$ anni \\
\hline 2b) Quel âge avez-vous? & $\ldots \ldots$ ans \\
\hline 2c) Welches Geschlecht haben Sie? & $\begin{array}{l}\text { O Männlich } \\
\text { O Weiblich }\end{array}$ \\
\hline 2c) What is your sex? & $\begin{array}{l}\text { O Male } \\
\text { O Female }\end{array}$ \\
\hline 2c) Qual è il Suo sesso? & $\begin{array}{l}\text { OMaschio } \\
\text { OFemmina }\end{array}$ \\
\hline 2c) Quel est votre sexe? & $\begin{array}{l}\text { O Masculin } \\
\text { O Féminin }\end{array}$ \\
\hline
\end{tabular}

Abbildung 14: Ausschnitt aus der multilingualen Version der Inventare für Lerner

Die Inventare wurden außerdem in jeder Sprache einzeln zur Verfügung gestellt, damit es für die Partner einfacher und übersichtlicher war, ihre individuellen Fragebögen zu erstellen.

\section{Phase 4: Erprobung und Ergebnisbericht}

Auf dem Projekttreffen im Mai 2005 wurde vereinbart, welche Partner welche Inventare für Lerner in ihren Kursen nutzen könnten. Alle Projektpartner gaben zu diesem Zeitpunkt an, die Inventare nutzen zu wollen, einige wollten vor Kursbeginn, während des Kurses und am Ende des Kurses die Inventare einsetzen, andere wollten zu Beginn und am Ende eine Evaluation durchführen, und manche planten, nur am Ende ihres Kurses die Lerner zu befragen. Die Projektpartner konnten die Inventare vollkommen selbstständig einsetzen oder auch Unterstützung von einem mit Evaluation erfahrenen Partner (Pädagogisches Seminar) bekommen. Diejenigen, die eine Selbstevaluation durchführen wollten, hatten folgende Arbeitsschritte zu tun:

- Auswahl der Fragen, die für ihren Kurs relevant sind.

- Erstellung eines Fragebogens.

- Einsatz und Auswertung der Fragebögen.

- Bericht über die Ergebnisse.

Die anderen Projektpartner, die sich für eine Fremdevaluation entschieden hatten, mussten lediglich bei der Auswahl der Fragen mitbestimmen, welche Fragen für ihren Kurs sinnvoll schienen.

Der Ergebnisbericht sollte zum letzten Projekttreffen im November 2005 vorliegen, damit die Ergebnisse in der Projektgruppe diskutiert werden konnten. Außerdem sollte mit den Ergebnissen und Erfahrungen der Erprobung eine gemeinsame Endversion der Inventare erarbeitet werden können. Es sollten auch Überlegungen angestellt werden, wie die Ergebnisse 
verbreitet werden könnten, um über die Internetseite von blinc Wissen über Lerner im Kontext von Blended Learning auf europäischer Ebene aufzubauen bzw. frei zugänglich zu machen.

\section{Phase 5: Gemeinsame Endversion und Verbreitung}

Im November 2005 fand das letzte Projekttreffen statt und es erfolgte eine Bestandsaufnahme, welche Partner welche Inventare eingesetzt hatten und welche Ergebnisse damit erzielt wurden. Es ergaben sich Diskussionen darüber, wie die Erfahrungen und Ergebnisse mit der Evaluation von verschiedensten Kursen genutzt werden könnten. Zum einen musste eine gemeinsame Endversion der Inventare bis zum Ende des Projektes erarbeitet werden, wozu die Daten des ersten Einsatzes verwendet wurden. Es sollte überprüft werden, ob sich die verschiedenen Fragenbereiche bewährt hatten, ob einzelne Fragen neu bzw. anders formuliert werden müssten und welche Rückmeldungen die Projektpartner von ihren Lernern zu den Fragebögen bekommen hatten. Zum anderen sollte auf dem Projekttreffen überlegt werden, wie die Inventare in der Zukunft genutzt werden könnten (z.B. freie Verbreitung der Inhalte über die Internetseite von blinc oder auch kommerzielle Nutzung).

Die Endversion der drei Inventare für Lerner umfasste einen Einführungstext für potenzielle Nutzer der Inventare zur Erklärung des Entstehungszusammenhanges, einen MusterEinleitungstext für die Lerner (Zielgruppe der Befragung) sowie Fragen zu fünf Themenbereichen:

- Kontext des Kurses.

- Angaben zur Person und Lernvoraussetzungen für e-Learning.

- Lernerfahrungen mit verschiedenen Lehrmethoden und Vorwissen.

- Ressourcen für e-Learning, Fähigkeiten und Wissen im Umgang mit dem Computer.

- Persönliche Motivation an dem Kurs teilzunehmen, Erwartungen und Ziele.

Die Bereiche sechs und sieben aus der ersten gemeinsamen Version der Inventare, in denen Platz für Vorschläge und Kommentare zum Kurs bzw. Fragebogen vorgesehen war, wurden herausgenommen, da zum einen die Erprobungsphase abgeschlossen war und zum anderen nicht besonders viele freie Kommentare von Lernern hinterlassen wurden. Im Folgenden werden die Fragenbereiche näher erläutert:

\section{Kontext des Kurses}

Die Fragen dieses Bereiches dienen dazu, Namen, Ort und Datum des Kurses zu dokumentieren. Dies erleichtert eine spätere Zuordnung der Ergebnisse, wenn z.B. mehrere Kurse ausgewertet werden. Außerdem ist die Dokumentation des Kurskontextes für den Vergleich 
von Daten auf europäischer Ebene unerlässlich. Dieser Teil kann auch von der Kursleitung selbst ausgefüllt werden, bevor die Teilnehmer den Bogen erhalten.

\section{Angaben zur Person und Lernvoraussetzungen für e-Learning}

Mit den Fragen dieses Bereiches sollen die Kursleiter Informationen darüber erhalten, wer an ihrem Kurs teilnimmt (Alter, Geschlecht, Bildungsstand, Beruf). Die Frage nach dem Namen oder einem Codenamen ist sinnvoll, wenn während des Kurses oder nach dem Kurs eine erneute Befragung durchgeführt werden soll. Es können dann „vorher- und nachher“ - Antworten verglichen und individuelle Lernprozesse nachvollzogen werden. Es ist auch möglich, den Lernern die ausgefüllten Fragebögen zurückzugeben, damit sie ihren eigenen Lernfortschritt (z.B. Erfahrung im Umgang mit dem Computer) nachprüfen können. Die Lerner haben so die Möglichkeit, ihre eigenen Antworten zu einem späteren Zeitpunkt nachzulesen. In diesem Fall könnte sich die Kursleitung die ausgefüllten Fragebögen kopieren und sie dann wieder aushändigen, wofür allerdings Codenummern benötigt würden.

Eine andere Möglichkeit ist, den Fragebogen anonym ausfüllen zu lassen und die Daten nur für die Kursevaluation (Dokumentation und Qualitätssicherung) zu verwenden. Dies sollte wohlüberlegt und ggf. in Abstimmung mit der Lerngruppe entschieden werden.

Mit dem zweiten Abschnitt der Fragen zur Person sollen die individuellen Lernbedingungen der Teilnehmer erhoben werden. Es geht hier vor allem darum herauszufinden, wie viel Zeit die Lerner nach eigener Einschätzung zur Verfügung haben, um eigenständig e-LearningTeile des Kurses zu bearbeiten, und wo sie lernen wollen bzw. können.

\section{Lernerfahrungen mit verschiedenen Lehrmethoden und Vorwissen}

Mit den Fragen dieses Bereiches werden Daten über die Lernerfahrungen mit verschiedenen Lehrmethoden gesammelt. Das Vorwissen der Lerner in Bezug auf die Seminarinhalte wird erfasst. Außerdem wird ermittelt, welche Methoden die Kursbesucher bereits kennen und wie innen diese Methoden gefallen haben. Falls sich z.B. ausschließlich Kursteilnehmer, die noch nicht mit e-Learning vertraut sind, in einem Kurs befinden, sollte der Umgang mit dem Computer für das Lernen entsprechend eingeführt werden.

\section{Ressourcen für e-Learning, Fähigkeiten und Wissen im Umgang mit dem Computer} Mit den Fragen dieses Bereiches werden Informationen darüber gesammelt, welche Ressourcen den Kursteilnehmern für e-Learning zur Verfügung stehen und wie viel Erfahrung sie im Umgang mit dem Computer haben. Die Teilnehmer sollen sich dabei selbst einschätzen. 


\section{Persönliche Motivation an dem Kurs teilzunehmen, Erwartungen und Ziele}

In diesem Teil wird nach den persönlichen Gründen und der Motivation der Teilnehmer, am Kurs teilzunehmen, gefragt. Es wird nach Zielen und Erwartungen, aber auch nach Sorgen der Lerner gefragt. Während des Kurses und auch am Ende kann durch eine erneute Befragung überprüft werden, ob die Erwartungen erfüllt wurden. Außerdem ist es möglich zu reagieren, wenn jemand Sorgen oder Ängste äußert.

In den Inventaren für Lerner variiert die Anzahl der Items, je nachdem, zu welchem Zeitpunkt sie eingesetzt werden sollen. So umfasst das Inventar für Lerner, das zu Beginn des Kurses eingesetzt werden kann, insgesamt 28 Items, ebenso wie das Inventar für Lerner, das während des Kurses verwendet werden kann. Das Inventar für Lerner, das am Ende eingesetzt werden kann, umfasst hingegen 42 Items, da noch einige Fragen zu der abschließenden Bewertung der e-Learning-Teile enthalten sind (z.B. Betreuung durch die Tutoren, Bedienbarkeit der Lernplattform). Der Aufbau der drei Inventare für Lerner ist jedoch gleich und die Inhalte einzelnen Bereiche sind nahezu identisch. Die folgende Tabelle gibt einen Überblick über die Inhalte der Inventare für Lerner: 


\begin{tabular}{|c|c|}
\hline Themenbereich & Ziele und Inhalte \\
\hline Einleitungstext & $\begin{array}{l}\text { Erklärungen für die Kursteilnehmer } \\
\text { - Zweck des Fragebogens } \\
\text { - Erwarteter Nutzen der Befragung } \\
\text { - Informationen dazu, wer den Fragebogen } \\
\text { entwickelt hat und ihn auswerten wird } \\
\text { - Hinweis auf Datenschutz } \\
\text { - Gewährleistung der Anonymität } \\
\text { - Dank für die Beteiligung }\end{array}$ \\
\hline 1. Kontext des Kurses & $\begin{array}{l}\text { Dokumentation von Kursdaten } \\
\text { - Kursname, Ort und Datum }\end{array}$ \\
\hline $\begin{array}{l}\text { 2. Demographische Daten der Lerner und } \\
\text { Lernvoraussetzungen für e-Learning }\end{array}$ & $\begin{array}{l}\text { Informationen über die Kursteilnehmer } \\
\text { - „Wer ist im Kurs?“ (Alter, Geschlecht, Kinder, } \\
\text { Nationalität, Bildungsstand, Beruf) } \\
\text { - Lernvoraussetzungen für e-Learning zu Hau- } \\
\text { se und am Arbeitsplatz (Lernort, Lernzeit, } \\
\text { Einschränkungen) }\end{array}$ \\
\hline $\begin{array}{l}\text { 3. Lernerfahrungen mit verschiedenen } \\
\text { Lehrmethoden und Vorwissen }\end{array}$ & $\begin{array}{l}\text { Voraussetzungen der Teilnehmer } \\
\text { - Lernerfahrungen mit Präsenzunterricht, } \\
\text { e-Learning, Projektlernen und Blended } \\
\text { Learning (Häufigkeit des Erlebens und Zu- } \\
\text { friedenheit mit den Methoden) } \\
\text { - Vorwissen in Bezug auf die Kursinhalte } \\
\text { - Erfahrungen mit selbstgesteuertem Lernen }\end{array}$ \\
\hline $\begin{array}{l}\text { 4. Ressourcen für e-Learning, Fähigkeiten } \\
\text { und Wissen im Umgang mit dem Computer }\end{array}$ & $\begin{array}{l}\text { Bedingungen für e-Learning } \\
\text { - Erfahrung im Umgang mit dem Computer } \\
\text { - Internetzugang } \\
\text { - Lernzeiten } \\
\text { - Hilfspersonen für e-Learning }\end{array}$ \\
\hline $\begin{array}{l}\text { 5. Persönliche Motivation an dem Kurs teil- } \\
\text { zunehmen, Erwartungen und Ziele }\end{array}$ & $\begin{array}{l}\text { Persönliche Einstellungen zum Kurs } \\
\text { - Motivation zur Kursteilnahme } \\
\text { - Erwartungen } \\
\text { - Befürchtungen }\end{array}$ \\
\hline 6. Platz für Vorschläge und Kommentare & $\begin{array}{l}\text { Interessen, Vorschläge und Wünsche } \\
\text { - Zukünftige Kursangebote } \\
\text { - Kommentare zum Kurs }\end{array}$ \\
\hline 7. Platz für Kommentare zum Fragebogen & $\begin{array}{l}\text { Rückmeldung zum Fragebogen } \\
\text { - Verständlichkeit der Fragen } \\
\text { - Länge des Fragebogens }\end{array}$ \\
\hline Dank & Dank für das Ausfüllen des Fragebogens \\
\hline
\end{tabular}

Tabelle 37: Übersicht über die Inhalte der Inventare für Lerner 
Die Inventare für Lerner wurden am Ende des Projektes eL3 auf den Internetseiten von der blinc-Gemeinschaft als pdf-Dokumente und als Word-Dokumente zur Verfügung gestellt. Auf diese Weise können die Inhalte der erarbeiteten Inventare von potenziellen Nutzern frei verwendet werden.

\subsubsection{Das Inventar für Autoren}

Zu Beginn der Planungen zur Entwicklung von Evaluationsinstrumenten für e-Learning und Blended Learning-Kurse wurden drei Zielgruppen identifiziert, wobei Autoren eine davon sind. Autoren wurden definiert als Personen, die e-Learning und Blended Learning-Kurse entwickeln. Für diese Zielgruppe ist es besonders wichtig, die Teilnehmer ihrer Kurse, die Lerner, zu kennen, damit sie das didaktische Design des Kurses entsprechend gestalten können. Im Gegensatz zu Kursen, in denen eine Lehrperson real mit den Lernern zusammen trifft, kann bei e-Learning und Blended Learning-Kursen das Design nicht spontan während der Interaktion verändert werden. Autoren sollten deshalb sorgfältig das didaktische Design des Kurses planen und Kriterien effektiver Lehre berücksichtigen.

\section{Phase 1: Recherche und Sammlung}

Es wurde mit einer Literaturrecherche zum Thema didaktische Gestaltung von e-Learning und Blended Learning-Kursen begonnen. Zusätzlich wurde im Internet recherchiert, welche Instrumente es zur Evaluation des didaktischen Designs von Kursen gibt. Es fand sich zwar Literatur zum Thema e-Learning - Didaktik (z.B. Schüpbach et al 2003, Swertz 2004, Meder 2006, Niegemann et al 2004), aber es gab keine Instrumente zur Evaluation des didaktischen Designs von e-Learning und Blended Learning-Kursen. Baumgartner (2003) weist aber auch darauf hin, dass es keine neue bzw. eigene Didaktik für e-Learning und Blended Learning geben müsse, sondern dass vielmehr bisher vorhandene Theorien und Ergebnisse aus der Didaktikforschung darauf angepasst werden müssten. Durch den Medieneinsatz sei nur die Komplexität erhöht, und es müssten die vorhandenen Theorien unter den neuen Bedingungen überprüft und weiterentwickelt werden. Demnach könnten Wissensinhalte und Erfahrungen aus der Unterrichtsforschung und der Didaktik auf e-Learning und Blended Learning-Kurse übertragen und für die Gestaltung des didaktischen Designs verwendet werden. Zum Begriff didaktisches Design siehe Flechsig (1987). An dieser Stelle sei nur kurz beschrieben, worum es dabei geht:

\footnotetext{
„Didaktisches Design im weitesten Sinne bezieht sich auf den Entwurf, die Planung, Gestaltung und Nutzung von Lernumwelten. Der Begriff bezeichnet somit Prozesse ebenso wie Produkte. Didaktisches Design ist insofern dem Design in der Architektur vergleichbar."
} 
Für den ersten Entwurf des Inventars für Autoren wurde auf die Arbeit von Lewis und Witlock (2003) zurückgegriffen, die betonen, wie wichtig es für Autoren von e-Learning und Blended Learning ist, ihre Zielgruppe - die Lerner - und deren Bedürfnisse zu kennen, um einen Kurs optimal zu gestalten. In traditionellen Lernumgebungen kann der Unterrichtsstil in der Interaktion mit den Lernern verändert und an die aktuelle Situation angepasst werden. Bei eLearning oder Blended Learning-Kursen wird das Lernen bzw. der Unterricht ohne einen Kontakt zu den Lernen geplant und ist danach relativ statisch. Deshalb sollten Autoren bzw. Gestalter von e-Learning oder Blended Learning-Kursen ihre Lerner sehr gut kennen und einige systematische Reflexionen über ihre Zielgruppe an den Anfang ihrer Arbeit stellen.

Für die Entwicklung des ersten Entwurfes des Inventars für Autoren wurden außerdem Elemente aus den Arbeiten von Flechsig und Haller (Flechsig 1983, 1990, 1996a, 1996b, Haller 1995, 1997, 2002, 2003) verwendet. Bereits Ende der 1980er Jahre hatten Karl-Heinz Flechsig und Hans-Dieter Haller damit begonnen, den Göttinger Katalog Didaktischer Modelle (Flechsig 1983, 1996) in einem computerbasierten System für die Gestaltung des didaktischen Designs von Kursen nutzbar zu machen. Mit dem Programm CEDID (Computerergänztes Didaktisches Design) legten sie ein System vor, das Lehrende bzw. didaktische Designer für die Planung, Dokumentation und Evaluierung von Kursen nutzen können:

\begin{abstract}
„Als sogenanntes tätigkeitsunterstützendes und wissensbasiertes System unterstützt es die Ausübung der Tätigkeiten didaktischen Designs, indem es den Design-Prozeß begleitet und didaktisches Wissen in interaktiver Weise zugänglich macht. Es entwickelt nicht nur das Expertenwissen, sondern auch den Experten weiter." (Flechsig, 1990, S.4)
\end{abstract}

Neben der Bereitstellung von Wissensbasen werden in CEDID verschiedene DesignOperationen unterstützt, wie etwa eine Kontextanalyse, die Auswahl Didaktischer Modelle, das Kursdesign und die Erprobung eines Kurses. Zu den Design-Operationen gehört auch die Design-Evaluierung. Das Ziel bei dieser Design-Evaluierung ist, die Qualität eines entwickelten Kurses im Sinne einer „Inspektions-Evaluierung“ zu überprüfen. Im Gegensatz zu einer „Wirkungs-Evaluierung“ geht es bei der „Inspektions-Evaluierung“ darum, die Qualität eines Kurses gedanklich zu kontrollieren, bevor dieser durchgeführt wird.

„Ziel der Design-Evaluierung ist es nun, nach der Arbeit an den einzelnen Blöcken festzustellen, ob das ganze Stück stimmt. Dies geschieht, indem der Designer sich zunächst selbst mit einer Art Prüfliste gegenübertritt, um sozusagen aus der Perspektive des Kontrolleurs sein eigenes Werk zu betrachten." (Flechsig, 1990, S.47)

In CEDID werden sieben Gesichtspunkte bereitgestellt, unter denen die Bewertung der Qualität des didaktischen Designs erfolgen soll: 
1. Vielfalt

2. Ökonomie

3. Angepasstheit

4. Produktivität

5. Selbstmotivation

6. Persönlichkeitsbildung

7. Stimmigkeit

Für jeden Bereich gibt es etwa zehn Aussagen, mit denen geprüft werden kann, inwieweit dieses Kriterium bei der Erstellung des didaktischen Designs berücksichtigt wurde. Die Ergebnisse der Design-Evaluierung nach diesen Prüfkriterien sollen dem Anwender Hinweise auf Veränderungs- und Verbesserungsmöglichkeiten geben (vgl. Flechsig, 1990 S. 49). Diese Prüfkriterien wurden in dem ersten Entwurf des Autoreninventars aufgenommen und an den Projektkontext von eL3 bzw. e-Learning und Blended Learning angepasst.

In den ersten Entwurf des Autoreninventars wurden außerdem Kriterien wirksamer Lehre aufgenommen. Auf der Basis empirischer Forschungsliteratur entwickelte Haller (2003) einen Katalog, der folgende Prinzipien (sieben Säulen) für wirksames Lehren bzw. sieben Merkmale wirksamen Lehrens enthält:

1. Klarheit

2. Relevanz und Bezug

3. Struktur und Sequenz

4. Anschaulichkeit der Darstellungsformen

5. Aufgabenorientierung

6. Rückmeldung

7. Anregungen

Für jedes Prinzip wurden Aussagen formuliert anhand derer geprüft werden kann, inwiefern es bei der Planung und Gestaltung eines Lernangebots Berücksichtigung gefunden hat. Die Ergebnisse dieser Überprüfung sollen dem Autoren, der sein Lehrangebot überprüft, Hinweise auf Veränderungs- und Verbesserungsmöglichkeiten geben. Die Prinzipien für wirksames Lehren sollen sowohl für traditionellen Präsenzunterricht als auch für e-Learning und Blended Learning gelten. Es wurde angestrebt, die Liste der Aussagen mit den Projektpartnern im Rahmen des Projektes eL3 und darüber hinaus zu ergänzen bzw. zu erweitern

Insgesamt wurden für den ersten Entwurf des Autoreninventars die folgenden drei thematischen Schwerpunkte herausgearbeitet: 
Teil A Adressatenmerkmale

Leitfrage: Kenne ich meine Lerner und deren Bedürfnisse?

Teil B Qualität des Didaktischen Designs

Leitfrage: Habe ich Kriterien eines guten didaktischen Designs genügend berücksichtigt?

Teil C Kriterien wirksamer Lehre

Leitfrage: Habe ich Kriterien wirksamer Lehre genügend berücksichtigt?

\section{Phase 2: Diskussion und Austausch}

Während des Projekttreffens im September 2004 wurde, wie auch für die Zielgruppe der Lerner, eine Arbeitsgruppe gegründet, die bis zum Ende des Projektes speziell für die Entwicklung des Inventars für Autoren zuständig war. Die Gruppe bestand aus fünf Personen, die aus Schottland, Frankreich, Italien und Deutschland stammten, von ihrem beruflichen Kontext her in der Lehre und Gestaltung von Kursen in Universitäten und Bildungseinrichtungen tätig waren und auch Erfahrung mit der Evaluation ihrer eigenen Kurse hatten.

Zunächst diskutierte die Arbeitsgruppe während des Projekttreffens in Aberdeen über den ersten Entwurf des Inventars aus Phase 1 (siehe oben). Für die weitere Entwicklung konnte die Arbeitsgruppe eine projektinterne Lernplattform nutzen. Eine Person übernahm die Führung und Koordination der Gruppe, formulierte verschiedene Arbeitsschritte und stellte auf der Lernplattform die Materialien für die weitere Arbeit bereit. Die Gruppe sollte innerhalb von neun Monaten eine gemeinsame Version des Inventars für Autoren entwickeln. Der Entwicklungsprozess umfasste folgende Arbeitsschritte:

1. Präsentation eines ersten gemeinsamen Entwurfes des Inventars für Autoren nach der Diskussion der Arbeitsgruppe in Aberdeen.

2. Diskussion über die neue Version im Forum und Sammlung von Instrumenten der beteiligten Partner.

3. Präsentation einer überarbeiteten Version des Inventars.

4. Diskussion und Austausch im Forum.

5. Abschluss und gemeinsame Endversion (Masterversion in Englisch).

6. Endversion in verschiedenen Sprachen.

Nach dem transnationalen Projekttreffen in Aberdeen und den dortigen Diskussionen zum ersten Entwurf des Autoreninventars wurden die Anmerkungen der Arbeitsgruppenmitglieder vom Arbeitsgruppenleiter in das Autoreninventar eingearbeitet.

Die neue Version wurde auf der Lernplattform für alle einsehbar hinterlegt. Die Partner wurden per E-Mail und auf der Plattform gebeten, diese Version anzuschauen und eigene Materialien mit der vorhandenen Sammlung zu vergleichen. Die Arbeitsgruppe sollte Kommentare 
und Verbesserungsvorschläge zur aktuellen Version des Autoreninventars im Forum äußern, oder per E-Mail an den Arbeitsgruppenleiter schicken. Die folgende Abbildung zeigt die Anleitung für die Partner auf der Lernplattform, was sie für die Weiterentwicklung des Autoreninventars als nächstes in welchem zeitlichen Rahmen zu tun hatten und welches Material sie dafür verwenden sollten:

\section{Step 1: Presentation of the results of the workgroup from Aberdeen - Microsoft Internet Explorer}

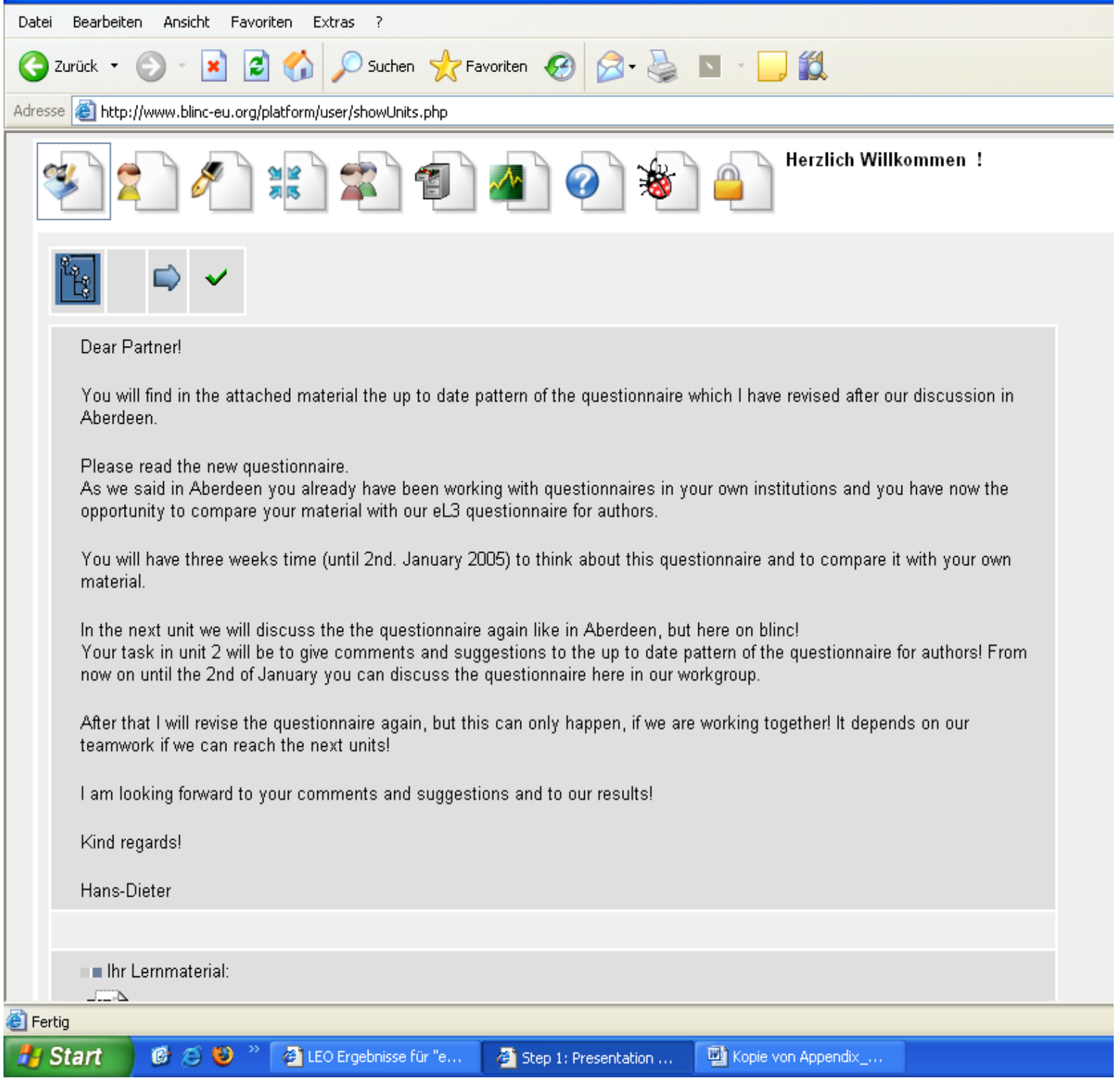

Abbildung 15: Anleitung für die Weiterentwicklung des Autoreninventars auf dem Lernmanagementsystem

Die Partner wurden gebeten, sich das Autoreninventar, das nach der Diskussion während des transnationalen Treffens in Aberdeen durch den Arbeitsgruppenleiter überarbeitet wurde, erneut anzuschauen und mit eigenen Materialien zu vergleichen. Sie hatten drei Wochen Zeit, sich den neuen Entwurf des Autoreninventars anzuschauen und mit vorhandenen Fragebögen oder Checklisten zu vergleichen, die sie bereits selbst verwendeten. Sie wurden 
gebeten, Kommentare und Vorschläge zur aktuellen Version des Autoreninventars im Forum zu hinterlassen.

In dieser Arbeitsphase hinterließen die Partner der Arbeitsgruppe für das Autoreninventar jedoch keine inhaltlich relevanten Beiträge im Forum und kontaktierten den Arbeitsgruppenleiter auch nicht per E-Mail.

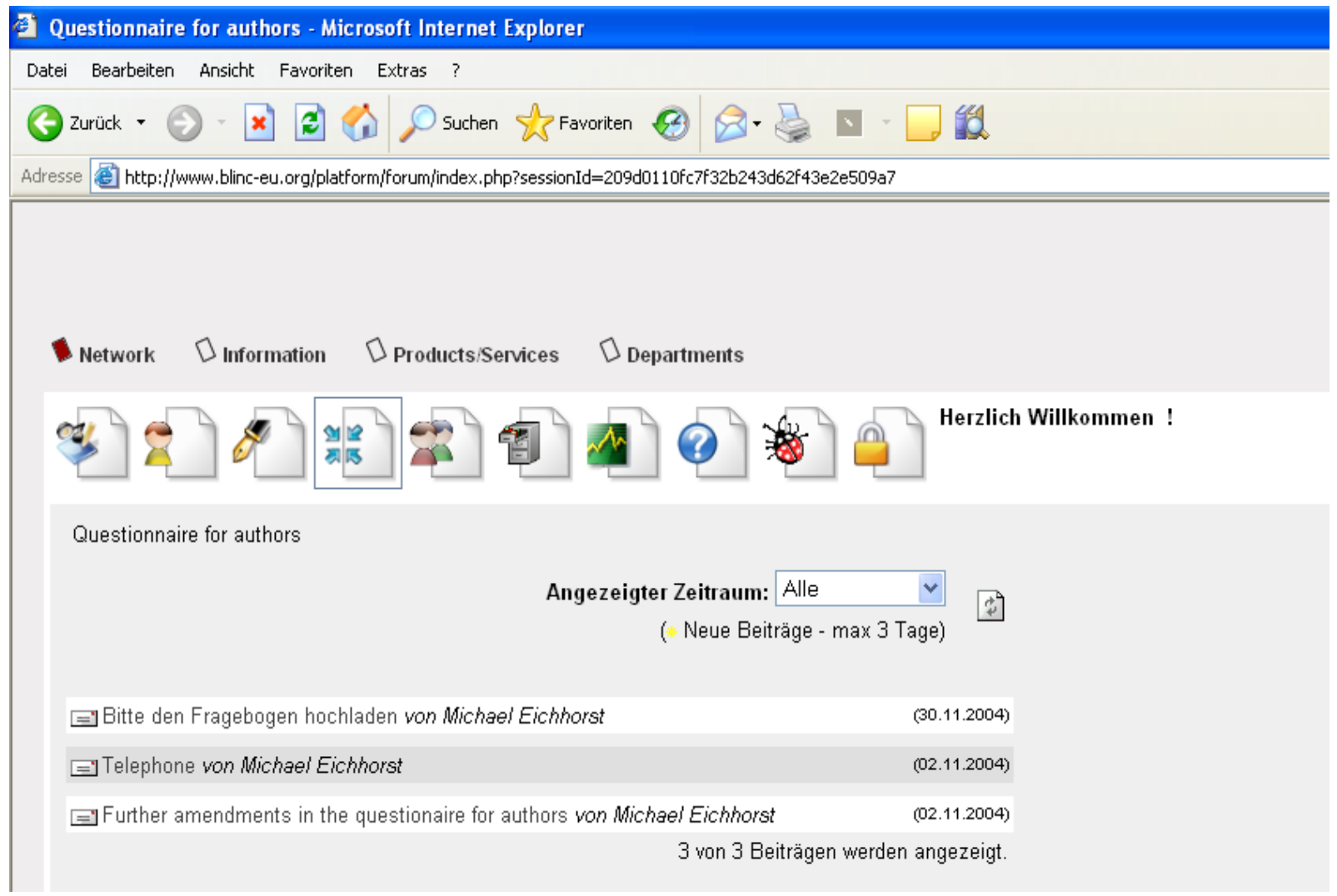

Abbildung 16: Beiträge der Arbeitsgruppe für das Autoreninventar im Forum

Während der darauf folgenden transnationalen Projekttreffen hatte die Arbeitsgruppe einige Stunden Zeit für die Weiterentwicklung des Autoreninventars, und bei diesen Gelegenheiten wurde auch diskutiert, es stellte sich aber heraus, dass die Projektpartner zu dem Thema didaktisches Design von e-Learning und Blended Learning Kursen zu diesem Zeitpunkt noch keine Instrumente entwickelt hatten, die sie hätten einbringen können, und dass sie außerdem relativ wenige Überlegungen zu diesem Thema angestellt hatten. Die Reflexion zu didaktischen Möglichkeiten und Kriterien für die Qualität eines didaktischen Designs war für die meisten Projektpartner neu und anspruchsvoll. Außerdem stellte die Arbeitssprache hin und wieder eine Herausforderung dar, wenn über komplexe Begrifflichkeiten diskutiert wurde.

Die Phase „Diskussion und Austausch“ dauerte insgesamt neun Monate, in denen sich die Arbeitsgruppe während der transnationalen Projekttreffen mit folgenden Themen beschäftigte: 
- Befragungszeitpunkte

- Inventar für reines e-Learning und/oder Blended Learning

- Papierbasierte Inventare versus online Befragung

- Begriffe: Kurs, Seminar, Programm, Bildungsmaßnahme

- Kursinhalt und Lernmethoden

- Kulturelle Unterschiede bei der Befragung

- Schriftgröße und Layout

- Umfang des Inventars

- Formulierung von Antwortvorgaben versus offene Fragen

- Copyright

Die Inhalte und Ergebnisse der Diskussionen werden im Folgenden beschrieben:

\section{Befragungszeitpunkte}

Die Arbeitsgruppe diskutierte, zu welchem Zeitpunkt das Autoreninventar genutzt werden sollte: Vor der Entwicklung eines e-Learning oder Blended Learning-Kurses, um die zusammengestellten Kriterien für die Planung nutzen zu können, oder nach der Entwicklung des didaktischen Designs eines Kurses, jedoch vor dessen Durchführung, oder erst nachdem der Kurs in der Praxis durchgeführt worden war, mit dem Ziel ggf. etwas zu verbessern.

\begin{tabular}{|l|l|}
\hline Zeitraum der Nutzung & Ziel \\
\hline Vor der Entwicklung des Kurses & $\begin{array}{l}\text { Wissen über Qualitätskriterien didaktischen De- } \\
\text { signs für die Planung von Kursen nutzen }\end{array}$ \\
\hline $\begin{array}{l}\text { Nach der Entwicklung des Kurses, } \\
\text { aber vor dessen Durchführung }\end{array}$ & $\begin{array}{l}\text { Qualitätskontrolle des didaktischen Designs von } \\
\text { entwickelten Kursen, um ggf. Nachbesserungen } \\
\text { vorzunehmen }\end{array}$ \\
\hline Nach der Durchführung des Kurses & Bestehende Kurse überprüfen und ggf. verbessern \\
\hline
\end{tabular}

Tabelle 38: Übersicht über verschiedene Möglichkeiten zur Nutzung des Autoreninventars

Die Arbeitsgruppe kam zu dem Ergebnis, dass jeder Zeitraum für eine Überprüfung des didaktischen Designs möglich sei. Für die Erprobung des Autoreninventars im Rahmen des Projektes eL3 schien jedoch vor allem die Überprüfung von bereits entwickelten und auch durchgeführten Kursen möglich zu sein. Die Projektpartner waren alle in der Lehre und Ausbildung tätig und erklärten, dass sie das Autoreninventar nutzen wollten, um das didaktische Design ihrer eigenen Kurse zu überprüfen. Zu einem späteren Zeitpunkt könnte das Autoreninventar für die Planung und Entwicklung von Kursen genutzt werden, jedoch müssten dazu Hintergrundinformationen zu den einzelnen Bereichen des Inventars zusammengestellt 
werden. Eine bloße Nutzung der Checklisten schien für Laien oder unerfahrene Designer bzw. Autoren von e-Learning und Blended Learning Kursen nach-Meinung der Arbeitsgruppenmitglieder zu komplex. Zu einem späteren Zeitpunkt könnte sich das Autoreninventar eignen, um Kurse oder Kursmaterialien zum Thema „Didaktisches Design - Planung, Durchführung und Evaluation von Blended Learning“ für Autoren zu entwickeln. Außerdem wurde die Idee entwickelt, ein Beratungsangebot auf der Basis des Autoreninventars von einem Experten der Projektgruppe auf europäischer Ebene über blinc anzubieten.

\section{Inventar für reines e-Learning und/oder Blended Learning}

In dem Projekt eL3 sollte es vor allem um e-Learning im Dritten Sektor in Europa gehen. Es stellte sich aber heraus, dass die meisten Projektpartner längerfristig Blended LearningKurse entwickeln und anbieten wollten, da reines e-Learning von ihren Zielgruppen häufig nicht besonders gut angenommen wurde (hohe Abbruchquoten, Unzufriedenheit und geringe Motivation). Eine Kombination von e-Learning und Präsenzveranstaltungen schien für die Partner in der Zukunft geeigneter als reines e-Learning. Das Autoreninventar sollte jedoch sowohl für e-Learning als auch für Blended Learning-Kurse nutzbar sein. Mit den Fragenbereichen des Autoreninventars ging es nicht um technische Aspekte, sondern vor allem um die Kenntnis der Zielgruppen seitens der Autoren und um Kriterien für die Qualität des didaktischen Designs und die Wirksamkeit der Lehre. Diese Bereiche lassen sich sowohl für eLearning als auch für Blended Learning nutzen, da es um das didaktische Design eines Lern- bzw. Lehrangebots an sich geht.

\section{Papierbasierte Inventare versus online-Befragung}

In der Arbeitsgruppe wurde überlegt, ob das Autoreninventar von den eL3-Partnern und zukünftigen Netzwerkpartnern online ausgefüllt werden sollte, oder ob eine Papierversion geeigneter wäre. Einerseits könnte bei einer online-Form eine automatische Auswertung erfolgen und es wäre eventuell sogar möglich, eine automatisierte Empfehlung zum didaktischen Design zu bekommen. Andererseits stellten einige Partner in Frage, ob Autoren von eLearning-Kursen bereit wären, das didaktische Design ihrer eigenen Kurse „öffentlich“ zu bewerten, oder ob sie es lieber selbst unabhängig und papierbasiert überprüfen würden. Die Arbeitsgruppenmitglieder entschieden, dass zunächst eine papierbasierte Form entwickelt und erprobt werden sollte, die jeder für sich nutzen könnte. Die Programmierung einer online-Form könnte später auf der Basis der Erfahrungen aus der Erprobungsphase im Rahmen von eL3 erfolgen. Es wurde auch vorgeschlagen, sowohl eine online-Befragung anzubieten als auch ein pdf-Dokument über die Internetseiten von blinc zur Verfügung zu stellen. 


\section{Begriffe: Kurs, Seminar, Programm, Bildungsmaßnahme}

Für die Überprüfung des Lehrangebots musste bei der Formulierung der Aussagen im Autoreninventar ein Begriff gefunden werden, der für möglichst viele verschiedene Formen passt. Manche Projektpartner boten Bildungsmaßnahmen an, die sich am besten als Kurse, Seminare oder Workshops bezeichnen lassen.

Einige Partner entwickelten aber auch ganze Ausbildungsprogramme, die mehrere Monate (z.B. ein Semester) oder sogar Jahre bzw. mehrere Semester umfassen. Für das Autoreninventar wurde der Begriff „Programm“ gewählt, da ein Programm umfassender ist als ein Kurs. Es sollte damit zum Ausdruck gebracht werden, dass sich auch komplexere Systeme und umfassendere Ausbildungsprogramme mit dem Autoreninventar hinsichtlich ihres didaktischen Designs überprüfen lassen.

\section{Kursinhalt und Lernmethoden}

Die Lernangebote der eL3-Projektpartner waren sehr verschieden, da diese aus unterschiedlichen Bereichen des Dritten Sektors in Europa stammten. Die Inventare sollten jedoch unabhängig vom Kursinhalt von allen Partnern und auch von zukünftigen Netzwerkpartnern genutzt werden können. Mit den drei Bereichen des Autoreninventars wurden Kriterien bereitgestellt, die generell für Lernangebote verwendet werden können. Der Fokus lag bei der Entwicklung auf e-Learning und Blended Learning, doch die Kriterien für gutes didaktisches Design und wirksame Lehre gelten für Lern- bzw. Lehrangebote an sich. Die Arbeitsgruppe war der Meinung, dass keine neuen Didaktiken für die Nutzung neuer Medien bzw. eLearning und Blended Learning entwickelt werden mussten, sondern dass bestehende didaktische Modelle genutzt werden konnten und ggf. nur an die Kontextbedingungen angepasst werden mussen.

\section{Kulturelle Unterschiede bei der Befragung}

In der Arbeitsgruppe wurde überlegt, ob es kulturelle Unterschiede in der Didaktik von eLearning und Blended Learning-Kursen gebe. Es könnte sein, dass unterschiedliche Praktiken und Gewohnheiten bei der Gestaltung in den Partnerländern eine Rolle spielten. Dieser Aspekt wurde in der weiteren Arbeit nicht berücksichtigt, sondern nur angemerkt. Auf der Basis der Daten aus der Erprobungsphase könnten sich eventuell Aussagen zu dieser Thematik formulieren lassen. Für die Entwicklung des Autoreninventars wurden vor allem Gemeinsamkeiten fokussiert.

\section{Schriftgröße und Layout}

Das Design des Autoreninventars sollte zu den Inventaren, die in den anderen Arbeitsgruppen entwickelt wurden, passen, d.h. visuell sollte deutlich werden, dass es zu einem System von Evaluationsinstrumenten gehört. Es sollten deshalb eine einheitliche Schrift und Schrift- 
größe gewählt und Überschriften grafisch einheitlich hervorgehoben werden. Die SchriftgröBe sollte nach Meinung der Gruppe 10-12 pt betragen, damit die Texte gut lesbar sind, und die einzelnen Themenbereiche sollten deutlich und übersichtlich voneinander getrennt erscheinen.

\section{Umfang des Inventars}

Im Gegensatz zum Lernerinventar, das besonders flexibel sein sollte, da es von den Partnern an sehr unterschiedliche Lernergruppen angepasst werden musste, konnte das Autoreninventar in seiner vollständigen Form eingesetzt werden. Eine Auswahl von Fragen aus dem Inventar schien hier nicht so notwendig wie bei den Inventaren für Lerner. Außerdem dient das Autoreninventar eher der Selbstkontrolle, wodurch die Motivation zum Beantworten und Ausfüllen der Checklisten nach Meinung der Arbeitsgruppe höher ist als bei Lernern, die am Ende eines Kurses einen Fragebogen ausfüllen sollen. Trotzdem sollte das Autoreninventar nicht zu lang sein, da dies abschreckend wirken könnte („Das muss ich alles in vollem Maße berücksichtigen, damit mein Kurs eine gute Qualität hat?!"). Eine gewisse Klarheit und Übersichtlichkeit sollte einladend wirken, sich mit den verschiedenen Fragenbereichen auseinander zu setzen und die Reflexion zum didaktischen Design anregen.

\section{Formulierung von Antwortvorgaben versus offene Fragen}

Die Arbeitsgruppe war sich einig, dass Antwortvorgaben zu einer besseren Vergleichbarkeit der Ergebnisse unterschiedlicher europäischer Projektpartner führen würde. Außerdem sollte das Autoreninventar den Nutzern vor allem (Selbst)-Prüfkriterien anbieten, anhand derer das didaktische Design eines e-Learning oder Blended Learning-Kurses kontrolliert werden kann. Offene Fragen seien an dieser Stelle ungeeignet, da es nicht darum gehen sollte, die Thematik zu explorieren, sondern das didaktische Design zu beurteilen.

\section{Copyright}

Das Autoreninventar wurde vor allem auf der Basis der Arbeiten von Flechsig und Haller (Flechsig 1983, 1990, 1996a, 1996b, Haller 1995, 1997, 2002, 2003) entwickelt. Von den Arbeitsgruppenmitgliedern kamen kaum bzw. keine maßgeblichen Impulse oder inhaltliche Beiträge. Es wurde während der Phase „Diskussion und Austausch“ nicht über das Copyright gesprochen. Im Projekt eL3 wurde mit der gesamten Gruppe während der transnationalen Treffen diskutiert, wie die Inventare genutzt werden könnten und wer das Copyright haben könnte oder sollte. Für das Autoreninventar sollten in jedem Fall die theoretischen Vorarbeiten genannt werden. Bei einer weiteren Verwendung sollten die Partner auf die entsprechenden Quellen verweisen. Es wurde entschieden, das Autoreninventar über die Internetseiten von blinc frei zur Verfügung zu stellen, mit der Angabe des Verantwortlichen für die Inhalte und mit dem Verweis auf den Entstehungszusammenhang. 


\section{Phase 3: Gemeinsamer Entwurf und Übersetzung}

Es wurde in den neun Monaten von September 2004 bis Mai 2005 kaum gemeinsam an dem Autoreninventar gearbeitet. Lediglich während der transnationalen Treffen brachten sich die Arbeitsgruppenmitglieder ein und machten vor allem zu sprachlichen Formulierungen einige Anmerkungen. Inhaltlich wurde nicht gemeinsam an dem Entwurf aus Phase 1 gearbeitet. Der Arbeitsgruppenleiter entwickelte das Inventar alleine weiter, indem er Aussagen zu den einzelnen Bereichen formulierte bzw. sie ergänzte und an e-Learning und Blended Learning anpasste. Außerdem wurde ein Einleitungstext verfasst, zum einen für das gesamte Inventar und zum anderen zu den einzelnen Bereichen. Es wurde erklärt, in welchem Kontext das Inventar entwickelt wurde, wozu die einzelnen Bereiche dienen und wie es zu verwenden ist. Auf dem transnationalen Treffen im Mai 2005 wurde das Autoreninventar allen Projektpartnern präsentiert. Es wurde vereinbart, dass die Partner das Autoreninventar in ihre jeweiligen Muttersprachen übersetzen, um es für eine Erprobung in unterschiedlichen europäischen Einrichtungen verwenden zu können.

Es erfolgte keine Übersetzung des Autoreninventars durch die Partner. Es vergingen einige Wochen und die Partner meldeten nichts an den Arbeitsgruppenleiter zurück. Es wurden keine Fragen gestellt und es wurde auch nicht um Unterstützung gebeten. Schließlich wurde von der Verfasserin an der Universität Göttingen im Sprachlehrzentrum die Übersetzung des Autoreninventars in die Sprachen Italienisch und Französisch in Auftrag gegeben. Dies hatte den Vorteil, dass die Qualität der Übersetzung garantiert war, da die Übersetzer professionell arbeiteten. Es war dem Arbeitsgruppenleiter und der Verfasserin nicht klar, warum die Projektpartner sich so zurückhaltend zeigten. Während des nächsten transnationalen Treffens wurde geäußert, dass die Übersetzung sehr aufwändig gewesen sei und keine zeitlichen und finanziellen Ressourcen dafür vorhanden seien. Manchen Partnern war auch der Nutzen des Autoreninventars nicht ganz deutlich, was zum Teil auch an Verständigungsproblemen und der Neuartigkeit des Themas lag.

\section{Phase 4: Erprobung \& Ergebnisbericht}

Zu Beginn der Entwicklung der Inventare hatten die Projektpartner vereinbart, die Inventare in ihren Einrichtungen einzusetzen, um sie in der Praxis zu erproben und Daten zu sammeln. Mit der Erprobung sollten zum einen Erkenntnisse über die Qualität und den Nutzen der Inventare erlangt werden, und zum anderen sollten die Daten genutzt werden, um Aussagen zur Praxis von e-Learning und Blended Learning im europäischen Gesundheits- und Sozialwesen treffen zu können. Dieses Wissen sollte dann im Netzwerk von blinc allen Mitgliedern frei zugänglich sein.

Einige Partner äußerten jedoch auf dem Treffen, dass sie das Autoreninventar nur als Checkliste zur Überprüfung und Qualitätskontrolle ihrer eigenen Kurse einsetzen wollten. Sie 
sprachen sich dagegen aus, die Daten in der Gruppe zu sammeln, sie wollten das Autoreninventar für sich allein erproben. Sie wollten das Autoreninventar zur Selbstevaluation (Inspektionsanalyse) und zur Reflexion ihrer Kurse nutzen und dann über ihre Erfahrungen damit auf dem nächsten transnationalen Treffen berichten. Auf dem letzten Projekttreffen im November 2005 berichteten die Partner mündlich über die Nutzung des Autoreninventars und damit verbundene Erfahrungen.

Sie brachten keine Ergänzungs- oder Verbesserungsvorschläge ein, meldeten aber zurück, dass die Inspektionsanalyse ihrer Kurse mit dem Autoreninventar viel Zeit in Anspruch genommen habe und die Inhalte recht komplex seien. Für „Praktiker“ sei es schwer, die Inhalte zu verstehen und umzusetzen, da das Autoreninventar sehr anspruchsvoll und „theoretisch“ sei. Für eine Verbreitung wäre es nach Meinung der Projektpartner nötig, eine Einführung oder Anleitung bereit zu stellen, die mehr Hintergrundinformationen zum Thema Didaktik enthalten sollte. Außerdem wurde der Wunsch geäußert, konkrete Hinweise für Verbesserungsmöglichkeiten für das didaktische Design eines Kurses in den einzelnen Bereichen bereit zu stellen, wenn sich durch die Inspektionsanalyse ergeben würde, dass dies nötig sei. Die Partner wollten genauer wissen, wie sie die Ergebnisse für die Verbesserung ihrer eLearning und Blended Learning-Kurse konkret nutzen könnten. Aufgrund der Rückmeldungen der Partner und der Ergebnisse aus der Erprobungsphase wurde beschlossen, das Autoreninventar durch einleitende Texte ausführlicher zu erklären und den Nutzen deutlicher herauszuarbeiten.

\section{Phase 5: Gemeinsame Endversion und Verbreitung}

Die Form des Autoreninventars aus Phase 3 blieb weitestgehend unverändert, aber die Einleitungstexte des Autoreninventars wurden durch den Arbeitsgruppenleiter überarbeitet und ergänzt, damit den el3-Partnern sowie potenziellen Anwendern die Nutzung erleichtert würde. Die Endversion des Autoreninventars, das im Rahmen von eL3 entwickelt wurde, umfasst drei Teile:

Teil A Adressatenmerkmale

Teil B Qualität des didaktischen Designs

Teil C Kriterien wirksamer Lehre

In Teil A geht es um die Merkmale der Adressaten, der Lerner. Die zentrale Frage ist, welche Kenntnisse Autoren und Produzenten von e-Learning und Blended-Learning-Kursen über „ihre“ Lernerinnen und Lerner haben und welche Informationen sie ggf. einholen sollten. Dieser Teil enthält Fragen zur Analyse der Charakteristika der Lerner und zum Kontext der Lernenden. Es werden verschiedene Aspekte aufgelistet, anhand derer die Autoren überprüfen können, was sie bereits über ihre Zielgruppe wissen und was sie ggf. noch in Erfahrung brin- 
gen müssen, um den Kurs optimal auf die Lerner abzustimmen. Der Bereich zur Analyse der Charakteristika der Lerner enthält folgende Aspekte:

- Allgemeine Merkmale der Lerner wie Alter, Geschlecht und körperliche oder mentale Merkmale mit Bedeutung für den Kurs.

- Bildungshintergrund und Berufsfelder der Lerner, d.h. vorhandene Qualifikationen, Berufe und Interessen.

- Kultureller Hintergrund und Einstellungen insbesondere gegenüber dem Lernen, z.B. Sprache und Herkunft, Bereitschaft zu neuen Aufgaben und Situationen oder Einstellung gegenüber elektronisch gestützten Lehrverfahren.

- Lernerfahrungen und Erwartungen, wie bisherige Erfahrungen mit verschiedenen Lehr-/Lernmethoden, individuelle Lernstile und Lernstrategien, Voraussetzungen im Wissen (Vorwissen, Qualifikation) oder Motive zum Lernen.

Der Bereich zur Analyse des Kontextes der Lernenden enthält Fragen zu folgenden Punkten:

- Lernort (Wo werden Sie lernen - zu Hause? am Arbeitsplatz? in Lernzentren?).

- Lernzeiten (Wie viel Zeit werden Sie für das Lernen aufbringen können? Wann werden Sie lernen (können)? Nachts? Während der Arbeitszeit? In kurzen oder langen Abständen?).

- Technische Ausstattung der Lernenden (Welche Ausstattung werden sie haben Hardware, Software, Netzwerkverbindungen, andere Ressourcen? Welche Art Internetanschluss werden sie haben/brauchen? Welche Schwierigkeiten könnten die Lerner erleben, z.B. in bezug auf den Zugang zur erforderlichen technischen Ausstattung?).

- Familiäre Bedingungen (Wie sind die familiären Bedingungen der Lerner? Kann dies einen Einfluss auf die möglichen Lernzeiten haben?).

- Unterstützung der Lernenden (Brauchen die Lerner eventuell Hilfe, um mit der Technologie umzugehen? Welche Unterstützung werden die Lerner haben? Tutoren, Trainer oder andere Personen? Wen? Wie? Qualifikationen der Tutoren? Wann sind sie erreichbar/verfügbar?).

Teil A des Autoreninventars kann als Checkliste verwendet werden, aus der hervorgeht, was bei der Gestaltung des Kurses bereits berücksichtigt wurde und was ggf. noch für die Durchführung wichtig sein könnte und in Erfahrung gebracht werden muss.

In Teil B des Autoreninventars geht es um die Bewertung der Qualität didaktischer Designs. Im Zentrum steht, wie Autoren und Produzenten von e-Learning und Blended-Learning- 
Kursen die Qualität des didaktischen Designs und der eingesetzten Materialien einschätzen. Es gibt in diesem Teil sieben Kriterien für eine gute Qualität des didaktischen Designs. Die Autoren können anhand dieser Kriterien überprüfen, inwiefern sie diese bei der Gestaltung bereits berücksichtigt haben und in welchen Punkten sie noch Veränderungen oder Verbesserungen vornehmen können. Jedes Kriterium beinhaltet sieben bis zehn Aussagen und eine sechsstufige Skala von "gar nicht“ bis „sehr viel“, auf der die Nutzer den Grad ihrer Zustimmung oder Ablehnung ausdrücken können. Die Nutzer können ihren Kurs bzw. ihr Programm auf dieser Skala zu jeder Aussage einstufen und sehen so, wie sehr sie diesen Aspekt bei der Gestaltung ihres Lehrangebots berücksichtigt haben. Hinsichtlich der Aspekte, die bisher „gar nicht“ oder nur „ein bisschen“ berücksichtigt wurden, sollten Veränderungen und Verbesserungen in Bezug auf das didaktische Design vorgenommen werden. Insgesamt kommt für jedes der sieben Kriterien ein Gesamtpunktwert heraus, der zeigt, inwiefern die einzelnen Kriterien berücksichtigt wurden. Die sieben Kriterien für ein gutes didaktisches Design sind die folgenden:

\section{Vielfalt der Inhalte, der Lernerpersönlichkeiten und der Methoden}

Dieses Kriterium beinhaltet, dass mehrere Sinne angesprochen werden, mehrere didaktische Modelle zum Einsatz kommen, Phasen der Anspannung und der Entspannung wechseln, individuelle und kollektive Tätigkeiten angeboten werden, angeleitetes und selbstständiges Lernen stattfindet und verschiedene Lernorte vorgesehen sind.

\section{2. Ökonomie im Sinne wirksamer Nutzung von Ressourcen}

Dieses Kriterium beinhaltet, dass möglichst einfache, preiswerte, wenig anfällige und ohnehin vorhandene Mittel und Medien verwendet werden, viel "Lehrzeit" in effektive Lernzeit umgesetzt wird, den Lernern ohne Mehraufwand zusätzliches Wissen vermittelt wird, nichts gelehrt wird, was die Lernenden schon gut wissen oder können, und dass bei den Lernern vorhandenes Wissen und Können genutzt wird.

\section{Anpassung an die gegebene/erwartete Situation}

Dieses Kriterium bedeutet, dass das Programm angepasst ist an die Sprachgewohnheiten der Lernenden und deren Vorwissen sowie an die Lernstile, die bei den Lernenden überwiegen, wie auch an die Lebenswelt und Lebensweise der Lernenden. Das Programm soll ferner angepasst sein an Ressourcen, mit denen die Lernenden vertraut sind, an Aufgaben, die sich aus dem Umfeld der Lernenden ergeben, und an die Kommunikationsformen, die von den Lernenden bevorzugt werden. Die zeitlichen Möglichkeiten der Lernenden und des Bezugssystems müssen ebenfalls berücksichtigt werden. 


\section{Produktivität und Nützlichkeit}

Dieses Kriterium beinhaltet, dass durch das Programm Schemata (z.B. verallgemeinerbare Methoden) geliefert werden, den Lernenden beim Umgang mit Arbeitsmitteln geholfen wird, Schlüsselqualifikationen vermittelt werden und Kontakte hergestellt oder verbessert werden (z.B. zu anderen Lernenden).

\section{Selbstmotivation für Lernende und Lehrende}

Dieses Kriterium beinhaltet, dass dem Lerner vor allem die positiven Effekte dessen, was er/sie bereits gelernt hat, gezeigt werden, Freude an körperlicher Bewegung beim Lernen berücksichtigt wird, Lernen in spielerischer Form möglich ist, auch auf gesellige Art und Weise gelernt werden kann, das Programm auch Erholungs- und Freizeitcharakter hat und ein Lernen ohne Angst vor Versagen gefördert wird.

\section{Persönlichkeitsbildung und Entwicklung der Lernerfähigkeiten}

Dieses Kriterium beinhaltet, dass das Programm die Kreativität der Lerner fördert, die Konfliktfähigkeit und die Kommunikationsfähigkeit der Lerner erhöht, innen Selbstwertgefühl vermittelt, die Entscheidungsfähigkeit der Lerner fördert und deren Verantwortungsgefühl erhöht.

\section{Stimmigkeit und Konsistenz}

Dieses Kriterium beinhaltet, dass in dem Programm in angemessener Weise die verschiedenen Komponenten des Blended Learning berücksichtigt wurden, Vorsorge dafür getroffen wurde, dass die Lehrenden selbst auch kompetent und routiniert im Umgang mit elektronischen Medien sind und keine gravierenden Widersprüche zwischen dem, was gesagt wird, und dem, was getan wird, auftreten. Außerdem sollte das Programm Lernaufgaben aufweisen, die mit den Praxisanforderungen übereinstimmen, den Lernenden genauso viel Handlungsspielraum wie in der beruflichen Praxis geben, und der Schwierigkeitsgrad der Lernanforderungen sollte gleichmäßig auf die ganze Lernzeit verteilt sein.

In Teil $C$ des Autoreninventars geht es um die Bewertung der Wirksamkeit des Lehrangebots. Die zentrale Frage ist, wie Autoren und Produzenten von Blended-Learning-Kursen die Wirksamkeit ihres didaktischen Designs bewerten. Es stehen sieben Prinzipien erfolgreicher Lehre für die Inspektions-Evaluierung bereit: 
1. Klarheit durch zuverlässige und verständliche Darstellungen

Dieses Prinzip beinhaltet, dass das Programm gekennzeichnet ist durch die Klarheit des Wissens und der Lernschritte bzw. des Lernprozesses sowie der Fragen und Aufgaben für die Lernenden. Außerdem sollten die Ziele und Beurteilungskriterien klar sein.

2. Relevanz und Bezug durch die Verteilung gültigen Wissens

Dieses Prinzip beinhaltet, dass das Programm Relevanz und Bezug aufweist im Hinblick auf das Fach bzw. den Beruf der Lernenden, den Forschungsstand, den Lernprozess, die späteren Prüfungen wie auch im Hinblick auf die Zukunft der Lernenden.

3. Struktur und Sequenz durch die Verteilung des Wissens in geordneten Maßen Dieses Prinzip beinhaltet, dass das Programm Bekanntes wieder aufgreift, Hauptpunkte betont werden, für ein gutes Zeit- und Raummanagement (Lernumgebungen) und Kontinuität gesorgt ist.

\section{Anschaulichkeit durch Lern- und Denkhilfen}

Dieses Prinzip beinhaltet, dass innerhalb des Programms für Anschaulichkeit gesorgt wurde durch Visualisierung (Bilder, Folien etc.), Beispiele, Analogien, direkte Erfahrungen der Lernenden und Anstöße zum Nachdenken an die Lernenden.

5. Anwendung durch die Entwicklung von Aufgaben und den Transfer in die Praxis

Dieses Prinzip beinhaltet, dass das Programm gut definierte Aufgabenstellungen aufweist, Möglichkeiten des Übens und der Überprüfung des Wissens in der Praxis geboten werden sowie Einblicke in die praktische Anwendung und Nützlichkeit vorhanden sind.

6. Rückmeldung durch Informationen zum Lernprozess, zur Entwicklung und zum Ertrag

Dieses Prinzip beinhaltet, dass das Programm Rückmeldung bietet durch Lob und Tadel, Produkte, Zertifikate/Bescheinigungen und die natürliche Umgebung („natura docet").

7. Weitere Anreize durch Gesichtspunkte, die über das unmittelbare Wissen und Lernen hinausgehen

Dieses Prinzip beinhaltet, dass das Programm zusätzliche Anregungen durch Berücksichtigung der Interessen und Bedürfnisse der Lernenden liefert und zusätzliche 
Anregungen durch Begeisterung der Lehrenden sowie durch feine Ironie und Selbstkritik gegeben werden.

Die folgende Tabelle gibt einen Gesamtüberblick über das Autoreninventar.

\begin{tabular}{|c|c|c|}
\hline Teil A & Teil B & Teil C \\
\hline $\begin{array}{l}\text { Informationen über Adressa- } \\
\text { tenmerkmale }\end{array}$ & $\begin{array}{l}\text { Bewertung der Qualität des } \\
\text { didaktischen Designs }\end{array}$ & $\begin{array}{l}\text { Bewertung der Wirksamkeit des } \\
\text { Kurses }\end{array}$ \\
\hline $\begin{array}{l}\text { Leitfrage: } \\
\text { Kenne ich meine Lerner und } \\
\text { deren Bedürfnisse? }\end{array}$ & $\begin{array}{l}\text { Leitfrage: } \\
\text { Welche Kriterien für eine gute } \\
\text { Qualität des didaktischen De-- } \\
\text { signs habe ich wie stark berück- } \\
\text { sichtigt? }\end{array}$ & $\begin{array}{l}\text { Leitfrage: } \\
\text { In welchem Ausmaß habe ich Kri- } \\
\text { terien wirksamer Lehre in meinem } \\
\text { Kurs berücksichtigt? }\end{array}$ \\
\hline $\begin{array}{l}\text { Ergebnis nach der Evaluation: } \\
\text { Was sollte ich noch in Erfahrung } \\
\text { bringen, um den Kurs optimal } \\
\text { auf die Adressaten abzustim- } \\
\text { men? }\end{array}$ & $\begin{array}{l}\text { Ergebnis nach der Evaluation: } \\
\text { In welchen Bereichen kann ich } \\
\text { die Qualität meines didaktischen } \\
\text { Designs optimieren? }\end{array}$ & $\begin{array}{l}\text { Ergebnis nach der Evaluation: } \\
\text { In welchen Bereichen kann ich } \\
\text { meinen Kurs hinsichtlich der Wirk- } \\
\text { samkeit meiner Lehre optimieren? }\end{array}$ \\
\hline $\begin{array}{l}\text { 1. Analyse der Charakteristika } \\
\text { der Lerner } \\
\text { - Allgemeine Merkmale } \\
\text { - Bildungshintergrund und Be- } \\
\text { rufsfelder der Lerner } \\
\text { - Kultureller Hintergrund und } \\
\text { Einstellungen insb. gegen- } \\
\text { über dem Lernen } \\
\text { - Lernerfahrungen und Erwar- } \\
\text { tungen } \\
\end{array}$ & $\begin{array}{l}\text { 1. Vielfalt der Inhalte, der } \\
\text { Lernerpersönlichkeiten und der } \\
\text { Methoden }\end{array}$ & $\begin{array}{l}\text { 1. Klarheit } \\
\text { Zuverlässige und verständliche } \\
\text { Darstellungen }\end{array}$ \\
\hline \multirow[t]{6}{*}{$\begin{array}{l}\text { 2. Analyse des Kontextes der } \\
\text { Lernenden } \\
\text { - Lernorte } \\
\text { - Lernzeiten } \\
\text { - Ressourcen für e-Learning } \\
\text { - Sozialer Kontext } \\
\text { - Unterstützungsbedarf }\end{array}$} & $\begin{array}{l}\text { 2. Ökonomie im Sinne wirksamer } \\
\text { Nutzung von Ressourcen }\end{array}$ & $\begin{array}{l}\text { 2. Relevanz und Bezug: } \\
\text { Gültiges Wissen vermitteln }\end{array}$ \\
\hline & $\begin{array}{l}\text { 3. Anpassung an die gegebe- } \\
\text { ne/erwartete Situation }\end{array}$ & $\begin{array}{l}\text { 3. Struktur und Sequenz: } \\
\text { Wissen in geordneten Maßen ver- } \\
\text { mitteln }\end{array}$ \\
\hline & 4. Produktivität und Nützlichkeit & $\begin{array}{l}\text { 4. Anschaulichkeit: } \\
\text { Lern- und Denkhilfen nutzen }\end{array}$ \\
\hline & $\begin{array}{l}\text { 5. Selbstmotivation für Lernende } \\
\text { und Lehrende }\end{array}$ & $\begin{array}{l}\text { 5. Anwendung: } \\
\text { Entwicklung von Aufgaben } \\
\text { Transfer in die Praxis }\end{array}$ \\
\hline & $\begin{array}{l}\text { 6. Persönlichkeitsbildung und } \\
\text { Entwicklung der Lernerfähigkei- } \\
\text { ten }\end{array}$ & $\begin{array}{l}\text { 6. Rückmeldung: } \\
\text { Informationen zum Lernprozess, } \\
\text { zur Entwicklung und zum Ertrag }\end{array}$ \\
\hline & 7. Stimmigkeit und Konsistenz & $\begin{array}{l}\text { 7. Weitere Anreize: } \\
\text { Gesichtspunkte, die über das un- } \\
\text { mittelbare Wissen und Lernen } \\
\text { hinausgehen anregen }\end{array}$ \\
\hline
\end{tabular}

Tabelle 39: Übersicht über das Autoreninventar

Das Inventar für Autoren wurde am Ende des Projektes eL3 über die Internetseiten von blinc als pdf-Dokument und als Word-Dokument einer breiteren Öffentlichkeit zugänglich gemacht und steht seitdem zur freien Verfügung. 


\subsubsection{Das Inventar für Entscheidungsträger}

Zu Beginn der Planungen zur Entwicklung von Evaluationsinstrumenten für e-Learning und Blended Learning-Kurse wurden drei Zielgruppen identifiziert, wobei Entscheidungsträger eine davon sind. Entscheidungsträger wurden definiert als Personen, die verantwortlich für die Ausbildung und Weiterbildung von Personal und Mitarbeitern in verschiedenen Einrichtungen im Dritten Sektor sind. Entscheidungsträger entscheiden, welche Weiterbildungsangebote und Weiterbildungsmöglichkeiten es in ihrer Einrichtung geben soll und ob e-Learning und Blended Learning-Kurse implementiert werden. Für diese Zielgruppe ist es besonders wichtig zu wissen, welche Bedingungen für bzw. gegen e-Learning und Blended Learning sprechen. Sie müssen über Vor- und Nachteile sowie Kosten, Nutzen und Effizienz von eLearning und Blended Learning-Kursen informiert sein, um angemessene Entscheidungen treffen zu können.

\section{Phase 1: Recherche und Sammlung}

Bei der Recherche nach Literatur wurden keine speziellen Handbücher oder Handreichungen für Entscheider gefunden und auch bei der Sichtung von Materialien im Internet wurden keine frei verfügbaren Instrumente gefunden, sondern nur kommerzielle Beratungsangebote. Auf der Basis der Arbeiten von Flechsig (1990) und Haller (1995, 2002) wurde deshalb zu Beginn des Projektes eine Aufstellung von relevanten Fragenbereichen verfasst. Es wurde eine Sammlung von Schlüsselfragen für Personen, die vor einer Entscheidung für eine eLearning bzw. Blended Learning-Maßnahme oder ein traditionelles Kurssystem stehen, erstellt. Acht Themenbereiche wurden erarbeitet:

1. Beschreibung der Situation (Was ist gegeben?)

2. Ziele und Absichten des Trägers (Warum sollte Blended-Learning oder e-Learning eingeführt werden?)

3. Beschreibung der Lernenden (Wer soll lernen?)

4. Beschreibung der Lernumgebung (Wo sollen sie lernen?)

5. Beschreibung der Anforderungen (Was soll das Ergebnis sein?)

6. Beschreibung der Inhalte (Was sollen sie lernen?)

7. Erwartete Ergebnisse

8. Organisationsentwicklung und Integration 
Zu jedem Themenbereich wurden Fragen entwickelt, die Entscheider unterstützen sollen zu reflektieren, ob die Kontextbedingungen eher für e-Learning oder Blended Learning oder ein traditionelles Kurssystem bzw. traditionelle Weiterbildungsmaßnahmen sprechen

\section{Phase 2: Diskussion und Austausch}

Während des Projekttreffens im September 2004 wurde, wie auch für die Zielgruppe der Lerner und Autoren, eine Arbeitsgruppe gegründet, die bis zum Ende des Projektes speziell für die Entwicklung des Inventars für Entscheidungsträger zuständig war. Die Gruppe bestand aus fünf Personen, die aus Schottland, Frankreich, Italien und Deutschland stammten, von ihrem beruflichen Kontext her in der Lehre und Gestaltung von Kursen an Universitäten und in Bildungseinrichtungen tätig waren und auch Erfahrung mit der Evaluation ihrer eigenen Kurse hatten.

Zunächst diskutierte die Arbeitsgruppe während des Projekttreffens in Aberdeen über den ersten Entwurf des Inventars bzw. die Themensammlung für das Inventar aus Phase 1 (siehe oben). Für die weitere Entwicklung konnte die Arbeitsgruppe eine projektinterne Lernplattform nutzen. Eine Person übernahm die Führung und Koordination der Gruppe, die innerhalb von neun Monaten eine gemeinsame Version des Inventars für Entscheidungsträger entwickeln sollte. Der Leiter der Gruppe entwarf einen Kurs auf der Lernplattform, um die weitere Arbeit zu strukturieren und zu dokumentieren. Der erste Schritt umfasste eine Einführung für die Gruppenmitglieder und eine Beschreibung des Vorhabens. Die folgende Abbildung zeigt die erste Bildschirmseite des Kurses auf der Lernplattform. 


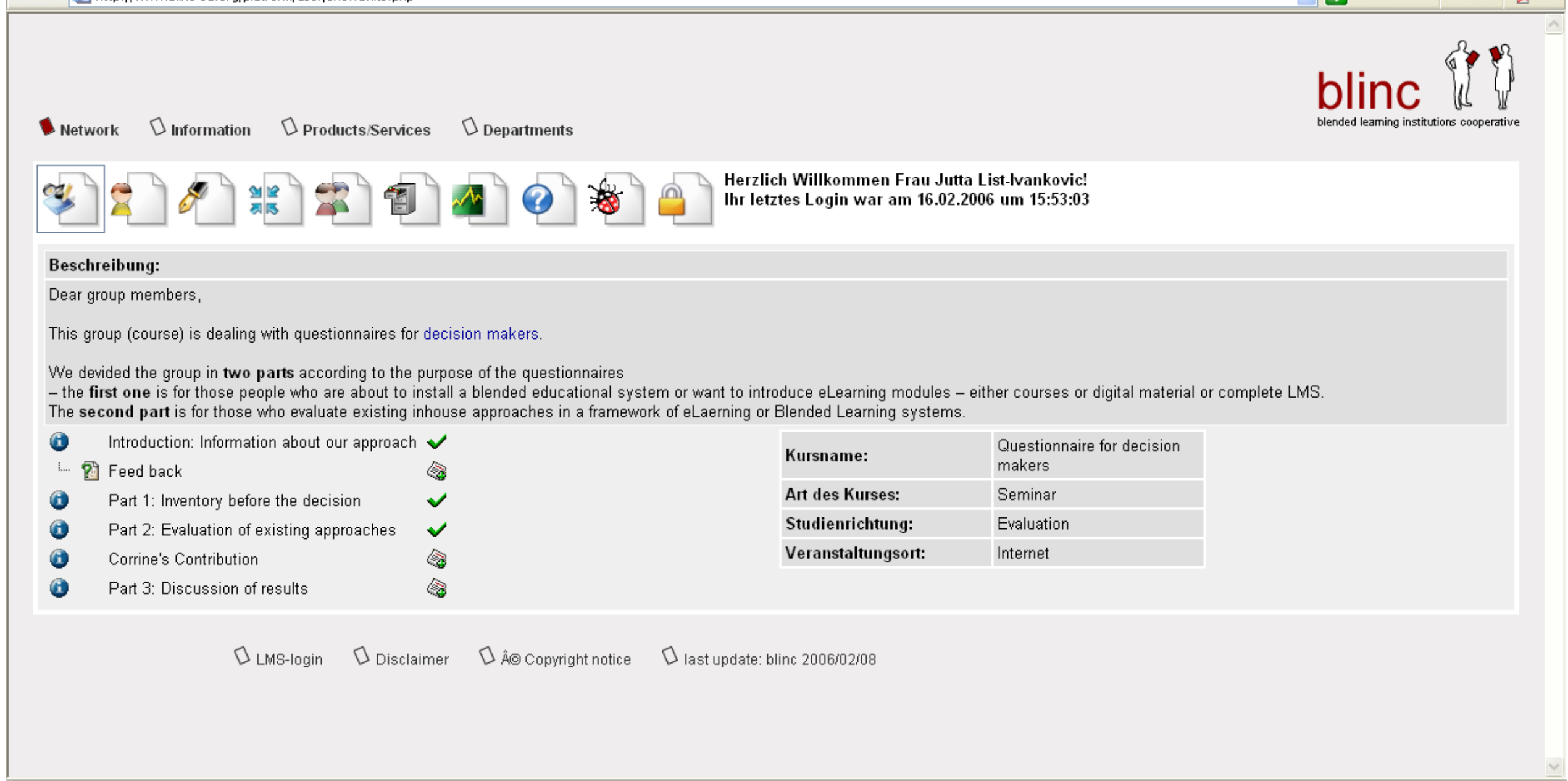


Der Gruppenleiter stellte nach dem transnationalen Treffen in Aberdeen zunächst nochmals in einer Einleitung zum Kurs das Vorhaben dar, gemeinsam ein Inventar für Entscheidungsträger zu entwickeln. Er beschrieb, dass zwei Inventare entwickelt werden sollten, eines, das Personen vor der Einführung eines e-Learning oder Blended Learning-Kurses verwenden können, und ein anderes, das Personen zur Evaluation bereits bestehender Systeme nutzen können. Der Gruppenleiter beschrieb die zentrale Idee der Inventare und die erwarteten Ergebnisse der Gruppenarbeit. Er bat die Arbeitsgruppenmitglieder, ihre Ideen und Vorschläge im Forum auf der Lernplattform oder per Mail in einem vorgegebenen zeitlichen Rahmen einzubringen, um eine gemeinsame Version für das nächste transnationale Treffen vorbereiten zu können. Die folgende Abbildung zeigt den Einführungstext für die Arbeitsgruppe „Inventare für Entscheidungsträger" auf der Lernplattform:

Introduction: Information about our approach - Microsoft Internet Explorer

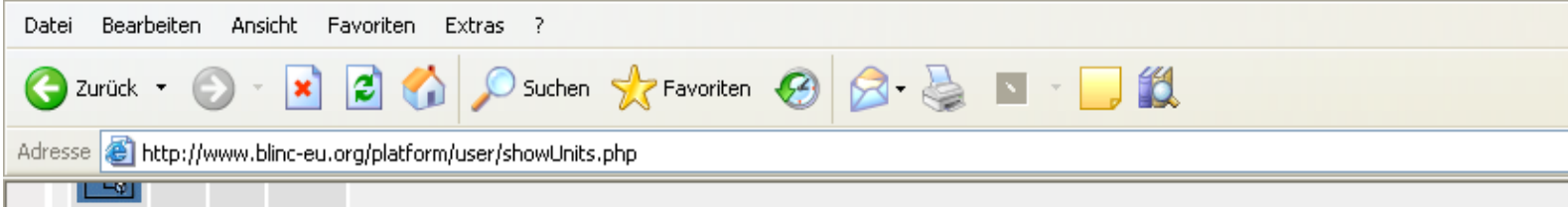

Intention of the questionnaire according to the first group session in Aberdeen:

We want to establish a Checklist for managing personnel to introduce or to improve BL-Systems

We want to elaborate an inventory of decisive questions that a decision maker must ask to get a reasonable approach and to find out the right system for his needs.

We want to show possible advantages for users of BL services (competition and economic advantages).

2 Approaches:

We will work on questionnaires for those who are about to introduce a system and, on the other hand, for those who want to evaluate their experiences with eLearning and Blended Learning approaches.

Methodology:

The central problem is that there is no standardized situation of the decision makers.

They all work in different institutions (like administrations, enterprises, social organisations manufactures etc.) with very different numbers of staff (5-5.000) and learning environments, with different business areas (health, environment, social services, insurances, Universities, private education companies etc) and with very different thematic fields (expert knowledge courses in health and social care, management courses, soft skills, team leading competencies, etc.)

Therefore we decided to work with so called inventories and not with fixed questionnaires.

We want to identify the major classes of content (patterns) and develop questions for these patterns. The users can take the questions that fit to their individual situation.

Expected outcome:
The basic pattern of the inventories shall reflect the decisive areas for the decision makers - from the equipment of the
enterprise to the possible economic implications connected with the introduction of computer based programmed learning.

There shall be 2 inventories - one for the analysis of the situation before the introduction and one for the evaluation of existing Blended Learning approaches.

Methodology

We prepared basic patterns for the structure of inventories. They are the classes for the questions. Up to the middle of January we would like to ask you to add your ideas to the questionnaires. You can post your ideas in the forum or send messages in the groups (Sorry here you cannot attach files because it's only meant to exchange short messages)

Up to the end of January we will then try put together all your contributions and prepare the meeting in February.

할 Fertig

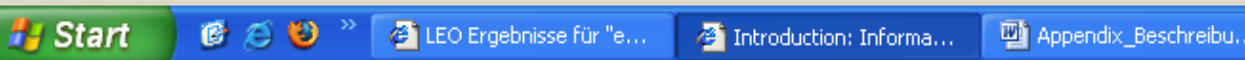

Abbildung 18: Einführungstext für die Arbeitsgruppe „Inventare für Entscheidungsträger“ auf der Lernplattform 
In dem Kurs auf der Lernplattform folgten nach der Einführung zwei Lerneinheiten, in denen die Arbeitsgruppenmitglieder eine Beschreibung der Situation von Entscheidungsträgern vor bzw. nach der Einführung eines e-Learning oder Blended Learning-Kurses erhielten. Es wurde außerdem eine konkrete Aufgabe gestellt. Die Arbeitsgruppenmitglieder wurden gebeten, sich die vorhandene Grundstruktur des Inventars für Entscheidungsträger anzuschauen und sich Gedanken dazu zu machen. Die bisher erarbeitete Themen- und Fragensammlung wurde in einem Word-Dokument auf der Lernplattform bereitgestellt. Die Partner konnten sich das Dokument herunterladen und sollten dann ihre Ideen und Vorschläge dazu im Forum oder per Mail äußern. Die folgende Abbildung zeigt die Lerneinheit zum Inventar für Entscheidungsträger, das vor der Einführung eines e-Learning oder Blended Learning-Systems genutzt werden kann.

\section{Part 1: Inventory before the decision - Microsoft Internet Explorer}

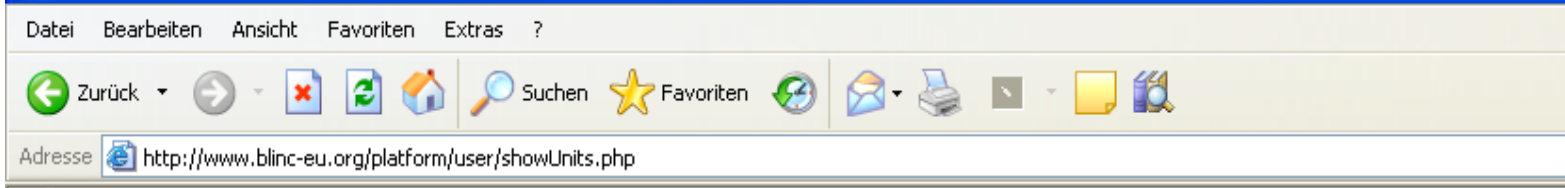

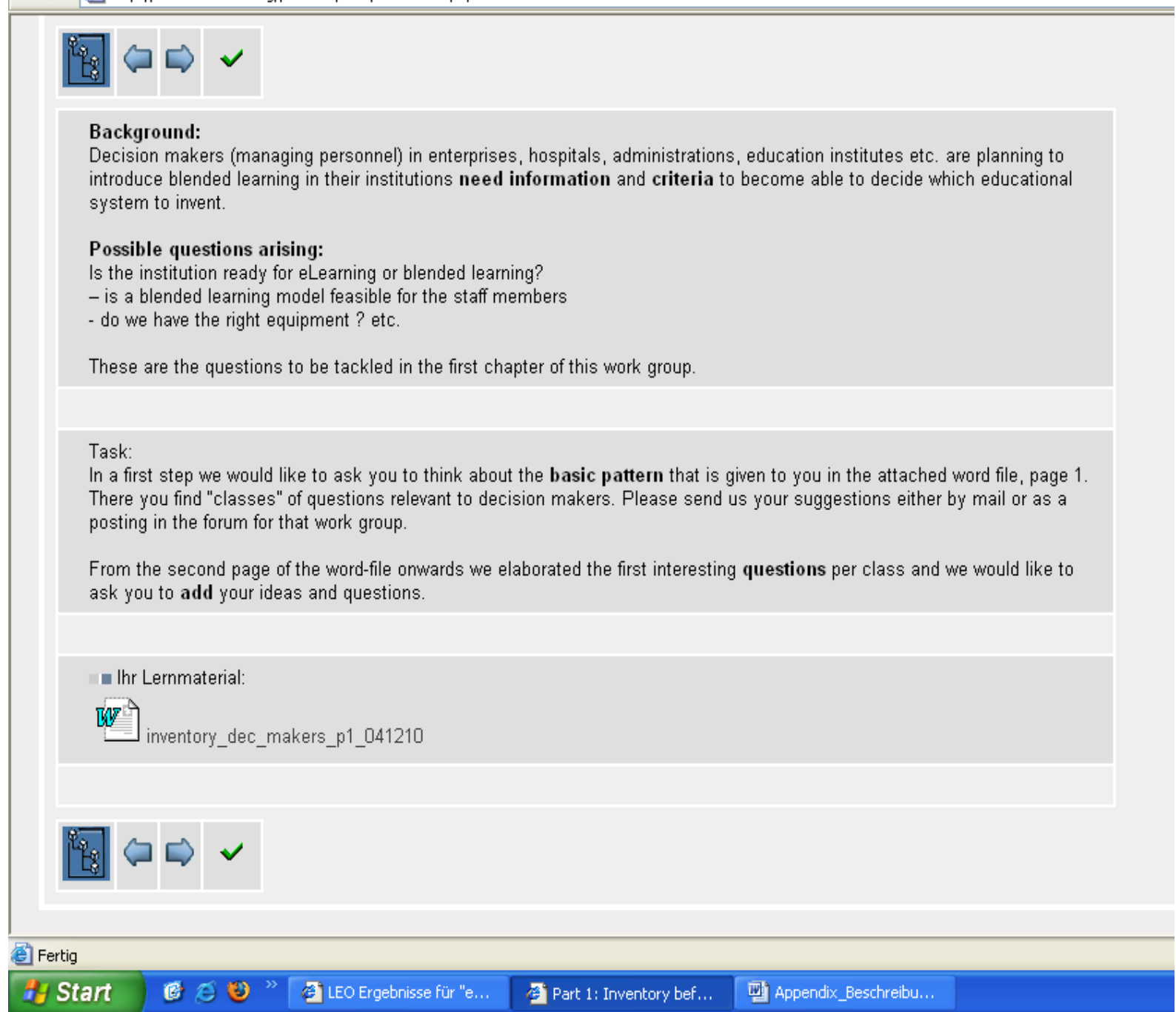

Abbildung 19: Lerneinheit zum Inventar für Entscheidungsträger vor der Einführung eines e-Learning oder Blended Learning-Systems 
Ein Arbeitsgruppenmitglied schickte dem Projektleiter ein Dokument per E-Mail. Alle Partner sollten die Gelegenheit haben, das Dokument einzusehen, um es für die Arbeit an dem Inventar für Entscheidungsträger nutzen zu können. Zu diesem Zweck erstellte der Arbeitsgruppenleiter eine eigene Lerneinheit innerhalb des Kurses auf der Lernplattform. Er informierte die Arbeitsgruppe darüber, dass ein Mitglied Material aus Frankreich für die gemeinsame Arbeit zur Verfügung gestellt hatte. Er bat die Teilnehmer, das pdf-Dokument zu lesen und über die Integration des Ansatzes in die Inventare zu diskutieren. Die folgende Abbildung zeigt die Lerneinheit:

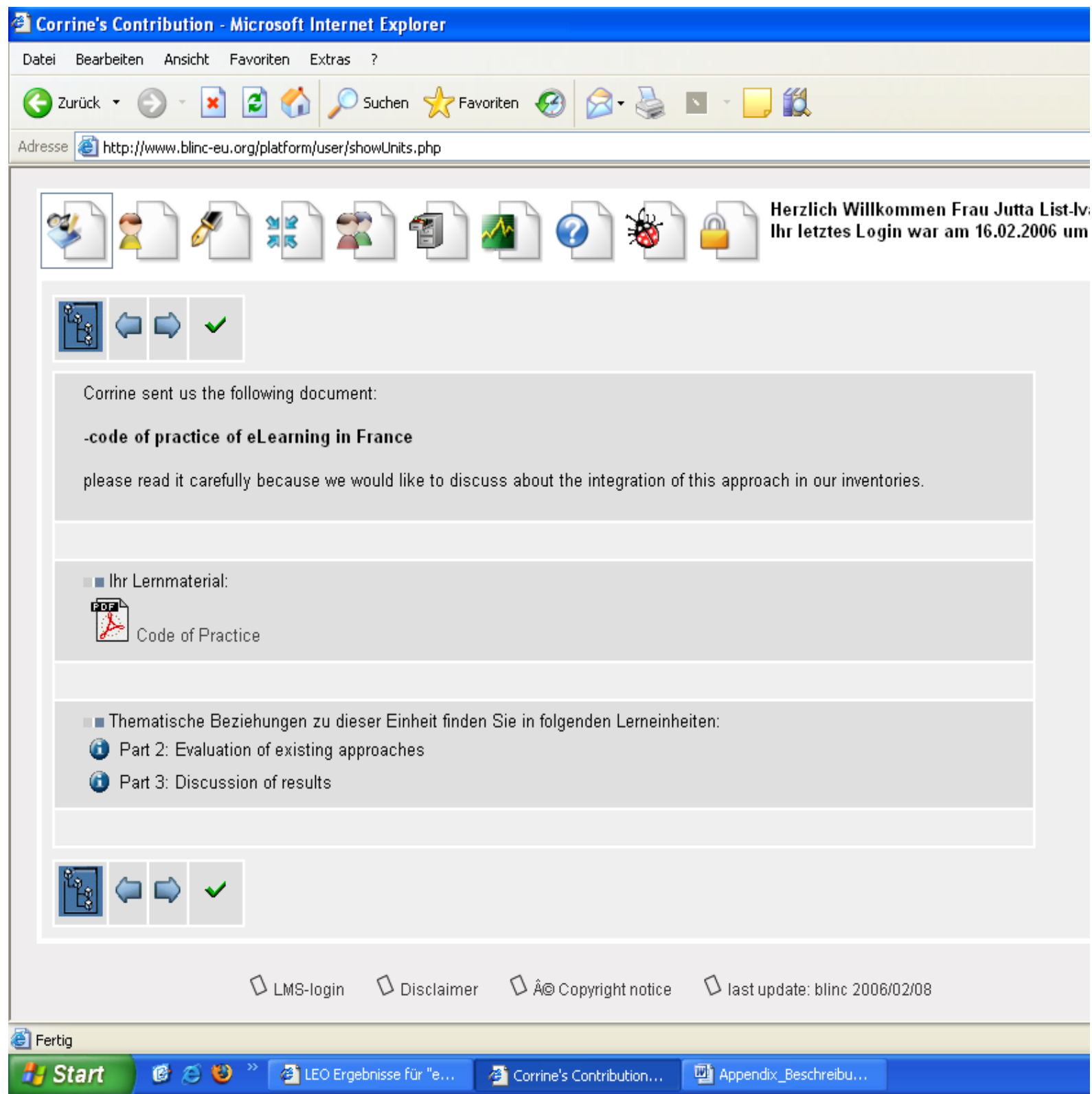

Abbildung 20: Lerneinheit im Kurs Inventare für Entscheidungsträger mit Material eines Arbeitsgruppenmitglieds

Die Arbeitsgruppenmitglieder hatten die Aufgabe, sich die hinterlegten Materialien in den einzelnen Lerneinheiten anzuschauen und ihre Ideen und Vorschläge für eine gemeinsame Version eines Inventars für Entscheidungsträger einzubringen. Sie sollten sich im Forum auf 
der Lernplattform äußern oder ihre Beiträge per E-Mail an den Arbeitsgruppenleiter schicken. Die folgende Abbildung zeigt die Beiträge von Arbeitsgruppenmitgliedern im Forum:

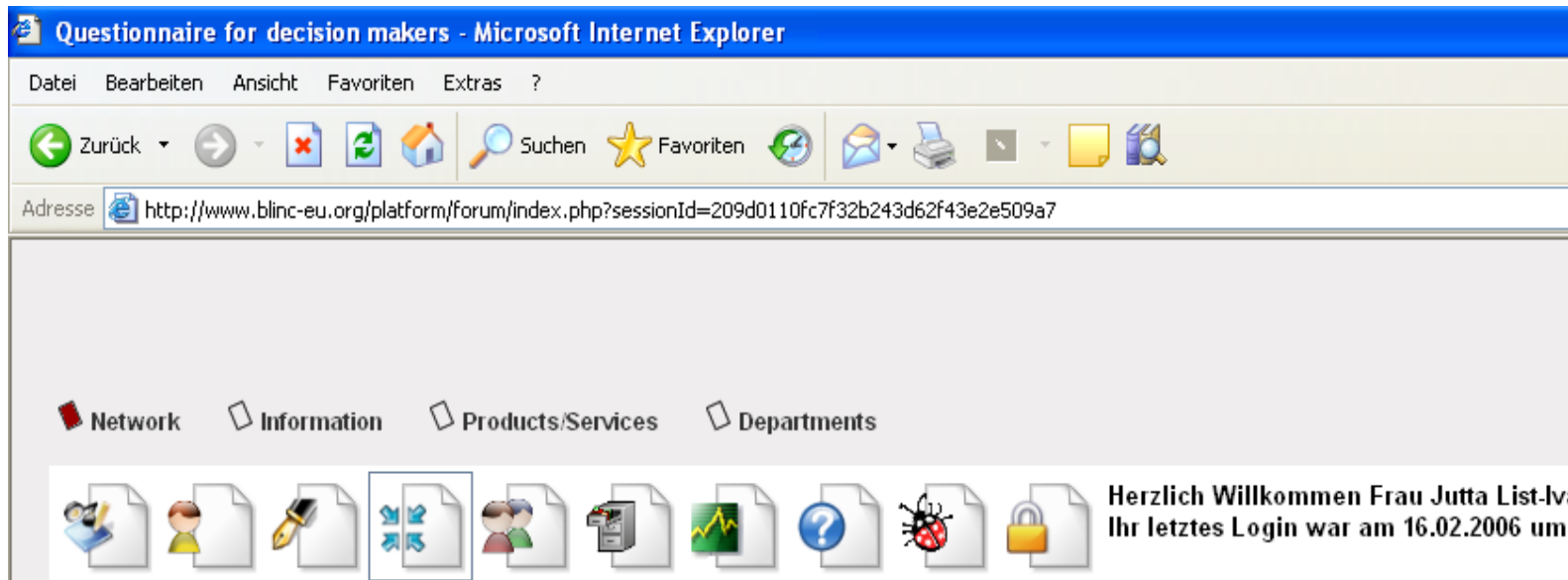

Questionnaire for decision makers

$$
\begin{aligned}
& \text { Angezeigter Zeitraum: Alle } \\
& \text { ( Neue Beiträge - max } 3 \text { Tage) }
\end{aligned}
$$

\begin{tabular}{|c|c|}
\hline$\Xi \mathrm{ACT}$ von Stellan Hansson & $(04.02 .2006)$ \\
\hline$\Xi$ hello von Cláudia Berjano & $(04.02 .2006)$ \\
\hline$\Xi$ some proposals for modification of part 2 von Hans-Dieter Haller & $(26.01 .2005)$ \\
\hline E some proposal for rmodification of part 1 von Hans-Dieter Haller & (26.01.2005) \\
\hline$\Xi$ some proposal for rmodification of part 1 von Hans-Dieter Haller & (26.01.2005) \\
\hline E some proposal for rmodification of part 1 von Hans-Dieter Haller & (26.01.2005) \\
\hline$\Xi$ Fragebogen Entscheider von Hans-Dieter Haller & $(12.12 .2004)$ \\
\hline$\equiv R$ Re: Fragebogen Entscheider von & (13.12.2004) \\
\hline$\equiv$ Bitte en Fragebogen hochladen von Michael Eichhorst & $(30.11 .2004)$ \\
\hline$\Xi$ Re: Bitte en Fragebogen hochladen von Tim Schoize & $(10.12 .2004)$ \\
\hline
\end{tabular}

10 von 10 Beiträgen werden angezeigt.

Fertig

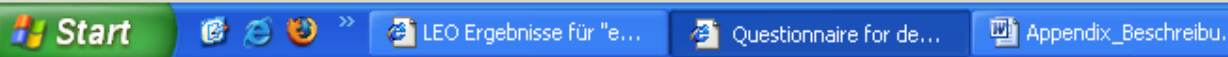

Abbildung 21: Beiträge der Arbeitsgruppe für die Inventare für Entscheidungsträger

Zu Beginn der gemeinsamen Arbeit an dem Inventar für Entscheidungsträger mittels der projektinternen Lernplattform beteiligten sich einige Partner im Forum. Einzelne Partner hinterließen Beiträge, die von den anderen Gruppenmitgliedern gelesen werden konnten. Insgesamt wurde aber recht wenig über die Lernplattform kommuniziert.

Während der transnationalen Treffen hatte die Arbeitsgruppe einige Stunden Zeit für die Weiterentwicklung der Inventare für Entscheidungsträger. In diesen Präsenzzeiten wurde auch diskutiert, und die Partner brachten ihre Ideen ein. Es stellte sich heraus, dass es für die Partner interessant war, bestimmte Schlüsselfragen zu diskutieren und Erfahrungen auszutauschen. 
Der Nutzen bzw. die weitere Verwendung der Inventare für Entscheidungsträger waren für manche Partner aber nicht ganz eindeutig. Es stellte sich die Frage, ob die Partner die Inventare selbst, für ihre eigenen e-Learning und Blended Learning-Systeme, nutzen könnten, oder ob sie mit den Inhalten zu Experten würden, die andere zukünftige Netzwerkpartner beraten könnten. Manche Partner waren vor allem an den Inhalten, dem Wissens- und Erfahrungsaustausch auf europäischer Ebene interessiert, und andere Partner sahen den Nutzen der Inventare eher unter dem Aspekt einer zukünftigen kommerziellen Nutzung in Form von Beratungen und Evaluationen.

Im Rahmen von eL3 war es das Ziel, die Inventare für Entscheidungsträger erst einmal gemeinsam zu entwickeln. Die weiteren Nutzungsmöglichkeiten stellten sich als vielfältig dar. Zunächst ging es vor allem um den Erfahrungsaustausch und die gemeinsame Entwicklung von Inhalten und Wissensbeständen, die dann von den Netzwerkpartnern individuell genutzt werden könnten.

Die Phase „Diskussion und Austausch“ dauerte insgesamt neun Monate, in denen sich die Arbeitsgruppe insbesondere während der transnationalen Projekttreffen mit folgenden Themen beschäftigte:

- Befragungszeitpunkte

- Inventare für reines e-Learning und/oder Blended Learning

- Papierbasierte Inventare versus online Befragung

- Schriftgröße und Layout

- Umfang der Inventare

- Formulierung von Antwortvorgaben versus offene Fragen

- Copyright

Die Diskussionsinhalte und -ergebnisse werden im Folgenden näher beschrieben:

\section{Befragungszeitpunkte}

Bereits bei den ersten Diskussionen während des transnationalen Treffens in Aberdeen kam die Arbeitsgruppe zu dem Ergebnis, dass zwei Inventare für Entscheidungsträger benötigt würden. Eine Version sollte von Personen genutzt werden können, die vor der Entscheidung stehen, ob sie ein e-Learning bzw. Blended Learning-System einführen wollen, und die andere Version sollte von Personen verwendet werden können, die bereits e-Learning bzw. Blended Learning-Kurse anbieten. 


\begin{tabular}{|l|l|}
\hline Zeitraum der Nutzung & Ziel \\
\hline $\begin{array}{l}\text { Vor der Einführung eines e-Learning } \\
\text { oder Blended Learning-Systems }\end{array}$ & $\begin{array}{l}\text { Überprüfung, ob ein e-Learning oder Blended } \\
\text { Learning-System geeignet ist für das Unternehmen }\end{array}$ \\
\hline $\begin{array}{l}\text { Nach der Einführung eines e-Learning } \\
\text { oder Blended Learning-Systems }\end{array}$ & Evaluation eines bestehenden Systems \\
\hline \\
Tabelle 40: Übersicht über verschiedene Möglichkeiten der Nutzung der Inventare für Entschei- \\
dungsträger
\end{tabular}

Insbesondere die Version, die vor der Einführung eines e-Learning oder Blended LearningSystems eingesetzt werden kann, würde sich auch für eine Beratung anbieten. In der Zukunft könnten eL3 Projektpartner über die Internetseiten von blinc Entscheidungsträgern Informationen und Beratungsdienste anbieten. Mit den Schlüsselfragen aus dem Inventar könnte im Gespräch oder auch schriftlich herausgearbeitet werden, ob e-Learning bzw. Blended Learning in einem Unternehmen eine geeignete Weiterbildungsmethode ist oder nicht. Mit den Antworten zu den einzelnen Fragenbereichen könnte ermittelt werden, welche Gegebenheiten für bzw. gegen die Implementierung neuer Methoden sprechen. Die Version, die für ein bestehendes System genutzt werden kann, wäre nach Meinung der Arbeitsgruppe ebenfalls geeignet, um Beratungen durchzuführen. Der Schwerpunkt würde hierbei darauf liegen, die Angemessenheit und Effektivität eines bestehenden e-Learning bzw. Blended Learning-Systems zu überprüfen und zu reflektieren.

Inventare für reines e-Learning und/oder Blended Learning

Zu Beginn des Projektes eL3 war vorgesehen, dass es vor allem um e-Learning im Dritten Sektor gehen sollte. Im Laufe des Projektes wurde jedoch deutlich, dass ein großer Teil der Projektpartner auch Blended Learning-Kurse für ihre Zielgruppen anboten und längerfristig auch ein größeres Potenzial in solchen Angeboten sahen. Nach den Erfahrungen der Projektpartner eignet sich reines e-Learning nur für bestimmte Wissensinhalte, ist jedoch meistens eher als eine Ergänzung zu traditionellen Bildungsmaßnahmen oder Ausbildungsprogrammen zu sehen. Die Kombination von e-Learning und traditionellen Lernformen in Blended Learning-Systemen ist nach Meinung der Arbeitsgruppe sinnvoll und förderlich für Lernprozesse. In modernen Unternehmen und Bildungseinrichtungen sollten Entscheidungsträger deshalb sowohl e-Learning als auch Blended Learning-Systeme für ihre Mitarbeiter bzw. Klienten anbieten. Es ist jedoch zu prüfen, ob auch tatsächlich günstige Bedingungen dafür gegeben sind. Die Inventare für Entscheidungsträger sollten nach Meinung der Arbeitsgruppe sowohl für reines e-Learning aber auch für Blended Learning-Systeme verwendet werden können. 


\section{Papierbasierte Inventare versus online Befragung}

Die Arbeitsgruppe diskutierte, ob die Inventare für Entscheidungsträger von den Nutzern online ausgefüllt werden sollten oder ob eine schriftliche Befragung günstiger sei. Bei einer online-Form könnten einerseits die Daten in einer Datenbank gesammelt werden und es könnte eine automatische Auswertung erfolgen. Es könnten sogar Empfehlungen auf der Basis der Daten gegeben werden (was spricht für bzw. gegen e-Learning oder Blended Learning). Andererseits war fraglich, ob potenzielle Nutzer ihre Daten preisgeben würden oder lieber für sich allein überprüfen möchten, ob günstige Bedingungen für e-Learning oder Blended Learning in ihrem Unternehmen gegeben sind. Die Arbeitsgruppe kam zu dem Ergebnis, dass zunächst eine papierbasierte Form der Inventare erarbeitet und erprobt werden sollte. Zu einem späteren Zeitpunkt könnte ein online-System auf der Basis der Erfahrungen aus der Erprobungsphase entwickelt werden. Es sei auch möglich, ein Beratungsangebot als Dienstleistung über blinc anzubieten, doch zunächst müssten sich die Fragenbereiche in der Praxis bewährt haben.

\section{Schriftgröße und Layout}

Das Design der Inventare für Entscheidungsträger sollte zu den Inventaren, die in den anderen Arbeitsgruppen entwickelt wurden, passen. Es sollte auch visuell deutlich werden, dass es zu einem System von Evaluationsinstrumenten gehört. Zu diesem Zweck wurde eine einheitliche Schrift und Schriftgröße gewählt und Überschriften der einzelnen Fragenbereiche wurden grafisch einheitlich hervorgehoben. Die Schriftgröße sollte nach Meinung der Gruppe 10-12 pt betragen, damit die Texte gut lesbar sind, außerdem sollten die einzelnen Themenbereiche deutlich und übersichtlich voneinander getrennt erscheinen.

\section{Umfang der Inventare}

Der Umfang der Inventare für Entscheidungsträger sollte nach Meinung der Arbeitsgruppe nicht zu groß sein. Die Zielgruppe der Entscheidungsträger zeichne sich dadurch aus, dass sie relativ zügig zu Ergebnissen und Entscheidungen kommen will. Es sollte mit möglichst wenig Zeitaufwand durch das Abwägen von Vor- und Nachteilen bzw. die Ermittlung von gegebenen Bedingungen des Unternehmens deutlich werden, ob sich e-Learning oder Blended Learning eignet oder nicht. Die Seitenzahl der Inventare sollte möglichst gering gehalten werden, um potenzielle Nutzer nicht abzuschrecken, gleichzeitig sollte es aber auch so umfangreich sein, dass wesentliche Aspekte, die für die Entscheidungen wichtig sind, enthalten sind, da eine Einführung von e-Learning bzw. Blended Learning-Systemen z.T. mit hohen Kosten verbunden ist. 


\section{Formulierung von Antwortvorgaben versus offene Fragen}

Eine Formulierung von Antwortvorgaben biete nach Meinung der Arbeitsgruppe zum einen den Vorteil, dass das Inventar zügiger ausgefüllt werden könne, als wenn Antworten von den Nutzern ausformuliert werden müssten. Zum anderen sei durch vorgegebene Antwortmöglichkeiten eine Vergleichbarkeit der Ergebnisse auf europäischer Ebene leichter, als wenn Texte analysiert und ausgewertet müssten. Insbesondere durch die Mehrsprachigkeit der Projektpartner würde dies einen erheblichen zeitlichen und finanziellen Aufwand bedeuten. Mit den Inventaren für Entscheidungsträger sollte es auch vor allem darum gehen, Argumente pro und contra e-Learning und Blended Learning-Systeme offen zu legen, auf deren Basis dann Entscheidungen getroffen werden können. Es ging nicht darum zu explorieren und zu beschreiben, wie z.B. das Unternehmen aufgebaut ist, welche Lernkultur herrscht etc., sondern eher Fakten zu benennen.

\section{Copyright}

Die Inventare für Entscheidungsträger wurden auf der Basis der Arbeiten von Flechsig (1990) und Haller (1995, 2002) entwickelt. Von den Arbeitsgruppenmitgliedern kamen kaum bzw. keine maßgeblichen Impulse oder inhaltliche Beiträge. Es wurde während der Phase „Diskussion und Austausch“ nicht über das Copyright gesprochen. Im Projekt eL3 wurde aber mit der gesamten Gruppe während der transnationalen Treffen diskutiert, wie die Inventare genutzt werden könnten, und wer das Copyright haben sollte. Für die Inventare für Entscheidungsträger sollten in jedem Fall die theoretischen Vorarbeiten und der Entstehungszusammenhang des Projektes eL3 genannt werden. Bei einer weiteren Verwendung sollten die Nutzer auf die entsprechenden Quellen verweisen. Es wurde entschieden, die Inventare über die Internetseiten von blinc frei zu Verfügung zu stellen.

\section{Phase 3: Gemeinsamer Entwurf und Übersetzung}

In den neun Monaten von September 2004 bis Mai 2005 (Phase 2) wurde bis auf ein paar Initiativen zu Beginn der gemeinsamen Arbeit kaum an den Inventaren für Entscheidungsträger gewirkt. Während der transnationalen Treffen wurde zu den Inventaren effektiv diskutiert, doch in den Zeiten, in denen über die Lernplattform zusammen gearbeitet und kommuniziert werden sollte, war die Beteiligung der Partner sehr gering. Der Arbeitsgruppenleiter stellte die erarbeiteten Inhalte auf dem transnationalen Treffen im Mai 2005 vor, und die gesamte Projektgruppe hatte Gelegenheit, Vorschläge und Verbesserungsmöglichkeiten einzubringen. Es wurde vereinbart, dass die Partner die Inventare, die bis dahin in Englisch und Deutsch vorlagen, übersetzen, um sie dann in ihren Einrichtungen zu erproben. Für die Bereitstellung eines gemeinsamen Entwurfes der Inventare nach den Diskussionen und die Übersetzung durch die Arbeitsgruppenmitglieder waren zwei Monate vorgesehen. In dieser Zeit erfolgte jedoch keine Übersetzung ins Italienische und Französische. 


\section{Phase 4: Erprobung und Ergebnisbericht}

Für die Erprobungsphase waren fünf Monate vorgesehen, in denen die Projektpartner die Inventare für Entscheidungsträger in ihren Einrichtungen verwenden sollten. Auf dem transnationalen Treffen im Mai 2005 hatten alle Projektpartner zugesagt, die Inventare erproben zu wollen. Die Partner gaben jedoch an, dass sie die Inventare vor allem zur Reflexion der Angemessenheit von e-Learning bzw. Blended Learning in ihren Einrichtungen verwenden wollten. Auf dem letzten transnationalen Treffen im November 2005 berichteten die Partner dann mündlich über die Erfahrungen mit den Inventaren. Lediglich ein Partner hatte eine Version der Inventare für Entscheidungsträger tatsächlich verwendet bzw. erprobt und die Daten projektintern zur Verfügung gestellt.

Die Projektgruppe diskutierte auf dem letzten Projekttreffen über die Inventare für Entscheidungsträger und es wurden einige, vor allem sprachliche, Verbesserungsvorschläge eingebracht. Die Partner meldeten zurück, dass die Anwendung der Inventare recht aufwändig und der direkte Nutzen nicht deutlich genug sei. Es sei nicht klar, wie mit den Ergebnissen umgegangen werden könne, wie sie konkret zu nutzen seien. Einige Partner waren der Meinung, dass eine reine Reflexion zu der Thematik, ob e-Learning oder Blended Learning für ein Unternehmen geeignet sei, den Anwendern nicht besonders viel nutzen würde. Sie waren der Meinung, dass ein Ausfüllen des Inventars direkt zu Empfehlungen führen müsse, um die Anwendung in der Praxis attraktiv zu machen. Außerdem wäre eine Einführung zum Thema e-Learning und Blended Learning als Bestandteil der Inventare für Entscheidungsträger wichtig, damit sich die Nutzer einen Überblick verschaffen können, bevor sie die Fragen beantworten und auswerten.

\section{Phase 5: Gemeinsame Endversion und Verbreitung}

Die Form der Inventare für Entscheidungsträger aus Phase 3 blieb weitestgehend unverändert, nur die Einleitungstexte wurden überarbeitet und ergänzt und einige Begriffe erläutert, damit den eL3-Partnern sowie potenziellen Anwendern die Nutzung erleichtert würde. AuBerdem wurden zu vielen Fragen Kommentare eingefügt, die den Anwendern erläutern, was eine Antwortoption konkret für die Praxis bedeutet, und wie diese Gegebenheit sich im Hinblick auf die Entscheidung für oder gegen e-Learning und Blended Learning auswirkt. Die Endversion der zwei Inventare für Entscheidungsträger, die im Rahmen von eL3 entwickelt wurden, umfassen acht Bereiche:

\section{Beschreibung der Situation (Was ist gegeben?)}

In diesem Bereich geht es um eine Bestandsaufnahme der gegebenen Situation. Es wird ermittelt, ob die Lerner sich in Gebäuden befinden, die zentral oder dezentral liegen, ob die Arbeits- und Geschäftsbereiche, aus denen die Lerner kommen, sehr verschieden sind, wie groß die Anzahl der Lerner und der Kurswiederholungen ist, welchen Status die Lerner in der 
Hierarchie des Unternehmens haben, und wie viele Sprachen die Mitarbeiter sprechen bzw. sprechen müssen.

\section{Ziele und Absichten des Trägers (Warum sollte Blended Learning oder e-Learning eingeführt werden?)}

Im diesem Teil geht es darum zu ermitteln, welche ökonomischen Gründe seitens des Unternehmens für die Einführung von e-Learning oder Blended Learning bestehen. Es wird die Frage gestellt, wie wichtig z.B. die Verringerung der Ausgaben für Bildung/Weiterbildung oder die Verringerung der Ausfallzeiten für Weiterbildungsmaßnahmen sind, oder welchen Stellenwert die Verbesserung der Lernergebnisse oder der Lernumgebung hat. Es wird auch die Frage gestellt, wie wichtig eine Standardisierung von Bildungsmaßnahmen für das Unternehmen ist.

\section{Beschreibung der Lernenden (Wer soll lernen?)}

Mit den Fragen dieses Bereiches sollen die Charakteristika der Zielgruppe - der Lerner erfasst werden. Zu diesem Zweck werden Fragen zum Bildungs- und Qualifikationsniveau der Lerner sowie zu deren Alter und Geschlecht gestellt. Außerdem wird nach der Motivation und nach den Perspektiven der Lerner gefragt, nach deren IT-Kompetenzen und nach dem Lerninteresse bzw. Wert von Bildung.

\section{Beschreibung der Lernumgebung (Wo sollen sie lernen?)}

In diesem Bereich wird die Lernumgebung genauer definiert. Es geht um die Bereiche für berufliches Lernen, die Lernkultur in dem Unternehmen, die technische Ausstattung sowie um die Beschaffenheit der Lern- und Arbeitsplätze. Außerdem wird nach den sprachlichen Anforderungen an die Lerner gefragt.

\section{Beschreibung der Anforderungen (Was soll das Ergebnis sein?)}

Mit diesem Teil des Inventars für Entscheidungsträger soll ermittelt werden, wie klar definiert die Absichten und Pläne des Bildungsträgers im Hinblick auf die Wissens- und Lernorganisation sind und mit welcher Deutlichkeit die Kompetenzanforderungen und Lernziele beschrieben werden. Außerdem wird erfragt, wie klar definiert die Qualifikationsangebote für Mitarbeiter und das Bewertungssystem für Weiterbildung sind.

\section{Beschreibung der Inhalte (Was sollen sie lernen?)}

Mit den Fragen aus diesem Teil des Inventars soll erfasst werden, um welche thematischen Bereiche es sich handelt und wie hoch der Anteil verschiedener Wissensarten ist (Methodisches Wissen, Hintergrundwissen, Erklärungswissen, Handlungswissen und Antizipatorisches Wissen). 


\section{Erwartete Ergebnisse}

In diesem Bereich wird abgefragt, welche Ergebnisse von der Einführung eines e-Learning oder Blended Learning-Systems erwartet werden. Es wird thematisiert, ob die Organisation der Weiterbildungen in der Einrichtung bzw. dem Unternehmen grundlegend oder nur einigermaßen neu gestaltet, oder ob sie möglichst wenig verändert werden soll. Außerdem wird ermittelt, welchen Stellenwert verschiedene Weiterbildungsformen und Lernmaterialien haben (z.B. webbasierte Bildungsangebote und digitale Lernmaterialien).

\section{Organisationsentwicklung und Integration}

Unter den Perspektiven der Organisationsentwicklung und Integration wird die Frage gestellt, wie vielfältig die weiteren Nutzungsmöglichkeiten für die Bildungsangebote sind und ob es Möglichkeiten zur internen Verbreitung gibt. Außerdem wird nach Perspektiven für die Integration der Bildung bzw. Weiterbildungen in Qualitäts-Management-Systeme gefragt.

Die folgende Tabelle gibt einen Gesamtüberblick über das Inventar für Entscheidungsträger: Das Inventar für Entscheidungsträger wurde am Ende des Projektes eL3 über die Internetseiten von blinc als pdf- Dokument und als Word-Dokument einer breiteren Öffentlichkeit frei zur Verfügung gestellt. 


\begin{tabular}{|c|c|}
\hline Fragenbereich & Themen/ Fragen zu... \\
\hline $\begin{array}{l}\text { 1. Beschreibung der Situation } \\
\text { (Status quo: Was ist gegeben?) }\end{array}$ & $\begin{array}{l}\text { - Koordinaten der Einrichtung/des Unternehmens } \\
\text { - Arbeits- oder Geschäftsbereiche } \\
\text { - Anzahl der Mitarbeiter/Lerner } \\
\text { - Hierarchie (Management, Arbeiter etc.) } \\
\text { - Sprachen, Internationalität des Unternehmens }\end{array}$ \\
\hline $\begin{array}{l}\text { 2. Ziele und Absichten des Trägers } \\
\text { (Warum sollte Blended Learning o- } \\
\text { der e-Learning eingeführt werden?) }\end{array}$ & $\begin{array}{l}\text { - Ökonomische Gründe } \\
\text { - Verbesserung der Lernergebnisse } \\
\text { - Verbesserung der Qualität des Lernens } \\
\text { - Standardisierung von Weiterbildungen }\end{array}$ \\
\hline $\begin{array}{l}\text { 3. Beschreibung der Lernenden } \\
\text { (Wer soll lernen?) }\end{array}$ & $\begin{array}{l}\text { - Bildung- und Qualifikationsniveau } \\
\text { - Alter } \\
\text { - Geschlecht } \\
\text { - Arbeits- und Lernzeit } \\
\text { - Motivation der Lerner (z.B. Belohnungen) } \\
\text { - Perspektiven der Lerner } \\
\text { - Weitere Zielgruppen (z.B. bei Geschäftspartnern) } \\
\text { - Dispositionen der Lerner: } \\
\text { - IT-Kompetenzen } \\
\text { - Lerninteresse/Wert von Bildung }\end{array}$ \\
\hline $\begin{array}{l}\text { 4. Beschreibung der Lernumgebung } \\
\text { (Wo sollen sie lernen?) }\end{array}$ & $\begin{array}{l}\text { - Bereiche beruflichen Lernens } \\
\text { - Lernkultur } \\
\text { - Technische Ausstattung } \\
\text { - Lernplätze und Arbeitsplätze } \\
\text { - Verschiedene Sprachen der Lernenden }\end{array}$ \\
\hline $\begin{array}{l}\text { 5. Beschreibung der Anforderungen } \\
\text { (Was soll das Ergebnis sein?) }\end{array}$ & $\begin{array}{l}\text { - Absichten und Pläne des Bildungsträgers } \\
\text { - Kompetenzanforderungen und Lernziele } \\
\text { - Qualifikationsangebote für Mitarbeiter } \\
\text { - Bewertungssystem für Weiterbildung }\end{array}$ \\
\hline $\begin{array}{l}\text { 6. Beschreibung der Inhalte } \\
\text { (Was sollen sie lernen?) }\end{array}$ & $\begin{array}{l}\text { - Thematische Bereiche/Inhalte } \\
\text { - Methodisches Wissen } \\
\text { - Hintergrundwissen } \\
\text { - Erklärungswissen } \\
\text { - Handlungswissen } \\
\text { - Antizipatorisches Wissen }\end{array}$ \\
\hline 7. Erwartete Ergebnisse & $\begin{array}{l}\text { - Gestaltung und Organisation der Weiterbildung in der } \\
\text { Einrichtung/dem Unternehmen } \\
\text { - Webbasierte Bildungsangebote } \\
\text { - e-Learning-Kurse } \\
\text { - Blended Learning-Kurse } \\
\text { - Digitale Lernmaterialien } \\
\text { - CBT's oder WBT's } \\
\text { - Tutoringmaßnahmen } \\
\text { - Schulungen der internen Weiterbildungsleiter und } \\
\text { Trainer/Dozenten }\end{array}$ \\
\hline $\begin{array}{l}\text { 8. Organisationsentwicklung und } \\
\text { Integration }\end{array}$ & $\begin{array}{l}\text { - Weitere Nutzungsmöglichkeiten der Bildungsangebote } \\
\text { - Möglichkeiten zur internen Dissemination von Bil- } \\
\text { dungsangeboten } \\
\text { - Möglichkeiten der Integration der Bil- } \\
\text { dung/Weiterbildung in Qualitäts-Management- } \\
\text { Systeme }\end{array}$ \\
\hline
\end{tabular}

Tabelle 41: Übersicht Inventar für Entscheidungsträger 


\subsection{Erprobung der Inventare: Fragebogen zum Abschluss des Pro- jektes}

Zum Abschluss des Projektes eL3 wurden die Partner per Fragebogen befragt (siehe Anhang), um zu ermitteln, wer welche Inventare in welcher Form verwendet hat und welche Erfahrungen damit gemacht wurden. Es war geplant, dass alle Partner die gemeinsam entwickelten Inventare benutzen und gewissermaßen "testen“, um sie danach ggf. zu überarbeiten und zu verbessern oder womöglich in der bestehenden Form über die Internetplattform "blinc" auch anderen zur Verfügung zu stellen. Mit diesem Pretest sollten die Praxistauglichkeit, die Handhabbarkeit und der Nutzen überprüft werden.

Auf dem vierten transnationalen Treffen in Siena im Mai 2005 wurden die Inventare, die in den Arbeitsgruppen erarbeitet waren, der gesamten Projektgruppe präsentiert und es wurde vereinbart, wer die in englischer Sprache verfassten Inventare übersetzt und die Ergebnisse auswertet. Die meisten Partner wollten ihre Daten selbst auswerten und nicht, dass es ein anderen Partner tut (etwa das Team des Pädagogischen Seminars). Es wurden erste Ideen formuliert, wie die Ergebnisse genutzt werden können. Einige Partner (mit universitärem Hintergrund) wollten vor allem Publikationen auf der Basis der ersten Erfahrungen mit den Inventaren erarbeiten und veröffentlichen, und andere Partner (Bildungseinrichtungen und Bildungsanbieter) wollten eher auf der Grundlage des ersten Einsatzes Beratungen für andere europäische Partner anbieten und damit gewissermaßen eine Dienstleistung oder ein Produkt auf den Markt bringen. Es wurde vereinbart, dass alle Partner die Inventare in ihren Einrichtungen benutzen und die Daten bzw. die Erfahrungen damit auf dem letzten transnationalen Treffen in Marseille im November 2005 zusammengetragen und ausgewertet würden (zum Ablauf des Projektes siehe auch Tabelle 9).

Am Ende des Projektes ergab sich bei der Gruppendiskussion auf dem letzten transnationalen Treffen und anhand der Befragung folgendes Bild: Nicht alle Partner haben überhaupt die Inventare benutzt (obwohl dies vereinbart war und zugesichert wurde). Einige Partner haben einzelne Inventare benutzt, wussten aber nicht, wie sie die erhobenen Daten auswerten sollten, und andere Partner haben Inventare genutzt und die Daten auch selbst ausgewertet. Für die meisten blieb es unklar, was sie mit den ermittelten Ergebnissen anfangen sollten, und sie wünschten sich eine Beratung oder Unterstützung bei der Klärung von entstandenen Fragen. Einige Partner fanden den Einsatz der Inventare anregend und haben auf der Grundlage der erhobenen Daten oder der (Selbst)reflexion über ihre Bildungsangebote neue Ideen entwickelt und Einsichten bekommen. Für die meisten Partner war der Einsatz der Inventare anscheinend komplizierter und schwieriger als gedacht. Die folgende Tabelle zeigt in der Übersicht, welche Partner welche Inventare verwendet haben (zu den Einrichtungen der Partner siehe Abschnitt 5.1.2): 


\begin{tabular}{|c|c|c|c|}
\hline Partner & $\begin{array}{l}\text { Art der Einrichtung } \\
\text { und Land }\end{array}$ & Einsatz welcher Inventare & $\begin{array}{l}\text { Erhobene Daten/ Fall- } \\
\text { zahlen }\end{array}$ \\
\hline 1 & $\begin{array}{l}\text { Krankenhaus } \\
\text { Deutschland }\end{array}$ & $\begin{array}{l}\text { Entscheidungsträger } \\
\text { Inventar für Lerner } 3\end{array}$ & $\begin{array}{l}\mathrm{n}=24 \\
\mathrm{n}=17\end{array}$ \\
\hline 2 & $\begin{array}{l}\text { Krankenhaus } \\
\text { Italien }\end{array}$ & Kein Inventar benutzt & - \\
\hline 3 & $\begin{array}{l}\text { Krankenhaus } \\
\text { Frankreich }\end{array}$ & Kein Inventar benutzt & - \\
\hline 4 & $\begin{array}{l}\text { Bildungsanbieter } \\
\text { Deutschland }\end{array}$ & $\begin{array}{l}\text { Autoreninventar } \\
\text { Inventar für Lerner1, } 2 \text { und } 3\end{array}$ & $\begin{array}{l}\mathrm{n}=1 \\
\mathrm{n}=9\end{array}$ \\
\hline 5 & $\begin{array}{l}\text { Bildungsanbieter } \\
\text { Italien }\end{array}$ & Inventar für Lerner 3 & $\mathrm{n}=17$ \\
\hline 6 & $\begin{array}{l}\text { Bildungsanbieter } \\
\text { Frankreich }\end{array}$ & Kein Inventar benutzt & - \\
\hline 7 & $\begin{array}{l}\text { Bildungsanbieter } \\
\text { Frankreich }\end{array}$ & Inventar für Lerner 3 & - \\
\hline 8 & $\begin{array}{l}\text { Universität } \\
\text { Schottland }\end{array}$ & $\begin{array}{l}\text { Inventar für Lerner 1, } 2 \text { und } 3 \\
\text { Einsatz scheiterte an der Uni- } \\
\text { versitätsleitung, aber beste- } \\
\text { hende Fragebögen wurden } \\
\text { ergänzt }\end{array}$ & - \\
\hline 9 & $\begin{array}{l}\text { Universität } \\
\text { Frankreich }\end{array}$ & Inventar für Lerner 3 & $\mathrm{n}=32$ \\
\hline
\end{tabular}

Tabelle 42: Übersicht, welche Partner welche Inventare benutzt haben

Insgesamt bleibt festzuhalten, dass fünf von neun Partnern die Inventare bzw. einen Teil der Inventare benutzt haben (siehe Tabelle Partner Nr. 1, 4, 5, 7 und 9). Die anderen vier Partner (siehe Tabelle Partner Nr. 2, 3, 6 und 8) haben keine Inventare eingesetzt, genauer gesagt: drei haben keine Inventare genutzt und ein Partner wollte die Inventare verwenden, bekam aber nicht die Erlaubnis innerhalb der Institution. Die Gründe bei den drei Partnern, die gar kein Inventar benutzt haben, bleiben unklar, in der Gruppendiskussion wurde zugesichert, dass die Inventare zu einem späteren Zeitpunkt noch verwendet werden würden.

Bei denjenigen, die Inventare benutzt haben, bestand nach den Rückmeldungen der Partner Interesse am Austausch zu Erfahrungen mit der Evaluation auf der Grundlage der Inventare, es war aber auch ein Bedarf an Unterstützung bei der Durchführung der Befragungen vorhanden. Alle fünf Partner haben eine eigene Auswahl von Fragen aus den Inventaren, die sie verwendet haben, getroffen und eine individuelle Form eines Fragebogens erstellt. Die Fragebögen wurden entweder per E-Mail an die Kursteilnehmer geschickt (als WordDokument) und von den Befragten ausgefüllt ebenfalls per E-Mail zurückgeschickt, oder es wurden papierbasierte Fragebögen während eines Kurses ausgeteilt und wieder eingesammelt (paper-pencil-Form). Von den fünf Partnern, die Inventare benutzt haben, schickten vier die erhobenen Daten in Form von ausgefüllten Fragebögen an die Verfasserin (entweder per E-Mail oder per Post). Das Ziel war, die Daten zunächst einmal zu sammeln, auszuwerten und zu vergleichen. Dabei ergaben sich jedoch einige Schwierigkeiten: 
1. Die Kurskontexte waren sehr verschieden, so dass ein Vergleich der Daten nicht ohne weiteres möglich war bzw. stark berücksichtigt werden musste.

2. Die Zielgruppen waren heterogen (Studenten, Pflegepersonal, Leitungskräfte).

3. Die Daten lagen in unterschiedlichen Sprachen vor (Französisch, Italienisch und Deutsch) - die Verfasserin konnte die Daten (die offenen Fragen) in Französisch und Italienisch nicht auswerten.

4. Die Durchführung der Befragungen wurde sehr unterschiedlich gehandhabt, so dass dies auch die Ergebnisse beeinflussen könnte (die Studenten wurden verpflichtet, den Fragebogen auszufüllen, und es wurde innen angedroht, den „Schein“ nicht zu bekommen, wenn sie es nicht tun würden, bei den Pflegekräften wurde in einem Fall eine Gruppe befragt, die nicht einmal in einem Kurs zusammen kam, sondern einfach über die Stationsleitung befragt wurde bzw. gebeten wurde, einen Fragebogen auszufüllen, da die Daten für ein Projekt gebraucht würden).

Es wurde deutlich, dass einige Partner (die Praxispartner) Unterstützung bei der Durchführung der Evaluation brauchten, sie äußerten dies auch bei der Befragung. Es zeigte sich, dass sowohl beim Einsatz der Inventare (Auswahl der Fragen, Gestaltung des individuellen Fragebogens und Durchführung der Befragung) als auch bei der Auswertung (Analyse der Daten, Ergebnisauswertung, Verwendung der Ergebnisse) Hilfestellungen nötig wären. Andere Partner (mit universitärem Hintergrund) waren durchaus in der Lage, die Erhebungen nach gängigen Regeln durchzuführen und die Daten auszuwerten, dafür waren sie weniger bereit, die Daten mit den anderen „zu teilen“, d.h. einen Einblick in ihre Untersuchungen zu gewähren. Insgesamt blieb fraglich, wie die Daten aller Partner weiter genutzt werden könnten bzw. wie die Ergebnisse ausgewertet und in einer Sprache auch anderen zugänglich gemacht werden könnten. Das "Teilen der Daten" stellte sich als ein aus verschiedenen Gründen sensibles Thema heraus:

1. Institutionelle Bedingungen

Vorgesetzte mussten einwilligen in eine Befragung, d.h. sie mussten umfassend informiert werden und die Befragung sinnvoll finden, unterstützen, genehmigen bzw. durchführen.

2. Einblick in die Arbeit

Für einige Partner war es offenbar ein Problem bzw. unangenehm, "sich in die Karten schauen zu lassen“. Sie wollten nicht, dass andere Personen Einblicke in ihre Arbeit haben, und wollten "die Fäden auf jeden Fall in den eigenen Händen behalten“. 


\section{Datenschutz}

Eine Veröffentlichung von Daten ist aus Datenschutzgründen nicht ohne weiteres möglich. Ergebnisse müssten (z.B. im Internet auf der Plattform blinc, aber auch im Projekt eL3) in einer entsprechenden Form (anonymisiert) oder in einem geschützten Bereich dargestellt werden.

Ein weiteres Thema war nach den Rückmeldungen der Partner, wie die Daten und die Ergebnisse weiter genutzt werden könnten. Einerseits war es für die Partner von Interesse, Instrumente zur Evaluation ihrer Kurse zu haben (die Fragebögen auf der Grundlage der Inventare), und andererseits wollten sie aber auch Erfahrungen austauschen und von den anderen lernen. Es war für die Partner im Projekt eL3 sehr wertvoll, Einblicke in die Arbeit von anderen zu bekommen und dadurch die eigene Arbeit neu zu betrachten (siehe Ergebnisse der Prozessevaluation im Unterkapitel 6.2).

Zusammenfassend kann mit der Befragung der Partner zum Abschluss des Projektes festgehalten werden, dass etwa die Hälfte der Partner einzelne Inventare benutzt, auf dieser Grundlage eine Auswahl von Fragen getroffen und so individuelle Fragebögen eingesetzt hat. Dabei wurden die Fragebögen per E-Mail verschickt und von den Befragten auch wieder per E-Mail zurückgeschickt, oder es wurden papierbasierte Fragebögen ausgeteilt, handschriftlich ausgefüllt und anschließend wieder eingesammelt. Die Inventare sind nach Meinung der Partner, die sie verwendet haben, nützlich. Es werden nach ihrer Meinung jedoch mehr Hintergrundinformationen benötigt, z.B. um auch Vorgesetzten oder den Befragten mehr Informationen geben zu können. Die Handhabbarkeit der Inventare muss nach den Erfahrungen der Partner ebenfalls verbessert werden. Es wird Unterstützung bei der Durchführung des Fragebogeneinsatzes und auch bei der Auswertung gebraucht. Bei dem Ausfüllen der Fragebögen müssten auch die Befragten häufig Hilfestellungen bekommen, so dass ein persönlicher Ansprechpartner bei den Befragungen wichtig ist, der sich mit den Inventaren bzw. den auf dieser Grundlage erstellten Fragebögen auskennt. Die Befragten meldeten teilweise zurück, dass sie den Sinn der Befragung gerne kennen würden und auch wissen möchten, was mit den Daten weiterhin passiert. Die weitere Verwendung der Ergebnisse war auch für die eL3-Partner ein Thema, das der Klärung bedarf. Es müsste eine Form gefunden werden, von der die einzelnen Partner ebenso wie die Gruppe bzw. die spätere blincGemeinschaft, die über das Projekt hinaus bestehen soll, profitieren können. Es müsste eine Basis geschaffen werden, wo Erfahrungen und Ergebnisse ausgetauscht werden können, ohne dass ein beteiligter Partner kontrolliert oder abgewertet werden könnte, sondern gute Praxis veröffentlicht und verbreitet werden würde. Da die Interessen der Partner sehr heterogen waren - je nach institutionellem Hintergrund - müssten verschiedene Möglichkeiten geschaffen werden, wie z.B. Raum für Publikationen, aber auch für Produkte oder Dienstleis- 
tungen, die angeboten werden können. Zum Ende des Projektes zeigte sich, dass es viele offene Fragen zu klären gab, die für eine weitere Verwendung und Nutzung der Inventare entscheidend sein würden.

Nach dieser allgemeinen Auswertung der Erprobungsphase wird in den nächsten Abschnitten auf der Grundlage der Berichte der Partner während des letzten transnationalen Treffens genauer dargestellt, wie die einzelnen Inventare genutzt wurden.

\subsubsection{Die Inventare für Lerner}

Nachdem die Inventare für Lerner in der Arbeitsgruppe entwickelt wurden, sollte deren Leistungsfähigkeit im Gebrauch von der gesamten Projektgruppe erprobt werden. Die Ergebnisse und Erfahrungen, die mit dem ersten Einsatz der Inventare für Lerner gesammelt wurden, sollten genutzt werden, um die Fragenbereiche bzw. einzelne Fragen zu optimieren. Durch die Erhebungen mit den Inventaren für Lerner solle außerdem der Austausch von Erfahrungen im blinc-Netzwerk angeregt werden (z.B. Präsentation von gelungenen Blended Learning-Kursen, Darstellung von Erfolgsfaktoren für das Gelingen eines Kurses aus Sicht der Lerner).

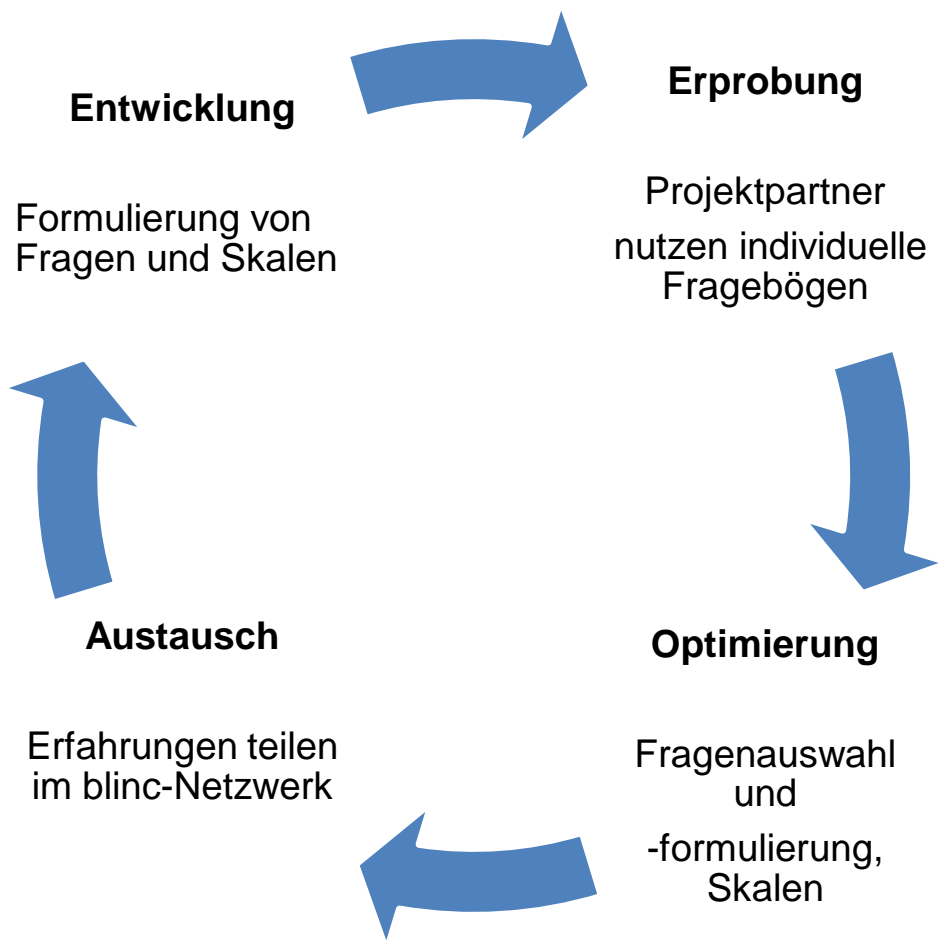

Abbildung 22: Verschiedene Phasen im Optimierungsprozess der Inventare für Lerner

Auf dem Projekttreffen im Mai 2005 wurde der gesamten Projektgruppe die englische Masterversion der drei Lernerinventare von der verantwortlichen Arbeitsgruppe vorgestellt. Die 
Inhalte wurden nochmals diskutiert bzw. erklärt, und es wurde entschieden, welche Projektpartner die Inventare in welchen Kursen einsetzen wollten. Das Ziel war, möglichst viele Kurse mit den Inventaren zu evaluieren und insgesamt auf eine möglichst hohe Anzahl von Lernern zu kommen, um Erfahrungen mit den Inventaren bzw. einzelnen Fragen zu sammeln. Nach dem ersten Einsatz der Inventare für Lerner hätten erste Aussagen zu Reliabilität und Validität der Instrumente getroffen werden können. Nahezu alle Projektpartner wollten die Inventare für Lerner in ihren Einrichtungen verwenden. Zu diesem Zeitpunkt gaben die Partner an, die Inventare in verschiedenen Kursen nutzen zu wollen, wobei die Anzahl der Lerner insgesamt etwa 750 betragen würde.

\begin{tabular}{|l|l|l|l|}
\hline $\begin{array}{l}\text { Partner } \\
\text { Einrichtung } \\
\text { Land }\end{array}$ & $\begin{array}{l}\text { Kurstitel bzw. } \\
\text { Kursthema oder Kursinhalt }\end{array}$ & Inventar & $\begin{array}{l}\text { Anzahl der } \\
\text { Lerner }\end{array}$ \\
\hline $\begin{array}{l}\text { Partner 1 } \\
\text { Bildungsanbieter } \\
\text { Deutschland }\end{array}$ & $\begin{array}{l}\text { "Fit for Change“ } \\
\text { Gesundheits- und Sozialwirtschaft }\end{array}$ & Inventar 2 & ca. 25 \\
\hline $\begin{array}{l}\text { Partner 1 } \\
\text { Bildungsanbieter } \\
\text { Deutschland }\end{array}$ & "Prokonsens“ & Inventar 1 & ca. 20 \\
\hline $\begin{array}{l}\text { Partner 2 } \\
\text { Krankenhaus } \\
\text { Deutschland }\end{array}$ & Weiterbildungen für Personal & Inventar 3 & ca. 200 \\
\hline $\begin{array}{l}\text { Partner 3 } \\
\text { Krankenhaus } \\
\text { Italien }\end{array}$ & Weiterbildungen für Personal & Inventar 3 & ca. 120 \\
\hline $\begin{array}{l}\text { Partner 4 } \\
\text { Universität } \\
\text { Italien }\end{array}$ & "Weblingua“ & Inventar 3 & ca. 100 \\
\hline $\begin{array}{l}\text { Partner 5 } \\
\text { Bildungsanbieter } \\
\text { Frankreich }\end{array}$ & Computerkurse mit ECDL-Zertifikat & Inventar 3 & ca. 200 \\
\hline $\begin{array}{l}\text { Partner 6 } \\
\text { Universität } \\
\text { Frankreich }\end{array}$ & "Tabaco“ & & \\
\hline $\begin{array}{l}\text { Partner 7 } \\
\text { Krankenhaus } \\
\text { Frankreich }\end{array}$ & Weinarerbildungen für Personal & Inventar 3 & ca. 35 \\
\hline $\begin{array}{l}\text { Partner 8 } \\
\text { Universität } \\
\text { Schottland }\end{array}$ & Seminar für Studenten & Inventar 3 & ca. 200 \\
\hline Insgesamt & Alle Kurse & Inventare & ca. 750 \\
\hline Tabelle 43: & Inventar 3 & ca. 30 \\
\hline
\end{tabular}

Tabelle 43: $\quad$ Geplanter Einsatz der Inventare für Lerner im Mai 2005

Die Inventare sollten zunächst in die Sprachen Deutsch, Italienisch und Französisch übersetzt werden. Für den gesamten Pool der Items aus den Inventaren für Lerner sollte es in jeder Sprache nur eine gemeinsame Übersetzung geben, um eine Vergleichbarkeit der Ergebnisse zu gewährleisten, deshalb war es die Aufgabe der Arbeitsgruppenleiterin, eine multilinguale Endversion der Inventare zu erarbeiten und den Projektpartnern zur Verfügung zu stellen. Auf dem Projekttreffen im Mai 2005 wurde die weitere Vorgehensweise genau vereinbart. Die nächsten Arbeitsschritte für die Projektpartner in Bezug auf den Einsatz der Inventare waren: 
a) Übersetzung der Inventare in die verschiedenen Sprachen durch die Partner.

b) Zusammenstellung und Kontrolle der Übersetzungen in einer multilingualen Version der Inventare für Lerner 1-3 durch die Verantwortliche der Arbeitsgruppe.

c) Bereitstellung der multilingualen Version für alle Partner über die gemeinsame Internetseite und durch Verschicken per E-Mail an jeden einzelnen Partner.

d) Auswahl der individuell relevanten Fragen für spezielle Kurszusammenhänge durch die Partner.

e) Erstellung eigener Fragebögen auf der Basis der Inventare.

f) Einsatz der Fragebögen in verschiedenen Kursen.

g) Eingabe der Daten in eine Excel-Tabelle, die von der Verantwortlichen der Arbeitsgruppe erstellt wurde oder alternativ Aushändigung der ausgefüllten Fragebögen per E-Mail oder Post.

h) Feedback zum ersten Einsatz der Inventare (Ausfüllen eines Fragebogens).

Während des Treffens im Mai 2005 zeichnete sich in den Diskussionen ab, dass manche Partner die Inhalte der Inventare für Lerner zwar nutzen wollten, aber nicht in dem eigentlich vorgesehenen Verfahren. Manche Partner wollten ihre eigenen bereits vorhandenen Instrumente (Fragebögen) durch einzelne Items aus den Inventaren für Lerner ergänzen. Sie wollten ihre „bewährten Instrumente“ nicht durch die Inventare ersetzen, sondern ergänzen. Diese Vorgehensweise konnte den Partnern nicht verboten werden, aber es war klar, dass dadurch ein Vergleich der Daten auf europäischer Ebene kaum möglich sein würde. Die Instrumente und damit erhobenen Daten wären so nicht vergleichbar (z.B. unterschiedlicher Aufbau des Fragebogens, andere Reihenfolge von Fragen). Ein anderer Projektpartner äuBerte Bedenken zum Einsatz der Inventare, da er sich nicht sicher sei, ob die Verantwortlichen seiner Einrichtung einverstanden wären, eine Befragung mit den Inventaren durchzuführen. Es müsste erst eine Erlaubnis eingeholt werden. Zu einem späteren Zeitpunkt wurde deutlich, dass eine Befragung aus forschungsethischen Gesichtspunkten abgelehnt wurde, da bereits verschiedene Evaluationen liefen und den Lernern nicht noch mehr Fragebögen zugemutet werden sollten. Es wurde dann von diesem Partner geplant, die Inventare in der Zukunft zu nutzen bzw. auf der Grundlage der Inhalte der Inventare neue Instrumente zu entwickeln. Insgesamt zeigte sich, dass die eL3-Projektpartner im Gegensatz zum ursprünglichen Plan (jeder testet die Inventare in mehreren Kursen) aus verschiedenen Gründen höchst individuell mit den Inventaren umgingen. Manche Partner hielten sich an den Plan, auf der Grundlage der Inventare einen eigenen Fragebogen zu erstellen und einzusetzen. Andere Partner integrierten einzelne Fragen aus den Inventaren in ihre bereits bestehenden Fragebögen, und wieder andere Partner verwendeten die Inhalte der Inventare als Diskussionsgrundlage, um neue Fragebögen zu entwickeln. ,Die Inventare wurden im Gegensatz zum ursprünglichen Plan also sehr unterschiedlich genutzt: 


\section{- als eigenständige Evaluationsinstrumente}

(mit allen bzw. einer Auswahl von Fragen aus dem Fragenpool),

- als Anregungs- und Ergänzungsmaterial

(mit einzelnen Fragen, die in bestehende Instrumente integriert wurden),

- als Diskussionsgegenstand

(zur Erarbeitung neuer Fragebögen),

- als Grundlage für weitere Erhebungsmethoden

(Inhalte wurden zur Erarbeitung des Interviewleitfadens verwendet).

Manche Partner waren bereit, nach dem ersten Einsatz der Inventare ihre Daten zur Verfügung zu stellen, aber die Mehrzahl erhob entweder gar keine Daten oder wollte bzw. durfte sie nicht in das Netzwerk geben. Einige Partner meldeten zurück, dass es zu aufwendig sei, die Daten in Excel zu übertragen, wollten diese Arbeit aber auch nicht dem verantwortlichen Projektpartner (Pädagogisches Seminar) überlassen. Das Mitteilen der Daten stellte sich als ein Problem heraus. Zusätzlich erschwerte die unterschiedliche Nutzung der Inventare immer mehr die Vergleichbarkeit der Daten. Die geplante Vorgehensweise entwickelte eine gewisse Eigendynamik. Bis zum letzten Projekttreffen im November 2005 wurden insgesamt folgende Daten mit den Inventaren für Lerner im Rahmen von eL3 erhoben und ausgewertet: 


\begin{tabular}{|c|c|c|c|c|}
\hline $\begin{array}{l}\text { Partner } \\
\text { Einrichtung } \\
\text { Land }\end{array}$ & $\begin{array}{l}\text { Kurstitel bzw. } \\
\text { Kursthema oder Kursinhalt }\end{array}$ & Inventar & $\begin{array}{l}\text { Anzahl der } \\
\text { Lerner }\end{array}$ & $\begin{array}{l}\text { Anzahl } \\
\text { ausgefüllter } \\
\text { Fragebögen }\end{array}$ \\
\hline $\begin{array}{l}\text { Partner } 1 \\
\text { Bildungsanbieter } \\
\text { Deutschland }\end{array}$ & $\begin{array}{l}\text { "Fit for Change“ } \\
\text { Managementfortbildung für Frauen in } \\
\text { Betrieben der Gesundheits- und Sozial- } \\
\text { wirtschaft }\end{array}$ & Inventar 2 & 24 & 9 \\
\hline $\begin{array}{l}\text { Partner 1 } \\
\text { Bildungsanbieter } \\
\text { Deutschland }\end{array}$ & $\begin{array}{l}\text { "Prokonsens“ } \\
\text { Konfliktmanagement im Krankenhaus }\end{array}$ & Inventar 1 & 15 & 14 \\
\hline $\begin{array}{l}\text { Partner } 2 \\
\text { Krankenhaus } \\
\text { Deutschland }\end{array}$ & Kein spezieller Kurs & Inventar 3 & 50 & 19 \\
\hline $\begin{array}{l}\text { Partner } 3 \\
\text { Krankenhaus } \\
\text { Italien }\end{array}$ & $\begin{array}{l}\text { Weiterbildungen Hygiene im Kranken- } \\
\text { haus }\end{array}$ & Inventar 3 & 120 & - \\
\hline $\begin{array}{l}\text { Partner } 4 \\
\text { Universität } \\
\text { Italien }\end{array}$ & $\begin{array}{l}\text { "Weblingua“ } \\
\text { Englischkurs für Studenten }\end{array}$ & Inventar 3 & 100 & 17 \\
\hline $\begin{array}{l}\text { Partner } 5 \\
\text { Bildungsanbieter } \\
\text { Frankreich }\end{array}$ & Computerkurse mit ECDL-Zertifikat & Inventar 3 & 172 & 80 \\
\hline $\begin{array}{l}\text { Partner } 6 \\
\text { Universität } \\
\text { Frankreich }\end{array}$ & $\begin{array}{l}\text { "Tabaco" } \\
\text { Seminar für Studenten }\end{array}$ & Inventar 3 & 32 & 32 \\
\hline $\begin{array}{l}\text { Partner } 7 \\
\text { Krankenhaus } \\
\text { Frankreich }\end{array}$ & Weiterbildungen für Personal & $\begin{array}{l}\text { Kein Ein- } \\
\text { satz }\end{array}$ & - & - \\
\hline $\begin{array}{l}\text { Partner } 8 \\
\text { Universität } \\
\text { Schottland }\end{array}$ & Seminar für Studenten & $\begin{array}{l}\text { Kein Ein- } \\
\text { satz }\end{array}$ & - & - \\
\hline Insgesamt & Alle Kurse & $\begin{array}{l}\text { Inventare } \\
1-3\end{array}$ & 513 & 171 \\
\hline
\end{tabular}

Tabelle 44: $\quad$ Tatsächlicher Einsatz der Inventare für Lerner bis November 2005

Insgesamt wurden im Rahmen von eL3 513 Lerner befragt, wobei 171 Lerner komplett ausgefüllte, d.h. brauchbare Fragebögen abgaben. Die Daten dieser ersten Befragung auf der Grundlage der Inventare lagen jedoch in unterschiedlicher Form vor. Ein Partner gab die Daten selbst in Excel ein und schickte eine Datei per E-Mail an die Verantwortliche der Arbeitsgruppe. Zwei Partner schickten ausgefüllte Fragebögen zur Auswertung an die Arbeitsgruppenleiterin (einmal per E-Mail und einmal per Post). Ein Partner kooperierte mit der Arbeitsgruppenleiterin und ließ von ihr die Erhebungen in seinen Kursen durchführen, und ein Partner schickte keine Daten oder Fragebögen, sondern einen Ergebnisbericht. Ein Partner hatte zwar 19 ausgefüllte Fragebögen erhalten, aber es gab keinen konkreten Kurs, sondern die Fragebögen wurden einfach so an Mitarbeiter verteilt. Die Beantwortung der Fragen fiel dementsprechend lückenhaft aus, manche Lerner fragten sogar in Kommentarfeldern nach dem Sinn der Befragung. Diese Daten wurden deshalb nicht ausgewertet. Dieser Fall verdeutlichte aber für das weitere Vorgehen, dass manche Partner Unterstützung für eine ordentlich durchgeführte Evaluation brauchten (z.B. eine Anleitung, wie Fragebögen verteilt werden sollten, wie Befragte instruiert werden können und wie man die Rücklaufquote erhöht). Die übrigen Partner schickten weder Daten noch Ergebnisberichte und setzten die 
Inventare für Lerner bis zum Ende des Projektes nicht ein. Insgesamt lagen bis November 2005 brauchbare Daten von 72 Lernern vor.

\begin{tabular}{|l|l|l|l|}
\hline $\begin{array}{l}\text { Partner } \\
\text { Einrichtung }\end{array}$ & $\begin{array}{l}\text { Anzahl ausgefüllter } \\
\text { Fragebögen }\end{array}$ & Vorgehensweise & Daten \\
\hline $\begin{array}{l}\text { Partner 1 } \\
\text { Bildungsanbieter } \\
\text { Deutschland }\end{array}$ & 9 & Papierbasiert & Eingabe in Excel \\
\hline $\begin{array}{l}\text { Partner 1 } \\
\text { Bildungsanbieter } \\
\text { Deutschland }\end{array}$ & 14 & Papierbasiert & Eingabe in Excel \\
\hline $\begin{array}{l}\text { Partner 2 } \\
\text { Krankenhaus } \\
\text { Deutschland }\end{array}$ & 19 & Papierbasiert & $\begin{array}{l}\text { Keine Auswertung, da } \\
\text { kein Kurszusammen- } \\
\text { hang! }\end{array}$ \\
\hline $\begin{array}{l}\text { Partner 3 } \\
\text { Krankenhaus } \\
\text { Italien }\end{array}$ & - & Keine Rückmeldung & Keine Daten \\
\hline $\begin{array}{l}\text { Partner 4 } \\
\text { Universität } \\
\text { Italien }\end{array}$ & 17 & $\begin{array}{l}\text { Online } \\
\text { (als Word-Dokument } \\
\text { bzw. Formular an die } \\
\text { Lerneinheit gehängt) }\end{array}$ & Eingabe in Excel \\
\hline $\begin{array}{l}\text { Partner 5 } \\
\text { Bildungsanbieter } \\
\text { Frankreich }\end{array}$ & 80 & Papierbasiert & $\begin{array}{l}\text { Ergebnisbericht } \\
\text { Zusammenfassung }\end{array}$ \\
\hline $\begin{array}{l}\text { Partner 6 } \\
\text { Universität } \\
\text { Frankreich }\end{array}$ & 32 & Papierbasiert & Eingabe in Excel \\
\hline $\begin{array}{l}\text { Partner 7 } \\
\text { Krankenhaus } \\
\text { Frankreich }\end{array}$ & - & Keine Rückmeldung & Keine Daten \\
\hline $\begin{array}{l}\text { Partner 8 } \\
\text { Universität } \\
\text { Schottland }\end{array}$ & - & Kein Einsatz & Keine Daten \\
\hline $\begin{array}{l}\text { Insgesamt } \\
\text { Tabell 45: }\end{array}$ & $\mathbf{n = 1 7 1}$ & $\begin{array}{l}\text { Eingabe von Daten } \\
\text { in Excel } \mathbf{n}=\mathbf{7 2}\end{array}$ \\
\hline
\end{tabular}

Tabelle 45: Übersicht über den Einsatz der Inventare und die endgültige Zahl verwendbarer Fragebögen bzw. Daten

Die Partner nutzten die Inventare für Lerner zu unterschiedlichen Zeiten im Kursverlauf. Ein Partner erhob Daten zu Beginn des Kurses, einer während des Kursverlaufs, und in drei Kursen wurde die Befragung am Ende durchgeführt. Es wurden also alle drei Inventare verwendet, wobei die meisten Partner auch schon in den Diskussionen auf dem Projekttreffen im Mai 2005 äußerten, dass eine Befragung am Ende eines Kurses für sie besonders wichtig und sinnvoll sei. Erhebungen am Anfang bzw. während des Kursverlaufs wurden von den meisten Partnern als zu aufwändig und zeitintensiv eingeschätzt (außer von den Partnern mit universitärem Hintergrund). Es wurden außerdem Bedenken geäußert, dass Lerner zu sehr strapaziert würden, wenn sie dreimal innerhalb eines Kurses Fragebögen ausfüllen müssen. Ein Partner entschied sich jedoch, über den gesamten Kursverlauf von einem Jahr die Inventare für Lerner zu nutzen (Kurs Prokonsens). Der Kurs fing aber erst im November 2005 an, so dass bis zum Ende des Projektes eL3 nur die erste Befragung am Anfang des Kurses durchgeführt werden konnte. 


\begin{tabular}{|l|l|l|l|}
\hline Inventar & $\begin{array}{l}\text { Anzahl der Kurse, in } \\
\text { denen die Inventare } \\
\text { eingesetzt wurden }\end{array}$ & $\begin{array}{l}\text { Anzahl vollständig aus- } \\
\text { gefüllter Fragebögen }\end{array}$ & Kurstitel \\
\hline Inventar 1 & 1 & $\mathrm{n}=14$ & Prokonsens \\
\hline Inventar 2 & 1 & $\mathrm{n}=9$ & Fit For Change \\
\hline Inventar 3 & 2 & $\mathrm{n}=32$ & $\begin{array}{l}\text { Tabaco } \\
\text { Weblingua }\end{array}$ \\
\hline Alle Inventare & 4 Kurse & $\mathrm{n}=72$ & \\
\hline
\end{tabular}

Tabelle 46: Vorhandene verwendbare Daten aus dem Pretest der Inventare für Lerner bis November 2005

Aus anderen Kursen, in denen Erhebungen auf der Basis der Inventare durchgeführt wurden, lagen keine Daten, dafür aber ein Ergebnisbericht vor. Ein eL3-Partner setzte die Inventare in verschiedenen Computerkursen am Ende des Kurses ein $(n=80)$. Ein anderer projektexterner Partner nutzte die Inventare in verschiedenen Kursen zum Thema „Nachwachsende Rohstoffe" ( $n=64)$ in den Jahren 2005 und 2006. Diese Daten standen aber am Ende des Projektes eL3 noch nicht zur Verfügung.

\begin{tabular}{|l|l|l|l|}
\hline Inventar & $\begin{array}{l}\text { Anzahl der Kurse, in } \\
\text { denen die Inventare } \\
\text { eingesetzt wurden }\end{array}$ & $\begin{array}{l}\text { Anzahl vollständig aus- } \\
\text { gefüllter Fragebögen }\end{array}$ & Kurstitel \\
\hline $\begin{array}{l}\text { Inventar 3 } \\
\text { Corinne }\end{array}$ & Kurse 2006 & $\mathrm{n}=80$ & Computerkurse ECDL \\
\hline $\begin{array}{l}\text { Inventar 3 } \\
\text { Veronika }\end{array}$ & 5 Kurse 2005-2006 & $\mathrm{n}=64$ & $\begin{array}{l}\text { NawaRo } \\
\text { Biogas }\end{array}$ \\
\hline
\end{tabular}

Tabelle 47: $\quad$ Einsatz Inventar für Lerner

Die Erfahrungen mit dem ersten Einsatz der Inventare sollten dennoch ausgewertet werden. Es wurde beschlossen, sich mehr auf die inhaltlichen Ergebnisse zu konzentrieren und diese zu nutzen, um die Wichtigkeit (Bedeutung) und Angemessenheit von einzelnen Fragenbereichen der Inventare für Lerner zu überprüfen und zu diskutieren. Die gemeinsame Entwicklung der Inventare war sehr aufwändig, aber erfolgreich, da am Ende des Projektes eL3 gemeinsam erarbeitete Instrumente in vier Sprachen vorlagen. Das Erheben und Mitteilen der Daten im Netzwerk stellte sich jedoch als eine Herausforderung dar, für die am Ende des Projektes eL3 noch nach Lösungen für die Zukunft gesucht wurde. Eine Idee, die auf dem letzten Projekttreffen im November 2005 diskutiert wurde, war, dass einzelne Partner Berichte zu ihren Kursevaluationen über blinc veröffentlichen könnten. Es sollten Themen herausgearbeitet oder Erfahrungen gebündelt werden, die dann als Wissensbasis und Diskussionsgrundlage zur Verfügung gestellt werden könnten. Dies ließe sich in einem Forum oder blog realisieren. Es könnte einen Hauptverantwortlichen für diesen Bereich (Evaluation von Blended Learning) geben, der die Beiträge koordiniert, d.h. dazu aufruft, sie durchliest und veröffentlicht, oder aber alle Netzwerkpartner könnten eigenständig Beiträge liefern. Eine weitere Idee war, dass die Evaluationen von Blended Learning-Kursen auf der Basis der Inventare 
von einzelnen Partnern als Dienstleistung über blinc angeboten werden könnte. Jeder Partner hatte einen eigenen Bereich zur Verfügung gestellt bekommen, in dem er sich und seine Arbeit präsentieren konnte. In diesen „Schaufenstern“ könnte auch Evaluation angeboten werden.

In der eL3 Projektgruppe wurde mehrheitlich der Wunsch geäußert, über blinc (d.h. die Internetseite, aber auch über zukünftige Projekte oder Treffen) Erfahrungen und gute Praxisbeispiele zu sammeln. Die Ergebnisse der Kursevaluationen sollten genutzt werden, um bestimmte Themen herauszuarbeiten und im Netzwerk gemeinsam nach Lösungsansätzen der verschiedenen Netzwerkpartner zu fragen. Auf dem Treffen im November 2005 wurde z.B. von mehreren Partnern berichtet, dass auf der Grundlage der erhobenen Daten die Bedeutsamkeit eines Tutors als Lernbegleiter im e-Learning sich als überaus wichtig herausgestellt habe, was bisher nicht so deutlich gewesen sei. Die Lerner hatten dies in den Fragebögen zurückgemeldet. Eine tutorielle Begleitung von Lernern bedeutet aber für die Organisatoren der Kurse einen erheblichen Mehraufwand. In der Gruppe wurde diskutiert, welche Erfahrungen zu diesem Thema vorlagen und wie einzelne Partner dieses „Problem“ lösen würden bzw. welche Ideen sie dazu hätten. Solche Diskussionen wurden von den Projektpartnern als sehr wertvoll und nützlich empfunden. Das Mitteilen der Daten stellte offenbar eine Hürde dar, aber der Austausch über Erfahrungen erwies sich als sehr hilfreich und anregend, um die eigene berufliche Praxis zu verbessern.

\subsubsection{Das Inventar für Autoren}

Das Inventar für Autoren, das in der Arbeitsgruppe erarbeitet worden war, wurde wie die Inventare für Lerner auf dem Projekttreffen im Mai 2005 der Projektgruppe vorgestellt. Das Ziel war, dass möglichst viele Autoren das Inventar benutzen würden, um seine Tauglichkeit und Handhabbarkeit zu erproben. Alle Partner versicherten zu diesem Zeitpunkt, das Inventar zumindest selbst ausprobieren zu wollen bzw. noch andere Autoren in ihren Institutionen einzubeziehen und sie das Inventar testen zu lassen. Zunächst musste das Inventar noch in die Sprachen Italienisch und Französisch übersetzt werden, damit eine multilinguale Form für alle Partner zur Verfügung stehen würde. Ähnlich wie bei den Inventaren für Lerner war es die Aufgabe des Arbeitsgruppenleiters, diese Arbeiten durchzuführen bzw. zu koordinieren.

Auf dem letzten Projekttreffen im November 2005 stellte sich heraus, dass nur ein Partner von den neun Partnern das Inventar für Autoren tatsächlich genutzt hatte. Die Anwendung des Inventars fand auf freiwilliger Basis statt, und so konnte auch kein Partner verpflichtet werden, das Inventar zu verwenden. Es war nun die Frage, woran es gelegen hatte, dass die Partner derart zurückhaltend waren. Wie schon bei der Entwicklung des Inventars für Auto- 
ren deutlich geworden war, war es für die meisten Partner sehr neu und ungewöhnlich, sich Gedanken zum didaktischen Design ihrer Kurse zu machen, und die Form der Reflexion auf der Grundlage des Inventars war ebenfalls eine sehr ungewohnte Praxis. Nach den Rückmeldungen der Partner wirkten nicht nur die Fragen, die in dem Inventar zusammengestellt waren, für sie befremdlich, sehr theoretisch, abstrakt und anspruchsvoll, sondern auch die Art, über die eigene Arbeit nachzudenken, war innen nicht vertraut. Diese Faktoren führten wohl dazu, dass die Partner das Inventar einfach nicht benutzten bzw. keine Daten zusammenkamen. Es traute sich jedoch auch keiner der Partner, nach Hilfestellungen oder Erklärungen zu fragen. Der eine Partner, der das Inventar benutzt hatte, war sehr begeistert und angeregt durch die Reflexion zu seinen Kursen, die er auf der Grundlage des Inventars für Autoren vollzogen hatte. Er meldete zurück, dass er völlig neue Sichtweisen und Erkenntnisse erworben hatte, nachdem er sich auf die zunächst kompliziert erscheinende Prozedur eingelassen hatte. Seiner Meinung nach müssten andere Praktiker eine Hilfestellung bekommen, etwa in Form einer Beratung, um das Potenzial des Inventars erkennen und nutzen zu können.

\subsubsection{Das Inventar für Entscheidungsträger}

Im Mai 2005 wurde das bis dahin in der Kleingruppe erarbeitete Inventar für Entscheidungsträger der gesamten Projektgruppe vorgestellt. Das Ziel war nun, dass möglichst viele Entscheidungsträger das Inventar benutzen und damit testen sollten, und alle Partner sicherten $z u$, dafür Sorge zu tragen. Zunächst musste noch eine Übersetzung ins Italienische und Französische erfolgen (Englisch und Deutsch lagen vor), damit die Partner das Inventar in ihren jeweiligen Institutionen ohne größeren Aufwand einsetzten könnten. Diese Aufgabe sollte von einzelnen Partnern übernommen und vom Arbeitsgruppenleiter koordiniert werden. Schon bei der Erarbeitung des Inventars für Entscheidungsträger war die Beteiligung der Partner sehr gering (siehe Kapitel 6.3.3), und auch im weiteren Verlauf schien das Interesse nicht besonders groß. Die Partner meldeten zurück, dass ihnen der Nutzen der Inventare nicht eindeutig sei und es für die weitere Verwendung so viele Möglichkeiten gäbe, dass innen nicht klar sei, worauf das Vorhaben hinauslaufe. Das Diskutieren innerhalb der Projektgruppe über Inhalte und Schlüsselfragen im Kontext von e-Learning und Blended Learning war für die Partner interessant, anregend und bereichernd, aber das Ausfüllen des Inventars war offenbar nicht besonders reizvoll. Es erfolgte auch keine Übersetzung ins Französische und Italienische, und nur ein Partner aus der Projektgruppe nutzte das Inventar für Entscheidungsträger in deutscher Sprache.

Dieser eine von neun Partnern, der das Inventar für Entscheidungsträger einsetzte, ging folgendermaßen vor: Er holte sich Unterstützung von der Leitungskraft der gesamten Einrichtung (ein Krankenhaus), und diese Person schickte den Fragebogen, der auf der Grundlage 
des Inventars erarbeitet worden war, mit einer Erklärung und der Bitte um das Ausfüllen an alle Kliniken der Kette in ganz Deutschland. Der Fragebogen wurde so an 30 Leitungskräfte bzw. Entscheidungsträger per E-Mail verschickt, und 24 Personen beteiligten sich an der Befragung. Die ausgefüllten Fragebögen wurden per E-Mail zurückgeschickt und auch an die Verfasserin weitergeleitet. Bei der Rückmeldung zum Inventareinsatz wurde angemerkt, dass Hilfestellungen nötig seien und sowohl die Befragten als auch derjenige, der die Befragung durchführte, mehr Informationen zum Inventar wie auch zum Thema e-Learning und Blended Learning benötigten. Außerdem sei das Inventar für Entscheidungsträger recht allgemein und abstrakt („theoretisch“) formuliert und ein Bezug zum Gesundheitssektor wäre wünschenswert. Für „Praktiker“ müsste das Inventar in einer Form vorliegen, die leichter zu handhaben sei, oder es müsste eine Person den Prozess begleiten und als Ansprechpartner eine Beratung und Unterstützung beim Ausfüllen und Auswerten bieten.

\subsection{Exkurs: Weitere Daten aus einer späteren Verwendung der In- ventare}

Im Unterkapitel 6.4 wurde deutlich, dass die Erprobungsphase der Inventare nicht wie erwartet verlief. Die Inventare waren in einem aufwändigen und über einen relativ langen Zeitraum von etwa einem Jahr in den Arbeitsgruppen entwickelt worden. Als die Inventare zur Nutzung bereit waren, näherte sich das Projektende und zahlreiche andere Aufgaben (neben der Inventarnutzung) standen an. Die Projektpartner mussten Berichte verfassen und Arbeitspakete abarbeiten. Diese Situation erwies sich als nicht ideal, um die Inventare zu verwenden. Die Motivation der meisten Partner, sich intensiv einzubringen, ließ nach, und nur wenige Partner - vor allem die Projektverantwortlichen - waren bestrebt, das Projekt zu einem guten Ende zu bringen und die im Projektantrag versprochenen Ergebnisse zusammenzustellen.

Zum Ende des Projektes eL3 war die Situation sowohl für die Projektleitung als auch die Verfasserin einigermaßen ernüchternd. Wesentliche Ziele wurden nicht optimal erreicht und es zeigte sich keine Möglichkeit, die Partner zu verpflichten, doch noch Inventare einzusetzen. Es fehlte nicht nur an Zeit, sondern offensichtlich mangelte es bei vielen der Partner auch an Möglichkeiten, die Inventare einzusetzen. Vermutlich hatten auch einige Partner nicht die notwendige Evaluationskompetenz, was sich ansatzweise bei den Ergebnissen der vorliegenden Forschungsarbeit andeutet. Mit der Befragung zum Abschluss des Projektes eL3 wurden aber auch einige Punkte deutlich, die dazu beitragen könnten, dass die Inventare zukünftig intensiver genutzt würden. Einige Partner brauchten Unterstützung bei dem Einsatz der Inventare, die in unterschiedlichen Formen realisierbar bzw. denkbar wären.

Für die Europäische Kommission musste zum Ende des Projektes (bzw. kurz danach) ein Abschlussbericht verfasst werden. Die vorhandenen Daten, die von den Partnern bis zum 
Projektende von eL3 erhoben worden waren, reichten jedoch nicht aus, um qualitativ hochwertige (oder auch nur akzeptable) Auswertungen durchzuführen. Aus diesem Grund entschied sich die Projektleitung nach Ende des Projektes, die Inventare in unterschiedlichen weiteren Kontexten einzusetzen, um wenigstens einige Daten zu erhalten und Auswertungen durchzuführen, mit denen ansatzweise gezeigt werden könnte, was mit den Inventaren möglich ist. In einem kleinen Team wurde auf der Grundlage der Daten ein Bericht verfasst und es wurden dazu Empfehlungen für verschiedene Zielgruppen (Entscheidungsträger, Autoren und Lerner) erarbeitet (vgl. Haller, List-Ivankovic \&. Scholze 2007). Der Bericht ist im Internet frei verfügbar unter http://www.blinc-eu.org/1203/ (Zugriff am 18.9.2012).

Es entstanden somit Daten zu folgenden Personengruppen:

1. Entscheidungsträger

Personen, die in verschiedenen Einrichtungen der Gesundheits- und Sozialwirtschaft wie bspw. Krankenhäusern, Bildungseinrichtungen oder Non-Profit-Organisations darüber entscheiden, welche Weiterbildungsmaßnahmen für verschiedene Zielgruppen, u.a. auch das Personal, angeboten werden.

2. Autoren

Personen, die e-Learning und Blended Learning-Kurse entwickeln und gestalten (sowohl was die Inhalte als auch die technischen Aspekte anbelangt).

3. Lerner

Personen, die e-Learning oder Blended Learning-Kurse als Teilnehmer besuchen

Die folgende Tabelle zeigt, wie viele Personen der verschiedenen Gruppen auf der Grundlage der Inventare bei der späteren Befragung (nach Ende des Projektes eL3) befragt wurden:

\begin{tabular}{|l|l|l|}
\hline Entscheidungsträger & Autoren & Lerner \\
\hline $\mathrm{n}=53$ & $\mathrm{n}=67$ & $\mathrm{n}=205$ \\
\hline
\end{tabular}

Tabelle 48: Übersicht über die Fallzahlen bei der späteren Verwendung der Inventare nach Abschluss des Projektes eL3

Im Folgenden werden kursorisch einige der dabei festgestellten Ergebnisse und der sich daran anschließenden Überlegungen wiedergegeben. Interessant dürfte dabei vor allem die Gegenüberstellung von Ergebnissen zu den verschiedenen Inventaren und damit auch zu verschiedenen Zielgruppen der Befragungen sein (insbesondere: was wissen und erwarten die Entscheidungsträger hinsichtlich der Einführung von e-Learning und wie ist die Situation von Teilnehmern an solchen Maßnahmen?). 
- Lerner in e-Learning-Angeboten berichteten über einen Zeitmangel an ihrem Arbeitsplatz bezüglich der Möglichkeit, e-Learning hier durchzuführen, sowie andererseits über Störungen beim Lernen zu Hause. 69\% lernten am Abend und 25\% noch in der Nacht. Demgegenüber war für Entscheidungsträger der wichtigste Grund bei der Einführung von e-Learning Kostenersparnisse dadurch zu erreichen, dass Mitarbeiter nicht Arbeitszeit für auswärtige Weiterbildungsangebote in Anspruch nähmen. Von den Autoren wussten (nach eigenen Angaben) nur 33\% etwas über die Zeitbedingungen und nur $22 \%$ etwas über die Lernschwierigkeiten ihrer Lerner.

- Es bestanden Korrelationen zwischen den Kenntnissen von Autoren (im weitesten Sinne Weiterbildnern) über ihre Lerner und deren Lernsituation und der Selbstbewertung ihrer didaktischen Designs, d.h. wer viel über seine Lerner wusste, neigte auch dazu, sein Angebot besser zu bewerten.

- Eine Faktorenanalyse zur Selbstbewertung der didaktischen Designs (Bildungsangebote) von Autoren ergab als primären Faktor die Orientierung an der Lernerpersönlichkeit sowie als weitere Faktoren die Orientierung an den fachlichen Inhalten und die Ausgestaltung (,Handlung“) des e-Learning.

- Für Entscheidungsträger ist die Intention, e-Learning einzuführen, vor allem damit verbunden, Kosten zu reduzieren. Einerseits sollen Kosten erspart werden, die durch die Abwesenheit von Personal vom Arbeitsplatz während der Weiterbildung entstehen, und andererseits sollen die Kosten für Weiterbildungsmaßnahmen reduziert werden oder wegfallen. Damit ist die Vorstellung verbunden, dass beim e-Learning einmal erarbeitete Kurse und Materialien immer wieder verwendet werden könnten. Am wenigsten interessiert bzw. motiviert die Entscheidungsträger die Verbesserung der Lernumgebung für ihr Personal oder die Förderung der Kommunikation innerhalb der Einrichtung.

- Die befragten Autoren verfügten nach eigener Einschätzung über ein relativ hohes Wissen über ihre Lerner, was allgemeine Merkmale wie z.B. Alter, Geschlecht, Berufsgruppen, Sprachfähigkeiten oder die Motivation zum Lernen anbelangt. Sie wissen hingegen wenig über ihre Lerner (die Zielgruppen ihrer Angebote) in beispielsweise folgenden Bereichen: generelle Kompetenzen, bevorzugte Lernmethoden, Lernstile und Lernstrategien, verfügbare Zeit und Ressourcen für das Lernen, Lernzeiten, familiäre Bedingungen oder Schwierigkeiten, die mit dem Lernen verbunden sein könnten. Es wird deutlich, dass ein großer Teil der Autoren nicht über genügend Wissen über die Zielgruppen ihrer Kurse und deren Kontexte verfügt. Vermutlich ist 
es für diese Autoren schwierig, „passende“ Lernangebote zu entwickeln, zumindest besteht die Gefahr, dass sie nicht optimal auf die Zielgruppe abgestimmt sind.

- Für die Lerner ist die größte Schwierigkeit, die Zeit für das e-Learning aufzubringen. Meistens müssen die Lernzeiten in die Freizeit verlegt werden, da am Arbeitsplatz im beruflichen Alltag aus verschiedenen Gründen keine Möglichkeit zum Lernen besteht. Im häuslichen Umfeld zeigen sich jedoch ebenfalls zahlreiche Schwierigkeiten wie etwa die technische Ausstattung oder fehlende Zeit (die meisten geben an, am späten Abend oder nachts zu lernen, was eine zusätzliche Belastung darstellt und nicht zu mehr Freude am Lernen beiträgt).

- Ein Großteil der befragten Lerner bevorzugt Blended Learning gegenüber e-Learning, da Präsenzzeiten und der Kontakt zu anderen Lernern als wertvoll und motivationsfördernd erlebt werden. Die Mischung von Methoden wird ebenfalls positiv bewertet.

- Für die Gruppe der Lerner ist die Begleitung durch einen Tutor beim e-Learning ein wesentliches Kriterium für den Lernerfolg. Sie empfinden die Anleitung durch einen Tutor und die Möglichkeit, einen Ansprechpartner zu haben, als sehr wichtig. Bevorzugte Kontaktmöglichkeiten sind E-Mails und Telefongespräche.

- Ein wesentliches Erfolgskriterium für die Lerner ist die technische Ausstattung beim e-Learning. Hier zeigte sich bei den Befragungen, dass einige Lerner weder zu Hause noch am Arbeitsplatz gute Bedingungen für das e-Learning haben (z.B. keinen Internetzugang). Manche Lerner sind auch nicht besonders vertraut mit technischen Verfahren bzw. es fehlen innen die Kompetenzen, die für das e-Learning jedoch wichtig wären. Hier wäre eine Begleitung durch einen Tutor ebenfalls wichtig oder förderlich.

Auf der Basis der Ergebnisse, von denen hier nur einige ausschnittsweise dargestellt wurden, wurde eine sogenannte „Blended Learning Matrix“ erarbeitet und veröffentlicht (vgl. Haller, List-Ivankovic \& Scholze 2007, S.56). Diese Matrix sollte zum einen zur Auswertung und Zusammenstellung der Ergebnisse aus der Befragung dienen und zum anderen als Planungsinstrument für Blended Learning-Angebote genutzt werden können. Die Ergebnisse der Befragungen der verschiedenen Zielgruppen (Entscheidungsträger, Autoren und Lerner) wurden in die Matrix übergetragen und Empfehlungen daraus abgeleitet. Die Inventare wurden so genutzt, um Wissen zu generieren, zugänglich und nutzbar zu machen.

Die oben dargestellten Befunde weisen darauf hin, dass mit den im Projekt eL3 entwickelten Inventaren auch Möglichkeiten verbunden sind (und zu interessanten Einsichten führen 
könnten), weitere Forschungen über die Entstehungsbedingungen von Bildungsangeboten durchzuführen. Es ist ja nach wie vor nicht bekannt, welche Interessen, Kenntnisse, Erfahrungen und Erwartungen die Personen leiten, welche Bildungsangebote initiieren, planen und schließlich ausführen. 


\section{Zusammenfassung und Diskussion}

Evaluation ist in der Bildungspraxis heutzutage, im Jahre 2012, ein ziemlich alltäglicher Vorgang, um die Qualität der Bildungsangebote zu sichern oder nachzuweisen, und wird mit einer gewissen Selbstverständlichkeit betrachtet. In der pädagogischen Praxis tätige Personen finden dafür jedoch kaum Instrumente oder Hilfestellungen und sehen sich nur dem Druck unterlegen und dem Anspruch gegenüber, ihre Bildungsmaßnahmen zu evaluieren. Im wissenschaftlichen Kontext gelten klare Regeln und Standards, nach denen Evaluationen durchgeführt werden müssen, die sich in der Praxis meistens nicht erfüllen lassen. An diesem Problem setzt die vorliegende Forschungsarbeit an: Es war das Ziel, gemeinsam mit Bildungspraktikern (Lehrkräften, Dozenten, Kursdesignern) und Bildungstheoretikern (Forschern, Wissenschaftlern) Evaluationsinstrumente zu entwickeln und zu erproben, um die Evaluationspraxis in den Bildungsprojekten der beteiligten Akteure zu unterstützen und zu verbessern. Dafür wurde der Ansatz von Inventaren entworfen, auf deren Grundlage die Beteiligten selbständig ihre Bildungsangebote evaluieren sollten. Inventare sind im Vergleich zu Fragebögen oder Tests komplexere Instrumente, die aus mehreren Teilen bestehen oder mehrere Skalen beinhalten. Im Kontext dieser Arbeit sind sie als Basis für eine qualitativ möglichst hochwertige Evaluation bzw. Selbstevaluation von Bildungsprojekten in unterschiedlichen beruflichen Feldern in verschiedenen europäischen Ländern entwickelt, eingesetzt und erprobt worden. Die Funktion der Evaluation ist dabei nicht die Kontrolle, sondern die Verbesserung der beruflichen Praxis gewesen, für eine Wissenserweiterung, einen Erkenntnisgewinn, die Reflexion des beruflichen Handelns sowie den Dialog mit anderen Bildungspraktikern. Die Ergebnisse und Erfahrungen der Evaluationen mit den Inventaren sollten auf freiwilliger Basis in einem europäischen Netzwerk ausgetauscht werden und so zu einer hohen Qualität der Bildungsangebote der Partner beitragen.

Die Verfasserin arbeitete als wissenschaftliche Mitarbeiterin in dem von der europäischen Kommission im Rahmen der e-Learning-Initiative geförderten Projekt eL3 (eLearning Project Cluster for Third System Organisations in Europe), das in dieser Arbeit als Fallbeispiel dient. Das Forschungsvorgehen wird der Aktionsforschung zugeordnet und erforderte ein hohes $\mathrm{Maß}$ an Flexibilität. Die beteiligten Praxispartner wurden gleichberechtigt in den Forschungsprozess einbezogen, und ihre Bedarfe und Erwartungen prägten die Forschungsarbeit entscheidend. Die Forschungsmethoden wurden mit den Partnern abgestimmt und Ergebnisse in der Projektgruppe transparent gemacht. Als Methoden kamen verschiedene Methoden zum Einsatz, die trianguliert wurden, um ein breiteres, umfassenderes Bild von dem Untersuchungsfeld zu bekommen, als wenn nur eine Methode verwendet würde. Die Kombination 
von qualitativen und quantitativen Verfahren sollte ebenfalls zu einem erweiterten Bild beitragen.

Zu Beginn des Projektes wurde ein Fragebogen eingesetzt, um die einzelnen Bildungsangebote der Projektpartner zu erfassen und zu analysieren. Das Ziel war hier zunächst eine Exploration zur Bildungs- und Evaluationspraxis der Partner. Die Ergebnisse wurden ausgewertet und auf einem transnationalen Treffen in der Gruppe vorgestellt. In Bezug auf die erste Fragestellung, was die Partner bisher zur Evaluation ihrer Bildungsprojekte taten, stellte sich heraus, dass die Evaluationspraxis der Projektpartner sehr unterschiedlich war. Es waren sowohl die Anzahl als auch die Kombination der verwendeten Methoden zur Evaluation verschieden. Das Spektrum reichte von der Nutzung einer Methode (z.B. Fragebogen) bis zur Kombination verschiedener Methoden (z.B. mündliche Befragung, Tests und informelle Rückmeldungen). Zur genaueren Analyse wurden die eL3-Projektpartner je nach ihren institutionellen Hintergründen in drei Gruppen eingeteilt und die Evaluationspraxis wurde gruppenweise betrachtet, um zu prüfen, ob sich Unterschiede ergaben bzw. das „ForschungPraxis-Dilemma“ zeigte (siehe Unterkapitel 4.3). Die Gruppen bestanden aus Personen aus Universitäten, Krankenhäusern und Bildungsanbietern. Es wurde deutlich, dass sich die Evaluationspraxis in den unterschiedlichen Kontexten unterschied und sich auch das ForschungPraxis-Dilemma zeigte:

In der Gruppe der Universitäten („die Theoretiker“) wurden deutlich mehr Methoden eingesetzt und kombiniert als in den anderen beiden Gruppen, wobei die Einhaltung wissenschaftlicher Standards üblich und selbstverständlich war. Bei den Partnern mit wissenschaftlichem Hintergrund bestand außerdem vor allem Interesse am Austausch von Erfahrungen und Wissensinhalten bzw. neuem Wissen. Das Reflektieren der eigenen beruflichen Praxis und der Evaluationspraxis wurde als wertvoll und hilfreich gesehen. Der Ansatz der Inventare wurde als spannend und lohnenswert aufgenommen, aber es wurden auch Kritik und Bedenken geäußert, ob wissenschaftliche Standards ausreichend berücksichtigt bzw. eingehalten werden könnten. Es bestand jedoch die Bereitschaft, sich auf das aufwändige Verfahren einzulassen, vor allem mit der Aussicht auf neue Impulse für die eigene Arbeit. Es wurde aber auch der Wunsch nach einer Auswertung der Entwicklung und Erprobung der Inventare geäußert und eine wissenschaftliche Begleitung begrüßt.

In der Gruppe der Bildungsanbieter und der Krankenhäuser („die Praktiker“) wurden zu Beginn des Projektes eL3 weniger Methoden eingesetzt. Der Einsatz erfolgte außerdem nach bestem Wissen und Gewissen, die Qualität der Erhebungen sollte gut sein, die Einhaltung wissenschaftlicher Standards war eher sekundär, da aufwändige Verfahren „Zeit und Geld“ kosten. Evaluationen wurden vor allem mit dem Ziel durchgeführt, die Qualität der eigenen 
Bildungsangebote „nach außen“ zu zeigen und zu verdeutlichen. Die Evaluation hatte insbesondere eine Legitimationsfunktion und nicht eine Erkenntnisfunktion oder Dialog- bzw. Lernfunktion (siehe Unterkapitel 1.3). Die bloße Reflexion der eigenen beruflichen Praxis wurde als wenig wertvoll erachtet oder es wurde darauf hingewiesen, dass im beruflichen Alltag dafür einfach keine Zeit vorhanden sei. Es bestand primär der Wunsch nach der Vereinfachung von Evaluationsverfahren sowie dem Austausch von Erfahrungen mit anderen Praktikern. Evaluationen sollen eine gute Qualität haben - also nicht "quick and dirty" aber "quick and easy" und vor allem praktikabel und nützlich sein.

Mit diesen Ergebnissen zeigte sich bereits zu Beginn des Projektes eL3, dass die Verständnisse von Evaluation und auch die Erwartungen an Evaluationen unterschiedlich waren. Die Bedarfe der Partner konnten auch nicht unbedingt auf einen Nenner gebracht werden. Diese Heterogenität der Partner zeigte sich bereits zu Beginn des Projektes als eine Stärke und zugleich als eine Schwäche und besonders als eine Herausforderung für die Entwicklung und Erprobung der Inventare. Der Ansatz wurde mit großem Interesse aufgenommen, es wurde jedoch schnell deutlich, dass unterschiedliche Arbeitsweisen und Interessen in der Gruppe vorhanden waren. Was allen Partnern unabhängig ihres institutionellen Hintergrundes gemeinsam war, war das Interesse und die Neugier, in einem „geschützten Raum“ (dem Projekt, in dem man über einen Zeitraum von zwei Jahren zusammen arbeitet und Vertrauen entstehen kann) einen Einblick in die Arbeit der anderen zu bekommen und Erfahrungen auszutauschen. Auf dieser Basis schien auch die Inventarentwicklung und Erprobung lohnenswert und erfolgversprechend, trotz aller Unterschiede.

Als weitere Methode wurde über den gesamten Projektverlauf von zwei Jahren eine fragebogenbasierte Prozessevaluation durchgeführt. Die Ziele waren hierbei vor allem die Qualitätssicherung der transnationalen Zusammenarbeit, die Dokumentation und Sichtbarmachung der Projektfortschritte sowie eine Beteiligungsförderung und Stärkung der Arbeitskultur. Durch die interne formative Evaluation sollten alle Partner die Gelegenheit bekommen, sich regelmäßig in einem relativ offenen, aber geschützten Rahmen strukturiert zum Projektfortschritt und ihrer Zufriedenheit bzw. Unzufriedenheit mit der gemeinsamen Arbeit zu äußern. Die Prozessevaluation sollte auch als „Frühwarnsystem“ dienen, falls sich Probleme oder Schwächen zeigen sollten und Optimierungsbedarfe bestehen würden. Auf diese Weise könnten rechtzeitig Weichenstellungen vorgenommen werden.

Im Hinblick auf die Fragestellungen dieser Forschungsarbeit wurden die Ergebnisse der Prozessevaluation genutzt, um zu untersuchen, wie die Partner die Zusammenarbeit im Projekt erlebten, welche positiven und negativen Aspekte sie äußerten, was sie schwierig fanden und was für wertvoll erachteten. Ein Fokus der Auswertungen lag auf Äußerungen zur Entwicklung und Erprobung der Inventare, wobei die Fragen im Fragebogen zur Prozessevaluation bewusst offen formuliert waren, um die Partner nicht zu beeinflussen. 
Die Ergebnisse zeigen, dass die Partner insgesamt mit dem Projektfortschritt über den gesamten Zeitraum zufrieden waren. Allerdings wurde der Kontakt zwischen den Partnern nach 11 Monaten Projektlaufzeit noch als „weniger gut“ bewertet. Der kontinuierliche Kontakt zwischen den Partnern und eine regelmäßige Kommunikation waren zentrale Herausforderungen, die auch den Entwicklungsprozess der Inventare beeinflussten. Das Projekt brauchte eine relativ lange Anlaufphase, es dauerte alles wesentlich länger als geplant, und erst nach etwa der Hälfte der Projektlaufzeit kam die Gruppenarbeit richtig in Gang. Für die Entwicklung der Inventare schien aber eine gute Basis vorhanden zu sein, da die Partner insgesamt sehr zufrieden mit der Zusammenstellung der Projektgruppe und den Projektinhalten waren. Die transnationalen Treffen wurden jedes Mal als sehr positiv bewertet und zu diesen Zeiten wurde auch gemeinsam viel geschafft, es wurden Fortschritte gemacht und Unklarheiten beseitigt (wenn vorhanden), was die Zufriedenheit erhöhte und die Gruppe stärkte. Als besonders positive Aspekte des Projektes eL3 nannten die Partner das Treffen von verschiedenartigen Menschen, den Austausch von Wissen und Erfahrungen sowie das Voneinanderlernen. Außerdem wurde es als wertvoll erlebt, einen Einblick in die Arbeit von anderen zu bekommen, Ideen zu teilen und die berufliche Praxis gemeinsam zu reflektieren. Die Vielfalt der Partner ist dabei ein Potenzial angesehen worden, das für alle gewinnbringend genutzt werden konnte und als sehr positiv bewertet worden. Als negative Aspekte nannten die Partner, dass unterschiedliche Ziele vorhanden seien, Ziele und Rollen unklar blieben und wenige Aktivitäten außerhalb der transnationalen Treffen stattfänden. Eine gute und regelmäßige Kommunikation und Interaktion stellte sich als eine der zentralen Herausforderungen für die Zusammenarbeit dar. Außerdem wurde negativ bemerkt, dass sich einige Partner stärker (bzw. weniger) beteiligten als andere; die Webseite, die den Partnern u.a. mit dem Lernmanagementsystem als Basis für die Inventarentwicklung dienen sollte, wurde zu jedem Erhebungszeitpunkt bemängelt. Mit diesen Ergebnissen zeigten sich Schwierigkeiten, die verdeutlichen, dass die Inventarentwicklung in der transnationalen Gruppe keine leichte Aufgabe war und den Partnern auch einiges abverlangte. Außerhalb der transnationalen Treffen mussten die Partner sich neben ihrer beruflichen Praxis und über die Landesgrenzen hinaus für die Kleingruppenarbeit und die Kommunikation auf der Internetplattform engagieren was einige mehr und andere weniger taten. Die Interessen und Bedarfe waren verschieden, was die Komplexität des Entwicklungsprozesses der Inventare noch erhöhte. Dennoch hat die Arbeit an den Inventaren den Partnern Impulse und Perspektiven für die eigene Arbeit gegeben, wie sie in der Prozessevaluation zurückmeldeten. Die Diskussionen rund um das Thema Evaluation sowie die Erarbeitung der Inventare hat bei den Partnern Reflexionsprozesse in Gang gesetzt und ihr Bild von Evaluation verändert. Das Reflektieren der eigenen beruflichen Praxis auf der Grundlage der Inventare wurde als „pädagogische Technik“ erlebt und positiv aufgenommen. 
Die Entwicklung der Inventare hat einen erheblich längeren Zeitraum der Projektlaufzeit in Anspruch genommen als geplant, und die Erprobungsphase gegen Ende des Projektes stellte einige Partner vor Fragen, wie sie die Inventare nun direkt nutzen könnten. In den Rückmeldungen zeigte sich, dass die Partner daran interessiert waren zu erfahren, wie die anderen die Inventare verwendeten. Außerdem waren wohl unterschiedliche Erwartungen hinsichtlich der zukünftigen Verwendung der Inventare vorhanden: Die einen wollten die Inventare „als Produkte vermarkten“ oder Dienstleistungen dazu anbieten (etwa die Durchführung der Evaluation), andere wollten Ergebnisse bzw. Erfahrungen mit europäischen Projektpartnern im Netzwerk „blinc“ auf freiwilliger Basis austauschen, und wieder andere wollten Ergebnisse aufarbeiten und Publikationen dazu veröffentlichen. Für eine weitere Zusammenarbeit über die Projektlaufzeit hinaus hätte eine Form gefunden werden müssen, um diesen verschiedenen Ansprüchen gerecht zu werden. In der Erprobungsphase innerhalb des Projektes eL3 wurden die Inventare von den Partnern genutzt, schließlich bestand auch eine gewisse Verpflichtung im Rahmen des geförderten Projektes, jedoch nicht von allen gleichermaßen. Einige Partner konnten mit dem Ansatz mehr anfangen als andere bzw. waren eher in der Lage, selbständig Evaluationen von der Erhebung bis zur Auswertung und Berichtserstellung durchzuführen. Insgesamt scheint der Ansatz der Inventare praktikabel und nützlich, es müssten jedoch noch einige Dinge für die weitere Verwendung geklärt werden oder Formen gefunden werden, wie alle Partner die Inventare gewinnbringend einsetzen könnten. Die weitere Zusammenarbeit war am Ende des Projektes fraglich. Die Produkte auch die Inventare - könnten von allen gleichermaßen benutzt werden, die Handhabung bliebe jedoch jedem selbst überlassen. Diese Offenheit oder Freiheit wurde unterschiedlich erlebt. Die einen fanden es gut wie es war und die anderen wünschten sich Hilfestellungen für ihre Evaluationen bzw. mehr Hintergrundwissen und Anleitungen. Für das Netzwerk „blinc“ stellte sich die Frage, wie der von den Partnern als wertvoll geschätzte Kontakt zu anderen europäischen Bildungspraktikern (und Bildungstheoretikern) und der Austausch von Erfahrungen gestaltet werden könnte, wie die Kommunikation und Interaktion strukturiert und organisiert werden sollte, damit ein offener, aber dennoch geschützter Raum für das Teilen von Wissen und Erfahrungen gelingen kann.

Die Entwicklung der Inventare sollte durch ein Lernmanagementsystem erleichtert werden. Die Gruppenleiter konnten hier die Zusammenarbeit strukturieren, alle Partner konnten Dokumente hinterlegen und im Forum diskutieren bzw. Beiträge für die anderen hinterlassen. Für diese Forschungsarbeit wurden die Aktivitäten der Partner dokumentiert, womit ein nichtreaktives Verfahren zum Einsatz kam, das die Kommunikation und den Prozess der Entstehung der Inventare nicht beeinflussen sollte. Den Partnern war jedoch bewusst, dass ihre Aktivitäten „Spuren hinterlassen“ und von der Verfasserin mitverfolgt würden. In Bezug zu der Fragestellung, ob gemeinsam in dem transnationalen Netzwerk Inventare entwickelt 
werden könnten zeigte sich, dass dies möglich war. Über den Zeitraum von eineinhalb Jahren wurden Inventare für verschiedene Zielgruppen (Lerner, Autoren und Entscheider) in Arbeitsgruppen erarbeitet, wobei nicht in allen Gruppen mit gleicher Intensität gearbeitet wurde. Dennoch waren am Ende des Projektes für jede Zielgruppe Inventare vorhanden und damit war etwas Innovatives erschaffen worden, denn für diese Zielgruppen waren bis dahin nach den Recherchen zu Beginn des Projektes eL3 noch keine Evaluationsinstrumente in dieser Form veröffentlicht. Die Entwicklung der Inventare in einer transnationalen Gruppe wurde erfolgreich durchgeführt.

Zum Abschluss des Projektes wurde erneut ein Fragebogen eingesetzt, mit dem erfasst werden sollte, wie die Partner die entwickelten Inventare erprobt und genutzt hatten. Auf dieser Grundlage sollte zum Ende des Projektes ein Resümee gezogen werden und das weitere Vorgehen geplant werden können. Für die Fragestellung, ob die gemeinsam entwickelten Inventare von den Partnern auch genutzt wurden, ergab sich folgendes Bild: Fünf von neun Partnern haben die Inventare bzw. einzelne Inventare benutzt. Die anderen vier Partner haben keine Inventare eingesetzt, ein Partner bekam keine Erlaubnis innerhalb der Institution dazu, und die anderen drei gaben an, die Inventare zu einem späteren Zeitpunkt nutzen zu wollen. Diejenigen, die Inventare benutzt haben, trafen eine Auswahl von Fragen aus dem Itempool und stellten auf dieser Basis einen individualisierten Fragebogen zusammen, der zum jeweiligen Kurskontext passte. Die Fragebögen wurden entweder per E-Mail verschickt (als Word-Dokument), von den Befragten ausgefüllt und per E-Mail zurückgeschickt, oder papierbasiert in Kursen ausgeteilt und wieder eingesammelt (paper-pencilForm). Von den fünf Partnern, die Inventare benutzt haben, schickten vier die erhobenen Daten auch an die Verfasserin, wobei deutlich wurde, dass einige Partner Hilfestellungen bei der Durchführung der Evaluationen brauchten. Die Partner äußerten auch bei der Befragung selbst, dass sie Unterstützung bei der Benutzung der Inventare benötigten - von der Zusammenstellung der individuellen Fragen über den gesamten Ablauf der Evaluation und bis zur Berichterstattung (Verteilung der Fragebögen, das Einsammeln und Auswerten). Vor allem die Praxispartner wünschten sich für die Zukunft eine Anleitung bzw. einen Ansprechpartner. Mit der Erprobungsphase zeigten sich weitere Schwierigkeiten: Es wurde deutlich, wie verschieden die Kurskontexte und die Zielgruppen der Partner waren, und dass auch die verschiedenen Sprachen eine Hürde darstellten, die Ergebnisse der Evaluationen auf der Grundlage der Inventare zusammenzutragen und gemeinsam auszuwerten. Außerdem wurden die Evaluationen so unterschiedlich durchgeführt, dass ein Vergleich der Daten ebenfalls schwierig war, da die Art der Befragung die Ergebnisse durchaus beeinflusst haben kann. Ein weiteres sensibles Thema kam bei der Erprobungsphase der Inventare deutlich zum Vorschein: Das Teilen bzw. Mitteilen der Daten. Besonders bei den Partnern mit wissenschaftlichem Hintergrund war eine ausgesprochene Vorsicht und Zurückhaltung vorhanden. 
Sie wiesen darauf hin, dass mehrere Aspekte dabei eine Rolle spielten wie etwa der Datenschutz und auch Institutionelle Bedingungen. Für eine Veröffentlichung von Daten - auch in einem geschützten Rahmen innerhalb des Projektes - müssten Vorgesetzte einwilligen, die Befragungen unterstützen und genehmigen. Mit einem Einblick in die Daten der anderen hätten die Partner auch einen Einblick in die Qualität der Arbeit; die meisten Partner wollten sich ungern oder gar nicht „in die Karten schauen lassen“.

Zum Ende des Projektes eL3 ergab sich auf der Grundlage der Befragung folgendes Resümee: Die Partner bewerteten die Inventare als nützlich, jedoch ist die Handhabbarkeit und Praxistauglichkeit verbesserungswürdig. Die meisten Partner gaben an, dass sie Unterstützung in unterschiedlichen Formen für die Durchführung von Evaluationen auf der Grundlage der Inventare benötigten. Zunächst wurden mehr Hintergrundinformationen zu den Inventaren gewünscht (auch, um beispielsweise Vorgesetzte über den Inventareinsatz zu informieren und zu gewinnen). Weiterhin wurde Unterstützung gewünscht zur konkreten Durchführung der Evaluationen (Fragebogeneinsatz und Fragebogenauswertung). Schließlich war die weitere Verwendung der Ergebnisse ein offenes Thema, das der Klärung bedurfte. Es müsste eine Form gefunden werden, mit der die Partner ihre Ergebnisse im Netzwerk teilen können - aber nicht zum Selbstzweck, sondern als Basis für einen Austausch von Erfahrungen bzw. eine Veröffentlichung von guter Praxis. In der blinc-Gemeinschaft müssten verschiedene Formen und Möglichkeiten geschaffen werden, damit sich die unterschiedlichen Partner mit ihren unterschiedlichen Erwartungen und Bedarfen einbringen möchten und alle davon profitieren können. Zum Ende des Projektes eL3 war die weitere Nutzung der Inventare fraglich, da für viele Fragen der Partner noch keine Antworten vorhanden waren.

Insgesamt lassen sich auf der Basis aller eingesetzten Methoden folgende Ergebnisse im Hinblick auf die Fragestellungen dieser Forschungsarbeit festhalten: Die Evaluationspraxis der Projektpartner war zu Beginn des Projektes eL3 sehr unterschiedlich. Außerdem waren sehr verschiedenartige Vorstellungen von und Erwartungen an Evaluationen vorhanden und es zeigte sich auch das Forschung-Praxis-Dilemma in der Projektgruppe. Bei allen Partnern bestand der Wunsch nach Austausch von Erfahrungen und guter Praxis sowie Unterstützung im Bereich der Evaluation von Bildungsprojekten. Das Modell der Inventare wurde in der Gruppe sehr positiv aufgenommen, und die Entwicklung der Inventare in dem transdisziplinären Netzwerk verlief zwar etwas schleppend aber dennoch erfolgreich. Nach zwei Dritteln der Projektlaufzeit waren Inventare gemeinsam für verschiedene Zielgruppen entwickelt worden, die in so einer Form bisher einzigartig sind. Die Partner wurden durch die Arbeit an den Inventaren angeregt und bekamen neue Impulse für ihre Arbeit. Bei der Erprobungsphase zeigten sich viele Schwierigkeiten, die zuvor nicht als solch große Hürden erschienen waren. 
Nur etwa die Hälfte der Partner nutzte die Inventare überhaupt, und dabei ergaben sich noch viele Probleme, die vorher nicht offensichtlich waren. Nach dem Ende des Projektes eL3 ist sehr deutlich, dass für eine weitere Verwendung der Inventare Hilfestellungen und Unterstützung notwendig sind, wenn sie in der Praxis tatsächlich genutzt werden sollen. Das Potenzial für eine sinnvolle, praxistaugliche und gewinnbringende Nutzung ist vorhanden, jedoch müssten Formen der Zusammenarbeit bzw. für den Umgang mit Ergebnissen gefunden werden.

In diesem Zusammenhang kann kritisch diskutiert werden, ob der Ansatz der Inventare in der Praxis tatsächlich umsetzbar ist bzw. wie dies gelingen könnte. Die meisten Partner hatten (aus wissenschaftlicher Perspektive) erhebliche Defizite, was die Kompetenzen zur Durchführung von Evaluationen anbelangte. Dies konnte so nicht vorher gesehen werden. Außerdem konnten die Partner nicht dazu gezwungen werden, die Inventare zu benutzen. Die Freiwilligkeit der Nutzung und die Bereitschaft, die Daten bzw. Ergebnisse auch mit anderen zu teilen, gehören zum Ansatz der Inventare dazu. Wenn nun aber die meisten Partner zwar die Inventare benutzen wollten, um ihre eigene Evaluationspraxis zu verbessern, aber aus welchen Gründen auch immer anderen keinen Einblick in die Ergebnisse der Erhebungen erlaubten, dann funktionierte der ursprüngliche Ansatz nicht. Da die Inventare aber flexibel genutzt werden können, war und ist es auch möglich, dass sie einfach über die Internetplattform frei zur Verfügung gestellt werden - bleibt die Frage, ob sie dann auch tatsächlich genutzt werden, wenn sich schon im Rahmen des Projektes el3 so massive Probleme zeigen. Die größte Herausforderung war im Projekt eL3 wohl die Heterogenität der Partner und damit verbunden so unterschiedliche Erwartungen und Bedarfe an Evaluationen. Es gab jedoch eine Gemeinsamkeit bei allen Partnern, unabhängig vom institutionellen Kontext, unterschiedlichen Arbeitsweisen und Ansprüchen: Das Interesse am Austausch und am Dialog mit anderen Praxispartnern.

An dieser Stelle könnte angeknüpft werden, um die Arbeit mit den Inventaren erfolgreicher zu gestalten. Über das Netzwerk blinc ist eine Rahmen gegeben, der für einen weiteren Austausch geeignet sein könnte. Die Kommunikation müsste allerdingst strukturiert und angeleitet werden, wie die Ergebnisse dieser Forschungsarbeit zeigen. Wenn in dem Netzwerk eine organisierte Form von Evaluationen bzw. Selbstevaluationen auf der Grundlage der Inventare angeboten würde, könnte dies der Schlüssel zu einer intensiveren Nutzung sein. Wie die Ergebnisse der Befragungen zeigen, brauchen die meisten Partner Unterstützung bei der Durchführung ihrer Evaluationen. Dies könnte in schriftlicher Form erfolgen, z.B. durch Materialien bzw. Hintergrundinformationen und Anleitungen zum Einsatz der Inventare, aber auch durch personelle Hilfestellungen in Form von Beratungen oder sogar Dienstleistungen (dass eine kompetente Person die Evaluation mit den Inventaren professionell durchführt). An dieser Stelle wären vielleicht Interviews mit den eL3-Partnern zum Ende des Projektes oder 
eine gewisse Zeit danach eine geeignete Form gewesen, um mehr in die Tiefe zu gehen und genauere Bedarfe der Partner zu ermitteln bzw. Ideen zu sammeln, wie die Nutzung der Inventare gefördert werden könnte. Zum Ende des Projektes eL3 bestand ein relativ hoher Druck, die Aufgaben zu erfüllen, Angefangenes zu beenden, Berichte zu schreiben und sich selbst dabei dem Mittelgeber möglichst gut darzustellen. Diese Situation ist u.U. nicht die beste, um die Idee der Inventare zu realisieren und lebendig werden zu lassen. So gesehen zeigten sich mit der Erprobungsphase Schwierigkeiten, die für eine weitere Nutzung der Inventare gelöst werden müssten, aber auch Potenziale, die genutzt werden könnten. Es wurde jedenfalls deutlich, dass die Inventare keine Selbstläufer sind, sondern vielmehr sehr komplexe Instrumente, deren Nutzung der Unterstützung bedarf.

Die Daten und ermittelten Befunde aus der späteren Verwendung der Inventare (siehe Unterkapitel 6.5) zeigen, dass mit den Inventaren Möglichkeiten verbunden sind, weitere Forschungen durchzuführen, die zu interessanten und für die Praxispartner nützlichen Ergebnissen führen könnten. Es wurde deutlich, dass auch Planungsinstrumente wie die „Blended Learning Matrix" auf der Basis des Inventareinsatzes entwickelt und veröffentlicht werden können, was den Bedarfen der eL3-Partner nach Anregungen für ihre e-Learning und Blended Learning-Angebote sowie dem Interesse am Teilen von Wissen beispielsweise sehr gerecht werden könnte (nach den Ergebnissen der Prozessevaluation - siehe Unterkapitel 6.2). Die Inventare könnten so genutzt werden, um gemeinsam erarbeitetes Wissen zu generieren, zugänglich und nutzbar zu machen.

Hinzuweisen ist an dieser Stelle noch darauf, dass das Projekt eL3 das erste in einer Reihe weiterer Projekte in Zusammenarbeit von BUPnet, blinc und dem Pädagogischen Seminar der Universität Göttingen war. In diesen EU-Projekten, die im Folgenden kurz beschrieben werden, wurden die Erfahrungen von eL3 genutzt, um die Zusammenarbeit der Projektgruppe zu verbessern. Erfolgreiche Methoden - wie etwa die Prozessevaluation - wurden übernommen und neuere technische Möglichkeiten zur Unterstützung der kontinuierlichen Kommunikation zwischen den Projektpartnern eingesetzt. Außerdem wurden verschiedene Materialien und Hintergrundinformationen zu Produkten der Projekte entwickelt, um Partnern, die Unterstützung oder Informationen benötigten, entgegenzukommen.

Im Zeitraum von 10/2004 bis 12/2006 war das Pädagogische Seminar einer von neun Partnern im EU-Projekt „INTEGRATION - Interkultureller Dialog für eine multikulturelle Gesellschaft in Europa“, das durch das Programm Sokrates/Grundtivg 1 der EU gefördert wurde (siehe dazu: www.integration-eu.org, Zugriff 24.9.2012). Das Projektziel von INTEGRATION war die Verbesserung der interkulturellen Kompetenzen von Personal aus sozialen und Gesundheitseinrichtungen sowie die ihrer jeweiligen Zielgruppe(n). Im Zentrum stand dabei u.a. 
das Erarbeiten eines gemeinsamen europäischen Weges im Umgang mit interkulturellen Lehr- und Lernsituationen. Im Projekt INTEGRATION wurde eine Prozessevaluation nach dem Muster, wie es im Rahmen dieser Forschungsarbeit entwickelt wurde, durchgeführt, da sich die Methode bewährt hatte, um Projektfortschritte zu dokumentieren und sichtbar zu machen (was wiederum einen positiven Effekt auf die Projektgruppe hatte).

Im Zeitraum von 10/2005 bis 09/2007 fand das EU-Projekt „ABCD- Advanced Blended Learning Competencies and Didactics“, gefördert durch das Programm Sokrates/Grundtivg 1.1 der EU, statt, in dem das Pädagogische Seminar einer von acht Partnern war. Das Hauptziel war die Förderung der Verbreitung von gemischten Lernformen (Blended Learning) in der Fort- und Weiterbildung in Europa. Zu den Projektzielen gehörte u.a. auch die Entwicklung eines Schulungskurses für Trainer, die ihre Kursangebote in gemischten Lernformen (Präsenzlernen, e-Learning, Projekte) gestalten und anbieten wollen, ferner die Durchführung einzelner Schulungsmodule auf nationaler Ebene sowie ein Testlauf des gesamten Kurses auf europäischer Ebene. In diesem EU-Projekt wurde ebenfalls eine Prozessevaluation nach dem Muster aus dem Projekt eL3 durchgeführt.

Im Zeitraum von 10/2005 bis 09/2007 war das Pädagogische Seminar Hauptverantwortlicher Partner im EU-Projekt „ACT! Active Citizenship Training“, das von der EU durch das Programm Sokrates Action 6.1.2-6.2 gefördert wurde (siehe dazu auch: http://www.act-eu.org/, Zugriff am 24.9.2012). Zu den Zielen des Projektes gehörten u.a. die Entwicklung eines gemeinsamen Systems zur Bewertung der Effekte von informellen Lernangeboten auf das staatsbürgerschaftliche Verhalten in Einrichtungen der Gesundheits- und Sozialwirtschaft in Europa sowie die Bewertung lokaler Projekte mit Hilfe dieses Bewertungssystems. Außerdem sollte ein Experten-Netzwerks zum Thema "Evaluation von bürgerschaftlichen Kompetenzen in informellen Lernprojekten" aufgebaut werden. Im Rahmen dieses Projektes entstand eine Dissertation am Pädagogischen Seminar der Universität Göttingen (vgl. Scholze 2009). Die Kommunikation der Projektpartner wurde über eine Konferenzplattform (einen Online-Raum) zwischen den transnationalen Projekttreffen regelmäßig gefördert. Die zwischenzeitliche Kommunikation wirkte sich positiv auf die Arbeit, die Projektfortschritte und die Projektgemeinschaft aus, was zu einem sehr erfolgreichen Projekt beitrug. Hinweise auf die positiven Effekte der regelmäßigen Kommunikation finden sich in der Dissertation von Scholze (2009). Im Rahmen des Projektes ACT! (und mehreren Folgeprojekten, die dann allerdings nicht mehr mit dem Pädagogischen Seminar als Partner durchgeführt wurden) wurde ein Evaluationsverfahren mit computergestützten Anleitungen zur Erstellung von eigenen Referenzsystemen entwickelt und eingesetzt. Die technischen Möglichkeiten erleichtern den heutigen und zukünftigen Nutzern (nicht nur den Projektpartnern) die Anwendung des Evaluationsverfahrens. Außerdem wurde eine Sammlung von Evaluationstools und Anweisungen von der Projektgruppe erarbeitet und für potentielle Nutzer frei zur Verfügung gestellt. 
Mit diesen Erläuterungen wird deutlich, dass die Erfahrungen aus dem Projekt eL3 trotz der Schwierigkeiten in der Erprobungsphase der Inventare genutzt werden konnten, um in weiteren EU-Projekten die Zusammenarbeit der Partner sowie die Produktentwicklung zu unterstützen und damit zum Erfolg der Projekte beizutragen 


\section{Fazit und Ausblick}

Im Hinblick auf die erste Fragestellung dieser Arbeit kann festgehalten werden, dass die Evaluationspraxis in dem Fallbeispiel des EU-Projektes eL3 zu Beginn des Projektes sehr unterschiedlich war. Sowohl das Verständnis von Evaluation und damit verbundene Erwartungen an Evaluationen als auch die Art der Durchführung waren sehr verschieden. Es zeigte sich auch das Forschung-Praxis-Dilemma (vgl. Unterkapitel 4.3), d.h. in der Praxis Tätige konnten wissenschaftliche Standards und Regeln nicht einhalten und führten Evaluationen nur nach nicht transparenten Kriterien und subjektiven Vorstellungen durch.

Die Praktiker wünschten sich Unterstützung für die Evaluation ihrer Bildungsprojekte vor allem, um die Qualität ihrer Angebote zu verbessern. Auf dieser Grundlage wurde der Ansatz von Inventaren entworfen (vgl. Kapitel 5). In dieser Forschungsarbeit wurde untersucht, ob gemeinsam in dem transnationalen europäischen Netzwerk Inventare als Evaluationsinstrumente entwickelt werden können, und ob diese Inventare dann auch genutzt werden.

Als zentrales Ergebnis kann festgehalten werden, dass die Inventare gemeinsam von den europäischen Projektpartnern entwickelt werden konnten. Am Ende des Projektes lagen Inventare für drei verschiedene Zielgruppen vor (Lerner, Autoren und Entscheider), die in dieser Form bisher einzigartig sind. Die Projektpartner bekamen bereits durch den Entwicklungsprozess der Inventare, die in Arbeitsgruppen erarbeitet wurden, wertvolle Anregungen für ihre berufliche Praxis und hatten großes Interesse an der Nutzung und am Austausch von Erfahrungen in dem Netzwerk. Bei der Erprobungsphase der Inventare zeigten sich dann aber verschiedene Probleme, die so nicht vorhersehbar waren. Zum einen benutzten nicht alle Partner die Inventare zur Evaluation ihrer Bildungsprojekte, wobei die Gründe zum Teil nicht eindeutig waren. Mit den Ergebnissen der Befragung zum Abschluss des Projektes wurde nur deutlich, dass die Partner teilweise keine Erlaubnis von Vorgesetzten bekamen, eine Evaluation auf der Grundlage der Inventare durchzuführen und teilweise die Inventare zu einem späteren Zeitpunkt einsetzen wollten. Zum anderen zeigten sich bei den Partnern, die Inventare benutzten, Schwierigkeiten beim Einsatz und Defizite in der Durchführung der Evaluation. Die Gründe hierfür waren verschiedene, es kann jedoch konstatiert werden, dass einigen Partnern die nötige Evaluationskompetenz fehlte. Die Inventare sind als sehr komplexe Evaluationsinstrumente keine Selbstläufer. Die Partner brauchen nach den Ergebnissen der Untersuchung Unterstützung bei der Durchführung der Evaluation auf der Basis der Inventare und zwar auf mehreren Ebenen. Einige Praxispartner forderten mehr Hintergrundinformationen (auch für Vorgesetzte) und eine Anleitung, wie sie die Evaluation durchführen sollen. Für die Datenauswertung wurde ebenfalls eine Hilfestellung gewünscht. Manche Partner wollten die Inventare bzw. die Inhalte auch nur als Anregung verwenden, um beste- 
hende Instrumente oder Verfahren zu ergänzen. Alle Projektpartner hatten aber unabhängig von ihren unterschiedlichen Arbeitsweisen, Ansprüchen und beruflichen Kontexten ein groBes Interesse am Austausch von Erfahrungen und Wissen. An dieser Stelle kann nun auf der Basis der vorliegenden Forschungsergebnisse angesetzt werden. Vier Aspekte könnten für eine zukünftige erfolgreichere Nutzung der Inventare als Evaluationsinstrumente für Bildungsprojekte in verschiedenen europäischen Ländern förderlich sein:

\section{Information und Materialien zu den Inventaren}

Dem Inventaransatz liegt ein bestimmtes Evaluationsverständnis zugrunde, das beispielsweise in schriftlicher Form ausgearbeitet und beigefügt werden könnte. Die Evaluation hat vor allem eine Erkenntnisfunktion und eine Dialog- bzw. Lernfunktion und nicht, wie häufig von den Partnern erwartet, eine Kontrollfunktion oder Legitimitätsfunktion (siehe dazu auch Kapitel1.3). Außerdem könnten Informationen zum Entwicklungsprozess der Inventare verfasst werden, um zu verdeutlichen, wer sie mit welcher Absicht erarbeitet hat. Darüber hinaus könnten weitere Materialien wie etwa kurze Filmsequenzen, in denen Partner darüber berichten, wie sie die Inventare benutzt haben, anregend und nützlich für Praxispartner sein, die die Inventare in ihren Einrichtungen nutzen wollen. Eine gewisse „Marketingstrategie“ könnte hier hilfreich sein, das Produkt (die Inventare) interessant zu machen und einen Einsatz zu fördern. Die Hintergrundinformationen und Materialien könnten auch Vorgesetzten, die dem Inventareinsatz zustimmen müssen, vorgelegt bzw. gezeigt werden. Über die Internetplattform „blinc“ könnten sämtliche Produkte, Dokumente und Materialien frei verfügbar gemacht werden.

\section{Sozialer Kontakt zu anderen europäischen Partnern}

Die Projektpartner im Projekt eL3 haben immer wieder betont, wie wichtig und wertvoll innen der Kontakt zu anderen europäischen Bildungspraktikern ist, und dass allein der Austausch von Wissen und Erfahrungen die eigene berufliche Praxis bereichert. Es könnte also eine organisierte Form des Kontaktes z.B. in Form von Treffen (Konferenzen mit vielen Partnern, aber auch eine Art „Austausch“ - d.h. ein Partner besucht einen anderen und bekommt einen Einblick in dessen Arbeit) hilfreich sein, um den Einsatz der Inventare zu fördern. Denkbar sind auch kontinuierliche Besprechungen über Online-Räume. Die Nutzung moderner Medien ermöglicht dabei eine synchrone Kommunikation. Ein Problem im Projekt eL3 war nach den Rückmeldungen der Partner die asynchrone Kommunikation über das Lernmanagementsystem. Eine synchrone Form der Zusammenarbeit kann hier ein Schlüssel sein, um die Effektivität der Arbeit und die Motivation zur Beteiligung zu optimieren. Die Vertrauensbasis, die durch das Projekt eL3 bereits vorhanden ist, stellt hier eine wichtige Voraussetzung dar. Die Partner kennen sich bereits und die Sorge, sich zu sehr in die Karten schauen zu lassen ist verringert. Es besteht vielmehr der Wunsch zu erfahren, was es bei den ande- 
ren neues gibt. Das Internet mit seinen technischen Möglichkeiten bietet hier eine Basis, um auch über die Projektlaufzeit hinaus in Kontakt zu bleiben.

\section{Unterstützung bei der Durchführung der Evaluation oder externe Evaluation}

Die meisten Praxispartner wünschten sich für die Zukunft eine Unterstützung bei der Durchführung der Evaluation z.B. in Form einer Beratung zu Beginn des Vorhabens. Hier könnte ein persönlicher Ansprechpartner und Experte für die Inventare dienlich sein. Wichtig ist auch dabei eine gewisse Vertrauensbasis, die durch das Projekt eL3 schon vorhanden ist. Die Erhebung und Auswertung von Daten mittels statistischer Verfahren sind für viele Praxispartner schwierig, so dass eine kompetente Person diese Schritte der Evaluation gut übernehmen oder begleiten könnte. Eine Befragung der eL3 Partner mittels Interviews könnte noch genauere Auskünfte darüber geben, welche Formen der Unterstützung für die Partner hilfreich und annehmbar wären. Im gemeinsamen Netzwerk „blinc“ wäre es auch denkbar, dass eine externe Evaluation von Bildungsprojekten auf der Basis der Inventare als Dienstleistung angeboten würde.

\section{Stärkung der Evaluationskompetenz durch Training und Weiterbildung}

Die Evaluationskompetenz der Partner könnte durch ein Trainingsangebot oder eine Weiterbildung gestärkt werden, weil dann vermutlich die Evaluationen auf der Grundlage der Inventare erfolgreicher wären. Die Deutsche Gesellschaft für Evaluation (DeGEval) hat Empfehlungen für die Aus- und Weiterbildung in der Evaluation herausgegeben (DeGEval 2004 und 2008) und Anforderungsprofile an Evaluatorinnen und Evaluatoren erstellt. Die Evaluationskompetenz besteht nach der DeGEval aus einem Set an Wissen, Fertigkeiten und Haltungen, welches eine Person befähigt, eine Evaluation den Evaluationsstandards (DeGeval 2002) entsprechend auszuführen. Es werden vier Kompetenzfelder beschrieben, die für eine Aus- bzw. Weiterbildung in der Evaluation wichtig sind:

1. Theorie und Geschichte der Evaluation

2. Methodenkompetenzen

3. Organisations- und Feldkenntnisse

4. Sozial- und Selbst-Kompetenzen

Bei der Gestaltung einer Weiterbildung oder eines Training für die Projektpartner könnten diese Kompetenzfelder eine Orientierung sein, um die Qualität des Angebotes zu sichern. Die DeGEval (2011) gibt weiterhin Empfehlungen für die didaktische Gestaltung der Ausund Weiterbildung in der Evaluation heraus, die ebenfalls als eine Grundlage bei der Entwicklung eines speziell auf die Bedarfe der Partner abgestimmten Weiterbildungsangebotes genutzt werden könnten. 
In einer weiterführenden Untersuchung könnten auf der Basis von Interviews die Bedarfe der Partner in Bezug auf eine Unterstützung bei der Evaluation erforscht und ermittelt werden. Es könnte auch nachgefragt werden, ob die hier vorgeschlagenen Maßnahmen dazu beitragen würden, dass die Inventare häufiger genutzt würden und es könnte geklärt werden, was die genauen Motive bzw. Bedingungen für eine Nutzung der Inventare sind, um dann diese Voraussetzungen schaffen zu können. Danach müsste eine erneute Erprobungsphase erfolgen und geprüft werden, ob die Inventare tatsächlich intensiver genutzt werden.

Insgesamt bleibt festzuhalten, dass die Inventare die Reflexion des beruflichen Handelns anregen können und ein vielfältiges Potenzial besitzen, die Evaluationspraxis der beteiligten Partner positiv zu verändern. Für eine sehr gute Qualität von Evaluationen auf der Basis der Inventare können zukünftig noch verschiedene Maßnahmen ergriffen werden, die Bildungspraktiker unterstützen, Standards einzuhalten und ihre Evaluationskompetenzen zu steigern.

Schließlich ist darauf hinzuweisen, dass ein wichtiger methodologischer Aspekt aufzugreifen wäre, wenn eine Weiterentwicklung des Inventaransatzes in Angriff genommen werden sollte: eine sukzessive Standardisierung der Inventare dadurch, dass bei den verschiedenen Verwendungen entstandene Daten gesammelt werden und zur Kennzeichnung der einzelnen Items genutzt werden. Dieser Aspekt war von vornherein als wünschenswerter Ertrag der Inventare des Projektes eL3 bedacht gewesen, konnte aber wegen der geringen Erprobungen nicht umgesetzt werden. Gleichwohl besteht die Aussicht weiterhin, dass die Dokumentation verschiedener Parameter eines Inventars sinnvoll sein könnte, um den Nutzern bei ihren Verwendungen Informationen zu liefern, wie die Befunde zu interpretieren sind. 


\section{Tabellenverzeichnis}

Tabelle 1: Der Begriff Evaluation im alltäglichen und wissenschaftlichen Sprachgebrauch (erstellt von der Verfasserin in Anlehnung an Kromrey 2001, S.108)

Tabelle 2: Gemeinsamkeiten unterschiedlicher Definitionen von Evaluation (erstellt von der Verfasserin)

Tabelle 3: Beteiligte bei der Evaluation einer Weiterbildungsmaßnahme und deren Ziele und Motive (erstellt von der Verfasserin in Anlehnung an Wottawa und Thierau 2003, S.57)

Tabelle 4: Funktionen von Evaluation nach verschiedenen Autoren (erstellt von der Verfasserin) 22

Tabelle 5: Phasen von Programm Evaluation nach Madaus und Stufflebeam (vgl. 2000, S.3) _ 30

Tabelle 6: Evaluationskultur in verschiedenen Ländern (Quelle: Furubo, Rist und Sandahl 2002, S.10)

Tabelle 7: $\quad$ Aufteilung von Ländern in Abhängigkeit von internen und externen Faktoren, die einen starken oder schwachen Einfluss auf die Entwicklung der Evaluationskultur haben (Quelle: Furubo Rist und Sandahl 2002, S.21)

Tabelle 8: Typen Expertenorientierter Evaluation nach Fitzpatrick, Sanders und Worthen (übersetzt von der Verfasserin)

Tabelle 9: Übersicht über den Projektverlauf 132

Tabelle 10: Ziele einer Prozessevaluation 153

Tabelle 11: Beispiel für die Zusammenfassung von Antworten der Projektpartner auf die Frage „What have you liked so far in this project?" 155

Tabelle 12: Beispiel für die Zusammenfassung der Antworten in Kategorien 155

Tabelle 13: Frage zur Bestandsaufnahme der Evaluationspraxis zu Beginn des Projektes eL3:

"What kind of evaluation monitoring processes have been used so far in your project?" 160

Tabelle 14: Antworten der eL3- Partner auf die Frage:" What kind of evaluation monitoring processes have been used so far in your project?" $(n=9)$ 160

Tabelle 15: Erläuterungen der Partner zur verwendeten Evaluationsmethode „Tests/Selbsttests/Leistungsnachweise innerhalb des Projektes“ 161

Tabelle 16: Erläuterungen der Partner zur Evaluationsmethode „Prüfungen/Leistungsnachweise außerhalb des Projektes“ 162

Tabelle 17: Erläuterungen der Partner zur Evaluationsmethode "Feedback von anderen" 162

Tabelle 18: Erläuterungen der Partner zur Evaluationsmethode „Formale Befragung der Lerner“ 162

Tabelle 19: $\quad$ Übersicht über die Kombination von Methoden bei der Evaluationspraxis der einzelnen Projektpartner im Projekt eL3 $(n=9)$ 163

Tabelle 20: $\quad$ Verwendete Evaluationsmethoden in der Gruppe der Bildungsanbieter im Projekt eL3 164

Tabelle 21: Verwendete Evaluationsmethoden in der Gruppe der Krankenhäuser im Projekt eL3 165

Tabelle 22: Verwendete Evaluationsmethoden in der Gruppe der Universitäten im Projekt eL3 165 
Tabelle 23: Ergebnisse der Prozessevaluation auf die Frage „How do you like the progression of the eL3-network?"

Tabelle 24: Ergebnisse der Prozessevaluation auf die Frage "Considered all in all are you satisfied with the eL3 project?" 169

Tabelle 25: Ergebnisse der Prozessevaluation auf die Frage „What have you liked so far?“ _ 171

Tabelle 26: $\quad$ Ergebnisse der Prozessevaluation auf die Frage „What have you disliked so far?" 173

Tabelle 27: Ergebnisse der Prozessevaluation auf die Frage „In your opinion what needs improvement?" 175

Tabelle 28: Ergebnisse der Prozessevaluation auf die Frage „What positive effects has the eL3 network for you until now?" 177

Tabelle 29: Ergebnisse der Prozessevaluation auf die Frage „Do you have any perspectives or a fresh impetus which you can use or realize for your own project?" 178

Tabelle 30: $\quad$ Ergebnisse der Prozessevaluation auf die Frage „Do you have any perspectives or a fresh impetus which you can use or realize for your own project? - If yes, please describe in short?" 179

Tabelle 31: Ergebnisse der Prozessevaluation auf die Frage "Do you want to have any more information?" 179

Tabelle 32: $\quad$ Ergebnisse der Prozessevaluation auf die Frage „Do you want to have any more information? - If yes, what kind of information would you like?" 180

Tabelle 33: Ergebnisse der Prozessevaluation auf die Frage „What do you expect or wish for the further work of the eL3-network?" 182

Tabelle 34: Überblick über den Entwicklungszeitraum und die Entwicklungsphasen der Inventare im Projekt eL3 186

Tabelle 35: Ü̈bersicht über die Entwicklungsschritte der Inventare im Rahmen von eL3___ 187

Tabelle 36: Möglicher Evaluationsverlauf für die Befragung von Lernern __ 195

Tabelle 37: Übersicht über die Inhalte der Inventare für Lerner ___ 208

Tabelle 38: Übersicht über verschiedene Möglichkeiten zur Nutzung des Autoreninventars__ 215

Tabelle 39: Übersicht über das Autoreninventar 225

Tabelle 40: Übersicht über verschiedene Möglichkeiten der Nutzung der Inventare für Entscheidungsträger 234

Tabelle 41: Übersicht Inventar für Entscheidungsträger 240

Tabelle 42: Übersicht, welche Partner welche Inventare benutzt haben 242

Tabelle 43: Geplanter Einsatz der Inventare für Lerner im Mai 2005 246

Tabelle 44: Tatsächlicher Einsatz der Inventare für Lerner bis November 2005 249

Tabelle 45: Übersicht über den Einsatz der Inventare und die endgültige Zahl verwendbarer Fragebögen bzw. Daten 250

Tabelle 47: $\quad$ Einsatz Inventar für Lerner 251

Tabelle 48: Übersicht über die Fallzahlen bei der späteren Verwendung der Inventare nach Abschluss des Projektes eL3 255 


\section{Abbildungsverzeichnis}

Abbildung 1 Funktionen von Evaluation (Quelle: Stockmann 2006a, S.21) 17

Abbildung 2: Methodisches Vorgehen nach dem Modell der Vierten Generation nach Guba und Lincoln (1989) 71

Abbildung 3: Der Evaluationsbaum nach Alkin und Christie (2004) 73

Abbildung 4: Struktur des Fachs Erziehungswissenschaft (erstellt von der Verfasserin in Anlehnung an Lenzen 1994, S.38) 100

Abbildung 5: Evaluation von Fahrrädern, Krankentransporten und Unterricht 106

Abbildung 6: Triangulation verschiedener qualitativer Methoden (Quelle: Flick 2008b S.41) 148

Abbildung 7: Basisdesigns zur Verbindung von qualitativer und quantitativer Forschung (Quelle: Miles und Huberman 1994, S.41 in Flick 2008b, S.80) 148

Abbildung 8: Phasen der Prozessevaluation im Projektverlauf 156

Abbildung 9: Übersicht des Kurses für die Arbeitsgruppe „Inventare für Lerner“ auf der Lernplattform 190

Abbildung 10: Sammlung von Instrumenten der Partner im Kurs für die Arbeitsgruppe der Inventare für Lerner 191

Abbildung 11: Verschiedene Versionen des Lernerinventars im Kurs auf dem Lernmanagementsystem 192

Abbildung 12: Ausschnitt aus den Beiträgen der Arbeitsgruppenmitglieder zum Inventar der Lerner im Forum 193

Abbildung 13: Mögliche Onlineform von dynamischen Inventaren für Lerner mit einer Datenbank im Hintergrund 198

Abbildung 14: Ausschnitt aus der multilingualen Version der Inventare für Lerner 204

Abbildung 15: Anleitung für die Weiterentwicklung des Autoreninventars auf dem Lernmanagementsystem 213

Abbildung 16: Beiträge der Arbeitsgruppe für das Autoreninventar im Forum 214

Abbildung 17: Übersicht des Kurses für die Arbeitsgruppe „Inventare für Entscheidungsträger" auf der Lernplattform 228

Abbildung 18: Einführungstext für die Arbeitsgruppe „Inventare für Entscheidungsträger“ auf der Lernplattform 229

Abbildung 19: Lerneinheit zum Inventar für Entscheidungsträger vor der Einführung eines e-Learning oder Blended Learning-Systems 230

Abbildung 20: Lerneinheit im Kurs Inventare für Entscheidungsträger mit Material eines Arbeitsgruppenmitglieds 231

Abbildung 21: Beiträge der Arbeitsgruppe für die Inventare für Entscheidungsträger 232

Abbildung 22: Verschiedene Phasen im Optimierungsprozess der Inventare für Lerner 245 


\section{Literaturverzeichnis}

Alkin, M. C. (1969). Evaluation Theory Development. In: Evaluation Comment, Band 2, S. 27.

Alkin, M. C. (Hrsg.) (2004a). Evaluation Roots: Tracing Theorists` Views and Influences. Thousand Oaks: Sage.

Alkin, M. C. (2004b). Comparing Evaluation Points of View. In Alkin, M. C. (Hrsg.). Evaluation Roots: Tracing Theorists` Views and Influences. Thousand Oaks: Sage. S. 3-11.

Alkin, M. C. \& Christie, C. (2004). An Evaluation Tree. In Alkin, M. C. (Hrsg.). Evaluation Roots: Tracing Theorists`Views and Influences. Thousand Oaks: Sage. S. 12-65.

Altrichter, H. (1990). Ist das noch Wissenschaft? Darstellung und wissenschaftstheoretische Diskussion einer von Lehrern betriebenen Aktionsforschung. München: Profil.

Altrichter, H. (1999). Evaluation als Alltäglichkeit, als Profession und als Interaktion. In: Thonhauser, J. \& Patry, J.-L. (Hrsg.). Evaluation im Bildungsbereich. Wissenschaft und Praxis im Dialog. Studien zur Bildungsforschung \& Bildungspolitik, Band 22. Innsbruck-Wien: Studienverlag.

Altrichter, H. \& Posch, P. (2007). Lehrerinnen und Lehrer erforschen ihren Unterricht: Unterrichtsentwicklung und Unterrichtsevaluation durch Aktionsforschung. Bad Heilbrunn: Klinkhardt.

Baumert, J, Lehmann, R., Lehrke, M., Schmitz, B., Clausen, M., Hosenfeld, I., Köller, O. \& Neubrand, J. (1997). TIMMS. Mathematisch-naturwissenschaftlicher Unterricht im internationalen Vergleich. Deskriptive Befunde. Opladen: Leske + Budrich.

Baumert, J., Bos, W., \& Lehmann, R. (Hrsg.) (2000a): TIMSS/II. Dritte Internationale Mathematik- und Naturwissenschaftsstudie - Mathematische und naturwissenschaftliche Bildung am Ende der Schullaufbahn. Bd. 1: Mathematische und naturwissenschaftliche Grundbildung am Ende der Pflichtschulzeit. Opladen: Leske + Budrich.

Baumert, J., Bos, W., \& Lehmann, R. (Hrsg.) (2000b): TIMSS/III: Dritte Internationale Mathematik- und Naturwissenschaftsstudie - Mathematische und naturwissenschaftliche Bildung am Ende der Schullaufbahn. Bd. 2: Mathematische und naturwissenschaftliche Grundbildung am Ende der gymnasialen Oberstufe. Opladen: Leske + Budrich.

Baumgartner, P. (2003). Didaktik, E-Learning-Strategien, Softwarewerkzeuge und Standards - Wie passt das zusammen? In: Franzen, M. Mensch und E-Learning. Beiträge zur Didaktik und darüber hinaus. Aarau, Sauerländer: 9-25.

Bertelsmann Stiftung (2007-2011). Die gute gesunde Kita gestalten. Materialien zum Projekt: „Kitas bewegen“. Vier Broschüren zum Prozess der Organisationsentwicklung. Gütersloh. Im Internet unter: www.gute-gesunde-kita.de

Beywl, W. \& Bestvater, H. (1998). Selbst-Evaluation in pädagogischen und sozialen Arbeitsfeldern. Ergänzung und Alternative zur Fremdevaluation. In: Bundesvereinigung Kulturelle Jugendbildung e.V. (Hrsg.). Qualitätssicherung durch Evaluation. Konzepte, Methoden, Ergebnisse - Impulse für die kulturelle Kinder- und Jugendbildung. Schriftenreihe der Bundesvereinigung Kulturelle Jugendbildung, Band 46. Remscheid: Topprint.

Blankertz, H. (Hrsg.) (1978). Die Theorie-Praxis-Diskussion in der Erziehungswissenschaft. Zeitschrift für Pädagogik. 15. Beiheft. Weinheim und Basel: Beltz. 
Bloom, B. S., Engelhart, M. D., Furst, E. J., Hill, W. H. \& Krathwohl, D. R. (1956). Taxonomy of Educational Objectives. Handbook I: Cognitive Domain. New York: David McKay.

Bloom, B. S., Krathwohl, D. R. \& Masia, B. B. (1964). Taxonomy of Educational Objectives. The Classification of Educational Goals. Handbook II: Affective Domain. New York: David McKay.

Böttcher, W., Holtappels, H. G. \& Brohm, M. (Hrsg.) (2006). Evaluation im Bildungswesen. Eine Einführung in Grundlagen und Praxisbeispiele. Weinheim und München: Juventa Verlag.

Borg, I. \& Staufenbiel, T. (2007). Lehrbuch Theorien und Methoden der Skalierung. 4., vollständig überarbeitete und erweiterte Auflage. Bern: Verlag Hans Huber.

Bortz, J. \& Döring, N. (1995). Forschungsmethoden und Evaluation für Sozialwissenschaftler. 2. Auflage. Berlin u.a.: Springer.

Bortz, J. \& Döring, N. (2006). Forschungsmethoden und Evaluation für Human- und Sozialwissenschaftler. 4., überarbeitete Auflage. Berlin u.a.: Springer.

Bos, W., Lankes, E.-M., Prenzel, M., Schwippert, K., Valtin, R. \& Walther, G. (Hrsg.) (2003). Erste Ergebnisse aus IGLU. Schülerleistungen am Ende der vierten Jahrgangsstufe im internationalen Vergleich. Münstzer u.a.: Waxmann.

Bos, W., Bonsen, M. Baumert, J. Prenzel, M., Selter, C. \& Walther, G (Hrsg.) (2008): TIMSS 2007. Mathematische und naturwissenschaftliche Kompetenzen von Grundschulkindern in Deutschland im internationalen Vergleich. Münster u.a. Waxmann.

Buhren, C. G., Killus, D. \& Müller, S. (2002). Wege und Methoden der Selbstevaluation. Ein praktischer Leitfaden für Schulen. 5. Auflage. Dortmund: IFS-Verlag.

Buhren, C.G. (2007). Selbstevaluation in Schule und Unterricht: Ein Leitfaden für Lehrkräfte und Schulleitungen. Köln:Luchterhand.

Bühner, M. (2006). Einführung in die Test- und Fragebogenkonstruktion. 2., aktualisierte Auflage. München: Pearson.

Bundesministerium für Familie, Senioren, Frauen und Jugend (1995-2001) (Hrsg.). Materialien zur Qualitätssicherung in der Kinder- und Jugendhilfe. 36 Bände. Im Internet abrufbar unter: http://www.qs-kompendium.de (zuletzt geprüft am 18.4.2011).

Bundesvereinigung Kulturelle Jugendbildung e.V. (Hrsg.) (1998). Qualitätssicherung durch Evaluation. Konzepte, Methoden, Ergebnisse - Impulse für die kulturelle Kinder und Jugendbildung. Remscheid.

Campbell, D. T. (1957). Factors relevant to the Validity of Experiments in Social Settings. In: Psychological Bulletin, 54, S. 297-312.

Campbell, D. T. \& Fiske, D. W. (1959). Convergent and Discriminant Validation by the Multitrait-Multimethod Matrix. In: Psychological Bulletin, 56, S.81-105.

Campbell, D. T. \& Stanley, J. C. (1966). Experimental and Quasi-experimental Designs. Boston, M.A.: Houghton Mifflin.

Chelimsky, E. (1997a). Thoughts for a New Evaluation Society. In: Evaluation. Volume 3 (1). S. 97-118. Thousand Oaks: Sage. 
Chelimsky, E. (1997b). The Coming Transformation in Evaluation. In: Chelimsky, E. \& Shadish, W. R. (Ed.). Evaluation for the 21st Century. A Handbook. Thousand Oaks u.a.: Sage.

Chelimsky, E. (2006). The Purpose of Evaluation in a Democratic Society. In: Shaw, I. F., Greene, J. C. \& Melvin, M. (Hrsg.). The Sage Handbook of Evaluation. S. 33-55.

Chen, H. \& Rossi, P. (1983). Evaluating with Sense: The Theory-Driven Approach. In: Evaluation Review, 7, S. 283-302.

Chen, H. \& Rossi, P. (1987). The Theory-Driven Approach to Validity. In: Evaluation and Programm Planning, 10, S. 95-103.

Chen, H. (1990). Theory-Driven Evaluations. Newbury Park: Sage.

Cousins, J. B. \& Earl, L. M. (1992). The Case for Participatory Evaluation. In: Educational Evaluation and Policy Analysis, 14, (4), S. 397-418.

Cousins, J. B. \& Earl, L. M. (Hrsg.) (1995). Participatory Evaluation in Education: Studies in Evaluation Use and Organizational Learning. London: Falmer.

Cronbach, L. J. (1963). Course improvement through evaluation. In: Teachers College Record, 64, S. $672-683$.

Cronbach, L. J. \& Associates (1980). Toward Reform of Program Evaluation: Aims, Methods, and Institutional Arrangements. San Francisco: Jossey-Bass.

Cronbach, L. J. (1982). Designing Evaluations of Educational and Social Programs. San Francisco: Jossey-Bass.

Cronbach, L. J. (2000). Course Improvement Through Evaluation. In: Stufflebeam, D. L., Madaus, G. F. \& Kellaghan, T. (2000) (Hrsg.). Evaluation Models. Viewpoints on Educational and Human Services Evaluation. Second Edition. Boston u.a.: Kluwer. S. 235-247

Datta, L.-E. (2006). The Practice of Evaluation: Challenges and New Directions. In: Shaw, I. F., Greene, J. C. \& Melvin, M. (Ed.). The Sage Handbook of Evaluation. Thousand Oaks: Sage.

Denzin, N. K. (1970). The Research Act. Chicago: Aldine.

Denzin, N. K. (1989). The Research Act. 3. Edition. Englewood Cliffs, N. J.: Prentice Hall.

Derlien, H.-U. (1990). Genesis and Structure of Evaluation Efforts in Comparative Perspective. In: Rist, R. C. (Ed.). Program Evaluation and the Management of Government. Patterns and Prospects across Eight Nations. New Brunswick and London: Transaction Publishers. S. 147-175.

Deutsche Gesellschaft für Evaluation e.V. (2002). Standards für Evaluation. Köln/Alfter.

Deutsche Gesellschaft für Evaluation e.V. (2004a). Empfehlungen zur Anwendung der Standards für Evaluation im Handlungsfeld der Selbstevaluation. Alfter.

Deutsche Gesellschaft für Evaluation e.V. (2004b). Empfehlungen für die Aus- und Weiterbildung in der Evaluation. Alfter.

Dohmen, G. (2001). Das informelle Lernen. Die internationale Erschließung einer bisher vernachlässigten Grundform menschlichen Lernens für das lebenslange Lernen aller. Bonn: BMBF. 
Dorenkamp L. (1999). Evaluation als Steuerungs- und Führungsinstrument für (berufliche) Schulen. In: Dorenkamp L. und Lauks, K. (Hrsg.). Schulentwicklung durch Bildungsinnovationen: Ergebnisse eines internationalen Symposiums zum Modellversuch DOPKAU (S. 181190). Rostock: Universität Rostock, Lehrstuhl für Wirtschaftspädagogik, Forschungsgruppe Mecklenburg-Vorpommern.

Ehlers, U.-D. (2004). Qualität im E-Learning: Empirische Grundlegung und Modellkonzeption einer subjektorientierten Qualitätsentwicklung. Dissertation. Bielefeld

European Commission (1996). Evaluation: Concrete Steps towards Best Practice across the Commission. Communication adopted on 8 May 1996. Brussels. SEC (96) 659.

European Commission (1997). Evaluating EU Expenditure Programs: A Guide for Ex Post and Interim Evaluations. Luxembourg: Office for Official Publications of the EC.

European Commission (1999a). Evaluation Review 1998. Internal document. Brussels: ECDirectorate General for Budgets

European Commission (1999b). Good Practice Guidelines for the Management of Evaluation Function. Information note to the Commission. Brussels: SEC.

European Commission (1999c). Evaluation Design and Management. In MEANS Collection. Volume 1. Luxembourg: Office for Official Publications of the EC.

Fatke, R. (Hrsg.) (1997). Forschungs- und Handlungsfelder der Pädagogik. Zeitschrift für Pädagogik. 36. Beiheft. Beltz: Weinheim und Basel.

Fetterman, D. (1994). Empowerment Evaluation. In: Evaluation Practice, 15 (1), S. 1-15

Fetterman, D., Kaftarian, S. J. \& Wandersman, A. (Hrsg.) (1996a). Empowerment Evaluation: Knowledge and Tools for Self-Assessment and Accountability. Thousand Oaks, CA: Sage.

Fetterman, D. (1996b). Empowerment Evaluation: An Introduction to Theory and Practice. In: Fetterman, D., Kaftarian, S. J. \& Wandersman, A. (Hrsg.). Empowerment Evaluation: Knowledge and Tools for Self-Assessment and Accountability. Thousand Oaks, CA: Sage.

Fetterman, D. (1998). Empowerment Evaluation and Accreditation in Higher Education. In: Chelimsky, E. \& Shadish, W. (Hrsg.). Evaluation for the 21st Century: A Handbook. Thousand Oaks, CA: Sage. S. 381-395.

Fetterman, D. (2000). Foundations of Empowerment Evaluation. Thousand Oaks, CA: Sage.

Fetterman, D. (2004). Branching out or standing on a limb? Looking at our roots for insight. In: Alkin, M. C. (Hrsg.) (2004a). Evaluation Roots: Tracing Theorists`Views and Influences. Thousand Oaks: Sage, S. 304-318.

Fitzpatrick, J. L., Sanders, J. R. \& Worthen, B.R. (2004). Program Evaluation. Alternative Approaches and Practical Guidelines. 3. Auflage. Boston u.a.: Pearson.

Flechsig, K.-H. (1975). Forschungsschwerpunkte im Bereich der Unterrichtstechnologie. In: Roth, H. \& Friedrich, D. (Hrsg.). Bildungsforschung. Probleme - Perspektiven - Prioritäten. Stuttgart: Ernst Klett Verlag.

Flechsig, K.-H. \& Haller, H.-D. (1975). Einführung in didaktisches Handeln: Ein Lehrbuch für Einzel- und Gruppenarbeit. Stuttgart: Klett. 
Flechsig, K.-H. (1983). Der Göttinger Katalog Didaktischer Modelle. Theoretische und methodologische Grundlagen. Göttingen: Zentrum für Didaktische Studien.

Flechsig, K.-H. (1987). Didaktisches Design: Neue Mode oder neues Entwicklungsstadium der Didaktik? Internes Arbeitspapier 6/1987. Institut für Kommunikationswissenschaften der Georg-August-Universität Göttingen, Abteilung „Allgemeine Didaktik und Unterrichtsforschung (ADU).

Flechsig, K.-H. (1990). Einführung in CEDID. Ein tätigkeits-unterstützendes und wissensbasiertes System für computer-ergänztes Didaktisches Design. Verfügbar unter: www.gkdm.de/EinfCEDID.doc

Flechsig, K.-H. (1996a). Kleines Handbuch Didaktischer Modelle. Eichenzell: Neuland: Verlag für lebendiges Lernen.

Flechsig, K.-H. (1996b). Historical and Philosophical Backgrounds of Instructional Design. A European View.

Verfügbar unter: http://www.ikkconsult.de/internearbeitspapiere/1994\%202.pdf

Flick, U. (2008a). Design und Prozess qualitativer Forschung. In: Flick, U., von Kardoff, E. \& Steinke, I. (Hrsg.). Qualitative Forschung. Ein Handbuch. Reinbek bei Hamburg: Rowohlt Taschenbuch Verlag $\mathrm{GmbH}$.

Flick, U. (2008b). Triangulation. Eine Einführung. 2. Auflage. Wiesbaden: Verlag für Sozialwissenschaften.

Flick, U., von Kardoff, E. Steinke, I. (2008). Was ist qualitative Forschung. Einleitung und Überblick. In Flick, U., von Kardoff, E. \& Steinke, I. (Hrsg.). Qualitative Forschung. Ein Handbuch. Reinbek bei Hamburg: Rowohlt Taschenbuch Verlag GmbH.

Friedrichs, J. (1990). Methoden empirischer Sozialforschung. 14. Auflage. Opladen: Westdeutscher Verlag GmbH.

Furubo, J.E., Rist, R. C. \& Sandahl, R. (Ed.) (2002). International Atlas of Evaluation. New Brunswick and London: Transaction Publishers.

Galuske, M. (2009). Methoden der Sozialen Arbeit. Eine Einführung. 8. Auflage. Weinheim und München: Juventa Verlag.

Giesecke, H. (1994). Einführung in die Pädagogik. Weinheim und München: Juventa.

Giesecke, H. (1996). Pädagogik als Beruf. Grundformen pädagogischen Handelns. Weinheim und München: Juventa.

Gstetter, P. (1995). Handlungsforschung. In: Flick, U., Von Kardorff, E., Keupp, H., Von Rosenstiel, L. \& Wolff, S. (Hrsg.). Handbuch Qualitative Sozialforschung. Grundlagen, Konzepte, Methoden und Anwendungen. 2. Auflage. Weinheim: Beltz Psychologie Verlags Union.

Greenwood, D. J. \& Levin, M. (2007). Introduction to Action Research: Socisl Research for Social Change. 2. Ed. Thousand Oaks: Sage Publications.

Guba, E. \& Lincoln, Y. (1981). Effective Evaluation. Improving the Usefulness of Evaluation Results Through Responsive and Naturalistic Approaches. San Francisco: Jossey-Bass.

Guba, E. \& Lincoln, Y. (1989). Fourth Generation Evaluation. Newbury Park u.a.: Sage. 
Haag, F. Krüger, H., Schwärzel, W., Wildt, J. (Hrsg.) (1972). Aktionsforschung. Forschungsstrategien, Forschungsfelder und Forschungspläne. München: Juventa.

Haller, H.D. (1995). Wissensorganisation mit CEWID, einem wissensorientierten und tätigkeitsunterstützenden System. In: N. Meder/P.Jaenecke/W. Schmitz-Esser (Hrsg.): Konstruktion und Retrieval von Wissen. Frankfurt/M., INDEKS Verlag, S. 14-21.

Haller, H.D. (1997). Alternative Instructional Models and Knowledge-Organization and Design-Support With CEDID. In: Tennyson, R., Schott, F., Seel, N.M., Dijkstra, S. (eds.). Instructional Design: International Perspectives, Vol.1: Theory, Research, and Models. Mahwah, New Jersey/London. Lawrence Erlbaum, Associates, p. 371-379.

Haller, H.D. (2002). E-Learning und didaktische Vielfalt. In: Riekhof, H.C. \& Schüle, H. (Hrsg.). E-Learning in der Praxis. Strategien, Konzepte, Fallstudien. Wiesbaden: Gabler, S. 229-252.

Haller, H.D. (2003). Sieben Merkmale wirksamen Lehrens. Verfügbar unter: wwwuser.gwdg.de/ hhaller/WebdidaktikSiebenSäulen.doc

Haller, H.D. (2005). Evaluation von Bildungsprojekten: Grundlegende Konzeptüberlegungen und Begründung für einen Ansatz kontinuierlicher Sammlungen von Inventaren. Im Internet verfügbar unter: http://www.blinc-eu.org/uploads/media/Eval_bg_HDH_0605_de.pdf

Haller, H.-D., List-Ivankovic, J. \& Scholze, T. (2007). Blended Learning in the European 3rd Sector. Evaluation report developed in the framework of the project eL3: eLearning Project Cluster for Third System Organisations in Europe funded by the European Commission via the Initiative eLearning. Im Internet verfügbar unter: http://www.blinc-eu.org/1203/ (Zugriff am 18.9.2012).

Hamel, J. (1993). Case Study Methods. London: Sage.

Harvey, L. \& Green, D. (2000). Qualität definieren. Fünf unterschiedliche Ansätze. In: Helmke, H., Hornstein, W. \& Terhart, E. (Hrsg.) (2000). Qualität und Qualitätssicherung im Bildungsbereich: Schule, Sozialpädagogik, Hochschule. Zeitschrift für Pädagogik. 41. Beiheft. Weinheim und Basel: Beltz.

Heiner, M. (1988). Von der forschungsorientierten zur praxisorientierten Selbstevaluation. Entwurf eines Konzeptes. In: Heiner, M. (Hrsg.). Selbstevaluation in der sozialen Arbeit. Fallbeispiele zur Dokumentation und Reflexion beruflichen Handelns. S. 7-40. Freiburg: Lambertus.

Heiner, M. (1994). Selbstevaluation als Qualifizierung in der Sozialen Arbeit. Freiburg: Lambertus.

Heinze, T., Müller, E., Stickelmann, B., Zinnecker, J. (1975). Handlungsforschung im pädagogischen Feld. München: Juventa.

Heinze, T. (2001). Qualitative Sozialforschung. Einführung, Methodologie und Forschungspraxis. München, Wien: R. Oldenbourg Verlag.

Hellstern, G.-M. \& Wollmann, H. (1984). Evaluierung und Evaluierungsforschung - ein Entwicklungsbericht. In: Hellstern, G.-M. \& Wollmann, H. (Hrsg.). Handbuch zur Evaluierungsforschung Band 1. Opladen: Westdeutscher Verlag.

Helmke, H., Hornstein, W. \& Terhart, E. (Hrsg.) (2000a). Qualität und Qualitätssicherung im Bildungsbereich. Zur Einleitung in das Beiheft. In: Helmke, H., Hornstein, W. \& Terhart, E. 
(Hrsg.) (2000). Qualität und Qualitätssicherung im Bildungsbereich: Schule, Sozialpädagogik, Hochschule. Zeitschrift für Pädagogik. 41. Beiheft. Weinheim und Basel: Beltz.

Helmke, H., Hornstein, W. \& Terhart, E. (Hrsg.) (2000b). Qualität und Qualitätssicherung im Bildungsbereich: Schule, Sozialpädagogik, Hochschule. Zeitschrift für Pädagogik. 41. Beiheft. Weinheim und Basel: Beltz.

Juna, J. (Hrsg.) (2002). Selber forschen - selber verändern: Lehrerinnen und Lehrer verändern ihren Unterricht durch Aktionsforschung. Innsbruck: Studien-Verlag.

Joint Committee on Standards for Educational Evaluation (1981). Standards for Evaluations of Educational Programs, Projects and Materials. New York: McGraw-Hill.

Joint Committee on Standards for Educational Evaluation (1994). The Program Evaluation Standards. How to assess Evaluations of Educational Programs. Thousand Oaks, CA: Sage.

Joint Commitee on Standards for Educational Evaluation (2006). Sanders, J. R. (Hrsg.) Handbuch der Evaluationsstandards. Die Standards des „Joint Commitee on Standards for Educational Evaluation". 3. Auflage. Opladen: Leske \& Budrich.

Kempfert, G. \& Rolff, H.-G. (2005). Qualität und Evaluation. Ein Leitfaden für Pädagogisches Qualitätsmanagement. 4., überarbeitete und erweiterte Auflage. Weinheim und Basel: Beltz Verlag.

Klein, H.-J. (Hrsg.) (1991). Evaluation als Instrument der Ausstellungsplanung. Karlsruher Schriften zur Besucherforschung, Heft1. Karlsruhe: Rothirsch.

Kirchhoff, S., Kuhnt, S., Lipp, P. \& Schlawin, S. (2006). Der Fragebogen. Datenbasis, Konstruktion und Auswertung. 3., überarbeitete Auflage. Wiesbaden: VS Verlag für Sozialwissenschaften.

Klafki, W. (1973). Handlungsforschung im Schulfeld. In: Zeitschrift für Pädagogik 19, S. 487515.

Klein, H.-J. (Hrsg.) (1991). Evaluation als Instrument der Ausstellungsplanung. Karlsruher Schriften zur Besucherforschung, Heft 1. Karlsruhe: Rohrhirsch.

Klieme, E. \& Tippelt, R. (Hrsg.) (2008). Qualitätssicherung im Bildungswesen. Eine aktuelle Zwischenbilanz. Zeitschrift für Pädagogik. 53. Beiheft. Weinheim und Basel: Beltz.

Klieme, E., Artelt, C., Hartig, J., Jude, N., Köller, O., Prenzel, M., Schneider, W. \& Stanat, P. (Hrsg.) (2010). PISA 2009: Bilanz nach einem Jahrzehnt. Münster u.a.: Waxmann.

König, J. (2007). Einführung in die Selbstevaluation. Ein Leitfaden zur Bewertung der Praxis Sozialer Arbeit. 2., neu überarbeitete Auflage. Freiburg: Lambertus.

Krathwohl, D. R., Bloom, B. S. \& Masia, B. B. (1964). Taxonomiy of Educational Objectives. The Classification of Educational Goals. Handbook II: Affective Domain. New York: David McKay.

Kromrey, Helmut. (2001). Evaluation - ein vielschichtiges Konzept. Begriff und Methodik von Evaluierung und Evaluationsforschung. Empfehlungen für die Praxis. In: Sozialwissenschaften und Berufspraxis, Bd.24 (2), S. 105-131.

Krüger, H.-H. (1999). Einführung in Theorien und Methoden der Erziehungswissenschaft. 2. durchgesehene Auflage. Opladen. Leske und Budrich. 
Krüger, H.-H. \& Rauschenbach, T. (Hrsg.) (1997). Einführung in die Arbeitsfelder der Erziehungswissenschaft. 2. durchgesehene Auflage. Opladen: Leske und Budrich.

Krüger, H.-H. \& Helsper, W. (Hrsg.) (1998). Einführung in Grundbegriffe und Grundfragen der Erziehungswissenschaft. 3. durchgesehene Auflage. Opladen: Leske und Budrich.

Kümmel, F. (1978). Zur Bestimmung der Formel: Pädagogik als „Theorie einer Praxis“. In: Blankertz, H. (Hrsg.). Die Theorie-Praxis-Diskussion in der Erziehungswissenschaft. Zeitschrift für Pädagogik. 15. Beiheft. Weinheim und Basel: Beltz.

Kuper, H. (2005). Evaluation im Bildungssystem. Eine Einführung. Grundriss der Pädagogik/Erziehungswissenschaft. Band 28. Herausgegeben von Helsper, W., Kade, J., Lüders, C. \& Radtke, F.-O..Stuttgart: Kohlhammer.

Leeuw, F. L., Toulemonde, J. \& Brouwers, A. (1999). Evaluation Activities in Europe : A Quick Scan of the Market in 1998. Evaluation No.5/1999. S. 487-496.

Leeuw, F. L. (2006). Evaluation in Europe. In: Stockmann, R. (Hrsg.). Evaluationsforschung. Grundlagen und ausgewählte Forschungsfelder. 3. Auflage. Münster u.a.: Waxmann. S. 6484.

Lenzen, D. (Hrsg.) (1994). Erziehungswissenschaft. Ein Grundkurs. Reinbek bei Hamburg: Rowohlt.

Leutner, D. (1999). Evaluation aus Sicht der kritisch-empirischen Forschung. In: Thonhauser, J. \& Patry, J.-L. (Hrsg.). Evaluation im Bildungsbereich. Wissenschaft und Praxis im Dialog. Studien zur Bildungsforschung \& Bildungspolitik, Band 22. Innsbruck-Wien: Studienverlag.

Lewin, K. (1946). Action Research and Minority Problems. Journal of Social Issues, 1946, 2, S. 34-46.

Lewin, K. (1953). Tat-Forschung und Minderheitenprobleme. In: K. Lewin, Die Lösung sozialer Konflikte. Ausgewählte Abhandlungen über Gruppendynamik. Bad Neuheim: ChristianVerlag. S. 278-298 (Amerikanisches Original: Resolving Social Conflicts. Selected Papers on Group Dynamics, New York: Haper, 1948).

Lewis, R., Whitlock, Q. (2003). How to Plan and Manage an E-Learning Programme. Gower Publishing Limited.

Lienert, G. A. \& Raatz, U. (1998). Testaufbau und Testanalyse. 6. Auflage. Weinheim: Psychologie Verlags Union.

Lincoln, Y. S. \& Guba, E. G. (1985). Naturalistic Inquiry. Beverly Hills, CA: Sage.

Livingstone, D. W. (1999). Informelles Lernen in der Wissensgesellschaft. In: Arbeitsgemeinschaft Qualifikations-Entwicklungs-Management (Hrsg.): Kompetenz für Europa - Wandel durch Lernen - Lernen im Wandel. Referate auf dem internationalen Fachkongress Berlin 1999, QUEM-Report 60. S.65-92.

Livingstone, D. W. (2006). Informal Learning: Conceptual Distinctions and Preliminary Findings. In: Bekerman, Z., Burbules, N., Nicholas, C., Silberman-Keller, D. (Hrsg.). Learning in Places. The Informal Education Reader. New York: Peter Lang Publishing. S.202-226.

Lück, H. E. (2001). Kurt Lewin. Eine Einführung in sein Werk. Weinheim und Basel: BeltzVerlag. 
Madaus, G.F. \& Stufflebeam, D. L. (2000). Programm Evaluation: A Historical Overview. In: Stufflebeam, D. L., Madaus, G. F. \& Kellaghan, T. (Hrsg.). Evaluation Models. Viewpoints on Educational and Human Services Evaluation. Second Edition. Boston, Dordrecht, London: Kluwer Academic Publishers. S. 3-18.

Marrow, A. J. (2002). Kurt Lewin. Leben und Werk. Weinheim und Basel: Beltz-Verlag. (Erstausgabe in deutsch 1977, Original 1969: The Practical Theorist. The Life and Work of Kurt Lewin).

Mayring, P. (2000). Qualitative Inhaltsanalyse. Grundlagen und Techniken (siebte Auflage, erste Auflage 1983). Weinheim: Deutscher Studien Verlag.

Meder, N. (Hrsg) (2006). Web-Didaktik. Eine neue Didaktik webbasierten, vernetzten Lernens. Reihe: Wissen und Bildung im Internet. Band 2. Bielefeld: Bertelsmann Verlag GmbH \& Co. KG.

Mertens, D. (1998). Research methods in education and psychology: Integrating diversity with quantitative and qualitative approaches. Thousand Oaks, CA: Sage.

Mertens, D. (2006). Institutionalizing Evaluation in the United States of Amerika. In: Stockmann, R. (Hrsg.) (2006). Evaluationsforschung. Grundlagen und ausgewählte Forschungsfelder. 3. Auflage. Münster u.a.:Waxmann. S. 47-63.

Miles, M. B. \& Huberman, A. M. (1994). Qualitative Data Analysis: A Sourcebook of New Methods. 2. Edition: Newbury Park. Sage.

Moser, H. (1975). Aktionsforschung als kritische Theorie der Sozialwissenschaften. München: Kösel.

Moser, H. (1999). Selbstevaluation. Einführung für Schulen und andere soziale Institutionen. Zürich: Pestalozzianum.

Mullis, I. V. S., Martin, M. O., Gonzales, E. J. \& Kennedy, A. M. (2003). PIRLS 2001. International Report. IEA`s Study of Reading Literacy in Primary School in 35 Countries. Chestnut Hill, MA: Boston College.

Mummendey, H. D. (1987). Die Fragebogen-Methode. Göttingen, Toronto, Zürich: Hogrefe Verlag.

Niegemann, H. M., Hessel, S., Hochscheid-Mauel, D., Aslanski, K., Deimann, M. \& Kreuzberger, G. (2004). Kompendium E-Learning. Berlin, Heidelberg, New York: Springer.

OECD (Hrsg.) (2011a). Bildung auf einen Blick: OECD-Indikatoren. Bielefeld: Bertelsmann.

OECD (Hrsg.) (2011b). PISA 2009. Vol.1-5. Bielefeld: Bertelsmann.

Overwien, B. (2005). Stichwort: Informelles Lernen. In: Zeitschrift für Erziehungswissenschaft, Heft 3, S.339-355.

Overwien, B. (2006). Informelles Lernen in der internationalen Diskussion. In: Rauschenbach, T., Düx, W. \& Sass, E. (Hrsg.). Informelles Lernen im Jugendalter eine vernachlässigte Dimension in der Binlungsdebatte. Weinheim, S.35-62.

Overwien, B. (2009). Informelles Lernen. Definitionen und Forschungsansätze. In: Brodowski, M., Devers-Kanoglu, U., Overwien, B., Rohs, M., Salinger, S. \& Walser, M. (Hrsg.). Informelles Lernen und Bildung für eine nachhaltige Entwicklung. Beiträge aus Theorie und Praxis. Opladen: Barbara Budrich.

Patton, M. Q. (1978). Utilization-Focused Evaluation. Beverly Hills, CA: Sage. 
Patton, M. Q. (1986). Utilization-Focused Evaluation (2nd edition). Beverly Hills, CA: Sage.

Patton, M. Q. (1997). Utilization-Focused Evaluation: The New Century Text (3rd edition). Thousand Oaks, CA: Sage.

Patton, M. Q. (2008). Utilization-Focused Evaluation. 4th edition. Thousand Oaks, CA: Sage.

Patton, M. Q. (2010). Developmental Evaluation: Applying Complexity Concepts to Enhance Innovation and Use. Guilford Press

Pieper, H.-R. (1977). Theorie, Praxis, Technik und Altag: Wissenstheoretische Grundlage der Aktionsforschung. Hamburg. Dissertation.

Pollitt, C. (1998). Evaluation in Europe: Boom or Bubble? Evaluation No.4 (2). S. 214-224.

Porst, R. (2000). Question Wording - Zur Formulierung von Fragebogen-Fragen. ZUMA How-to-Reihe, Nr.2. Im Internet verfügbar unter: http://www.gesis.org/fileadmin/upload/forschung/publikationen/gesis_reihen/howto/howto2rp.pdf (Zugriff am 29.2.2012)

Porst, R. (2008). Fragebogen. Ein Arbeitsbuch. Wiesbaden: VS Verlag für Sozialwissenschaften.

Preskill, H. \& Torres, R. T. (Hrsg.) (2001). The Learning dimension of Evaluation Use. In: New Directions for Evaluation (Vol. 88). San Francisco: Jossey-Bass.

Provus, M. (1971). Discrepancy Evaluation. Berkeley, CA: McCutcheon.

Ragin, C. C. \& Becker, H. S. (Hrsg.) (1992). What is a case? Exploring the foundation of social inquiry. Cambridge: University Press.

Rappaport, J. (1987). Terms of Empowerment / Exemplars of Prevention: Toward a Theory for Community Psychology. In: American Journal of Community Psychology, 15 (2), S. 121148.

Rist, R. C. (Ed.) (1990). Program Evaluation and the Management of Government. Patterns and Prospects across Eight Nations. New Brunswick and London: Transaction Publishers.

Rohrmann, B. (1978). Empirische Studien zur Entwicklung von Antwortskalen für die Sozialwissenschaftliche Forschung. In: Zeitschrift für Sozialpsychologie, 9, S. 222-245.

Rossi, P. H., Freeman H. E. \& Wright, S. R. (1979). Evaluation. A Systematic Approach. Beverly Hills, CA: Sage.

Rossi, P. H., Freeman H. E., Hofmann, G. (1988). Programm-Evaluation. Einführung in die Methoden angewandter Sozialforschung. Stuttgart: Enke.

Rossi, P. H. \& Freeman H. E. (1993). Evaluation. A Systematic Approach. 5. Auflage. Newbury Park: Sage

Rossi, P. H., Lipsey, M. \& Freeman H. E. (2004). Evaluation. A Systematic Approach. 7. Auflage. Thousand Oaks u.a.: Sage.

Rost, J. (2004). Lehrbuch Testtheorie - Testkonstruktion. Zweite, vollständig überarbeitete und erweiterte Auflage. Bern, Göttingen, Toronto, Seattle: Verlag Hans Huber.

Scholze, T. (2009). Evidencing the Impact of Informal Learning on Active Citizenship in European Projects. Göttingen: Cuvillier Verlag. Zugl.:Univ. Diss., 2009. 
Schratz, M., Jakobson, L. B., MacBeath, J. \& Meuret, D. (2002). Serena, oder: Wie Menschen ihre Schule verändern: Schulentwicklung und Selbstevaluation in Europa. Innsbruck u.a.: Studienverlag

Schulmeister, R. (2003). Lernplattformen für das virtuelle Lernen. München, Wien: Oldenbourg.

Schüpbach, E., Guggenbühl, U., Krehl, C., Siegenthaler, H. \& Kaufmann-Hayoz, R. (2003). Didaktischer Leitfaden für E-Learning. Bern: h.e.p.-Verlag.

Scriven, M. (1967). The Methodology of Evaluation. In: Tyler, R. W., Gagne, R. M. \& Scriven, M. (Ed.). Perspectives on Curriculum Evaluation. AERA Monograph Series on Curriculum Evaluation, Volume 1. S. 39-83.

Scriven, M. (1972). Die Methodologie der Evaluation. In: Wulf, C. (Hrsg.). Evaluation. Beschreibung und Bewertung von Unterricht, Curricula und Schulversuchen. München: Piper. S. 60-91.

Scriven, M. (1973). Goal-free Evaluation. In: House, E. (Ed.). School Evaluation. The Politics and Process. Berkeley, CA: McCutchan.

Scriven, M. (1974). Evaluation Perspectives and Procedures. In: Popham, J. W. (Hrsg.). Evaluation in Education: Current Application. Berkeley, CA: McCutcheon. S. 3-93.

Scriven, M. (1991). Evaluation Thesaurus. Newbury Park u.a.: Sage.

Scriven, M. (1991b). Key Evaluation Checklist. In: Scriven, M.:Evaluation Thesaurus. Newbury Park u.a.: Sage

Scriven, M. (2002). Evaluation Thesaurus. 4th Edition. Newbury Park u.a.: Sage.

Scriven, M. (2007a). Key Evaluation Checklist. Online erhältlich unter: http://www.wmich.edu/evalctr/archive_checklists/kec_feb07.pdf

Scriven, M. (2007b). The Logic and methodology of Checklists. Online erhältlich unter: http://www.wmich.edu/evalctr/archive_checklists/papers/logic\&methodology_dec07.pdf

Shadish, W., Cook, T. \& Leviton, L. (1991). Foundations of Program Evaluation: Theories of Practice. Newburg Park, Ca: Sage.

Sensi, D. \& Cracknell, B. (1991). Inquiri into Evaluation Practices in the Commission. In: MONITOR Collection. Luxembourg: Office for Official Publications of the EC.

Skowronek, H., Schmied, D. (1977). Forschungstypen und Forschungsstrategien in der Erziehungswissenschaft). Hamburg: Hoffmann und Campe.

Spranger, E. (1962). Das Gesetz der ungewollten Nebenwirkungen in der Erziehung. Heidelberg: Quelle und Meyer.

Stake, R. E. (1967). The Countenance of Educational Evaluation. Teachers College Record, 68 , S. $523-540$.

Stake, R. E. (1969). Evaluation Design, Instrumentation, Data Collection and Analysis of Data. In: davis, J. L. (Hrsg.). Educational Evaluation. Columbus, $\mathrm{OH}$ : State Superintendent of Public Instruction.

Stake, R. E. (1972). Responsive Evaluation. Unpublished manuscript. 
Stake, R. E. (1975a). Program Evaluation, particulary Responsive Evaluation. (Occasional Paper No.5) Kalamazoo: Western Michigan University Evaluation Center.

Stake, R. E. (1975b). Evaluating the Arts in Education. A Responsive Approach. Columbus, $\mathrm{OH}$ : Merill.

Stake, R. E. (1978). The Case Study Method in Social Inquiry. In: Educational Researcher, 7, S. 5-8.

Stake, R. E. (1980). Program Evaluation, particulary Responsive Evaluation. In: Dockrell, W. B. \& Hamilton, D. (Hrsg.). Rethinking Educational Research. London: Hodeder \& Stoughton.

Stake, R. E. (1981). Case Study Methodology: An Epistemological Advocacy. In: Welch, W. (Hrsg.). Case Study Methodology in Educational Evaluation. Minneapolis: Minnesota Research and Evaluation Center.

Stake, R. E. (1988). Case Study Methods in Educational Research: Seeking Sweet Water. In: Jaeger, R. M. (Hrsg.). Complementary Methods for Research in Education. Washington, DC: American Educational Research Association.

Stake, R. E. (1994). Case Studies. In: Denzin, N. K. \& Lincoln, Y. S. (Hrsg.). Handbook of Qualitative Research. Thousand Oaks, CA: Sage.

Stake, R. E. (1995). The Art of Case Study Research. Thousand Oaks, CA: Sage.

Stake, R. E. (2000). A Modest Commitment to the Promotion of Democracy. In: Ryan, K. E. \& DeStefano, L. (Hrsg.). Evaluation as a Democratic Process. Promoting Inclusion, Dialogue, and Deliberation. New Directions for Evaluation, 85, S. 97-106. San Francisco: Jossey-Bass.

Stockmann, R. (2006a). Evaluation in Deutschland. In: Stockmann, R. (Hrsg.). Evaluationsforschung. Grundlagen und ausgewählte Forschungsfelder. 3. Auflage. Münster u.a.: Waxmann. S. 15-46.

Stockmann, R. (Hrsg.). (2006b). Evaluationsforschung. Grundlagen und ausgewählte Forschungsfelder. Sozialwissenschaftliche Evaluationsforschung Band 1. 3. Auflage. Münster u.a.:Waxmann.

Stockmann, R. (2006c). Evaluation und Qualitätsmanagement. Eine Grundlage für wirkungsorientiertes Qualitätsmanagement. Sozialwissenschaftliche Evaluationsforschung Band 5. Münster u.a.:Waxmann.

Stockmann, R. (2006d). Qualitätsmanagement und Evaluation im Vergleich. In: Böttcher, W., Holtappels, H.G. \& Brohm, M. (Hrsg): Evaluation im Bildungswesen. Eine Einführung in Grundlagen und Praxisbeispiele. S.23-38. Weinheim und München: Juventa

Stockmann, Reinhard (Hrsg.). (2007). Handbuch zur Evaluation. Eine praktische Handlungsanleitung. Münster u.a.: Waxmann.

Stockmann, R. \& Meyer, W. (2010). Evaluation. Eine Einführung. Opladen und Bloomfield Hills: Verlag Barbara Budrich.

Stufflebeam, D. L. et. al. (1971a). Educational Evaluation and Decision Making. Phi Delta Kappa National Study Committee on Evaluation. Itasca.

Stufflebeam, D. L. (1971). The Relevance of the CIPP Evaluation Modell for the Educational Accountability. In: Journal of Research and Development in Education, Band 5, S.19-25. 
Stufflebeam, D. L. (1972). Evaluation als Entscheidungshilfe. In: Wulf, C. (Hrsg.). Evaluation. Beschreibung und Bewertung von Unterricht, Curricula und Schulversuchen. München: Piper. S. 113-145.

Stufflebeam, D. L. (1983). The CIPP Model for Program Evaluation. In: Madaus, G. F., Scriven, M. \& Stufflebeam, D. L. (Hrsg.). Evaluation models. Viewpoints on Educational and Human Services Evaluation. Boston: Kluwer: Nijhoff, S. 117-141.

Stufflebeam, D. L., Madaus, G. F. \& Kellaghan, T. (2000) (Hrsg.). Evaluation Models. Viewpoints on Educational and Human Services Evaluation. Second Edition. Boston u.a.: Kluwer.

Suchman, E. A. (1967). Evaluative Research. New York: Russell Sage Foundation.

Summa, H. \& Toulemonde, J. (2002). Evaluation in the European Union: Addressing Complexity and Ambiguity. In: Furubo, J.E., Rist, R. C. \& Sandahl, R. (Ed.). International Atlas of Evaluation. New Brunswick and London: Transaction Publishers.

Swertz, C. (2004). Didaktisches Design. Ein Leitfaden für den Aufbau hypermedialer Lernsysteme mit der Webdidaktik. Reihe Wissen und Bildung im Internet, Band 4. Bielefeld: Bertelsmann.

Thonhauser, J. \& Patry, J.-L. (Hrsg.) (1999). Evaluation im Bildungsbereich. Wissenschaft und Praxis im Dialog. Innsbruck, Wien: Studien Verlag.

Tyler, R. W. (1934). Constructing Achievement Tests. Columbus, OH: Ohio State University Bureau of Educational Research.

Tyler, R. W. (1949). Basic Principles of Curriculum and Instruction. Chicago: University of Chicago Press.

Tyler, R. W. (2000). A Rationale for Program Evaluation. In: Stufflebeam, D. L., Madaus, G. F. \& Kellaghan, T. (Hrsg.). Evaluation Models. Viewpoints on Educational and Human Services Evaluation. Second Edition. Boston, Dordrecht, London: Kluwer Academic Publishers. S. 87-96.

Von Saldern, M. (Hrsg.) (2010). Selbstevaluation von Schule: Hintergrund - Durchführung Kritik. Schule in Deutschland 2. Norderstedt: Books on Demand.

Von Spiegel, H. (1993). Aus Erfahrung Lernen. Qualifizierung durch Selbstevaluation. Münster: Votum Verlag.

Webb, E. J., Campbell, D. T., Schwartz, R. D. \& Sechrest, L. (1966). Unobtrusive Measures: Nonreactive Research in the Social Sciences. Chicago: Rand McNally.

Webb, E. J., Campbell, D. T., Schwartz, R. D. \& Sechrest, L. (1975). Nichtreaktive Meßverfahren. Weinheim und Basel: Beltz Verlag.

Weiss, C. H. (1972). Evaluation Research. Methods of Assessing Program Effectiveness. Prentice Hall, Inc., Englewood Cliffs, N.J.

Weiss, C. H. (1973). Where politics and Evaluation Research meet. In: Evaluation, 1, S. 3745.

Weiss, C. H. (1974). Evaluierungsforschung. Opladen: Westdeutscher Verlag.

Weiss, C. H. (1998). Evaluation. New Jersey: Prentice Hall. 
Wellenreuther, M. (1982). Grundkurs: Empirische Forschungsmethoden für Pädagogen, Psychologen, Soziologen. Königstein/Ts.: Athenäum.

Wellhöfer, P. R. (1997). Grundstudium Sozialwissenschaftliche Methoden und Arbeitsweisen. Eine Einführung für Sozialwissenschaftler und Sozialarbeiter/-pädagogen. 2. überarbeitete und erweiterte Auflage. Stuttgart: Enke.

Will, H. und Blickhan, C. (1987). Evaluation als Intervention. In: Will, H., Winteler, A. und Krapp, A. (Hrsg.). Evaluation in der beruflichen Aus- und Weiterbildung. Konzepte und Strategien (S.43-59). Heidelberg: Sauer.

Wottawa, Heinrich \& Thierau, Heike (2003). Lehrbuch Evaluation. 3., korrigierte Auflage. Bern: Huber.

Wulf, C. (Hrsg.) (1972). Evaluation. Beschreibung und Bewertung von Unterricht, Curricula und Schulversuchen. München: Piper.

Yin, R.K. (2009). Case Study Research Design and Methods. 4. Edition.Thousand Oaks, California: Sage. (zuerst 1989).

Zürcher, R. (2007). Informelles Lernen und der Erwerb von Kompetenzen: Theoretische, didaktische und politische Aspekte. Materialien zur Erwachsenenbildung, Nr.2. Bundesministerium für Unterricht, Kunst und Kultur. Wien. Im Internet: http://www.erwachsenenbildung.at/services/publikationen/materialien_zur_eb/nr2_2007_infor melles_lernen.pdf (Zugriff am 30.1.2012) 


\section{Anhang}

Inhaltsverzeichnis

1. Fragebogen zur Bestandsaufnahme 292

2. Partnerprofile 304

3. Fragebogen Prozessevaluation 306

4. Information zu den blinc-Inventaren und blinc-Fragebögen 307

5. Information zur Anwendung der Inventare für Lerner 308

6. Inventare für Lerner 311

6.1 Inventar für Lerner zu Beginn eines Kurses 314

6.2 Inventar für Lerner während eines Kurses 339

6.3 Inventar für Lernen zum Abschluss eines Kurses 365

7. Inventar für Autoren und Produzenten 391

8. Inventar für Entscheidungsträger 422

9. Fragebogen zum Abschluss des Projektes 436 


\section{Fragebogen zur Bestandsaufnahme}

\section{Questionnaire for the Project-Synopsis of the Partners of eL3 and for the preparation of the Evaluation of the eL3-Network Version October2004}

\section{Indications for the purpose and the composition of this questionnaire:}

In the framework of the evaluation that will accompany eL3 the following questionnaire prepares a first description of the single projects. This description should serve as the basis for a synopsis of the participating projects.

For present purposes the term "project" means those measures that have been introduced to enable elearning in your institution. Then, the "eL3-network" is the means of bringing together your project and those of all network partners. By placing your project into the eL3-network we are able to disseminate and share practices.

If you think that the questions don not capture clealry what you are intending or actually doing, please, add your comments, descriptions and/or documents etc.

\section{Main Terms:}

"eLearning" means an electronically saved and conducted training educational or development programmme, this might be via internet or intranet (online), via programmes, documents etc. on computers and/or their data carriers CD, DVD etc. (offline).

"Blended learning" means a mixture of eLearning ("virtual part") and presence lessons and/or traditional courses.

\section{Evaluation of the project itself:}

As we decided during the meeting in Goslar, it is not our task nor our intention to evaluate the single projects or even their entire institution. But in order to be able to evaluate the eL3network, we firstly need partner specific information in order to develop further the eL3network.

If you think that you might will need help or tips for your own internal evaluation, we should like to try to give you directions within the bounds of our knowledge and possibilities. But you have to carry out all the rest by yourselves, e.g. the collection and evaluation of data. You are not obliged to provide us with the results of your internal (own) evaluation.

\section{Authors of this questionnaire:}

This questionnaire has been designed by Prof. Dr.Hans-Dieter Haller and Jutta List in cooperation with Dr. Bernice West

Prof. Dr. Hans-Dieter Haller

Managing Director of the Pedagogical Seminar of the Georg-August-University Göttingen

E-mail: hhaller@gwdg.de

Postal Address:

Baurat-Gerber-Str. 4/6

D-37073 Göttingen

Tel./FAX + 49 / (0) 551/39 9348

Jutta List, M.A.

Assistant at the Pedagogical Seminar of the Georg-August-University Göttingen

E-Mail: jist@gwdg.de

Dr. Bernice West

School of Nursing and Midwifery, Robert Gordon University Aberdeen

E-Mail: b.west@rgu.ac.uk 


\section{Part A: Profile of your institution and specific e Learning project}

\section{Basic Data Regarding the Project}

1.1 Name of the institution:

1.2 Address and email:

1.3 Contact person:

1.4 Who developed the project you are reporting here?

1.5 Who is the mainly responsible for the project (staff member)?

Is this Fulltime? YES NO:

If Part-time how many hours per week:

1.6 How is the project financed?

By whom?

For how long has finance been secured?

How much money has been obtained?

1.7 Is this project part of a bigger or another programme?

$\square$ Yes $\quad \square$ No

If yes, in which programme is the project included?

\section{Educational process and Operational/Institutional Management}

2.1 Which methods of education/further training/teaching are used in your institution?

2.2 For how long have you been offering eLearning in your organisation/institution? 
2.3 Is eLearning offered in addition to other educational techniques??

$\square$ Yes $\quad \square$ No

2.4 Is eLearning offered as an exclusive training opportunity?

$\square$ Yes $\quad \square$ No

2.5 Is blended learning used?

$\square$ Yes $\quad \square$ No

If yes, approximately what proportion of the programme is delivered by

Virtual techniques: $\quad \% \quad$ Classroom contact lessons or Presence les-

sons: $\quad \%$

2.5.1 How is blended learning generally delivered in your organisation/institution?

(Please, describe in short, e.g. presence phases, self-guided learning, virtual conference etc.)

2.5.2 What is the respective amount of time is allocated to each teaching or learning style?

2.6 methods of education/further trainind/teaching

Which approaches to (methods of) education/training do you mainly use within your organisation/institution?

\section{Questions Regarding Specific Project Contents}

3.1 Please, describe in short the eLearning-project, you are bringing to the eL3 programme?.

(Content/main topics):

3.2 What are the main objectives

3.3 What are the secondary objectives of the project?

3.4 What are the learning aims (competence, content, learning targets)? 


\begin{tabular}{|l||l|l|l|l|}
\hline 3.5 How important are the following different types of knowledge to your project ? \\
\hline Acting knowledge & $\square$ very important & $\square$ important & $\square$ less important & $\square$ unimportant \\
\hline Interpretation knowledge & $\square$ very important & $\square$ important & $\square$ less important & $\square$ unimportant \\
\hline Orientation knowledge & $\square$ very important & $\square$ important & $\square$ less important & $\square$ unimportant \\
\hline Source knowledge & $\square$ very important & $\square$ important & $\square$ less important & $\square$ unimportant \\
\hline
\end{tabular}

Acting knowledge:

Knowledge which refers to the real acting of people (practices, techniques, methods and strategies) ("Know-how" = "known how"). In this context the term "competence" or "skills" are used. Acting knowledge can refer to traditional acting, which is passed on by cultural tradition and which is ("naturally") used without questioning. But it can also refer to rational and intentional acting, where practices are based on rational understanding (e.g. scientific insights).

Acting knowledge includes

Knowledge about reasonable purposes and objectives,

Knowledge about the conditions which make a certain acting sensible,

Knowledge about procedures und operations,

Knowledge about means and tools,

Knowledge about control procedures and quality criteria,

Knowledge about possible dangers.

\section{Interpretation knowledge:}

Knowledge that bears answers to the question why something is as it is ("know why"). "Interpretation and explanation knowledge" is understood as theoretical (scientific-theoretical or "everydaytheoretical") knowledge. Interpretation and explanation knowledge is knowledge about how to interpret and explain oneself or others one's own or external experience. Therefore, explanation knowledge always presupposes experience, not necessarily external experience, it could also be " internal" experience. Interpretation knowledge results from reflection, the " thinking about" perceptions, acts and events.

Interpretation and explanation knowledge is generally available in kind of integral interpretation patterns ("world pictures", "philosophies", "ideas of man" etc.)

As well as in kind of elemental interpretation patterns (terms, models, theories, typologies, classifications etc.).

\section{Orientation knowledge:}

Knowledge that is gained in order to find one's way in the world and/or in a specific area, without getting active in a specific way. Most of school knowledge is orientation knowledge, but also knowledge which is passed on via the mass media. Someone who does have orientation knowledge knows more or less that a certain issue does exist, but he doesn't necessarily know what to do about it. He often also has a first attitude towards this issue, knows if it is something good or bad, without being able to give reasons for this. In the best cases he can classify this matter into a "mental drawer". In short: He knows words, names, events, dates, terms and links them to each other and with emotional attitudes. Orientation knowledge can be subdivided in

Single facts (names, events, findings, attributes, dates),

Terms (denotations for classified amounts of single facts),

Schemes (systems and organizing principle) as well as

Attitudes (moral concepts and judgements).

\section{Source knowledge:}

Knowledge about sources of information. "Known where" often is as important as "known what" or "known how". The acquirement of source knowledge includes the getting to know of

Persons (contact persons, experts, concerned),

Institutions (firms, associations, organisations, schools etc.),

Documentations (archives, libraries),

Conferences and meetings,

Networks (Exchange of experience-circles),

Handbooks, dictionaries and reference books,

Textbooks and special literature,

AV-media (films, videos, tapes),

Software, data bases and expert systems,

Running research projects,

Journals and sequels and

"Grey material" (e.g. business reports and manuscripts). 


\section{Questions Regarding the Background to the Project Development}

4.1 Why has the project been developed?

$\square$ Dissatisfaction with practice so far

$\square$ Expected cost saving

$\square$ To offer innovative ways of learning

$\square$ Other reasons ( Please specify)

4.2 What are the expected effects?

$\square$ Better qualification of the staff/learners

$\square$ Cost savings

$\square$ Time savings

$\square$ Other effects/other use (Please specify)

\section{Questions Regarding the Target Group}

5.1 For whom has the project been developed, or who are the target users of the project?

5.2 What is the estimated size of the target group?

5.3 Please provide details of the potential users 5.3.1 Age/age group or range:

5.3.2 Qualifications:

5.3.3 Professional experience:

5.3.4 Learning requirements:

5.3.5 Motives of the learner:

5.4 How many persons are actually enrolled on the project at the moment? 
5.5 How do the learners get to know about the project (Please, tick all the relevant answers)

$\square$ Advertisement

If yes, what kind?(e.g. flyer, internet, ads etc)

$\square$ In the course of lesson/further training

$\square$ Verbal hints $\quad \square$ Written hints/documents regarding the project

$\square$ By superiors/charges ")

If yes, by whom?

$\square$ Others:

5.6 Do the users have to pay anything for the utilisation?

$\square$ Yes

$\square$ No

If yes, how much?

5.7 Is the offer accessible to external users?

$\square$ Yes $\quad \square$ No

If yes, in what way?

5.8 Is it planned to offer the project for other users?

$\square$ Yes $\quad \square$ No

If yes, in what way? (e.g. sale/free access for other users)

\section{Questions Regarding the Learning Place and Learning Environment}

6.1 How do learners gain access to the project? (Please, tick all the relevant answers)

$\square$ In their institution (at their working place)

$\square$ In their institution (in a special room, PC-studio or other)

$\square$ In your institution

$\square$ At their own PC at home

$\square$ Other possibilities:(please specify)

6.2 To which of the learning environments do learners have access? (Please, tick all the relevant answers)

$\square$ A room especially equipped within the organisation/institution

$\square$ Private rooms (e.g. at home)

$\square$ Others: (please specify) 
6.3 What do users need to get access to the project and/or what do they have to do?

(Please, tick all the relevant answers)

$\square$ PC with internet access

$\square$ Login/registration of personal data

$\square$ Technical requirements

If yes, which (for example a special programme on the PC; please specify)

$\square$ Costs for the use (for example payment for the project)

$\square$ Others (please specify)

6.4 Which competences are required to be able to gain access to the project? (Please, tick all the relevant answers)

$\square$ Common knowledge about the handling of a PC

If yes, which?

$\square$ Knowledge of a specific programme

If yes, which?

$\square$ Other competences

If yes, which?

6.5 At what times do learners usually gain access to the project? (Please, tick all the relevant answers)

$\square$ Normal working hours

$\square$ Standby time

$\square$ Leisure time

$\square$ Others:

6.6 What are the anticipated learning hours for each learner?Weekly (hours)

6.7 Are there any additional learning materials?

$\square$ Yes $\quad \square$ No

If yes, which (e.g. text books, videos, cassettes, documents, simulators etc.) 


\section{Questions Regarding the Methodology and Didactics of the programme}

\begin{tabular}{|l|l|l|l|l|}
\hline 7.1 Which of the following methods of teaching are used in the project? \\
\hline Expository teaching & $\square$ regularly & $\square$ occasionally & $\square$ rarely & $\square$ not at all \\
\hline Task-oriented teaching & $\square$ regularly & $\square$ occasionally & $\square$ rarely & $\square$ not at all \\
\hline Discovering teaching & $\square$ regularly & $\square$ occasionally & $\square$ rarely & $\square$ not at all \\
\hline
\end{tabular}

Expository teaching:

Descriptive teaching like e.g. ex-cathedra teaching, lecture, reading, programmed lessons, CBT.

Task-oriented teaching:

After having got an introduction the learners are given a task which they have to solve.

The task is clearly defined and there is a not-ambiguous solution to it.

Discovering teaching:

A problem is described to the learners and they have to elaborate solutions themselves. Material has to be independently organised and a solution strategy has to be developed (e.g. project teaching).

7.2. How do you call the teaching method(s) of your eLearning project?

7.3 What didactic approaches form the basis of the program?

7.4 Who supervises learners? (Please, tick all the relevant answers)

Authors who have developed the project.

$\square$ Tutors who have been trained to supervise the learners.

$\square$ Traditional teachers who are co-supervisors to the project.

$\square$ Others $\quad$ Please specify

7.4.1 How many of the following persons are involved in supervising learners?
Authors
Tutors
Traditional teachers
Others

7.4.2 How many hours per week are needed for the supervision of learners?
Authors: hours
Tutors: hours
Traditional teachers: hours
Others: hours 
7.4.3 If there are tutors:

Where are these tutors trained?

How are the tutors trained?

Which competences do tutors have ?

\section{Questions Regarding the time-frame of the Project}

8.1 For how long has the project been in use, or when will it be introduced?

8.2 What plans are there for further development of the project? 


\section{Questions Regarding the Past Evaluation of the Project}

9.1 What kind of evaluation or monitoring processes have been used so far in the project? (Please, tick all the relevant answers)

$\square$ Informal feedback from learners

If yes, is this usually done $\square$ Verbally $\square$ In written

Please give an summary of these results.

$\square$ Tests/self-tests/efficiency statements

If yes, which?

$\square$ Exams/efficiency statements outside of the programme

If yes, which?

$\square$ Feedback of others (e.g. superiors/charges/other institutions)

If yes, which?

$\square$ Formal questioning of learners

If yes, in what way?

$\square$ Questionnaire $\square$ Interviews $\quad \square$ Others:

Any results already available?

$\square$ Yes $\quad \square$ No

(If yes, please, give details)

9.2 Do users or learners on your project obtain certificates/ or a grade on completion of the programme?

$\square$ Yes $\quad \square$ No

If yes, which?

9.3 What kind(s) of monitoring or quality assurance techniques are used for the project? 


\section{Part B: Experience with the own eLearning-Project and "Visions"}

1. What kind of experience have you had so far with your project?

Positive:

Negative:

2. What has worked well and what has not?

Worked well

Not worked well

3. How have the learners received the project so far? (Please tick all the relevant answers)

$\square$ Very satisfied $\square$ satisfied $\square$ not satisfied $\square$ very dissatisfied

Don't know

Please give details

4. Have you noticed anything that needs to be improved?

5. What are you planning for the further development of your project?

6. What is your "vision" for your project?

7. What do you expect of the eL3-network? 


\section{How do you judge the progressof the eL3-network so far?}

\begin{tabular}{|l|l|l|l|l|}
\hline Contact with partners & $\square$ very good & $\square$ good & $\square$ less good & $\square$ bad \\
\hline Management/Project management & $\square$ very good & $\square$ good & $\square$ less good & $\square$ bad \\
\hline Transnational Meeting & $\square$ very good & $\square$ good & $\square$ less good & $\square$ bad \\
\hline
\end{tabular}

9. What have you liked so far about the EL3 project?

10. What have you dislike about the EL3 project and why?

11. What do you suggest for the future?

\section{Part C: Feedback on the questionnaire}

1. What questions have been missed?

2 Which questions have been difficult to answer?

3. What else would you like to tell us? (Suggestions/praise/criticism)

\section{THANK YOU VERY MUCH FOR HAVING COMPLETED THIS QUESTIONNAIRE!!!}

Kind regards

Prof. Dr. Hans-Dieter Haller and Jutta List 


\section{Partnerprofile}

FOTO of the Institution or the person, who is involved in eL3

\section{Background information}

Name of the contact person:

Address:

Telephone:

E-Mail:

Synopsis of the type of work/education/research (specific areas of expertise):

\section{Reported project}

\section{Aims of the project}

\section{Background and reasons for the development of the project}

Reasons:

Expected effects:

\section{Target client group}

Target group:

Size:

Age group:

Qualifications:

Professional experience:

Learning requirements:

Motives of the learner:

How the learners get to know the project:

Costs for the learners: 


\section{Learning environment}

Learning places:

Access requirements:

Competences of the learners:

Additional material:

\section{Educational support}

Methods of teaching:

Didactic reflections:

Supervising the learners:

Training of the tutors:

\section{Time frame of the project}

Start of the poject:

End of the project:

\section{Evaluation and experiences so far}

Evaluation:

Experiences:

(:)

:

(:)

Proven well has:

(:)

Not proven well has:

루

What needs improvement:

10. Future plans

tr

is

㶦 


\section{Fragebogen Prozessevaluation}

Dear partner of the eL3-network,

With the following questions we want to get a feedback from you concerning the progression of our eL3-project.. This is only an internal evaluation and we will not jugde your work or comments. Our aim is to find out if you are satisfied or if there is something that in your opinion must be changed or optimized. Your answers and the answers of your partners will lead to a collection of statements about our common work which we will present during our next transnational meeting. Thank you for taking part in this.

Jutta and Hans-Dieter

\begin{tabular}{|l|l|l|l|l|}
\hline 1. How do you judge the project progression of the eL3-network? \\
\hline Contacts with partners & $\square$ very good & $\square$ good & $\square$ less good & $\square$ bad \\
\hline Management/Project management & $\square$ very good & $\square$ good & $\square$ less good & $\square$ bad \\
\hline Transnational Meeting & $\square$ very good & $\square$ good & $\square$ less good & $\square$ bad \\
\hline
\end{tabular}

2. Considered all in all are you satisfied with the eL3 project?

$\square$ Very satisfied $\quad \square$ Satisfied $\square$ It's O.K. $\square$ Not satisfied $\square$ Not at all satisfied
Reasons:

3. What have you liked so far?

4. What did you dislike so far?

5. In your opinion what needs improvement?

6. What are possible solutions or ways for this improvement?

7. What positive effects has the eL3 network for you until now?

8. Do you have any perspectives or a fresh impetus which you can use or realize for your own projects?

$\square$ Yes $\quad \square$ No

If yes, please describe in short:

9. After the last meeting do you know what your partners are doing in their e-learning projects?

$\square$ Yes, exactly $\square$ Yes, approximately $\square$ Could be better $\square$ No, not exactly $\square$ No, not at all

10. Do you want to have any more information? $\square$ Yes $\square$ No

If yes, what kind of information would you like?

11. The working language in the eL3 network is English...how do you get along? Your point of view:

12. What do you expect or wish for the further work of the eL3-network?

13. What else would you like to tell us (critic, commend, opinions, ideas etc.)?

Thank you very much for your feedback! 


\section{Information zu den blinc-Inventaren und blinc-Fragebögen}

In einem Netzwerk von Partnern aus Frankreich, Italien, Schottland und Deutschland wurden im Rahmen des von der Europäischen Kommission geförderten Projekts „eL3“ verschiedene Materialien für die Evaluation von elearning und Blended Learning entwickelt. Das Hauptziel des Projektes „eL3“ (eLearning Project Cluster for Third System Organisations in Europe) ist die Verbreitung von gemischten Lernformen (Blended Learning) in Betrieben der europäischen Gesundheits- und Sozialwirtschaft. Informationen über das Projekt erhalten Sie auf unserer Homepage www.blinc-eu.org .

Zunächst wurden Zielgruppen identifiziert, die im Kontext von elearning und Blended Learning von Evaluationsinstrumenten profitieren können. Das Projektteam entschied sich für

- Lerner, die in elearning oder Blended Learning Kursen lernen.

- Autoren und Produzenten, die elearning und Blended Learning Angebote entwickeln

- Entscheidungsträger, die vor der Entscheidung stehen, elearning oder Blended Learning in einer Institution einzuführen

Über einen Zeitraum von zwei Jahren erarbeiteten die Partner des Projektes in Arbeitsgruppen gemeinsam Evaluationsinstrumente. Zunächst wurden Entwürfe auf der Basis bisheriger wissenschaftlicher Erkenntnisse erarbeitet, die dann mit den Praxiserfahrungen der Projektpartner erweitert und verfeinert wurden. Aufgrund der Unterschiedlichkeit der Bildungsangebote von den Projektpartnern wurde das Konzept der Inventare entwickelt. Die Blinc-Inventare stellen eine Sammlung von Fragen dar, die für die Evaluation von elearning und Blended Learning genutzt werden können. Dabei sind nicht alle Fragen für alle Nutzer von gleicher Relevanz. Die Nutzer müssen vielmehr selbst aussuchen, welche Fragen in ihrem Kontext relevant sind. Der Vorteil, den die Inventare bieten ist, dass den Nutzern eine Komposition sinnvoller Fragen rund um elearning und Blended Learning zur Verfügung steht, aus dem sie auswählen können. Das Problem in der Praxis der Evaluation von elearning und Blended Learning ist bisher, dass häufig „selbstgebastelte Instrumente“ zum Einsatz kommen und jeder auf sich allein gestellt ist. Mit den Inventaren wird den Nutzern eine Basis angeboten, die von verschiedenen Partnern (Wissenschaftlern, Dozenten, Bildungsplanern) gemeinsam erarbeitet wurde. Es wird angestrebt die Ergebnisse der Befragungen für die Gemeinschaft der Inventarnutzer zugänglich zu machen. Auf diese Weise können Untersuchungsergebnisse und Erfahrungen geteilt werden und zur Verbesserung der Praxis von elearning und Blended Learning beitragen.

Wir möchte Sie einladen die Inventare und Fragebögen zu nutzen. Feedback und Verbesserungsvorschläge sind jederzeit willkommen.

Ihr blinc-Team. 


\section{Information zur Inventaranwendung}

\section{Information zur Anwendung der Inventare für Lerner}

Die Inventare richten sich an Personen, die elearning oder Blended Learning anbieten, z.B. Autoren, Tutoren, Entscheidungsträger oder Wissenschaftler, die ihr Bildungsangebot durch begleitende Evaluation verbessern wollen.

Die vorliegenden Inventare für Lernerfragebögen wurde in einem Netzwerk von Partnern des EU-Projektes eL3 entwickelt. Das Hauptziel des Projekts "eL3" (eLearning Project Cluster for Third System Organisations in Europe) ist die Verbreitung von gemischten Lernformen (Blended Learning) in Betrieben der europäischen Gesundheits- und Sozialwirtschaft.

Im Rahmen der zentralen Arbeitspakete Analyse, Vernetzung und Verbreitung soll eL3 zur Verbesserung der Qualität in der beruflichen Bildung beitragen und den Akteuren in diesen Berufsfeldern Handlungs- und Entscheidungshilfen für einen Einsatz von innovativen Lernformen liefern. In dem Projekt kooperieren 10 Organisationen aus Kultur-, Gesundheits- und Sozialwirtschaft.

Das Wissen und die Erfahrungen der Partner aus den verschiedenen Institutionen gingen ein in eine Sammlung von Checklisten und Inventaren rund um elearning und Blended Learning. Es wurden gemeinsam Instrumente für Entscheidungsträger, Autoren und Lerner erarbeitet.

Für die Befragung von Kursteilnehmern bzw. Lernern wurden drei Inventare entwickelt:

1. Das Inventar für Lernerfragebögen zu Beginn eines Kurses.

2. Das Inventar für Lernerfragebögen während des Kursverlaufes

3. Das Inventar für Lernerfragebögen am Ende eines Kurses

Die Inventare können einzeln als auch aufeinander folgend eingesetzt werden und dienen dazu, Informationen über die Kursteilnehmer zusammenzustellen, um das Kursangebot optimal auf die jeweilige Gruppe abzustimmen.

Gründe für die Entwicklung der Inventare sind u.a. folgende Aspekte:

- Die manchmal geringe Akzeptanz der Lerner in Bezug auf elearning.

- Die manchmal hohe Abbruchquote (Kurse werden nicht zu Ende geführt).

- Die manchmal schwierige Kommunikation und Interaktion zwischen Lehrenden und Lernern.

Wir haben versucht, Fragen zu formulieren, die generell für die Befragung von Lernern verwendbar sind und die zugleich speziell auf einzelne Kurse abgestimmt sind. Wir haben zentrale Bereiche erarbeitet (siehe unten), die unserer Meinung nach elementar sind, um relevante Informationen über die Teilnehmer eines Kurses zu erhalten. In jedem Bereich haben wir Fragen zusammengestellt, die mehr oder weniger wichtig für Ihren speziellen Kurs sein können. Aus dieser Sammlung von Fragen dürfen Sie selbst die Fragen aussuchen, die für Ihren speziellen Kurszusammenhang relevant sind, Sie können auch weitere Fragen formulieren und gestalten so Ihren individuellen Fragebogen. Mit den Inventaren stellen wir Ihnen 
die in unserem Netwerk erarbeiteten Instrumente zur freien Verfügung. Über Rückmeldungen und Erfahrungen mit den Inventaren sind wir dankbar und werden sie in die Weiterentwicklung einfließen lassen.

\section{Die zentralen Bereiche sind:}

\section{Kontext des Kurses}

Die Fragen dieses Bereiches dienen dazu, Namen, Ort und Datum des Kurses zu dokumentieren. Dies erleichtert eine spätere Zuordnung der Ergebnisse, wenn Sie z.B. mehrere Kurse auswerten. Sie können diesen Teil auch selbst ausfüllen, bevor die Teilnehmer den Bogen erhalten.

\section{Angaben zur Person}

Mit den Fragen dieses Bereiches sollen Sie Informationen darüber erhalten, "wer" in Ihrem Kurs ist (Alter, Geschlecht, Bildungsstand, Beruf). Die Frage nach dem Namen oder einem Codenamen ist sinnvoll, wenn Sie z.B. nach dem Kurs eine erneute Befragung durchführen wollen. Sie können dann "vorher- und nachher" - Antworten vergleichen und so den individuellen Lernprozess besser nachvollziehen. Eine andere Möglichkeit ist, den Fragebogen anonym ausfüllen zu lassen. Bitte entscheiden Sie dies wohlüberlegt und ggf. in Abstimmung mit Ihrer Lerngruppe!

Mit dem zweiten Abschnitt der Fragen zur Person erfahren Sie mehr über die individuellen Lernbedingungen der Teilnehmer. Hier werden aber „private“ bzw. „persönliche“ Informationen erfragt und deshalb sollte die Beantwortung dieser Fragen freiwillig sein. Die Antworten können Ihnen helfen, den Kurs optimal auf die Gruppe abzustimmen.

\section{Lernerfahrungen und Vorwissen}

Mit den Fragen dieses Bereiches erfahren Sie mehr über die Lernerfahrungen mit verschiedenen Lehrmethoden. Sie gewinnen auch Informationen zum Vorwissen der Beteiligten in bezug auf die Seminarinhalte. Sie ermitteln, welche Methoden Ihre Kursbesucher bereits kennen und wie ihnen diese Methoden gefallen haben. Falls sich z.B. ausschließlich Kursteilnehmer, die noch nicht mit elearning vertraut sind, in Ihrem Kurs befinden, sollten Sie die Computerarbeit dementsprechend einführen. 
Mit den Fragen dieses Bereiches erhalten Sie Informationen über die Ressourcen Ihrer Kursteilnehmer für elarning, deren PC-Wissen und Erfahrung im Umgang mit dem Computer. Die Teilnehmer sollen sich dabei selbst einschätzen.

Bitte stellen Sie nur die Fragen, die in Ihrem Kontext nützlich und sinnvoll sind. Bei der Einschätzung der eigenen Fähigkeiten mit verschiedenen Programmen sollten Sie nur diejenigen abfragen, die für Ihren Kurszusammenhang wichtig sind.

5. Persönliche Motivation, an diesem Kurs teilzunehmen, Erwartungen und Ziele

Mit den Fragen dieses Bereiches erfahren Sie mehr über die persönlichen Gründe und die Motivation der Teilnehmer, am Kurs teilzunehmen. Es können Ziele, Erwartungen, aber auch Ängste und Sorgen der Lerner niedergeschrieben werden. Während des Kurses und auch am Ende können Sie durch eine erneute Befragung überprüfen, ob die Erwartungen erfüllt wurden. Außerdem ist es möglich zu reagieren, wenn jemand Sorgen oder Ängste äußert.

\section{Platz für Vorschläge, Kommentare und Fragen}

In diesem Bereich haben die Kursteilnehmer "Platz" für Vorschläge und Wünsche bezüglich des zukünftigen Kursangebotes, die Ihnen bei der Planung neuer Kurse helfen. Außerdem haben die Befragten die Gelegenheit, Kommentare und Fragen zu äußern.

Der Fragebogen sollte den Kursteilnehmern im Kurs ausgeteilt werden, wenn Sie sich für eine papierbasierte Version entschieden haben. Es ist unserer Meinung nach sehr wichtig, genügend Zeit und Raum für das Ausfüllen des Fragebogens zur Verfügung zu stellen.

Sie sollten Ihren Kursteilnehmern versichern, dass Sie vertraulich mit den Antworten umgehen und diese nur zur Verbesserung des jetzigen oder eines späteren Kursangebotes verwenden werden.

Zum Abschluss sollten Sie sich unbedingt bei Ihren Kursteilnehmern für das Ausfüllen des Fragebogens bedanken.

Wir halten es für sinnvoll, dass die Teilnehmer ihren Fragebogen behalten und ihrem Tutor oder Dozenten eine Kopie geben. Auf diese Weise können die Lerner selbst zu einem späteren Zeitpunkt ihre Antworten einsehen. Bitte sorgen Sie dafür, dass ein Kopierer in der Nähe ist, damit sie die Fragebögen unmittelbar einsammeln können. 
6. Inventare für Lerner

Workgroup:

Filippo Del Casino, Jutta List-Ivanković, Corinne Rudelle, Colin Macduff

Translations:

English:

Bernice West, Neil Johnson

German:

Jutta List-Ivanković

Italian:

Filippo del Casino, Loredana Marasco

French:

Elisabeth Belarbi, Giséle Massol, Emeric Pividori 


\section{Examples for introductions for the learners from different eL3-partners:}

\section{German}

Liebe Kursteilnehmerin, lieber Kursteilnehmer, Herzlich willkommen in diesem Kurs! Mit diesem Fragebogen möchten wir Informationen über die Menschen erhalten, die unsere Kurse besuchen - also über Sie!

Uns interessieren Ihre persönlichen Voraussetzungen, Ihre Wünsche und Bedürfnisse. Ihre Antworten werden uns helfen, den Kurs optimal auf Ihre Erwartungen und Ihr Vorwissen abzustimmen.

Wir bitten Sie, uns einige Informationen über sich, Ihre Erfahrung mit verschiedenen Lernmethoden sowie über Ihre Erwartungen in diesem Kurs zu geben. Wir werden Ihre Antworten nur für die Kursauswertung und -vorbereitung innerhalb unserer Einrichtung verwenden.

Der Fragebogen wurde in einem Netzwerk von Partnern aus Frankreich, Italien, Schottland und Deutschland ("blinc") im Rahmen des von der Europäischen Kommission geförderten Projekts „eL3“ entwickelt. Das Hauptziel des Projektes „eL3“ (eLearning Project Cluster for Third System Organisations in Europe) ist die Verbreitung von gemischten Lernformen (Blended Learning) in Betrieben der europäischen Gesundheits- und Sozialwirtschaft. Informationen über das Projekt erhalten Sie auf unserer Homepage www.blinc-eu.org .

Wir freuen uns über Ihren Besuch!

Vielen Dank für Ihre Beteiligung!

\section{English:}

Dear learner,

This questionnaire has been designed to gather information about learners on the course, their personal circumstances, their learning experiences and their resources for elearning. Your answers will help us to develop courses in the future and support your current learning needs. Please take your time to complete the six sections in the questionnaire. Once completed, we recommend that you take a copy for yourself and submit the completed questionnaire to your tutor. We are interested in your expectations, experiences, aims and concerns so please be frank.

Finally we plan to collate the information gathered from the questionnaires for our own evaluation purposes and as such individual's names will not be identified.

Thank you very much! 


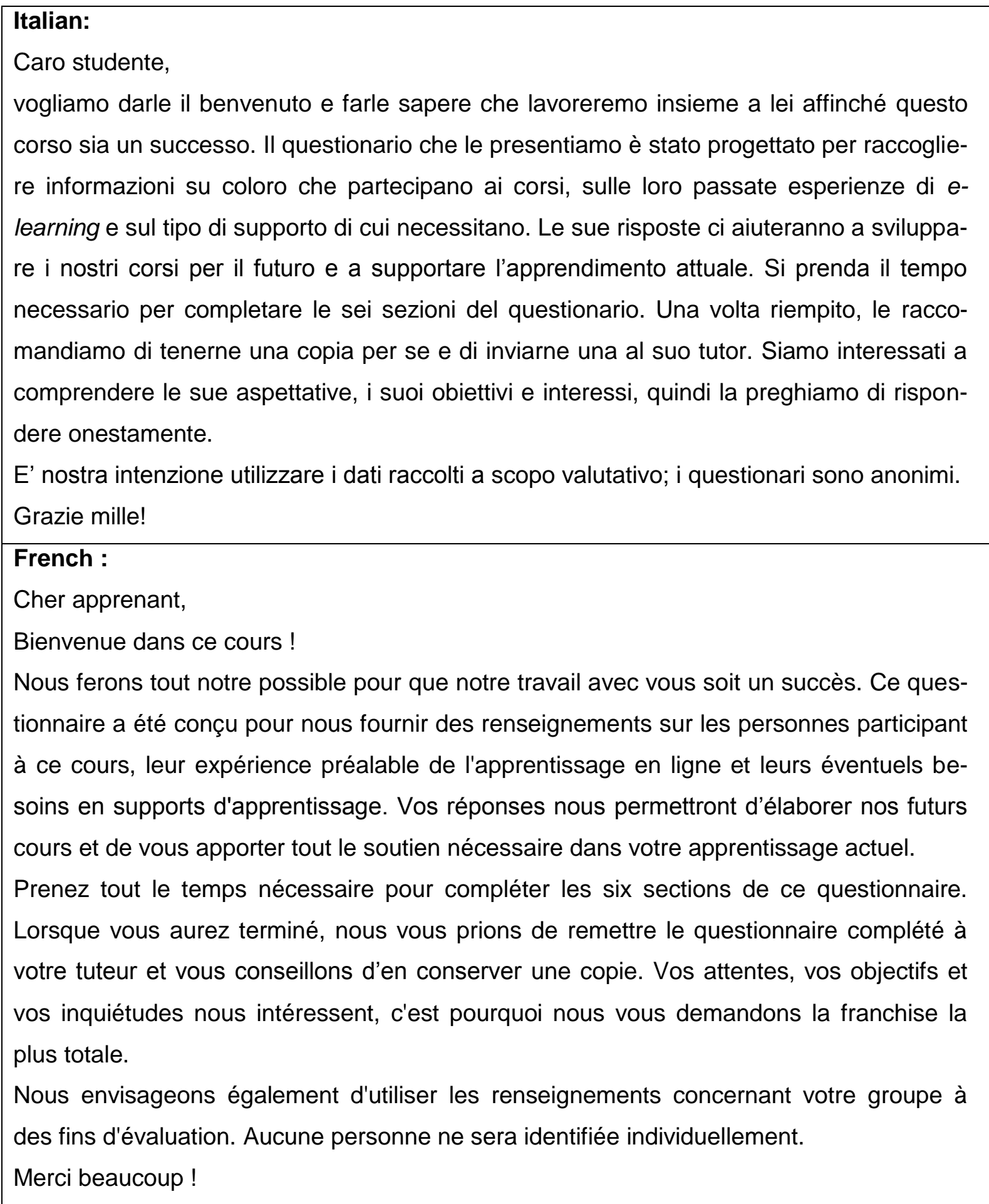




\subsection{Inventar für Lerner zu Beginn eines Kurses}

\section{Kontext des Kurses}

Bitte schreiben Sie Ihre Antworten in die rechte Spalte.

\section{Context of the course}

Please write your responses in the boxes on the right side.

1. Contesto del corso

Per favore scriva le sue risposte negli spazi grigi a destra.

1. Contexte du cours

Veuillez inscrire vos réponses dans les cases de droite.

\begin{tabular}{|l|l|}
\hline Fragen & Antworten \\
\hline Questions & Responses \\
\hline Domande & Risposte \\
\hline Questions & Réponses \\
\hline
\end{tabular}

1a) Name des Kurses?

1a) What is the title of your course?

1a) Qual è il titolo del suo corso?

1a) Quel est l'intitulé de votre cours ?

1b) Wo findet der Kurs statt?

1b) Where does the course take place?

1b) Dove si svolge il corso?

1b) Où le cours a-t-il lieu ?

1c) Das heutige Datum?

1c) What is today's date?

1c) Qual è la data di oggi?

1c) Quelle est la date d'aujourd'hui ? 
2. Angaben zur Person

\section{Personal Characteristics of learners}

\section{Scheda personale dello studente}

\section{Renseignements personnels sur les apprenants}

\begin{tabular}{|l|l|}
\hline Fragen & Antworten \\
\hline Questions & Responses \\
\hline Domande & Risposte \\
\hline Questions & Réponses \\
\hline
\end{tabular}

2a) Ihr Name oder ein Codename

Für eine erneute Befragung zu einem späteren Zeitpunkt möchten wir Sie gerne wieder erkennen, gleichzeitig möchten wir jedoch Ihre Anonymität wahren. Deshalb bitten wir Sie, einen Codenamen bzw. eine Codenummer anzugeben. Bitte geben Sie nacheinander den Geburtstag und den Geburtsmonat von zwei Menschen an, die Ihnen besonders nahe stehen.

Hier ein Beispiel:

Partner 01.03. (Geburtstag. Geburtsmonat) Mutter 12.12. (Geburtstag. Geburtsmonat) Der Codename wäre demnach: 01031212 2a) Your name or codename?

2a) II vostro nome o pseudonimo?

2a) Quel est votre nom ou votre pseudonyme?

\begin{tabular}{|l}
\hline 2b) Wie alt sind Sie? \\
\hline 2b) How old are you? \\
\hline 2b) Quanti anni ha? \\
\hline 2b) Quel âge avez-vous?
\end{tabular}




\begin{tabular}{|l|l|}
\hline 2c) Welches Geschlecht haben Sie? & $\begin{array}{l}\text { O Männlich } \\
\text { O Weiblich }\end{array}$ \\
\hline 2c) What is your sex? & $\begin{array}{l}\text { O Male } \\
\text { O Female }\end{array}$ \\
\hline 2c) Sesso & $\begin{array}{l}\text { OMaschio } \\
\text { OFemmina }\end{array}$ \\
\hline 2c) Quel est votre sexe ? & $\begin{array}{l}\text { O Masculin } \\
\text { O Féminin }\end{array}$ \\
\hline
\end{tabular}

\begin{tabular}{|l|l|}
\hline 2d) Haben Sie Kinder? & $\begin{array}{l}\text { O Ja } \\
\text { O Nein }\end{array}$ \\
\hline 2d) Do you have children? & $\begin{array}{l}\text { O Yes } \\
\text { O No }\end{array}$ \\
\hline 2d) Ha figli? & $\begin{array}{l}\text { O Si } \\
\text { O No }\end{array}$ \\
\hline 2d) Avez-vous des enfants ? & $\begin{array}{l}\text { O Oui } \\
\text { O Non }\end{array}$ \\
\hline
\end{tabular}

\begin{tabular}{|l|l|}
\hline 2e) Welche Nationalität haben Sie? & \\
\hline 2e) What is your nationality? & \\
\hline 2e) Di che nazionalità è? & \\
\hline 2e) Quelle est votre nationalité ? & \\
\hline
\end{tabular}

2f) Welchen höchsten Allgemeinbildenden Abschluss haben Sie?

2f) What is your highest educational qualification?

2f) Quale titolo di studio/istruzione?

2f) Quel est votre niveau d'étude ?

$2 g)$ Welchen Beruf haben Sie?

$2 \mathrm{~g}$ ) What is your current job title or occupation?

$2 \mathrm{~g})$ Qual è il suo grado di professione?

$2 \mathrm{~g})$ Quelle est votre profession? 
2h) In welcher Einrichtung/Firma oder in welchem Betrieb arbeiten Sie?

2h) In which institution do you work?

2h) Presso quale istituzione lavora?

2h) Quel est l'intitulé de votre emploi actuel ?

2i) Wo wollen Sie Ihr elearning, d.h. elektronisch gestütztes Lernen, durchführen?

(Bitte alle zutreffenden Antworten ankreuzen.)

Zu Hause

O An meinem Arbeitsplatz

O Andere Orte (Bitte erläutern.)

2i) Where do you do your elearning?

Ot home

At my working place

O Others (please specify):

2i) Dove vorrebbe fare il corso elearning?

\section{O A casa}

O Sul posto di lavoro

Altro (specificare):

2i) Où souhaitez-vous suivre votre apprentissage en ligne?

O À mon domicile

O Sur mon lieu de travail

O Ailleurs (précisez) :

\begin{tabular}{|c|c|c|c|c|}
\hline $\begin{array}{l}\text { 2j) Wieviel Zeit haben Sie in der Regel } \\
\text { pro Woche zu Hause für elearning? } \\
\text { (Zutreffendes bitte ankreuzen.) }\end{array}$ & $\begin{array}{l}\text { O Ich habe } \\
\text { zu Hause } \\
\text { sehr viel } \\
\text { Zeit } \\
\text { für elearn- } \\
\text { ing. }\end{array}$ & $\begin{array}{l}\text { O Ich habe } \\
\text { zu Hause } \\
\text { viel Zeit } \\
\text { für elearn- } \\
\text { ing. }\end{array}$ & $\begin{array}{l}\text { O Ich habe } \\
\text { zu Hause } \\
\text { wenig Zeit } \\
\text { für elearn- } \\
\text { ing. }\end{array}$ & $\begin{array}{l}\text { O Ich habe } \\
\text { zu Hause } \\
\text { gar keine } \\
\text { Zeit } \\
\text { für elearn- } \\
\text { ing. }\end{array}$ \\
\hline $\begin{array}{l}\text { 2j) How do you feel about your personal } \\
\text { situation at home concerning the time } \\
\text { available for elearning? (Please tick the } \\
\text { relevant statement) }\end{array}$ & $\begin{array}{l}\text { I have } \\
\text { A lot of time } \\
\text { available at } \\
\text { home for my } \\
\text { elearning }\end{array}$ & $\begin{array}{l}\text { O I have } \\
\text { Some time } \\
\text { available at } \\
\text { home for my } \\
\text { elearning }\end{array}$ & $\begin{array}{l}\text { I have } \\
\text { Little time } \\
\text { available at } \\
\text { home for my } \\
\text { elearning }\end{array}$ & $\begin{array}{l}\text { O I have } \\
\text { No time } \\
\text { available at } \\
\text { home for my } \\
\text { elearning }\end{array}$ \\
\hline $\begin{array}{l}\text { 2j) Considerando la sua situazione per- } \\
\text { sonale a casa, quanto tempo pensa di } \\
\text { avere per l'e-learning ? (con il pc ed il } \\
\text { collegamento in rete) (segna } \\
\text { l'affermazione che ritiene più adatta) }\end{array}$ & $\begin{array}{l}\square \text { Ho molto } \\
\text { tempo a } \\
\text { casa per l' } \\
\text { e-learning. }\end{array}$ & $\begin{array}{l}\square \text { Ho ab- } \\
\text { bastanza } \\
\text { tempo a } \\
\text { casa per l' } \\
\text { e-learning. }\end{array}$ & $\begin{array}{l}\square \text { Ho poco } \\
\text { tempo a } \\
\text { casa per l' } \\
\text { e-learning. }\end{array}$ & $\begin{array}{l}\square \text { Non ho } \\
\text { tempo a } \\
\text { casa per l' } \\
\text { e-learning. }\end{array}$ \\
\hline
\end{tabular}


2j) Comment voyez-vous votre situation personnelle à votre domicile en ce qui concerne le temps dont vous disposez pour l'apprentissage en ligne ? (Veuillez cocher la réponse correspondante)
O Je dispose de beaucoup de temps à mon domicile pour mon apprentissage en ligne

O Je dis-
pose
d'un certain
temps à
mon domi-
cile pour
mon ap-
prentissage
en ligne

O Je dispose de peu de temps à mon domicile pour mon apprentissage en ligne
O Je ne dispose pas de temps à mon domicile pour mon apprentissage en ligne 2k) Wieviele Stunden haben Sie zu Hause pro Woche für elearning?

2k) How many hours per week do you have for your elearning?

2k) Quante ore a settimana ha per l' elearning? (con il pc ed il collegamento in rete)

2k) De combien d'heures hebdomadaires disposez-vous pour votre apprentissage en ligne?
Ich habe ..... Stunden pro Woche zu Hause zur Verfügung für elearning.

I have hours per week at home for elearning. ore la settimana per l' e-learning. heures hebdomadaires à mon domicile pour mon apprentissage en ligne.

\begin{tabular}{|l|l|}
\hline $\begin{array}{l}\text { 2l) Gibt es zu Hause irgendwelche Ein- } \\
\text { schränkungen, wenn Sie mit dem Compu- } \\
\text { ter arbeiten wollen? }\end{array}$ & $\begin{array}{l}\text { O Nein } \\
\text { O Ja (Bitte erläutern): }\end{array}$ \\
\hline $\begin{array}{l}\text { 2l) Are there any restrictions at home with } \\
\text { regard to using a computer } \\
\text { (e.g. no computer at home; other family } \\
\text { members have priority) }\end{array}$ & $\begin{array}{l}\text { O No } \\
\text { O Yes (please specify): }\end{array}$ \\
\hline $\begin{array}{l}\text { 2l) Ci sono limitazioni a casa riguardo } \\
\text { all'uso del computer } \\
\text { (es. non ha il computer a casa; altri mem- } \\
\text { bri della famiglia hanno la priorità)? }\end{array}$ & $\square$ No (specificare): \\
\hline $\begin{array}{l}\text { 2l) Existe-t-il des contraintes à votre do- } \\
\text { micile concernant l'utilisation d'un ordina- } \\
\text { teur? } \\
\text { (par exemple pas d'ordinateur ; d'autres } \\
\text { membres de la famille sont prioritaires) }\end{array}$ & \begin{tabular}{l} 
\\
\hline
\end{tabular} \\
\hline
\end{tabular}




\begin{tabular}{|c|c|c|c|c|}
\hline $\begin{array}{l}\text { 2m) Wieviel Zeit haben Sie, um an die- } \\
\text { sem Kurs teilzunehmen? } \\
\text { (Zutreffendes bitte ankreuzen.) }\end{array}$ & $\begin{array}{l}\text { O Ich habe } \\
\text { sehr viel } \\
\text { Zeit, } \\
\text { um an die- } \\
\text { sem Kurs } \\
\text { teilzu- } \\
\text { nehmen }\end{array}$ & $\begin{array}{l}\text { O Ich habe } \\
\text { viel Zeit, } \\
\text { um an die- } \\
\text { sem Kurs } \\
\text { teilzu- } \\
\text { nehmen. }\end{array}$ & $\begin{array}{l}\text { O Ich habe } \\
\text { wenig Zeit, } \\
\text { um an die- } \\
\text { sem Kurs } \\
\text { teilzu- } \\
\text { nehmen. }\end{array}$ & $\begin{array}{l}\text { O lch habe } \\
\text { gar keine } \\
\text { Zeit, } \\
\text { um an die- } \\
\text { sem Kurs } \\
\text { teilzu- } \\
\text { nehmen. }\end{array}$ \\
\hline $\begin{array}{l}2 \mathrm{~m}) \text { How do you feel about your personal } \\
\text { situation at home concerning the time } \\
\text { available for taking part in this course? } \\
\text { (Please tick the relevant statement) }\end{array}$ & $\begin{array}{l}\text { I have } \\
\text { A lot of time } \\
\text { available at } \\
\text { home for } \\
\text { taking part } \\
\text { in this } \\
\text { course }\end{array}$ & $\begin{array}{l}\text { O I have } \\
\text { Some time } \\
\text { available at } \\
\text { home for } \\
\text { taking part } \\
\text { in this } \\
\text { course }\end{array}$ & $\begin{array}{l}\text { O I have } \\
\text { Little time } \\
\text { available at } \\
\text { home for } \\
\text { taking part } \\
\text { in this } \\
\text { course }\end{array}$ & $\begin{array}{l}\text { I have } \\
\text { No time } \\
\text { available at } \\
\text { home for } \\
\text { taking part } \\
\text { in this } \\
\text { course }\end{array}$ \\
\hline $\begin{array}{l}2 \mathrm{~m} \text { ) Considerando la sua situazione per- } \\
\text { sonale a casa, quanto tempo pensa di } \\
\text { avere per seguire questo corso? (segna } \\
\text { l'affermazione che ritiene più adatta) }\end{array}$ & $\begin{array}{l}\square \text { Ho molto } \\
\text { tempo a } \\
\text { casa per } \\
\text { seguire } \\
\text { questo cor- } \\
\text { so }\end{array}$ & $\begin{array}{l}\square \text { Ho tem- } \\
\text { po abba- } \\
\text { stanza a } \\
\text { casa per } \\
\text { seguire } \\
\text { questo cor- } \\
\text { so }\end{array}$ & $\begin{array}{l}\square \text { Ho poco } \\
\text { tempo a } \\
\text { casa per } \\
\text { seguire } \\
\text { questo cor- } \\
\text { so }\end{array}$ & $\begin{array}{l}\square \text { Non ho } \\
\text { tempo a } \\
\text { casa per } \\
\text { seguire } \\
\text { questo cor- } \\
\text { so }\end{array}$ \\
\hline $\begin{array}{l}2 \mathrm{~m}) \text { Quelle est votre situation personnelle } \\
\text { à votre domicile concernant le temps dont } \\
\text { vous disposez pour participer à ce } \\
\text { cours? (Veuillez cocher la réponse cor- } \\
\text { respondante) }\end{array}$ & $\begin{array}{l}\text { O Je dis- } \\
\text { pose } \\
\text { de beau- } \\
\text { coup de } \\
\text { temps à } \\
\text { mon domi- } \\
\text { cile pour } \\
\text { participer à } \\
\text { ce cours }\end{array}$ & $\begin{array}{l}\text { O Je dis- } \\
\text { pose } \\
\text { d'un certain } \\
\text { temps à } \\
\text { mon domi- } \\
\text { cile pour } \\
\text { participer à } \\
\text { ce cours }\end{array}$ & $\begin{array}{l}\text { O Je dis- } \\
\text { pose } \\
\text { de peu de } \\
\text { temps à } \\
\text { mon domi- } \\
\text { cile pour } \\
\text { participer à } \\
\text { ce cours }\end{array}$ & $\begin{array}{l}\text { J Je ne } \\
\text { dispose } \\
\text { pas de } \\
\text { temps à } \\
\text { mon domi- } \\
\text { cile pour } \\
\text { participer à } \\
\text { ce cours }\end{array}$ \\
\hline
\end{tabular}

2n) Wieviele Stunden haben Sie pro Woche zur Verfügung, um an diesem Kurs teilzunehmen?

2n) How many hours per week do you have at home for taking part in this course?

2n) Quante ore la settimana ha a casa per seguire tutti gli elementi di questo corso?

2n) De combien d'heures hebdomadaires disposez-vous à votre domicile pour participer à ce cours ?
Ich habe ..... Stunden pro Woche zur Verfügung, um an diesem Kurs teilzunehmen.

I have hours per week at home for taking part in this course.

Ho ....... ore la settimana a casa per seguire questo corso.

Je dispose de heures hebdomadaires à mon domicile pour participer à ce cours. 


\begin{tabular}{|c|c|c|c|c|}
\hline $\begin{array}{l}\text { 20) Wieviel Zeit haben Sie in der Regel } \\
\text { pro Woche an Ihrem Arbeitsplatz, die } \\
\text { Sie für elearning nutzen können? } \\
\text { (Zutreffendes bitte ankreuzen.) }\end{array}$ & $\begin{array}{l}\text { O lch habe } \\
\text { an meinem } \\
\text { Arbeitsplatz } \\
\text { sehr viel } \\
\text { Zeit } \\
\text { für elearn- } \\
\text { ing. }\end{array}$ & $\begin{array}{l}\text { O Ich habe } \\
\text { an meinem } \\
\text { Arbeitsplatz } \\
\text { viel Zeit } \\
\text { für elearn- } \\
\text { ing. }\end{array}$ & $\begin{array}{l}\text { O Ich habe } \\
\text { an meinem } \\
\text { Arbeitsplatz } \\
\text { wenig Zeit } \\
\text { für elearn- } \\
\text { ing. }\end{array}$ & $\begin{array}{l}\text { Ich habe } \\
\text { an meinem } \\
\text { Arbeitsplatz } \\
\text { gar keine } \\
\text { Zeit } \\
\text { für elearn- } \\
\text { ing. }\end{array}$ \\
\hline $\begin{array}{l}\text { 20) How do you feel about your personal } \\
\text { situation at your working place concerning } \\
\text { the time available for elearning? (Please } \\
\text { tick the relevant statement) }\end{array}$ & $\begin{array}{l}\text { O I have } \\
\text { A lot of time } \\
\text { available at } \\
\text { my working } \\
\text { place for my } \\
\text { elearning }\end{array}$ & $\begin{array}{l}\text { O I have } \\
\text { Some time } \\
\text { available at } \\
\text { my working } \\
\text { place for my } \\
\text { elearning }\end{array}$ & $\begin{array}{l}\text { O I have } \\
\text { Little time } \\
\text { available at } \\
\text { my working } \\
\text { place for my } \\
\text { elearning }\end{array}$ & $\begin{array}{l}\text { O I have } \\
\text { No time } \\
\text { available at } \\
\text { my working } \\
\text { place for my } \\
\text { elearning }\end{array}$ \\
\hline $\begin{array}{l}\text { 2o) Considerando la sua situazione per- } \\
\text { sonale sul posto di lavoro, quanto tempo } \\
\text { pensa di avere per l'e-learning ? (con il pc } \\
\text { ed il collegamento in rete) (segna } \\
\text { l'affermazione che ritiene più adatta) }\end{array}$ & $\begin{array}{l}\text { Ho molto } \\
\text { tempo sul } \\
\text { lavoro per } \\
\text { l'e-learning }\end{array}$ & $\begin{array}{l}\text { Ho abba- } \\
\text { stanza tem- } \\
\text { po sul lavo- } \\
\text { ro per l' e- } \\
\text { learning }\end{array}$ & $\begin{array}{l}\text { O Ho poco } \\
\text { tempo sul } \\
\text { lavoro per } \\
\text { l'e-learning }\end{array}$ & $\begin{array}{l}\text { O Non ho } \\
\text { tempo sul } \\
\text { lavoro per } \\
\text { l'e-learning }\end{array}$ \\
\hline $\begin{array}{l}\text { 2o) Quelle est votre situation personnelle } \\
\text { sur votre lieu de travail concernant le } \\
\text { temps dont vous disposez pour l'appren- } \\
\text { tissage en ligne? (Veuillez cocher la ré- } \\
\text { ponse correspondante) }\end{array}$ & $\begin{array}{l}\text { O Je dis- } \\
\text { pose } \\
\text { de beau- } \\
\text { coup de } \\
\text { temps sur } \\
\text { mon lieu de } \\
\text { travail pour } \\
\text { mon ap- } \\
\text { prentissage } \\
\text { en ligne }\end{array}$ & $\begin{array}{l}\text { O Je dis- } \\
\text { pose } \\
\text { d'un certain } \\
\text { temps sur } \\
\text { mon lieu de } \\
\text { travail pour } \\
\text { mon ap- } \\
\text { prentissage } \\
\text { en ligne }\end{array}$ & $\begin{array}{l}\text { O Je dis- } \\
\text { pose } \\
\text { de peu de } \\
\text { temps sur } \\
\text { mon lieu de } \\
\text { travail pour } \\
\text { mon ap- } \\
\text { prentissage } \\
\text { en ligne }\end{array}$ & $\begin{array}{l}\text { O Je ne } \\
\text { dispose } \\
\text { pas de } \\
\text { temps sur } \\
\text { mon lieu de } \\
\text { travail pour } \\
\text { mon ap- } \\
\text { prentissage } \\
\text { en ligne }\end{array}$ \\
\hline
\end{tabular}

2p) Wieviele Stunden haben Sie an Ihrem Arbeitsplatz pro Woche für elearning?

2p) How many hours per week do you have for your elearning at your working place?

2p) Quante ore a settimana ha per l'elearning sul posto di lavoro?

2p) De combien d'heures hebdomadaires disposez-vous pour votre apprentissage en ligne sur votre lieu de travail ?
Ich habe ..... Stunden pro Woche an meinem Arbeitsplatz zur Verfügung für elearning.

I have hours per week at my working place for elearning.

Ho ....... ore la settimana per l' e-learning sul posto di lavoro.

Je dispose de heures hebdomadaires sur mon lieu de travail pour l'apprentissage en ligne.

\begin{tabular}{|l|l|}
\hline $\begin{array}{l}\text { 2q) Gibt es an Ihrem Arbeitsplatz ir- } \\
\text { gendwelche Einschränkungen, wenn Sie } \\
\text { mit dem Computer arbeiten wollen? }\end{array}$ & $\begin{array}{l}\text { O Nein } \\
\text { O Ja (Bitte erläutern): }\end{array}$ \\
\hline $\begin{array}{l}\text { 2q) Are there any restrictions at your } \\
\text { working place with regard to using a } \\
\text { computer }\end{array}$ & $\begin{array}{l}\text { O No } \\
\text { O Yes (please specify): }\end{array}$ \\
\hline
\end{tabular}


2q) Ci sono limitazioni sul posto di lavoro riguardo l'uso del computer?

(es. Può usare il computer solo per questioni di lavoro; non ci sono computer, c'è un firewall, il livello di accesso ad internet, ecc.)

2q) Existe-t-il des contraintes sur votre lieu de travail concernant l'utilisation d'un ordinateur? $\square$ No

$\square$ Si (specificare):

O Non

O Oui (précisez) : 2r) Wieviele Stunden bekommen Sie von Ihrem Arbeitgeber pro Woche, um an diesem Kurs teilzunehmen?

2r) How many hours per week do you have for taking part in this course at your working place?

2r) Quante ore la settimana ha sul posto di lavoro per seguire tutti gli elementi di questo corso?

2r) De combien d'heures hebdomadaires disposez-vous pour participer à ce cours sur votre lieu de travail?
Ich bekomme ..... Stunden pro Woche von meinem Arbeitgeber, um an diesem Kurs teilzunehmen.

I have hours per week at my working place for taking part in this course.

Ho ore la settimana sul posto di lavoro per seguire questo corso.

Je dispose de heures hebdomadaires sur mon lieu de travail pour participer à ce cours. 


\section{Lernerfahrungen und Vorwissen}

3. Learning experiences in this course

3. Passate esperienze di apprendimento e conoscenze precedenti

3. Acquis en termes d'apprentissage et de connaissances

Im Folgenden sind einige Lehrmethoden aufgeführt. Bitte kreuzen Sie an, wie häufig Sie die einzelnen in der Vergangenheit außerhalb der Schule erlebt haben und schreiben Sie bitte auch Beispiele bzw. Erklärungen oder Kommentare in die Zeile darunter.

Several learning methods are listed below. Please indicate how often you have been involved in using each method in the past (tick one box as appropriate), and add any examples or explanatory comments in the spaces below. Please indicate also on the right side how you liked these methods.

Di seguito trova elencati diversi metodi di apprendimento. Indichi quelli che ha sperimentato frequentemente in passato (segni solo la risposta più appropriata) e aggiunga esempi o commenti negli spazi sottostanti. A destra indichi il suo grado di soddisfazione relativa a ciascun metodo di apprendimento.

Plusieurs méthodes d'apprentissage vous sont présentées ci-dessous. Veuillez indiquer combien de fois vous avez utilisé chaque méthode dans le passé (cochez la case correspondante), et veuillez donner des exemples ou des commentaires dans les cases prévues à cet effet. Indiquez à droite votre satisfaction vis-à-vis de la méthode d'apprentissage.
Bitte kreuzen Sie auf der rechten Seite an, wie Ihnen die jeweilige Methode gefallen hat.

\begin{tabular}{|c|c|c|c|c|c|c|}
\hline \multirow[b]{2}{*}{$\begin{array}{l}\text { 3a) Frontalunterricht } \\
\text { (Das Lernen findet in } \\
\text { einen Klassenraum statt } \\
\text { und eine Lehrperson ist } \\
\text { anwesend.) }\end{array}$} & \multicolumn{3}{|c|}{ Häufigkeit des Erlebens } & \multicolumn{3}{|c|}{ Zufriedenheit mit der Methode } \\
\hline & $\begin{array}{l}\text { O } \\
\text { Noch } \\
\text { nie }\end{array}$ & $\begin{array}{c}\text { O } \\
\text { Einmal }\end{array}$ & $\begin{array}{c}\bigcirc \\
\text { Häufiger }\end{array}$ & $\begin{array}{l}\text { O } \\
\ldots \text { hat mir } \\
\text { gut } \\
\text { gefallen }\end{array}$ & $\begin{array}{c}\text { O } \\
\text {... hat mir } \\
\text { mittelmäßig } \\
\text { gefallen }\end{array}$ & $\begin{array}{c}\text { O } \\
\text {... hat mir } \\
\text { nicht ge- } \\
\text { fallen }\end{array}$ \\
\hline
\end{tabular}

Platz für Kommentare und Erklärungen: 


\begin{tabular}{|c|c|c|c|c|c|c|}
\hline $\begin{array}{l}\text { 3a) In-person attend- } \\
\text { ance (e.g. where you go } \\
\text { to a classroom to learn) }\end{array}$ & $\begin{array}{c}\text { O } \\
\text { Often }\end{array}$ & $\begin{array}{c}\mathrm{O} \\
\text { At least } \\
\text { once }\end{array}$ & $\begin{array}{c}\text { O } \\
\text { Never }\end{array}$ & $\begin{array}{c}\mathrm{O} \\
\text { I liked it }\end{array}$ & $\begin{array}{c}\mathrm{O} \\
\mathrm{I} \text { joined it }\end{array}$ & $\begin{array}{l}\mathrm{O} \\
\text { I did not } \\
\text { like it }\end{array}$ \\
\hline \multicolumn{7}{|l|}{ Comment: } \\
\hline & \multicolumn{3}{|c|}{ Frequenza } & \multicolumn{3}{|c|}{ Grado di soddisfazione } \\
\hline & Mai & $\begin{array}{c}\text { Almeno } \\
\text { una volta }\end{array}$ & Spesso & $\begin{array}{c}\text { Mi è } \\
\text { piaciuto } \\
\text { molto }\end{array}$ & Non so & $\begin{array}{l}\text { Non mi è } \\
\text { piaciuto }\end{array}$ \\
\hline $\begin{array}{l}\text { 3a) Presenza in aula } \\
\text { (Es. lezioni frontali in } \\
\text { classe) }\end{array}$ & $\square$ & $\square$ & $\square$ & $\square$ & $\square$ & $\square$ \\
\hline \multicolumn{7}{|l|}{ Commenti: } \\
\hline & \multicolumn{3}{|c|}{ Fréquence d'apprentissage } & \multicolumn{3}{|c|}{ Satisfaction vis-à-vis de la méthode } \\
\hline & $\begin{array}{l}\text { Ja- } \\
\text { mais }\end{array}$ & $\begin{array}{l}\text { Au moins } \\
\text { une fois }\end{array}$ & Souvent & $\begin{array}{l}\text { J'ai beau- } \\
\text { coup ap- } \\
\text { précié }\end{array}$ & Sans avis & $\begin{array}{c}\text { Je n'ai } \\
\text { pas aimé }\end{array}$ \\
\hline $\begin{array}{l}\text { 3a) Enseignement en } \\
\text { présentiel } \\
\text { (Vous vous rendez dans } \\
\text { une salle pour y suivre } \\
\text { un cours) }\end{array}$ & $\mathrm{O}$ & $\mathrm{O}$ & $\mathrm{O}$ & $\mathrm{O}$ & O & $\mathrm{O}$ \\
\hline
\end{tabular}

\begin{tabular}{|c|c|c|c|c|c|c|}
\hline \multirow[b]{2}{*}{$\begin{array}{l}\text { 3b) Fernunterricht } \\
\text { (Materialien und Aufga- } \\
\text { ben werden zugeschickt } \\
\text { oder ausgeteilt und zu } \\
\text { Hause bearbeitet.) }\end{array}$} & \multicolumn{3}{|c|}{ Häufigkeit des Erlebens } & \multicolumn{3}{|c|}{ Zufriedenheit mit der Methode } \\
\hline & $\begin{array}{c}\mathrm{O} \\
\text { Noch } \\
\text { nie }\end{array}$ & $\begin{array}{c}\text { O } \\
\text { Einmal }\end{array}$ & $\begin{array}{c}\bigcirc \\
\text { Häufiger }\end{array}$ & $\begin{array}{l}\text { O } \\
\ldots \text { hat mir } \\
\text { gut } \\
\text { gefallen }\end{array}$ & $\begin{array}{c}\text { O } \\
\text {... hat mir } \\
\text { mittelmäßig } \\
\text { gefallen }\end{array}$ & $\begin{array}{c}\text { O } \\
\text {... hat mir } \\
\text { nicht ge- } \\
\text { fallen }\end{array}$ \\
\hline
\end{tabular}

Platz für Kommentare und Erklärungen:

\begin{tabular}{|l|c|c|c|c|c|c|}
\hline & \multicolumn{2}{|c|}{} & & & \\
\hline $\begin{array}{l}\text { 3b) Distance/open } \\
\text { learning (e.g. where you } \\
\text { are given paper-based } \\
\begin{array}{l}\text { materials to work through } \\
\text { at your own place) }\end{array}\end{array}$ & $\begin{array}{c}\text { Often } \\
\text { Ot least } \\
\text { once }\end{array}$ & $\begin{array}{c}\text { Never } \\
\text { I liked it }\end{array}$ & I joined it & $\begin{array}{c}\text { I did not } \\
\text { like it }\end{array}$ \\
\hline
\end{tabular}

Comment: 


\begin{tabular}{|l|c|c|c|c|c|c|c|}
\hline & \multicolumn{2}{|l|}{ Frequenza } & \multicolumn{2}{l|}{ Grado di soddisfazione } \\
\hline & Mai & $\begin{array}{c}\text { Almeno una } \\
\text { volta }\end{array}$ & Spesso & & $\begin{array}{c}\text { Mi è } \\
\text { piaciuto } \\
\text { molto }\end{array}$ & Non so & $\begin{array}{c}\text { Non mi è } \\
\text { piaciuto }\end{array}$ \\
\hline $\begin{array}{l}\text { 3b) Apprendimento } \\
\text { a distanza/open lear- } \\
\text { ning } \\
\text { (Es. quando vengono } \\
\text { consegnate delle di- } \\
\text { spense su cui lavorare } \\
\text { per conto proprio) }\end{array}$ & $\square$ & $\square$ & $\square$ & $\square$ & $\square$ & $\square$ \\
\hline
\end{tabular}

Commenti:

\begin{tabular}{|l|c|c|c|c|c|c|}
\hline & \multicolumn{2}{|c|}{ Fréquence d'apprentissage } & \multicolumn{2}{c|}{ Satisfaction vis-à-vis de la méthode } \\
\hline & $\begin{array}{c}\text { Ja- } \\
\text { mais }\end{array}$ & $\begin{array}{c}\text { Au moins } \\
\text { une fois }\end{array}$ & Souvent & $\begin{array}{c}\text { J'ai beau- } \\
\text { coup ap- } \\
\text { précié }\end{array}$ & Sans avis & $\begin{array}{c}\text { Je n'ai } \\
\text { pas aimé }\end{array}$ \\
\hline $\begin{array}{l}\text { 3b)Apprentissage ou- } \\
\text { vert/à distance } \\
\text { (Par exemple, on vous } \\
\text { remet des documents } \\
\text { papier pour travailler } \\
\text { chez vous) }\end{array}$ & 0 & 0 & 0 & 0 & 0 & 0 \\
\hline
\end{tabular}

Commentaires :

\begin{tabular}{|c|c|c|c|c|c|c|}
\hline \multirow[b]{2}{*}{$\begin{array}{l}\text { 3c) elearning } \\
\text { (Lernmaterialien und } \\
\text { Aktivitäten werden elekt- } \\
\text { ronisch/mit dem Compu- } \\
\text { ter vermittelt.) }\end{array}$} & \multicolumn{3}{|c|}{ Häufigkeit des Erlebens } & \multicolumn{3}{|c|}{ Zufriedenheit mit der Methode } \\
\hline & $\begin{array}{c}\mathrm{O} \\
\text { Noch } \\
\text { nie }\end{array}$ & $\begin{array}{c}\mathrm{O} \\
\text { Einmal }\end{array}$ & $\begin{array}{c}\bigcirc \\
\text { Häufiger }\end{array}$ & $\begin{array}{c}\text { O } \\
\ldots \text { hat mir } \\
\text { gut } \\
\text { gefallen }\end{array}$ & $\begin{array}{c}\text { O } \\
\text {... hat mir } \\
\text { mittelmäßig } \\
\text { gefallen }\end{array}$ & $\begin{array}{l}\text { O } \\
\ldots . \text { hat mir } \\
\text { nicht ge- } \\
\text { fallen }\end{array}$ \\
\hline
\end{tabular}

Platz für Kommentare und Erklärungen:

\begin{tabular}{|l|c|c|c|c|c|c|}
\hline & \multicolumn{2}{|c|}{} & & & \\
\hline $\begin{array}{l}\text { 3c) E-learning (e.g. } \\
\text { where learning materials } \\
\text { and activities are ac- } \\
\begin{array}{l}\text { cessed electronically by } \\
\text { conoputer) }\end{array}\end{array}$ & $\begin{array}{c}\text { Often } \\
\text { At least } \\
\text { once }\end{array}$ & $\begin{array}{c}\text { Never } \\
\text { I liked it }\end{array}$ & $\begin{array}{c}\text { I joined it } \\
\text { I did not } \\
\text { like it }\end{array}$ \\
\hline
\end{tabular}

Comment: 


\begin{tabular}{|l|c|c|c|c|c|c|c|}
\hline & \multicolumn{2}{|l|}{ Frequenza } & \multicolumn{3}{l|}{ Grado di soddisfazione } \\
\hline & Mai & $\begin{array}{c}\text { Almeno una } \\
\text { volta }\end{array}$ & Spesso & $\begin{array}{c}\text { Mi è } \\
\text { piaciuto } \\
\text { molto }\end{array}$ & Non so & $\begin{array}{c}\text { Non mi è } \\
\text { piaciuto }\end{array}$ \\
\hline $\begin{array}{l}\text { 3c) E-learning } \\
\text { (Es. quando accede al } \\
\text { materiale didattico e alle } \\
\text { attività elettronicamente } \\
\text { tramite computer) }\end{array}$ & $\square$ & $\square$ & $\square$ & $\square$ & $\square$ & $\square$ \\
\hline Commenti: & $\square$ & $\square$ & $\square$ & $\square$ \\
\hline
\end{tabular}

Commenti:

\begin{tabular}{|l|c|c|c|c|c|c|}
\hline & \multicolumn{2}{|c|}{ Fréquence d'apprentissage } & \multicolumn{2}{|c|}{ Satisfaction vis-à-vis de la méthode } \\
\hline & $\begin{array}{c}\text { Ja- } \\
\text { mais }\end{array}$ & $\begin{array}{c}\text { Au moins } \\
\text { une fois }\end{array}$ & Souvent & $\begin{array}{c}\text { J'ai beau- } \\
\text { coup ap- } \\
\text { précié }\end{array}$ & $\begin{array}{c}\text { Sans avis } \\
\text { pas n'aimé } \\
\text { paime }\end{array}$ \\
\hline $\begin{array}{l}\text { 3c) Apprentissage en } \\
\text { ligne } \\
\text { (Par exemple, lorsque } \\
\text { les supports et activités } \\
\text { d'apprentissage sont } \\
\text { disponibles par informa- } \\
\text { tique) }\end{array}$ & 0 & 0 & 0 & 0 & 0 & 0 \\
\hline
\end{tabular}

Commentaires :

\begin{tabular}{|c|c|c|c|c|c|c|}
\hline \multirow[b]{2}{*}{$\begin{array}{l}\text { 3d) Projektlernen } \\
\text { (Eine vorgegebene Auf- } \\
\text { gabe wird auf individuelle } \\
\text { Art und Weise gelöst. Es } \\
\text { wird z.B. ein eigenes } \\
\text { Projekt im Rahmen eines } \\
\text { Kurses entwickelt.) }\end{array}$} & \multicolumn{3}{|c|}{ Häufigkeit des Erlebens } & \multicolumn{3}{|c|}{ Zufriedenheit mit der Methode } \\
\hline & $\begin{array}{l}\text { O } \\
\text { Noch } \\
\text { nie }\end{array}$ & $\begin{array}{c}\bigcirc \\
\text { Einmal }\end{array}$ & $\begin{array}{c}\bigcirc \\
\text { Häufiger }\end{array}$ & $\begin{array}{c}\text { O } \\
\ldots \text { hat mir } \\
\text { gut } \\
\text { gefallen }\end{array}$ & $\begin{array}{c}\text { O } \\
\text {... hat mir } \\
\text { mittelmäßig } \\
\text { gefallen }\end{array}$ & $\begin{array}{c}\text { O } \\
\text {... hat mir } \\
\text { nicht ge- } \\
\text { fallen }\end{array}$ \\
\hline
\end{tabular}

Platz für Kommentare und Erklärungen:

\begin{tabular}{|l|c|c|c|c|c|c|}
\hline & \multicolumn{2}{|c|}{} & & \\
\hline $\begin{array}{l}\text { 3d) Project learning } \\
\text { (e.g. where you work } \\
\text { individually on a given } \\
\text { task) }\end{array}$ & $\begin{array}{c}\text { O } \\
\text { Often }\end{array}$ & $\begin{array}{c}\text { Ot least } \\
\text { once }\end{array}$ & $\begin{array}{c}\text { Never } \\
\text { Iliked it }\end{array}$ & $\begin{array}{c}\text { O } \\
\text { I joined it }\end{array}$ & $\begin{array}{c}\text { I did not } \\
\text { like it }\end{array}$ \\
\hline
\end{tabular}

Comment: 


\begin{tabular}{|l|c|c|c|c|c|c|c|}
\hline & \multicolumn{2}{|l|}{ Frequenza } & & \multicolumn{2}{l|}{ Grado di soddisfazione } \\
\hline & Mai & $\begin{array}{c}\text { Almeno una } \\
\text { volta }\end{array}$ & Spesso & $\begin{array}{c}\text { Mi è } \\
\text { piaciuto } \\
\text { molto }\end{array}$ & Non so & $\begin{array}{c}\text { Non mi è } \\
\text { piaciuto }\end{array}$ \\
\hline $\begin{array}{l}\text { 3d) Project learning } \\
\text { (Es. quando deve realiz- } \\
\text { zare un suo progetto } \\
\text { come base del corso) }\end{array}$ & $\square$ & $\square$ & $\square$ & $\square$ & $\square$ & $\square$ \\
\hline Commenti: & $\square$ & $\square$ & $\square$ & $\square$ \\
\hline
\end{tabular}

Commenti:

\begin{tabular}{|l|c|c|c|c|c|c|}
\hline & \multicolumn{2}{|c|}{ Fréquence d'apprentissage } & \multicolumn{2}{|c|}{ Satisfaction vis-à-vis de la méthode } \\
\hline & $\begin{array}{c}\text { Ja- } \\
\text { mais }\end{array}$ & $\begin{array}{c}\text { Au moins } \\
\text { une fois }\end{array}$ & Souvent & $\begin{array}{c}\text { J'ai beau- } \\
\text { coup ap- } \\
\text { précié }\end{array}$ & $\begin{array}{c}\text { Sans avis } \\
\text { pas aimé }\end{array}$ \\
\hline $\begin{array}{l}\text { 3d) Apprentissage par } \\
\text { projet } \\
\text { (Par exemple, lorsque } \\
\text { vous devez élaborer } \\
\text { votre propre projet qui } \\
\text { servira de base au } \\
\text { cours) }\end{array}$ & 0 & 0 & 0 & 0 & 0 & \\
\hline
\end{tabular}

Commentaires :

\begin{tabular}{|c|c|c|c|c|c|c|}
\hline \multirow[b]{2}{*}{$\begin{array}{l}\text { 3e) Blended Learning } \\
\text { (Kombination von elearn- } \\
\text { ing und anderen Lern- } \\
\text { formen wie z.B. Frontal- } \\
\text { unterricht und Projektler- } \\
\text { nen ) }\end{array}$} & \multicolumn{3}{|c|}{ Häufigkeit des Erlebens } & \multicolumn{3}{|c|}{ Zufriedenheit mit der Methode } \\
\hline & $\begin{array}{c}\text { O } \\
\text { Noch } \\
\text { nie }\end{array}$ & $\begin{array}{c}\mathrm{O} \\
\text { Einmal }\end{array}$ & $\begin{array}{c}\bigcirc \\
\text { Häufiger }\end{array}$ & $\begin{array}{l}\text { O } \\
\ldots \text { hat mir } \\
\text { gut } \\
\text { gefallen }\end{array}$ & $\begin{array}{c}\text { O } \\
\text {... hat mir } \\
\text { mittelmäßig } \\
\text { gefallen }\end{array}$ & $\begin{array}{c}\mathrm{O} \\
\ldots \text { hat mir } \\
\text { nicht ge- } \\
\text { fallen }\end{array}$ \\
\hline
\end{tabular}

Platz für Kommentare und Erklärungen:

\begin{tabular}{|l|c|c|c|c|c|c|}
\hline & \multicolumn{2}{|c|}{} & & \\
\hline $\begin{array}{l}\text { 3e) Blended learning } \\
\text { (e.g. where any of the } \\
\text { above mentioned meth- } \\
\text { odsare used concurrently } \\
\text { within a learningpro- } \\
\text { gramme) }\end{array}$ & $\begin{array}{c}\text { Often } \\
\text { Ot least } \\
\text { once }\end{array}$ & $\begin{array}{c}\text { Never } \\
\text { At lear }\end{array}$ & & $\begin{array}{c}\text { O } \\
\text { I liked it }\end{array}$ & $\begin{array}{c}\text { O joined it } \\
\text { I did not } \\
\text { like it }\end{array}$ \\
\hline
\end{tabular}

Comment:

\begin{tabular}{|l|c|c|c|c|c|c|c|}
\hline \multicolumn{2}{|l|}{ Frequenza } & & \multicolumn{2}{l|}{ Grado di soddisfazione } \\
\hline & Mai & $\begin{array}{c}\text { Almeno una } \\
\text { volta }\end{array}$ & Spesso & $\begin{array}{c}\text { Mi è } \\
\text { piaciuto } \\
\text { molto }\end{array}$ & Non so & $\begin{array}{c}\text { Non mi è } \\
\text { piaciuto }\end{array}$ \\
\hline
\end{tabular}




\begin{tabular}{|c|c|c|c|c|c|c|}
\hline $\begin{array}{l}\text { 3e) Blended learning } \\
\text { (Es. quando i metodi } \\
\text { sopra indicati sono utiliz- } \\
\text { zati in maniera integrata } \\
\text { all'interno del corso) } \\
\text { WebLingu@ con tutor è } \\
\text { un corso ‘blended lear- } \\
\text { ning'. }\end{array}$ & $\square$ & $\square$ & $\square$ & $\square$ & $\square$ & $\square$ \\
\hline \multicolumn{7}{|l|}{ Commenti: } \\
\hline & \multicolumn{3}{|c|}{ Fréquence d'apprentissage } & \multicolumn{3}{|c|}{ Satisfaction vis-à-vis de la méthode } \\
\hline & $\begin{array}{l}\text { Ja- } \\
\text { mais }\end{array}$ & $\begin{array}{l}\text { Au moins } \\
\text { une fois }\end{array}$ & Souvent & $\begin{array}{l}\text { J'ai beau- } \\
\text { coup ap- } \\
\text { précié }\end{array}$ & Sans avis & $\begin{array}{l}\text { Je n'ai } \\
\text { pas aimé }\end{array}$ \\
\hline $\begin{array}{l}\text { 3e) Apprentissage } \\
\text { mixte } \\
\text { (Par exemple, lorsque le } \\
\text { cours est élaboré à partir } \\
\text { des différentes méthodes } \\
\text { présentées ci-dessus) }\end{array}$ & $\mathrm{O}$ & $\mathrm{O}$ & $\mathrm{O}$ & $\mathrm{O}$ & $\mathrm{O}$ & O \\
\hline
\end{tabular}

\begin{tabular}{|c|c|c|c|c|}
\hline $\begin{array}{l}\text { 3f) Wie schätzen Sie Ihr } \\
\text { Vorwissen bezüglich der } \\
\text { Inhalte dieses Kurses ein? } \\
\text { Mein Vorwissen ist... } \\
\text { (Bitte ankreuzen.) }\end{array}$ & sehr gut & O gut & Oeher gering & nicht vorhanden \\
\hline $\begin{array}{l}\text { 3f) Your previous knowledge } \\
\text { concerning the content of } \\
\text { this course ist... }\end{array}$ & O Very good & O Good & O Less good & O Bad \\
\hline $\begin{array}{l}\text { 3f) Come valuteresti le vo- } \\
\text { streconoscenze in relazione } \\
\text { ai contenuti di questo corso? }\end{array}$ & Molto buone & O Buone & $\begin{array}{l}\text { O Soddisfacen- } \\
\text { ti }\end{array}$ & O Scarse \\
\hline $\begin{array}{l}\text { 3f) Comment évalueriez- } \\
\text { vous vos acquis en matière } \\
\text { de connaissances concer- } \\
\text { nant le contenu de ce cours } \\
?\end{array}$ & Très bon & O Bon & O Moyen & O Faible \\
\hline
\end{tabular}




\begin{tabular}{|l|l|}
\hline $\begin{array}{l}\text { 3g) Haben Sie schonmal versucht, ganz } \\
\text { selbstständig zu lernen (z.B. mit einer CD- } \\
\text { Rom oder einem Selbstlernbuch)? }\end{array}$ & $\begin{array}{l}\text { O Ja } \\
\text { O Nein }\end{array}$ \\
\hline $\begin{array}{l}\text { 3g) Did you ever try to learn all by yourself } \\
\text { (for example with a CD-Rom or a self-study- } \\
\text { book)? }\end{array}$ & $\begin{array}{l}\text { O Yes } \\
\text { O No }\end{array}$ \\
\hline $\begin{array}{l}\text { 3g) Ha mai fatto esperienza di apprendimento } \\
\text { autonomo? } \\
\begin{array}{l}\text { (Per esempio con un cd-rom o un libro per } \\
\text { l'auto-apprendimento)? }\end{array}\end{array}$ & $\square$ Ni \\
\hline $\begin{array}{l}\text { 3g) Avez-vous déjà essayé d'apprendre par } \\
\text { vos propres moyens ? } \\
\text { (Par exemple avec un CD-ROM ou un livre } \\
\text { auto-didactique) }\end{array}$ & \begin{tabular}{l} 
O Oui \\
\hline
\end{tabular} \\
\hline
\end{tabular}

\begin{tabular}{|l|l|}
\hline $\begin{array}{l}\text { 3h) Wenn Sie „Ja“ geantwortet haben, welche } \\
\text { Materialien haben Sie genutzt? }\end{array}$ & $\begin{array}{l}\text { O CD-Rom } \\
\text { O Bücher } \\
\text { O Anderes (Bitte erläutern): }\end{array}$ \\
\hline $\begin{array}{l}\text { 3h) If you answered "Yes“, which kind of ma- } \\
\text { terial did you use? }\end{array}$ & $\begin{array}{l}\text { O CD-Rom } \\
\text { O Books } \\
\text { O Others (please specify) }\end{array}$ \\
\hline $\begin{array}{l}\text { 3h) Se ha risposto "Si", che tipo di materiali } \\
\text { ha utilizzato? } \\
\text { Segna le caselle che ritiene adatte }\end{array}$ & $\square$ CD-Rom \\
& $\square$ Libri \\
& $\square$ corso internet \\
\hline $\begin{array}{l}\text { 3h) Si oui, quel type de support avez-vous } \\
\text { utilisé ? } \\
\text { Veuillez cocher la réponse correspondante }\end{array}$ & $\begin{array}{l}\square \text { CD-ROM } \\
\text { O Livres } \\
\text { O Autres } \\
\text { (Précisez) }\end{array}$ \\
\hline
\end{tabular}

3i) Wie bewerten Sie heute diese Lernform?

Das selbständige Lernen war erfolgreich.

O Das selbständige Lernen war zufrieden stellend.

O Das selbständige Lernen war nicht zufrieden stellend.

O Das selbständige Lernen war schwierig.

3i) What was your experience with this kind of learning?

O It was a success.

O It was ok.

It was not satisfying.

It was difficult. 


\begin{tabular}{|l|l|}
\hline $\begin{array}{l}\text { 3i) Come valuta la sua esperienza riguardo a } \\
\text { questo tipo di apprendimento? } \\
\text { E stato... }\end{array}$ & $\begin{array}{l}\square \text { E' stata un successo } \\
\square \text { E' stata frustrante } \\
\square \text { Altro (specificare) }\end{array}$ \\
\hline $\begin{array}{l}\text { 3i) Comment qualifieriez-vous votre expé- } \\
\text { rience avec ce type d'apprentissage? }\end{array}$ & O Réussite \\
& $\begin{array}{l}\text { O Frustration } \\
\text { O Autres (précisez) }\end{array}$ \\
\hline
\end{tabular}

3j) Bitte beschreiben Sie kurz Ihre Erfahrungen mit dem selbstständigen Lernen:

3j) Please decribe in short your experiences with self-directed learning:

3j) In breve descrive la sua esperienza di apprendimento auto-diretto:

3j) Veuillez décrire ou expliquer brièvement vos expériences en matière d'apprentissage auto-dirigé : 


\section{Ressourcen für elearning, Fähigkeiten und Wissen im Umgang mit dem Computer}

4. Resources for elearning computer skills and knowledge

4. Risorse per l'e-learning: abilità e conoscenza dell'uso del computer

4. Ressources en termes de compétences et connaissances informatiques pour l'apprentissage ligne

Mit den folgenden Fragen möchten wir Ihr PC-Wissen ermitteln. Bitte antworten Sie ganz ehrlich, wir wollen Ihren persönlichen Kenntnisstand ermitteln, um den Kurs nach Ihren Fähigkeiten zu gestalten (Sie brauchen keine Bedenken zu haben, falls Sie noch nicht so geübt sind im Umgang mit dem Computer!).

The following questions aim to gather information about your computer knowledge and skills.

Le facciamo le seguenti domande per avere più informazioni sulle tue capacità e conoscenza dell'uso del computer.

Les questions suivantes nous permettront de mieux connaître vos acquis en termes de connaissances et de compétences en informatique.

\begin{tabular}{|l|l|}
\hline $\begin{array}{l}\text { 4a) Haben Sie Erfahrung im Um- } \\
\text { gang mit Computern? }\end{array}$ & $\begin{array}{l}\text { O Ja, viel Erfahrung } \\
\text { O Ja, etwas Erfahrung } \\
\text { O Nein, keine Erfahrung }\end{array}$ \\
\hline $\begin{array}{l}\text { 4a) Do you have any previous expe- } \\
\text { rience of using a computer? }\end{array}$ & $\begin{array}{l}\text { O Yes, a lot } \\
\text { O Yes, a little } \\
\text { O No }\end{array}$ \\
\hline $\begin{array}{l}\text { 4a) Ha mai usato il computer prima } \\
\text { d'ora? }\end{array}$ & $\begin{array}{l}\text { O Si, molto } \\
\text { O Si, un po' } \\
\text { O No }\end{array}$ \\
\hline $\begin{array}{l}\text { 4a) Possédez-vous l'expérience de } \\
\text { l'utilisation d'un ordinateur? }\end{array}$ & $\begin{array}{l}\text { O Oui, une grande expérience } \\
\text { O Oui, une petite expérience } \\
\text { O Non }\end{array}$ \\
\hline
\end{tabular}

4b) Wofür nutzen Sie bisher Computer?

4b) What do you use a computer for?

4b) Per che cosa utilizza il computer?

4b) À quelles fins utilisez-vous un ordinateur?

4c) Bitte schätzen Sie Ihre Fähigkeiten und Erfahrung im Umgang mit Folgendem ein:

4c) How much experience do you have in using the following software programmes?

4c) Quanta esperienza ha nell'uso dei seguenti software?

4c) Quelle est votre expérience en matière d'utilisation des logiciels suivants ? 


\begin{tabular}{|c|c|c|c|c|c|}
\hline & $\begin{array}{l}\text { Ich habe } \\
\text { bereits viel } \\
\text { Erfahrung }\end{array}$ & $\begin{array}{l}\text { Ich habe be- } \\
\text { reits etwas } \\
\text { Erfahrung }\end{array}$ & $\begin{array}{l}\text { Ich habe } \\
\text { wenig Er- } \\
\text { fahrung }\end{array}$ & $\begin{array}{l}\text { Ich habe } \\
\text { keine Erfah- } \\
\text { rung }\end{array}$ & $\begin{array}{l}\text { Den Begriff } \\
\text { kenne ich } \\
\text { nicht }\end{array}$ \\
\hline & $\begin{array}{c}\text { I have } \\
\text { a lot experi- } \\
\text { ence }\end{array}$ & $\begin{array}{c}\text { I have } \\
\text { some experi- } \\
\text { ence }\end{array}$ & $\begin{array}{l}\text { I have } \\
\text { a little expe- } \\
\text { rience }\end{array}$ & $\begin{array}{c}\text { I have } \\
\text { no experi- } \\
\text { ence }\end{array}$ & \\
\hline & $\begin{array}{l}\text { Ho molta } \\
\text { esperienza }\end{array}$ & $\begin{array}{l}\text { Ho qualche } \\
\text { esperienza }\end{array}$ & $\begin{array}{c}\text { Ho poca } \\
\text { esperienza }\end{array}$ & $\begin{array}{c}\text { Non ho } \\
\text { esperienza }\end{array}$ & \\
\hline & $\begin{array}{l}\text { J'ai beau- } \\
\text { coup d'ex- } \\
\text { périence }\end{array}$ & $\begin{array}{l}\text { J'ai une } \\
\text { certaine ex- } \\
\text { périence }\end{array}$ & $\begin{array}{c}\text { J'ai peu } \\
\text { d'ex- } \\
\text { périence }\end{array}$ & $\begin{array}{c}\text { Je n'ai } \\
\text { aucune ex- } \\
\text { périence }\end{array}$ & \\
\hline $\begin{array}{l}\text { Textverarbeitungs- } \\
\text { programme (z.B. Word) } \\
\text { Text programmes } \\
\text { (e.g. Word) } \\
\text { Programmi di scrittura } \\
\text { (es. Word) } \\
\text { Traitement de texte } \\
\text { (par exemple, Word) }\end{array}$ & $\mathrm{O}$ & O & 0 & 0 & 0 \\
\hline $\begin{array}{l}\text { Kalkulations-Software } \\
\text { (z.B. Excel) } \\
\text { Calculation Software } \\
\text { (E.g. Excel) } \\
\text { Fogli di Calcolo } \\
\text { (es. Excel) } \\
\text { Logiciel de calcul } \\
\text { (Par exemple, Excel) }\end{array}$ & O & 0 & 0 & 0 & 0 \\
\hline $\begin{array}{l}\text { Datenbanken } \\
\text { (z.B. Access oder dbase) } \\
\text { Databases } \\
\text { (Access, dbase) } \\
\text { Banche dati } \\
\text { (Access, dbase) } \\
\text { Bases de données } \\
\text { (Access, dbase) }\end{array}$ & 0 & 0 & 0 & 0 & 0 \\
\hline
\end{tabular}




\begin{tabular}{|c|c|c|c|c|c|}
\hline $\begin{array}{l}\text { Präsentations-Software } \\
\text { (z.B. Power Point) } \\
\text { Presentation software } \\
\text { (E.g. Power Point) } \\
\text { Software per presenta- } \\
\text { zioni } \\
\text { (es. Power Point) } \\
\text { Logiciel de présentation } \\
\text { (Par exemple, Power } \\
\text { Point) }\end{array}$ & 0 & 0 & 0 & 0 & 0 \\
\hline $\begin{array}{l}\text { Internet } \\
\text { (surfen, recherchieren) } \\
\text { Internet browsing } \\
\text { Navigazione in Internet } \\
\text { Navigateur Internet }\end{array}$ & 0 & 0 & 0 & 0 & 0 \\
\hline $\begin{array}{l}\text { E-Mail } \\
\text { E-Mailing } \\
\text { Posta Elettronica } \\
\text { Courrier électronique }\end{array}$ & 0 & 0 & 0 & 0 & 0 \\
\hline $\begin{array}{l}\text { Webdesign } \\
\text { Web designing } \\
\text { Web designing } \\
\text { Conception de pages } \\
\text { Web }\end{array}$ & 0 & 0 & 0 & 0 & 0 \\
\hline
\end{tabular}




\begin{tabular}{|c|c|c|c|c|c|}
\hline $\begin{array}{l}\text { Webpublishing } \\
\text { Web publishing } \\
\text { Web publishing } \\
\text { Publication de pages } \\
\text { Web }\end{array}$ & 0 & O & 0 & 0 & 0 \\
\hline Networking & 0 & 0 & 0 & 0 & 0 \\
\hline $\begin{array}{l}\text { Kommentare: } \\
\text { Comments: } \\
\text { Commenti: } \\
\text { Commentaires : }\end{array}$ & & & & & \\
\hline
\end{tabular}

4d) Wo haben Sie Zugang zu einem Computer mit Internet-Anschluss, den Sie für den Kurs nutzen können?

(Bitte alle zutreffenden Antworten ankreuzen.)

4d) Where do you have access to a computer with internet access that you can use for this learning programme?
Zu Hause

Am Arbeitsplatz

Andere Orte (bitte erläutern):

4d) Dove ha la possibilità di usare internet per il corso?

At home

At your working place

O Other places (Please specify)

O A casa

posto di lavoro

O luoghi diversi

4d) Où avez-vous la possibilité d'utiliser l'ordinateur pour le cours?

O À mon domicile

O Sur mon lieu de travail

O Ailleurs (précisez):

\begin{tabular}{|l|l|}
\hline $\begin{array}{l}\text { 4e) Was für einen Internet-Anschluss werden } \\
\text { Sie für diesen Kurs nutzen? }\end{array}$ & $\begin{array}{l}\text { O Schnelle Telefonleitung (z.B. TDSL/ADSL) } \\
\text { O Langsame Telefonleitung (Modem) } \\
\text { O Weiß nicht }\end{array}$ \\
\hline 4e) What kind of internet access do you use? & $\begin{array}{l}\text { O High speed } \\
\text { O Low speed } \\
\text { O Don`t know }\end{array}$ \\
\hline 4e) Che tipo di collegamento a internet usa? & $\begin{array}{l}\square \text { Alta velocità } \\
\square \text { Bassa velocità } \\
\square \text { Non so }\end{array}$ \\
\hline
\end{tabular}




\begin{tabular}{|l|l}
\hline 4e) Quel type de connexion Internet utilise- & O Haut débit \\
rez-vous? & O Bas débit \\
& O Ne sait pas
\end{tabular}

4f) Welche Zeit werden Sie wahrscheinlich meistens für das elearning nutzen?

(Bitte ankreuzen.)

O Zwischen 8 und 12 Uhr

O Zwischen 12 und $18 \mathrm{Uhr}$

O Zwischen 18 und $22 \mathrm{Uhr}$

O Nach 22 Uhr

Bitte erläutern Sie die Gründe für Ihre Antwort:

4f) When do you most often spend time elearning?

Daytime

Ovening

O Night

Please describe the reasons for your answer.

di fare più spesso e-learning?

Giorno

$\square$ Sera

Notte

Perché?:

4f) À quel moment de la journée envisagezvous d'utiliser le plus souvent votre méthode d'apprentissage en ligne?

O Journée

O Soir

O Nuit

Veuillez indiquer les raisons motivant votre réponse.

4g) Wieviel Zeit planen Sie pro

Woche für das elearning ein?

(Bitte ankreuzen.)

\begin{tabular}{|c|} 
Mehr als \\
10 Stun- \\
den pro \\
Woche
\end{tabular}

\begin{tabular}{|c|} 
Zwischen \\
5 und 10 \\
Stunden \\
pro Woche
\end{tabular}

0

Weniger als 5 Stunden pro Woche

Platz für Kommentare, Erklärungen und andere Pläne:

4g) How much time per week do you spend on your elearning programme?

\section{O More than 10 hours per week}

\begin{tabular}{|c|c|c|c|}
$\begin{array}{c}\text { Between } 5 \\
\text { and } 10 \\
\text { hours } \\
\text { per week }\end{array}$ & $\begin{array}{c}\text { Less than } \\
\text { hours per } \\
\text { week }\end{array}$ & $\begin{array}{c}\text { O will de- } \\
\text { cide every } \\
\text { week }\end{array}$ & $\begin{array}{c}\text { I have no } \\
\text { idea }\end{array}$ \\
\hline
\end{tabular}


Comments:

\begin{tabular}{|c|c|c|c|c|c|}
\hline $\begin{array}{l}\text { 4g) Quanto tempo alla setti- } \\
\text { mana usa il suo program- } \\
\text { ma/piattaforma di e-learning? }\end{array}$ & $\begin{array}{l}\square \\
\text { Più di } 10 \\
\text { ore la set- } \\
\text { timana }\end{array}$ & $\begin{array}{l}\square \\
\text { Tra le } 5 \text { e } \\
\text { le } 10 \text { ore la } \\
\text { settimana }\end{array}$ & $\begin{array}{c}\square \\
\text { Meno di } \\
\text { cinque ore } \\
\text { la settimana }\end{array}$ & $\begin{array}{l}\square \\
\text { Deciderò } \\
\text { ogni volta }\end{array}$ & $\begin{array}{l}\square \\
\text { Non ho } \\
\text { idea }\end{array}$ \\
\hline \multicolumn{6}{|l|}{ Commenti: } \\
\hline $\begin{array}{l}4 \mathrm{~g} \text { ) Combien de temps par } \\
\text { semaine utilisez-vous votre } \\
\text { programme d'apprentissage } \\
\text { en ligne }\end{array}$ & $\begin{array}{c}O \\
\text { Plus de } \\
10 \text { heures } \\
\text { par se- } \\
\text { maine }\end{array}$ & $\begin{array}{c}0 \\
\text { Entre } 5 \text { et } \\
10 \text { heures } \\
\text { par se- } \\
\text { maine }\end{array}$ & $\begin{array}{l}\text { O } \\
\text { Moins de } \\
5 \text { heures } \\
\text { par semaine }\end{array}$ & $\begin{array}{c}\text { O } \\
\text { Je dé- } \\
\text { ciderai } \\
\text { chaque } \\
\text { semaine }\end{array}$ & $\begin{array}{l}\text { Je n'en ai } \\
\text { aucune } \\
\text { idée }\end{array}$ \\
\hline
\end{tabular}

Commentaires :

\begin{tabular}{|l|l|}
\hline $\begin{array}{l}\text { 4h) Haben Sie jemanden, der Ihnen beim } \\
\text { elearning behilflich sein kann? }\end{array}$ & $\begin{array}{l}\text { O Ja } \\
\text { O Nein } \\
\text { O Weiß nicht }\end{array}$ \\
\hline $\begin{array}{l}\text { 4h) Do you have anyone who provides local } \\
\text { support for your e-learning? }\end{array}$ & $\begin{array}{l}\text { O Yes } \\
\text { O No } \\
\text { O Unsure }\end{array}$ \\
\hline $\begin{array}{l}\text { 4h) Ha qualcuno a livello locale che per l'e- } \\
\text { learning può aiutarla? }\end{array}$ & $\square$ Si \\
$\square$ No \\
$\square$ Non so
\end{tabular}

4i) Wenn Sie mit „Ja“ geantwortet haben, wer könnte Ihnen helfen?

(Sie können mehrere Antworten ankreuzen.)

O Freunde

O Partner

O Kollegen

O Vorgesetzte

O Andere Personen (Bitte erläutern.):

4i) If you answered, "Yes", who may help you? (Please tick all the relevant answers)

Friends

O Partner

O Colleagues

O Officials

Others: (Please specify) 


\begin{tabular}{|l|l|}
\hline $\begin{array}{l}\text { 4i) Se ha risposto "Si", chi la può aiutare? } \\
\text { (segna la risposta che ritiene più adatta) }\end{array}$ & $\square$ Amici \\
& $\square$ Partner \\
& $\square$ Colleghi \\
& $\square$ Altri : (specificare) \\
\hline $\begin{array}{l}\text { 4i) Si "Oui", qui peut vous aider (Veuillez co- } \\
\text { cher toutes les réponses correspondantes) }\end{array}$ & O Amis \\
& O Partenaire \\
& $\begin{array}{l}\text { O Collègues } \\
\text { O Responsables } \\
\text { O Autres : (Précisez) }\end{array}$ \\
\hline
\end{tabular}

\begin{tabular}{|l|l|}
\hline $\begin{array}{l}\text { 4j) Wenn Sie mit „Nein“ geantwortet haben, } \\
\text { brauchen Sie eventuell Hilfe? }\end{array}$ & $\begin{array}{l}\text { O Ja } \\
\text { O Nein } \\
\text { O Weiß nicht }\end{array}$ \\
\hline $\begin{array}{l}\text { 4j) If you answered “No“, do you need some } \\
\text { help? }\end{array}$ & $\begin{array}{l}\text { O Yes } \\
\text { O No } \\
\text { O Don` t know }\end{array}$ \\
\hline $4 j)$ Se ha risposto „No“, ha bisogno di aiuto? & $\begin{array}{l}\text { O Si } \\
\text { O No } \\
\text { O Non lo so }\end{array}$ \\
\hline $4 j)$ Si „Non“, avez-vous besoin d'aide? & $\begin{array}{l}\text { O Oui } \\
\text { O Non } \\
\text { O Je ne sais pas }\end{array}$ \\
\hline
\end{tabular}


5. Persönliche Motivation an diesem Kurs teilzunehmen, Erwartungen und Ziele

5. Personal motivation for doing this course, expectations and aims

5. Motivazioni personali, aspettative e obiettivi in relazione al corso

5. Motivations personnelles pour suivre ce cours, attentes et objectifs

\begin{tabular}{|c|c|}
\hline $\begin{array}{l}\text { 5a) Warum nehmen Sie an diesem Kurs teil? } \\
\text { (Bitte alle zutreffenden Antworten ankreu- } \\
\text { zen.) }\end{array}$ & $\begin{array}{l}\text { Interesse am Thema } \\
\text { Qualifikation } \\
\text { Zertifikat } \\
\text { Pflicht/Vorgabe } \\
\text { Anderes (Bitte erläutern): }\end{array}$ \\
\hline 5a) Why did you join this course? & $\begin{array}{l}\text { Interest in the topic } \\
\text { Qualification } \\
\text { Certification } \\
\text { Obligation } \\
\text { Others (please specify): }\end{array}$ \\
\hline 5a) Perché segue questo corso? & $\begin{array}{l}\text { Interesse al tema } \\
\text { Qualificazione } \\
\text { Certificato } \\
\text { Obbligo } \\
\text { Altro (specificare): }\end{array}$ \\
\hline 5a) Pourquoi participez-vous à ce cours? & $\begin{array}{l}\text { Intérêt thématique } \\
\text { Qualification } \\
\text { Certificat } \\
\text { Devoir } \\
\text { Autres (précisez): }\end{array}$ \\
\hline
\end{tabular}

5b) Welchen Lerngewinn versprechen Sie sich von diesem Kurs?

5b) What are your personal learning objectives?

5b) Quali sono i suoi principali obiettivi di apprendimento?

5b) Quels sont vos principaux objectifs d'apprentissage?

5c) Welche Erwartungen haben Sie in Bezug auf diesen Kurs?

5c) What expectations do you have about the course?

5c) Quali sono le sue aspettative riguardo a questo corso?

5c) Qu'attendez-vous de ce cours ? 
5d) Welche Befürchtungen haben Sie in Bezug auf diesen Kurs?

$5 d)$ What worries/fears do you have about the course?

5d) Quali sono le sue preoccupazioni o paure riguardo a questo corso?

$5 d)$ Quelles inquiétudes/craintes éprouvez-vous vis-à-vis de ce cours ?

\section{Platz für Vorschläge, Kommentare und Fragen}

6. Open space for suggestions comments and questions

6. Spazio per suggerimenti, commenti e domande

6. Espace libre pour vos suggestions, commentaires et questions

6a) An welchen anderen Kursthemen hätten Sie Interesse?

6a) What other courses would you like to see offered in the future?

6a) Quali altri corsi di lingue le piacerebbe fossero offerti in futuro?

6a) Quels autres cours souhaiteriez-vous voir proposer à l'avenir ?

6b) Wenn Sie noch Kommentare oder Fragen haben, die Sie uns in Bezug auf den Kurs mitteilen wollen, bitte nutzen Sie diesen Platz:

6b) If you have any other comments or questions that you would like to share regarding the forthcoming course please write them here.

6b) Se ha ancora commenti o domande riguardo al corso che sta per iniziare, li riporta qui.

6b) Indiquez ici tout commentaire ou question que vous souhaiteriez faire partager concernant le cours à venir.

Wir bedanken uns ganz herzlich dafür,

dass Sie sich die Zeit genommen haben, den Fragebogen auszufüllen!

Thank you very much

for taking the time to complete this questionnaire!

Grazie mille per aver completato il questionario!

Merci beaucoup pour avoir pris le temps de répondre à ce questionnaire ! 


\subsection{Inventar für Lerner während eines Kurses}

Workgroup:

Filippo Del Casino, Jutta List-Ivanković, Corinne Rudelle, Colin Macduff

Translations:

English:

Bernice West, Neil Johnson

German:

Jutta List-Ivanković

Italian:

Filippo del Casino, Loredana Marasco

French:

Elisabeth Belarbi, Giséle Massol, Emeric Pividori 
Examples for introductions for the learners from different eL3-partners:

\section{German:}

Liebe Kursteilnehmerin, lieber Kursteilnehmer,

Mit diesem Fragebogen möchten wir Informationen über die Menschen erhalten, die unsere Kurse besuchen - also über Sie!

Wir sind interessiert an Ihren Lernbedingungen und Ihren Lernerfahrungen in diesem Kurs. Ihre Antworten werden uns bei der aktuellen und zukünftigen Kursplanung helfen.

Wir bitten Sie, uns einige Informationen über sich, Ihre Erfahrung mit den verschiedenen Lernmethoden sowie über Ihre Erwartungen in diesem Kurs zu geben. Wir werden Ihre Antworten nur für die Kursauswertung innerhalb unserer Einrichtung verwenden.

Der Fragebogen wurde in einem Netzwerk von Partnern aus Frankreich, Italien, Schottland und Deutschland ("blinc") im Rahmen des von der Europäischen Kommission geförderten Projekts „el3“ entwickelt. Das Hauptziel des Projektes „el3“ (eLearning Project Cluster for Third System Organisations in Europe) ist die Verbreitung von gemischten Lernformen (Blended Learning) in Betrieben der europäischen Gesundheits- und Sozialwirtschaft.

Informationen über das Projekt erhalten Sie auf unserer Homepage www.blinc-eu.org . Wir freuen uns über Ihren Besuch!

Vielen Dank für Ihre Beteiligung! 


\section{Information for learners}

Dear learner,

This questionnaire has been designed to gather information about learners on the course, their personal circumstances, their learning experiences and their resources for elearning. Your answers will help us to develop courses in the future and support your current learning needs. Please take your time to complete the six sections in the questionnaire. Once completed, we recommend that you take a copy for yourself and submit the completed questionnaire to your tutor. We are interested in your expectations, experiences, aims and concerns so please be frank.

Finally we plan to collate the information gathered from the questionnaires for our own evaluation purposes and as such individual's names will not be identified.

Thank you very much!

Caro studente,

vogliamo darle il benvenuto e farle sapere che lavoreremo insieme a lei affinché questo corso sia un successo. Il questionario che le presentiamo è stato progettato per raccogliere informazioni su coloro che partecipano ai corsi, sulle loro passate esperienze di e-learning e sul tipo di supporto di cui necessitano. Le sue risposte ci aiuteranno a sviluppare i nostri corsi per il futuro e a supportare l'apprendimento attuale. Si prenda il tempo necessario per completare le sei sezioni del questionario. Una volta riempito, le raccomandiamo di tenerne una copia per se e di inviarne una al suo tutor. Siamo interessati a comprendere le sue aspettative, i suoi obiettivi e interessi, quindi la preghiamo di rispondere onestamente.

E' nostra intenzione utilizzare i dati raccolti a scopo valutativo; i questionari sono anonimi. Grazie mille!

Cher apprenant,

Bienvenue dans ce cours!

Nous ferons tout notre possible pour que notre travail avec vous soit un succès. Ce questionnaire a été conçu pour nous fournir des renseignements sur les personnes participant à ce cours, leur expérience préalable de l'apprentissage en ligne et leurs éventuels besoins en supports d'apprentissage. Vos réponses nous permettront d'élaborer nos futurs cours et de vous apporter tout le soutien nécessaire dans votre apprentissage actuel.

Prenez tout le temps nécessaire pour compléter les six sections de ce questionnaire. Lorsque vous aurez terminé, nous vous prions de remettre le questionnaire complété à votre tuteur et vous conseillons d'en conserver une copie. Vos attentes, vos objectifs et vos inquiétudes nous intéressent, c'est pourquoi nous vous demandons la franchise la plus totale. Nous envisageons également d'utiliser les renseignements concernant votre groupe à des fins d'évaluation. Aucune personne ne sera identifiée individuellement.

Merci beaucoup! 


\section{Kontext des Kurses}

Bitte schreiben Sie Ihre Antworten in die rechte Spalte.

\section{Context of the course}

Please write your responses in the boxes on the right side.

\section{Contesto del corso}

Per favore scrivi le tue risposte negli spazi grigi a destra.

1. Contexte du cours

Veuillez inscrire vos réponses dans les cases de droite.

\begin{tabular}{|l|l}
\hline Fragen & Antworten \\
Questions & Response \\
Domande & Risposte \\
Questions & Réponses
\end{tabular}

1a) Name des Kurses?

1a) What is the title of your course?

1a) Qual è il titolo del suo corso?

1a) Quel est l'intitulé de votre cours ?

1b) Wo findet der Kurs statt?

1b) Where does the course take place?

1b) Dove si svolge il corso?

1b) Où le cours a-t-il lieu ?

1c) Das heutige Datum?

1c) What is today's date?

1c) Qual è la data di oggi?

1c) Quelle est la date d'aujourd'hui ? 


\section{Angaben zur Person}

2. Personal Characteristics of learners

2. Scheda personale dello studente

2. Renseignements personnels sur les apprenants

\begin{tabular}{|l|l|}
\hline Fragen & Antworten \\
Questions & Response \\
Domande & Risposte \\
Questions & Réponses \\
\hline
\end{tabular}

2a) Ihr Name oder ein Codename

Für eine erneute Befragung zu einem späteren Zeitpunkt möchten wir Sie gerne wieder erkennen, gleichzeitig möchten wir jedoch Ihre Anonymität wahren. Deshalb bitten wir Sie, einen Codenamen bzw. eine Codenummer anzugeben. Bitte geben Sie nacheinander den Geburtstag und den Geburtsmonat von zwei Menschen an, die Ihnen besonders nahe stehen.

Hier ein Beispiel:

Partner 01.03. (Geburtstag. Geburtsmonat) Mutter 12.12. (Geburtstag. Geburtsmonat) Der Codename wäre demnach: 01031212

2a) Your name or codename?

2a) II vostro nome o pseudonimo?

2a) Quel est votre nom ou votre pseudonyme?

2b) Wie alt sind Sie?

2b) How old are you?

2b) Quanti anni ha?

2b) Quel âge avez-vous ? 
2c) Welches Geschlecht haben Sie?

2c) What is your sex?

2c) Sesso:

2c) Quel est votre sexe ?
Oännlich

O Weiblich

O Male

O Female

OMaschio

OFemmina

O Masculin

O Féminin 2d) Haben Sie Kinder?

2d) Do you have children?

2d) Ha figli?

2d) Avez-vous des enfants ?
O Ja
O Nein
O Yes
O No
$\mathrm{OSi}$
O No
O Oui
O Non

2e) Welche Nationalität haben Sie?

2e) What is your nationality?

2e) Di che nazionalità è?

2e) Quelle est votre nationalité ?

2f) Welchen höchsten Allgemeinbildenden Abschluss haben Sie?

2f) What is your highest educational qualification?

2f) Quale titolo di studio/istruzione?

2f) Quel est votre niveau d'étude ?

2g) Welchen Beruf haben Sie?

$2 \mathrm{~g}$ ) What is your current job title or occupation?

$2 \mathrm{~g})$ Qual è il suo grado di professione?

$2 \mathrm{~g})$ Quelle est votre profession? 
2h) In welcher Einrichtung/Firma oder

in welchem Betrieb arbeiten Sie?

2h) In which institution do you work?

2h) Presso quale istituzione lavora?

2h) Quel est l'intitulé de votre emploi actuel ?

Die Beantwortung der folgenden Fragen ist freiwillig. Wir stellen diese Fragen, weil wir etwas mehr über Ihre persönlichen Lern-Umstände wenn Sie zu Hause oder am Arbeitsplatz sind erfahren wollen (je nachdem, wo Sie lernen).

Please feel to answer the following questions. We ask these questions because we want to know about various issues that may affect your learning either at your home or at your work place. This information will help us adapt the course to suit the personal circumstances of learners

Per favore risponda alle seguenti domande. Le facciamo queste domande perché vogliamo sapere quali problemi possono incidere sul suo apprendimento sia a casa che sul posto di lavoro. Queste informazioni ci saranno utili per adattare il corso alle sue condizioni di apprendimento.

Merci de bien vouloir répondre aux questions suivantes. Nous posons ces questions car nous souhaiterions connaître les problèmes susceptibles d'affecter votre apprentissage à votre domicile ou sur votre lieu de travail. Ces informations nous aideront à adapter le cours aux cas personnels des apprenants.

\begin{tabular}{|l|l|}
\hline $\begin{array}{l}\text { 2i) Wo führen Sie elearning, d.h. elektro- } \\
\text { nisch gestütztes Lernen durch? }\end{array}$ & $\begin{array}{l}\text { O Zu Hause } \\
\text { O An meinem Arbeitsplatz } \\
\text { O Andere Orte (Bitte erläutern.) }\end{array}$ \\
\hline 2i) Where do you do your elearning? & $\begin{array}{l}\text { O At home } \\
\text { O At my work place } \\
\text { O Others (please specify): }\end{array}$ \\
\hline $\begin{array}{l}\text { 2i) Dove vorrebbe fare il corso e- } \\
\text { learning? }\end{array}$ & $\begin{array}{l}\text { O A casa } \\
\text { O Sul posto di lavoro } \\
\text { O Altro (specificare): }\end{array}$ \\
\hline $\begin{array}{l}\text { 2i) Où souhaitez-vous suivre votre ap- } \\
\text { prentissage en ligne ? }\end{array}$ & $\begin{array}{l}\text { O À mon domicile } \\
\text { O Sur mon lieu de travail } \\
\text { O Ailleurs (précisez) : }\end{array}$ \\
\hline
\end{tabular}

2j) Wieviel Zeit haben Sie in der Regel pro Woche zu Hause für elearning?

(Zutreffendes bitte ankreuzen.)

\begin{tabular}{|l|l|l|}
\hline O Ich habe & O Ich habe & O Ich habe \\
zu Hause & zu Hause & zu Hause \\
sehr viel & viel Zeit & wenig Zeit \\
Zeit & für elearn- & für elearn- \\
für elearn- & ing. & ing. \\
ing. & & \\
\hline
\end{tabular}

O Ich habe
zu Hause
gar keine
Zeit
für elearn-
ing.


2j) How do you feel about your personal situation at home concerning the time available for elearning? (Please tick the relevant statement)

2j) Considerando la sua situazione personale a casa, quanto tempo pensa di avere per l'e-learning ? (con il pc ed il collegamento in rete) (segna l'affermazione che ritieni più adatta)

2j) Comment voyez-vous votre situation personnelle à votre domicile en ce qui concerne le temps dont vous disposez pour l'apprentissage en ligne ? (Veuillez cocher la réponse correspondante)
O I have

A lot of time available at home for my elearning

$\square$ Ho molto tempo a casa per l' e-learning.

\section{O I have} Some time available at home for my elearning
O I have Little time available at home for my elearning

$\square \mathrm{Ho} a b-$ bastanza tempo a casa per l' e-learning.

O Je dispose de beaucoup de temps à mon domicile pour mon apprentissage en ligne
O Je dispose d'un certain temps à mon domicile pour mon apprentissage en ligne e-learning.
O Je dispose de peu de temps à mon domicile pour mon apprentissage en ligne
I have No time available at home for my elearning

Non ho tempo a casa per l' e-learning.
O Je ne dispose pas de temps à mon domicile pour mon apprentissage en ligne

\begin{tabular}{|l|l|}
\hline $\begin{array}{l}\text { 2k) Wieviele Stunden haben Sie zu Hau- } \\
\text { se pro Woche für elearning? }\end{array}$ & $\begin{array}{l}\text { Ich habe ..... Stunden pro Woche zu Hause zur Verfü- } \\
\text { gung für elearning. }\end{array}$ \\
\hline $\begin{array}{l}\text { 2k) How many hours per week do you } \\
\text { have for your elearning? }\end{array}$ & I have ......... hours per week at home for elearning. \\
\hline $\begin{array}{l}\text { 2k) Quante ore la settimana ha per l' e- } \\
\text { learning? (con il pc ed il collegamento in } \\
\text { rete) }\end{array}$ & Ho ....... ore la settimana per l' e-learning. \\
\hline $\begin{array}{l}\text { 2k) De combien d'heures hebdomadaires } \\
\text { disposez-vous pour votre apprentissage } \\
\text { en ligne? }\end{array}$ & $\begin{array}{l}\text { Je dispose de ........ heures hebdomadaires à mon do- } \\
\text { micile pour mon apprentissage en ligne. }\end{array}$ \\
\hline
\end{tabular}

\begin{tabular}{|l|l|}
\hline $\begin{array}{l}\text { 2l) Gibt es zu Hause irgendwelche Ein- } \\
\text { schränkungen, wenn Sie mit dem Compu- } \\
\text { ter arbeiten wollen? }\end{array}$ & $\begin{array}{l}\text { O Nein } \\
\text { O Ja (Bitte erläutern): }\end{array}$ \\
\hline $\begin{array}{l}\text { 2l) Are there any restrictions at home with } \\
\text { regard to using a computer } \\
\begin{array}{l}\text { (e.g. no computer at home; other family } \\
\text { members have priority) }\end{array}\end{array}$ & $\begin{array}{l}\text { O No } \\
\text { O Yes (please specify): }\end{array}$ \\
\hline $\begin{array}{l}\text { 2l) Ci sono limitazioni a casa riguardo } \\
\text { all'uso del computer } \\
\text { (es. non ha il computer a casa; altri mem- } \\
\text { bri della famiglia hanno la priorità)? }\end{array}$ & $\square$ No (specificare): \\
\hline
\end{tabular}


2I) Existe-t-il des contraintes à votre domicile concernant l'utilisation d'un ordinateur?

Non

(par exemple pas d'ordinateur; d'autres membres de la famille sont prioritaires)

Oui (précisez) :

\begin{tabular}{|c|c|c|c|c|}
\hline $\begin{array}{l}\text { 2m) Wieviel Zeit haben Sie, um an die- } \\
\text { sem Kurs teilzunehmen? } \\
\text { (Zutreffendes bitte ankreuzen.) }\end{array}$ & $\begin{array}{l}\text { O Ich habe } \\
\text { sehr viel } \\
\text { Zeit, } \\
\text { um an die- } \\
\text { sem Kurs } \\
\text { teilzu- } \\
\text { nehmen } \\
\end{array}$ & $\begin{array}{l}\text { I Ich habe } \\
\text { viel Zeit, } \\
\text { um an die- } \\
\text { sem Kurs } \\
\text { teilzu- } \\
\text { nehmen. }\end{array}$ & $\begin{array}{l}\text { Ich habe } \\
\text { wenig Zeit, } \\
\text { um an die- } \\
\text { sem Kurs } \\
\text { teilzu- } \\
\text { nehmen. }\end{array}$ & $\begin{array}{l}\text { O Ich habe } \\
\text { gar keine } \\
\text { Zeit, } \\
\text { um an die- } \\
\text { sem Kurs } \\
\text { teilzu- } \\
\text { nehmen. }\end{array}$ \\
\hline $\begin{array}{l}2 \mathrm{~m}) \text { How do you feel about your personal } \\
\text { situation at home concerning the time } \\
\text { available for taking part in this course? } \\
\text { (Please tick the relevant statement) }\end{array}$ & $\begin{array}{l}\text { I have } \\
\text { A lot of time } \\
\text { available at } \\
\text { home for } \\
\text { taking part } \\
\text { in this } \\
\text { course }\end{array}$ & $\begin{array}{l}\text { O I have } \\
\text { Some time } \\
\text { available at } \\
\text { home for } \\
\text { taking part } \\
\text { in this } \\
\text { course }\end{array}$ & $\begin{array}{l}\text { I have } \\
\text { Little time } \\
\text { available at } \\
\text { home for } \\
\text { taking part } \\
\text { in this } \\
\text { course }\end{array}$ & $\begin{array}{l}\text { I I have } \\
\text { No time } \\
\text { available at } \\
\text { home for } \\
\text { taking part } \\
\text { in this } \\
\text { course }\end{array}$ \\
\hline $\begin{array}{l}2 \mathrm{~m} \text { ) Considerando la sua situazione per- } \\
\text { sonale a casa, quanto tempo pensa di } \\
\text { avere per seguire questo corso? (segna } \\
\text { l'affermazione che ritieni più adatta) }\end{array}$ & $\begin{array}{l}\square \text { Ho molto } \\
\text { tempo a } \\
\text { casa per } \\
\text { seguire } \\
\text { questo cor- } \\
\text { so }\end{array}$ & $\begin{array}{l}\square \text { Ho tem- } \\
\text { po abba- } \\
\text { stanza a } \\
\text { casa per } \\
\text { seguire } \\
\text { questo cor- } \\
\text { so }\end{array}$ & $\begin{array}{l}\square \text { Ho poco } \\
\text { tempo a } \\
\text { casa per } \\
\text { seguire } \\
\text { questo cor- } \\
\text { so }\end{array}$ & $\begin{array}{l}\square \text { Non ho } \\
\text { tempo a } \\
\text { casa per } \\
\text { seguire } \\
\text { questo cor- } \\
\text { so }\end{array}$ \\
\hline $\begin{array}{l}2 \mathrm{~m}) \text { Quelle est votre situation personnelle } \\
\text { à votre domicile concernant le temps dont } \\
\text { vous disposez pour participer à ce } \\
\text { cours? (Veuillez cocher la réponse cor- } \\
\text { respondante) }\end{array}$ & $\begin{array}{l}\text { O Je dis- } \\
\text { pose } \\
\text { de beau- } \\
\text { coup de } \\
\text { temps à } \\
\text { mon domi- } \\
\text { cile pour } \\
\text { participer à } \\
\text { ce cours }\end{array}$ & $\begin{array}{l}\text { O Je dis- } \\
\text { pose } \\
\text { d'un certain } \\
\text { temps à } \\
\text { mon domi- } \\
\text { cile pour } \\
\text { participer à } \\
\text { ce cours }\end{array}$ & $\begin{array}{l}\text { O Je dis- } \\
\text { pose } \\
\text { de peu de } \\
\text { temps à } \\
\text { mon domi- } \\
\text { cile pour } \\
\text { participer à } \\
\text { ce cours }\end{array}$ & $\begin{array}{l}\text { Je ne } \\
\text { dispose } \\
\text { pas de } \\
\text { temps à } \\
\text { mon domi- } \\
\text { cile pour } \\
\text { participer à } \\
\text { ce cours }\end{array}$ \\
\hline
\end{tabular}

2n) Wieviele Stunden haben Sie pro Woche zur Verfügung, um an diesem Kurs teilzunehmen?

2n) How many hours per week do you have at home for taking part in this course?

2n) Quante ore la settimana ha a casa per seguire tutti gli elementi di questo corso?
Ich habe ..... Stunden pro Woche zur Verfügung, um an diesem Kurs teilzunehmen.

I have hours per week at home for taking part in this course.

Ho ....... ore la settimana a casa per seguire questo corso. 
2n) De combien d'heures hebdomadaires disposez-vous à votre domicile pour participer à ce cours ?
Je dispose de heures hebdomadaires à mon domicile pour participer à ce cours.

\begin{tabular}{|c|c|c|c|c|}
\hline $\begin{array}{l}\text { 2o) Wieviel Zeit haben Sie in der Regel } \\
\text { pro Woche an Ihrem Arbeitsplatz, die } \\
\text { Sie für elearning nutzen können? } \\
\text { Bitte geben Sie Ihr persönliches Empfin- } \\
\text { den an. } \\
\text { (Zutreffendes bitte ankreuzen.) }\end{array}$ & $\begin{array}{l}\text { O Ich habe } \\
\text { an meinem } \\
\text { Arbeitsplatz } \\
\text { sehr viel } \\
\text { Zeit } \\
\text { für elearn- } \\
\text { ing. }\end{array}$ & $\begin{array}{l}\text { O Ich habe } \\
\text { an meinem } \\
\text { Arbeitsplatz } \\
\text { viel Zeit } \\
\text { für elearn- } \\
\text { ing. }\end{array}$ & $\begin{array}{l}\text { O Ich habe } \\
\text { an meinem } \\
\text { Arbeitsplatz } \\
\text { wenig Zeit } \\
\text { für elearn- } \\
\text { ing. }\end{array}$ & $\begin{array}{l}\text { O Ich habe } \\
\text { an meinem } \\
\text { Arbeitsplatz } \\
\text { gar keine } \\
\text { Zeit } \\
\text { für elearn- } \\
\text { ing. }\end{array}$ \\
\hline $\begin{array}{l}\text { 2o) How do you feel about your personal } \\
\text { situation at your working place concerning } \\
\text { the time available for elearning? (Please } \\
\text { tick the relevant statement) }\end{array}$ & $\begin{array}{l}\text { O I have } \\
\text { A lot of time } \\
\text { available at } \\
\text { my working } \\
\text { place for my } \\
\text { elearning }\end{array}$ & $\begin{array}{l}\text { O I have } \\
\text { Some time } \\
\text { available at } \\
\text { my working } \\
\text { place for my } \\
\text { elearning }\end{array}$ & $\begin{array}{l}\text { O I have } \\
\text { Little time } \\
\text { available at } \\
\text { my working } \\
\text { place for my } \\
\text { elearning }\end{array}$ & $\begin{array}{l}\text { O I have } \\
\text { No time } \\
\text { available at } \\
\text { my working } \\
\text { place for my } \\
\text { elearning }\end{array}$ \\
\hline $\begin{array}{l}\text { 2o) Considerando la sua situazione per- } \\
\text { sonale sul posto di lavoro, quanto tempo } \\
\text { pensa di avere per l'e-learning ? (con il pc } \\
\text { ed il collegamento in rete) (segna } \\
\text { l'affermazione che ritieni più adatta) }\end{array}$ & $\begin{array}{l}\text { Ho molto } \\
\text { tempo sul } \\
\text { lavoro per } \\
\text { l'e-learning }\end{array}$ & $\begin{array}{l}\text { O Ho abba- } \\
\text { stanza tem- } \\
\text { po sul lavo- } \\
\text { ro per l' e- } \\
\text { learning }\end{array}$ & $\begin{array}{l}\text { Ho poco } \\
\text { tempo sul } \\
\text { lavoro per } \\
\text { l'e-learning }\end{array}$ & $\begin{array}{l}\text { O Non ho } \\
\text { tempo sul } \\
\text { lavoro per } \\
\text { l'e-learning }\end{array}$ \\
\hline $\begin{array}{l}\text { 2o) Quelle est votre situation personnelle } \\
\text { sur votre lieu de travail concernant le } \\
\text { temps dont vous disposez pour l'appren- } \\
\text { tissage en ligne? (Veuillez cocher la ré- } \\
\text { ponse correspondante) }\end{array}$ & $\begin{array}{l}\text { O Je dis- } \\
\text { pose } \\
\text { de beau- } \\
\text { coup de } \\
\text { temps sur } \\
\text { mon lieu de } \\
\text { travail pour } \\
\text { mon ap- } \\
\text { prentissage } \\
\text { en ligne }\end{array}$ & $\begin{array}{l}\text { O Je dis- } \\
\text { pose } \\
\text { d'un certain } \\
\text { temps sur } \\
\text { mon lieu de } \\
\text { travail pour } \\
\text { mon ap- } \\
\text { prentissage } \\
\text { en ligne }\end{array}$ & $\begin{array}{l}\text { O Je dis- } \\
\text { pose } \\
\text { de peu de } \\
\text { temps sur } \\
\text { mon lieu de } \\
\text { travail pour } \\
\text { mon ap- } \\
\text { prentissage } \\
\text { en ligne }\end{array}$ & $\begin{array}{l}\text { O Je ne } \\
\text { dispose } \\
\text { pas de } \\
\text { temps sur } \\
\text { mon lieu de } \\
\text { travail pour } \\
\text { mon ap- } \\
\text { prentissage } \\
\text { en ligne }\end{array}$ \\
\hline
\end{tabular}

2p) Wieviele Stunden haben Sie an Ihrem Arbeitsplatz pro Woche für elearning?

2p) How many hours per week do you have for your elearning at your working place?

$2 p)$ Quante ore la settimana ha per l'elearning sul posto di lavoro?

$2 p)$ De combien d'heures hebdomadaires disposez-vous pour votre apprentissage en ligne sur votre lieu de travail ?
Ich habe ..... Stunden pro Woche an meinem Arbeitsplatz zur Verfügung für elearning.

I have hours per week at my working place for elearning.

Ho ore la settimana per l' e-learning sul posto di lavoro.

Je dispose de heures hebdomadaires sur mon lieu de travail pour l'apprentissage en ligne. 


\begin{tabular}{|l|l|}
\hline $\begin{array}{l}\text { 2q) Gibt es an Ihrem Arbeitsplatz ir- } \\
\text { gendwelche Einschränkungen, wenn Sie } \\
\text { mit dem Computer arbeiten wollen? }\end{array}$ & $\begin{array}{l}\text { O Nein } \\
\text { O Ja (Bitte erläutern): }\end{array}$ \\
\hline $\begin{array}{l}\text { 2q) Are there any restrictions at your } \\
\text { working place with regard to using a } \\
\text { computer }\end{array}$ & $\begin{array}{l}\text { O No } \\
\text { O Yes (please specify): }\end{array}$ \\
\hline $\begin{array}{l}\text { 2q) Ci sono limitazioni sul posto di lavoro } \\
\text { riguardo l'uso del computer? } \\
\text { (es. Può usare il computer solo per que- } \\
\text { stioni di lavoro; non ci sono computer, c'è } \\
\text { un firewall, il livello di accesso ad internet, } \\
\text { ecc.) }\end{array}$ & $\square$ Si (specificare): \\
\hline $\begin{array}{l}\text { 2q) Existe-t-il des contraintes sur votre } \\
\text { lieu de travail concernant l'utilisation d'un } \\
\text { ordinateur ? }\end{array}$ & O Non \\
\hline
\end{tabular}

\begin{tabular}{|l|l|}
\hline $\begin{array}{l}\text { 2r) Wieviele Stunden bekommen Sie von } \\
\text { Ihrem Arbeitgeber pro Woche, um an } \\
\text { diesem Kurs teilzunehmen? }\end{array}$ & $\begin{array}{l}\text { Ich bekomme ..... Stunden pro Woche von meinem Ar- } \\
\text { beitgeber, um an diesem Kurs teilzunehmen. }\end{array}$ \\
\hline $\begin{array}{l}\text { 2r) How many hours per week do you } \\
\text { have for taking part in this course at your } \\
\text { working place? }\end{array}$ & $\begin{array}{l}\text { I have ........ hours per week at my working place for } \\
\text { taking part in this course. }\end{array}$ \\
\hline $\begin{array}{l}\text { 2r) Quante ore la settimana ha sul posto } \\
\text { di lavoro per seguire tutti gli elementi di } \\
\text { questo corso? }\end{array}$ & $\begin{array}{l}\text { Ho ....... ore la settimana sul posto di lavoro per seguire } \\
\text { questo corso. }\end{array}$ \\
\hline $\begin{array}{l}\text { 2r) De combien d'heures hebdomadaires } \\
\text { disposez-vous pour participer à ce cours } \\
\text { sur votre lieu de travail ? }\end{array}$ & $\begin{array}{l}\text { Je dispose de ......... heures hebdomadaires sur mon lieu } \\
\text { de travail pour participer à ce cours. }\end{array}$ \\
\hline
\end{tabular}




\section{Lernerfahrungen in diesem Kurs}

3. Learning experiences in this course

3. Esperienze di apprendimento in questo corso

3. Expériences en termes d'apprentissage de ce cours

Im Folgenden sind einige Lehrmethoden aufgeführt, die Sie in diesem Kurs erleben. Bitte geben Sie an, wie Ihnen diese Methoden gefallen (bitte ankreuzen, was für Sie zutrifft).

Several learning methods that you may have expereinced during this course are listed below. Please indicate how you like the single methods (tick one box as appropriate).

Di seguito sono elencati alcuni metodi di apprendimento che ha incontrato in questo corso. Per favore indichi come considera ciascun metodo (segna la casella che ritieni più adatta).

Le tableau ci-dessous présente une liste d'outils et méthodes pédagogiques qui ont été utilisés pendant cette formation. Veuillez indiquer votre degré d'appréciation de chacun d'entre eux (cochez la case correspondante).

\begin{tabular}{|c|c|c|c|c|c|}
\hline $\begin{array}{l}\text { 3a) Präsenzunterricht } \\
\text { (Das Lernen findet in einen } \\
\text { Klassenraum statt und } \\
\text { eine Lehrperson ist anwe- } \\
\text { send.) }\end{array}$ & $\begin{array}{l}O \text { Gefällt } \\
\text { mir sehr } \\
++\end{array}$ & $\begin{array}{l}\text { O Gefällt } \\
\text { mir } \\
+\end{array}$ & $\begin{array}{l}\text { O Finde ich } \\
\text { mittelmäßig } \\
\text { o }\end{array}$ & $\begin{array}{l}\bigcirc \text { Gefällt } \\
\text { mir nicht } \\
\text { - }\end{array}$ & $\begin{array}{l}\text { O Gefällt mir } \\
\text { gar nicht } \\
--\end{array}$ \\
\hline \multicolumn{6}{|l|}{ Gründe für Ihre Antwort: } \\
\hline $\begin{array}{l}\text { 3a) In-person attendance } \\
\text { (E.g. where we meet in a } \\
\text { classroom to learn) }\end{array}$ & $\begin{array}{l}\text { O I like it very } \\
\text { much } \\
++\end{array}$ & $\begin{array}{l}\text { O I like } \\
\text { it } \\
+\end{array}$ & $\begin{array}{l}\text { O It`s OK } \\
\text { for me } \\
0\end{array}$ & $\begin{array}{l}\text { I do not } \\
\text { like it } \\
\text { - }\end{array}$ & $\begin{array}{l}\text { O I do not like it } \\
\text { at all } \\
\text {-- }\end{array}$ \\
\hline \multicolumn{6}{|l|}{ Reasons for your answer: } \\
\hline $\begin{array}{l}\text { 3a) Presenza in aula } \\
\text { (Es. lezioni frontali in clas- } \\
\text { se) }\end{array}$ & $\begin{array}{l}\square \text { Mi piace } \\
\text { molto } \\
++\end{array}$ & $\begin{array}{l}\square \mathrm{Mi} \\
\text { piace } \\
+ \\
\end{array}$ & $\begin{array}{l}\square \text { Per me } \\
\text { va bene } \\
\text { o }\end{array}$ & $\begin{array}{l}\square \text { Non mi } \\
\text { piace } \\
-\end{array}$ & $\begin{array}{l}\square \text { Non mi pia- } \\
\text { ce affatto } \\
\text {-- }\end{array}$ \\
\hline
\end{tabular}




\begin{tabular}{|c|c|c|c|c|c|}
\hline $\begin{array}{l}\text { 3a) Enseignement en } \\
\text { présentiel } \\
\text { (réunion étudiant- } \\
\text { enseignant en salle pour } \\
\text { l'enseignement) }\end{array}$ & $\begin{array}{l}\text { O J'ai beau- } \\
\text { coup apprécié }\end{array}$ & $\begin{array}{l}\text { O J'ai } \\
\text { apprécié }\end{array}$ & $\begin{array}{l}\text { Cela } \\
\text { m'était égal }\end{array}$ & $\begin{array}{l}\text { O Je n'ai } \\
\text { pas ap- } \\
\text { précié }\end{array}$ & $\begin{array}{l}\text { Je n'ai pas } \\
\text { du tout apprécié }\end{array}$ \\
\hline \multicolumn{6}{|l|}{ Commentaires : } \\
\hline \multicolumn{6}{|c|}{ 3b) Was gefällt Ihnen besonders am bzw. am Präsenzunterricht? } \\
\hline \multicolumn{6}{|c|}{ 3b) What do you like especially about in-person attendance? } \\
\hline \multicolumn{6}{|c|}{ 3b) Che cosa le piace di più della presenza in aula? } \\
\hline \multicolumn{6}{|c|}{ 3b) Qu'avez-vous particulièrement apprécié dans l'enseignement en présentiel ? } \\
\hline
\end{tabular}

3c) Was bereitet Ihnen Schwierigkeiten beim Präsenzunterricht?

3c) What causes difficulties with in-person attendance?

3c) Quali difficoltà comporta la presenza in aula?

3c) Quels ont été vos problèmes avec l'enseignement en présentiel ?

\begin{tabular}{|c|c|c|c|c|c|}
\hline $\begin{array}{l}\text { 3d) Fernunterricht } \\
\text { (Materialien und Aufgaben } \\
\text { werden zugeschickt und zu } \\
\text { Hause bearbeitet.) }\end{array}$ & $\begin{array}{l}\text { G Gefällt } \\
\text { mir sehr } \\
++\end{array}$ & $\begin{array}{l}\text { O Gefällt } \\
\text { mir } \\
+\end{array}$ & $\begin{array}{l}\text { Finde ich } \\
\text { mittelmäßig } \\
\text { o }\end{array}$ & $\begin{array}{l}\text { O Gefällt } \\
\text { mir nicht } \\
\text { - }\end{array}$ & $\begin{array}{l}\text { O Gefällt mir } \\
\text { gar nicht } \\
\text {-- }\end{array}$ \\
\hline \multicolumn{6}{|l|}{ Gründe für Ihre Antwort: } \\
\hline $\begin{array}{l}\text { 3d)Distance/open learn- } \\
\text { ing } \\
\text { (E.g. where you are given } \\
\text { paper-based materials to } \\
\text { work through at your own } \\
\text { place) }\end{array}$ & $\begin{array}{l}\text { O I like it very } \\
\text { much }\end{array}$ & $\begin{array}{l}\text { O I like } \\
\text { it }\end{array}$ & $\begin{array}{l}\text { O It`s OK } \\
\text { for me }\end{array}$ & $\begin{array}{l}\text { O I do not } \\
\text { like it }\end{array}$ & $\begin{array}{l}\text { O I do not like it } \\
\text { at all }\end{array}$ \\
\hline \multicolumn{6}{|l|}{ Reasons for your answer: } \\
\hline $\begin{array}{l}\text { 3d) Apprendimento a } \\
\text { distanza/open learning } \\
\text { (es.materiale inviato per } \\
\text { lavorare a casa) }\end{array}$ & $\begin{array}{l}\square \text { Mi piace } \\
\text { molto } \\
++ \\
\end{array}$ & $\begin{array}{l}\square \mathrm{Mi} \\
\text { piace } \\
+\end{array}$ & $\begin{array}{l}\square \text { Per me } \\
\text { va bene } \\
\text { o }\end{array}$ & $\begin{array}{l}\square \text { Non mi } \\
\text { piace } \\
-\end{array}$ & $\begin{array}{l}\square \text { Non mi pia- } \\
\text { ce affatto } \\
\text {-- }\end{array}$ \\
\hline \multicolumn{6}{|l|}{ Motivi della sua risposta: } \\
\hline $\begin{array}{l}\text { 3d) Apprentissage ou- } \\
\text { vert/à distance } \\
\text { (par exemple, lorsque l'on } \\
\text { vous a remis des docu- } \\
\text { ments pour travailler chez } \\
\text { vous) }\end{array}$ & $\begin{array}{l}\text { J'ai beau- } \\
\text { coup apprécié }\end{array}$ & $\begin{array}{l}\text { O J'ai } \\
\text { apprécié }\end{array}$ & $\begin{array}{l}\text { O Cela } \\
\text { m'était égal }\end{array}$ & $\begin{array}{l}\text { O Je n'ai } \\
\text { pas ap- } \\
\text { précié }\end{array}$ & $\begin{array}{l}\text { O Je n'ai pas } \\
\text { du tout apprécié }\end{array}$ \\
\hline
\end{tabular}


3e) Was gefällt Ihnen besonders am Fernunterricht?

3e) What do you like especially about distance/open learning?

3e) Che cosa le piace di più nell' apprendimento a distanza?

3e) Qu'avez-vous particulièrement apprécié dans l'apprentissage ouvert/à distance ?

3f) Was bereitet Ihnen Schwierigkeiten beim Fernunterricht?

3f) What causes difficulties with distance/open learning?

3f) Quali sono le difficoltà per lei nell' apprendimento a distanza?

3f) Quels ont été vos problèmes dans l'apprentissage ouvert/à distance ?

\begin{tabular}{|c|c|c|c|c|c|}
\hline $\begin{array}{l}\text { 3g) elearning } \\
\text { (Lernmaterialien und Akti- } \\
\text { vitäten werden elektro- } \\
\text { nisch/mit dem Computer } \\
\text { vermittelt.) }\end{array}$ & $\begin{array}{l}\text { O Gefällt } \\
\text { mir sehr } \\
++\end{array}$ & $\begin{array}{l}\text { ○ Gefällt } \\
\text { mir } \\
+\end{array}$ & $\begin{array}{l}\text { O Finde ich } \\
\text { mittelmäßig } \\
\text { o }\end{array}$ & $\begin{array}{l}\text { O Gefällt } \\
\text { mir nicht } \\
\text { - }\end{array}$ & $\begin{array}{l}\text { O Gefällt mir } \\
\text { gar nicht } \\
\text {-- }\end{array}$ \\
\hline \multicolumn{6}{|l|}{ Gründe für Ihre Antwort: } \\
\hline $\begin{array}{l}3 \mathrm{~g}) \text { Elearning } \\
\text { (E.g. where learning mate- } \\
\text { rials and activities are ac- } \\
\text { cessed electronically by } \\
\text { computer) }\end{array}$ & $\begin{array}{l}\text { O I like it very } \\
\text { much }\end{array}$ & $\begin{array}{l}\text { O I like } \\
\text { it }\end{array}$ & $\begin{array}{l}\text { It's OK } \\
\text { for me }\end{array}$ & $\begin{array}{l}\text { O I do not } \\
\text { like it }\end{array}$ & $\begin{array}{l}\text { O I do not like it } \\
\text { at all }\end{array}$ \\
\hline \multicolumn{6}{|l|}{ Reasons for your answer: } \\
\hline $\begin{array}{l}\text { 3d) E-learning } \\
\text { (Es. quando accede al } \\
\text { materiale didattico e alle } \\
\text { attività elettronicamente } \\
\text { tramite computer) }\end{array}$ & $\begin{array}{l}\square \text { Mi piace } \\
\text { molto }\end{array}$ & $\begin{array}{l}\square \mathrm{Mi} \\
\text { piace }\end{array}$ & $\begin{array}{l}\square \text { Per me } \\
\text { va bene }\end{array}$ & $\begin{array}{l}\square \text { Non mi } \\
\text { piace }\end{array}$ & $\begin{array}{l}\square \text { Non mi pia- } \\
\text { ce affatto }\end{array}$ \\
\hline \multicolumn{6}{|l|}{ Motivi della tua risposta: } \\
\hline $\begin{array}{l}\text { 3g) Apprentissage en } \\
\text { ligne } \\
\text { (lorsque les supports } \\
\text { d'enseignement étaient } \\
\text { disponibles sur internet) }\end{array}$ & $\begin{array}{l}\text { J'ai beau- } \\
\text { coup apprécié }\end{array}$ & $\begin{array}{l}\text { O J'ai } \\
\text { apprécié }\end{array}$ & $\begin{array}{l}\text { O Cela } \\
\text { m'était égal }\end{array}$ & $\begin{array}{l}\text { O Je n'ai } \\
\text { pas ap- } \\
\text { précié }\end{array}$ & $\begin{array}{l}\text { O Je n'ai pas } \\
\text { du tout apprécié }\end{array}$ \\
\hline
\end{tabular}

Commentaires: 
3h) Was gefällt Ihnen besonders am elearning?

3h) What do you like especially about elearning?

3h) Che cosa le piace di più dell'e-learning?

3h) Qu'avez-vous particulièrement apprécié dans l'enseignement en ligne ?

3i) Was bereitet Ihnen Schwierigkeiten beim elearning?

3i) What causes difficulties with elearning?

3i) Quali difficoltà comporta l'e-learning?

3i) Quels ont été vos problèmes dans l'enseignement en ligne ?

\begin{tabular}{|c|c|c|c|c|c|}
\hline $\begin{array}{l}\text { 3j) Projektunterricht } \\
\text { (Eine vorgegebene Aufga- } \\
\text { be wird auf selbstgewählte } \\
\text { Art und Weise gelöst und } \\
\text { es wird z.B. ein eigenes } \\
\text { Projekt im Rahmen eines } \\
\text { Kurses entwickelt.) }\end{array}$ & $\begin{array}{l}\text { Gefällt } \\
\text { mir sehr } \\
++\end{array}$ & $\begin{array}{l}\text { O Gefällt } \\
\text { mir } \\
+\end{array}$ & $\begin{array}{l}\text { F Finde ich } \\
\text { mittelmäßig } \\
\text { o }\end{array}$ & $\begin{array}{l}\text { O Gefällt } \\
\text { mir nicht } \\
\text { - }\end{array}$ & $\begin{array}{l}\text { O Gefällt mir } \\
\text { gar nicht } \\
--\end{array}$ \\
\hline \multicolumn{6}{|l|}{ Gründe für Ihre Antwort: } \\
\hline $\begin{array}{l}\text { 3j) Project learning } \\
\text { (E.g. where you have to } \\
\text { carry out your own project } \\
\text { as the basis of the course) }\end{array}$ & $\begin{array}{l}\text { O I like it very } \\
\text { much }\end{array}$ & $\begin{array}{l}\text { O I like } \\
\text { it }\end{array}$ & $\begin{array}{l}\text { O It`s OK } \\
\text { for me }\end{array}$ & $\begin{array}{l}\text { O I do not } \\
\text { like it }\end{array}$ & $\begin{array}{l}\text { O I do not like it } \\
\text { at all }\end{array}$ \\
\hline \multicolumn{6}{|l|}{ Reasons for your answer: } \\
\hline $\begin{array}{l}\text { 3j) Insegnamento proget- } \\
\text { to } \\
\text { (es. lavorare su un proprio } \\
\text { progetto) }\end{array}$ & $\begin{array}{l}\square \text { Mi piace } \\
\text { molto } \\
++\end{array}$ & $\begin{array}{l}\square \mathrm{Mi} \\
\text { piace } \\
+ \\
\end{array}$ & $\begin{array}{l}\square \text { Per me } \\
\text { va bene } \\
\text { o }\end{array}$ & $\begin{array}{l}\square \text { Non mi } \\
\text { piace } \\
-\end{array}$ & $\begin{array}{l}\square \text { Non mi pia- } \\
\text { ce affatto } \\
--\end{array}$ \\
\hline \multicolumn{6}{|l|}{ Motivi della sua risposta: } \\
\hline $\begin{array}{l}\text { 3j) Enseignement au pro- } \\
\text { jet } \\
\text { (p.ex. travailler sur des } \\
\text { projets/ être responsable) }\end{array}$ & $\begin{array}{l}\text { O J'ai beau- } \\
\text { coup apprécié }\end{array}$ & $\begin{array}{l}\text { O J'ai } \\
\text { apprécié }\end{array}$ & $\begin{array}{l}\text { O Cela } \\
\text { m'était égal }\end{array}$ & $\begin{array}{l}\text { O Je n'ai } \\
\text { pas ap- } \\
\text { précié }\end{array}$ & $\begin{array}{l}\text { Je n'ai pas } \\
\text { du tout apprécié }\end{array}$ \\
\hline
\end{tabular}


3k) Was gefällt Ihnen besonders am Projektunterricht?

3k) What do you like especially about project learning?

3k) Che cosa le piace nell' insegnamento progetto?

3k) Qu'avez-vous particulièrement apprécié dans l'enseignement au projet?

3I) Was bereitet Ihnen Schwierigkeiten beim Projektunterricht?

3I) What causes difficulties wit project learning?

31) Quali difficoltà comporta l'insegnamento progetto?

3l) Quels ont été vos problèmes dans l'enseignement au projet?

\begin{tabular}{|c|c|c|c|c|c|}
\hline $\begin{array}{l}3 \mathrm{~m} \text { ) Blended Learning } \\
\text { (Kombination von elearn- } \\
\text { ing und anderen Lehrfor- } \\
\text { men.) }\end{array}$ & $\begin{array}{l}\text { O Gefällt } \\
\text { mir sehr } \\
++\end{array}$ & $\begin{array}{l}\text { O Gefällt } \\
\text { mir } \\
+\end{array}$ & $\begin{array}{l}\text { F Finde ich } \\
\text { mittelmäßig } \\
\text { o }\end{array}$ & $\begin{array}{l}\text { O Gefällt } \\
\text { mir nicht } \\
\text { - }\end{array}$ & $\begin{array}{l}\text { O Gefällt mir } \\
\text { gar nicht } \\
--\end{array}$ \\
\hline \multicolumn{6}{|l|}{ Gründe für Ihre Antwort: } \\
\hline $\begin{array}{l}3 \mathrm{~m}) \text { Blended learning } \\
\text { (E.g. where any of the } \\
\text { methods mentioned above } \\
\text { were used concurrently } \\
\text { within one course }\end{array}$ & $\begin{array}{l}\text { O I like it very } \\
\text { much }\end{array}$ & $\begin{array}{l}\text { O I like } \\
\text { it }\end{array}$ & $\begin{array}{l}\text { O It`s OK } \\
\text { for me }\end{array}$ & $\begin{array}{l}\text { O I do not } \\
\text { like it }\end{array}$ & $\begin{array}{l}\text { O I do not like it } \\
\text { at all }\end{array}$ \\
\hline \multicolumn{6}{|l|}{ Reasons for your answer: } \\
\hline $\begin{array}{l}\text { 3m)Blended learning } \\
\text { WebLingu@ con tutor è un } \\
\text { corso 'blended learning'. } \\
\text { (Es. quando i metodi sopra } \\
\text { indicati sono utilizzati in } \\
\text { maniera integrata } \\
\text { all'interno del corso) }\end{array}$ & $\begin{array}{l}\square \text { Mi piace } \\
\text { molto }\end{array}$ & $\begin{array}{l}\square \mathrm{Mi} \\
\text { piace }\end{array}$ & $\begin{array}{l}\square \text { Per me } \\
\text { va bene }\end{array}$ & $\begin{array}{l}\square \text { Non mi } \\
\text { piace }\end{array}$ & $\begin{array}{l}\square \text { Non mi pia- } \\
\text { ce affatto }\end{array}$ \\
\hline \multicolumn{6}{|l|}{ Motivi della sua risposta: } \\
\hline $\begin{array}{l}3 \mathrm{~m}) \text { Blended Learning } \\
\text { (combination de plusieurs } \\
\text { méthodes à la fois) }\end{array}$ & $\begin{array}{l}\text { J J'ai beau- } \\
\text { coup apprécié }\end{array}$ & $\begin{array}{l}\text { O J'ai } \\
\text { apprécié }\end{array}$ & $\begin{array}{l}\text { O Cela } \\
\text { m'était égal }\end{array}$ & $\begin{array}{l}\text { O Je n'ai } \\
\text { pas ap- } \\
\text { précié }\end{array}$ & $\begin{array}{l}\text { Je n'ai pas } \\
\text { du tout apprécié }\end{array}$ \\
\hline \multicolumn{6}{|l|}{ Commentaires : } \\
\hline
\end{tabular}


3n) Was gefällt Ihnen besonders am Blended Learning?

3n) What do you like especially about blended learning?

3n) Che cosa le piace di più del blended learning?

3n) Qu'avez-vous particulièrement apprécié dans l'enseignement blended learning?

3o) Was bereitet Ihnen Schwierigkeiten beim Blended Learning?

3o) What causes difficulties with blended learning?

3o) Quali difficoltà comporta il blended learning ?

3o) Quels ont été vos problèmes dans l'enseignement blended learning? 


\section{Ressourcen für elearning, Fähigkeiten und Wissen im Umgang mit dem Computer}

\section{Resources for elearning computer skills and knowledge}

4. Risorse per l'e-learning - abilità e conoscenza dell'uso del computer.

4. Ressources en termes de compétences et connaissances informatiques pour l'apprentissas ligne

Mit den folgenden Fragen möchten wir Ihr PC-Wissen ermitteln. Bitte antworten Sie ganz ehrlich, wir wollen Ihren persönlichen Kenntnisstand ermitteln, um den Kurs nach Ihren Fähigkeiten zu gestalten (sie brauchen keine Bedenken zu haben, falls Sie noch nicht so geübt sind im Umgang mit dem Computer!).

The following questions aim to gather information about your computer knowledge and skills.

Le facciamo le seguenti domande per avere più informazioni sulle sue capacità e conoscenza dell'uso del computer.

Les questions suivantes nous permettront de mieux connaître vos acquis en termes de connaissances et de compétences en informatique. Répondez en toute franchise, nous ne cherchons qu'à évaluer les problèmes que vous avez pu rencontrer pendant la formation.

\begin{tabular}{|l|l|}
\hline $\begin{array}{l}\text { 4a) Haben Sie Erfahrung im Um- } \\
\text { gang mit Computern? }\end{array}$ & $\begin{array}{l}\text { O Ja, viel Erfahrung } \\
\text { O Ja, etwas Erfahrung } \\
\text { O Nein, keine Erfahrung }\end{array}$ \\
\hline $\begin{array}{l}\text { 4a) Do you have any previous expe- } \\
\text { rience of using a computer? }\end{array}$ & $\begin{array}{l}\text { O Yes, a lot } \\
\text { O Yes, a little } \\
\text { O No }\end{array}$ \\
\hline $\begin{array}{l}\text { 4a) Ha mai usato il computer prima } \\
\text { d'ora? }\end{array}$ & $\begin{array}{l}\text { O Si, molto } \\
\text { O Si, un po' } \\
\text { O No }\end{array}$ \\
\hline $\begin{array}{l}\text { 4a) Possédez-vous l'expérience de } \\
\text { l'utilisation d'un ordinateur? }\end{array}$ & $\begin{array}{l}\text { O Oui, une grande expérience } \\
\text { O Oui, une petite expérience } \\
\text { O Non }\end{array}$ \\
\hline
\end{tabular}

4b) Wofür nutzen Sie bisher Computer?

4b) What do you use a computer for?

4b) Per che cosa utilizza il computer

4b) À quelles fins utilisez-vous un ordinateur?

4c) Bitte schätzen Sie Ihre Fähigkeiten und Erfahrung im Umgang mit Folgendem ein:

4c) How much experience do you have in using the following software programmes?

4c) Quanta esperienza ha nell'uso dei seguenti software?

4c) Quelle est votre expérience en matière d'utilisation des logiciels suivants ?

\begin{tabular}{|c|c|c|c|c|c|}
\hline & $\begin{array}{c}\text { Ich habe } \\
\text { bereits viel } \\
\text { Erfahrung }\end{array}$ & $\begin{array}{c}\text { Ich habe be- } \\
\text { reits etwas } \\
\text { Erfahrung }\end{array}$ & $\begin{array}{c}\text { Ich habe } \\
\text { wenig Er- } \\
\text { fahrung }\end{array}$ & $\begin{array}{c}\text { Ich habe } \\
\text { keine Erfah- } \\
\text { rung }\end{array}$ & $\begin{array}{c}\text { Den Be- } \\
\text { griff kenne } \\
\text { ich nicht }\end{array}$ \\
\hline
\end{tabular}




\begin{tabular}{|c|c|c|c|c|c|}
\hline & $\begin{array}{l}\text { I have } \\
\text { a lot experi- } \\
\text { ence }\end{array}$ & $\begin{array}{l}\text { I have } \\
\text { some experi- } \\
\text { ence }\end{array}$ & $\begin{array}{l}\text { I have } \\
\text { a little expe- } \\
\text { rience }\end{array}$ & $\begin{array}{l}\text { I have } \\
\text { no experi- } \\
\text { ence }\end{array}$ & \\
\hline & $\begin{array}{c}\text { Ho molta } \\
\text { esperienza }\end{array}$ & $\begin{array}{l}\text { Ho qualche } \\
\text { esperienza }\end{array}$ & $\begin{array}{c}\text { Ho poca } \\
\text { esperienza }\end{array}$ & $\begin{array}{c}\text { Non ho } \\
\text { esperienza }\end{array}$ & \\
\hline & $\begin{array}{l}\text { J'ai beau- } \\
\text { coup d'ex- } \\
\text { périence }\end{array}$ & $\begin{array}{l}\text { J'ai une } \\
\text { certaine ex- } \\
\text { périence }\end{array}$ & $\begin{array}{c}\text { J'ai peu } \\
\text { d'ex- } \\
\text { périence }\end{array}$ & $\begin{array}{c}\text { Je n'ai } \\
\text { aucune ex- } \\
\text { périence }\end{array}$ & \\
\hline $\begin{array}{l}\text { Textverarbeitungs- } \\
\text { programme (z.B. Word) } \\
\text { Text programmes } \\
\text { (e.g. Word) } \\
\text { Programmi di scrittura } \\
\text { (es. Word) } \\
\text { Traitement de texte } \\
\text { (par exemple, Word) }\end{array}$ & 0 & 0 & 0 & 0 & 0 \\
\hline $\begin{array}{l}\text { Kalkulations-Software } \\
\text { (z.B. Excel) } \\
\text { Calculation Software } \\
\text { (E.g. Excel) } \\
\text { Fogli di Calcolo } \\
\text { (es. Excel) } \\
\text { Logiciel de calcul } \\
\text { (Par exemple, Excel) }\end{array}$ & 0 & 0 & 0 & 0 & 0 \\
\hline $\begin{array}{l}\text { Datenbanken } \\
\text { (z.B. Access oder dbase) } \\
\text { Databases } \\
\text { (Access, dbase) } \\
\text { Banche dati } \\
\text { (Access, dbase) } \\
\text { Bases de données } \\
\text { (Access, dbase) }\end{array}$ & 0 & 0 & 0 & 0 & 0 \\
\hline
\end{tabular}




\begin{tabular}{|c|c|c|c|c|c|}
\hline $\begin{array}{l}\text { Präsentations-Software } \\
\text { (z.B. Power Point) } \\
\text { Presentation software } \\
\text { (E.g. Power Point) } \\
\text { Software per presenta- } \\
\text { zioni } \\
\text { (es. Power Point) } \\
\text { Logiciel de présentation } \\
\text { (Par exemple, Power } \\
\text { Point) }\end{array}$ & 0 & 0 & 0 & 0 & 0 \\
\hline $\begin{array}{l}\text { Internet } \\
\text { (surfen, recherchieren) } \\
\text { Internet browsing } \\
\text { Navigazione in Internet } \\
\text { Navigateur Internet }\end{array}$ & 0 & 0 & 0 & 0 & 0 \\
\hline $\begin{array}{l}\text { E-Mail } \\
\text { E-Mailing } \\
\text { Posta Elettronica } \\
\text { Courrier électronique }\end{array}$ & 0 & 0 & 0 & 0 & 0 \\
\hline $\begin{array}{l}\text { Webdesign } \\
\text { Web designing } \\
\text { Web designing } \\
\text { Conception de pages } \\
\text { Web }\end{array}$ & 0 & 0 & 0 & 0 & 0 \\
\hline
\end{tabular}




\begin{tabular}{|c|c|c|c|c|c|}
\hline $\begin{array}{l}\text { Webpublishing } \\
\text { Web publishing } \\
\text { Web publishing } \\
\text { Publication de pages } \\
\text { Web }\end{array}$ & 0 & 0 & 0 & 0 & 0 \\
\hline Networking & 0 & 0 & 0 & 0 & 0 \\
\hline $\begin{array}{l}\text { Kommentare: } \\
\text { Comments: } \\
\text { Commenti: } \\
\text { Commentaires: }\end{array}$ & & & & & \\
\hline
\end{tabular}

\begin{tabular}{|l|l|}
\hline $\begin{array}{l}\text { 4d) Wo haben Sie Zugang zu einem Compu- } \\
\text { ter mit Internet-Anschluss, den Sie für den } \\
\text { Kurs nutzen? }\end{array}$ & $\begin{array}{l}\text { O Zu Hause } \\
\text { O Am Arbeitsplatz } \\
\text { O Andere Orte (bitte erläutern): }\end{array}$ \\
$\begin{array}{l}\text { (Bitte alle zutreffenden Antworten ankreu- } \\
\text { zen.) }\end{array}$ & $\begin{array}{l}\text { O At home } \\
\text { O At your working place } \\
\text { O Other places (Please specify) }\end{array}$ \\
\hline $4 d)$ & $\begin{array}{l}\text { O A casa } \\
\text { O Posto di lavoro } \\
\text { O Luoghi diversi }\end{array}$ \\
\hline $\begin{array}{l}\text { 4d) Dove ha la possibilità di usare internet } \\
\text { per il corso? }\end{array}$ & $\begin{array}{l}\text { O À mon domicile } \\
\text { O Sur mon lieu de travail } \\
\text { O Ailleurs (précisez): }\end{array}$ \\
\hline $\begin{array}{l}\text { 4d) Où avez-vous la possibilité d'utiliser } \\
\text { l'ordinateur pour le cours? }\end{array}$ & \\
\hline
\end{tabular}

4e) Was für einen Internet-Anschluss nutzen Sie für diesen Kurs?

\begin{tabular}{|l|}
\hline $4 e)$ What kind of internet access do you use? \\
\hline $4 e)$ Che tipo di collegamento a internet usa? \\
\hline
\end{tabular}

O Schnelle Telefonleitung (z.B. TDSL/ADSL)

$O$ Langsame Telefonleitung (Modem)

Weiß nicht

O High speed

O Low speed

O Don`t know

$\square$ Alta velocità

Bassa velocità

Non so 


\begin{tabular}{|l|l|}
\hline $\begin{array}{l}\text { 4e) Quel type de connexion Internet utilise- } \\
\text { rez-vous? }\end{array}$ & O Haut débit \\
& O Bas débit \\
& O Ne sait pas \\
\hline
\end{tabular}

\begin{tabular}{|c|c|}
\hline $\begin{array}{l}\text { 4f) Welche Zeit werden Sie wahrscheinlich } \\
\text { meistens für das elearning nutzen? } \\
\text { (Bitte ankreuzen.) }\end{array}$ & $\begin{array}{l}\text { Zwischen } 8 \text { und } 12 \text { Uhr } \\
\text { Zwischen } 12 \text { und } 18 \text { Uhr } \\
\text { Zwischen } 18 \text { und } 22 \text { Uhr } \\
\text { Nach } 22 \text { Uhr }\end{array}$ \\
\hline \multicolumn{2}{|l|}{ Bitte erläutern Sie die Gründe für Ihre Antwort: } \\
\hline $\begin{array}{l}\text { 4f) When do you most often spend time } \\
\text { elearning? }\end{array}$ & $\begin{array}{l}0 \\
0 \\
0 \\
0\end{array}$ \\
\hline \multicolumn{2}{|l|}{ Please describe the reasons for your answer. } \\
\hline $\begin{array}{l}\text { 4f) In quale momento della giornata prevede } \\
\text { di fare più spesso e-learning? }\end{array}$ & $\begin{array}{ll}\square & \text { Dalle ore } 8 \text { alle ore } 12 \\
\square & \text { Dalle ore } 12 \text { alle ore } 18 \\
\square & \text { Dalle ore } 18 \text { alle ore } 22 \\
\square & \text { Dopo le ore } 22\end{array}$ \\
\hline \multicolumn{2}{|l|}{ Perché?: } \\
\hline $\begin{array}{l}\text { 4f) À quel moment de la journée envisagez- } \\
\text { vous d'utiliser le plus souvent votre méthode } \\
\text { d'apprentissage en ligne? }\end{array}$ & $\begin{array}{l}\text { De } 8 \text { à } 12 \text { heures } \\
\text { De } 12 \text { à } 18 \text { heures } \\
\text { De } 18 \text { à } 22 \text { heures } \\
\text { Après } 22 \text { heures }\end{array}$ \\
\hline
\end{tabular}

\begin{tabular}{|c|c|c|c|c|c|c|}
\hline $\begin{array}{l}4 \mathrm{~g}) \text { Wieviel Zeit nutzen Sie pro } \\
\text { Woche für elearning? } \\
\text { (Bitte ankreuzen.) }\end{array}$ & $\begin{array}{l}\text { O } \\
\text { Mehr als } \\
10 \text { Stun- } \\
\text { den pro } \\
\text { Woche }\end{array}$ & $\begin{array}{l}\text { O } \\
\text { Zwischen } \\
5 \text { und } 10 \\
\text { Stunden } \\
\text { pro Woche }\end{array}$ & $\begin{array}{l}\text { O } \\
\text { Weniger als } 5 \\
\text { Stunden pro } \\
\text { Woche }\end{array}$ & \multicolumn{2}{|c|}{$\begin{array}{c}\text { O } \\
\text { Ich werde } \\
\text { jede Woche } \\
\text { neu ent- } \\
\text { scheiden }\end{array}$} & $\begin{array}{l}\text { O } \\
\text { Ich } \\
\text { weiß } \\
\text { es } \\
\text { nicht }\end{array}$ \\
\hline \multicolumn{7}{|l|}{ Kommentare: } \\
\hline $\begin{array}{l}4 \mathrm{~g}) \text { How much time per week } \\
\text { do you spend on your elearn- } \\
\text { ing programme? }\end{array}$ & $\begin{array}{l}\text { O } \\
\text { More than } \\
10 \text { hours } \\
\text { per week }\end{array}$ & $\begin{array}{l}\text { O } \\
\text { Between } 5 \\
\text { and } 10 \\
\text { hours } \\
\text { per week }\end{array}$ & $\begin{array}{c}\text { O } \\
\text { Less than } \\
5 \text { hours per } \\
\text { week }\end{array}$ & $\begin{array}{l}\text { O } \\
\text { I will de- } \\
\text { cide every } \\
\text { week }\end{array}$ & & $\begin{array}{l}\text { O } \\
\text { ave no } \\
\text { dea }\end{array}$ \\
\hline
\end{tabular}




\begin{tabular}{|c|c|c|c|c|c|}
\hline $\begin{array}{l}\text { 4g) Quanto tempo alla setti- } \\
\text { mana usa il suo program- } \\
\text { ma/piattaforma di e-learning? }\end{array}$ & $\begin{array}{l}\square \\
\text { Più di } 10 \\
\text { ore la set- } \\
\text { timana }\end{array}$ & $\begin{array}{l}\square \\
\text { Tra le } 5 \text { e } \\
\text { le } 10 \text { ore la } \\
\text { settimana }\end{array}$ & $\begin{array}{c}\square \\
\text { Meno di } \\
\text { cinque ore } \\
\text { la settimana }\end{array}$ & $\begin{array}{l}\square \\
\text { Deciderò } \\
\text { ogni volta }\end{array}$ & $\begin{array}{l}\square \\
\text { Non ho } \\
\text { idea }\end{array}$ \\
\hline \multicolumn{6}{|l|}{ Commenti: } \\
\hline $\begin{array}{l}\text { 4g) Combien de temps par } \\
\text { semaine utilisez-vous votre } \\
\text { programme d'apprentissage } \\
\text { en ligne }\end{array}$ & $\begin{array}{c}\bigcirc \\
\text { Plus de } \\
10 \text { heures } \\
\text { par se- } \\
\text { maine } \\
\end{array}$ & $\begin{array}{c}\quad 0 \\
\text { Entre } 5 \text { et } \\
10 \text { heures } \\
\text { par se- } \\
\text { maine } \\
\end{array}$ & $\begin{array}{c}\text { O } \\
\text { Moins de } \\
5 \text { heures } \\
\text { par semaine }\end{array}$ & $\begin{array}{c}\text { O } \\
\text { Je dé- } \\
\text { ciderai } \\
\text { chaque } \\
\text { semaine }\end{array}$ & $\begin{array}{l}\text { O } \\
\text { Je n'en ai } \\
\text { aucune } \\
\text { idée }\end{array}$ \\
\hline
\end{tabular}

\begin{tabular}{|l|l|}
\hline $\begin{array}{l}\text { 4h) Haben Sie jemanden, der Ihnen beim } \\
\text { elearning behilflich ist? }\end{array}$ & $\begin{array}{l}\text { O Ja } \\
\text { O Nein } \\
\text { O Weiß nicht }\end{array}$ \\
\hline $\begin{array}{l}\text { 4h) Do you have anyone who provides local } \\
\text { support for your e-learning? }\end{array}$ & $\begin{array}{l}\text { O Yes } \\
\text { O No } \\
\text { O Unsure }\end{array}$ \\
\hline $\begin{array}{l}\text { 4h) Ha qualcuno a livello locale che per l'e- } \\
\text { learning può aiutarla? }\end{array}$ & $\begin{array}{l}\square \text { Si } \\
\square \text { No } \\
\square \text { Non so }\end{array}$ \\
\hline $\begin{array}{l}\text { 4h) Une personne peut-elle vous apporter un } \\
\text { soutien à domicile pendant votre apprentis- } \\
\text { sage en ligne ? }\end{array}$ & $\begin{array}{l}\text { O Oui } \\
\text { O Non } \\
\text { O Ne suis pas sûr(e) }\end{array}$ \\
\hline
\end{tabular}

\begin{tabular}{|l|l|}
\hline 4i) Wenn Sie mit „Ja“ geantwortet haben, wer & O Freunde \\
hilft Ihnen? & O Partner \\
(Sie können mehrere Antworten ankreuzen.) & O Kollegen \\
& O Vorgesetzte \\
& O Andere Personen (Bitte erläutern.): \\
\hline $\begin{array}{l}\text { 4i) If you answered, "Yes”, who helps you? } \\
\text { (Please tick all the relevant answers) }\end{array}$ & O Friends \\
& O Partner \\
& O Colleagues \\
& O Officials \\
& O Others: (Please specify) \\
\hline $\begin{array}{l}\text { 4i) Se ha risposto "Si”, chi la può aiutare? } \\
\text { (segna la risposta che ritiene più adatta) }\end{array}$ & $\square$ Amici \\
& $\square$ Partner \\
& $\square$ Colleghi \\
& $\square$ Altri : (specificare) \\
\hline
\end{tabular}




\begin{tabular}{|l|l|}
\hline $\begin{array}{l}\text { 4i) Si "Oui", qui peut vous aider (Veuillez co- } \\
\text { cher toutes les réponses correspondantes) }\end{array}$ & O Amis \\
& O Partenaire \\
& O Collègues \\
& O Responsables \\
& O Autres : (Précisez) \\
\hline
\end{tabular}

\begin{tabular}{|l|l|}
\hline $\begin{array}{l}\text { 4j) Wenn Sie mit „Nein“ geantwortet haben, } \\
\text { brauchen Sie eventuell Hilfe? }\end{array}$ & $\begin{array}{l}\text { O Ja } \\
\text { O Nein } \\
\text { O Weiß nicht }\end{array}$ \\
\hline $\begin{array}{l}\text { 4j) If you answered, “No”, would you need } \\
\text { help? }\end{array}$ & $\begin{array}{l}\text { O Yes } \\
\text { O No } \\
\text { O Don`t know }\end{array}$ \\
\hline $4 j)$ Se ha risposto „No“, ha bisogno di aiuto? & $\begin{array}{l}\text { O Si } \\
\text { O No } \\
\text { O Non lo so }\end{array}$ \\
\hline $4 j)$ Si „Non“, avez-vous besoin d'aide? & $\begin{array}{l}\text { O Oui } \\
\text { O Non } \\
\text { O Je ne sais pas }\end{array}$ \\
\hline
\end{tabular}


5. Persönliche Motivation an diesem Kurs teilzunehmen, Erwartungen und Ziele

5. Personal motivation for doing this course, expectations and aims

5. Motivazioni personali, aspettative e obiettivi in relazione al corso

5. Motivations personnelles pour suivre ce cours, attentes et objectifs

\begin{tabular}{|c|c|}
\hline $\begin{array}{l}\text { 5a) Warum nehmen Sie an diesem Kurs teil? } \\
\text { (Bitte alle zutreffenden Antworten ankreu- } \\
\text { zen.) }\end{array}$ & $\begin{array}{l}\text { O Interesse am Thema } \\
\text { O Qualifikation } \\
\text { O Zertifikat } \\
\text { O Pflicht/Vorgabe } \\
\text { O Anderes (Bitte erläutern): }\end{array}$ \\
\hline 5a) Why did you join this course?: & $\begin{array}{l}\text { Interest in the topic } \\
\text { Q Qualification } \\
\text { O Certification } \\
\text { Obligation } \\
\text { Others (please specify): }\end{array}$ \\
\hline 5a) Perché segue questo corso? & $\begin{array}{l}\text { O Interesse al tema } \\
\text { Qualificazione } \\
\text { Certificato } \\
\text { Obbligo } \\
\text { Altro (specificare): }\end{array}$ \\
\hline 5a) Pourquoi participez-vous à ce cours? & $\begin{array}{l}\text { Intérêt thématique } \\
\text { Qualification } \\
\text { Certificat } \\
\text { Devoir } \\
\text { Autres (précisez): }\end{array}$ \\
\hline
\end{tabular}

5b) Welchen Lerngewinn versprechen Sie sich von diesem Kurs?

5b) What are your personal learning objectives?

5b) Quali sono i suoi principali obiettivi di apprendimento?

5b) Quels sont vos principaux objectifs d'apprentissage ?

5c) Welche Erwartungen haben Sie in Bezug auf diesen Kurs?

5c) What expectations do you have about the course?

5c) Quali sono le sue aspettative riguardo a questo corso?

5c) Qu'attendez-vous de ce cours ?

5d) Welche Befürchtungen haben Sie in Bezug auf diesen Kurs?

$5 d)$ What worries/fears do you have about the course?

5d) Quali sono le sue preoccupazioni o paure riguardo a questo corso?

$5 d)$ Quelles inquiétudes/craintes éprouvez-vous vis-à-vis de ce cours ? 


\section{Platz für Vorschläge, Kommentare und Fragen}

6. Open space for suggestions comments and questions

6. Spazio per suggerimenti, commenti e domande

6. Espace libre pour vos suggestions, commentaires et questions

6a) An welchen anderen Kursthemen hätten Sie Interesse?

6a) What other courses would you like to see offered in the future?

6a) Quali altri corsi di lingue le piacerebbe fossero offerti in futuro?

6a) Quels autres cours souhaiteriez-vous voir proposer à l'avenir?

6b) Wenn Sie noch Kommentare oder Fragen haben, die Sie uns in Bezug auf den Kurs mitteilen wollen, bitte nutzen Sie diesen Platz:

6b) If you have any other comments or questions that you would like to share regarding the forthcoming course please write them here.

6b) Se ha altri commenti o domande da condividere riguardo al corso che stai per iniziare, li riporta qui

6b) Indiquez ici tout commentaire ou question que vous souhaiteriez faire partager concernant le cours à venir.

Wir bedanken uns ganz herzlich dafür,

dass Sie sich die Zeit genommen haben,

den Fragebogen auszufüllen!

Thank you very much

for taking the time to complete this questionnaire!

Grazie mille per aver completato il questionario!

Merci beaucoup pour avoir pris le temps de répondre à ce questionnaire ! 


\subsection{Inventar für Lernen zum Abschluss eines Kurses}

Workgroup:

Filippo Del Casino, Jutta List-Ivanković, Corinne Rudelle, Colin Macduff

Translations:

English:

Bernice West, Neil Johnson

German:

Jutta List-Ivanković

Italian:

Filippo del Casino, Loredana Marasco

French:

Elisabeth Belarbi, Giséle Massol, Emeric Pividori 
Examples for introductions for the learners from different eL3-partners:

\section{German:}

Liebe Kursteilnehmerin, lieber Kursteilnehmer,

Mit diesem Fragebogen möchten wir Informationen über die Menschen erhalten, die unsere Kurse besuchen - also über Sie!

Wir sind interessiert an Ihren Lernbedingungen und Ihren Lernerfahrungen in diesem Kurs. Ihre Antworten werden uns bei der zukünftigen Kursplanung helfen.

Wir bitten Sie, uns einige Informationen über sich und Ihre Erfahrung mit den verschiedenen Lernmethoden in diesem Kurs zu geben. Wir werden Ihre Antworten nur für die Kursauswertung innerhalb unserer Einrichtung verwenden.

Der Fragebogen wurde in einem Netzwerk von Partnern aus Frankreich, Italien, Schottland und Deutschland ("blinc") im Rahmen des von der Europäischen Kommission geförderten Projekts „eL3“ entwickelt. Das Hauptziel des Projektes „eL3“ (eLearning Project Cluster for Third System Organisations in Europe) ist die Verbreitung von gemischten Lernformen (Blended Learning) in Betrieben der europäischen Gesundheits- und Sozialwirtschaft.

Informationen über das Projekt erhalten Sie auf unserer Homepage www.blinc-eu.org . Wir freuen uns über Ihren Besuch!

Vielen Dank für Ihre Beteiligung! 


\section{Information for learners}

Dear learner,

This questionnaire has been designed to gather information about learners on the course, their personal circumstances, their learning experiences and their resources for elearning. Your answers will help us to develop courses in the future and support your current learning needs. Please take your time to complete the six sections in the questionnaire. Once completed, we recommend that you take a copy for yourself and submit the completed questionnaire to your tutor. We are interested in your expectations, experiences, aims and concerns so please be frank.

Finally we plan to collate the information gathered from the questionnaires for our own evaluation purposes and as such individual's names will not be identified.

Thank you very much!

\section{Informazioni per gli studenti}

Caro studente,

il questionario che ti presentiamo è stato progettato per raccogliere informazioni su coloro che partecipano ai corsi, sulle loro condizioni di apprendimento, sulle loro esperienze di apprendimento in questo corso e sulle risorse per l'e-learning. Vogliamo sapere se ha gradito i diversi metodi di apprendimento che ha sperimentato in questo corso e se ha raggiunto i suoi obiettivi. Le sue risposte ci aiuteranno a sviluppare i nostri corsi per il futuro. Si prenda il tempo necessario per completare le sei sezioni del questionario. Una volta riempito, le raccomandiamo di tenerne una copia per se e di inviarne una al suo tutor. Siamo interessati a comprendere le sue aspettative, i suoi obiettivi e interessi , quindi le preghiamo di rispondere onestamente.

E' nostra intenzione utilizzare i dati raccolti a scopo valutativo; i questionari sono anonimi. Grazie mille!

Ce questionnaire a été conçu pour nous renseigner sur les personnes qui ont participé à cette formation, le contexte de leur apprentissage, leurs expériences en termes d'apprentissage dans le cadre de ce diplôme et leurs moyens d'apprentissage en ligne.

Nous souhaiterions savoir comment vous évaluez les différentes méthodes qui vous ont été proposées dans ce diplôme et si vous avez atteint vos objectifs.

Vos réponses nous permettront d'améliorer les futurs diplômes.

Prenez tout le temps nécessaire pour remplir les sept sections de ce questionnaire. Enfin, nous envisageons d'utiliser les renseignements concernant votre groupe à des fins d'évaluation et aucune personne ne sera identifiée individuellement.

Merci beaucoup! 


\section{Kontext des Kurses}

Bitte schreiben Sie Ihre Antworten in die rechte Spalte.

\section{Context of the course}

Please write your responses in the boxes on the right side.

1. Contesto del corso

Per favore scriva le tue risposte negli spazi grigi a destra.

1. Contexte du cours

Veuillez inscrire vos réponses dans les cases de droite.

\begin{tabular}{|l|l|}
\hline Fragen & Antworten \\
Questions & Response \\
Domande & Risposte \\
Questions & Réponses \\
\hline
\end{tabular}
1a) Name des Kurses?
1a) What is the title of your course?
1a) Qual è il titolo del suo corso?
1a) Quel est l'intitulé de votre cours ?

1b) Wo findet der Kurs statt?

1b) Where does the course take place?

1b) Dove si svolge il corso?

1b) Où le cours a-t-il lieu?

1c) Das heutige Datum?

1c) What is today's date?

1c) Qual è la data di oggi?

1c) Quelle est la date d'aujourd'hui ? 


\section{Angaben zur Person}

2. Personal Characteristics of learners

2. Scheda personale dello studente

2. Renseignements personnels sur les apprenants

\begin{tabular}{|l|l|}
\hline Fragen & Antworten \\
Questions & Response \\
Domande & Risposte \\
Questions & Réponses \\
\hline
\end{tabular}

\begin{tabular}{|l|l|}
\hline 2a) Ihr Name oder ein Codename & \\
Für eine erneute Befragung zu einem spä- & \\
teren Zeitpunkt möchten wir Sie gerne wie- & \\
der erkennen, gleichzeitig möchten wir je- & \\
doch Ihre Anonymität wahren. Deshalb bit- & \\
ten wir Sie, einen Codenamen bzw. eine & \\
Codenummer anzugeben. Bitte geben Sie & \\
nacheinander den Geburtstag und den Ge- & \\
burtsmonat von zwei Menschen an, die & \\
Ihnen besonders nahe stehen. \\
Hier ein Beispiel: \\
Partner 01.03. (Geburtstag. Geburtsmonat) & \\
Mutter 12.12. (Geburtstag. Geburtsmonat) & \\
Der Codename wäre demnach: 01031212 & \\
2a) Your name or codename? & \\
2a) II vostro nome o pseudonimo? & \\
2a) Quel est votre nom ou votre pseudo- & \\
nyme? & \\
\hline
\end{tabular}

2b) Wie alt sind Sie?

2b) How old are you?

2b) Quanti anni ha?

2b) Quel âge avez-vous ?

2c) Welches Geschlecht haben Sie?

2c) What is your sex?

2c) Sesso

2c) Quel est votre sexe ?
Männlich
O Weiblich
O Male
O Female
OMaschio
OFemmina
O Masculin
O Féminin 


\begin{tabular}{l|l} 
2d) Haben Sie Kinder? & O Ja \\
2d) Do you have children? & O Nein \\
2d) Ha figli? & O Yes \\
2d) Avez-vous des enfants ? & O No \\
& O Si \\
& O No \\
& O Oui \\
& O Non
\end{tabular}

2e) Welche Nationalität haben Sie?

2e) What is your nationality?

2e) Di che nazionalità è?

2e) Quelle est votre nationalité ?

2f) Welchen höchsten Allgemeinbildenden Abschluss haben Sie?

2f) What is your highest educational qualification?

2f) Quale titolo di studio/istruzione?

2f) Quel est votre niveau d'étude ?

2g) Welchen Beruf haben Sie?

$2 \mathrm{~g}$ ) What is your current job title or occupation?

$2 \mathrm{~g})$ Qual è il tuo grado di professione ?

$2 \mathrm{~g})$ Quelle est votre profession?

2h) In welcher Einrichtung/Firma oder in welchem Betrieb arbeiten Sie?

2h) In which institution do you work?

2h) Presso quale istituzione lavora?

2h) Quel est l'intitulé de votre emploi actuel ? 
Die Beantwortung der folgenden Fragen ist freiwillig. Wir stellen diese Fragen, weil wir etwas mehr über Ihre persönlichen Lern-Umstände zu Hause oder am Arbeitsplatz erfahren wollen (je nachdem, wo Sie gelernt haben).

Please feel to answer the following questions. We ask these questions because we want to know about various issues that may affect your learning either at your home or at your work place. This information will help us adapt the course to suit the personal circumstances of learners

Per favore risponda alle seguenti domande. Le facciamo queste domande perché vogliamo sapere quali problemi possono incidere sul suo apprendimento sia a casa che sul posto di lavoro. Queste informazioni ci saranno utili per adattare il corso alle sue condizioni di apprendimento.

Merci de bien vouloir répondre aux questions suivantes. Nous posons ces questions car nous souhaiterions connaître les problèmes susceptibles d'affecter votre enseignement à domicile ou sur votre lieu de travail. Ces informations nous aideront à adapter les enseignements aux cas personnels des étudiants.

\begin{tabular}{|l|l|}
\hline $\begin{array}{l}\text { 2i) Wo haben Sie elearning, } \\
\text { d.h. elektronisch gestütztes Ler- } \\
\text { nen/Lernen mit dem Computer, durchge- } \\
\text { führt? }\end{array}$ & $\begin{array}{l}\text { O Zu Hause } \\
\text { O An meinem Arbeitsplatz } \\
\text { O Andere Orte (Bitte erläutern.) }\end{array}$ \\
\hline 2i) Where did you do your elearning? & O At home \\
& O At my working place \\
& O Others (please specify): \\
\hline 2i) Dove ha fatto e-learning? & $\square$ A casa \\
& $\square$ Sul posto di lavoro \\
& $\square$ Altro (specificare): \\
\hline $\begin{array}{l}\text { 2i) D'où avez-vous accédé à cette base } \\
\text { d'enseignement en ligne? }\end{array}$ & O De mon domicile \\
& O De mon lieu de travail \\
& O Autres (précisez) : \\
\hline
\end{tabular}

\begin{tabular}{|l|l|l|l|l|}
\hline $\begin{array}{l}\text { 2j) Wieviel Zeit hatten Sie in der Regel } \\
\text { pro Woche zu Hause für elearning? } \\
\text { (Zutreffendes bitte ankreuzen.) }\end{array}$ & $\begin{array}{l}\text { O Ich hatte } \\
\text { zu Hause } \\
\text { sehr viel } \\
\text { Zeit } \\
\text { für elearn- } \\
\text { ing. }\end{array}$ & $\begin{array}{l}\text { O Ich hatte } \\
\text { zu Hause } \\
\text { viel Zeit } \\
\text { für elearn- } \\
\text { ing. }\end{array}$ & $\begin{array}{l}\text { O Ich hatte } \\
\text { zu Hause } \\
\text { wenig Zeit } \\
\text { für elearn- } \\
\text { ing. }\end{array}$ & $\begin{array}{l}\text { O Ich hatte } \\
\text { zu Hause } \\
\text { gar keine } \\
\text { Zeit } \\
\text { für elearn- } \\
\text { ing. }\end{array}$ \\
\hline $\begin{array}{l}\text { 2j) How did you feel about your personal } \\
\text { situation at home concerning the time you } \\
\text { had for elearning? (Please tick the rele- } \\
\text { vant statement) }\end{array}$ & $\begin{array}{l}\text { O I had } \\
\text { A lot of time } \\
\text { available at } \\
\text { home for my } \\
\text { elearning }\end{array}$ & $\begin{array}{l}\text { O I had } \\
\text { Some time } \\
\text { available at } \\
\text { home for my } \\
\text { elearning }\end{array}$ & $\begin{array}{l}\text { O I had } \\
\text { Little time } \\
\text { available at } \\
\text { home for my } \\
\text { elearning }\end{array}$ & $\begin{array}{l}\text { O I had } \\
\text { available at } \\
\text { home for my } \\
\text { elearning }\end{array}$ \\
\hline $\begin{array}{l}\text { 2j) Considerando la sua situazione per- } \\
\text { sonale a casa, quanto tempo pensa di } \\
\text { avere avuto per l'e-learning ? (con il pc ed } \\
\text { il collegamento in rete) (segna } \\
\text { l'affermazione che ritiene più adatta) }\end{array}$ & $\begin{array}{l}\square \text { Ho avuto } \\
\text { molto tempo } \\
\text { a casa per } \\
\text { l'e-learning. }\end{array}$ & $\begin{array}{l}\square \text { Ho avu- } \\
\text { to abba- } \\
\text { stanza tem- } \\
\text { po a casa } \\
\text { per l' e- } \\
\text { learning. }\end{array}$ & $\begin{array}{l}\square \text { Ho avu- } \\
\text { to poco } \\
\text { tempo a } \\
\text { casa per l' } \\
\text { e-learning }\end{array}$ & $\begin{array}{l}\text { Non ho } \\
\text { avuto tempo casa per l' } \\
\text { e-learning }\end{array}$ \\
\hline
\end{tabular}




\begin{tabular}{|l|l|l|l|l|}
\hline 2j) Qu'avez-vous pensé de votre situation & O J'ai dis- & O J'ai dis- & O J'ai dis- & O Je n'ai \\
personnelle à votre domicile en ce qui & posé & posé & posé & pas disposé \\
concerne le temps dont vous avez dispo- & de beau- & d'un certain & de peu de & de temps à \\
sé pour l'enseignement en ligne? & coup de & temps à & temps à & mon domi- \\
(Veuillez cocher la réponse correspon- & temps à & mon domi- & mon domi- & cile pour \\
dante) & mon domi- & cile pour & cile pour & l'enseignem \\
& cile pour & l'enseignem & l'enseignem & ent en ligne \\
& l'enseignem & ent en ligne & ent en ligne & \\
& ent en ligne & & & \\
& & & & \\
\hline
\end{tabular}

2k) Wieviele Stunden hatten Sie zu Hause pro Woche für Ihr elearning?

Ich hatte ..... Stunden pro Woche zu Hause zur Verfügung für elearning.

2k) How many hours per week did you have at home for your elearning?

I had hours per week at home for elearning.

$2 \mathrm{k}$ ) Quante ore la settimana aveva per l' e-learning? (con il pc ed il collegamento in rete)

2k) De combien d'heures hebdomadaires avez-vous disposé pour votre enseignement en ligne à votre domicile dans le dernier mois ?

Avevo ore la settimana per l' e-learning.

J'ai disposé de ... heures hebdomadaires à mon domicile pour l'enseignement en ligne dans le dernier mois.

2I) Gab es zu Hause irgendwelche Einschränkungen, wenn Sie mit dem Computer arbeiten wollten?

O Nein

O Ja (Bitte erläutern.):

2l) Were there any restrictions at home with regard to using a computer

(e.g. no computer at home; other family

O No

Y Yes (please specify): members have priority)

2I) Ci sono state limitazioni a casa riguardo all'uso del computer?

( es. non aveva il computer a casa; altri membri della famiglia avevano la priorità)

2l) Avez-vous connu des contraintes à votre domicile concernant l'utilisation d'un ordinateur?

(par exemple, pas d'ordinateur ; d'autres membres de la famille étaient prioritaires...)

O Non

Oui (précisez)

\begin{tabular}{|l|l|l|l|l|}
\hline 2m) Wieviel Zeit hatten sie, um an diesem & O Ich hatte & O Ich hatte & O Ich hatte & O Ich hatte \\
Kurs teilzunehmen? & sehr viel & viel Zeit, & wenig Zeit, & gar keine \\
(Zutreffendes bitte ankreuzen.) & Zeit, & um an die- & um an die- & Zeit, \\
& um an die- & sem Kurs & sem Kurs & um an die- \\
& sem Kurs & teilzuneh- & teilzuneh- & sem Kurs \\
& teilzuneh- & men. & men. & $\begin{array}{l}\text { teilzuneh- } \\
\text { men. }\end{array}$ \\
& men. & & & \\
\hline
\end{tabular}




\begin{tabular}{|c|c|c|c|c|}
\hline $\begin{array}{l}2 \mathrm{~m}) \text { How did you feel about your personal } \\
\text { situation at home, concerning the time } \\
\text { you had for taking part in this course? } \\
\text { (Please tick the relevant statement) }\end{array}$ & $\begin{array}{l}\text { O I had } \\
\text { A lot of time } \\
\text { available at } \\
\text { home for } \\
\text { taking part } \\
\text { in this } \\
\text { course }\end{array}$ & $\begin{array}{l}\text { O I had } \\
\text { Some time } \\
\text { available at } \\
\text { home for } \\
\text { taking part } \\
\text { in this } \\
\text { course }\end{array}$ & $\begin{array}{l}\text { O I had } \\
\text { Little time } \\
\text { available at } \\
\text { home for } \\
\text { taking part } \\
\text { in this } \\
\text { course }\end{array}$ & $\begin{array}{l}\text { O I had } \\
\text { No time } \\
\text { available at } \\
\text { home for } \\
\text { taking part } \\
\text { in this } \\
\text { course }\end{array}$ \\
\hline $\begin{array}{l}\text { 2m) Considerando la sua situazione per- } \\
\text { sonale a casa, quanto tempo pensa di } \\
\text { aver avuto per seguire questo corso? } \\
\text { (segna l'affermazione che ritiene più adat- } \\
\text { ta) }\end{array}$ & $\begin{array}{l}\square \text { Ho avuto } \\
\text { molto tempo } \\
\text { a casa per } \\
\text { seguire } \\
\text { questo cor- } \\
\text { so }\end{array}$ & $\begin{array}{l}\square \text { Ho avu- } \\
\text { to abba- } \\
\text { stanza } \\
\text { tempo a } \\
\text { casa per } \\
\text { seguire } \\
\text { questo cor- } \\
\text { so }\end{array}$ & $\begin{array}{l}\square \text { Ho avuto } \\
\text { poco tempo } \\
\text { a casa per } \\
\text { seguire } \\
\text { questo cor- } \\
\text { so }\end{array}$ & $\begin{array}{l}\square \text { Non ho } \\
\text { avuto tempo } \\
\text { a casa per } \\
\text { seguire } \\
\text { questo cor- } \\
\text { so }\end{array}$ \\
\hline $\begin{array}{l}\text { 2m) Quelle était votre situation person- } \\
\text { nelle à votre domicile concernant le } \\
\text { temps dont vous avez disposé pour étu- } \\
\text { dier ce cours de tabacologie? (Veuillez } \\
\text { cocher la réponse correspondante) }\end{array}$ & $\begin{array}{l}\text { O J'ai dis- } \\
\text { posé } \\
\text { de beau- } \\
\text { coup de } \\
\text { temps à } \\
\text { mon domi- } \\
\text { cile pour } \\
\text { étudier ce } \\
\text { cours }\end{array}$ & $\begin{array}{l}\text { O J'ai dis- } \\
\text { posé } \\
\text { d'un certain } \\
\text { temps à } \\
\text { mon domi- } \\
\text { cile pour } \\
\text { étudier ce } \\
\text { cours }\end{array}$ & $\begin{array}{l}\text { O J'ai dis- } \\
\text { posé } \\
\text { de peu de } \\
\text { temps à } \\
\text { mon domi- } \\
\text { cile pour } \\
\text { étudier ce } \\
\text { cours }\end{array}$ & $\begin{array}{l}\text { O Je n'ai } \\
\text { pas disposé } \\
\text { de temps à } \\
\text { mon domi- } \\
\text { cile pour } \\
\text { étudier ce } \\
\text { cours }\end{array}$ \\
\hline
\end{tabular}

2n) Wieviele Stunden hatten Sie pro Woche zur Verfügung, um an diesem Kurs teilzunehmen?

2n) How many hours per week did you have at home for taking part in this course?

2n) Quante ore la settimana aveva a casa per seguire questo corso?

2n) De combien d'heures hebdomadaires avez vous disposé à votre domicile pour étudier ce cours de tabacologie pendant le dernier mois?
Ich hatte ..... Stunden pro Woche zur Verfügung, um an diesem Kurs teilzunehmen.

I had hours per week at home for taking part in this course.

Avevo corso.

ore la settimana a casa per seguire questo

J'ai disposé de ... heures hebdomadaires à mon domicile pour étudier ce cours de tabacologie pendant le dernier mois.

\begin{tabular}{|l|l|l|l|l|}
\hline $\begin{array}{l}\text { 2o) Wieviel Zeit hatten Sie in der Regel } \\
\text { pro Woche an Ihrem Arbeitsplatz, die } \\
\text { Sie für elearning nutzen konnten? }\end{array}$ & $\begin{array}{l}\text { O Ich hatte } \\
\text { an meinem } \\
\text { Arbeitsplatz } \\
\text { sehr viel } \\
\text { (Zutreffendes bitte ankreuzen.) }\end{array}$ & $\begin{array}{l}\text { O Ich hatte } \\
\text { an meinem } \\
\text { Arbeitsplatz } \\
\text { viel Zeit } \\
\text { für elearn- } \\
\text { ing elearn- } \\
\text { ing. }\end{array}$ & $\begin{array}{l}\text { O Ich habe } \\
\text { an meinem } \\
\text { Arbeitsplatz } \\
\text { wenig Zeit } \\
\text { für elearn- } \\
\text { ing. }\end{array}$ & $\begin{array}{l}\text { O Ich habe } \\
\text { an meinem } \\
\text { Arbeitsplatz } \\
\text { gar keine } \\
\text { Zeit } \\
\text { für elearn- } \\
\text { ing. }\end{array}$ \\
\hline $\begin{array}{l}\text { 2o) How did you feel about your personal } \\
\text { situation at your work place, concerning } \\
\text { the time you had for elearning? (Please } \\
\text { tick the relevant statement) }\end{array}$ & $\begin{array}{l}\text { O I had } \\
\text { available at } \\
\text { my work } \\
\text { place for my } \\
\text { elearning }\end{array}$ & $\begin{array}{l}\text { O I had } \\
\text { Some time } \\
\text { available at } \\
\text { my work } \\
\text { place for my } \\
\text { elearning }\end{array}$ & $\begin{array}{l}\text { O I had } \\
\text { Little time } \\
\text { available at } \\
\text { my work } \\
\text { place for my } \\
\text { elearning }\end{array}$ & $\begin{array}{l}\text { No time } \\
\text { available at } \\
\text { my work } \\
\text { place for my } \\
\text { elearning }\end{array}$ \\
\hline
\end{tabular}




\begin{tabular}{|c|c|c|c|c|}
\hline $\begin{array}{l}\text { 2o) Considerando la sua situazione per- } \\
\text { sonale sul posto di lavoro, quanto tempo } \\
\text { pensa di aver avuto per l'e-learning? } \\
\text { (segna l'affermazione che ritiene più adat- } \\
\text { ta) }\end{array}$ & $\begin{array}{l}\square \text { Ho avuto } \\
\text { molto tempo } \\
\text { sul lavoro } \\
\text { per l' e- } \\
\text { learning }\end{array}$ & $\begin{array}{l}\square \text { Ho avuto } \\
\text { abbastanza } \\
\text { tempo sul } \\
\text { lavoro per l' } \\
\text { e-learning }\end{array}$ & $\begin{array}{l}\square \text { Ho avuto } \\
\text { poco tempo } \\
\text { sul lavoro } \\
\text { per l' e- } \\
\text { learning }\end{array}$ & $\begin{array}{l}\square \text { Non ho } \\
\text { avuto tempo } \\
\text { sul lavoro } \\
\text { per l' e- } \\
\text { learning } \\
\end{array}$ \\
\hline $\begin{array}{l}\text { 2o) Quelle était votre situation person- } \\
\text { nelle sur votre lieu de travail concernant } \\
\text { le temps dont vous avez disposé pour } \\
\text { l'enseignement en ligne du diplôme de } \\
\text { tabacologie? (Veuillez cocher la réponse } \\
\text { correspondante) }\end{array}$ & $\begin{array}{l}\text { O J'ai dis- } \\
\text { posé } \\
\text { de beau- } \\
\text { coup de } \\
\text { temps sur } \\
\text { mon lieu de } \\
\text { travail pour } \\
\text { l'enseignem } \\
\text { ent en ligne }\end{array}$ & $\begin{array}{l}\text { O J'ai dis- } \\
\text { posé } \\
\text { d'un certain } \\
\text { temps sur } \\
\text { mon lieu de } \\
\text { travail pour } \\
\text { l'enseignem } \\
\text { ent en ligne }\end{array}$ & $\begin{array}{l}\text { O J'ai dis- } \\
\text { posé } \\
\text { de peu de } \\
\text { temps sur } \\
\text { mon lieu de } \\
\text { travail pour } \\
\text { l'enseignem } \\
\text { ent en ligne }\end{array}$ & $\begin{array}{l}\text { O Je n'ai } \\
\text { pas disposé } \\
\text { de temps } \\
\text { sur mon lieu } \\
\text { de travail } \\
\text { pour } \\
\text { l'enseignem } \\
\text { ent en ligne }\end{array}$ \\
\hline
\end{tabular}

\begin{tabular}{|l|l|}
\hline $\begin{array}{l}2 p) \text { Wieviele Stunden hatten Sie an Ih- } \\
\text { rem Arbeitsplatz pro Woche für elearn- } \\
\text { ing? }\end{array}$ & $\begin{array}{l}\text { Ich hatte ..... Stunden pro Woche an meinem Arbeits- } \\
\text { platz zur Verfügung für elearning. }\end{array}$ \\
\hline $\begin{array}{l}2 p) \text { How many hours per week did you } \\
\text { have for your elearning at your work } \\
\text { place? }\end{array}$ & $\begin{array}{l}\text { I had ......... hours per week at my work place for elearn- } \\
\text { ing. }\end{array}$ \\
\hline $\begin{array}{l}2 p) \text { Quante ore la settimana aveva per l' } \\
\text { e-learning sul posto di lavoro? }\end{array}$ & $\begin{array}{l}\text { Avevo __ ore la settimana per l' e-learning sul posto di } \\
\text { lavoro. }\end{array}$ \\
\hline $\begin{array}{l}2 p) \text { De combien d'heures hebdomadaires } \\
\begin{array}{l}\text { avez-vous disposé pour cet enseigne- } \\
\text { ment en ligne sur votre lieu de tra- } \\
\text { vail dans le dernier mois? }\end{array}\end{array}$ & $\begin{array}{l}\text { J'ai disposé de ... heures hebdomadaires sur mon lieu } \\
\text { de travail pour l'enseignement en ligne. }\end{array}$ \\
\hline
\end{tabular}

\begin{tabular}{|l|l|}
\hline $\begin{array}{l}\text { 2q) Gab es an Ihrem Arbeitsplatz ir- } \\
\text { gendwelche Einschränkungen, wenn Sie } \\
\text { mit dem Computer arbeiten wollten? }\end{array}$ & $\begin{array}{l}\text { O Nein } \\
\text { O Ja (Bitte erläutern) }\end{array}$ \\
\hline $\begin{array}{l}\text { 2q) Were there any restrictions at your } \\
\text { work place with regard to using a com- } \\
\text { puter }\end{array}$ & $\begin{array}{l}\text { O No } \\
\text { O Yes (please specify): }\end{array}$ \\
\hline $\begin{array}{l}\text { 2q) Ci sono state limitazioni sul posto di } \\
\text { lavoro riguardo all'uso del computer? (es. }\end{array}$ & \\
$\begin{array}{l}\text { Poteva usare il computer solo per que- } \\
\text { stioni di lavoro; non c'erano computer, } \\
\text { c'era un firewall, limitato livello di accesso } \\
\text { ad internet, ecc.) }\end{array}$ & $\square$ No \\
\hline $\begin{array}{l}\text { 2q) Avez-vous connu des contraintes sur } \\
\text { votre lieu de travail concernant l'utilisation } \\
\text { d'un ordinateur? } \\
\text { (par exemple, utilisation de l'ordinateur } \\
\text { pour le travail uniquement ; pas d'ordina- } \\
\text { teur sur le lieu de travail) }\end{array}$ & \\
\hline $\begin{array}{l}\text { 2r) Wurden Sie von der Arbeit freigestellt, } \\
\text { um an diesem Kurs teilzunehmen? }\end{array}$ & O Non \\
& O Nein (précisez) : \\
& O Ja, immer \\
\hline
\end{tabular}




\begin{tabular}{|c|c|c|c|c|}
\hline $\begin{array}{l}2 r \text { ) How did you feel about your personal } \\
\text { situation at your work place concerning } \\
\text { the time you had for taking part in this } \\
\text { course? (Please tick the relevant state- } \\
\text { ment) }\end{array}$ & $\begin{array}{l}\text { O I had } \\
\text { A lot of time } \\
\text { available at } \\
\text { my work } \\
\text { place for } \\
\text { taking part } \\
\text { in this } \\
\text { course }\end{array}$ & $\begin{array}{l}\text { O I had } \\
\text { Some time } \\
\text { available at } \\
\text { my work } \\
\text { place for } \\
\text { taking part } \\
\text { in this } \\
\text { course }\end{array}$ & $\begin{array}{l}\text { O I had } \\
\text { Little time } \\
\text { available at } \\
\text { my work } \\
\text { place for } \\
\text { taking part } \\
\text { in this } \\
\text { course }\end{array}$ & $\begin{array}{l}\text { O I had } \\
\text { No time } \\
\text { available at } \\
\text { my work } \\
\text { place for } \\
\text { taking part } \\
\text { in this } \\
\text { course }\end{array}$ \\
\hline $\begin{array}{l}\text { 2r) Considerando la sua situazione per- } \\
\text { sonale sul posto di lavoro, quanto tempo } \\
\text { pensa di aver avuto per seguire tutti gli } \\
\text { elementi di questo corso? (segna } \\
\text { l'affermazione che le sembra più adatta) }\end{array}$ & $\begin{array}{l}\square \text { Ho avuto } \\
\text { molto tempo } \\
\text { sul lavoro } \\
\text { per seguire } \\
\text { questo cor- } \\
\text { so }\end{array}$ & $\begin{array}{l}\square \text { Ho avuto } \\
\text { abbastanza } \\
\text { tempo sul } \\
\text { lavoro per } \\
\text { seguire } \\
\text { questo cor- } \\
\text { so }\end{array}$ & $\begin{array}{l}\square \text { Ho avuto } \\
\text { poco tempo } \\
\text { sul lavoro } \\
\text { per seguire } \\
\text { questo cor- } \\
\text { so }\end{array}$ & $\begin{array}{l}\square \text { Non ho } \\
\text { avuto tempo } \\
\text { sul lavoro } \\
\text { per seguire } \\
\text { questo cor- } \\
\text { so }\end{array}$ \\
\hline $\begin{array}{l}\text { 2r) Quelle était votre situation personnelle } \\
\text { sur votre lieu de travail concernant le } \\
\text { temps dont vous avez disposé pour étu- } \\
\text { dier ce cours dans le dernier mois? } \\
\text { (Veuillez cocher la réponse correspon- } \\
\text { dante) }\end{array}$ & $\begin{array}{l}\text { O J'ai dis- } \\
\text { posé } \\
\text { de beau- } \\
\text { coup de } \\
\text { temps sur } \\
\text { mon lieu de } \\
\text { travail pour } \\
\text { étudier ce } \\
\text { cours }\end{array}$ & $\begin{array}{l}\text { O J'ai dis- } \\
\text { posé } \\
\text { d'un certain } \\
\text { temps sur } \\
\text { mon lieu de } \\
\text { travail pour } \\
\text { étudier ce } \\
\text { cours }\end{array}$ & $\begin{array}{l}\text { O J'ai dis- } \\
\text { posé } \\
\text { de peu de } \\
\text { temps sur } \\
\text { mon lieu de } \\
\text { travail pour } \\
\text { étudier ce } \\
\text { cours }\end{array}$ & $\begin{array}{c}\text { O Je n'ai } \\
\text { pas disposé } \\
\text { de temps } \\
\text { sur mon lieu } \\
\text { de travail } \\
\text { pour étudier } \\
\text { ce cours }\end{array}$ \\
\hline
\end{tabular}

2s) Wieviele Stunden bekamen Sie von Ihrem Arbeitgeber pro Woche, um an diesem Kurs teilzunehmen?

2s) How many hours per week did you have for taking part in this course at your work place?

2s) Quante ore la settimana aveva sul posto di lavoro per seguire questo corso?

2s) De combien d'heures hebdomadaires avez-vous disposé pour étudier ce cours sur votre lieu de travail dans le dernier mois?
Ich hatte ..... Stunden pro Woche von meinem Arbeitgeber zur Verfügung, um an diesem Kurs teilzunehmen.

I had hours per week at my work place for taking part in this course.

Avevo ore la settimana sul posto di lavoro per seguire questo corso.

J'ai disposé de ... heures hebdomadaires sur mon lieu de travail pour étudier ce cours. 


\section{Lernerfahrungen in diesem Kurs}

3. Learning experiences in this course

3. Esperienze di apprendimento in questo corso

3. Expériences en termes d'apprentissage de ce cours

Im Folgenden sind einige Lernmethoden aufgeführt, die Sie in diesem Kurs erlebten. Bitte geben Sie an, wie Ihnen diese Methoden gefallen haben (bitte ankreuzen, was für Sie zutrifft).

Several learning methods that you have experienced in this course are listed below. Please indicate how you liked the single methods (tick one box as appropriate).

Di seguito sono elencati alcuni metodi di apprendimento che ha incontrato in questo corso. Per favore indichi come considera ciascun metodo (segna la casella che ritiene più adatta).

Le tableau ci-dessous présente une liste d'outils et méthodes pédagogiques qui ont été utilisés pendant cette formation. Veuillez indiquer votre degré d'appréciation de chacun d'entre eux (cochez la case correspondante).

\begin{tabular}{|c|c|c|c|c|c|}
\hline $\begin{array}{l}\text { 3a) Präsenzunterricht } \\
\text { (Das Lernen findet in einen } \\
\text { Klassenraum statt und } \\
\text { eine Lehrperson ist anwe- } \\
\text { send.) }\end{array}$ & \multirow[t]{2}{*}{$\begin{array}{l}\text { O Gefiel mir } \\
\text { sehr } \\
++\end{array}$} & \multirow[t]{2}{*}{$\begin{array}{l}\text { O Gefiel } \\
\text { mir } \\
+\end{array}$} & \multirow[t]{2}{*}{$\begin{array}{l}\text { O Fand ich } \\
\text { mittelmäßig } \\
\text { o }\end{array}$} & $\begin{array}{l}\text { O Gefiel } \\
\text { mir nicht } \\
-\end{array}$ & $\begin{array}{l}\text { O Gefiel mir } \\
\text { gar nicht }\end{array}$ \\
\hline \multicolumn{3}{|l|}{ Gründe für Ihre Antwort: } & & & \\
\hline $\begin{array}{l}\text { 3a) In-person attendance } \\
\text { (E.g. where we met in a } \\
\text { classroom to learn) }\end{array}$ & $\begin{array}{l}\text { O I liked it } \\
\text { very much } \\
++\end{array}$ & $\begin{array}{l}\text { O I liked } \\
\text { it } \\
+\end{array}$ & $\begin{array}{l}\text { It was OK } \\
\text { for me } \\
\text { o }\end{array}$ & $\begin{array}{l}\text { O I did } \\
\text { not like it } \\
-\end{array}$ & $\begin{array}{l}\text { I did not like } \\
\text { it at all } \\
\text {-- }\end{array}$ \\
\hline \multicolumn{6}{|l|}{ Reasons for your answer: } \\
\hline $\begin{array}{l}\text { 3a) Presenza in aula } \\
\text { (Es. lezioni frontali in clas- } \\
\text { se) }\end{array}$ & $\begin{array}{l}\square \text { Mi è pia- } \\
\text { ciuto molto } \\
++\end{array}$ & $\begin{array}{l}\square \text { Mi è } \\
\text { piaciuto } \\
+\end{array}$ & $\begin{array}{l}\square \text { Per me } \\
\text { andava be- } \\
\text { ne } \\
\text { o }\end{array}$ & $\begin{array}{l}\square \text { Non mi } \\
\text { è piaciuto } \\
-\end{array}$ & $\begin{array}{l}\square \text { Non mi è } \\
\text { piaciuto affatto } \\
--\end{array}$ \\
\hline \multicolumn{6}{|l|}{ Motivi della sua risposta: } \\
\hline $\begin{array}{l}\text { 3a) Enseignement en } \\
\text { présentiel } \\
\text { (réunion étudiant- } \\
\text { enseignant en salle pour } \\
\text { l'enseignement) }\end{array}$ & $\begin{array}{l}1 \text { O J'ai } \\
\text { beaucoup } \\
\text { apprécié }\end{array}$ & $\begin{array}{l}2 \text { O J'ai } \\
\text { apprécié }\end{array}$ & $\begin{array}{l}3 \text { ○ Cela } \\
\text { m'était égal }\end{array}$ & $\begin{array}{l}4 \text { O Je } \\
\text { n'ai pas } \\
\text { apprécié }\end{array}$ & $\begin{array}{l}5 \bigcirc \text { Je n'ai pas du } \\
\text { tout apprécié }\end{array}$ \\
\hline \multicolumn{6}{|l|}{ Commentaires : } \\
\hline
\end{tabular}

3b) Was gefiel Ihnen besonders am Präsenzunterricht"?

3b) What did you like especially about in-person attendance?

$3 \mathrm{~b})$ Che cosa le è piaciuto di più della presenza in aula?

3b) Qu'avez-vous particulièrement apprécié dans l'enseignement en présentiel ? 
3c) Was bereitete Ihnen Schwierigkeiten beim Präsenzunterricht?

3c) What caused you difficulties with in-person attendance?

3c) Quali difficoltà comportava la presenza in aula?

3c) Quels ont été vos problèmes avec l'enseignement en présentiel ?

\begin{tabular}{|c|c|c|c|c|c|}
\hline $\begin{array}{l}\text { 3d) Fernunterricht } \\
\text { (Materialien und Aufgaben } \\
\text { werden zugeschickt und zu } \\
\text { Hause bearbeitet.) }\end{array}$ & $\begin{array}{l}\text { O Gefiel mir } \\
\text { sehr } \\
++\end{array}$ & $\begin{array}{l}\text { O Gefiel } \\
\text { mir } \\
+\end{array}$ & $\begin{array}{l}\text { O Fand ich } \\
\text { mittelmäßig } \\
\text { o }\end{array}$ & $\begin{array}{l}\text { O Gefiel } \\
\text { mir nicht } \\
\text { - }\end{array}$ & $\begin{array}{l}\text { O Gefiel mir } \\
\text { gar nicht } \\
--\end{array}$ \\
\hline \multicolumn{6}{|l|}{ Gründe für Ihre Antwort: } \\
\hline $\begin{array}{l}\text { 3d)Distance/open learn- } \\
\text { ing } \\
\text { (E.g. where you were giv- } \\
\text { en paper-based materials } \\
\text { to work through at your } \\
\text { own place) }\end{array}$ & $\begin{array}{l}\text { O I liked it } \\
\text { very much }\end{array}$ & $\begin{array}{l}\text { O I liked } \\
\text { it }\end{array}$ & $\begin{array}{l}\text { O It was OK } \\
\text { for me }\end{array}$ & $\begin{array}{l}\text { O I did } \\
\text { not like it }\end{array}$ & $\begin{array}{l}\text { I did not like } \\
\text { it at all }\end{array}$ \\
\hline \multicolumn{6}{|l|}{ Reasons for your answer: } \\
\hline $\begin{array}{l}\text { 3d) Insegnamento a di- } \\
\text { stanza/open learning } \\
\text { (Es. quando vengono con- } \\
\text { segnate delle dispense su } \\
\text { cui lavorare per conto pro- } \\
\text { prio) }\end{array}$ & $\begin{array}{l}\square \text { Mi è pia- } \\
\text { ciuto molto } \\
++\end{array}$ & $\begin{array}{l}\square \text { Mi è } \\
\text { piaciuto } \\
+\end{array}$ & $\begin{array}{l}\square \text { Per me } \\
\text { andava be- } \\
\text { ne } \\
\text { o }\end{array}$ & $\begin{array}{l}\square \text { Non mi } \\
\text { è piaciuto } \\
\text { - }\end{array}$ & $\begin{array}{l}\square \text { Non mi è } \\
\text { piaciuto affatto } \\
\text {-- }\end{array}$ \\
\hline \multicolumn{6}{|l|}{ Motivi della sua risposta: } \\
\hline $\begin{array}{l}\text { 3d) Apprentissage ou- } \\
\text { vert/à distance } \\
\text { (par exemple, lorsque } \\
\text { l'on vous a remis des } \\
\text { documents pour travailler } \\
\text { chez vous) }\end{array}$ & $\begin{array}{l}1 \bigcirc \text { J'ai } \\
\text { beaucoup } \\
\text { apprécié }\end{array}$ & $\begin{array}{l}2 \text { ○ J'ai } \\
\text { apprécié }\end{array}$ & $\begin{array}{l}3 \bigcirc \text { Cela } \\
\text { m'était égal }\end{array}$ & $\begin{array}{l}4 \text { ○ Je } \\
\text { n'ai pas } \\
\text { apprécié }\end{array}$ & $\begin{array}{l}5 \bigcirc \text { Je n'ai pas du } \\
\text { tout apprécié }\end{array}$ \\
\hline
\end{tabular}

3e) Was gefiel Ihnen besonders am Fernunterricht?

3e) What did you like especially about distance/open learning?

3e) Che cosa le è piaciuto nell'insegnamento a distanza?

3e) Qu'avez-vous particulièrement apprécié dans l'apprentissage ouvert/à distance ?

3f) Was bereitete Ihnen Schwierigkeiten beim Fernunterricht?

3f) What caused you difficulties with distance/open learning?

3f) Quali sono le difficoltà per lei nell' insegnamento a distanza?

3f) Quels ont été vos problèmes dans l'apprentissage ouvert/à distance ?

\begin{tabular}{|l|l|l|l|l|l|}
\hline $\begin{array}{l}\text { 3g) elearning } \\
\text { (Lernmaterialien und Akti- } \\
\text { vitäten werden elektro- } \\
\text { nisch/mit dem Computer } \\
\text { vermittelt.) }\end{array}$ & $\begin{array}{l}\text { O Gefiel mir } \\
\text { sehr } \\
++\end{array}$ & $\begin{array}{l}\text { O Gefiel } \\
\text { mir } \\
+\end{array}$ & $\begin{array}{l}\text { O Fand ich } \\
\text { mittelmäßig } \\
0\end{array}$ & $\begin{array}{l}\text { O Gefiel } \\
\text { mir nicht } \\
-\end{array}$ & $\begin{array}{l}\text { O Gefiel mir } \\
\text { gar nicht } \\
--\end{array}$ \\
\hline Grüdelur & & & & \\
\hline
\end{tabular}

Gründe für Ihre Antwort: 


\begin{tabular}{|c|c|c|c|c|c|}
\hline $\begin{array}{l}\text { 3g) Elearning } \\
\text { (E.g. where learning mate- } \\
\text { rials and activities were } \\
\text { accessed electronically by } \\
\text { computer) }\end{array}$ & $\begin{array}{l}\text { I l liked it } \\
\text { very much }\end{array}$ & $\begin{array}{l}\text { O I liked } \\
\text { it }\end{array}$ & $\begin{array}{l}\text { It was OK } \\
\text { for me }\end{array}$ & $\begin{array}{l}\text { O I did } \\
\text { not like it }\end{array}$ & $\begin{array}{l}\text { I did not like } \\
\text { it at all }\end{array}$ \\
\hline \multicolumn{6}{|l|}{ Reasons for your answer: } \\
\hline $\begin{array}{l}\text { 3g) E-learning } \\
\text { ( Es. quando accedeva al } \\
\text { materiale didattico e alle } \\
\text { attività elettronicamente } \\
\text { tramite computer) }\end{array}$ & $\begin{array}{l}\square \text { Mi è pia- } \\
\text { ciuto molto }\end{array}$ & $\begin{array}{l}\square \text { Mi è } \\
\text { piaciuto }\end{array}$ & $\begin{array}{l}\square \text { Per me } \\
\text { andava be } \\
\text { ne }\end{array}$ & $\begin{array}{l}\square \text { Non } \\
\text { mi è pia- } \\
\text { ciuto }\end{array}$ & $\begin{array}{l}\square \text { Non mi è } \\
\text { piaciuto affatto }\end{array}$ \\
\hline \multicolumn{6}{|l|}{ Motivi della sua risposta: } \\
\hline $\begin{array}{l}\text { 3g) Apprentissage en } \\
\text { ligne } \\
\text { (lorsque les supports } \\
\text { d'enseignement étaient } \\
\text { disponibles sur internet) }\end{array}$ & $\begin{array}{l}\text { O J'ai } \\
\text { beaucoup } \\
\text { apprécié }\end{array}$ & $\begin{array}{l}\text { O J'ai } \\
\text { apprécié }\end{array}$ & $\begin{array}{l}\text { O Cela } \\
\text { m'était égal }\end{array}$ & $\begin{array}{l}\text { O Je n'ai } \\
\text { pas ap- } \\
\text { précié }\end{array}$ & $\begin{array}{l}\text { O Je n'ai pas du } \\
\text { tout apprécié }\end{array}$ \\
\hline
\end{tabular}

3h) Was gefiel Ihnen besonders am elearning?

3h) What did you like especially about elearning?

3h) Che cosa le è piaciuto di più dell'e-learning?

3h) Qu'avez-vous particulièrement apprécié dans l'enseignement en ligne ?

3i) Was bereitete Ihnen Schwierigkeiten beim elearning?

3i) What caused you difficulties with elearning?

3i) Quali difficoltà comportava l'e-learning?

3i) Quels ont été vos problèmes dans l'enseignement en ligne ?

\begin{tabular}{|c|c|c|c|c|}
\hline $\begin{array}{l}\text { 3j) Wie fanden Sie die von } \\
\text { der Lernplattform angebo- } \\
\text { tenen Funktionen? }\end{array}$ & $\begin{array}{l}\text { O Einfach zu } \\
\text { bedienen, ich } \\
\text { brauchte keine } \\
\text { Erklärungen } \\
\text { von meinem } \\
\text { Tutor }\end{array}$ & $\begin{array}{l}\text { O Mittelmäßig } \\
\text { zu bedienen, } \\
\text { ich brauchte } \\
\text { Hilfe von mei- } \\
\text { nem Tutor, um } \\
\text { mit den wich- } \\
\text { tigsten Funkti- } \\
\text { onen umgehen } \\
\text { zu können. }\end{array}$ & $\begin{array}{l}\text { O Schwierig zu } \\
\text { bedienen, trotz } \\
\text { der Hilfestellung } \\
\text { durch meinen } \\
\text { Tutor hatte ich } \\
\text { oft Schwierigkei- } \\
\text { ten mit den } \\
\text { Funktionen. }\end{array}$ & O Weiß nicht \\
\hline $\begin{array}{l}\text { 3j) How did you find the } \\
\text { different tools/activities } \\
\text { available on the elearning } \\
\text { platform? }\end{array}$ & $\begin{array}{l}\text { E Easy to use, } \\
\text { I did not need } \\
\text { explanations } \\
\text { from the tutor }\end{array}$ & $\begin{array}{l}\text { O Moderate to } \\
\text { use, I needed } \\
\text { the tutor's help } \\
\text { to command } \\
\text { the main func- } \\
\text { tions }\end{array}$ & $\begin{array}{l}\text { O Difficult to } \\
\text { use, in spite of } \\
\text { the tutor's ex- } \\
\text { planations, } \\
\text { I often had diffi- } \\
\text { culties with the } \\
\text { tools }\end{array}$ & $\begin{array}{l}\text { O Others } \\
\text { (Please specify) }\end{array}$ \\
\hline $\begin{array}{l}\text { 3j) Come ha trovato i di- } \\
\text { versi strumenti disponibili } \\
\text { sulla piattaforma? }\end{array}$ & $\begin{array}{l}\square \text { Facili da } \\
\text { usare, non ave- } \\
\text { vo bisogno del- } \\
\text { le spiegazioni } \\
\text { del tutor }\end{array}$ & $\begin{array}{l}\square \text { Abbastanza } \\
\text { semplici, ho } \\
\text { avuto bisogno } \\
\text { del tutor per le } \\
\text { funzioni princi- } \\
\text { pali. }\end{array}$ & $\begin{array}{l}\text { Difficili da } \\
\text { usare, nono- } \\
\text { stante l'aiuto del } \\
\text { tutor, ho avuto } \\
\text { spesso problemi } \\
\text { con gli strumen- } \\
\text { ti. }\end{array}$ & $\begin{array}{l}\square \text { Altro } \\
\text { (specificare) }\end{array}$ \\
\hline
\end{tabular}




\begin{tabular}{|l|l|l|l|l|}
\hline $\begin{array}{l}\text { 3j) Quelle est votre opinion } \\
\text { concernant les activités/les } \\
\text { outils disponibles sur la } \\
\text { plate-forme? }\end{array}$ & $\begin{array}{l}\text { O Facile à } \\
\text { user, je n'avais } \\
\text { pas besoin } \\
\text { d'explications }\end{array}$ & $\begin{array}{l}\text { O Assez facile } \\
\text { à user, j'avais } \\
\text { besoin d'aide } \\
\text { de la part de } \\
\text { mon tutor }\end{array}$ & $\begin{array}{l}\text { O Difficile à } \\
\text { user, malgré } \\
\text { l'aide de mon } \\
\text { tutor, j'avais } \\
\text { souvent des } \\
\text { problèmes avec } \\
\text { les outils }\end{array}$ & $\begin{array}{l}\text { O Autres } \\
\text { (précisez) }\end{array}$ \\
& & & \\
& & & \\
\hline
\end{tabular}

\begin{tabular}{|l|l|}
\hline $\begin{array}{l}\text { 3k) Welche Kontaktmöglichkeit zu Ihrem Tu- } \\
\text { tor haben Sie bevorzugt? } \\
\text { (Bitte ankreuzen.) }\end{array}$ & O Electronic Mail (E-Mail) \\
& O Intefon \\
& O Chat \\
& O Forum \\
& O Anderes (Bitte erläutern.) \\
\hline $\begin{array}{l}\text { 3k) What was your favourite way to contact } \\
\text { your tutor? }\end{array}$ & O Electronic Mail \\
& O Phone \\
& O Internetphone with a headset \\
& O Chat \\
& O Forum \\
& O Others (Please specify) \\
\hline & $\square$ Posta Elettronica \\
3k) In che modo preferiva contattare il tuo & $\square$ Telefono \\
tutor? & $\square$ Chat \\
& $\square$ Forum \\
\hline 3k) Quels moyens préférez vous pour contac- & O Altro (specificare) \\
ter vos enseignants à distance (hors de la & O Téléphone \\
présence en salle) ? & O Chat \\
& O Forum \\
& O Autres (Précisez) \\
\hline
\end{tabular}

\begin{tabular}{|l|l|}
\hline 3I) Wie oft haben Sie pro Woche mit Ihrem & O Weniger als einmal pro Woche \\
Tutor Kontakt gehabt? & O Einmal pro Woche \\
& O Zweimal pro Woche \\
O Dreimal pro Woche & O Mehr als dreimal pro Woche \\
\hline 3I) How often did you contact your tutor? & O Once a week \\
& O Twice a week \\
& O Three times a week \\
& O Others (please specify) \\
\hline 3I) Con quale frequenza contattava il tutor? & $\square$ Mai \\
& $\square$ Una volta la settimana \\
& $\square$ Due volte la settimana \\
& $\square$ Tre volte la settimana \\
\hline 3I) Combien de fois avez-vous contacté vos & O Jamais die tre volte la settimana \\
enseignants à distance pendant cette forma- & O Une fois par semaine \\
tion (hors de la présence en salle)? & O Deux fois par semaine \\
& O Trois fois par semaine \\
& Plus de trois fois par semaine \\
\hline
\end{tabular}




\begin{tabular}{|c|c|c|c|c|c|}
\hline $\begin{array}{l}3 m) \text { Sie empfanden Ih- } \\
\text { ren Tutor als... }\end{array}$ & $\begin{array}{l}\text { O } \\
\text { Sehr hilfs- } \\
\text { bereit }\end{array}$ & $\begin{array}{l}\mathrm{O} \\
\text { Hilfsbereit }\end{array}$ & $\begin{array}{l}\text { O } \\
\text { Einigermas- } \\
\text { sen hilfsbereit }\end{array}$ & $\begin{array}{l}\text { O } \\
\text { Nicht sehr } \\
\text { hilfsbereit }\end{array}$ & $\begin{array}{l}\text { Weiß nicht } \\
\text { W }\end{array}$ \\
\hline $\begin{array}{l}3 \mathrm{~m}) \text { You found that your } \\
\text { tutor was... }\end{array}$ & $\begin{array}{l}\text { V Very } \\
\text { receptive }\end{array}$ & $\begin{array}{l}\text { O Recepti- } \\
\text { ve }\end{array}$ & $\begin{array}{l}\text { O Quite re- } \\
\text { ceptive }\end{array}$ & $\begin{array}{l}\text { O Not very } \\
\text { receptive }\end{array}$ & $\begin{array}{l}\text { O Others } \\
\text { (Please } \\
\text { specify) }\end{array}$ \\
\hline 3m) I tutori erano & $\begin{array}{l}\square \text { Molto } \\
\text { disponibile }\end{array}$ & $\square$ Disponi- & $\begin{array}{l}\square \text { Abbas- } \\
\text { tanza dispo- } \\
\text { nibile }\end{array}$ & $\begin{array}{l}\square \text { Poco dis- } \\
\text { ponibile }\end{array}$ & $\begin{array}{l}\square \text { Altro } \\
\text { (specificare) }\end{array}$ \\
\hline $\begin{array}{l}3 m \text { ) Les tutors/moniteurs } \\
\text { ont été }\end{array}$ & $\begin{array}{l}\text { Très } \\
\text { disponib- } \\
\text { les }\end{array}$ & $\begin{array}{l}\text { Disponib- } \\
\text { les }\end{array}$ & $\begin{array}{l}\text { Assez dis- } \\
\text { ponnibles }\end{array}$ & $\begin{array}{l}\text { Peu dispo- } \\
\text { nbles }\end{array}$ & $\begin{array}{l}\text { Autres } \\
\text { (précisez) }\end{array}$ \\
\hline
\end{tabular}

\begin{tabular}{|c|c|c|c|c|c|}
\hline $\begin{array}{l}\text { 3n) Wie bewerten Sie die } \\
\text { Leistungen Ihres Tutors? } \\
\text { Mit der Arbeit meines } \\
\text { Tutors bin ich... }\end{array}$ & $\begin{array}{l}\text { Sehr zufrie- } \\
\text { den }\end{array}$ & $\begin{array}{l}\text { O } \\
\text { Zufrieden }\end{array}$ & $\begin{array}{l}\text { Einigerma- } \\
\text { ssen zufrie- } \\
\text { den }\end{array}$ & $\begin{array}{l}\text { Unzufrieden } \\
\text { Unzula }\end{array}$ & $\begin{array}{l}\text { Weiß nicht } \\
\text { Went }\end{array}$ \\
\hline $\begin{array}{l}\text { 3n) How satisfied are } \\
\text { you with the feedback } \\
\text { given from your tutor? }\end{array}$ & $\begin{array}{l}\text { O Very sa- } \\
\text { tisfactory }\end{array}$ & $\begin{array}{l}\text { O Satisfac- } \\
\text { tory }\end{array}$ & $\begin{array}{l}\text { O Quite satis- } \\
\text { factory }\end{array}$ & $\begin{array}{l}\text { O Unsatisfac- } \\
\text { tory }\end{array}$ & $\begin{array}{l}\text { O Others } \\
\text { (Please } \\
\text { specify) }\end{array}$ \\
\hline $\begin{array}{l}\text { 3n) Come valuta il lavoro } \\
\text { del suo tutor? }\end{array}$ & $\begin{array}{l}\square \text { Molto } \\
\text { soddisfa- } \\
\text { centi }\end{array}$ & $\begin{array}{l}\square \text { Soddis- } \\
\text { facente }\end{array}$ & $\begin{array}{l}\square \text { Abbas- } \\
\text { tanza soddis- } \\
\text { facenti }\end{array}$ & $\begin{array}{l}\square \text { Insoddis- } \\
\text { facente }\end{array}$ & $\begin{array}{l}\square \text { Altro } \\
\text { (specifi- } \\
\text { care) }\end{array}$ \\
\hline $\begin{array}{l}\text { 3n) Comment avez-vous } \\
\text { trouvez le travail des } \\
\text { tutors/moniteurs? }\end{array}$ & $\begin{array}{l}\text { Très satis- } \\
\text { faisant }\end{array}$ & Satisfaisant & $\begin{array}{l}\text { Assez satis- } \\
\text { faisant }\end{array}$ & Insatisfaisant & $\begin{array}{l}\text { Autres } \\
\text { (précisez) }\end{array}$ \\
\hline
\end{tabular}

\begin{tabular}{|l|l|}
\hline $\begin{array}{l}\text { 3o) Würden Sie nach diesem Kurs wieder an } \\
\text { einem elearning-Kurs teilnehmen? }\end{array}$ & $\begin{array}{l}\text { O Ja } \\
\text { O Nein } \\
\text { O Weiß nicht } \\
\end{array}$ \\
O Anderes (Bitte erläutern.) \\
\hline $\begin{array}{l}\text { 3o) After this course: Would you join an } \\
\text { elearning course or programme again? }\end{array}$ & O Yes \\
& O No \\
& O Don`t know \\
& O Others (please specify) \\
\hline $\begin{array}{l}\text { 3o) Dopo questo corso: Seguirebbe ancora } \\
\text { un corso di lingue e-learning? }\end{array}$ & $\square$ Si \\
& $\square$ No \\
& $\square$ Non lo so \\
\hline $\begin{array}{l}\text { 3o) Après ce cours: Fréquenteriez-vous en- } \\
\text { core un cours de langue e-learning? }\end{array}$ & O Oui \\
& O Non \\
& O Je ne sais pas \\
& O Autres (précisez) \\
\hline
\end{tabular}




\begin{tabular}{|c|c|c|c|c|c|c|}
\hline $\begin{array}{l}\text { 3p) Projektunterricht } \\
\text { (Eine vorgegebene Aufga- } \\
\text { be wird auf selbstgewählte } \\
\text { Art und Weise gelöst und } \\
\text { es wird z.B. ein eigenes } \\
\text { Projekt im Rahmen eines } \\
\text { Kurses entwickelt.) }\end{array}$ & \multicolumn{2}{|c|}{$\begin{array}{l}\text { O Gefiel mir } \\
\text { sehr } \\
++\end{array}$} & $\begin{array}{l}\text { O Gefiel } \\
\text { mir } \\
+\end{array}$ & $\begin{array}{l}\text { O Fand ich } \\
\text { mittelmäßig } \\
\text { o }\end{array}$ & $\begin{array}{l}\text { O Gefiel } \\
\text { mir nicht } \\
\text { - }\end{array}$ & $\begin{array}{l}\text { O Gefiel mir } \\
\text { gar nicht } \\
--\end{array}$ \\
\hline \multicolumn{7}{|l|}{ Gründe für Ihre Antwort: } \\
\hline $\begin{array}{l}\text { 3p) Project learning } \\
\text { (E.g. where you had to } \\
\text { carry out your own project } \\
\text { at the basis of the course) }\end{array}$ & \multicolumn{2}{|c|}{$\begin{array}{l}\text { O I liked it } \\
\text { very much }\end{array}$} & $\begin{array}{l}\text { O I liked } \\
\text { it }\end{array}$ & $\begin{array}{l}\text { It was OK } \\
\text { for me }\end{array}$ & $\begin{array}{l}\text { O I did } \\
\text { not like it }\end{array}$ & $\begin{array}{l}\text { I I did not like } \\
\text { it at all }\end{array}$ \\
\hline \multicolumn{7}{|l|}{ Reasons for your answer: } \\
\hline \multicolumn{2}{|l|}{$\begin{array}{l}\text { 3p) Insegnamento progetto } \\
\text { (es. lavorare su un proprio } \\
\text { progetto) }\end{array}$} & $\begin{array}{l}\square \text { Mi è } \\
\text { piaciuto } \\
\text { molto }\end{array}$ & $\begin{array}{l}\square \text { Mi è } \\
\text { piaciuto }\end{array}$ & $\begin{array}{l}\square \text { Per me } \\
\text { andava be- } \\
\text { ne }\end{array}$ & $\begin{array}{l}\square \text { Non mi } \\
\text { è piaciuto }\end{array}$ & $\begin{array}{l}\square \text { Non mi è } \\
\text { piaciuto affatto }\end{array}$ \\
\hline \multicolumn{7}{|l|}{ Motivi della sua risposta: } \\
\hline \multicolumn{2}{|c|}{$\begin{array}{l}\text { 3p) Enseignement au projet } \\
\text { (p.ex. travailler sur des projets/ } \\
\text { être responsable) }\end{array}$} & $\begin{array}{l}\text { O J'ai } \\
\text { beaucoup } \\
\text { apprécié }\end{array}$ & $\begin{array}{l}\text { O J'ai } \\
\text { apprécié }\end{array}$ & $\begin{array}{l}\text { O Cela } \\
\text { m'était égal }\end{array}$ & $\begin{array}{l}\text { O Je n'ai } \\
\text { pas ap- } \\
\text { précié }\end{array}$ & $\begin{array}{l}\text { O Je n'ai pas } \\
\text { du tout apprécié }\end{array}$ \\
\hline
\end{tabular}

3q) Was gefiel Ihnen besonders am Projektunterricht?

$3 q)$ What did you like especially about project learning?

$3 q)$ Che cosa le è piacuto di più nell'insegnamento progetto?

$3 q)$ Qu'avez-vous particulièrement apprécié dans l'enseignement au projet?

3r) Was bereitete Ihnen Schwierigkeiten beim Projektunterricht?

3r) What caused you difficulties with project learning?

3r) Quali difficoltà comporta l'insegnamento progetto?

3r) Quels ont été vos problèmes dans l'enseignement au projet?

\begin{tabular}{|c|c|c|c|c|c|}
\hline $\begin{array}{l}\text { 3s) Blended Learning } \\
\text { (Kombination von elearn- } \\
\text { ing und anderen Lehrfor- } \\
\text { men.) }\end{array}$ & $\begin{array}{l}\text { O Gefiel mir } \\
\text { sehr } \\
++\end{array}$ & $\begin{array}{l}\text { O Gefiel } \\
\text { mir } \\
+\end{array}$ & $\begin{array}{l}\text { O Fand ich } \\
\text { mittelmäßig } \\
\text { o }\end{array}$ & $\begin{array}{l}\text { O Gefiel } \\
\text { mir nicht } \\
\text { - }\end{array}$ & $\begin{array}{l}\text { O Gefiel mir } \\
\text { gar nicht } \\
--\end{array}$ \\
\hline \multicolumn{6}{|l|}{ Gründe für Ihre Antwort: } \\
\hline $\begin{array}{l}\text { 3s) Blended learning } \\
\text { (E.g. where any of the } \\
\text { methods mentioned above } \\
\text { were used in combination) }\end{array}$ & $\begin{array}{l}\text { O I liked it } \\
\text { very much }\end{array}$ & $\begin{array}{l}\text { I liked } \\
\text { it }\end{array}$ & $\begin{array}{l}\text { It was OK } \\
\text { for me }\end{array}$ & $\begin{array}{l}\text { O I did } \\
\text { not like it }\end{array}$ & $\begin{array}{l}\text { I did not like } \\
\text { it at all }\end{array}$ \\
\hline
\end{tabular}




\begin{tabular}{|c|c|c|c|c|c|}
\hline $\begin{array}{l}\text { 3s) Blended learning - II cor- } \\
\text { so WebLingu@ con tutor è un } \\
\text { corso 'blended'. } \\
\text { (Es. quando i metodi sopra } \\
\text { indicati erano utilizzati in ma- } \\
\text { niera integrata all'interno del } \\
\text { corso) }\end{array}$ & $\begin{array}{l}\square \text { Mi è } \\
\text { piaciuto } \\
\text { molto }\end{array}$ & $\begin{array}{l}\square \text { Mi è } \\
\text { piaciuto }\end{array}$ & $\begin{array}{l}\square \text { Per me } \\
\text { andava be- } \\
\text { ne }\end{array}$ & $\begin{array}{l}\square \text { Non mi } \\
\text { è piaciuto }\end{array}$ & $\begin{array}{l}\square \text { Non mi è } \\
\text { piaciuto affatto }\end{array}$ \\
\hline \multicolumn{6}{|l|}{ Motivi della sua risposta: } \\
\hline $\begin{array}{l}\text { 3s) Blended Learning } \\
\text { (combination de plusieurs mé- } \\
\text { thodes à la fois) }\end{array}$ & $\begin{array}{l}\text { O J'ai } \\
\text { beaucoup } \\
\text { apprécié }\end{array}$ & $\begin{array}{l}\text { O J'ai } \\
\text { apprécié }\end{array}$ & $\begin{array}{l}\text { O Cela } \\
\text { m'était égal }\end{array}$ & $\begin{array}{l}\text { O Je n'ai } \\
\text { pas ap- } \\
\text { précié }\end{array}$ & $\begin{array}{l}\text { O Je n'ai pas } \\
\text { du tout apprécié }\end{array}$ \\
\hline
\end{tabular}

3t) Was gefiel Ihnen besonders am Blended Learning?

3t) What did you like especially about blended learning?

3t) Che cosa le è piaciuto di più del blended learning?

3t) Qu'avez-vous particulièrement apprécié dans l'enseignement blended learning?

3u) Was bereitete Ihnen Schwierigkeiten beim Blended Learning?

3u) What caused you difficulties with blended learning?

3u) Quali difficoltà comportava il blended learning?

3u) Quels ont été vos problèmes dans l'enseignement blended learning? 
4. Ressourcen für elearning, Fähigkeiten und Wissen im Umgang mit dem Computer

4. Resources for elearning computer skills and knowledge

4. Risorse per l'e-learning: abilità e conoscenza dell'uso del computer

4. Ressources en termes de compétences et connaissances informatiques pour l'apprentissage ligne

Mit den folgenden Fragen möchten wir Ihr PC-Wissen ermitteln. Bitte antworten Sie ganz ehrlich, wir wollen Ihren persönlichen Kenntnisstand ermitteln, um den Kurs nach Ihren Fähigkeiten zu gestalten (Sie brauchen keine Bedenken zu haben, falls Sie noch nicht so geübt sind im Umgang mit dem Computer!).

The following questions aim to gather information about your computer knowledge and skills.

Le facciamo le seguenti domande per avere più informazioni sulle abilità e conoscenza del computer già in suo possesso. Per favore sia onesto, vogliamo solo renderci conto se ha avuto problemi durante il corso. Inoltre vogliamo sapere se ha imparato qualcosa in questo corso (è per noi un feedback).

Les questions suivantes nous permettront de mieux connaître vos acquis en termes de connaissances et de compétences en informatique. Répondez en toute franchise, nous ne cherchons qu'à évaluer les problèmes que vous avez pu rencontrer pendant la formation.

\begin{tabular}{|l|l|}
\hline $\begin{array}{l}\text { 4a) Haben Sie Erfahrung im Um- } \\
\text { gang mit Computern? }\end{array}$ & $\begin{array}{l}\text { O Ja, viel Erfahrung } \\
\text { O Ja, etwas Erfahrung } \\
\text { O Nein, keine Erfahrung }\end{array}$ \\
\hline $\begin{array}{l}\text { 4a) Do you have any previous expe- } \\
\text { rience of using a computer? }\end{array}$ & $\begin{array}{l}\text { O Yes, a lot } \\
\text { O Yes, a little } \\
\text { O No }\end{array}$ \\
\hline $\begin{array}{l}\text { 4a) Ha mai usato il computer prima } \\
\text { d'ora? }\end{array}$ & $\begin{array}{l}\text { O Si, molto } \\
\text { O Si, un po' } \\
\text { O No }\end{array}$ \\
\hline $\begin{array}{l}\text { 4a) Possédez-vous l'expérience de } \\
\text { l'utilisation d'un ordinateur? }\end{array}$ & $\begin{array}{l}\text { O Oui, une grande expérience } \\
\text { O Oui, une petite expérience } \\
\text { O Non }\end{array}$ \\
\hline
\end{tabular}

4b) Wofür nutzen Sie bisher Computer?

4b) What do you use a computer for?

4b) Per che cosa utilizza il computer?

4b) À quelles fins utilisez-vous un ordinateur?

4c) Bitte schätzen Sie Ihre Fähigkeiten und Erfahrung im Umgang mit Folgendem ein:

4c) How much experience do you have in using the following software programmes?

4c) Quanta esperienza ha nell'uso dei seguenti software?

4c) Quelle est votre expérience en matière d'utilisation des logiciels suivants ?

\begin{tabular}{|c|c|c|c|c|c|}
\hline & $\begin{array}{c}\text { Ich habe } \\
\text { bereits viel } \\
\text { Erfahrung }\end{array}$ & $\begin{array}{c}\text { Ich habe be- } \\
\text { reits etwas } \\
\text { Erfahrung }\end{array}$ & $\begin{array}{c}\text { Ich habe } \\
\text { wenig Er- } \\
\text { fahrung }\end{array}$ & $\begin{array}{c}\text { Ich habe } \\
\text { keine Erfah- } \\
\text { rung }\end{array}$ & $\begin{array}{c}\text { Den Begriff } \\
\text { kenne ich } \\
\text { nicht }\end{array}$ \\
\hline $\begin{array}{c}\text { I have } \\
\text { a lot experi- } \\
\text { ence }\end{array}$ & $\begin{array}{c}\text { I have } \\
\text { some experi- } \\
\text { ence }\end{array}$ & $\begin{array}{c}\text { I have } \\
\text { a little expe- } \\
\text { rience }\end{array}$ & $\begin{array}{c}\text { I have } \\
\text { no experi- } \\
\text { ence }\end{array}$ & \\
\hline
\end{tabular}




\begin{tabular}{|c|c|c|c|c|c|}
\hline & $\begin{array}{c}\text { Ho molta } \\
\text { esperienza } \\
\text { J'ai beau- } \\
\text { coup d'ex- } \\
\text { périence }\end{array}$ & $\begin{array}{c}\text { Ho qualche } \\
\text { esperienza } \\
\text { J'ai une } \\
\text { certaine ex- } \\
\text { périence }\end{array}$ & $\begin{array}{c}\text { Ho poca } \\
\text { esperienza } \\
\text { J'ai peu } \\
\text { d'ex- } \\
\text { périence } \\
\end{array}$ & $\begin{array}{c}\begin{array}{c}\text { Non ho } \\
\text { esperienza }\end{array} \\
\begin{array}{c}\text { Je n'ai } \\
\text { aucune ex- } \\
\text { périence }\end{array} \\
\end{array}$ & \\
\hline $\begin{array}{l}\text { Textverarbeitungs- } \\
\text { programme (z.B. Word) } \\
\text { Text programmes } \\
\text { (e.g. Word) } \\
\text { Programmi di scrittura } \\
\text { (es. Word) } \\
\text { Traitement de texte } \\
\text { (par exemple, Word) }\end{array}$ & 0 & O & 0 & 0 & 0 \\
\hline $\begin{array}{l}\text { Kalkulations-Software } \\
\text { (z.B. Excel) } \\
\text { Calculation Software } \\
\text { (E.g. Excel) } \\
\text { Fogli di Calcolo } \\
\text { (es. Excel) } \\
\text { Logiciel de calcul } \\
\text { (Par exemple, Excel) }\end{array}$ & $\bigcirc$ & $\bigcirc$ & 0 & 0 & 0 \\
\hline $\begin{array}{l}\text { Datenbanken } \\
\text { (z.B. Access oder dbase) } \\
\text { Databases } \\
\text { (Access, dbase) } \\
\text { Banche dati } \\
\text { (Access, dbase) } \\
\text { Bases de données } \\
\text { (Access, dbase) }\end{array}$ & $\mathrm{O}$ & $\bigcirc$ & 0 & 0 & 0 \\
\hline $\begin{array}{l}\text { Präsentations-Software } \\
\text { (z.B. Power Point) } \\
\text { Presentation software } \\
\text { (E.g. Power Point) } \\
\text { Software per presenta- } \\
\text { zioni } \\
\text { (es. Power Point) } \\
\text { Logiciel de présentation } \\
\text { (Par exemple, Power } \\
\text { Point) }\end{array}$ & 0 & 0 & 0 & 0 & 0 \\
\hline
\end{tabular}




\begin{tabular}{|c|c|c|c|c|c|}
\hline $\begin{array}{l}\text { Internet } \\
\text { (surfen, recherchieren) } \\
\text { Internet browsing } \\
\text { Navigazione in Internet } \\
\text { Navigateur Internet }\end{array}$ & $\mathrm{O}$ & $\mathrm{O}$ & $\mathrm{O}$ & 0 & $\mathrm{O}$ \\
\hline $\begin{array}{l}\text { E-Mail } \\
\text { E-Mailing } \\
\text { Posta Elettronica } \\
\text { Courrier électronique }\end{array}$ & $\mathrm{O}$ & $\mathrm{O}$ & $\mathrm{O}$ & $\mathrm{O}$ & $\mathrm{O}$ \\
\hline $\begin{array}{l}\text { Webdesign } \\
\text { Web designing } \\
\text { Web designing } \\
\text { Conception de pages } \\
\text { Web }\end{array}$ & $\mathrm{O}$ & $\mathrm{O}$ & $\mathrm{O}$ & $\mathrm{O}$ & $\mathrm{O}$ \\
\hline $\begin{array}{l}\text { Webpublishing } \\
\text { Web publishing } \\
\text { Web publishing } \\
\text { Publication de pages } \\
\text { Web }\end{array}$ & $\mathrm{O}$ & $\mathrm{O}$ & $\mathrm{O}$ & $\mathrm{O}$ & $\mathrm{O}$ \\
\hline Networking & $\mathrm{O}$ & 0 & 0 & 0 & $\mathrm{O}$ \\
\hline $\begin{array}{l}\text { Kommentare: } \\
\text { Comments: } \\
\text { Commenti: } \\
\text { Commentaires }\end{array}$ & & & & & \\
\hline
\end{tabular}

\begin{tabular}{|l|l|}
\hline $\begin{array}{l}\text { 4d) Wo hatten Sie Zugang zu einem Compu- } \\
\text { ter mit Internet-Anschluss, den sie für den } \\
\text { Kurs genutzt haben? } \\
\text { (Bitte alle zutreffenden Antworten ankreu- } \\
\text { zen.) }\end{array}$ & $\begin{array}{l}\text { O Zu Hause } \\
\text { O Am Arbeitsplatz } \\
\text { O Andere Orte (Bitte erläutern.) }\end{array}$ \\
\hline 4d) & $\begin{array}{l}\text { O At home } \\
\text { O At your working place } \\
\text { O Other places (Please specify) }\end{array}$ \\
\hline $\begin{array}{l}\text { 4d) Dove ha la possibilità di usare internet } \\
\text { per il corso? }\end{array}$ & $\begin{array}{l}\text { O A casa } \\
\text { O posto di lavoro } \\
\text { O luoghi diversi }\end{array}$ \\
\hline $\begin{array}{l}\text { 4d) Où avez-vous la possibilité d'utiliser } \\
\text { l'ordinateur pour le cours? }\end{array}$ & $\begin{array}{l}\text { O À mon domicile } \\
\text { O Sur mon lieu de travail } \\
\text { O Ailleurs (précisez): }\end{array}$ \\
\hline
\end{tabular}




\begin{tabular}{|l|l|}
\hline $\begin{array}{l}\text { 4e) Was für einen Internet-Anschluss haben } \\
\text { Sie für diesen Kurs genutzt? }\end{array}$ & $\begin{array}{l}\text { O Schnelle Telefonleitung (z.B. TDSL/ADSL) } \\
\text { O Langsame Telefonleitung (Modem) } \\
\text { O Weiß nicht }\end{array}$ \\
\hline 4e) What kind of internet access did you use? & $\begin{array}{l}\text { O High speed } \\
\text { O Low speed } \\
\text { O Don`t know }\end{array}$ \\
\hline 4e) Che tipo di collegamento a internet usa? & $\square$ Alta velocità \\
& $\begin{array}{l}\text { Bassa velocità } \\
\square \text { Non so }\end{array}$ \\
\hline $\begin{array}{l}\text { 4e) Quel type de connexion Internet utilise- } \\
\text { rez-vous? }\end{array}$ & $\begin{array}{l}\text { O Haut débit } \\
\text { O Bas débit } \\
\text { O Ne sait pas }\end{array}$ \\
\hline
\end{tabular}

\begin{tabular}{|c|c|}
\hline $\begin{array}{l}\text { 4h) Welche Zeit haben Sie meistens für } \\
\text { elearning genutzt? } \\
\text { (Bitte ankreuzen.) }\end{array}$ & $\begin{array}{l}\text { Z Zwischen } 8 \text { und } 12 \text { Uhr } \\
\text { Zwischen } 12 \text { und } 18 \text { Uhr } \\
\text { Zwischen } 18 \text { und } 22 \text { Uhr } \\
\text { Nach } 22 \text { Uhr }\end{array}$ \\
\hline \multicolumn{2}{|l|}{ Bitte erläutern Sie die Gründe für Ihre Antwort: } \\
\hline $\begin{array}{l}\text { 4h) At what time did you most often do } \\
\text { elearning? }\end{array}$ & $\begin{array}{l}\text { O Daytime } \\
\text { O Evening } \\
\text { O Night }\end{array}$ \\
\hline \multicolumn{2}{|l|}{ Please describe reasons for your answer. } \\
\hline $\begin{array}{l}\text { 4h) In quale momento della giornata faceva } \\
\text { e-learning? }\end{array}$ & $\begin{array}{l}\square \text { Giorno } \\
\square \text { Sera } \\
\square \text { Notte } \\
\end{array}$ \\
\hline \multicolumn{2}{|l|}{ Perché? } \\
\hline $\begin{array}{l}\text { 4h) À quel moment de la journée avez-vous } \\
\text { utilisé l'enseignement en ligne sur internet? }\end{array}$ & $\begin{array}{l}\text { O Journée } \\
\text { O Soir } \\
\text { O Nuit }\end{array}$ \\
\hline
\end{tabular}

\begin{tabular}{|c|c|c|c|c|c|c|}
\hline $\begin{array}{l}\text { 4i) Wieviele Stunden pro Wo- } \\
\text { che haben Sie für elearning } \\
\text { genutzt? } \\
\text { Was schätzen Sie... } \\
\text { (Bitte ankreuzen.) }\end{array}$ & $\begin{array}{l}\text { Mehr als } \\
10 \text { Stun- } \\
\text { den pro } \\
\text { Woche }\end{array}$ & $\begin{array}{l}\text { O } \\
\text { Zwischen } \\
5 \text { und } 10 \\
\text { Stunden } \\
\text { pro Woche }\end{array}$ & $\begin{array}{l}\text { Weniger als } \\
\text { Stunden pro } \\
\text { Woche }\end{array}$ & \multicolumn{2}{|c|}{$\begin{array}{c}\text { O } \\
\text { Ich ent- } \\
\text { scheide jede } \\
\text { Woche neu }\end{array}$} & $\begin{array}{l}\text { O } \\
\text { Ich } \\
\text { weiß } \\
\text { es } \\
\text { nicht }\end{array}$ \\
\hline \multicolumn{7}{|c|}{ Platz für Kommentare und Erklärungen und andere Angaben: } \\
\hline $\begin{array}{l}\text { 4i) How much time per week } \\
\text { did you spend using your } \\
\text { elearning programme }\end{array}$ & $\begin{array}{l}\text { O } \\
\text { More than } \\
10 \text { hours } \\
\text { per week }\end{array}$ & $\begin{array}{l}\text { O } \\
\text { Between } 5 \\
\text { and } 10 \\
\text { hours } \\
\text { per week }\end{array}$ & $\begin{array}{c}\text { O } \\
\text { Less than } \\
5 \text { hours per } \\
\text { week }\end{array}$ & $\begin{array}{l}\text { O } \\
\text { I will de- } \\
\text { cide every } \\
\text { week }\end{array}$ & & $\begin{array}{l}\text { O } \\
\text { ave no } \\
\text { dea }\end{array}$ \\
\hline \multicolumn{7}{|l|}{ Comments: } \\
\hline $\begin{array}{l}\text { 4i) Quanto tempo alla settima- } \\
\text { na ha usato la piattaforma } e^{-} \\
\text {learning? }\end{array}$ & $\begin{array}{l}\square \\
\text { Più di } 10 \\
\text { ore a setti- } \\
\text { mana }\end{array}$ & $\begin{array}{l}\square \\
\text { Tra le } 5 \text { e } \\
\text { le } 10 \text { ore a } \\
\text { settimana }\end{array}$ & \begin{tabular}{c|}
$\square$ \\
Meno di \\
cinque ore a \\
settimana \\
\end{tabular} & $\begin{array}{c}\square \\
\text { Decidevo } \\
\text { ogni volta }\end{array}$ & & $\begin{array}{l}\square \\
\text { on ho } \\
\text { dea }\end{array}$ \\
\hline \multicolumn{7}{|l|}{ Commenti: } \\
\hline
\end{tabular}




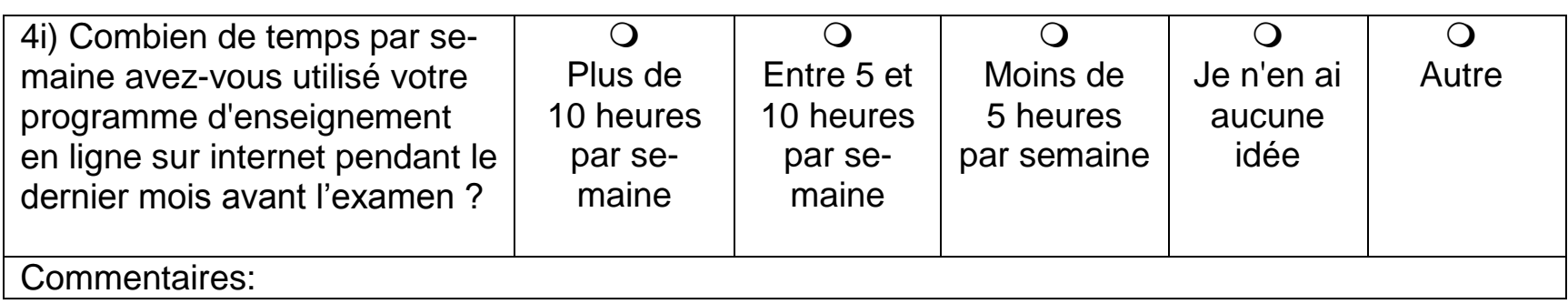

\begin{tabular}{|l|l|}
\hline $\begin{array}{l}\text { 4j) Hatten Sie jemanden, der innen beim } \\
\text { elearning behilflich war? }\end{array}$ & $\begin{array}{l}\text { O Ja } \\
\text { O Nein } \\
\text { O Weiß nicht }\end{array}$ \\
\hline $\begin{array}{l}\text { 4j) Did you have anyone who provided local } \\
\text { support for your e-learning? }\end{array}$ & $\begin{array}{l}\text { O Yes } \\
\text { O No } \\
\text { O Unsure }\end{array}$ \\
\hline $\begin{array}{l}\text { 4j) Aveva qualcuno a livello locale che per l'e- } \\
\text { learning poteva aiutarla? }\end{array}$ & $\square$ Si \\
& $\square$ No \\
\hline $\begin{array}{l}\text { 4j) Quelqu'un vous a t-il aidé pendant votre } \\
\text { enseignement en ligne? }\end{array}$ & $\begin{array}{l}\text { O Oui } \\
\text { O Non } \\
\text { O Ne suis pas sûr(e) }\end{array}$ \\
\hline
\end{tabular}

\begin{tabular}{|l|l|}
\hline $\begin{array}{l}\text { 4k) Wenn sie mit „Ja“ geantwortet haben, wer } \\
\text { hat Ihnen geholfen? } \\
\text { (Sie können mehrere Antworten ankreuzen.) }\end{array}$ & $\begin{array}{l}\text { O Freunde } \\
\text { O Partner } \\
\text { O Kollegen } \\
\text { O Vorgesetzte } \\
\text { O Andere Personen (Bitte erläutern.) }\end{array}$ \\
\hline $\begin{array}{l}\text { 4k) If you answered, "Yes", who helped you? } \\
\text { (Please tick all the relevant answers) }\end{array}$ & O Friends \\
& O Partner \\
& O Colleagues \\
& O Officials \\
& O Others: (Please specify) \\
\hline $\begin{array}{l}\text { 4k) Se ha risposto "Si", chi le poteva aiutare? } \\
\text { (segna la risposta che ritiene più adatta) }\end{array}$ & $\square$ Amici \\
& $\square$ Partner \\
& $\square$ Colleghi \\
\hline 4k) Si "Oui", qui vous a aidé ? (Veuillez co- \\
cher toutes les réponses correspondantes)
\end{tabular}

\begin{tabular}{|c|c|}
\hline $\begin{array}{l}\text { 4j) Wenn Sie mit „Nein“ geantwortet haben, } \\
\text { hätten Sie Hilfe gebraucht? }\end{array}$ & $\begin{array}{l}\text { O Ja } \\
\text { Nein } \\
\text { Weiß nicht }\end{array}$ \\
\hline $4 j)$ & $\begin{array}{l}\text { O Yes } \\
\text { O No } \\
\text { O Don`t know }\end{array}$ \\
\hline $\begin{array}{l}\text { 4j) Se ha risposto „No“, avrebbe avuto biso- } \\
\text { gno d'aiuto? }\end{array}$ & $\begin{array}{l}\text { O Si } \\
\text { O No } \\
\text { Non so }\end{array}$ \\
\hline 4j) Si „Non“, auriez-vous eu besoin d'aide? & $\begin{array}{l}\text { O Oui } \\
\text { O Non } \\
\text { O Je ne sais pas }\end{array}$ \\
\hline
\end{tabular}


5. Persönliche Motivation an diesem Kurs teilzunehmen, Erwartungen und Ziele

5. Personal motivation for doing this course, expectations and aims

5. Motivazioni personali e progressi conseguiti in relazione al corso.

5. Motivations personnelles pour suivre cette formation, atteinte des objectifs

\begin{tabular}{|c|c|}
\hline $\begin{array}{l}\text { 5a) Warum haben Sie an diesem Kurs teilge- } \\
\text { nommen? } \\
\text { (Sie können mehrere Antworten ankreuzen.) }\end{array}$ & $\begin{array}{l}\text { O Interesse am Thema } \\
\text { O Qualifikation } \\
\text { O Zertifikat } \\
\text { O Pflicht/Vorgabe } \\
\text { O Anderes (Bitte erläutern.) }\end{array}$ \\
\hline 5a) Why did you join this course? & $\begin{array}{l}\text { Interest in the topic } \\
\text { Qualification } \\
\text { Certification } \\
\text { Obligation } \\
\text { Others (please specify): }\end{array}$ \\
\hline 5a) Perché ha seguito questo corso? & $\begin{array}{l}\text { Interesse al tema } \\
\text { Qualificazione } \\
\text { Certificato } \\
\text { Obbligo } \\
\text { Altro (specificare): }\end{array}$ \\
\hline $\begin{array}{l}\text { 5a) Pourquoi avez-vous participé à cette for- } \\
\text { mation? }\end{array}$ & $\begin{array}{l}\text { Intérêt thématique } \\
\text { Qualification } \\
\text { Certificat } \\
\text { Devoir } \\
\text { Autres (précisez): }\end{array}$ \\
\hline
\end{tabular}

5b) Haben sich Ihre Erwartungen an diesen Kurs erfüllt?

5b) Did you reach your learning objectives by the end of the course?

5b) Ha raggiunto gli obiettivi che lei aspettava alla fine del corso?

5b) A la fin de cette formation, avez-vous atteint les objectifs d'enseignement que vous visiez?

\begin{tabular}{|l|}
\hline Ja \\
O Nein \\
O Teils/teils \\
O Yes \\
O No \\
O Si \\
O No \\
O Oui \\
O Non \\
\hline
\end{tabular}

5c) Welche Gründe gibt es für die Erfüllung oder Nichterfüllung Ihrer Erwartungen?

5c) What were the reasons for reaching or not reaching this?

5c) Quali sono state le ragioni per cui li ha raggiunti o non li ha raggiunti?

5c) Pour quelles raisons ? 


\begin{tabular}{|c|c|c|c|c|c|}
\hline $\begin{array}{l}\text { 5d) Sind Sie zufrieden mit dem } \\
\text { Kurs? } \\
\text { (Bitte ankreuzen.) }\end{array}$ & $\begin{array}{c}\text { O } \\
\text { Sehr zufrie- } \\
\text { den }\end{array}$ & $\begin{array}{c}\text { O } \\
\text { Eher zu- } \\
\text { frieden }\end{array}$ & $\begin{array}{c}O \\
\text { Teils/teil } \\
s\end{array}$ & $\begin{array}{c}O \\
\text { Eher unzu- } \\
\text { frieden }\end{array}$ & $\begin{array}{l}\text { O } \\
\text { Völlig un- } \\
\text { zufrieden }\end{array}$ \\
\hline \multicolumn{2}{|c|}{ 5d) Are you satisfied with the course? } & \multicolumn{4}{|l|}{$\begin{array}{l}\mathrm{O} \text { Yes } \\
\mathrm{O} \text { No }\end{array}$} \\
\hline \multicolumn{2}{|l|}{ 5d) È soddisfatto di questo corso? } & \multicolumn{4}{|l|}{$\begin{array}{l}\mathrm{OSi} \\
\mathrm{O} \mathrm{No}\end{array}$} \\
\hline \multicolumn{2}{|c|}{ 5d) Étes-vous satisfait(e) de cette formation ? } & \multicolumn{4}{|l|}{$\begin{array}{l}\text { O Oui } \\
\text { O Non }\end{array}$} \\
\hline
\end{tabular}

5e) Welches sind die Gründe für die Zufriedenheit bzw. Unzufriedenheit mit dem Kurs?

$5 \mathrm{e})$ What are the reasons for this satisfaction or disatisfaction?

$5 e)$ Quali sono le ragioni della sua soddisfazione o insoddisfazione?

5e) Pour quelles raisons?

5f) Was hat Ihnen am besten gefallen in diesem Kurs?

5f) What did you like most in the course?

$5 f)$ Che cosa le è piaciuto di più di questo corso?

5f) Qu'avez-vous tout particulièrement apprécié dans cette formation ?

$5 \mathrm{~g})$ Was war für Sie die größte Hilfe beim Lernen?

$5 \mathrm{~g})$ What was the most beneficial thing you have learned?

$5 \mathrm{~g}$ ) Che cosa le ha aiutato di più nell'apprendimento?

$5 \mathrm{~g})$ Quelle a été votre plus grande aide dans cette formation ?

5h) Was hat Ihnen in diesem Kurs nicht gefallen?

$5 \mathrm{~h})$ What did you dislike in the course?

$5 \mathrm{~h})$ Che cosa non le è piaciuto del corso?

5h) Qu'est-ce que vous n'avez pas apprécié dans cette formation ?

5i) Was hat Ihnen in diesem Kurs Schwierigkeiten bereitet?

5i) What caused you difficulties?

5i) Che cosa le ha causato difficoltà?

5i) Quels ont été vos problèmes ?

5j) Was hat Ihnen in diesem Kurs gefehlt?

5j) What did you miss in this course?

5 j) Che cosa le è mancato in questo corso?

5j) Qu'est-ce qui vous a manqué pendant cette formation ?

5k) Welche Vorschläge haben Sie, um die Qualität des Kurses in der Zukunft zu verbessern?

$5 \mathrm{k}$ ) What suggestions do you have to improve the course in the future?

$5 \mathrm{k})$ Quali suggerimenti ha per migliorare questo corso in futuro?

5k) Que proposeriez-vous pour améliorer cette formation à l'avenir? 
6. Platz für Vorschläge, Kommentare und Fragen

6. Open space for suggestions comments and questions

6. Spazio per suggerimenti, commenti e domande

6. Espace libre pour vos suggestions, commentaires et questions

6a) An welchen anderen Kursthemen hätten Sie Interesse?

6a) What other courses would you like to see offered in the future?

6a) Quali altri corsi di lingue le piacerebbe fossero offerti in futuro?

6a) Quels autres cours souhaiteriez-vous voir proposer à l'avenir?

6b) Wenn Sie noch Kommentare oder Fragen haben, die Sie uns in Bezug auf den Kurs mitteilen wollen, bitte nutzen Sie diesen Platz:

6b) If you have any other comments or questions that you would like to share regarding the forthcoming course please write them here.

6b) Se ha altri commenti o domande da condividere riguardo al corso che stai per iniziare, li riporta qui.

6b) Indiquez ici tout commentaire ou question que vous souhaiteriez faire partager concernant le cours à venir.

Wir bedanken uns ganz herzlich dafür,

dass Sie sich die Zeit genommen haben,

den Fragebogen auszufüllen!

Thank you very much

for taking the time to complete this questionnaire!

Grazie mille per aver completato il questionario!

Merci beaucoup pour avoir pris le temps de répondre à ce questionnaire! 


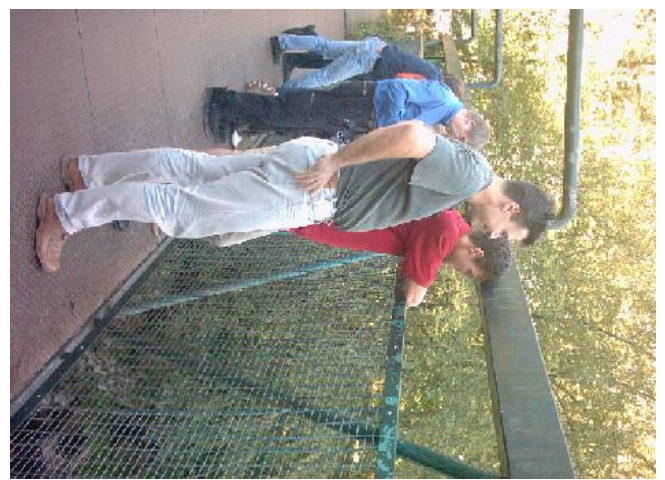

\section{Inventory authors/producers Inventar Autoren/Produzenten Inventario autori/produttori Inventaire auteurs/producteurs}
responsable:
Hans-Dieter Haller verantwortlich: responsabile: responsable:

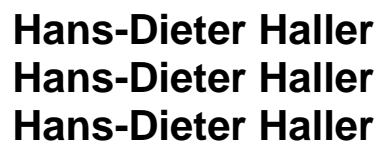

multilinguale version multilinguale Fassung versione di multilinguale version plurilingue
English e-learning, blended learning
Deutsch e-Learning, Blended Learning
Italiano insegnamento elettronico, apprendimento diverso
Français apprentissage électronique, apprentissage mixte

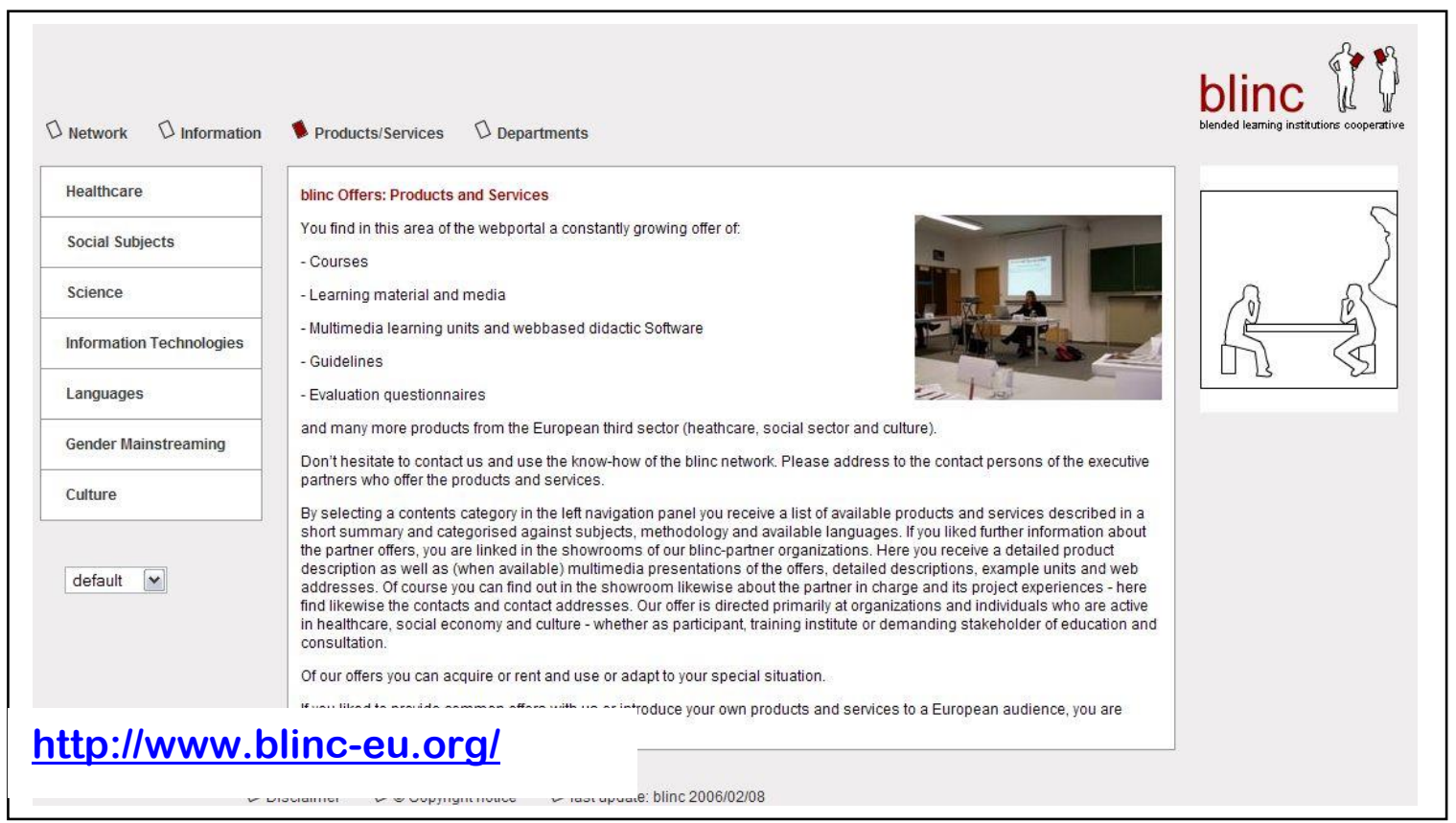


Self-evaluation inventory for authors and producers of e-learning.

Inventar zur Selbstevaluation für Autoren und Produzenten von e-Learning.

Inventario dell'auto-valutazione per l'insegnamento elettronico

Inventaire d'une auto-évaluation pour auteurs et producteurs d'apprentissage électronique.

The inventory consists of three parts:

Das Inventar besteht aus drei Teilen:

Inventario diviso in tre parti

L'inventaire est composé de trois parties:

1. The first part should help producers and authors of e-learning and blended learning ${ }^{7}$ courses to check if they know their target group.

1. Der erste Teil soll Produzenten und Autoren von e-Learning oder Blended Learning ${ }^{1}$ helfen zu überprüfen, ob sie ihre Zielgruppe kennen.

1. La prima parte dovrà aiutare i produttori dell'insegnamento elettronico a verificare si conoscono bene i loro gruppi di obiettivi.

1. La première partie devrait aider les auteurs et producteurs d'apprentissage électronique ${ }^{1}$ de verifier s'ils connaissent bien leur groupe en vue.

2. The second part of this self-test is concerned with questions about the design of instructional programmes.

2. Der zweite Teil dieses Selbst-Tests enthält Fragen zum didaktischen Design von Lehr-/Lernmaterialien.

2. La seconda parte di questa auto-valutazione contiene domande riguardando il disegno didattico dei materiali.

2. La deuxième partie de cette auto-évaluation contient des questions concernant le design didactique du programme d'enseignement.

3. The third part leads to criteria that may be decisive for the effectiveness of a programme.

3. Der dritte Teil besteht aus Kriterien, die für die Effektivität eines Programms relevant sind

3. La terza parte dimostra le tre caratteristiche importanti per l'efficacia di un programma.

3. La troisième partie montre des critères significatifs pour l'efficacité d'un programme.

\footnotetext{
7 "Blended learning" is meant as a course or learning programme with e-learning, traditional "face-to-face learning and often practice-learning.

7 "Blended learning" wird verstanden als Mischung aus e-Learning, Präsenzlernen und oft auch Praxisprojekten.

7 "Blended learning / L'apprendimento misto" s'intende come combinazione dell'apprendimento, della presenza nell'insegnamento e spesso dei progetti pratici.

${ }^{1}$ Le mode d'apprentissage mixte (appelé aussi Blended Learning en anglais) désigne l'utilisation conjointe du eLearning et du mode classique d'apprentissage appelé souvent « présentiel ». En général, l'apprenant va ainsi alterner entre des sessions à distance en ligne et des sessions en face-à-face avec le ou les formateurs.
} 
This inventory was compiled by eL3-project partners on the basis of former concepts of Karl-Heinz Flechsig and Hans-Dieter Haller as well as other authors which are cited and is free for your adoption and usage if this source is quoted.

Dieses Inventar wurde von eL3-Projektpartnern auf der Grundlage früherer Entwürfe von Karl-Heinz Flechsig und Hans-Dieter Haller sowie zitierten Autoren entwickelt und steht bei Verweisen auf die Quelle frei zur Verfügung und Nutzung.

Questo inventario è stato sviluppato coi partner del progetto el3 sulla base di progetti precedenti di Karl-Heinz Flechsig e Hans-Dieter Haller così come altri autori citati; l'inventario è a libera disposizione riferendosi sempre alle fonti.

Cet inventaire fut développé par les partenaires du projet el3 sur la base d'anciens projets de Karl-Heinz Flechsig et Hans-Dieter Haller ainsi que par d'autres auteurs cités; il est à votre disposition et utilisation en vous référant à la source.

A. Do I know my learners and their needs?

A. Kenne ich meine Lerner und deren Bedürfnisse?

A. Conosco i miei studenti e i loro bisogni?

A. Est-ce que je connais mes apprenants et leurs besoins?

Especially for the providers and designers of e-learning it is very important to consider the target audience, i.e. the learners. In traditional learning environments the teachers or trainers can easily adjust their style in the interaction with the learners. In elearning settings the trainer or tutor has to communicate with learners whom he/she will perhaps never see or whom he/she will not see while they are learning. That is one of the main reasons why it is necessary to know the learners very well or why designers of e-learning should do some research or systematic reflections about the target group and criteria of good instructional designs and products when planning and producing a programme. "Who are my learners and what are their needs?" therefore is the first important question when designing an e-learning programme (see Lewis \& Whitlock 20038, p.3).

Be aware that these questions or aspects are to be regarded in their relation to $e$ learning/blended learning and specialities of the programme in question.

Für Anbieter und Gestalter von e-Learning ist es besonders wichtig, ihre Zielgruppe die Lerner - zu kennen. In traditionellen Lernumgebungen kann der Lehrer oder Trainer seinen Unterrichtsstil in der Interaktion mit den Lernern verändern und ihn anpassen an die aktuelle Situation. Bei e-Learning-Szenarien muss er mit Lernern kommunizieren, die er womöglich nie sehen wird bzw. zu denen er beim Lernen keinen Kontakt hat. Dies ist einer der wesentlichen Gründe, warum Gestalter von e-Learning ihre Lerner sehr gut kennen sollten bzw. warum sie einige Untersuchungen oder systematische Reflexionen über die Zielgruppe anstellen sollten, wenn sie planen, e-Learning einzuführen oder Programme produzieren wollen. "Wer sind meine Lerner und was sind ihre Bedürfnisse?" ist

\footnotetext{
${ }^{8}$ Roger Lewis, Quentin Whitlock: How to Plan and Manage an E-learning Programme, Gower Publishing Limited, January, 2003
} 
deshalb die erste wichtige Frage bei der Gestaltung eines e-Learning-Programms (vgl. Lewis \& Whitlock 2003, p.3).

Bitte berücksichtigen Sie, dass die folgenden Fragen oder Aspekte in ihrer Beziehung zu den Besonderheiten des betreffenden e-Learning oder Blended Learning-Kurses gesehen werden müssen.

Per tutti quelli che presentano e producono l'insegnamento elettronico è importante conoscere i loro gruppi di obiettivi. Nelle situazioni classici d'insegnamento l'istruttore può variare il suo stile d' interazione con gli studenti nell'attuale situazione. Negli scenari d'insegnamento elettronico l'insegnante dovrà communicare con studenti che lui prababilmente non vedrà mai oppure non entrerà in contatto. Questo è uno dei motivi più importanti per cui l'organizzatore dell'insegnamento elettronico dovrà conoscere bene i suoi studenti oppure per cui certe indagini o reflessioni sistematiche sui gruppi di obiettivi dovranno essere fatti se vuole eseguire o produrre questo programma. "Chi sono $i$ miei allievi e quali sono i loro bisogno?" è la prima domanda per l'organizzazione del programma.

Tenga presente che le seguenti domande o aspetti devono essere visti in relazione con le caratteristiche speciali dello stesso apprendimento elettronico o apprendimento misto.

Pour ceux qui présentent et produisent l'apprentissage électronique, il est particulièrement important de connaître leur groupe individual d'apprenants. Dans des situations classiques d'instruction l'enseignant ou l'entraîneur peut modifier ou adapter son mode d'enseignement à la situation actuelle en interaction avec l'apprenant. Dans la situation de l'apprentissage électronique il doit communiquer avec des apprenants qu'il ne verra probablement jamais respectivement avec lesquels il n'entre pas en contact direct pendant leurs cours. Voilà la raison essentielle pourquoi les producteurs de l'apprentissage électronique devraient très bien connaître leurs apprenants respectivement pourquoi ils devraient étudier et réflechir systematiquement concernants leurs apprenants dès qu'ils ont l'intention d'introduire l'apprentissage électronique ou de produire des programmes. Voilà donc la première question importante en formant un programme pour apprentissage électronique: Qui sont mes étudiants et leurs besoins?

Veuillez svp prendre en consideration que les questions suivantes ou aspects devraient être vus en relation avec la particularité des programmes apprentissage électronique en questions. 
A1. Analysis of learner characteristics

A1. Analyse der Charakteristika der Lerner

\section{A1. Analisi delle caratteristiche degli studenti}

A1. Analyse des caractéristiques des apprenants

\section{General features:}

Allgemeine Merkmale:

\section{Caratteristiche generali:}

Caractéristiques générales:

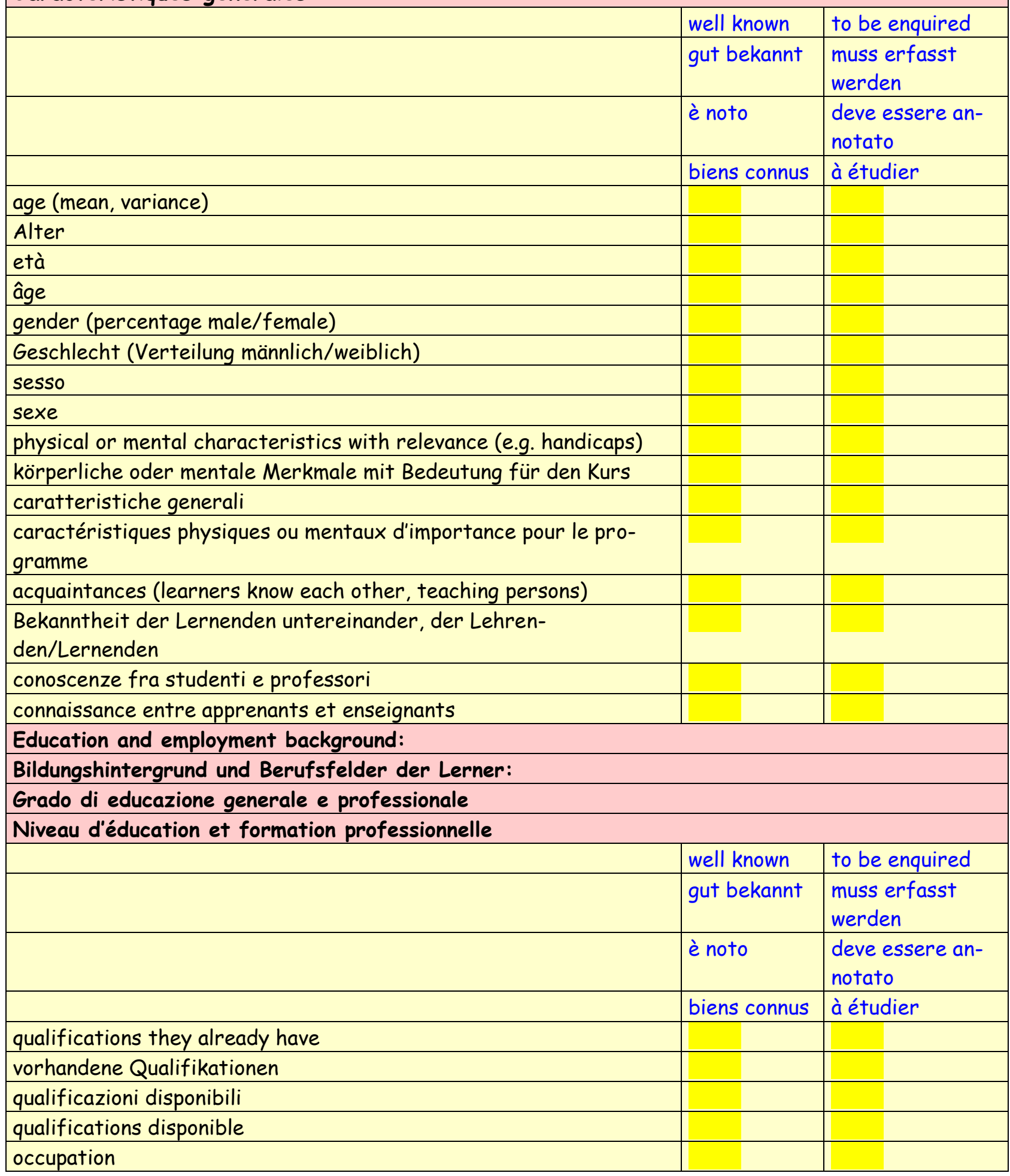




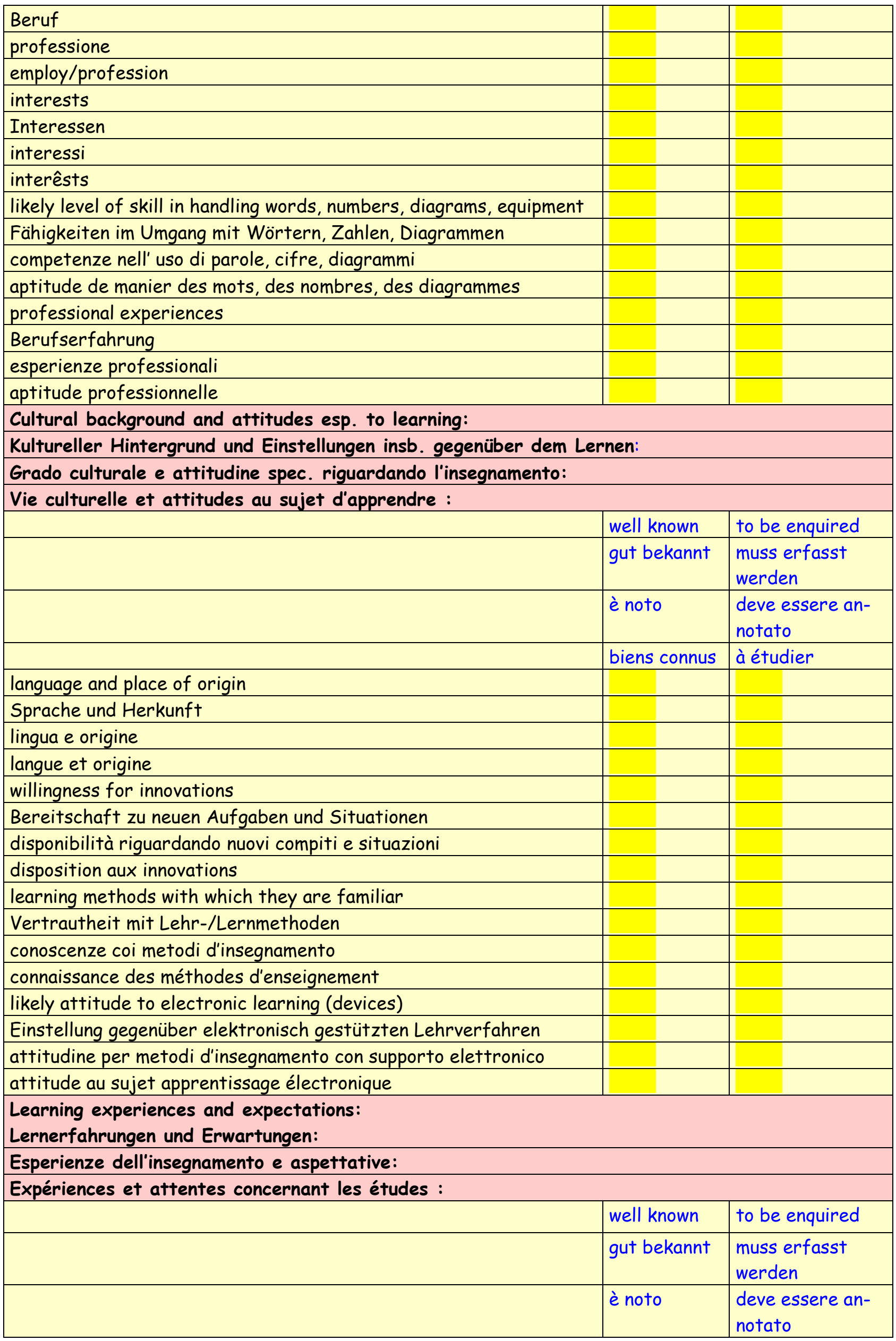




\begin{tabular}{|l|l|l|}
\hline & biens connus & à étudier \\
\hline $\begin{array}{l}\text { learning experiences so far (different methods of learn- } \\
\text { ing/competence) }\end{array}$ & & \\
\hline bisherige Erfahrungen mit verschiedenen Lehr-/Lernmethoden & & \\
\hline esperienze fino ad oggi con metodi d'insegnamento diversi & & \\
\hline $\begin{array}{l}\text { expériences jusqu'à présent avec les méthodes différentes } \\
\text { d'enseignement }\end{array}$ & & \\
\hline individual learning styles and learning strategies & & \\
\hline individuelle Lernstile und Lernstrategien & & \\
\hline modi e strategie individuali per studiare & & \\
\hline styles et stratégies individuels d'apprendre & & \\
\hline prerequisites in knowledge (previous knowledge/qualification) & & \\
\hline Voraussetzungen im Wissen (Vorwissen, Qualifikation) & & \\
\hline conoscenze & & \\
\hline connaissances & & \\
\hline preferences in special learning arrangements & & \\
\hline Vorlieben für bestimmte Lernarrangements & & \\
\hline preferenze per precisi arrangiamenti di apprenzione & & \\
\hline $\begin{array}{l}\text { préférences pour certains arrangements concernant le processus } \\
\text { d'apprendre }\end{array}$ & & \\
\hline motives for learning & & \\
\hline Motive zum Lernen & & \\
\hline motivi per imparare & & \\
\hline motives pour apprendre & & \\
\hline expected results & & \\
\hline erwartete Ergebnisse & & \\
\hline risultati aspettati & & \\
\hline résultats attendus & & \\
\hline
\end{tabular}

\begin{tabular}{|c|c|c|}
\hline A2. Analysis of learners' contexts & $\downarrow$ well known & $\downarrow$ to be inquired \\
\hline A2. Analyse des Kontextes der Lernenden & $\downarrow$ gut bekannt & $\downarrow$ muss erfasst werden \\
\hline A2. Risultati aspettati & Vè noto & $\downarrow$ deve essere annotato \\
\hline A2. Analyse du contexte des apprenants & Wbiens connus & ฟà étudier \\
\hline $\begin{array}{l}\text { 1) Where will they learn (at home? at work? at learning } \\
\text { centers?) }\end{array}$ & & \\
\hline $\begin{array}{l}\text { 1) Wo werden sie lernen (zu Hause? am Arbeitsplatz) in } \\
\text { Lernzentren?) }\end{array}$ & & \\
\hline 1) Dove si svolgerà l'insegnamento? & & \\
\hline 1) Où les apprenants vont-ils apprendre? & & \\
\hline 2) How much time will they be able to spend for learning? & & \\
\hline $\begin{array}{l}\text { 2) Wie viel Zeit werden Sie für das Lernen aufbringen } \\
\text { können? }\end{array}$ & & \\
\hline 2) Quanto tempo ha disponibile per imparare? & & \\
\hline 2) Combien de temps leur reste-t-il pour apprendre? & & \\
\hline $\begin{array}{l}\text { 3) What equipment will they have (hardware? software? } \\
\text { network connection? other resources?) }\end{array}$ & & \\
\hline $\begin{array}{l}\text { 3) Welche Ausstattung werden sie haben (Hardware, } \\
\text { Software, Netzwerkverbindungen, andere Ressourcen)? }\end{array}$ & & \\
\hline
\end{tabular}


3) Quanto tempo ha disponibile per imparare?

3) Quel équipement auront-ils (matériel, logiciel, jonctions du réseau, d'autres ressources)?

4) What kind of internet access will they have/need?

4) Welche Art Internetanschluss werden sie haben/brauchen?

4) Quanto tempo ha disponibile per imparare?

4) Il leur faut quel raccordement de l'internet?

5) When will they learn? (at night? during working hours? with short or long during intervals?)

5) Wann werden sie lernen (können)? (Nachts? Während der Arbeitszeit? In kurzen oder langen Abständen?)

5) Quanto tempo ha disponibile per imparare?

5) Quand vont-ils apprendre (la nuit? pendant les heures du travail ? par intervalles courts ou longs ??

6) What are their family circumstances?

6) Wie sind die familiären Bedingungen der Lerner?

6) Come sono i condizioni familiari?

6) Quel est l'environnement familial des apprenants ?

7) What difficulties may they experience (for example in gaining access to the necessary technology)?

7) Welche Schwierigkeiten könnten die Lerner erleben (z.B. in bezug auf den Zugang zur erforderlichen technischen Ausstattung)?

7) Quali difficoltà di studiare portrebbero esserci?

7) Quelles pourront être les difficultés (p.ex. concernant la disponibilité d'une technologie nécessaire)?

8) Do they need some training to get started with the technology?

8) Brauchen die Lerner eventuell Hilfe, um mit der Technologie umzugehen?

8) Gli studenti hanno bisogno di aiutio nell'uso delle tecnologie?

8) Les apprenants ont-ils peut-être besoin d'aide pour se servir de la technologie?

9) What kind of support will the learners have? (tutors/trainers or others: who? how? qualifications of the tutors? when are they available)

9) Welche Unterstützung werden die Lerner haben? (Tutoren, Trainer oder andere Personen? Wen? Wie? Qualifikationen der Tutoren? Wann sind sie erreichbar/verfügbar?)

9) Quale supporto avranno gli studenti? (chi?come? qualificazioni die tutori?)

9) Quel sera le soutient accordé aux apprenants (conseillers pédagogiques, entraîneurs ou d'autres personnes, qui, comment, qualifications des conseillers, quand est-ce qu'on peut les joindre)? 


\section{B. Questions to think about the design of your e-learning programme}

B. Fragen zum didaktischen Design des e-Learning-Programms

B. Domande sul disegno didattico del programma d'insegnamento

B. Questions concernant le design didactique du programme apprentissage électronique

The didactic design of your e-learning programme is the basis on which your target group will be able to learn. As a producer of e-learning it is you to plan how to make learning possible. You will think about the contents and required competencies and the ways to offer your knowledge to the learners or manage this. Of course there are many ways of teaching and each subject can be presented in various ways. Below we give you seven criteria of didactic designs, i.e. plans and products within an e-learning programme. You can use them to reflect your didactic design and to check if you thought about these questions when planning or offering your e-learning programme.

Das didaktische Design Ihres e-Learning-Angebotes ist für Ihre Lerner eine wesentliche Voraussetzung dafür, wie sie lernen werden. Als Autor oder Produzent von eLearning sind Sie also jemand, der einen Lernprozess ermöglichen soll. Sie denken über Inhalte, über erforderliche Kompetenzen und die Wege nach, wie dieses Wissen den Lernern und Lernerinnen angeboten werden soll und diese Kompetenzen von ihnen erreicht werden können. Sicherlich, es gibt verschiedene Wege zu unterrichten, und jedes Fach kann unterschiedliche Lernanforderungen präsentieren.

Untenstehend benennen wir sieben Kriterien für didaktische Designs, also für Pläne und Produkte für ein e-Learning-Programm. Sie können sie nutzen, um Ihr didaktisches Design selbst zu überdenken und abzuschätzen, ob Sie diese Fragen überhaupt und hinreichend berücksichtigt haben bei der Planung und Umsetzung Ihres Programms.

Il disegno didattico della vostra offerta dell'insegnamento elettronico è un lato essenziale per come gli studenti potranno imparare. Come autore e produttore di questo programma siete qualcuno che rende possibile un processo d'insegnamento. Lei penserà al concetto, alle competenze necessarie e ai modi per arrivare a farsi che gli studenti imparino e raggiungano queste competenze. Certo esistono varie metodi per insegnare ed ogni materia può presentare diverse bisogni di studiare.

In seguito nominiamo sette criteri per il disegno didattico. Lei può usarli per verificare se nel suo disegno didattico ha tenuto conto di queste domande.

Le design didactique de votre programme apprentissage électronique est pour vos apprenants une condition essentielle pour leur manière d'apprendre. C'est donc vous le producteur du programme apprentissage électronique qui rend possible l'apprentissage. Bien sûr, il y a des modes différentes d'enseignement, et chaque matière pourra exiger différamment.

Sur les pages suivantes, nous nommons sept critères pour des designs didactique, donc pour des plans et des produits pour un programme apprentissage électronique. Vous pouvez vous en servir pour réfléchir vous-même sur votre designs didactique et pour contrôler si vous avez pensé suffisamment à ces questions en projetant et réalisant votre programme. 
B1. Variety: of contents, learner personality, methods

B1. Vielfalt: der Inhalte, der Lernerpersönlichkeiten, der Methoden

B1. Varietà: dei contesti, degli studenti, dei metodi

B1. Diversité des contenus, des personalités des apprenants, des méthodes

\begin{tabular}{|c|c|c|c|c|c|c|}
\hline My/Our programme ... & $\begin{array}{l}\text { not at } \\
\text { all } \\
---\end{array}$ & $\begin{array}{l}\text { just a } \\
\text { little } \\
--\end{array}$ & $\begin{array}{l}\text { quite a } \\
\text { bit } \\
\text { - }\end{array}$ & $\begin{array}{l}\text { rather } \\
\text { much } \\
+\end{array}$ & $\begin{array}{l}\text { much } \\
++\end{array}$ & $\begin{array}{l}\text { very } \\
\text { much } \\
+++\end{array}$ \\
\hline Mein/Unser Programm ... & gar nicht & $\begin{array}{l}\text { ein biss- } \\
\text { chen } \\
\text {-- }\end{array}$ & $\begin{array}{l}\text { einiger- } \\
\text { maßen } \\
\text { - }\end{array}$ & $\begin{array}{l}\text { ziemlich } \\
+\end{array}$ & $\begin{array}{l}\text { viel } \\
++\end{array}$ & $\begin{array}{l}\text { sehr } \\
\text { viel } \\
+++\end{array}$ \\
\hline Il mio/Nostro programma ... & $\begin{array}{l}\text { niente } \\
---\end{array}$ & un pò & $\begin{array}{l}\text { suffi- } \\
\text { ciente } \\
\text { - }\end{array}$ & $\begin{array}{l}\text { abba- } \\
\text { stanza } \\
+\end{array}$ & $\begin{array}{l}\text { molto } \\
++\end{array}$ & $\begin{array}{l}\text { tantis- } \\
\text { simo } \\
+++\end{array}$ \\
\hline Mon/Notre programme ... & $\begin{array}{l}\text { pas du } \\
\text { tout } \\
---\end{array}$ & un peu & $\begin{array}{l}\text { passa- } \\
\text { blement } \\
\text { - }\end{array}$ & $\begin{array}{l}\text { assez } \\
+\end{array}$ & beaucoup & $\begin{array}{l}\text { énor- } \\
\text { mément } \\
+++\end{array}$ \\
\hline $\begin{array}{l}\text { 1) addresses to several senses (vision, } \\
\text { hearing, touch, smelling, equilibrium). }\end{array}$ & $\mathrm{O}$ & $\mathrm{O}$ & $\mathrm{O}$ & O & O & $\mathrm{O}$ \\
\hline $\begin{array}{l}\text { 1) spricht mehrere Sinne an (Sehsinn, } \\
\text { Hörsinn, Tastsinn, Riechsinn, Gleich- } \\
\text { gewichtssinn). }\end{array}$ & $\mathrm{O}$ & $\mathrm{O}$ & $\mathrm{O}$ & O & $\mathrm{O}$ & $\mathrm{O}$ \\
\hline $\begin{array}{l}\text { 1) Stimola i sensi (vedere, sentire, } \\
\text { tatto, odorare, equilibrio) }\end{array}$ & $\mathrm{O}$ & O & $\mathrm{O}$ & O & O & $\mathrm{O}$ \\
\hline $\begin{array}{l}\text { 1) stimule plusieurs sens (la vue, l'ouiie., } \\
\text { le toucher, l'odorat, l'équilibre). }\end{array}$ & $\mathrm{O}$ & $\mathrm{O}$ & $\mathrm{O}$ & O & O & $\mathrm{O}$ \\
\hline $\begin{array}{l}\text { 2) uses several didactic meth- } \\
\text { ods/models. }\end{array}$ & $\mathrm{O}$ & $\mathrm{O}$ & $\mathrm{O}$ & O & O & O \\
\hline $\begin{array}{l}\text { 2) bringt mehrere didaktische Modelle } \\
\text { zum Einsatz. }\end{array}$ & $\mathrm{O}$ & $\mathrm{O}$ & $\mathrm{O}$ & O & O & O \\
\hline 2) Porta diversi modelli didattici & $\mathrm{O}$ & $\mathrm{O}$ & $\mathrm{O}$ & $\mathrm{O}$ & $\mathrm{O}$ & $\mathrm{O}$ \\
\hline $\begin{array}{l}\text { 2) utilise plusieurs modèles didac- } \\
\text { tiques. }\end{array}$ & O & O & O & O & O & O \\
\hline $\begin{array}{l}\text { 3) matches content to delivery meth- } \\
\text { ods }\end{array}$ & $\mathrm{O}$ & $\mathrm{O}$ & $\mathrm{O}$ & O & O & O \\
\hline 3) stimmt in Form und Inhalt überein & $\mathrm{O}$ & $\mathrm{O}$ & $\mathrm{O}$ & $\mathrm{O}$ & $\mathrm{O}$ & $\mathrm{O}$ \\
\hline 3) Forma e contesto compiacciono & $\mathrm{O}$ & $\mathrm{O}$ & $\mathrm{O}$ & $\mathrm{O}$ & $\mathrm{O}$ & $\mathrm{O}$ \\
\hline $\begin{array}{l}\text { 3) correspond dans ses formes aux } \\
\text { contenus. }\end{array}$ & $\mathrm{O}$ & $\mathrm{O}$ & $\mathrm{O}$ & O & O & O \\
\hline 4) addresses to reason and emotion. & $\mathrm{O}$ & $\mathrm{O}$ & $\mathrm{O}$ & $\mathrm{O}$ & $\mathrm{O}$ & $\mathrm{O}$ \\
\hline 4) spricht Verstand und Gefühl an. & $\mathrm{O}$ & $\mathrm{O}$ & $\mathrm{O}$ & $\mathrm{O}$ & $\mathrm{O}$ & $\mathrm{O}$ \\
\hline 4) Stimola la ragione e le sensazioni & $\mathrm{O}$ & $\mathrm{O}$ & $\mathrm{O}$ & $\mathrm{O}$ & $\mathrm{O}$ & $\mathrm{O}$ \\
\hline $\begin{array}{l}\text { 4) s'adresse et à la raison et à } \\
\text { l'emotion. }\end{array}$ & $\mathrm{O}$ & $\mathrm{O}$ & $\mathrm{O}$ & O & O & $\mathrm{O}$ \\
\hline $\begin{array}{l}\text { 5) changes between phases of tension } \\
\text { and relaxation. }\end{array}$ & $\mathrm{O}$ & O & $\mathrm{O}$ & $\mathrm{O}$ & O & O \\
\hline $\begin{array}{l}\text { 5) wechselt Phasen der Anspannung } \\
\text { und der Entspannung. }\end{array}$ & $\mathrm{O}$ & $\mathrm{O}$ & $\mathrm{O}$ & O & O & O \\
\hline 5) Alterna le fasi di tensione e relax & $\mathrm{O}$ & $\mathrm{O}$ & $\mathrm{O}$ & $\mathrm{O}$ & $\mathrm{O}$ & $\mathrm{O}$ \\
\hline $\begin{array}{l}\text { 5) alterne entre des phase de tension } \\
\text { et de détente. }\end{array}$ & $\mathrm{O}$ & $\mathrm{O}$ & $\mathrm{O}$ & O & O & O \\
\hline
\end{tabular}




\begin{tabular}{|c|c|c|c|c|c|c|}
\hline $\begin{array}{l}\text { 6) includes individual and collaborative } \\
\text { activities. }\end{array}$ & $\mathrm{O}$ & $\mathrm{O}$ & $\mathrm{O}$ & $\mathrm{O}$ & 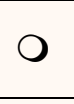 & $\mathrm{O}$ \\
\hline $\begin{array}{l}\text { 6) bietet individuelle und kollektive } \\
\text { Tätigkeiten an. }\end{array}$ & $\mathrm{O}$ & O & $\mathrm{O}$ & $\mathrm{O}$ & $\mathrm{O}$ & $\mathrm{O}$ \\
\hline 6) offre pratiche individuali e colletive & $\mathrm{O}$ & $\mathrm{O}$ & $\mathrm{O}$ & $\mathrm{O}$ & $\mathrm{O}$ & $\mathrm{O}$ \\
\hline $\begin{array}{l}\text { 6) offre des activités individuelles et } \\
\text { collectives. }\end{array}$ & O & O & O & $\mathrm{O}$ & O & $\mathrm{O}$ \\
\hline $\begin{array}{l}\text { 7) motivates learners with different } \\
\text { ways of learning. }\end{array}$ & $\mathrm{O}$ & $\mathrm{O}$ & $\mathrm{O}$ & $\mathrm{O}$ & $\mathrm{O}$ & $\mathrm{O}$ \\
\hline $\begin{array}{l}\text { 7) regt Lerner mit unterschiedlichen } \\
\text { Lernstilen an. }\end{array}$ & $\mathrm{O}$ & O & $\mathrm{O}$ & $\mathrm{O}$ & $\mathrm{O}$ & $\mathrm{O}$ \\
\hline $\begin{array}{l}\text { 7) stili d'insegnamento diversi stimola- } \\
\text { no gli studenti }\end{array}$ & $\mathrm{O}$ & $\mathrm{O}$ & O & $\mathrm{O}$ & $\mathrm{O}$ & $\mathrm{O}$ \\
\hline $\begin{array}{l}\text { 7) motive des apprenants avec des } \\
\text { styles d'apprentissage différents. }\end{array}$ & $\mathrm{O}$ & O & O & $\mathrm{O}$ & $\mathrm{O}$ & $\mathrm{O}$ \\
\hline $\begin{array}{l}\text { 8) has opportunities to learn in other } \\
\text { situations than sitting on a chair }\end{array}$ & O & $\mathrm{O}$ & $\mathrm{O}$ & $\mathrm{O}$ & $\mathrm{O}$ & $\mathrm{O}$ \\
\hline $\begin{array}{l}\text { 8) bietet auch andere Lerngelegenhei- } \\
\text { ten als nur auf einem Stuhl sitzend. }\end{array}$ & O & $\mathrm{O}$ & $\mathrm{O}$ & $\mathrm{O}$ & $\mathrm{O}$ & $\mathrm{O}$ \\
\hline $\begin{array}{l}\text { 8) stimola gli studenti ad imparare non } \\
\text { solo stando seduti }\end{array}$ & $\mathrm{O}$ & O & $\mathrm{O}$ & $\mathrm{O}$ & $\mathrm{O}$ & $\mathrm{O}$ \\
\hline $\begin{array}{l}\text { 8) offre aussi d'autre situations } \\
\text { d'apprendre que d'être assis sur une } \\
\text { chaise. }\end{array}$ & O & O & O & $\mathrm{O}$ & $\mathrm{O}$ & $\mathrm{O}$ \\
\hline $\begin{array}{l}\text { 9) changes between guided and self- } \\
\text { directed learning }\end{array}$ & O & $\mathrm{O}$ & $\mathrm{O}$ & $\mathrm{O}$ & $\mathrm{O}$ & $\mathrm{O}$ \\
\hline $\begin{array}{l}\text { 9) wechselt angeleitetes und selbsttä- } \\
\text { tiges Lernen. }\end{array}$ & $\mathrm{O}$ & $\mathrm{O}$ & $\mathrm{O}$ & $\mathrm{O}$ & $\mathrm{O}$ & $\mathrm{O}$ \\
\hline $\begin{array}{l}\text { 9) Alterna fra insegnamento guidato e } \\
\text { autonomo }\end{array}$ & $\mathrm{O}$ & O & O & $\mathrm{O}$ & $\mathrm{O}$ & $\mathrm{O}$ \\
\hline $\begin{array}{l}\text { 9) alterne entre un apprentissage gui- } \\
\text { dé et autonome. }\end{array}$ & $\mathrm{O}$ & O & O & O & $\mathrm{O}$ & $\mathrm{O}$ \\
\hline $\begin{array}{l}\text { 10) allows for different learning loca- } \\
\text { tions. }\end{array}$ & $\mathrm{O}$ & $\mathrm{O}$ & $\mathrm{O}$ & $\mathrm{O}$ & $\mathrm{O}$ & $\mathrm{O}$ \\
\hline 10) sieht verschiedene Lernorte vor. & $\mathrm{O}$ & $\mathrm{O}$ & $\mathrm{O}$ & $\mathrm{O}$ & 0 & 0 \\
\hline $\begin{array}{l}\text { 10) Riguar do diversi luoghi } \\
\text { d'insegnamento }\end{array}$ & 0 & 0 & O & 0 & $\mathrm{O}$ & 0 \\
\hline \multirow[t]{2}{*}{$\begin{array}{l}\text { 10) propose des lieux d'apprentissage } \\
\text { différents. }\end{array}$} & 0 & 0 & O & O & $\mathrm{O}$ & 0 \\
\hline & $\star 1$ & $\star 2$ & $\star 3$ & ${ }^{*} 4$ & $\star 5$ & ${ }^{\star} 6$ \\
\hline
\end{tabular}


B2. Economy: in the sense of efficacious use of resources

B2. Ökonomie: im Sinne wirksamer Nutzung von Ressourcen

B2. economia: nel senso dell'uso efficace degli risorsi

B2. Économie: au sens d'un usage efficace des ressources

\begin{tabular}{|c|c|c|c|c|c|c|}
\hline My/Our programme ... & $\begin{array}{l}\text { not at } \\
\text { all } \\
--\end{array}$ & $\begin{array}{l}\text { just a } \\
\text { little } \\
--\end{array}$ & $\begin{array}{l}\text { quite a } \\
\text { bit } \\
-\end{array}$ & $\begin{array}{l}\text { rather } \\
\text { much } \\
+\end{array}$ & $\begin{array}{l}\text { much } \\
++\end{array}$ & $\begin{array}{l}\text { very } \\
\text { much } \\
+++\end{array}$ \\
\hline Mein/Unser Programm ... & $\begin{array}{l}\text { gar } \\
\text { nicht } \\
---\end{array}$ & $\begin{array}{l}\text { ein biss- } \\
\text { chen } \\
--\end{array}$ & $\begin{array}{l}\text { einiger- } \\
\text { maßen } \\
-\end{array}$ & $\begin{array}{l}\text { ziemlich } \\
+\end{array}$ & $\begin{array}{l}\text { viel } \\
++\end{array}$ & $\begin{array}{l}\text { sehr viel } \\
+++\end{array}$ \\
\hline Il mio/Nostro programma... & niente & un pò & $\begin{array}{l}\text { suf- } \\
\text { fieciente } \\
-\end{array}$ & $\begin{array}{l}\text { abba- } \\
\text { stanza } \\
+\end{array}$ & $\begin{array}{l}\text { molto } \\
++\end{array}$ & $\begin{array}{l}\text { tantis- } \\
\text { simo } \\
+++\end{array}$ \\
\hline Mon/Notre programme & $\begin{array}{l}\text { pas du } \\
\text { tout } \\
---\end{array}$ & un peu & $\begin{array}{l}\text { passa- } \\
\text { blement } \\
-\end{array}$ & $\begin{array}{l}\text { assez } \\
+ \\
\end{array}$ & $\begin{array}{l}\text { beaucoup } \\
++\end{array}$ & $\begin{array}{l}\text { énormé- } \\
\text { ment } \\
+++\end{array}$ \\
\hline $\begin{array}{l}\text { 1) avoids counterproductive effects } \\
\text { like a negative attitude towards } \\
\text { learning. }\end{array}$ & $\mathrm{O}$ & $\mathrm{O}$ & $\mathrm{O}$ & $\mathrm{O}$ & $\mathrm{O}$ & $\mathrm{O}$ \\
\hline $\begin{array}{l}\text { 1) vermeidet kontraproduktive Effek- } \\
\text { te wie negative Einstellungen zum } \\
\text { Lernen. }\end{array}$ & $\mathrm{O}$ & $\mathrm{O}$ & $\mathrm{O}$ & $\mathrm{O}$ & $\mathrm{O}$ & $\mathrm{O}$ \\
\hline $\begin{array}{l}\text { 1) Evita effetti contraproduttivi come } \\
\text { attegiamenti negativi riguardando lo } \\
\text { studio }\end{array}$ & $\mathrm{O}$ & $\mathrm{O}$ & $\mathrm{O}$ & $\mathrm{O}$ & $\mathrm{O}$ & $\mathrm{O}$ \\
\hline $\begin{array}{l}\text { 1) évite des effets malfaisants } \\
\text { comme des attitudes négatives envers } \\
\text { le processus d'apprendre, }\end{array}$ & $\mathrm{O}$ & O & $\mathrm{O}$ & $\mathrm{O}$ & $\mathrm{O}$ & $\mathrm{O}$ \\
\hline $\begin{array}{l}\text { 2) uses as far as possible simple and } \\
\text { cheap means and media which are } \\
\text { little susceptible and that are availa- } \\
\text { ble anyway. }\end{array}$ & $\mathrm{O}$ & $\mathrm{O}$ & $\mathrm{O}$ & $\mathrm{O}$ & $\mathrm{O}$ & $\mathrm{O}$ \\
\hline $\begin{array}{l}\text { 2) verwendet möglichst einfache, } \\
\text { preiswerte, wenig anfällige und ohne- } \\
\text { hin vorhandene Mittel und Medien. }\end{array}$ & $\mathrm{O}$ & $\mathrm{O}$ & $\mathrm{O}$ & $\mathrm{O}$ & $\mathrm{O}$ & $\mathrm{O}$ \\
\hline $\begin{array}{l}\text { 2) Usa come possibile materiali e } \\
\text { mezzi facili, economici, resistenti e } \\
\text { disponibili in ogni modo }\end{array}$ & $\mathrm{O}$ & $\mathrm{O}$ & $\mathrm{O}$ & $\mathrm{O}$ & $\mathrm{O}$ & $\mathrm{O}$ \\
\hline $\begin{array}{l}\text { 2) emploie-si possible- des moyens et } \\
\text { médias simples, pas chers, solides et } \\
\text { déjà disponibles. }\end{array}$ & $\mathrm{O}$ & $\mathrm{O}$ & $\mathrm{O}$ & $\mathrm{O}$ & $\mathrm{O}$ & $\mathrm{O}$ \\
\hline $\begin{array}{l}\text { 3) converts much "teaching time" into } \\
\text { effective learning time }\end{array}$ & $\mathrm{O}$ & $\mathrm{O}$ & $\mathrm{O}$ & $\mathrm{O}$ & $\mathrm{O}$ & $\mathrm{O}$ \\
\hline $\begin{array}{l}\text { 3) setzt viel "Lehrzeit" in effektive } \\
\text { Lernzeit um. }\end{array}$ & $\mathrm{O}$ & $\mathrm{O}$ & $\mathrm{O}$ & $\mathrm{O}$ & $\mathrm{O}$ & 0 \\
\hline $\begin{array}{l}\text { 3) Trasforma molto tempo } \\
\text { dell'insegnamento nell'imparo effetti- } \\
\text { vo }\end{array}$ & 0 & 0 & 0 & 0 & 0 & 0 \\
\hline $\begin{array}{l}\text { 3) transforme une grande partie du } \\
\text { temps d'enseignement en temps }\end{array}$ & 0 & 0 & 0 & 0 & 0 & 0 \\
\hline
\end{tabular}




\begin{tabular}{|c|c|c|c|c|c|c|}
\hline d'apprentissage effectif. & & & & & & \\
\hline $\begin{array}{l}\text { 4) leads to learning outcomes that are } \\
\text { close to the aims }\end{array}$ & O & O & O & $\mathrm{O}$ & $\mathrm{O}$ & 0 \\
\hline $\begin{array}{l}\text { 4) lässt tatsächlich das lernen, was } \\
\text { gelernt werden soll. }\end{array}$ & O & O & O & $\mathrm{O}$ & $\mathrm{O}$ & O \\
\hline $\begin{array}{l}\text { 4) lascia per imparare, quale deve } \\
\text { essere imparato }\end{array}$ & O & O & O & $\mathrm{O}$ & $\mathrm{O}$ & O \\
\hline $\begin{array}{l}\text { 4) fait apprendre effectivement ce } \\
\text { qu'il faut apprendre. }\end{array}$ & O & O & O & $\mathrm{O}$ & $\mathrm{O}$ & 0 \\
\hline $\begin{array}{l}\text { 5) imparts additional knowledge with- } \\
\text { out greater efforts by the learners }\end{array}$ & O & O & O & $\mathrm{O}$ & $\mathrm{O}$ & O \\
\hline $\begin{array}{l}\text { 5) vermittelt ohne Mehraufwand für } \\
\text { die Lernenden zusätzliches Wissen. }\end{array}$ & O & O & O & $\mathrm{O}$ & $\mathrm{O}$ & O \\
\hline $\begin{array}{l}\text { 5) media per gli studenti conoscenze } \\
\text { supplementare senza dispendio sup- } \\
\text { plementare }\end{array}$ & O & O & O & $\mathrm{O}$ & $\mathrm{O}$ & $\mathrm{O}$ \\
\hline $\begin{array}{l}\text { 5) communique aux apprenants des } \\
\text { connaissances supplémentaires sans } \\
\text { un surcroît de travail. }\end{array}$ & O & O & O & $\mathrm{O}$ & $\mathrm{O}$ & $\mathrm{O}$ \\
\hline 6) avoids learning disorders & $\mathrm{O}$ & $\mathrm{O}$ & $\mathrm{O}$ & $\mathrm{O}$ & $\mathrm{O}$ & $\mathrm{O}$ \\
\hline 6) vermeidet Lernstörungen. & $\mathrm{O}$ & $\mathrm{O}$ & $\mathrm{O}$ & $\mathrm{O}$ & $\mathrm{O}$ & $\mathrm{O}$ \\
\hline 6) Evita problemi d'imparare & $\mathrm{O}$ & $\mathrm{O}$ & $\mathrm{O}$ & $\mathrm{O}$ & $\mathrm{O}$ & $\mathrm{O}$ \\
\hline $\begin{array}{l}\text { 6) évite que les processus } \\
\text { d'apprendre sont dérangés. }\end{array}$ & O & O & O & $\mathrm{O}$ & $\mathrm{O}$ & O \\
\hline 7) uses means which are trouble-free & $\mathrm{O}$ & $\mathrm{O}$ & $\mathrm{O}$ & $\mathrm{O}$ & $\mathrm{O}$ & $\mathrm{O}$ \\
\hline $\begin{array}{l}\text { 7) nutzt Mittel, die nicht störanfällig } \\
\text { sind. }\end{array}$ & O & O & O & $\mathrm{O}$ & $\mathrm{O}$ & O \\
\hline 7) Usa materiali che sono durevole & $\mathrm{O}$ & $\mathrm{O}$ & $\mathrm{O}$ & $\mathrm{O}$ & $\mathrm{O}$ & $\mathrm{O}$ \\
\hline 7) utilise des moyens non-troublants. & $\mathrm{O}$ & $\mathrm{O}$ & $\mathrm{O}$ & $\mathrm{O}$ & $\mathrm{O}$ & $\mathrm{O}$ \\
\hline $\begin{array}{l}\text { 8) does not teach things as new that } \\
\text { are already known to most learners }\end{array}$ & O & O & O & $\mathrm{O}$ & $\mathrm{O}$ & $\mathrm{O}$ \\
\hline $\begin{array}{l}\text { 8) lehrt nichts, was die Lernenden } \\
\text { schon gut wissen oder können. }\end{array}$ & O & $\mathrm{O}$ & $\mathrm{O}$ & $\mathrm{O}$ & $\mathrm{O}$ & O \\
\hline $\begin{array}{l}\text { 8) Non insegna niente di quello che gli } \\
\text { studenti già sanno }\end{array}$ & O & O & O & $\mathrm{O}$ & $\mathrm{O}$ & O \\
\hline $\begin{array}{l}\text { 8) n'enseigne rien ce que les appre- } \\
\text { nants savent ou connaissent déjà bien. }\end{array}$ & O & O & 0 & 0 & 0 & 0 \\
\hline $\begin{array}{l}\text { 9) builds up on the given knowledge } \\
\text { and abilities of the learners }\end{array}$ & 0 & 0 & 0 & 0 & 0 & 0 \\
\hline $\begin{array}{l}\text { 9) nutz† das bei den Lernern vorhan- } \\
\text { dene Wissen und Können. }\end{array}$ & 0 & 0 & 0 & 0 & 0 & 0 \\
\hline $\begin{array}{l}\text { 9) usa le già aquisite competenze de- } \\
\text { gli studenti }\end{array}$ & 0 & 0 & 0 & 0 & 0 & 0 \\
\hline $\begin{array}{l}\text { 9) profite des connaissances déjà } \\
\text { existantes. }\end{array}$ & 0 & 0 & 0 & 0 & 0 & 0 \\
\hline $\begin{array}{l}\text { 10) has undergone a risk assessment } \\
\text { for potential failure }\end{array}$ & 0 & 0 & 0 & 0 & 0 & 0 \\
\hline $\begin{array}{l}\text { 10) ist hinsichtlich möglichen Versa- } \\
\text { gens geprüft worden. }\end{array}$ & 0 & 0 & 0 & 0 & 0 & 0 \\
\hline $\begin{array}{l}\text { 10) è stato verificato considerando } \\
\text { possibile fallimenti }\end{array}$ & 0 & 0 & 0 & 0 & 0 & 0 \\
\hline
\end{tabular}


10) a été contrôlé à l'égard d'une dé-

faillance possible.

\begin{tabular}{|l|l|l|l|l|l|}
\hline & & & & & \\
& & 0 & 0 & 0 & 0 \\
$* 1$ & $\star_{2}$ & $\star_{3}$ & $\star_{4}$ & $\star_{5}$ & ${ }^{*} 6$ \\
\hline
\end{tabular}

B3. Adjustment: to the given/expected situation

B3. Anpassung: an die gegebene/erwartete Situation

B3. Adattazione: alla situazione data/aspettativa

B3. Adaptation: à la situation donnée ou attendue

\begin{tabular}{|c|c|c|c|c|c|c|}
\hline $\begin{array}{l}\text { My/Our programme is adjusted } \\
\ldots\end{array}$ & not at all & $\begin{array}{l}\text { just a } \\
\text { little } \\
--\end{array}$ & $\begin{array}{l}\text { quite a } \\
\text { bit } \\
-\end{array}$ & $\begin{array}{l}\text { rather } \\
\text { much } \\
+\end{array}$ & $\begin{array}{l}\text { much } \\
++\end{array}$ & $\begin{array}{l}\text { very } \\
\text { much } \\
+++\end{array}$ \\
\hline $\begin{array}{l}\text { Mein/Unser Programm ist ange- } \\
\text { passt ... }\end{array}$ & gar nicht & $\begin{array}{l}\text { ein biss- } \\
\text { chen } \\
\text {-- }\end{array}$ & $\begin{array}{l}\text { einiger- } \\
\text { maßen } \\
\text { - }\end{array}$ & $\begin{array}{l}\text { ziemlich } \\
+\end{array}$ & $\begin{array}{l}\text { viel } \\
++\end{array}$ & $\begin{array}{l}\text { sehr } \\
\text { viel } \\
+++\end{array}$ \\
\hline $\begin{array}{l}\text { Il mio/Nostro programma è a- } \\
\text { datto... }\end{array}$ & niente & $\begin{array}{l}\text { un pò } \\
-\end{array}$ & $\begin{array}{l}\text { suffi- } \\
\text { ciente } \\
-\end{array}$ & $\begin{array}{l}\text { abba- } \\
\text { stanza } \\
+\end{array}$ & $\begin{array}{l}\text { molto } \\
++\end{array}$ & $\begin{array}{l}\text { tantis- } \\
\text { simo } \\
+++\end{array}$ \\
\hline $\begin{array}{l}\text { Mon/Notre programme est } \\
\text { adapté ... }\end{array}$ & $\begin{array}{l}\text { pas du } \\
\text { tout } \\
-\cdots\end{array}$ & un peu & $\begin{array}{l}\text { passa- } \\
\text { blement } \\
-\end{array}$ & $\begin{array}{l}\text { assez } \\
+ \\
\end{array}$ & $\begin{array}{l}\text { beaucoup } \\
++ \\
+\end{array}$ & $\begin{array}{l}\text { énor- } \\
\text { mément } \\
+++\end{array}$ \\
\hline $\begin{array}{l}\text { 1) to the language habits of the } \\
\text { learners. }\end{array}$ & $\mathrm{O}$ & $\mathrm{O}$ & $\mathrm{O}$ & $\mathrm{O}$ & $\mathrm{O}$ & $\mathrm{O}$ \\
\hline $\begin{array}{l}\text { 1) an die Sprachgewohnheiten der } \\
\text { Lernenden. }\end{array}$ & $\mathrm{O}$ & $\mathrm{O}$ & $\mathrm{O}$ & $\mathrm{O}$ & $\mathrm{O}$ & $\mathrm{O}$ \\
\hline 1) ai modi di parlare degli studenti & $\mathrm{O}$ & $\mathrm{O}$ & $\mathrm{O}$ & $\mathrm{O}$ & $\mathrm{O}$ & $\mathrm{O}$ \\
\hline $\begin{array}{l}\text { 1) aux habitudes et usages de langue } \\
\text { des apprenants. }\end{array}$ & $\mathrm{O}$ & $\mathrm{O}$ & $\mathrm{O}$ & $\mathrm{O}$ & $\mathrm{O}$ & $\mathrm{O}$ \\
\hline $\begin{array}{l}\text { 2) to the previous knowledge of the } \\
\text { learners }\end{array}$ & $\mathrm{O}$ & $\mathrm{O}$ & $\mathrm{O}$ & $\mathrm{O}$ & $\mathrm{O}$ & $\mathrm{O}$ \\
\hline 2) an das Vorwissen der Lernenden. & $\mathrm{O}$ & $\mathrm{O}$ & $\mathrm{O}$ & $\mathrm{O}$ & $\mathrm{O}$ & $\mathrm{O}$ \\
\hline $\begin{array}{l}\text { 2) alle conoscenze già note a gli stu- } \\
\text { denti }\end{array}$ & $\mathrm{O}$ & $\mathrm{O}$ & $\mathrm{O}$ & $\mathrm{O}$ & $\mathrm{O}$ & $\mathrm{O}$ \\
\hline 2) aux connaissances des apprenants. & $\mathrm{O}$ & $\mathrm{O}$ & $\mathrm{O}$ & $\mathrm{O}$ & $\mathrm{O}$ & $\mathrm{O}$ \\
\hline $\begin{array}{l}\text { 3) to the ways of learning predomi- } \\
\text { nant among the learners }\end{array}$ & $\mathrm{O}$ & $\mathrm{O}$ & O & 0 & 0 & 0 \\
\hline $\begin{array}{l}\text { 3) an die Lernstile, die bei den Ler- } \\
\text { nenden überwiegen. }\end{array}$ & O & 0 & 0 & 0 & 0 & 0 \\
\hline $\begin{array}{l}\text { 3) ai stili d'imparare corrisponente } \\
\text { alla maggior parte degli studenti }\end{array}$ & 0 & 0 & 0 & 0 & 0 & 0 \\
\hline $\begin{array}{l}\text { 3) aux styles d'apprentissage domi- } \\
\text { nants chez les apprenants. }\end{array}$ & 0 & 0 & 0 & 0 & 0 & 0 \\
\hline $\begin{array}{l}\text { 4) to the environment and the way of } \\
\text { living (life styles) of the learners. }\end{array}$ & 0 & $\mathrm{O}$ & 0 & 0 & 0 & 0 \\
\hline $\begin{array}{l}\text { 4) an die Lebenswelt und Lebenswei- } \\
\text { se der Lernenden. }\end{array}$ & 0 & 0 & 0 & 0 & 0 & 0 \\
\hline $\begin{array}{l}\text { 4) all'ambiente e modi di vivere degli } \\
\text { studenti. }\end{array}$ & 0 & 0 & O & 0 & 0 & 0 \\
\hline
\end{tabular}




\begin{tabular}{|c|c|c|c|c|c|c|}
\hline $\begin{array}{l}\text { 4) à l'environnement et au mode de } \\
\text { vie des apprenants. }\end{array}$ & O & O & O & $\mathrm{O}$ & 0 & $\mathrm{O}$ \\
\hline 5) to the resilience of the learners. & $\mathrm{O}$ & $\mathrm{O}$ & $\mathrm{O}$ & $\mathrm{O}$ & $\mathrm{O}$ & $\mathrm{O}$ \\
\hline $\begin{array}{l}\text { 5) an die Belastbarkeit der Lernen- } \\
\text { den. }\end{array}$ & O & $\mathrm{O}$ & $\mathrm{O}$ & $\mathrm{O}$ & O & $\mathrm{O}$ \\
\hline $\begin{array}{l}\text { 5) alla capacità di sopoortare degli } \\
\text { studenti. }\end{array}$ & $\mathrm{O}$ & $\mathrm{O}$ & $\mathrm{O}$ & $\mathrm{O}$ & O & $\mathrm{O}$ \\
\hline $\begin{array}{l}\text { 5) à la capacité de charge des ap- } \\
\text { prenants. }\end{array}$ & O & $\mathrm{O}$ & $\mathrm{O}$ & $\mathrm{O}$ & O & $\mathrm{O}$ \\
\hline $\begin{array}{l}\text { 6) to resources that learners are } \\
\text { used to. }\end{array}$ & O & $\mathrm{O}$ & $\mathrm{O}$ & $\mathrm{O}$ & O & $\mathrm{O}$ \\
\hline $\begin{array}{l}\text { 6) an die Ressourcen, mit denen die } \\
\text { Lernenden vertraut sind. }\end{array}$ & $\mathrm{O}$ & $\mathrm{O}$ & $\mathrm{O}$ & $\mathrm{O}$ & O & $\mathrm{O}$ \\
\hline 6) alle risorse già note agli studenti. & $\mathrm{O}$ & $\mathrm{O}$ & $\mathrm{O}$ & $\mathrm{O}$ & $\mathrm{O}$ & $\mathrm{O}$ \\
\hline $\begin{array}{l}\text { 6) aux ressources que les apprenants } \\
\text { connaissent à fond. }\end{array}$ & $\mathrm{O}$ & $\mathrm{O}$ & $\mathrm{O}$ & $\mathrm{O}$ & 0 & 0 \\
\hline $\begin{array}{l}\text { 7) to those tasks which result from } \\
\text { the framework, that are asked in } \\
\text { practice. }\end{array}$ & 0 & 0 & $\mathrm{O}$ & $\mathrm{O}$ & 0 & 0 \\
\hline $\begin{array}{l}\text { 7) an die Aufgaben, die sich aus dem } \\
\text { Umfeld der Lernenden ergeben. }\end{array}$ & 0 & 0 & 0 & 0 & 0 & 0 \\
\hline $\begin{array}{l}\text { 7) Ai compiti risultanti dell'ambiente } \\
\text { degli studenti }\end{array}$ & 0 & 0 & 0 & 0 & 0 & 0 \\
\hline $\begin{array}{l}\text { 7) aux tâches qui résultent de la } \\
\text { situation des apprenants. }\end{array}$ & 0 & 0 & $\mathrm{O}$ & O & 0 & 0 \\
\hline $\begin{array}{l}\text { 8) to those ways of communication } \\
\text { which are preferred of the learners. }\end{array}$ & 0 & 0 & $\mathrm{O}$ & 0 & 0 & 0 \\
\hline $\begin{array}{l}\text { 8) an die Kommunikationsformen, die } \\
\text { von den Lernenden bevorzugt wer- } \\
\text { den. }\end{array}$ & 0 & 0 & 0 & 0 & 0 & 0 \\
\hline $\begin{array}{l}\text { 8) alle forme di communicazione pre- } \\
\text { ferite dagli studenti. }\end{array}$ & 0 & 0 & 0 & 0 & 0 & 0 \\
\hline $\begin{array}{l}\text { 8) aux manières de communiquer que } \\
\text { les apprenants préfèrent. }\end{array}$ & 0 & 0 & 0 & 0 & 0 & 0 \\
\hline $\begin{array}{l}\text { 9) to the time possibilities of the } \\
\text { learners and of the framework. }\end{array}$ & 0 & 0 & 0 & 0 & 0 & 0 \\
\hline $\begin{array}{l}\text { 9) an die zeitlichen Möglichkeiten } \\
\text { der Lernenden und des Bezugssys- } \\
\text { tems. }\end{array}$ & 0 & 0 & 0 & $\mathrm{O}$ & 0 & 0 \\
\hline $\begin{array}{l}\text { 9) al tempo disponibile degli studenti } \\
\text { ed il sistema }\end{array}$ & 0 & 0 & 0 & 0 & 0 & 0 \\
\hline $\begin{array}{l}\text { 9) aux possibilités de temps et du } \\
\text { système de référence. }\end{array}$ & 0 & 0 & 0 & 0 & 0 & 0 \\
\hline $\begin{array}{l}\text { 10) to the interests of the learners } \\
\text { and excites motivation. }\end{array}$ & 0 & 0 & 0 & 0 & 0 & 0 \\
\hline $\begin{array}{l}\text { 10) an die Interessen der Lernenden } \\
\text { und löst Motivationen aus. }\end{array}$ & 0 & 0 & 0 & 0 & 0 & 0 \\
\hline 10) Ai loro interessi e motivazioni. & $\mathrm{O}$ & $\mathrm{O}$ & $\mathrm{O}$ & $\mathrm{O}$ & $\mathrm{O}$ & $\mathrm{O}$ \\
\hline \multirow[t]{2}{*}{$\begin{array}{l}\text { 10) aux intérêts des apprenants et } \\
\text { provoque des motivations. }\end{array}$} & $\mathrm{O}$ & $\mathrm{O}$ & $\mathrm{O}$ & $\mathrm{O}$ & O & $\mathrm{O}$ \\
\hline & $\star_{1}$ & $\star 2$ & $\star 3$ & $* 4$ & $\star 5$ & *6 \\
\hline
\end{tabular}


B4. Productivity and utility

B4. Produktivität und Nützlichkeit

B4. Rendimento ed utilità

B4. Productivité et utilité

\begin{tabular}{|c|c|c|c|c|c|c|}
\hline My/Our programme ... & $\begin{array}{l}\text { not at } \\
\text { all } \\
-\end{array}$ & $\begin{array}{l}\text { just a } \\
\text { little } \\
--\end{array}$ & $\begin{array}{l}\text { quite a } \\
\text { bit } \\
-\end{array}$ & $\begin{array}{l}\text { rather } \\
\text { much } \\
+\end{array}$ & $\begin{array}{l}\text { much } \\
++\end{array}$ & $\begin{array}{l}\text { very } \\
\text { much } \\
+++\end{array}$ \\
\hline Mein/Unser Programm ... & $\begin{array}{l}\text { gar nicht } \\
\text {-.- }\end{array}$ & $\begin{array}{l}\text { ein biss- } \\
\text { chen } \\
--\end{array}$ & $\begin{array}{l}\text { einiger- } \\
\text { maßen } \\
-\end{array}$ & $\begin{array}{l}\text { ziemlich } \\
+\end{array}$ & $\begin{array}{l}\text { viel } \\
++\end{array}$ & $\begin{array}{l}\text { sehr } \\
\text { viel } \\
+++\end{array}$ \\
\hline Il mio/Nostro programma ... & $\begin{array}{l}\text { niente } \\
---\end{array}$ & un pò & $\begin{array}{l}\text { suffi- } \\
\text { ciente } \\
-\end{array}$ & $\begin{array}{l}\text { abba- } \\
\text { stanza } \\
+\end{array}$ & $\begin{array}{l}\text { molto } \\
++\end{array}$ & $\begin{array}{l}\text { tantis- } \\
\text { simo } \\
+++\end{array}$ \\
\hline Mon/Notre programme ... & $\begin{array}{l}\text { pas du } \\
\text { tout } \\
---\end{array}$ & $\begin{array}{l}\text { un peu } \\
--\end{array}$ & $\begin{array}{l}\text { passa- } \\
\text { blement } \\
-\end{array}$ & $\begin{array}{l}\text { assez } \\
+ \\
\end{array}$ & $\begin{array}{l}\text { beaucoup } \\
++\end{array}$ & $\begin{array}{l}\text { énor- } \\
\text { mément } \\
+++\end{array}$ \\
\hline $\begin{array}{l}\text { 1) supplies cognitive schemes (e.g. } \\
\text { methods that can be generalised). }\end{array}$ & $\mathrm{O}$ & $\mathrm{O}$ & $\mathrm{O}$ & $\mathrm{O}$ & O & $\mathrm{O}$ \\
\hline $\begin{array}{l}\text { 1) liefert Schemata ( } z . B \text {. verallge- } \\
\text { meinerbare Methoden). }\end{array}$ & $\mathrm{O}$ & $\mathrm{O}$ & $\mathrm{O}$ & $\mathrm{O}$ & O & $\mathrm{O}$ \\
\hline $\begin{array}{l}\text { 1) offre modelli (metodi che possono } \\
\text { essere generalizati) }\end{array}$ & $\mathrm{O}$ & $\mathrm{O}$ & $\mathrm{O}$ & $\mathrm{O}$ & $\mathrm{O}$ & $\mathrm{O}$ \\
\hline $\begin{array}{l}\text { 1) fournit des modèles (p.ex. des } \\
\text { méthodes à généraliser). }\end{array}$ & $\mathrm{O}$ & $\mathrm{O}$ & $\mathrm{O}$ & $\mathrm{O}$ & $\mathrm{O}$ & $\mathrm{O}$ \\
\hline $\begin{array}{l}\text { 2) helps the learners to gain more } \\
\text { routine with the use of reference } \\
\text { works. }\end{array}$ & $\mathrm{O}$ & O & $\mathrm{O}$ & $\mathrm{O}$ & $\mathrm{O}$ & $\mathrm{O}$ \\
\hline $\begin{array}{l}\text { 2) hilft den Lernenden beim Umgang } \\
\text { mit Arbeitsmitteln. }\end{array}$ & $\mathrm{O}$ & $\mathrm{O}$ & $\mathrm{O}$ & O & O & $\mathrm{O}$ \\
\hline 2) aiuta l'allievo nell'uso dei mezzi. & $\mathrm{O}$ & $\mathrm{O}$ & $\mathrm{O}$ & $\mathrm{O}$ & $\mathrm{O}$ & $\mathrm{O}$ \\
\hline $\begin{array}{l}\text { 2) aide les apprenants à manier les } \\
\text { moyens de travail. }\end{array}$ & $\mathrm{O}$ & $\mathrm{O}$ & $\mathrm{O}$ & $\mathrm{O}$ & $\mathrm{O}$ & $\mathrm{O}$ \\
\hline 3) offers a line of orientation. & $\mathrm{O}$ & $\mathrm{O}$ & $\mathrm{O}$ & $\mathrm{O}$ & $\mathrm{O}$ & $\mathrm{O}$ \\
\hline $\begin{array}{l}\text { 3) liefert einen Orientierungsrah- } \\
\text { men. }\end{array}$ & $\mathrm{O}$ & $\mathrm{O}$ & $\mathrm{O}$ & $\mathrm{O}$ & O & $\mathrm{O}$ \\
\hline $\begin{array}{l}\text { 3) offre una struttura di orienta- } \\
\text { mento. }\end{array}$ & $\mathrm{O}$ & $\mathrm{O}$ & $\mathrm{O}$ & $\mathrm{O}$ & O & O \\
\hline 3) fournit un cadre d'orientation. & $\mathrm{O}$ & $\mathrm{O}$ & $\mathrm{O}$ & $\mathrm{O}$ & $\mathrm{O}$ & $\mathrm{O}$ \\
\hline 4) imparts key qualifications. & $\mathrm{O}$ & $\mathrm{O}$ & $\mathrm{O}$ & $\mathrm{O}$ & $\mathrm{O}$ & $\mathrm{O}$ \\
\hline $\begin{array}{l}\text { 4) vermittelt Schlüsselqualifika- } \\
\text { tionen. }\end{array}$ & $\mathrm{O}$ & $\mathrm{O}$ & $\mathrm{O}$ & O & O & O \\
\hline 4) media chiave qualificazioni. & $\mathrm{O}$ & $\mathrm{O}$ & $\mathrm{O}$ & $\mathrm{O}$ & $\mathrm{O}$ & $\mathrm{O}$ \\
\hline $\begin{array}{l}\text { 4) communique des qualifications- } \\
\text { cléfs. }\end{array}$ & $\mathrm{O}$ & $\mathrm{O}$ & $\mathrm{O}$ & $\mathrm{O}$ & $\mathrm{O}$ & O \\
\hline $\begin{array}{l}\text { 5) creates and improves contacts } \\
\text { (e.g. to other learners, practition- } \\
\text { ers). }\end{array}$ & $\mathrm{O}$ & $\mathrm{O}$ & $\mathrm{O}$ & O & O & $\mathrm{O}$ \\
\hline $\begin{array}{l}\text { 5) stellt Kontakte her oder verbes- } \\
\text { sert sie (z.B. zu anderen Lernenden). }\end{array}$ & $\mathrm{O}$ & $\mathrm{O}$ & $\mathrm{O}$ & $\mathrm{O}$ & O & $\mathrm{O}$ \\
\hline $\begin{array}{l}\text { 5) apre contatti o li migliora (p.e. con } \\
\text { altri allievi). }\end{array}$ & $\mathrm{O}$ & $\mathrm{O}$ & $\mathrm{O}$ & O & O & O \\
\hline
\end{tabular}




\begin{tabular}{|c|c|c|c|c|c|c|}
\hline $\begin{array}{l}\text { 5) améliore les relations, p.ex. entre } \\
\text { les apprenants. }\end{array}$ & $\mathrm{O}$ & O & $\mathrm{O}$ & O & $\mathrm{O}$ & 0 \\
\hline $\begin{array}{l}\text { 6) conforms to appropriate regulato- } \\
\text { ry frameworks. }\end{array}$ & $\mathrm{O}$ & $\mathrm{O}$ & O & $\mathrm{O}$ & $\mathrm{O}$ & $\mathrm{O}$ \\
\hline $\begin{array}{l}\text { 6) entspricht den Anforderungen der } \\
\text { Kontrollinstanzen. }\end{array}$ & $\mathrm{O}$ & $\mathrm{O}$ & $\mathrm{O}$ & O & $\mathrm{O}$ & 0 \\
\hline $\begin{array}{l}\text { 6) corrisponda ai compiti e punti di } \\
\text { controllo. }\end{array}$ & O & $\mathrm{O}$ & O & $\mathrm{O}$ & $\mathrm{O}$ & O \\
\hline $\begin{array}{l}\text { 6) est conforme aux demandes de } \\
\text { l'organe e contrôle. }\end{array}$ & O & $\mathrm{O}$ & O & $\mathrm{O}$ & $\mathrm{O}$ & 0 \\
\hline 7) is fit for purpose. & $\mathrm{O}$ & $\mathrm{O}$ & $\mathrm{O}$ & $\mathrm{O}$ & $\mathrm{O}$ & $\mathrm{O}$ \\
\hline 7) ist auf den Zweck ausgerichtet. & $\mathrm{O}$ & $\mathrm{O}$ & $\mathrm{O}$ & $\mathrm{O}$ & $\mathrm{O}$ & $\mathrm{O}$ \\
\hline 7) è allineato allo scopo. & $\mathrm{O}$ & $\mathrm{O}$ & $\mathrm{O}$ & $\mathrm{O}$ & $\mathrm{O}$ & $\mathrm{O}$ \\
\hline \multirow[t]{2}{*}{ 7) poursuit un but. } & $\mathrm{O}$ & $\mathrm{O}$ & $\mathrm{O}$ & $\mathrm{O}$ & $\mathrm{O}$ & $\mathrm{O}$ \\
\hline & $\star_{1}$ & $\star 2$ & $\star 3$ & $\star 4$ & $\star_{5}$ & *6 \\
\hline
\end{tabular}

\begin{tabular}{|c|c|c|c|c|c|c|}
\hline \multicolumn{7}{|c|}{ B5. Self motivation: for teaching and learning persons } \\
\hline \multicolumn{7}{|c|}{ B5. Selbstmotivation: für Lernende und Lehrende } \\
\hline \multicolumn{7}{|c|}{ B5. Auto-motivazione: per gli studenti e gli istruttori } \\
\hline \multicolumn{7}{|c|}{ B5. Motivation de soi-même : pour apprenants et instructeurs } \\
\hline My/Our programme ... & $\begin{array}{l}\text { not at } \\
\text { all } \\
---\end{array}$ & $\begin{array}{l}\text { just a } \\
\text { little } \\
--\end{array}$ & $\begin{array}{l}\text { quite a } \\
\text { bit } \\
\text { - }\end{array}$ & $\begin{array}{l}\text { rather } \\
\text { much } \\
+\end{array}$ & $\begin{array}{l}\text { much } \\
++\end{array}$ & $\begin{array}{l}\text { very } \\
\text { much } \\
+++\end{array}$ \\
\hline Mein/Unser Programm ... & $\begin{array}{l}\text { gar nicht } \\
\text {-.- }\end{array}$ & $\begin{array}{l}\text { ein biss- } \\
\text { chen } \\
\text {-- }\end{array}$ & $\begin{array}{l}\text { einiger- } \\
\text { maßen } \\
-\end{array}$ & $\begin{array}{l}\text { ziemlich } \\
+\end{array}$ & $\begin{array}{l}\text { viel } \\
++\end{array}$ & $\begin{array}{l}\text { sehr } \\
\text { viel } \\
+++\end{array}$ \\
\hline II mio/Nostro programma ... & niente & un pò & $\begin{array}{l}\text { suffi- } \\
\text { ciente } \\
\text { - }\end{array}$ & $\begin{array}{l}\text { abba- } \\
\text { stanza } \\
+\end{array}$ & $\begin{array}{l}\text { molto } \\
++\end{array}$ & $\begin{array}{l}\text { tantis- } \\
\text { simo } \\
+++\end{array}$ \\
\hline Mon/Notre programme ... & $\begin{array}{l}\text { pas du } \\
\text { tout } \\
---\end{array}$ & un peu & $\begin{array}{l}\text { passa- } \\
\text { blement } \\
\text { - }\end{array}$ & $\begin{array}{l}\text { assez } \\
+\end{array}$ & beaucoup & $\begin{array}{l}\text { énor- } \\
\text { mément } \\
+++\end{array}$ \\
\hline $\begin{array}{l}\text { 1) shows the learner especially the } \\
\text { positive effects of what he/she has } \\
\text { already learnt }\end{array}$ & O & $\mathrm{O}$ & $\mathrm{O}$ & $\mathrm{O}$ & O & O \\
\hline $\begin{array}{l}\text { 1) zeigt dem Lerner vor allem die } \\
\text { positiven Effekte dessen, was er/sie } \\
\text { bereits gelernt hat. }\end{array}$ & O & $\mathrm{O}$ & $\mathrm{O}$ & $\mathrm{O}$ & O & O \\
\hline $\begin{array}{l}\text { 1) dimostra agli studenti positiva- } \\
\text { mente ciò che hanno imparato }\end{array}$ & $\mathrm{O}$ & O & O & $\mathrm{O}$ & O & O \\
\hline $\begin{array}{l}\text { 1) rend évident aux apprenants sur- } \\
\text { tout les effets positives de ce qu'ils } \\
\text { ont appris. }\end{array}$ & O & $\mathrm{O}$ & 0 & O & 0 & 0 \\
\hline $\begin{array}{l}\text { 2) takes the joy in physical exercise } \\
\text { during the learning process into ac- } \\
\text { count. }\end{array}$ & 0 & 0 & 0 & 0 & 0 & 0 \\
\hline $\begin{array}{l}\text { 2) berücksichtigt die Freude an kör- } \\
\text { perlicher Bewegung beim Lernen. }\end{array}$ & 0 & 0 & 0 & 0 & 0 & 0 \\
\hline $\begin{array}{l}\text { 2) considera la gioia nel movimento } \\
\text { fisico imparando. }\end{array}$ & 0 & 0 & 0 & 0 & 0 & 0 \\
\hline
\end{tabular}




\begin{tabular}{|c|c|c|c|c|c|c|}
\hline $\begin{array}{l}\text { 2) montre la joie du mouvement phy- } \\
\text { sique en apprenant. }\end{array}$ & 0 & 0 & 0 & 0 & 0 & 0 \\
\hline $\begin{array}{l}\text { 3) is a suitable basis for fruitful } \\
\text { questions. }\end{array}$ & 0 & 0 & 0 & 0 & 0 & 0 \\
\hline $\begin{array}{l}\text { 3) ist dazu geeignet, fruchtbare } \\
\text { Fragestellungen zu eröffnen. }\end{array}$ & 0 & 0 & 0 & 0 & 0 & 0 \\
\hline $\begin{array}{l}\text { 3) è adeguato per aprire nuovi impul- } \\
\text { si e domande. }\end{array}$ & 0 & 0 & 0 & 0 & 0 & 0 \\
\hline $\begin{array}{l}\text { 3) est capable de poser des ques- } \\
\text { tions fructueuses/productives. }\end{array}$ & 0 & 0 & 0 & 0 & 0 & 0 \\
\hline $\begin{array}{l}\text { 4) offers also learning in a playful } \\
\text { way }\end{array}$ & 0 & 0 & 0 & 0 & 0 & 0 \\
\hline $\begin{array}{l}\text { 4) ermöglicht auch Lernen in spiele- } \\
\text { rischer Form }\end{array}$ & 0 & 0 & 0 & 0 & 0 & 0 \\
\hline $\begin{array}{l}\text { 4) rende possibile d'imparare giocan- } \\
\text { do. }\end{array}$ & 0 & 0 & $\mathrm{O}$ & 0 & 0 & 0 \\
\hline $\begin{array}{l}\text { 4) permet l'apprentissage aussi d'une } \\
\text { façon legère. }\end{array}$ & 0 & 0 & 0 & 0 & 0 & 0 \\
\hline 5) means also learning in a social way & $\mathrm{O}$ & $\mathrm{O}$ & $\mathrm{O}$ & $\mathrm{O}$ & $\mathrm{O}$ & $\mathrm{O}$ \\
\hline $\begin{array}{l}\text { 5) ermöglicht auch, dass auf geselli- } \\
\text { ge Art und Weise gelernt wird. }\end{array}$ & $\mathrm{O}$ & $\mathrm{O}$ & $\mathrm{O}$ & $\mathrm{O}$ & $\mathrm{O}$ & O \\
\hline $\begin{array}{l}\text { 5) rende possibile il modo d'imparare } \\
\text { in compagnia. }\end{array}$ & $\mathrm{O}$ & $\mathrm{O}$ & $\mathrm{O}$ & $\mathrm{O}$ & $\mathrm{O}$ & O \\
\hline $\begin{array}{l}\text { 5) rend possible un apprentissage en } \\
\text { réunion avec d'autres. }\end{array}$ & $\mathrm{O}$ & $\mathrm{O}$ & $\mathrm{O}$ & $\mathrm{O}$ & $\mathrm{O}$ & O \\
\hline 6) bears recreational features. & $\mathrm{O}$ & $\mathrm{O}$ & $\mathrm{O}$ & $\mathrm{O}$ & $\mathrm{O}$ & $\mathrm{O}$ \\
\hline $\begin{array}{l}\text { 6) hat auch Erholungs- und Freizeit- } \\
\text { charakter. }\end{array}$ & $\mathrm{O}$ & $\mathrm{O}$ & O & O & O & O \\
\hline $\begin{array}{l}\text { 6) ha un carattere di riposo e tempo } \\
\text { libero. }\end{array}$ & $\mathrm{O}$ & $\mathrm{O}$ & $\mathrm{O}$ & O & O & 0 \\
\hline $\begin{array}{l}\text { 6) contribue aussi à la détente et } \\
\text { aux loisirs. }\end{array}$ & $\mathrm{O}$ & $\mathrm{O}$ & $\mathrm{O}$ & O & O & 0 \\
\hline $\begin{array}{l}\text { 7) is carried out without glancing too } \\
\text { much at a certification. }\end{array}$ & $\mathrm{O}$ & $\mathrm{O}$ & $\mathrm{O}$ & O & 0 & 0 \\
\hline $\begin{array}{l}\text { 7) verstärkt das Lernen ohne dau- } \\
\text { ernden Blick auf Zertifikate. }\end{array}$ & 0 & 0 & 0 & 0 & 0 & 0 \\
\hline $\begin{array}{l}\text { 7) rinforza il modi d'imparare senza } \\
\text { pensare sempre ai certificati. }\end{array}$ & 0 & 0 & 0 & 0 & 0 & 0 \\
\hline $\begin{array}{l}\text { 7) renforce l'apprentissage sans as- } \\
\text { piration permanente à un diplôme. }\end{array}$ & 0 & 0 & 0 & 0 & 0 & 0 \\
\hline $\begin{array}{l}\text { 8) is carried out without fearing to } \\
\text { fail. }\end{array}$ & 0 & 0 & 0 & 0 & 0 & 0 \\
\hline $\begin{array}{l}\text { 8) fördert das Lernen ohne Angst } \\
\text { vor Versagen. }\end{array}$ & 0 & 0 & 0 & 0 & 0 & 0 \\
\hline $\begin{array}{l}\text { 8) promouve il modo d'imparare senza } \\
\text { timore di fallimento. }\end{array}$ & 0 & 0 & 0 & 0 & 0 & 0 \\
\hline $\begin{array}{l}\text { 8) encourage l'apprentissage sans } \\
\text { peur de rater. }\end{array}$ & 0 & 0 & 0 & 0 & 0 & 0 \\
\hline $\begin{array}{l}\text { 9) promotes the learning rather in } \\
\text { competition with oneself and not to } \\
\text { others. }\end{array}$ & 0 & 0 & 0 & 0 & 0 & 0 \\
\hline
\end{tabular}




\begin{tabular}{|c|c|c|c|c|c|c|}
\hline $\begin{array}{l}\text { 9) fördert eher den Wettbewerb } \\
\text { der Lernenden mit sich selbst als } \\
\text { untereinander. }\end{array}$ & 0 & 0 & 0 & 0 & 0 & 0 \\
\hline $\begin{array}{l}\text { 9) rimouve la concorrenza più con se } \\
\text { stesso che con gli altri. }\end{array}$ & 0 & 0 & 0 & 0 & 0 & 0 \\
\hline $\begin{array}{l}\text { 9) aide à entrer en compétition plu- } \\
\text { tôt avec soi-même qu'avec d'autrui. }\end{array}$ & 0 & 0 & $O$ & 0 & 0 & 0 \\
\hline $\begin{array}{l}\text { 10) takes the joy in intellectu- } \\
\text { al/artistic games into account }\end{array}$ & 0 & 0 & 0 & 0 & 0 & 0 \\
\hline $\begin{array}{l}\text { 10) berücksichtigt die Freude am } \\
\text { intellektuellen Spiel. }\end{array}$ & 0 & 0 & 0 & 0 & 0 & 0 \\
\hline $\begin{array}{l}\text { 10) considera la gioia nel gioco intel- } \\
\text { lettuale. }\end{array}$ & 0 & 0 & 0 & 0 & 0 & 0 \\
\hline \multirow[t]{2}{*}{$\begin{array}{l}\text { 10) accentue la joie du jeu intelléc- } \\
\text { tuell. }\end{array}$} & 0 & 0 & 0 & 0 & 0 & 0 \\
\hline & $\star_{1}$ & $\star 2$ & $\star 3$ & $\star 4$ & $\star 5$ & *6 \\
\hline
\end{tabular}


B6. Development of learners' abilities

B6. Persönlichkeitsbildung: Entwicklung der Lernerfähigkeiten

B6. Sviluppo delle abilità degli studenti

B6. Développement personelle des aptitudes de l'apprentissage

\begin{tabular}{|c|c|c|c|c|c|c|}
\hline My/Our programme ... & $\begin{array}{l}\text { not at } \\
\text { all } \\
-\end{array}$ & $\begin{array}{l}\text { just a } \\
\text { little } \\
--\end{array}$ & $\begin{array}{l}\text { quite a } \\
\text { bit } \\
-\end{array}$ & $\begin{array}{l}\text { rather } \\
\text { much } \\
+\end{array}$ & $\begin{array}{l}\text { much } \\
++\end{array}$ & $\begin{array}{l}\text { very } \\
\text { much } \\
+++\end{array}$ \\
\hline Mein/Unser Programm ... & $\begin{array}{l}\text { gar nicht } \\
\text {--- }\end{array}$ & $\begin{array}{l}\text { ein biss- } \\
\text { chen } \\
\text {-- }\end{array}$ & $\begin{array}{l}\text { einiger- } \\
\text { maßen } \\
\text { - }\end{array}$ & $\begin{array}{l}\text { ziemlich } \\
+\end{array}$ & $\begin{array}{l}\text { viel } \\
++\end{array}$ & $\begin{array}{l}\text { sehr } \\
\text { viel } \\
+++\end{array}$ \\
\hline Il mio/Nostro programma ... & niente & un pò & $\begin{array}{l}\text { suffi- } \\
\text { ciente } \\
-\end{array}$ & $\begin{array}{l}\text { abba- } \\
\text { stanza } \\
+\end{array}$ & $\begin{array}{l}\text { molto } \\
++\end{array}$ & $\begin{array}{l}\text { tantis- } \\
\text { simo } \\
+++\end{array}$ \\
\hline Mon/Notre programme ... & $\begin{array}{l}\text { pas du } \\
\text { tout } \\
---\end{array}$ & $\begin{array}{l}\text { un peu } \\
-- \\
\end{array}$ & $\begin{array}{l}\text { passa- } \\
\text { blement } \\
\text { - }\end{array}$ & $\begin{array}{l}\text { assez } \\
+ \\
\end{array}$ & $\begin{array}{l}\text { beaucoup } \\
++\end{array}$ & $\begin{array}{l}\text { énor- } \\
\text { mément } \\
+++\end{array}$ \\
\hline $\begin{array}{l}\text { 1) increases the moral sensibility of } \\
\text { the learner (in regard to the sub- } \\
\text { ject, qualification). }\end{array}$ & $\mathrm{O}$ & $\mathrm{O}$ & $\mathrm{O}$ & $\mathrm{O}$ & O & $\mathrm{O}$ \\
\hline $\begin{array}{l}\text { 1) erhöht die moralische Sensibilität } \\
\text { der Lernenden (im Hinblick auf das } \\
\text { Thema, die Qualifikation), }\end{array}$ & $\mathrm{O}$ & $\mathrm{O}$ & $\mathrm{O}$ & $\mathrm{O}$ & $\mathrm{O}$ & $\mathrm{O}$ \\
\hline $\begin{array}{l}\text { 1) aumenta la sensibilità morale degli } \\
\text { studenti. }\end{array}$ & $\mathrm{O}$ & $\mathrm{O}$ & $\mathrm{O}$ & O & $\mathrm{O}$ & $\mathrm{O}$ \\
\hline $\begin{array}{l}\text { 1) augmente la sensibilité morale des } \\
\text { apprenants. }\end{array}$ & $\mathrm{O}$ & $\mathrm{O}$ & $\mathrm{O}$ & $\mathrm{O}$ & $\mathrm{O}$ & $\mathrm{O}$ \\
\hline $\begin{array}{l}\text { 2) increases the intelligence of the } \\
\text { learners. }\end{array}$ & $\mathrm{O}$ & $\mathrm{O}$ & $\mathrm{O}$ & $\mathrm{O}$ & O & $\mathrm{O}$ \\
\hline $\begin{array}{l}\text { 2) steigert die Intelligenz der Ler- } \\
\text { ner. }\end{array}$ & $\mathrm{O}$ & $\mathrm{O}$ & $\mathrm{O}$ & $\mathrm{O}$ & $\mathrm{O}$ & $\mathrm{O}$ \\
\hline $\begin{array}{l}\text { 2) aumenta l'intelligenza degli stu- } \\
\text { denti. }\end{array}$ & $\mathrm{O}$ & $\mathrm{O}$ & $\mathrm{O}$ & $\mathrm{O}$ & O & $\mathrm{O}$ \\
\hline $\begin{array}{l}\text { 2) renforce l'intelligence des appre- } \\
\text { nants. }\end{array}$ & $\mathrm{O}$ & $\mathrm{O}$ & O & $\mathrm{O}$ & $\mathrm{O}$ & $\mathrm{O}$ \\
\hline $\begin{array}{l}\text { 3) promotes the creativity of the } \\
\text { learners. }\end{array}$ & $\mathrm{O}$ & $\mathrm{O}$ & $\mathrm{O}$ & $\mathrm{O}$ & $\mathrm{O}$ & $\mathrm{O}$ \\
\hline fördert die Kreativität der Lerner. & $\mathrm{O}$ & $\mathrm{O}$ & $\mathrm{O}$ & $\mathrm{O}$ & $\mathrm{O}$ & $\mathrm{O}$ \\
\hline rimuove la creatività degli studenti & $\mathrm{O}$ & $\mathrm{O}$ & $\mathrm{O}$ & $\mathrm{O}$ & $\mathrm{O}$ & $\mathrm{O}$ \\
\hline $\begin{array}{l}\text { 3) 3) augmente la créativité des ap- } \\
\text { prenants. }\end{array}$ & $\mathrm{O}$ & $\mathrm{O}$ & $\mathrm{O}$ & $\mathrm{O}$ & $\mathrm{O}$ & O \\
\hline $\begin{array}{l}\text { 4) helps to diminish fears of the } \\
\text { learners. }\end{array}$ & $\mathrm{O}$ & $\mathrm{O}$ & $\mathrm{O}$ & $\mathrm{O}$ & O & $\mathrm{O}$ \\
\hline $\begin{array}{l}\text { 4) baut bei den Lernern Ängstlich- } \\
\text { keit ab. }\end{array}$ & $\mathrm{O}$ & $\mathrm{O}$ & $\mathrm{O}$ & $\mathrm{O}$ & $\mathrm{O}$ & $\mathrm{O}$ \\
\hline 4) diminuisce il timore degli studenti. & $\mathrm{O}$ & $\mathrm{O}$ & $\mathrm{O}$ & $\mathrm{O}$ & $\mathrm{O}$ & $\mathrm{O}$ \\
\hline 4) diminue la peur des apprenants. & $\mathrm{O}$ & $\mathrm{O}$ & $\mathrm{O}$ & $\mathrm{O}$ & $\mathrm{O}$ & $\mathrm{O}$ \\
\hline $\begin{array}{l}\text { 5) strengthens the conflict abilities } \\
\text { of the learners. }\end{array}$ & $\mathrm{O}$ & $\mathrm{O}$ & O & $\mathrm{O}$ & O & $\mathrm{O}$ \\
\hline $\begin{array}{l}\text { 5) stärkt die Konfliktfähigkeit der } \\
\text { Lerner. }\end{array}$ & $\mathrm{O}$ & $\mathrm{O}$ & $\mathrm{O}$ & $\mathrm{O}$ & $\mathrm{O}$ & 0 \\
\hline $\begin{array}{l}\text { 5) rinforza l'abilità di conflitto degli } \\
\text { studenti. }\end{array}$ & 0 & 0 & 0 & 0 & 0 & 0 \\
\hline
\end{tabular}




\begin{tabular}{|c|c|c|c|c|c|c|}
\hline $\begin{array}{l}\text { 5) renforce la capacité de resoudre } \\
\text { des conflits des apprenants. }\end{array}$ & $\mathrm{O}$ & $\mathrm{O}$ & $\mathrm{O}$ & $\mathrm{O}$ & $\mathrm{O}$ & O \\
\hline $\begin{array}{l}\text { 6) increases the communication abili- } \\
\text { ties of the learners. }\end{array}$ & $\mathrm{O}$ & $\mathrm{O}$ & $\mathrm{O}$ & $\mathrm{O}$ & $\mathrm{O}$ & O \\
\hline $\begin{array}{l}\text { 6) erhöht die Kommunikationsfähig- } \\
\text { keit der Lerner. }\end{array}$ & $\mathrm{O}$ & $\mathrm{O}$ & $\mathrm{O}$ & $\mathrm{O}$ & $\mathrm{O}$ & O \\
\hline $\begin{array}{l}\text { 6) aumenta il grado delle capacità di } \\
\text { communicazione degli studenti. }\end{array}$ & $\mathrm{O}$ & $\mathrm{O}$ & $\mathrm{O}$ & $\mathrm{O}$ & $\mathrm{O}$ & $\mathrm{O}$ \\
\hline $\begin{array}{l}\text { 6) contribue à la capacité de commu- } \\
\text { niquer des apprenants. }\end{array}$ & $\mathrm{O}$ & $\mathrm{O}$ & $\mathrm{O}$ & $\mathrm{O}$ & $\mathrm{O}$ & $\mathrm{O}$ \\
\hline $\begin{array}{l}\text { 7) gives the learners a better self- } \\
\text { esteem (self confidence). }\end{array}$ & O & O & $\mathrm{O}$ & $\mathrm{O}$ & $\mathrm{O}$ & O \\
\hline $\begin{array}{l}\text { 7) vermittelt den Lernern Selbst- } \\
\text { wertgefühl. }\end{array}$ & O & O & $\mathrm{O}$ & $\mathrm{O}$ & $\mathrm{O}$ & O \\
\hline 7) rinforza la sicurezza in se stessi. & $\mathrm{O}$ & $\mathrm{O}$ & $\mathrm{O}$ & $\mathrm{O}$ & $\mathrm{O}$ & $\mathrm{O}$ \\
\hline $\begin{array}{l}\text { 7) fournit aux apprenants la con- } \\
\text { fiance en soi. }\end{array}$ & $\mathrm{O}$ & $\mathrm{O}$ & $\mathrm{O}$ & $\mathrm{O}$ & $\mathrm{O}$ & O \\
\hline $\begin{array}{l}\text { 8) promotes the decision-making } \\
\text { ability of the learners. }\end{array}$ & $\mathrm{O}$ & $\mathrm{O}$ & $\mathrm{O}$ & $\mathrm{O}$ & $\mathrm{O}$ & 0 \\
\hline $\begin{array}{l}\text { 8) fördert die Entscheidungsfähig- } \\
\text { keit der Lerner. }\end{array}$ & $\mathrm{O}$ & O & $\mathrm{O}$ & $\mathrm{O}$ & O & $\mathrm{O}$ \\
\hline 8) rinforza le capacità di decidere. & $\mathrm{O}$ & $\mathrm{O}$ & $\mathrm{O}$ & $\mathrm{O}$ & $\mathrm{O}$ & $\mathrm{O}$ \\
\hline $\begin{array}{l}\text { 8) actrive la capacité de prendre des } \\
\text { décisions des apprenants. }\end{array}$ & $\mathrm{O}$ & $\mathrm{O}$ & $\mathrm{O}$ & $\mathrm{O}$ & $\mathrm{O}$ & O \\
\hline $\begin{array}{l}\text { 9) increases the sense of responsi- } \\
\text { bility of the learners. }\end{array}$ & $\mathrm{O}$ & $\mathrm{O}$ & $\mathrm{O}$ & $\mathrm{O}$ & O & $\mathrm{O}$ \\
\hline $\begin{array}{l}\text { 9) erhöht das Verantwortungsgefühl } \\
\text { der Lerner. }\end{array}$ & $\mathrm{O}$ & $\mathrm{O}$ & $\mathrm{O}$ & $\mathrm{O}$ & O & O \\
\hline $\begin{array}{l}\text { 9) aumenta la responsabilità degli } \\
\text { studenti. }\end{array}$ & $\mathrm{O}$ & $\mathrm{O}$ & $\mathrm{O}$ & $\mathrm{O}$ & $\mathrm{O}$ & 0 \\
\hline \multirow[t]{2}{*}{$\begin{array}{l}\text { 9) augmente le sens de la responsabi- } \\
\text { lité des apprenants. }\end{array}$} & $\mathrm{O}$ & $O$ & $\mathrm{O}$ & $\mathrm{O}$ & O & $\mathrm{O}$ \\
\hline & $\star_{1}$ & *2 & $\star 3$ & $\star 4$ & $* 5$ & *6 \\
\hline
\end{tabular}




\begin{tabular}{|c|c|c|c|c|c|c|}
\hline \multicolumn{7}{|l|}{ B7. Consistency } \\
\hline \multicolumn{7}{|l|}{ B7. Konsistenz } \\
\hline \multicolumn{7}{|l|}{ B7. Consistenza } \\
\hline \multicolumn{7}{|l|}{ B7. Consistance } \\
\hline My/Our programme ... & $\begin{array}{l}\text { not at } \\
\text { all } \\
---\end{array}$ & $\begin{array}{l}\text { just a } \\
\text { little } \\
--\end{array}$ & $\begin{array}{l}\text { quite a } \\
\text { bit } \\
-\end{array}$ & $\begin{array}{l}\text { rather } \\
\text { much } \\
+\end{array}$ & $\begin{array}{l}\text { much } \\
++\end{array}$ & $\begin{array}{l}\text { very } \\
\text { much } \\
+++\end{array}$ \\
\hline Mein/Unser Programm ... & $\begin{array}{l}\text { gar nicht } \\
\text {-- }\end{array}$ & $\begin{array}{l}\text { ein biss- } \\
\text { chen } \\
--\end{array}$ & $\begin{array}{l}\text { einiger- } \\
\text { maßen } \\
-\end{array}$ & $\begin{array}{l}\text { ziemlich } \\
+\end{array}$ & $\begin{array}{l}\text { viel } \\
++\end{array}$ & $\begin{array}{l}\text { sehr } \\
\text { viel } \\
+++\end{array}$ \\
\hline Il mio/Nostro programma ... & niente & $\begin{array}{l}\text { un pò } \\
--\end{array}$ & $\begin{array}{l}\text { suffi- } \\
\text { ciente } \\
-\end{array}$ & $\begin{array}{l}\text { abba- } \\
\text { stanza } \\
+\end{array}$ & $\begin{array}{l}\text { molto } \\
++\end{array}$ & $\begin{array}{l}\text { tantis- } \\
\text { simo } \\
+++\end{array}$ \\
\hline Mon/Notre programme ... & $\begin{array}{l}\text { pas du } \\
\text { tout } \\
---\end{array}$ & un peu & $\begin{array}{l}\text { passa- } \\
\text { blement } \\
\text { - }\end{array}$ & $\begin{array}{l}\text { assez } \\
+\end{array}$ & $\begin{array}{l}\text { beaucoup } \\
++\end{array}$ & $\begin{array}{l}\text { énor- } \\
\text { mément } \\
+++\end{array}$ \\
\hline $\begin{array}{l}\text { 1) shows a close distinc- } \\
\text { tion/distribution between the various } \\
\text { modi of blended learning on the basis } \\
\text { of their different functionalities and } \\
\text { possibilities. }\end{array}$ & $\mathrm{O}$ & $\mathrm{O}$ & $\mathrm{O}$ & $\mathrm{O}$ & $\mathrm{O}$ & O \\
\hline $\begin{array}{l}\text { 1) berücksichtigt in angemessener } \\
\text { Weise die verschiedenen Komponenten } \\
\text { des Blended Learning. }\end{array}$ & $\mathrm{O}$ & $\mathrm{O}$ & $\mathrm{O}$ & $\mathrm{O}$ & $\mathrm{O}$ & O \\
\hline $\begin{array}{l}\text { 1) tiene nel modo giusto i varii compo- } \\
\text { nenti del apprendimento diverso. }\end{array}$ & O & O & $\mathrm{O}$ & $\mathrm{O}$ & $\mathrm{O}$ & O \\
\hline $\begin{array}{l}\text { 1) tient convenablement compte des } \\
\text { parties composantes de } \\
\text { l'apprentissage mixte. }\end{array}$ & $\mathrm{O}$ & $\mathrm{O}$ & $\mathrm{O}$ & $\mathrm{O}$ & $\mathrm{O}$ & $\mathrm{O}$ \\
\hline $\begin{array}{l}\text { 2) takes care that the teaching people } \\
\text { themselves are experienced in han- } \\
\text { dling the electronic devices. }\end{array}$ & $\mathrm{O}$ & $\mathrm{O}$ & $\mathrm{O}$ & $\mathrm{O}$ & $\mathrm{O}$ & $\mathrm{O}$ \\
\hline $\begin{array}{l}\text { 2) trifft Vorsorge dafür, dass die } \\
\text { Lehrenden selbst auch kompetent und } \\
\text { routiniert im Umgang mit elektroni- } \\
\text { schen Medien sind. }\end{array}$ & $\mathrm{O}$ & $\mathrm{O}$ & $\mathrm{O}$ & $\mathrm{O}$ & $\mathrm{O}$ & $\mathrm{O}$ \\
\hline $\begin{array}{l}\text { 2) fa si che gli studenti abbiano una } \\
\text { certa competenza nel uso dei mezzi } \\
\text { elettronici. }\end{array}$ & $\mathrm{O}$ & $\mathrm{O}$ & $\mathrm{O}$ & $\mathrm{O}$ & $\mathrm{O}$ & $\mathrm{O}$ \\
\hline $\begin{array}{l}\text { 2) pourvoit au fait que les énseignants } \\
\text { eux-mêmes savent manier avec compé- } \\
\text { tence et routine les médias électro- } \\
\text { niques. }\end{array}$ & $\mathrm{O}$ & $\mathrm{O}$ & $\mathrm{O}$ & $\mathrm{O}$ & $\mathrm{O}$ & $\mathrm{O}$ \\
\hline $\begin{array}{l}\text { 3) has no serious discrepancies be- } \\
\text { tween what is said and what is done. }\end{array}$ & O & $\mathrm{O}$ & $\mathrm{O}$ & $\mathrm{O}$ & O & O \\
\hline $\begin{array}{l}\text { 3) weist keine gravierenden Wider- } \\
\text { sprüche zwischen dem, was gesagt } \\
\text { wird, und dem, was getan wird. }\end{array}$ & $\mathrm{O}$ & 0 & 0 & 0 & 0 & 0 \\
\hline $\begin{array}{l}\text { 3) non ha paradossi fra il dire ed il } \\
\text { fare. }\end{array}$ & 0 & 0 & 0 & 0 & O & 0 \\
\hline $\begin{array}{l}\text { 3) ne montre aucun désaccord entre } \\
\text { mots et action. }\end{array}$ & 0 & 0 & 0 & O & O & 0 \\
\hline
\end{tabular}




\begin{tabular}{|c|c|c|c|c|c|c|}
\hline $\begin{array}{l}\text { 4) takes care that the teaching people } \\
\text { "live" what they are imparting. }\end{array}$ & 0 & 0 & 0 & 0 & 0 & 0 \\
\hline $\begin{array}{l}\text { 4) trifft Vorsorge dafür, dass die } \\
\text { Lehrenden das "vorleben", was sie } \\
\text { inhaltlich vermitteln. }\end{array}$ & 0 & 0 & 0 & 0 & 0 & 0 \\
\hline $\begin{array}{l}\text { 4) fa si che gli istruttori vivono quello } \\
\text { che insegnano. }\end{array}$ & 0 & 0 & 0 & 0 & 0 & 0 \\
\hline $\begin{array}{l}\text { 4) réalise que les enseignants vivent ce } \\
\text { qu'ils enseignent. }\end{array}$ & 0 & 0 & 0 & 0 & 0 & 0 \\
\hline $\begin{array}{l}\text { 5) has a high quality of ver- } \\
\text { bal/audiovisual material. }\end{array}$ & 0 & 0 & 0 & 0 & 0 & 0 \\
\hline $\begin{array}{l}\text { 5) weist Lehrmaterialien und -texte } \\
\text { von beispielhafter Qualität auf. }\end{array}$ & 0 & 0 & 0 & 0 & 0 & 0 \\
\hline $\begin{array}{l}\text { 5) dimostra materiali e testi di grande } \\
\text { qualità. }\end{array}$ & 0 & 0 & 0 & $O$ & 0 & 0 \\
\hline $\begin{array}{l}\text { 5) prépare des matériaux et des } \\
\text { textes d'une qualité exemplaire. }\end{array}$ & 0 & 0 & 0 & $O$ & 0 & 0 \\
\hline $\begin{array}{l}\text { 6) delivers tasks which agree with the } \\
\text { practical requirements. }\end{array}$ & 0 & 0 & 0 & 0 & 0 & 0 \\
\hline $\begin{array}{l}\text { 6) weist Lernaufgaben auf, die mit den } \\
\text { Praxisanforderungen übereinstimmen. }\end{array}$ & 0 & 0 & 0 & 0 & 0 & 0 \\
\hline $\begin{array}{l}\text { 6) dispose des devoirs conformes aux } \\
\text { exigences de la pratique. }\end{array}$ & 0 & 0 & 0 & 0 & 0 & 0 \\
\hline $\begin{array}{l}\text { 7) gives learners as much room for } \\
\text { manoeuvre as in professional practice }\end{array}$ & 0 & 0 & 0 & 0 & 0 & 0 \\
\hline $\begin{array}{l}\text { 7) gibt den Lernenden genauso viel } \\
\text { Handlungsspielraum wie in der berufli- } \\
\text { chen Praxis. }\end{array}$ & 0 & 0 & $O$ & 0 & 0 & 0 \\
\hline $\begin{array}{l}\text { 7) da agli studenti lo stesso modo di } \\
\text { agire che in prattica professionale. }\end{array}$ & 0 & 0 & 0 & 0 & 0 & 0 \\
\hline $\begin{array}{l}\text { 7) permet aux apprenants autant de } \\
\text { champ libre comme dans la vie profes- } \\
\text { sionnelle. }\end{array}$ & 0 & 0 & 0 & 0 & 0 & 0 \\
\hline $\begin{array}{l}\text { 8) equally distributes the learning } \\
\text { difficulties, not clustering them only } \\
\text { to the beginning or to the end of the } \\
\text { programme. }\end{array}$ & 0 & 0 & 0 & 0 & 0 & 0 \\
\hline $\begin{array}{l}\text { 8) verteilt den Schwierigkeitsgrad der } \\
\text { Lernanforderungen auf die ganze } \\
\text { Lernzeit. }\end{array}$ & 0 & 0 & 0 & 0 & 0 & 0 \\
\hline $\begin{array}{l}\text { 8) distribuisce le difficoltà di appren- } \\
\text { zione per tutta la durata del corso. }\end{array}$ & 0 & 0 & 0 & 0 & 0 & 0 \\
\hline \multirow[t]{2}{*}{$\begin{array}{l}\text { 8) distribue les difficultés au cours du } \\
\text { programme entier sans les concentrer } \\
\text { au début ou à la fin. }\end{array}$} & 0 & 0 & 0 & 0 & 0 & 0 \\
\hline & $\star_{1}$ & $\star 2$ & $\star 3$ & $* 4$ & $\star 5$ & ${ }^{*} 6$ \\
\hline
\end{tabular}


C. Criteria to think about the efficacy of your e-learning programme

C. Kriterien der Wirksamkeit eines e-Learning-Programms

C. Criteri dell'efficacia di un programma elettronico

C. Critères pour évaluer l'efficacité d'un programme apprentissage électronique.

The following criteria reflect how efficacious a learning programme is expected to be. Effects will depend on many circumstances, and it is quite sure that only the practical try-out will prove them in last instance. But as we are here in an inspective evaluation the first judgment can be done by yourself.

Die folgenden Kriterien behandeln die Wirksamkeit eines Lernprogramms. Lernwirkungen hängen sicher von vielen Umständen ab, und es ist klar, dass sie letztlich erst bei der Umsetzung des Programms erfasst werden können. Aber in einer Inspektionsevaluation soll auch schon die eigenen Inaugenscheinnahme eine Rolle spielen.

Le seguenti criteri riguardano l'efficacia del programma. L'apprendimento dipende certamente di molti fattori ed è chiaro che possono essere annotati alla fine del programma. Ma una inspezione durante il corso può dare un aiuto nella valutazione finale.

Les critères suivants traitent de l'efficacité d'un programme. Les effets dépendent sûrement de plusieurs circonstances et il est clair qu'on ne puisse les saisir que lors de la réalisation du programme. Mais comme nous sommes ici dans une évaluation inspectatrice vous pouvez y porter un premier jugement par vous-mêmes. 
C1. Clarity: reliable and comprehensible performances

C1. Klarheit: zuverlässige und verständliche Darstellungen

C1. Chiarezza: chiara e sicura rappresentazione

C1. Clarté: présentation sûre et compréhensible

\begin{tabular}{|c|c|c|c|c|c|c|}
\hline My/Our programme ... & $\begin{array}{l}\text { not at } \\
\text { all } \\
-\end{array}$ & $\begin{array}{l}\text { just a } \\
\text { little } \\
--\end{array}$ & $\begin{array}{l}\text { quite a } \\
\text { bit } \\
-\end{array}$ & $\begin{array}{l}\text { rather } \\
\text { much } \\
+\end{array}$ & $\begin{array}{l}\text { much } \\
++\end{array}$ & $\begin{array}{l}\text { very } \\
\text { much } \\
+++\end{array}$ \\
\hline Mein/Unser Programm ... & $\begin{array}{l}\text { gar nicht } \\
\text {--- }\end{array}$ & $\begin{array}{l}\text { ein biss- } \\
\text { chen } \\
--\end{array}$ & $\begin{array}{l}\text { einiger- } \\
\text { maßen } \\
-\end{array}$ & $\begin{array}{l}\text { ziemlich } \\
+\end{array}$ & $\begin{array}{l}\text { viel } \\
++\end{array}$ & $\begin{array}{l}\text { sehr } \\
\text { viel } \\
+++\end{array}$ \\
\hline Il mio/Nostro programma ... & niente & un pò & $\begin{array}{l}\text { suffi- } \\
\text { ciente } \\
-\end{array}$ & $\begin{array}{l}\text { abba- } \\
\text { stanza } \\
+\end{array}$ & $\begin{array}{l}\text { molto } \\
++\end{array}$ & $\begin{array}{l}\text { tantis- } \\
\text { simo } \\
+++\end{array}$ \\
\hline Mon/Notre programme ... & $\begin{array}{l}\text { pas du } \\
\text { tout } \\
---\end{array}$ & un peu & $\begin{array}{l}\text { passa- } \\
\text { blement } \\
-\end{array}$ & $\begin{array}{l}\text { assez } \\
+ \\
\end{array}$ & $\begin{array}{l}\text { beaucoup } \\
++\end{array}$ & $\begin{array}{l}\text { énor- } \\
\text { mément } \\
+++\end{array}$ \\
\hline 1) of knowledge & $\mathrm{O}$ & $\mathrm{O}$ & $\mathrm{O}$ & $\mathrm{O}$ & $\mathrm{O}$ & $\mathrm{O}$ \\
\hline 1) des Wissens & $\mathrm{O}$ & $\mathrm{O}$ & $\mathrm{O}$ & $\mathrm{O}$ & $\mathrm{O}$ & $\mathrm{O}$ \\
\hline 1) del sapere & 0 & 0 & O & O & O & O \\
\hline 1) des connaissances & 0 & $\mathrm{O}$ & O & O & O & O \\
\hline 2) of learning steps/processes & $\mathrm{O}$ & $\mathrm{O}$ & $\mathrm{O}$ & $\mathrm{O}$ & $\mathrm{O}$ & $\mathrm{O}$ \\
\hline $\begin{array}{l}\text { 2) der Lernschritte/des Lernpro- } \\
\text { zesses }\end{array}$ & 0 & $\mathrm{O}$ & $\mathrm{O}$ & $\mathrm{O}$ & O & O \\
\hline $\begin{array}{l}\text { 2) dei fasi di apprendimento / del } \\
\text { processo }\end{array}$ & $\mathrm{O}$ & O & $\mathrm{O}$ & O & $\mathrm{O}$ & $\mathrm{O}$ \\
\hline 2) du processus d'apprentissage & $\mathrm{O}$ & $\mathrm{O}$ & $\mathrm{O}$ & $\mathrm{O}$ & $\mathrm{O}$ & $\mathrm{O}$ \\
\hline 3) of classification & $\mathrm{O}$ & $\mathrm{O}$ & $\mathrm{O}$ & $\mathrm{O}$ & $\mathrm{O}$ & $\mathrm{O}$ \\
\hline 3) der Klassifikation & $\mathrm{O}$ & $\mathrm{O}$ & $\mathrm{O}$ & $\mathrm{O}$ & $\mathrm{O}$ & $\mathrm{O}$ \\
\hline 3) della classificazione & $\mathrm{O}$ & $\mathrm{O}$ & $\mathrm{O}$ & $\mathrm{O}$ & $\mathrm{O}$ & $\mathrm{O}$ \\
\hline 3) de la classification & $\mathrm{O}$ & $\mathrm{O}$ & $\mathrm{O}$ & $\mathrm{O}$ & $\mathrm{O}$ & $\mathrm{O}$ \\
\hline $\begin{array}{l}\text { 4) of questions and assignments } \\
\text { to the learners }\end{array}$ & $\mathrm{O}$ & O & O & $\mathrm{O}$ & $\mathrm{O}$ & $\mathrm{O}$ \\
\hline $\begin{array}{l}\text { 4) der Fragen und Aufgaben für } \\
\text { die Lernenden }\end{array}$ & $\mathrm{O}$ & O & O & $\mathrm{O}$ & $\mathrm{O}$ & $\mathrm{O}$ \\
\hline $\begin{array}{l}\text { 4) delle domande e compiti per gli } \\
\text { studenti }\end{array}$ & $\mathrm{O}$ & O & O & $\mathrm{O}$ & $\mathrm{O}$ & $\mathrm{O}$ \\
\hline $\begin{array}{l}\text { 4) des questions et devoirs pour } \\
\text { les apprenants }\end{array}$ & $\mathrm{O}$ & O & O & $\mathrm{O}$ & $\mathrm{O}$ & $\mathrm{O}$ \\
\hline $\begin{array}{l}\text { 5) of aims and criteria for judg- } \\
\text { ments }\end{array}$ & $\mathrm{O}$ & O & O & $\mathrm{O}$ & $\mathrm{O}$ & $\mathrm{O}$ \\
\hline $\begin{array}{l}\text { 5) der Ziele und Beurteilungskri- } \\
\text { terien }\end{array}$ & $\mathrm{O}$ & O & $\mathrm{O}$ & O & $\mathrm{O}$ & $\mathrm{O}$ \\
\hline $\begin{array}{l}\text { 5) dello scopo e del parere degli } \\
\text { studenti }\end{array}$ & $\mathrm{O}$ & O & $\mathrm{O}$ & O & $\mathrm{O}$ & $\mathrm{O}$ \\
\hline $\begin{array}{l}\text { 5) des buts et des critères du } \\
\text { jugement }\end{array}$ & 0 & O & $\mathrm{O}$ & $\mathrm{O}$ & $\mathrm{O}$ & $\mathrm{O}$ \\
\hline $\begin{array}{l}\text { 6) of positions (authenticity, } \\
\text { teacher as model) }\end{array}$ & 0 & O & $\mathrm{O}$ & $\mathrm{O}$ & $\mathrm{O}$ & $\mathrm{O}$ \\
\hline $\begin{array}{l}\text { 6) der Positionen (Authentizität, } \\
\text { Lehrende als Modell) }\end{array}$ & 0 & O & $\mathrm{O}$ & $\mathrm{O}$ & $\mathrm{O}$ & 0 \\
\hline $\begin{array}{l}\text { 6) delle posizioni (autenticità, } \\
\text { l'insegnante come modello) }\end{array}$ & 0 & O & O & O & $\mathrm{O}$ & 0 \\
\hline \multirow[t]{2}{*}{$\begin{array}{l}\text { 6) des positions (authenticité, } \\
\text { enseignants comme modèles) }\end{array}$} & $\mathrm{O}$ & O & $\mathrm{O}$ & O & $\mathrm{O}$ & $\mathrm{O}$ \\
\hline & $\star 1$ & $\star 2$ & $\star 3$ & $\star 4$ & $\star 5$ & *6 \\
\hline
\end{tabular}


C2. Relevance and reference: imparting valid knowledge

C2. Relevanz und Bezug: gültiges Wissen verteilen

C2. Rilevanza ed attimenza: distribuizione del sapere

C2. Signification et référence:

\begin{tabular}{|c|c|c|c|c|c|c|}
\hline My/Our programme ... & $\begin{array}{l}\text { not at } \\
\text { all } \\
---\end{array}$ & $\begin{array}{l}\text { just a } \\
\text { little } \\
--\end{array}$ & $\begin{array}{l}\text { quite a } \\
\text { bit } \\
\text { - }\end{array}$ & $\begin{array}{l}\text { rather } \\
\text { much } \\
+\end{array}$ & $\begin{array}{l}\text { much } \\
++\end{array}$ & $\begin{array}{l}\text { very } \\
\text { much } \\
+++\end{array}$ \\
\hline Mein/Unser Programm ... & gar nicht & $\begin{array}{l}\text { ein biss- } \\
\text { chen } \\
\text {-- }\end{array}$ & $\begin{array}{l}\text { einiger- } \\
\text { maßen } \\
\text { - }\end{array}$ & $\begin{array}{l}\text { ziemlich } \\
+\end{array}$ & $\begin{array}{l}\text { viel } \\
++\end{array}$ & $\begin{array}{l}\text { sehr } \\
\text { viel } \\
+++\end{array}$ \\
\hline Il mio/Nostro programma ... & niente & un pò & $\begin{array}{l}\text { suffi- } \\
\text { ciente } \\
-\end{array}$ & $\begin{array}{l}\text { abba- } \\
\text { stanza } \\
+\end{array}$ & $\begin{array}{l}\text { molto } \\
++\end{array}$ & $\begin{array}{l}\text { tantis- } \\
\text { simo } \\
+++\end{array}$ \\
\hline Mon/Notre programme ... & $\begin{array}{l}\text { pas du } \\
\text { tout } \\
---\end{array}$ & un peu & $\begin{array}{l}\text { passa- } \\
\text { blement } \\
\text { - }\end{array}$ & $\begin{array}{l}\text { assez } \\
+\end{array}$ & beaucoup & $\begin{array}{l}\text { énor- } \\
\text { mément } \\
+++\end{array}$ \\
\hline 1) the subject/profession. & $\mathrm{O}$ & $\mathrm{O}$ & $\mathrm{O}$ & $\mathrm{O}$ & $\mathrm{O}$ & $\mathrm{O}$ \\
\hline 1) das Fach/den Beruf. & $\mathrm{O}$ & $\mathrm{O}$ & $\mathrm{O}$ & $\mathrm{O}$ & $\mathrm{O}$ & $\mathrm{O}$ \\
\hline 1) alla materia / alla professione & $\mathrm{O}$ & $\mathrm{O}$ & $\mathrm{O}$ & $\mathrm{O}$ & $\mathrm{O}$ & $\mathrm{O}$ \\
\hline 1) pour la matière et le métier. & $\mathrm{O}$ & $\mathrm{O}$ & $\mathrm{O}$ & $\mathrm{O}$ & $\mathrm{O}$ & $\mathrm{O}$ \\
\hline $\begin{array}{l}\text { 2) the research level (state of the } \\
\text { art). }\end{array}$ & $\mathrm{O}$ & $\mathrm{O}$ & $\mathrm{O}$ & O & O & O \\
\hline 2) den Forschungsstand. & $\mathrm{O}$ & $\mathrm{O}$ & $\mathrm{O}$ & $\mathrm{O}$ & $\mathrm{O}$ & $\mathrm{O}$ \\
\hline 2) à l'attuale scienza & $\mathrm{O}$ & $\mathrm{O}$ & $\mathrm{O}$ & $\mathrm{O}$ & $\mathrm{O}$ & $\mathrm{O}$ \\
\hline 2) le niveau des recherches. & $\mathrm{O}$ & $\mathrm{O}$ & $\mathrm{O}$ & $\mathrm{O}$ & $\mathrm{O}$ & $\mathrm{O}$ \\
\hline 3) the further learning process. & $\mathrm{O}$ & $\mathrm{O}$ & $\mathrm{O}$ & $\mathrm{O}$ & $\mathrm{O}$ & $\mathrm{O}$ \\
\hline 3) den weiteren Lernprozess. & $\mathrm{O}$ & $\mathrm{O}$ & $\mathrm{O}$ & $\mathrm{O}$ & $\mathrm{O}$ & $\mathrm{O}$ \\
\hline 3) al procedimento dell'insegnamento & $\mathrm{O}$ & $\mathrm{O}$ & $\mathrm{O}$ & $\mathrm{O}$ & $\mathrm{O}$ & $\mathrm{O}$ \\
\hline 3) le processus futur d'apprendre. & $\mathrm{O}$ & $\mathrm{O}$ & $\mathrm{O}$ & $\mathrm{O}$ & $\mathrm{O}$ & $\mathrm{O}$ \\
\hline 4) the forthcoming examinations. & $\mathrm{O}$ & $\mathrm{O}$ & $\mathrm{O}$ & $\mathrm{O}$ & $\mathrm{O}$ & $\mathrm{O}$ \\
\hline 4) die späteren Prüfungen. & $\mathrm{O}$ & $\mathrm{O}$ & $\mathrm{O}$ & $\mathrm{O}$ & $\mathrm{O}$ & $\mathrm{O}$ \\
\hline 4) ai prossimi esami & $\mathrm{O}$ & $\mathrm{O}$ & $\mathrm{O}$ & $\mathrm{O}$ & $\mathrm{O}$ & $\mathrm{O}$ \\
\hline 4) les examens ultérieurs. & $\mathrm{O}$ & $\mathrm{O}$ & $\mathrm{O}$ & $\mathrm{O}$ & $\mathrm{O}$ & $\mathrm{O}$ \\
\hline 5) the learners' situations & $\mathrm{O}$ & $\mathrm{O}$ & $\mathrm{O}$ & $\mathrm{O}$ & $\mathrm{O}$ & $\mathrm{O}$ \\
\hline 5) die Situation der Lernenden & $\mathrm{O}$ & $\mathrm{O}$ & $\mathrm{O}$ & $\mathrm{O}$ & $\mathrm{O}$ & $\mathrm{O}$ \\
\hline 5) alle situazione degli studenti & $\mathrm{O}$ & $\mathrm{O}$ & $\mathrm{O}$ & $\mathrm{O}$ & $\mathrm{O}$ & $\mathrm{O}$ \\
\hline 5) la situation des apprenants. & $\mathrm{O}$ & $\mathrm{O}$ & $\mathrm{O}$ & $\mathrm{O}$ & $\mathrm{O}$ & $\mathrm{O}$ \\
\hline 6) the learners' future. & $\mathrm{O}$ & $\mathrm{O}$ & $\mathrm{O}$ & $\mathrm{O}$ & $\mathrm{O}$ & $\mathrm{O}$ \\
\hline 6) die Zukunft der Lernenden. & $\mathrm{O}$ & $\mathrm{O}$ & $\mathrm{O}$ & $\mathrm{O}$ & $\mathrm{O}$ & $\mathrm{O}$ \\
\hline 6) al futuro degli studenti & $\mathrm{O}$ & $\mathrm{O}$ & $\mathrm{O}$ & $\mathrm{O}$ & $\mathrm{O}$ & $\mathrm{O}$ \\
\hline 6) l'avenir des apprenants. & $\mathrm{O}$ & $\mathrm{O}$ & $\mathrm{O}$ & $\mathrm{O}$ & $\mathrm{O}$ & $\mathrm{O}$ \\
\hline $\begin{array}{l}\text { 7) the society and subsystems (pro- } \\
\text { fessions e.g.). }\end{array}$ & $\mathrm{O}$ & $\mathrm{O}$ & $\mathrm{O}$ & 0 & O & $\mathrm{O}$ \\
\hline $\begin{array}{l}\text { 7) die Gesellschaft und ihre Subsys- } \\
\text { teme (Berufe etc.) }\end{array}$ & $\mathrm{O}$ & $\mathrm{O}$ & $\mathrm{O}$ & O & O & $\mathrm{O}$ \\
\hline 7) alle condizioni sociali & $\mathrm{O}$ & $\mathrm{O}$ & $\mathrm{O}$ & $\mathrm{O}$ & $\mathrm{O}$ & $\mathrm{O}$ \\
\hline \multirow[t]{2}{*}{$\begin{array}{l}\text { 7) la société et les sous-systèmes } \\
\text { (p.ex. professions). }\end{array}$} & $\mathrm{O}$ & $\mathrm{O}$ & $\mathrm{O}$ & $\mathrm{O}$ & O & O \\
\hline & $\star 1$ & *2 & *3 & $* 4$ & *5 & *6 \\
\hline
\end{tabular}


C3. Structure and sequential order: imparting knowledge "in measured doses"

C3. Struktur und Sequenz: Wissen in geordneten Maßen verteilen

C3. Struttura e sequenza: Distribuizione del sapere in modo ordinato

C3. Structure et séquence : distribuer les connaissances avec mesure

\begin{tabular}{|c|c|c|c|c|c|c|}
\hline My/Our programme ... & $\begin{array}{l}\text { not at } \\
\text { all } \\
--\end{array}$ & $\begin{array}{l}\text { just a } \\
\text { little } \\
--\end{array}$ & $\begin{array}{l}\text { quite a } \\
\text { bit } \\
\text { - }\end{array}$ & $\begin{array}{l}\text { rather } \\
\text { much } \\
+\end{array}$ & $\begin{array}{l}\text { much } \\
++\end{array}$ & $\begin{array}{l}\text { very } \\
\text { much } \\
+++\end{array}$ \\
\hline Mein/Unser Programm ... & $\begin{array}{l}\text { gar nicht } \\
-\end{array}$ & $\begin{array}{l}\text { ein biss- } \\
\text { chen } \\
--\end{array}$ & $\begin{array}{l}\text { einiger- } \\
\text { maßen } \\
-\end{array}$ & $\begin{array}{l}\text { ziemlich } \\
+\end{array}$ & $\begin{array}{l}\text { viel } \\
++\end{array}$ & $\begin{array}{l}\text { sehr } \\
\text { viel } \\
+++\end{array}$ \\
\hline Il mio/Nostro programma ... & niente & un pò & $\begin{array}{l}\text { suffi- } \\
\text { ciente } \\
-\end{array}$ & $\begin{array}{l}\text { abba- } \\
\text { stanza } \\
+\end{array}$ & $\begin{array}{l}\text { molto } \\
++\end{array}$ & $\begin{array}{l}\text { tantis- } \\
\text { simo } \\
+++\end{array}$ \\
\hline Mon/Notre programme ... & \begin{tabular}{|l} 
pas du \\
tout \\
---
\end{tabular} & un peu & $\begin{array}{l}\text { passa- } \\
\text { blement } \\
-\end{array}$ & $\begin{array}{l}\text { assez } \\
+ \\
\end{array}$ & $\begin{array}{l}\text { beaucoup } \\
++\end{array}$ & $\begin{array}{l}\text { énor- } \\
\text { mément } \\
+++\end{array}$ \\
\hline $\begin{array}{l}\text { 1) resumes what is already } \\
\text { known }\end{array}$ & O & O & O & $\mathrm{O}$ & $\mathrm{O}$ & $\mathrm{O}$ \\
\hline 1) greift Bekanntes wieder auf. & $\mathrm{O}$ & $\mathrm{O}$ & $\mathrm{O}$ & $\mathrm{O}$ & $\mathrm{O}$ & $\mathrm{O}$ \\
\hline $\begin{array}{l}\text { 1) riagrappa vecchie conoscen- } \\
\text { ze }\end{array}$ & $\mathrm{O}$ & O & $\mathrm{O}$ & $\mathrm{O}$ & $\mathrm{O}$ & $\mathrm{O}$ \\
\hline $\begin{array}{l}\text { 1) répète des sujets déjà con- } \\
\text { nus. }\end{array}$ & O & O & O & $\mathrm{O}$ & $\mathrm{O}$ & $\mathrm{O}$ \\
\hline $\begin{array}{l}\text { 2) gives accentuation of main } \\
\text { points }\end{array}$ & O & O & O & $\mathrm{O}$ & O & $\mathrm{O}$ \\
\hline 2) betont die Hauptpunkte. & $\mathrm{O}$ & $\mathrm{O}$ & $\mathrm{O}$ & $\mathrm{O}$ & $\mathrm{O}$ & $\mathrm{O}$ \\
\hline 2) sollecita i punti principali & $\mathrm{O}$ & $\mathrm{O}$ & $\mathrm{O}$ & $\mathrm{O}$ & $\mathrm{O}$ & $\mathrm{O}$ \\
\hline $\begin{array}{l}\text { 2) accentue les points les plus } \\
\text { importants. }\end{array}$ & O & O & $\mathrm{O}$ & O & $\mathrm{O}$ & $\mathrm{O}$ \\
\hline 3) gives time management. & $\mathrm{O}$ & $\mathrm{O}$ & $\mathrm{O}$ & $\mathrm{O}$ & $\mathrm{O}$ & $\mathrm{O}$ \\
\hline $\begin{array}{l}\text { 3) sorgt für gutes Zeitma- } \\
\text { nagement. }\end{array}$ & $\mathrm{O}$ & O & O & $\mathrm{O}$ & $\mathrm{O}$ & $\mathrm{O}$ \\
\hline $\begin{array}{l}\text { 3) fornisce una buona distrbui- } \\
\text { zione del tempo }\end{array}$ & $\mathrm{O}$ & O & O & $\mathrm{O}$ & $\mathrm{O}$ & $\mathrm{O}$ \\
\hline $\begin{array}{l}\text { 3) permet de biens manier } \\
\text { l'emploi du temps. }\end{array}$ & $\mathrm{O}$ & O & O & $\mathrm{O}$ & $\mathrm{O}$ & O \\
\hline $\begin{array}{l}\text { 4) gives space management } \\
\text { (learning environment) }\end{array}$ & $\mathrm{O}$ & O & O & $\mathrm{O}$ & O & $\mathrm{O}$ \\
\hline $\begin{array}{l}\text { 4) sorgt für gutes Raumma- } \\
\text { nagement (Lernumgebung). }\end{array}$ & 0 & O & 0 & $\mathrm{O}$ & $\mathrm{O}$ & $\mathrm{O}$ \\
\hline $\begin{array}{l}\text { 4) fornisce una buona distri- } \\
\text { buizione del luogo }\end{array}$ & $\mathrm{O}$ & O & O & $\mathrm{O}$ & O & $\mathrm{O}$ \\
\hline $\begin{array}{l}\text { 4) procure un environnement } \\
\text { agréable. }\end{array}$ & $\mathrm{O}$ & O & $\mathrm{O}$ & $\mathrm{O}$ & O & $\mathrm{O}$ \\
\hline 5) gives continuity & $\mathrm{O}$ & $\mathrm{O}$ & $\mathrm{O}$ & $\mathrm{O}$ & $\mathrm{O}$ & $\mathrm{O}$ \\
\hline 5) sorgt für Kontinuität. & $\mathrm{O}$ & $\mathrm{O}$ & $\mathrm{O}$ & $\mathrm{O}$ & $\mathrm{O}$ & $\mathrm{O}$ \\
\hline 5) fornisce la continuità & $\mathrm{O}$ & $\mathrm{O}$ & $\mathrm{O}$ & $\mathrm{O}$ & $\mathrm{O}$ & $\mathrm{O}$ \\
\hline \multirow[t]{2}{*}{ 5) fait gaffe à la continuité. } & 0 & 0 & 0 & 0 & 0 & 0 \\
\hline & $\star 1$ & $\star 2$ & $\star 3$ & $\star 4$ & $\star 5$ & *6 \\
\hline
\end{tabular}


C4. Representational forms of instruction: using aids for learning and thinking

C4. Anschaulichkeit: durch Lern- und Denkhilfen

C4. Panoramica dell' insegnamento e del pensiero

C4. Mettre en évidence: utiliser des aides pour apprendre

\begin{tabular}{|c|c|c|c|c|c|c|}
\hline $\begin{array}{l}\text { My/our programme is representational } \\
\text { through ... }\end{array}$ & $\begin{array}{l}\text { not at } \\
\text { all } \\
-\end{array}$ & $\begin{array}{l}\text { just a } \\
\text { little } \\
--\end{array}$ & $\begin{array}{l}\text { quite a } \\
\text { bit } \\
-\end{array}$ & $\begin{array}{l}\text { rather } \\
\text { much } \\
+\end{array}$ & $\begin{array}{l}\text { much } \\
++\end{array}$ & $\begin{array}{l}\text { very } \\
\text { much } \\
+++\end{array}$ \\
\hline $\begin{array}{l}\text { Mein/Unser Programm sorgt für Anschau- } \\
\text { lichkeit durch ... }\end{array}$ & gar nicht & $\begin{array}{l}\text { ein biss- } \\
\text { chen } \\
\text {-- }\end{array}$ & $\begin{array}{l}\text { einiger- } \\
\text { maßen } \\
\text { - }\end{array}$ & $\begin{array}{l}\text { ziemlich } \\
+\end{array}$ & $\begin{array}{l}\text { viel } \\
++\end{array}$ & $\begin{array}{l}\text { sehr } \\
\text { viel } \\
+++\end{array}$ \\
\hline $\begin{array}{l}\text { Il mio/Nostro programma fornisce una } \\
\text { panoramica per... }\end{array}$ & niente & un pò & $\begin{array}{l}\text { suffi- } \\
\text { ciente } \\
-\end{array}$ & $\begin{array}{l}\text { abba- } \\
\text { stanza } \\
+\end{array}$ & $\begin{array}{l}\text { molto } \\
++\end{array}$ & $\begin{array}{l}\text { tantis- } \\
\text { simo } \\
+++\end{array}$ \\
\hline $\begin{array}{l}\text { Mon/Notre programme mets en évidence } \\
\text { par... }\end{array}$ & $\begin{array}{l}\text { pas du } \\
\text { tout } \\
---\end{array}$ & un peu & $\begin{array}{l}\text { passa- } \\
\text { blement } \\
\text { - }\end{array}$ & $\begin{array}{l}\text { assez } \\
+ \\
\end{array}$ & $\begin{array}{l}\text { beaucoup } \\
++\end{array}$ & $\begin{array}{l}\text { énor- } \\
\text { mément } \\
+++\end{array}$ \\
\hline $\begin{array}{l}\text { 1) visualization (images, transparencies } \\
\text { e.g.). }\end{array}$ & $\mathrm{O}$ & $\mathrm{O}$ & $\mathrm{O}$ & $\mathrm{O}$ & $\mathrm{O}$ & $\mathrm{O}$ \\
\hline 1) Visualisierung (Bilder, Folien etc.). & $\mathrm{O}$ & $\mathrm{O}$ & $\mathrm{O}$ & $\mathrm{O}$ & $\mathrm{O}$ & $\mathrm{O}$ \\
\hline 1) la visualizzazione & $\mathrm{O}$ & $\mathrm{O}$ & $\mathrm{O}$ & $\mathrm{O}$ & $\mathrm{O}$ & $\mathrm{O}$ \\
\hline $\begin{array}{l}\text { 1) la visualisation (images, feuille transpa- } \\
\text { rente). }\end{array}$ & $\mathrm{O}$ & $\mathrm{O}$ & $\mathrm{O}$ & O & $\mathrm{O}$ & $\mathrm{O}$ \\
\hline 2) examples. & $\mathrm{O}$ & $\mathrm{O}$ & $\mathrm{O}$ & $\mathrm{O}$ & $\mathrm{O}$ & $\mathrm{O}$ \\
\hline 2) Beispiele. & $\mathrm{O}$ & $\mathrm{O}$ & $\mathrm{O}$ & $\mathrm{O}$ & $\mathrm{O}$ & $\mathrm{O}$ \\
\hline 2) gli esempi & $\mathrm{O}$ & $\mathrm{O}$ & $\mathrm{O}$ & $\mathrm{O}$ & $\mathrm{O}$ & $\mathrm{O}$ \\
\hline 2) des examples. & $\mathrm{O}$ & $\mathrm{O}$ & $\mathrm{O}$ & $\mathrm{O}$ & $\mathrm{O}$ & $\mathrm{O}$ \\
\hline 3) analogies. & $\mathrm{O}$ & $\mathrm{O}$ & $\mathrm{O}$ & $\mathrm{O}$ & $\mathrm{O}$ & $\mathrm{O}$ \\
\hline 3) Analogien. & $\mathrm{O}$ & $\mathrm{O}$ & $\mathrm{O}$ & $\mathrm{O}$ & $\mathrm{O}$ & $\mathrm{O}$ \\
\hline 3) le analogie & $\mathrm{O}$ & $\mathrm{O}$ & $\mathrm{O}$ & $\mathrm{O}$ & $\mathrm{O}$ & $\mathrm{O}$ \\
\hline 3) des analogies. & $\mathrm{O}$ & $\mathrm{O}$ & $\mathrm{O}$ & $\mathrm{O}$ & $\mathrm{O}$ & $\mathrm{O}$ \\
\hline 4) concrete experiences for learners. & $\mathrm{O}$ & $\mathrm{O}$ & $\mathrm{O}$ & $\mathrm{O}$ & $\mathrm{O}$ & $\mathrm{O}$ \\
\hline 4) direkte Erfahrungen der Lernenden. & $\mathrm{O}$ & $\mathrm{O}$ & $\mathrm{O}$ & $\mathrm{O}$ & $\mathrm{O}$ & $\mathrm{O}$ \\
\hline 4) le esperienze dirette degli studenti & $\mathrm{O}$ & $\mathrm{O}$ & $\mathrm{O}$ & $\mathrm{O}$ & $\mathrm{O}$ & $\mathrm{O}$ \\
\hline $\begin{array}{l}\text { 4) des expériences directes des appre- } \\
\text { nants. }\end{array}$ & $\mathrm{O}$ & $\mathrm{O}$ & $\mathrm{O}$ & $\mathrm{O}$ & O & $\mathrm{O}$ \\
\hline 5) reflection by the learners. & $\mathrm{O}$ & $\mathrm{O}$ & $\mathrm{O}$ & $\mathrm{O}$ & $\mathrm{O}$ & $\mathrm{O}$ \\
\hline $\begin{array}{l}\text { 5) Anstöße zum Nachdenken an die Ler- } \\
\text { nenden. }\end{array}$ & $\mathrm{O}$ & $\mathrm{O}$ & $\mathrm{O}$ & $\mathrm{O}$ & $\mathrm{O}$ & $\mathrm{O}$ \\
\hline 5) gli impulsi per pensare & $\mathrm{O}$ & $\mathrm{O}$ & $\mathrm{O}$ & $\mathrm{O}$ & $\mathrm{O}$ & $\mathrm{O}$ \\
\hline \multirow[t]{2}{*}{ 5) des initiatives à réflechir. } & $\mathrm{O}$ & $\mathrm{O}$ & $\mathrm{O}$ & $\mathrm{O}$ & $\mathrm{O}$ & $\mathrm{O}$ \\
\hline & $\star_{1}$ & $\star 2$ & $\star 3$ & $\star 4$ & $\star 5$ & *6 \\
\hline
\end{tabular}




\begin{tabular}{|c|c|c|c|c|c|c|}
\hline \multicolumn{7}{|l|}{ C5. Practising: application of knowledge } \\
\hline \multicolumn{7}{|l|}{ C5. Anwendung: } \\
\hline \multicolumn{7}{|l|}{ C5. Uso: } \\
\hline \multicolumn{7}{|c|}{ C5. application:utilisation des connaissances } \\
\hline My/our programme ... & $\begin{array}{l}\text { not at } \\
\text { all } \\
---\end{array}$ & $\begin{array}{l}\text { just a } \\
\text { little } \\
--\end{array}$ & $\begin{array}{l}\text { quite a } \\
\text { bit } \\
\text { - }\end{array}$ & $\begin{array}{l}\text { rather } \\
\text { much } \\
+\end{array}$ & $\begin{array}{l}\text { much } \\
++\end{array}$ & $\begin{array}{l}\text { very } \\
\text { much } \\
+++\end{array}$ \\
\hline Mein/Unser Programm ... & $\begin{array}{l}\text { gar } \\
\text { nicht } \\
---\end{array}$ & $\begin{array}{l}\text { ein } \\
\text { bisschen }\end{array}$ & $\begin{array}{l}\text { einiger- } \\
\text { maßen } \\
\text { - }\end{array}$ & $\begin{array}{l}\text { ziemlich } \\
+\end{array}$ & $\begin{array}{l}\text { viel } \\
++\end{array}$ & $\begin{array}{l}\text { sehr } \\
\text { viel } \\
+++\end{array}$ \\
\hline Il mio / nostro programma ... & $\begin{array}{l}\text { niente } \\
-\ldots\end{array}$ & un pò & $\begin{array}{l}\text { suffi- } \\
\text { ciente } \\
-\end{array}$ & $\begin{array}{l}\text { abbas- } \\
\text { tanza } \\
+\end{array}$ & $\begin{array}{l}\text { molto } \\
+\end{array}$ & $\begin{array}{l}\text { tantis- } \\
\text { simo } \\
+++\end{array}$ \\
\hline Mon/notre programme ... & $\begin{array}{l}\text { pas du } \\
\text { tout } \\
---\end{array}$ & un peu & $\begin{array}{l}\text { passab- } \\
\text { lement } \\
\text { - }\end{array}$ & $\begin{array}{l}\text { assez } \\
+\end{array}$ & $\begin{array}{l}\text { beau- } \\
\text { coup } \\
++\end{array}$ & $\begin{array}{l}\text { énormé } \\
\text { ment } \\
+++\end{array}$ \\
\hline 1) is proving knowledge in practice & $\mathrm{O}$ & $\mathrm{O}$ & $\mathrm{O}$ & $\mathrm{O}$ & $\mathrm{O}$ & $\mathrm{O}$ \\
\hline $\begin{array}{l}\text { 1) bietet Möglichkeiten der Überprü- } \\
\text { fung des Wissens in der Praxis. }\end{array}$ & $\mathrm{O}$ & O & $\mathrm{O}$ & $\mathrm{O}$ & $\mathrm{O}$ & O \\
\hline $\begin{array}{l}\text { 1) offre possibiltà di controllo } \\
\text { dell'insegnamento /sapere in pratica. }\end{array}$ & $\mathrm{O}$ & O & $\mathrm{O}$ & O & $\mathrm{O}$ & $\mathrm{O}$ \\
\hline $\begin{array}{l}\text { 1) rends possible la contrôle des con- } \\
\text { naissances en pratique. }\end{array}$ & $\mathrm{O}$ & 0 & $\mathrm{O}$ & $\mathrm{O}$ & $\mathrm{O}$ & $\mathrm{O}$ \\
\hline 2) has well defined assignments & $\mathrm{O}$ & $\mathrm{O}$ & $\mathrm{O}$ & $\mathrm{O}$ & $\mathrm{O}$ & $\mathrm{O}$ \\
\hline $\begin{array}{l}\text { 2) hat gut definierte Aufgabenstellun- } \\
\text { gen. }\end{array}$ & 0 & $\mathrm{O}$ & $\mathrm{O}$ & $\mathrm{O}$ & $\mathrm{O}$ & $\mathrm{O}$ \\
\hline 2) ha ben definiti compiti. & O & $\mathrm{O}$ & $\mathrm{O}$ & $\mathrm{O}$ & $\mathrm{O}$ & $\mathrm{O}$ \\
\hline 2) donne des devoirs biens définis. & $\mathrm{O}$ & $\mathrm{O}$ & $\mathrm{O}$ & $\mathrm{O}$ & $\mathrm{O}$ & $\mathrm{O}$ \\
\hline 3) has opportunities to train. & $\mathrm{O}$ & $\mathrm{O}$ & $\mathrm{O}$ & $\mathrm{O}$ & $\mathrm{O}$ & $\mathrm{O}$ \\
\hline 3) bietet Möglichkeiten des Übens. & $\mathrm{O}$ & $\mathrm{O}$ & $\mathrm{O}$ & $\mathrm{O}$ & $\mathrm{O}$ & $\mathrm{O}$ \\
\hline 3) offre possibilità di allenamento. & $\mathrm{O}$ & $\mathrm{O}$ & $\mathrm{O}$ & $\mathrm{O}$ & $\mathrm{O}$ & $\mathrm{O}$ \\
\hline 3) offre la possibilité d'entraînement, & 0 & $\mathrm{O}$ & $\mathrm{O}$ & $\mathrm{O}$ & $\mathrm{O}$ & $\mathrm{O}$ \\
\hline $\begin{array}{l}\text { 4) offers insight into the practical } \\
\text { application and usefulness }\end{array}$ & 0 & O & $\mathrm{O}$ & $\mathrm{O}$ & $\mathrm{O}$ & $\mathrm{O}$ \\
\hline $\begin{array}{l}\text { 4) gibt Einblicke in die praktische An- } \\
\text { wendung und Nützlichkeit. }\end{array}$ & 0 & $\mathrm{O}$ & $\mathrm{O}$ & $\mathrm{O}$ & $\mathrm{O}$ & $\mathrm{O}$ \\
\hline $\begin{array}{l}\text { 4) da possibilità di come usare nella } \\
\text { pratica. }\end{array}$ & 0 & 0 & 0 & 0 & 0 & 0 \\
\hline $\begin{array}{l}\text { 4) permet de vérifier l'application en } \\
\text { pratique et la valeur utile. }\end{array}$ & 0 & $\mathrm{O}$ & 0 & 0 & 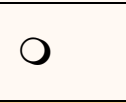 & 0 \\
\hline & ${ }^{* 1}$ & *2 & $\star 3$ & $\star 4$ & *5 & *6 \\
\hline
\end{tabular}


C6. Feedback: information about the learning process and one's personal status

C6. Rückmeldung: Informationen zum Lernprozess, zur Entwicklung und zum Ertrag

C6. Feedback: Informazioni sul processo d'insegnamento, dello sviluppo e del risultato

C6. Rétroaction: information sur le success dur processus $d^{*}$ apprendre

\begin{tabular}{|c|c|c|c|c|c|c|}
\hline $\begin{array}{l}\text { My/Our programme gives feedback } \\
\ldots\end{array}$ & $\begin{array}{l}\text { not at } \\
\text { all }\end{array}$ & $\begin{array}{l}\text { just a } \\
\text { little } \\
--\end{array}$ & $\begin{array}{l}\text { quite a } \\
\text { bit } \\
\text { - }\end{array}$ & $\begin{array}{l}\text { rather } \\
\text { much } \\
+\end{array}$ & $\begin{array}{l}\text { much } \\
++\end{array}$ & $\begin{array}{l}\text { very } \\
\text { muc } \\
h \\
+++\end{array}$ \\
\hline $\begin{array}{l}\text { Mein/Unser Programm bietet Rück- } \\
\text { meldung ... }\end{array}$ & gar nicht & $\begin{array}{l}\text { ein biss- } \\
\text { chen } \\
\text {-- }\end{array}$ & $\begin{array}{l}\text { einiger- } \\
\text { maßen } \\
-\end{array}$ & $\begin{array}{l}\text { ziemlich } \\
+\end{array}$ & $\begin{array}{l}\text { viel } \\
++\end{array}$ & $\begin{array}{l}\text { sehr } \\
\text { viel } \\
+++\end{array}$ \\
\hline $\begin{array}{l}\text { Il mio/Nostro programma offre } \\
\text { feedback... }\end{array}$ & niente & $\begin{array}{l}\text { un pò } \\
--\end{array}$ & $\begin{array}{l}\text { suffi- } \\
\text { ciente } \\
-\end{array}$ & $\begin{array}{l}\text { abba- } \\
\text { stanza } \\
+\end{array}$ & $\begin{array}{l}\text { molto } \\
++\end{array}$ & $\begin{array}{l}\text { tan- } \\
\text { tis- } \\
\text { simo } \\
+++\end{array}$ \\
\hline Mon/Notre programme répond ... & $\begin{array}{l}\text { pas du } \\
\text { tout } \\
---\end{array}$ & un peu & $\begin{array}{l}\text { passa- } \\
\text { blement } \\
\text { - }\end{array}$ & $\begin{array}{l}\text { assez } \\
+\end{array}$ & $\begin{array}{l}\text { beaucoup } \\
++\end{array}$ & $\begin{array}{l}\text { énor } \\
\text { mé- } \\
\text { ment } \\
+++\end{array}$ \\
\hline 1) through praise and reproach & $\mathrm{O}$ & $\mathrm{O}$ & $\mathrm{O}$ & $\mathrm{O}$ & $\mathrm{O}$ & $\mathrm{O}$ \\
\hline 1) durch Lob und Tadel. & $\mathrm{O}$ & $\mathrm{O}$ & $\mathrm{O}$ & $\mathrm{O}$ & $\mathrm{O}$ & $\mathrm{O}$ \\
\hline 1) per elogi e rimproveri. & $\mathrm{O}$ & $\mathrm{O}$ & $\mathrm{O}$ & $\mathrm{O}$ & $\mathrm{O}$ & $\mathrm{O}$ \\
\hline 1) par louage et critique. & $\mathrm{O}$ & $\mathrm{O}$ & $\mathrm{O}$ & $\mathrm{O}$ & $\mathrm{O}$ & $\mathrm{O}$ \\
\hline 2) by products & $\mathrm{O}$ & $\mathrm{O}$ & $\mathrm{O}$ & $\mathrm{O}$ & $\mathrm{O}$ & $\mathrm{O}$ \\
\hline 2) durch Produkte. & $\mathrm{O}$ & $\mathrm{O}$ & $\mathrm{O}$ & $\mathrm{O}$ & $\mathrm{O}$ & $\mathrm{O}$ \\
\hline 2) per i prodotti. & $\mathrm{O}$ & $\mathrm{O}$ & $\mathrm{O}$ & $\mathrm{O}$ & $\mathrm{O}$ & $\mathrm{O}$ \\
\hline 2) par des résultats. & $\mathrm{O}$ & $\mathrm{O}$ & $\mathrm{O}$ & $\mathrm{O}$ & $\mathrm{O}$ & $\mathrm{O}$ \\
\hline 3) by certifications & $\mathrm{O}$ & $\mathrm{O}$ & $\mathrm{O}$ & $\mathrm{O}$ & $\mathrm{O}$ & $\mathrm{O}$ \\
\hline 3) durch Zertifikate/Bescheinigungen. & $\mathrm{O}$ & $\mathrm{O}$ & $\mathrm{O}$ & $\mathrm{O}$ & $\mathrm{O}$ & $\mathrm{O}$ \\
\hline 3) per i certificati. & $\mathrm{O}$ & $\mathrm{O}$ & $\mathrm{O}$ & $\mathrm{O}$ & $\mathrm{O}$ & $\mathrm{O}$ \\
\hline 3) par des certificats. & $\mathrm{O}$ & $\mathrm{O}$ & $\mathrm{O}$ & $\mathrm{O}$ & $\mathrm{O}$ & $\mathrm{O}$ \\
\hline $\begin{array}{l}\text { 4) by the natural environment ("natura } \\
\text { docet") }\end{array}$ & $\mathrm{O}$ & O & O & O & O & $\mathrm{O}$ \\
\hline $\begin{array}{l}\text { 4) durch die natürliche Umgebung ("natu- } \\
\text { ra docet"). }\end{array}$ & $\mathrm{O}$ & O & O & O & $\mathrm{O}$ & $\mathrm{O}$ \\
\hline $\begin{array}{l}\text { 4) per l'ambiente naturale ("natura do- } \\
\text { cet"). }\end{array}$ & $\mathrm{O}$ & O & $\mathrm{O}$ & O & $\mathrm{O}$ & $\mathrm{O}$ \\
\hline \multirow[t]{2}{*}{$\begin{array}{l}\text { 4) par l'environnement naturel ("natura } \\
\text { docet"). }\end{array}$} & O & O & $\mathrm{O}$ & O & $\mathrm{O}$ & $\mathrm{O}$ \\
\hline & *1 & *2 & $\star 3$ & $\star 4$ & $\star 5$ & *6 \\
\hline
\end{tabular}


C7. Further stimulus: perspectives for more than mere knowledge and a more farreaching learning

C7. Weitere Anreize: Gesichtspunkte, die über das unmittelbare Wissen und Lernen hinausgehen

C7. Altri impulse: Aspetti che vanno oltre all'insegnamento

C7. Incitation des idées en plus

\begin{tabular}{|c|c|c|c|c|c|c|}
\hline $\begin{array}{l}\text { My/Our programme gives stim- } \\
\text { ulus ... }\end{array}$ & $\begin{array}{l}\text { not at } \\
\text { all } \\
---\end{array}$ & $\begin{array}{l}\text { just a } \\
\text { little } \\
--\end{array}$ & $\begin{array}{l}\text { quite a } \\
\text { bit } \\
-\end{array}$ & $\begin{array}{l}\text { rather } \\
\text { much } \\
+\end{array}$ & $\begin{array}{l}\text { much } \\
++\end{array}$ & $\begin{array}{l}\text { very } \\
\text { muc } \\
h \\
+++\end{array}$ \\
\hline $\begin{array}{l}\text { Mein/Unser Programm gibt zu- } \\
\text { sätzliche Anregungen .... }\end{array}$ & gar nicht & $\begin{array}{l}\text { ein biss- } \\
\text { chen } \\
--\end{array}$ & $\begin{array}{l}\text { einiger- } \\
\text { maßen } \\
-\end{array}$ & $\begin{array}{l}\text { ziemlich } \\
+\end{array}$ & $\begin{array}{l}\text { viel } \\
++\end{array}$ & $\begin{array}{l}\text { sehr } \\
\text { viel } \\
+++\end{array}$ \\
\hline $\begin{array}{l}\text { Il mio/Nostro programma da } \\
\text { ulteriori impulse... }\end{array}$ & niente & un pò & $\begin{array}{l}\text { suffi- } \\
\text { ciente } \\
-\end{array}$ & $\begin{array}{l}\text { abba- } \\
\text { stanza } \\
+\end{array}$ & $\begin{array}{l}\text { molto } \\
++\end{array}$ & $\begin{array}{l}\text { tan- } \\
\text { tis- } \\
\text { simo } \\
+++\end{array}$ \\
\hline $\begin{array}{l}\text { Mon/Notre programme incite } \\
\text { des idées en plus ... }\end{array}$ & $\begin{array}{l}\text { pas du } \\
\text { tout } \\
---\end{array}$ & un peu & $\begin{array}{l}\text { passa- } \\
\text { blement } \\
\text { - }\end{array}$ & $\begin{array}{l}\text { assez } \\
+\end{array}$ & $\begin{array}{l}\text { beaucoup } \\
++\end{array}$ & $\begin{array}{l}\text { énor } \\
\text { mé- } \\
\text { ment } \\
+++\end{array}$ \\
\hline $\begin{array}{l}\text { 1) by consideration of learners' } \\
\text { interests and needs }\end{array}$ & O & O & $\mathrm{O}$ & $\mathrm{O}$ & $\mathrm{O}$ & $\mathrm{O}$ \\
\hline $\begin{array}{l}\text { 1) durch Berücksichtigung der In- } \\
\text { teressen und Bedürfnisse der Ler- } \\
\text { nenden. }\end{array}$ & $\mathrm{O}$ & $\mathrm{O}$ & O & $\mathrm{O}$ & O & $\mathrm{O}$ \\
\hline $\begin{array}{l}\text { 1) considerando gli interessi e bi- } \\
\text { sogni degli studenti. }\end{array}$ & $\mathrm{O}$ & $\mathrm{O}$ & $\mathrm{O}$ & $\mathrm{O}$ & $\mathrm{O}$ & $\mathrm{O}$ \\
\hline $\begin{array}{l}\text { 1) en tenant compte des intérêts } \\
\text { et des besoins des apprenants. }\end{array}$ & $\mathrm{O}$ & $\mathrm{O}$ & $\mathrm{O}$ & $\mathrm{O}$ & $\mathrm{O}$ & $\mathrm{O}$ \\
\hline 2) by teachers' enthusiasm. & $\mathrm{O}$ & $\mathrm{O}$ & $\mathrm{O}$ & $\mathrm{O}$ & $\mathrm{O}$ & $\mathrm{O}$ \\
\hline $\begin{array}{l}\text { 2) durch Begeisterung der Lehren- } \\
\text { den. }\end{array}$ & $\mathrm{O}$ & $\mathrm{O}$ & $\mathrm{O}$ & $\mathrm{O}$ & $\mathrm{O}$ & $\mathrm{O}$ \\
\hline 2) per l'euforia degli insegnanti. & $\mathrm{O}$ & $\mathrm{O}$ & $\mathrm{O}$ & $\mathrm{O}$ & $\mathrm{O}$ & $\mathrm{O}$ \\
\hline $\begin{array}{l}\text { 2) par l'enthusiasme des einsei- } \\
\text { gnants. }\end{array}$ & $\mathrm{O}$ & $\mathrm{O}$ & $\mathrm{O}$ & $\mathrm{O}$ & $\mathrm{O}$ & $\mathrm{O}$ \\
\hline $\begin{array}{l}\text { 3) with a certain irony and self- } \\
\text { criticism }\end{array}$ & $\mathrm{O}$ & $\mathrm{O}$ & $\mathrm{O}$ & $\mathrm{O}$ & $\mathrm{O}$ & $\mathrm{O}$ \\
\hline $\begin{array}{l}\text { 3) durch feine Ironie und Selbst- } \\
\text { kritik. }\end{array}$ & $\mathrm{O}$ & $\mathrm{O}$ & $\mathrm{O}$ & $\mathrm{O}$ & $\mathrm{O}$ & $\mathrm{O}$ \\
\hline 3) per sottile ironia e auto-critica. & $\mathrm{O}$ & $\mathrm{O}$ & $\mathrm{O}$ & $\mathrm{O}$ & $\mathrm{O}$ & $\mathrm{O}$ \\
\hline \multirow[t]{2}{*}{$\begin{array}{l}\text { 3) par ironie fine et par autocri- } \\
\text { tique. }\end{array}$} & O & $\mathrm{O}$ & $\mathrm{O}$ & $\mathrm{O}$ & $\mathrm{O}$ & $\mathrm{O}$ \\
\hline & *1 & $\star 2$ & *3 & *4 & *5 & *6 \\
\hline
\end{tabular}




\section{Inventar für Entscheidungsträger}

\section{Inventory for decision makers}

Part 1: Preparing a decision on the adoption of Blended-Learning

Inventar für Entscheidungsträger

Teil 1: Vorbereitung einer Entscheidung zur Einrichtung einer Blended-Learning

Maßnahme

This is a first proposal for the key questions for decision makers before deciding upon the invention of Blended-Learning Systems or e-earning modules or deciding for a traditional course system:

This version is for trial; persons who are possible decision-makers on the adoption of Blended-Learning courses or e-Learning systems or who have already decided, are asked to fill out the inventory with a practical project in their mind and then give us remarks on the usefulness and comprehensibility of items.

Dieses ist ein erster Vorschlag für Schlüsselfragen für Personen, die vor einer Entscheidung für eine Blended-Learning Maßnahme oder ein traditionelles Kurssystem stehen.

Diese Version ist noch eine Probefassung. Personen, die mögliche Entscheidungsträger hinsichtlich der Annahme einer Blended-Learning oder e-Learning Maßnahme sind oder vielleicht eine solche Entscheidung getroffen haben, werden gebeten, das Inventar unter dem Gesichtspunkt eines praktischen Projekts auszufüllen und jeweils im Hinblick auf die Nützlichkeit und Verständlichkeit der Fragen zu kommentieren. 
Fragenbereiche:

1. Description of the entity: Status quo

(What is given?)

1. Beschreibung der Situation: Status quo

2. Intention of the management

(Why do you want to introduce BL or e-Learning?)

2. Ziele und Absichten

(Warum sollte Blended-Learning oder e_Learning eingeführt werden?)

3. Description of the learners

(Who shall learn?)

3. Beschreibung der Lerner

(Wer soll lernen?)

4. Description of the learning environment

(Where shall they learn?)

4. Beschreibung der Lernumgebung

(Wo sollen sie lernen?)

6. Description of the contents

(What shall they learn?)

6. Beschreibung der Inhalte

(Was sollen sie lernen?)

7. Expected outcome

7. Erwartete Ergebnisse

8. Organisational development and integration

8. Organisationsentwicklung und Integration 
1. Description of the entity: Status quo

(What is given?)

\section{Beschreibung der Situation: Status quo}

Coordinates of the enterprise (location)

1a) The buildings where learners stay are ..

\begin{tabular}{l|l|l} 
O centralized & $\begin{array}{l}\text { O mainly } \\
\text { centralized }\end{array}$ & $\begin{array}{l}\text { O decentral- } \\
\text { ized }\end{array}$ \\
\hline
\end{tabular}

Koordinaten der Einrichtung/des Unternehmens:

1a) Die Lerner befinden sich in Gebäuden, die ...

O zentral liegen
$O$ einigermaßen zentral liegen
O dezentral liegen

\section{Economic sector (business area)}

1b) Diversity of business areas/divisions where learners come from is... high

1c) Which are the business areas/divisions where learners come from? (Please list them)

1d) Number of learners?

1e) Number of cyles?

\section{Arbeits- oder Geschäftsbereiche:}

1b) Die Diversität der Arbeits-oder Geschäftsbereiche, aus denen die Lerner kommen ist...

1c) Welches sind die Arbeits- oder Geschäftsbereiche, aus denen die Lerner kommen? (Bitte auflisten)

1d) Anzahl der Lerner?

1e) Anzahl der Zyklen (Kurswiederholungen)?

Hierarchy (management, workers etc.)

1f) Diversity of learners' status is ...

$1 \mathrm{~g})$ Clustering of learners (working together in
groups) is

O very high

\begin{tabular}{|l|l|}
\hline $\begin{array}{l}\text { O rather } \\
\text { high }\end{array}$ & O low \\
\hline $\begin{array}{l}\text { O rather } \\
\text { high }\end{array}$ & O low \\
\hline
\end{tabular}

Hierarchie (Management, Arbeiter etc.):

1f) Der Status der Lerner in der Hierarchie ist ...

o sehr hoch 
1g) Die Lerner stammen aus eng zusammenar-

O sehr hoch

O einigermaßen hoch

gering

beitenden Gruppen...

Languages (European, international enterprise)

1h) Which languages do the learners need to speak in your organisation/institution?

1i) Which languages do the learners need to speak during their working time (with clients)?

Sprachen, Internationalität des Unternehmens:

\begin{tabular}{l|l|l|l|}
$\begin{array}{l}\text { 1h) Welche Sprache(n) sprechen die Lerner in } \\
\text { Ihrem Unternehmen/Ihrer Einrichtung? }\end{array}$ & $\begin{array}{l}\text { O eine Sprache } \\
\text { (ihre Mutters- } \\
\text { prache) }\end{array}$ & $\begin{array}{l}\text { O zwei } \\
\text { Sprachen }\end{array}$ & $\begin{array}{l}\text { O mehr } \\
\text { als } 2 \\
\text { Sprachen }\end{array}$ \\
\hline $\begin{array}{l}\text { 1i) Welche Sprache(n) sprechen die Lerner in } \\
\text { der Arbeitszeit (z.B. mit Klienten)? }\end{array}$ & $\begin{array}{l}\text { O eine Sprache } \\
\text { (ihre Mutters- } \\
\text { prache) }\end{array}$ & $\begin{array}{l}\text { O zwei } \\
\text { Sprachen }\end{array}$ & $\begin{array}{l}\text { O mehr } \\
\text { als } 2 \\
\text { Sprachen }\end{array}$ \\
\hline
\end{tabular}

x) Comments on usefulness and comprehensibility of items:

x) Kommentar zur Nützlichkeit und Verständlichkeit der Fragen: 


\section{Intention of the management \\ (Why do you want to introduce BL or e-Learning?) \\ 2. Ziele und Absichten \\ (Warum sollte Blended-Learning oder e_Learning eingeführt werden?)}

\begin{tabular}{|c|c|c|c|}
\hline \multicolumn{4}{|l|}{ Economic reasons: } \\
\hline $\begin{array}{l}\text { 2a) Reducing costs for educa- } \\
\text { tion/training activities }\end{array}$ & $\begin{array}{l}\text { O very im- } \\
\text { portant }\end{array}$ & O rather important & O not important \\
\hline $\begin{array}{l}\text { 2b) Reducing costs for ab- } \\
\text { sence of learners from work- } \\
\text { place }\end{array}$ & $\begin{array}{l}\text { O very im- } \\
\text { portant }\end{array}$ & O rather important & O not important \\
\hline \multicolumn{4}{|l|}{ Ökonomische Gründe: } \\
\hline $\begin{array}{l}\text { 2a) Verringerung der Ausgaben } \\
\text { für Bildung/Weiterbildung }\end{array}$ & O sehr wichtig & $\begin{array}{c}\text { O einigermaßen } \\
\text { wichtig }\end{array}$ & O nicht wichtig \\
\hline $\begin{array}{l}\text { 2b) Verringerung der Ausfall- } \\
\text { zeiten für Bildungs- } \\
\text { /Weiterbildungsmaßnahmen } \\
\text { (Lerner abwesend vom Ar- } \\
\text { beitsplatz) }\end{array}$ & O sehr wichtig & $\begin{array}{l}\text { O einigermaßen } \\
\text { wichtig }\end{array}$ & O nicht wichtig \\
\hline
\end{tabular}

\begin{tabular}{|l|c|c|c|}
\hline $\begin{array}{l}\text { 2c) Having quicker the bene- } \\
\text { fits of education/training (sav- } \\
\text { ing of learning time) }\end{array}$ & $\begin{array}{c}\text { O very im- } \\
\text { portant }\end{array}$ & O rather important & O not important \\
\hline $\begin{array}{l}\text { 2c) Schnellere Ergebnisse der } \\
\text { Bildungs- } \\
\text { Weiterbildungsmaßnahmen }\end{array}$ & O sehr wichtig & $\begin{array}{c}\text { O einigermaßen } \\
\text { wichtig }\end{array}$ & O nicht wichtig \\
\hline
\end{tabular}

\begin{tabular}{|l|c|c|c|}
\hline $\begin{array}{l}\text { 2d) Improving the quality (re- } \\
\text { sults) of learning } \\
\text { e) Improving the learning envi- } \\
\text { ronment }\end{array}$ & $\begin{array}{c}\text { O very im- } \\
\text { portant }\end{array}$ & O rather important & O not important \\
\hline $\begin{array}{l}\text { 2d) Verbesserung der Lerner- } \\
\text { gebnisse }\end{array}$ & O sehr wichtig & $\begin{array}{c}\text { O einigermaßen } \\
\text { wichtig }\end{array}$ & O nicht wichtig \\
\hline
\end{tabular}

\begin{tabular}{|l|l|c|c|}
\hline $\begin{array}{l}2 e) \text { Improving the learning } \\
\text { environment }\end{array}$ & $\begin{array}{l}\text { O very im- } \\
\text { portant }\end{array}$ & O rather important & O not important \\
\hline $\begin{array}{l}2 e) \text { Verbesserung der Lernum- } \\
\text { gebung (Lernatmospäre) }\end{array}$ & O sehr wichtig & $\begin{array}{l}\text { O einigermaßen } \\
\text { wichtig }\end{array}$ & O nicht wichtig \\
\hline
\end{tabular}




\begin{tabular}{|l|c|c|c|}
\hline $\begin{array}{l}\text { 2f) Standardized training re- } \\
\text { sults (comparability) }\end{array}$ & $\begin{array}{c}\text { O very im- } \\
\text { portant }\end{array}$ & O rather important & O not important \\
\hline $\begin{array}{l}\text { 2f) Standardisierung der Bil- } \\
\text { dung/Weiterbildung (Ver- } \\
\text { gleichbarkeit) }\end{array}$ & O sehr wichtig & $\begin{array}{c}\text { O einigermaßen } \\
\text { wichtig }\end{array}$ & O nicht wichtig \\
\hline
\end{tabular}

\begin{tabular}{|l|c|c|c|}
\hline $\begin{array}{l}\text { 2g) Different working places } \\
\text { (ODL) and improvement of } \\
\text { communication }\end{array}$ & $\begin{array}{c}\text { O very im- } \\
\text { portant }\end{array}$ & O rather important & O not important \\
\hline $\begin{array}{l}2 g) \text { Verschiedene Arbeitsplät- } \\
\text { ze und Verbesserung der } \\
\text { Kommunikation }\end{array}$ & O sehr wichtig & $\begin{array}{c}\text { O einigermaßen } \\
\text { wichtig }\end{array}$ & O nicht wichtig \\
\hline
\end{tabular}

\begin{tabular}{|l|c|c|c|}
\hline 2h) Customer care & $\begin{array}{c}\text { O very im- } \\
\text { portant }\end{array}$ & O rather important & O not important \\
\hline $\begin{array}{l}\text { 2h) Versorgung der Kund- } \\
\text { schaft }\end{array}$ & O sehr wichtig & $\begin{array}{c}\text { O einigermaßen } \\
\text { wichtig }\end{array}$ & O nicht wichtig \\
\hline
\end{tabular}

x) Comments on usefulness and comprehensibility of items:

x) Kommentar zur Nützlichkeit und Verständlichkeit der Fragen: 
3. Description of the learners

(Who shall learn?)

\section{Beschreibung der Lerner}

(Wer soll lernen?)

\section{Educational level}

\begin{tabular}{|c|c|c|c|}
\hline $\begin{array}{l}\text { 3a) What kind of educational } \\
\text { level do the learners have? }\end{array}$ & $\begin{array}{c}\text { O very homoge- } \\
\text { neous }\end{array}$ & $\begin{array}{c}\text { O fairly homogene- } \\
\text { ous }\end{array}$ & $\begin{array}{c}\text { O heterogene- } \\
\text { ous }\end{array}$ \\
\hline \multicolumn{4}{|c|}{ Bildung- und Qualifikationsniveau: } \\
\hline $\begin{array}{l}\text { 3a) Wie ist das Qualifikati- } \\
\text { onsniveau der Lerner? }\end{array}$ & O sehr homogen & $\begin{array}{c}\text { O einigermaßen ho- } \\
\text { mogen }\end{array}$ & O heterogen \\
\hline
\end{tabular}

\section{Age}

3b) The age of the learners is...

\section{$O$ very homoge-} neous

Alter

3b) Das Alter der Lerner ist
O sehr homogen heterogeneous ...

fairly homogeneous

\section{Sex}

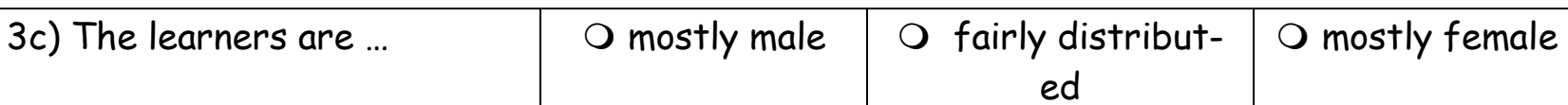
Geschlecht

\begin{tabular}{|l|c|c|c|}
\hline 3c) Die Lerner sind ... & $\begin{array}{c}\text { O überwiegend } \\
\text { männlich }\end{array}$ & O gemischt & $\begin{array}{c}\text { O überwiegend } \\
\text { weiblich }\end{array}$ \\
\hline
\end{tabular}

\section{Working and learning time}

3d) The working and learning times in my organisation are...

Arbeits - und Lernzeit:

3d) Arbeits- und Lernzeit sind
O strictly sepa- O sometimes mixed rated

O not separated ...
O sehr stark getrennt

\section{O einigermaßen} getrennt

O nicht getrennt

\section{Motivation der Lerner (e.g.incentives)}

$3 e)$ The motivation to learn is

o high

O average

O low

\section{Motivation der Lerner (z.B. Belohnungen)}

3e) Die Motivation zum Ler-

O hoch

O durchschnittlich

gering nen ist ... ...

\section{Perspectives of the learners (job security)}

\begin{tabular}{l|l} 
3f) The job security is ... & O high
\end{tabular}

Perspektiven der Lerner:

3f) Die Sicherheit der Ar-

beitsplätze ist ...

Other target groups (e.g. clients) 


\begin{tabular}{|l|l|l|l|}
\hline $\begin{array}{l}\text { 3g) How many other target } \\
\text { groups do you have for e- } \\
\text { learning or blended-learning } \\
\text { approaches? }\end{array}$ & O a lot & O some & O few \\
\hline Weitere Zielgruppen (z.B. bei Geschäftspartnern) & O wenige/keine \\
\hline $\begin{array}{l}\text { 3g) Wie viele andere Ziel- } \\
\text { gruppen gibt es, die möglich- } \\
\text { erweise auch auf Blended- } \\
\text { Learning, e-Learning umstellen } \\
\text { würden? }\end{array}$ & O viele & & \\
\hline
\end{tabular}

Learners' disposition (ability of self-learning)

\begin{tabular}{l|l|c|c|}
\hline $\begin{array}{l}\text { 3h) The ability of the learn- } \\
\text { ers } \\
\text { in self-learning is ... }\end{array}$ & O high & O average \\
\hline $\begin{array}{l}\text { Dispositionen der Lerner: } \\
\text { 3h)Die Fähigkeit der Lerner } \\
\text { zum Selbsttätigen Lernen ist } \\
\ldots\end{array}$ & O hoch & O durchschnittlich & O gering \\
\hline
\end{tabular}

\section{IT competencies}

3i) Learners' competencies with IT

\section{IT Kompetenzen}

3i) Kompetenzen der Lerner im Umgang mit Informationstechnologien sind...

\section{high}

Overage

O low

\begin{tabular}{|l|l|c|c|}
\hline $\begin{array}{l}\text { 3j) Learners' interest in fur- } \\
\text { ther education as a nice time } \\
\text { outside }\end{array}$ & O high & O lowerage \\
\hline $\begin{array}{l}\text { Lerninteresse/Wert von Bildung? } \\
\text { 3j) Erwartungen der Lerner } \\
\text { an Weiterbildung als "gehobe- } \\
\text { ne Freizeit" sind .... }\end{array}$ & O hoch & O durchschnittlich & O gering \\
\hline
\end{tabular}

x) Comments on usefulness and comprehensibility of items:

x) Kommentar zur Nützlichkeit und Verständlichkeit der Fragen: 
4. Description of the learning environment

(Where shall they learn?)

4. Beschreibung der Lernumgebung

(Wo sollen sie lernen?)

\begin{tabular}{|l|c|c|c|}
\hline $\begin{array}{l}\text { 4a) Fields of vocational train- } \\
\text { ing, special equipment for } \\
\text { practical learning }\end{array}$ & O high & O average & O low \\
\hline $4 a)$ & O hoch & O durchschnittlich & O gering \\
\hline
\end{tabular}

\begin{tabular}{|l|c|c|c|}
\hline $\begin{array}{l}\text { 4b) Learning culture (how of- } \\
\text { ten, regular training etc.) } \\
\text { homogeneous groups }\end{array}$ & O high & O average & O low \\
\hline 4b) & O hoch & O durchschnittlich & O gering \\
\hline
\end{tabular}

\begin{tabular}{|l|c|c|c|}
\hline $\begin{array}{l}\text { 4c) Computer equipment } \\
\text { internet availability }\end{array}$ & O high & O average & O low \\
\hline 4c) & O hoch & O durchschnittlich & O gering \\
\hline
\end{tabular}

\begin{tabular}{|l|c|c|c|}
\hline 4d) Place of learning & O high & O average & O low \\
\hline 4d) & O hoch & O durchschnittlich & O gering \\
\hline
\end{tabular}

\begin{tabular}{|l|c|c|c|}
\hline $\begin{array}{l}\text { 4e) Place of working } \\
\text { (e.g. several branches) }\end{array}$ & O high & O average & O low \\
\hline $4 e)$ & O hoch & O durchschnittlich & O gering \\
\hline
\end{tabular}

\begin{tabular}{|l|c|c|c|}
\hline $\begin{array}{l}\text { 4f) different languages of the } \\
\text { learners }\end{array}$ & O high & O average & O low \\
\hline $4 f)$ & O hoch & O durchschnittlich & O gering \\
\hline
\end{tabular}

\begin{tabular}{|l|c|c|c|}
\hline $\begin{array}{l}\text { 4g) Availability of learning } \\
\text { material, media }\end{array}$ & O high & O average & O low \\
\hline $4 g)$ & O hoch & O durchschnittlich & O gering \\
\hline
\end{tabular}

x) Comments on usefulness and comprehensibility of items:

x) Kommentar zur Nützlichkeit und Verständlichkeit der Fragen: 
5. Description of the learning and qualification demands

(What shall they learn for?)

5. Beschreibung der Anforderungen

(Was soll das Ergebnis sein?)

\begin{tabular}{|l|c|c|c|}
\hline $\begin{array}{l}\text { 5a) Visions of the enterprise } \\
\text { and derived visions for the } \\
\text { "learning enterprise" and a } \\
\text { knowledge management }\end{array}$ & $\begin{array}{c}\text { Oery clearly de- } \\
\text { fined }\end{array}$ & $\begin{array}{c}\text { O } \\
\text { rather clear }\end{array}$ & $\begin{array}{c}\text { not defined } \\
\text { na) }\end{array}$ \\
\hline
\end{tabular}

\begin{tabular}{|c|c|c|c|}
\hline $\begin{array}{l}\text { 5b) Demand on competencies } \\
\text { and learning objectives }\end{array}$ & $\begin{array}{c}\text { O } \\
\text { very clearly de- } \\
\text { fined }\end{array}$ & $\begin{array}{c}\mathrm{O} \\
\text { rather clear }\end{array}$ & not defined \\
\hline $\begin{array}{l}\text { 5b) Kompetenzanforderungen } \\
\text { und Lernziele sind ... }\end{array}$ & $\begin{array}{c}\text { O } \\
\text { sehr klar defin- } \\
\text { iert }\end{array}$ & $\begin{array}{c}\mathrm{O} \\
\text { einigermaßen klar } \\
\text { definiert }\end{array}$ & $\begin{array}{c}\text { O } \\
\text { nicht klar defin- } \\
\text { iert }\end{array}$ \\
\hline
\end{tabular}

\begin{tabular}{|l|c|c|c|}
\hline $\begin{array}{l}\text { 5c) Regular qualification } \\
\text { courses (Repeated) for staff } \\
\text { members }\end{array}$ & O exactly & O partly & O not at all \\
\hline $5 c)$ & 0 & 0 & 0 \\
\hline
\end{tabular}

5d) Regular qualification courses (repeated) for staff members

how often?

5d)

\begin{tabular}{|l|c|c|c|}
\hline $\begin{array}{l}\text { 5e) Point system (getting } \\
\text { credits) }\end{array}$ & O exactly & O partly & O not at all \\
\hline $5 e)$ & 0 & 0 & 0 \\
\hline
\end{tabular}

x) Comments on usefulness and comprehensibility of items:

x) Kommentar zur Nützlichkeit und Verständlichkeit der Fragen: 


\begin{tabular}{|l|}
\hline $\begin{array}{l}\text { 6. Description of the contents } \\
\text { (What shall the learn?) }\end{array}$ \\
$\begin{array}{l}\text { 6. Beschreibung der Inhalte } \\
\text { (Was sollen sie lernen?) }\end{array}$ \\
\hline
\end{tabular}

6a) Which thematic fields?

6a) Welche thematischen $\mathrm{Be}$ reiche/Inhalte?

\begin{tabular}{|l|c|c|c|}
\hline 6b) Methodological knowledge & O mostly & O partly & O not at all \\
\hline 6b) Methodisches Wissen & O sehr hoch & O einigermaßen & O sehr gering \\
\hline
\end{tabular}

\begin{tabular}{|l|c|c|c|}
\hline $\begin{array}{l}\text { 6c) Background or source } \\
\text { knowledge }\end{array}$ & O mostly & O partly & O not at all \\
\hline 6c) Hintergrundwissen & O sehr hoch & O einigermaßen & O sehr gering \\
\hline
\end{tabular}

\begin{tabular}{|l|c|c|c|}
\hline 6d) Interpretation knowledge & O mostly & O partly & O not at all \\
\hline 6d) Erklärungswissen & O sehr hoch & O einigermaßen & O sehr gering \\
\hline
\end{tabular}

\begin{tabular}{|l|c|c|c|}
\hline $\begin{array}{l}\text { 6e) Acting knowledge (compe- } \\
\text { tencies) }\end{array}$ & O mostly & O partly & O not at all \\
\hline 6e) Handlungswissen & O sehr hoch & O einigermaßen & O sehr gering \\
\hline
\end{tabular}

\begin{tabular}{|l|c|c|c|}
\hline $\begin{array}{l}\text { 6f) Level of anticipative } \\
\text { knowledge (things that will } \\
\text { have to be developed) }\end{array}$ & O very high & O some & O low \\
\hline $\begin{array}{l}\text { 6f) Antizipatorisches Wissen } \\
\text { (Wissen, das sich auf noch } \\
\text { nicht gut bekannte Sachver- } \\
\text { halte bezieht) }\end{array}$ & O sehr hoch & O einigermaßen & O sehr gering \\
\hline
\end{tabular}

x) Comments on usefulness and comprehensibility of items:

x) Kommentar zur Nützlichkeit und Verständlichkeit der Fragen: 


\section{Expected outcome}

\section{Erwartete Ergebnisse}

\begin{tabular}{|l|c|c|c|}
\hline $\begin{array}{l}\text { 7a) Educational management } \\
\text { in the enterprise }\end{array}$ & $\begin{array}{c}\text { O } \\
\text { should be quite } \\
\text { new organised }\end{array}$ & O partly changes & O no changes \\
\hline $\begin{array}{l}\text { 7a) Die Organisation der Wei- } \\
\text { terbildung soll in der Einrich- } \\
\text { tung/dem Unternehmen... }\end{array}$ & $\begin{array}{c}\text { grundlegend neu } \\
\text { gestaltet werden }\end{array}$ & $\begin{array}{c}\text { O einigermaßen } \\
\text { umgestaltet werden }\end{array}$ & $\begin{array}{c}\text { O möglichst } \\
\text { wenig verändert } \\
\text { werden }\end{array}$ \\
\hline
\end{tabular}

\begin{tabular}{|l|c|c|c|}
\hline $\begin{array}{l}\text { 7b) Web based Learning Man- } \\
\text { agement System }\end{array}$ & $\begin{array}{c}0 \\
\text { high preference }\end{array}$ & $\begin{array}{c}\text { may be, may not } \\
\text { very low prefer- } \\
\text { ence }\end{array}$ \\
\hline $\begin{array}{l}\text { 7b) Webbasierte Bild- } \\
\text { ungsangebote ... }\end{array}$ & $\begin{array}{c}\text { haben für uns } \\
\text { eine hohe Präfe- } \\
\text { renz }\end{array}$ & $\begin{array}{c}\text { können für uns sein, } \\
\text { müssen aber nicht } \\
\text { sein }\end{array}$ & $\begin{array}{c}\text { haben für uns } \\
\text { keine Präferenz }\end{array}$ \\
\hline
\end{tabular}

\begin{tabular}{|c|c|c|c|}
\hline 7c) e-Learning courses & $\begin{array}{c}O \\
\text { high preference }\end{array}$ & may be, may not & $\begin{array}{c}\text { O } \\
\text { very low prefer- } \\
\text { ence }\end{array}$ \\
\hline 7c) e-Learning Kurse & $\begin{array}{c}\text { O } \\
\text { haben für uns } \\
\text { eine hohe Präfe- } \\
\text { renz }\end{array}$ & $\begin{array}{c}O \\
\text { können für uns sein, } \\
\text { müssen aber nicht } \\
\text { sein }\end{array}$ & $\begin{array}{c}\text { O } \\
\text { haben für uns } \\
\text { keine Präferenz }\end{array}$ \\
\hline
\end{tabular}

\begin{tabular}{|c|c|c|c|}
\hline 7d) Blended-Learning courses & $\begin{array}{c}O \\
\text { high preference }\end{array}$ & may be, may not & $\begin{array}{c}\text { O } \\
\text { very low prefer- } \\
\text { ence }\end{array}$ \\
\hline 7d) Blended-Learning Kurse & $\begin{array}{c}\text { O } \\
\text { haben für uns } \\
\text { eine hohe Präfe- } \\
\text { renz }\end{array}$ & $\begin{array}{c}O \\
\text { können für uns sein, } \\
\text { müssen aber nicht } \\
\text { sein }\end{array}$ & $\begin{array}{c}\text { O } \\
\text { haben für uns } \\
\text { keine Präferenz }\end{array}$ \\
\hline
\end{tabular}

7e) Digital learning materials

\begin{tabular}{|c|c|c|}
\hline 0 & 0 & 0 \\
high preference & may be, may not & very low prefer- \\
\hline
\end{tabular}




\begin{tabular}{|l|c|c|c|}
\hline 7e) Digitale Lernmaterialien & 0 & 0 & ence \\
& $\begin{array}{c}\text { haben für uns } \\
\text { eine hohe Präfe- } \\
\text { renz }\end{array}$ & $\begin{array}{c}\text { können für uns sein, } \\
\text { müssen aber nicht } \\
\text { sein }\end{array}$ & $\begin{array}{c}\text { haben für uns } \\
\text { keine Präferenz }\end{array}$ \\
\hline
\end{tabular}

\begin{tabular}{|c|c|c|c|}
\hline $7 f) C B T^{\prime} s$ or WBT's & $\begin{array}{c}\mathrm{O} \\
\text { high preference }\end{array}$ & may be, may not & $\begin{array}{c}\text { O } \\
\text { very low prefer- } \\
\text { ence }\end{array}$ \\
\hline 7f) CBT's oder WBT's & $\begin{array}{c}\text { O } \\
\text { haben für uns } \\
\text { eine hohe Präfe- } \\
\text { renz }\end{array}$ & $\begin{array}{c}\text { O } \\
\text { können für uns sein, } \\
\text { müssen aber nicht } \\
\text { sein }\end{array}$ & $\begin{array}{c}\text { O } \\
\text { haben für uns } \\
\text { keine Präferenz }\end{array}$ \\
\hline
\end{tabular}

\begin{tabular}{|c|c|c|c|}
\hline 7g) Tutoring & $\begin{array}{c}O \\
\text { high preference }\end{array}$ & $\begin{array}{c}O \\
\text { may be, may not }\end{array}$ & $\begin{array}{c}\text { O } \\
\text { very low prefer- } \\
\text { ence }\end{array}$ \\
\hline 7g) Tutoringmaßnahmen & $\begin{array}{c}\text { O } \\
\text { haben für uns } \\
\text { eine hohe Präfe- } \\
\text { renz }\end{array}$ & $\begin{array}{c}\text { O } \\
\text { können für uns sein, } \\
\text { müssen aber nicht } \\
\text { sein }\end{array}$ & $\begin{array}{c}O \\
\text { haben für uns } \\
\text { keine Präferenz }\end{array}$ \\
\hline
\end{tabular}

\begin{tabular}{|l|c|c|c|}
\hline $\begin{array}{l}\text { 7h) Training of internal train- } \\
\text { ers }\end{array}$ & $\begin{array}{c}0 \\
\text { high preference }\end{array}$ & $\begin{array}{c}0 \\
\text { may be, may not }\end{array}$ & $\begin{array}{c}\text { very low prefer- } \\
\text { ence }\end{array}$ \\
\hline $\begin{array}{l}\text { 7h) Schulungen der internen } \\
\text { Weiterbildungsleiter und - } \\
\text { Trainer/Dozenten }\end{array}$ & $\begin{array}{c}\text { haben für uns } \\
\text { eine hohe Präfe- } \\
\text { renz }\end{array}$ & $\begin{array}{c}\text { können für uns sein, } \\
\text { müssen aber nicht } \\
\text { sein }\end{array}$ & $\begin{array}{c}\text { haben für uns } \\
\text { keine Präferenz }\end{array}$ \\
\hline
\end{tabular}

x) Comments on usefulness and comprehensibility of items:

x) Kommentar zur Nützlichkeit und Verständlichkeit der Fragen: 


\section{Organisational development and integration}

\section{Organisationsentwicklung und Integration}

\begin{tabular}{|l|c|c|c|}
\hline $\begin{array}{l}\text { 8a) Adaptability of contents } \\
\text { to be adapted to other } \\
\text { groups) }\end{array}$ & O high & O may be, may not & O low \\
\hline $\begin{array}{l}\text { 8a) Weitere Nutzungsmög- } \\
\text { lichkeiten der Bildungsange- } \\
\text { bote }\end{array}$ & 0 & 0 & O I \\
\hline
\end{tabular}

\begin{tabular}{|l|c|c|c|}
\hline $\begin{array}{l}\text { 8b) Internal dissemination } \\
\text { opportunities }\end{array}$ & O high & O may be, may not & O low \\
\hline $\begin{array}{l}\text { 8b) Möglichkeiten zur inter- } \\
\text { nen Dissemination von Bil- } \\
\text { dungsangeboten }\end{array}$ & 0 & 0 & 0 \\
\hline
\end{tabular}

\begin{tabular}{|l|c|c|c|}
\hline $\begin{array}{l}\text { 8c) Integration in Quality } \\
\text { Management Systems }\end{array}$ & O high & O may be, may not & O low \\
\hline $\begin{array}{l}\text { 8c) Integration der Bil- } \\
\text { dung/Weiterbildung in Quali- } \\
\text { täts-Management-Systeme ... }\end{array}$ & 0 & 0 & 0 \\
\hline
\end{tabular}

x) Comments on usefulness and comprehensibility of items:

x) Kommentar zur Nützlichkeit und Verständlichkeit der Fragen: 


\section{Fragebogen zum Abschluss des Projektes}

Dear partners,

as we decided during our meeting in Siena we would like to do a "pretest" with the inventories that have been developed in our network. You agreed to test some or at least one of the inventories in your institution. With this "inventory - feedback" I want to collect information about who used which inventory. I also want to know in short what you think about the instruments. I ask you only a few questions to assess the usefulness and manageability of the inventories. Please give me your feedback as soon as you used an inventory and send it via E-Mail to me (jlist@gwdg.de). In case that you used different inventories, would you please be so kind as to give me your feedback at any one time. I will present the results during our next (and last) transnational meeting.

Thank you very much for your cooperation!

\begin{tabular}{|l|l|}
$\begin{array}{l}\text { In this questionnaire you' II find on the left } \\
\text { side of the table a question or request. }\end{array}$ & $\begin{array}{l}\text {...and please fill in your answers on the right side } \\
\text { of the table. (Please click into the description field } \\
\text { and write your answer in it or tick the relevant an- } \\
\text { swer.) } \\
\downarrow\end{array}$
\end{tabular}

1. Please insert your name.

2. Please insert the date of today.

\begin{tabular}{l|l} 
3. Which inventory did you use? & $\square$ The inventory for authors \\
(Please tick the relevant.) & $\square$ The inventory for decision makers 1 \\
& $\square$ The inventory for decision makers 2 \\
$\square$ The inventory for learners "before" \\
$\square$ The inventory for learners "during" \\
$\square$ The inventory for learners "after"
\end{tabular}

4. Did you create an indivudual questionnaire out of the pool of questions in the inventory?

$\square$ Yes, I created my individual questionnaire.

(Please tick the relevant.)

$\square$ No, I used all the questions/the whole inventory.

5. Which questions did you delete?

(Plese insert the questions.)

6. Why did you delete these questions?

(Please tell us your reasons.) 
7. When did you use the inventory or questionnaire?

(Please insert the date or time period.)

8. In which course or courses did you use the inventory or questionnaire?

(Please insert the title of the course or titles of courses, if you used it in different courses.)

9. Which form of a questionnaire did you use?

(Please tick the relevant.)

10. How many persons have been in the course or in the courses?

(Please insert the number of persons.)

11. How many persons have been receiving a questionnaire?

(Please insert the number of persons.)

12. How many inventories or questionnaires did you retrieve (rate of return)?

(Please insert the number of questionnaires you got back.)

13. What do you think about the usefulness of the inventory/questionnaire?

14. What do you think about the manageability of the inventory?

15. What do you like about the inventory?

16. What do you dislike about the inventory?

17. What else would you like to tell us concerning the inventory or your individual questionnaire?

(Please insert your comments, suggestions, ideas and/or questions.) 
Name
Geburtsdatum
Geburtsort
Staatsangehörigkeit
Familienstand

Schulabschluss

Studium

Ab dem Wintersemester 1994/95

Ab dem Wintersemester 1995/96

\section{Berufstätigkeit}

06.2004 bis 09.2007

09.2008 bis 09.2010

01.2011 bis 11.2011

Ab 12.2011
Jutta Marianne List-Ivankovic

09.12.1974

Bad Gandersheim

Deutsch

Verheiratet, eine Tochter

Abitur 1994 an der IGS-Göttingen

Philosophie (1. Hauptfach)

Pädagogik (2. Hauptfach)

Pädagogik (Hauptfach)

Wirtschafts- und Sozialpsychologie (1.Nebenfach)

Volkskunde (2.Nebenfach)

Magisterarbeit zum Thema „Die Bindungstheorie als Grundlage einer Lernausstellung für Eltern“

Abschluss: Magister Artium am 15.1.2004 Note: „Sehr gut mit Auszeichnung“

Wissenschaftliche Hilfskraft bei Prof. Dr. Hans-Dieter Haller am Pädagogischen Seminar, Georg-August-Universität Göttingen

Bildungsreferentin in der Klimaanpassungsakademie Kassel (Landkreis Kassel, Volkshochschule)

Wissenschaftliche Mitarbeiterin bei Prof. Dr. Bernd Overwien Universität Kassel, Fachbereich Gesellschaftswissenschaften, Fachgebiet "Didaktik der politischen Bildung"

Elternzeit 


\section{Erklärung gemäß § 18}

Ich versichere, dass ich die eingereichte Dissertation „Evaluation von Bildungsprojekten auf der Grundlage von Inventaren - Entwicklung und Erprobung eines Ansatzes im Rahmen von europäischen Projekten" selbständig und ohne unerlaubte Hilfsmittel verfasst habe. Anderer als der von mir angegebenen Hilfsmittel und Schriften habe ich mich nicht bedient. Alle wörtlich oder sinngemäß den Schriften anderer Autoren entnommenen Stellen habe ich kenntlich gemacht. 